\title{
UAB
}

Universitat Autònoma de Barcelona

\section{Extreme particle acceleration in microquasar jets and pulsar wind nebulae with the MAGIC telescopes}

\author{
Alba Fernández Barral
}

ADVERTIMENT. L'accés als continguts d'aquesta tesi queda condicionat a l'acceptació de les condicions d'ús establertes per la següent llicència Creative Commons:

(c) (i) (\$) $\mathrm{http}: / /$ cat.creativecommons.org/?page_id=184

ADVERTENCIA. El acceso a los contenidos de esta tesis queda condicionado a la aceptación de las condiciones de uso establecidas por la siguiente licencia Creative Commons: (c) (i) @ $\Theta$ http://es.creativecommons.org/blog/licencias/

WARNING. The access to the contents of this doctoral thesis it is limited to the acceptance of the use conditions set by the following Creative Commons license: (c) (i) \&) $\mathrm{https}$ ://creativecommons.org/licenses/?lang=en 

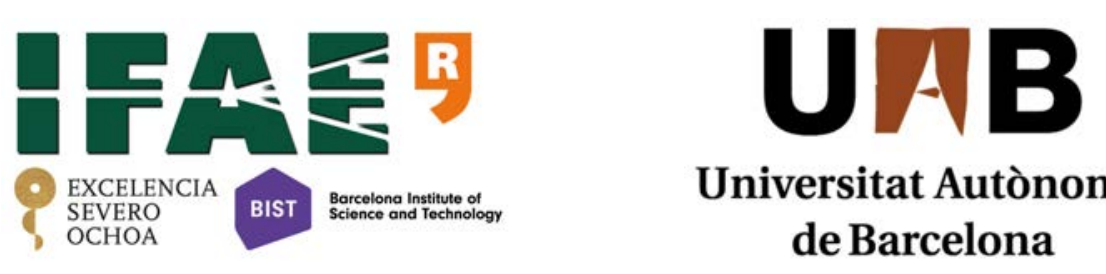

Universitat Autònoma de Barcelona

\title{
Extreme particle acceleration in microquasar jets and pulsar wind nebulae with the MAGIC telescopes
}

\author{
Alba Fernández Barral \\ Departament de Física \\ Universitat Autònoma de Barcelona
}

A thesis submitted for the degree of

PhilosophiaDoctor (PhD)

October 2017 
Director: Dr. Oscar Blanch Bigas IFAE

Edifici Cn, UAB

08193 Bellaterra (Barcelona), Spain

blanch@ifae.es
Tutor: Dr. Enrique Fernández Sánchez IFAE \& UAB

Edifici Cn, UAB

08193 Bellaterra (Barcelona), Spain enrique.fernandez@ifae.es

Day of defense: 6 October 2017 
Allà ets l'essència de tot. Pels crítics, els paradigmes i els cànons pels que es regien estan obsolets de cop. Que amb l'aura que tu desprens s'imposa, de forma urgent, fer una revisió dels clàssics. Que ets jove eternament.

Els amics de les arts Museu d'Història Natural

A mis padres, a BRAIS.

Os pertenece cada palabra aquí escrita. 
I herewith declare that I have produced this thesis without the prohibited assistance of third parties and without making use of aids other than those specified; notions taken over directly or indirectly from other sources have been identified as such. This paper has not previously been presented in identical or similar form to any other Spanish or foreign examination board.

The thesis work was conducted from September 2013 to October 2017 under the supervision of Oscar Blanch Bigas at Barcelona.

Barcelona, 6 October 2017. 


\begin{abstract}
Throughout our entire history, we humans have strived to unravel the mysteries with which the deep Universe challenges us. In our humble beginnings, this task was performed with our naked eyes, by gazing at the stars and planets and wondering how far away they were and how they moved in the night sky. For many centuries, only the visible Universe was reachable for us, but extraordinary achievements were accomplished despite the limited tools: we discovered, for example, that our planet was not the center of the Universe, owing to Nicolaus Copernicus' observations and his heliocentric model. From Copernicus' epoch up to now, the development of new technologies and the advancement of our own understanding of the Cosmos, allowed us to disentangle many riddles. Fortunately, this natural curiosity that leads us to improve never ends, and we face new questions that challenge our capacity as scientists. In the present thesis, I focus on a small fraction of this science: the gamma-ray astronomy. Within this field, I study particle acceleration and gamma-ray production mechanisms inside the relativistic jets displayed by the so-called microquasars and the shocks produced in Pulsar Wind Nebulae (PWNe).

In Part I of the thesis I present an introduction to the non-thermal Universe, delving into the mechanisms of production and absorption that govern the gamma-ray emission. I also introduce the MAGIC telescopes, from which the bulk of results in this thesis are obtained. Other detection techniques, such as those used by the HAWC Observatory and the Fermi-LAT satellite, are also introduced as results from both of them are used in the discussion of galactic sources included in this thesis.

The scientific achievements are encompassed in Part II and Part III. In the former, I discuss results from the three best microquasar candidates to emit Very-High-Energy (VHE) gamma rays: Cygnus X-1, Cygnus X-3 and V404 Cygni. I investigate them making use of MAGIC data during long-term campaigns or under flaring periods. Furthermore, in order to complement results at lower energies, I analyze Fermi-LAT data of Cygnus X-1, leading to the detection of the system in the High Energy (HE) regime. This constitutes the first firmly gamma-ray detection on a Black Hole (BH) binary system. Part III is focused on the study of PWNe. I analyze five sources of this type and set the results in the context of the TeV $[\mathrm{PWN}]$ population study performed by the High Energy Stereoscopic System (H.E.S.S.) Collaboration. Along with these results, I discuss the importance of the target photon field together with characteristic features of the pulsars hosted by these PWNe to emit gamma rays. In this thesis, I also present the first joint work between the HAWC Observatory and MAGIC, which opens the door to future synergy projects.
\end{abstract}


In Part IV, I present the technical work performed during my thesis for the future Cherenkov Telescope Array (CTA) instrument. I focus on the camera hardware for the Large Size Telescope (LST), working on the Quality Control (QC) for several subsystems, among which the Photomultiplier Tubes (PMTs), power supplies and trigger mezzanines stand out.

Finally, I summarize all the aforementioned results in a conclusion chapter. All the work developed during my thesis led to seven publications in scientific journals: two of them already published, two accepted by the corresponding journal and three currently under the revision of MAGIC and all implicated collaborations. 


\section{Resumen}

A lo largo de toda nuestra historia, los seres humanos nos hemos esforzado por descifrar los misterios con los que el Universo nos desafía. En nuestros humildes comienzos, esta tarea era realizada con nuestros ojos desnudos, mirando las estrellas y los planetas y preguntándonos qué tan lejos estaban y cómo se movían en el cielo nocturno. Durante muchos siglos, sólo el Universo visible fue accesible para nosotros, pero se obtuvieron logros extraordinarios a pesar de las limitadas herramientas: descubrimos, por ejemplo, que nuestro planeta no era el centro del Universo, gracias a las observaciones de Nicolaus Copernicus y su modelo heliocéntrico. Desde la época de Copernicus hasta ahora, el desarrollo de nuevas tecnologías y el avance de nuestra propia comprensión del Cosmos, nos ha permitido desentrañar mucho enigmas. Afortunadamente, esta curiosidad natural que nos lleva a mejorar nunca termina, y nos enfretamos a nuevas preguntas que desafían nuestra capacidad como científicos. En la presente tesis, me centro en una pequeña fracción de esta ciencia: la astronomía de rayos gamma. Dentro de este campo, estudio la aceleración de partículas y los mecanismos de producción de rayos gamma en los jets relativistas de los denominados microcuásares y en shocks producidos en pleriones.

En la Parte I de la tesis, presento una introducción al Universo no térmico, profundizando en los mecanismos de producción y absorción que gobiernan la emisión de rayos gamma. También introduzco los telescopios MAGIC, de los cuales se obtiene la mayor parte de los resultados de esta tesis. Otras técnicas de detección, como las empleadas en el Observatorio HAWC o por el satélite Fermi-LAT, son igualmente presentadas dado que sus resultados son empleados en la discusión de fuentes galácticas incluidas en esta tesis.

Los logros científicos están incluidos en la Parte II y Parte II. En el primera, discuto los resultados de los tres mejores candidatos microcuásares para emitir rayos gamma a muy altas energías: Cygnus X-1, Cygnus X-3 y V404 Cygni. Investigo todos ellos haciendo uso de los datos de MAGIC durante campañas observacionales de larga duración o durante periodos de alta actividad. Por otra parte, con el fin de complementar los resultados a energías más bajas, analizo los datos de Fermi-LAT de Cygnus X-1, lo cual condujo a la detección del sistema en el régime de altas energías. Esto constituye la primer detección firme de rayos gamma a altas energías de un sistema binario compuesto por un agujero negro. La Parte III se centra en el estudio de pleriones. Analizo cinco fuentes pertenecientes a esta clase y contextualizo los resultados dentro del estudio de población de pleriones realizado por la Colaboración H.E.S.S.. Haciendo uso de estos resultados, se discute la importancia 
de la densidad de fotones en el medio circundante junto con las características de los púlsares alojado por estos pleriones para la emisión de rayos gamma. En esta tesis, también presento el primer trabajo conjunto entre el Observatorio HAWC y MAGIC, que abre las puertas a futuros proyectos en sinergia.

La Parte IV incluye el trabajo técnico realizado durante mi tesis con el futuro CTA. Este trabajo está centrado en el hardware de la cámara de los telescopios de gran tamaño (LST, por sus siglas en inglés) del futuro instrumento CTA, que incluye el control de calidad de varios subsistemas, entre los que descatan los fotomultiplicadores, las fuentes de alimentación y el sistema de trigger.

Finalmente, resumo todos los anteriormente mencionados resultados en un capítulo de conclusiones. Todo el trabajo desarrollado durante mi tesis dio lugar a siete publicaciones en revistas científicas: dos ya publicadas, dos aceptadas por la correspondiente revista y tres actualmente bajo revisión de la Colaboración de MAGIC y otras colaboraciones implicadas. 


\section{Contents}

Contents

List of Figures

List of Tables

I Introduction to the non-thermal Universe 1

1 Cosmic rays and gamma-ray astrophysics 3

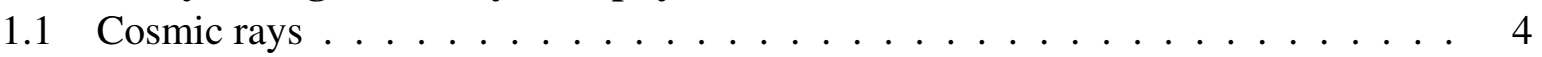

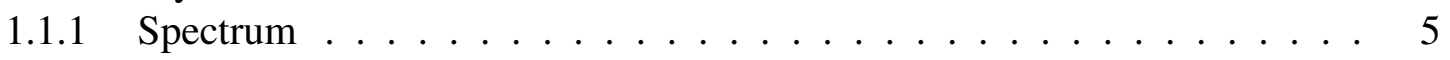

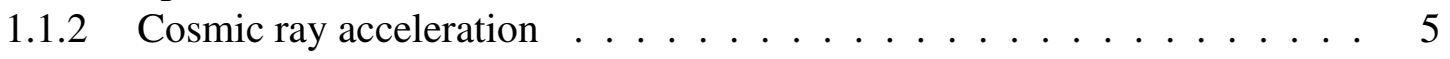

1.2 Gamma-ray astrophysics . . . . . . . . . . . . . . . . 7

1.2.1 Gamma-ray production . . . . . . . ........... 8

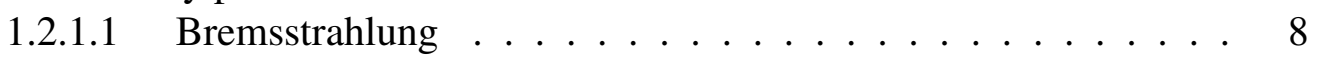

1.2.1.2 Electron/positron annihilation .............. 9

1.2 .1 .3 Pion decay . . . . . . . . . . . . . . 9

1.2.1.4 Synchrotron .................... 9

1.2.1.5 Inverse Compton . . . . . . . . . . . . . . . . . 11

1.2.2 Gamma-ray absorption . . . . . . . . . . . . . . . 11

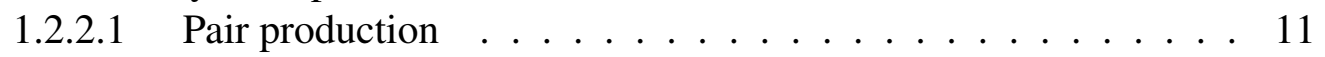

1.2 .3 Gamma-ray sources . . . . . . . . . . . . . . . 12

1.2.3.1 Galactic sources . . . . . . . . . . . . . . 12

1.2.3.2 Extragalactic sources ................ 13

1.2.4 Detection techniques . . . . . . . . . . . . . 13

2 Gamma-ray telescopes 17

2.1 Cherenkov light . . . . . . . . . . . . . . . . 17

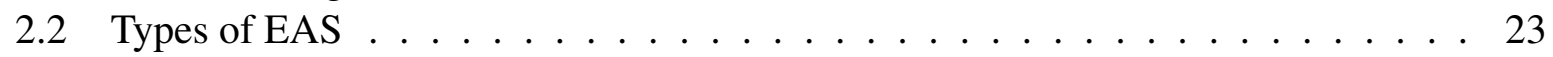

2.2.1 Electromagnetic showers . . . . . . . . . . . . . 23

2.2 .2 Hadronic showers . . . . . . . . . . . . . . . . . 24

2.3 Imaging atmospheric Cherenkov technique .............. 26

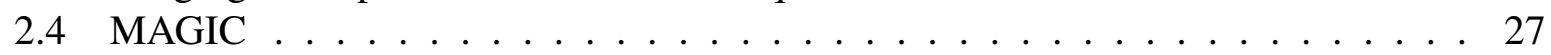




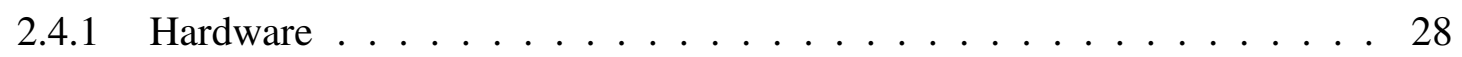

2.4.1.1 Alt-azimuth frame and drive system . . . . . . . . . . . . . 29

2.4.1.2 Mirrors and reflector . . . . . . . . . . . . . . 31

2.4.1.3 Pre- and post-upgrade camera . . . . . . . . . . . . 32

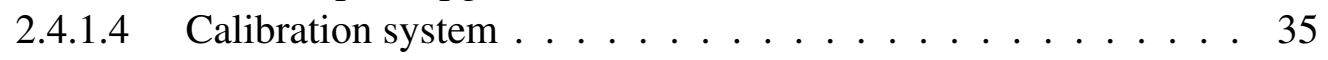

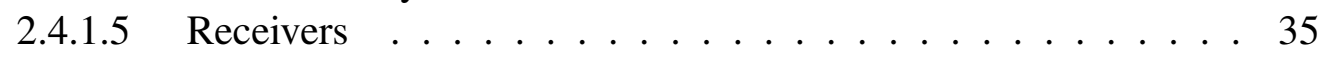

2.4.1.6 Trigger system .................. 35

2.4.1.7 Former and current readout systems . . . . . . . . . . . . 38

2.4.1.8 Other subsystems . . . . . . . . . . . . . . . 41

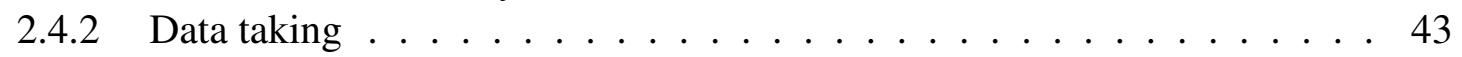

2.4.2.1 Observational pointing modes . . . . . . . . . . 44

2.4.2.2 Types of data . . . . . . . . . . . . . . . . . . . . . . . .

2.4.2.3 Safety limits . . . . . . . . . . . . . . 46

2.4 .3 Data analysis . . . . . . . . . . . . . . . . 48

2.4.3.1 Monte Carlo simulations . . . . . . . . . . . . . . . . . . . . .

2.4.3.2 Signal pre-processing . . . . . . . . . . . . 50

2.4.3.3 Image cleaning and Hillas parameters calculation . . . . . . 50

2.4.3.4 Data quality cuts . . . . . . . . . . . . . . 56

2.4.3.5 Stereo image parameters calculation .......... 5 57

2.4.3.6 $\gamma$ /hadron separation .................. 58

2.4.3.7 Arrival direction reconstruction . . . . . . . . . . . 60 60

2.4.3.8 Energy reconstruction, energy resolution and energy threshold 61

2.4.3.9 Signal extraction . . . . . . . . . . . . . . 663

2.4 .3 .10 Sensitivity .................... 65

2.4 .3 .11 Angular resolution . . . . . . . . . . . . 66 6 . . . . . .

2.4.3.12 Skymaps .................... 67 67

2.4.3.13 Spectrum and Light Curve . . . . . . . . . . . . . . . . . . . . . . . . . . . . .

2.4.3.14 Unfolding spectrum . . . . . . . . . . . . . 77

2.4 .3 .15 Upper limits . . . . . . . . . . . . . . . . . . . . . . . . . .

2.4.3.16 Systematic uncertainties . . . . . . . . . . . . 74

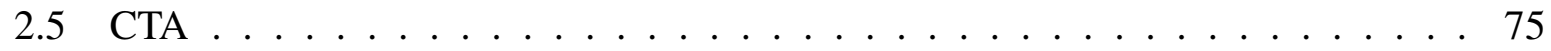

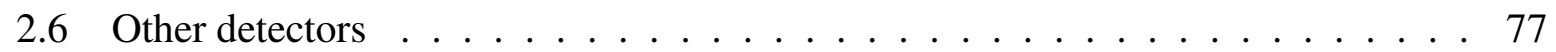

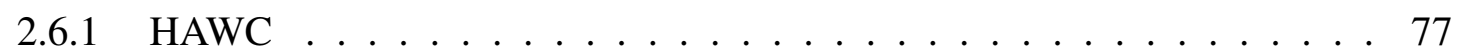

2.6 .2 Fermi-LAT . . . . . . . . . . . . . . . . . 79

2.6.2.1 Performance and analysis . . . . . . . . . 79

\section{Microquasars in the very high-energy gamma-ray regime 85}

3 Microquasars, binary systems with powerful jets 8

3.1 X-ray Binaries . . . . . . . . . . . . . . . . . . 87

3.1.1 High-Mass X-ray Binary Systems . . . . . . . . . . . . . . . 89

3.1.1.1 Be/X-ray binaries ............... . . . . . . 
3.1.1.2 Supergiant $X$-ray binaries $\ldots \ldots \ldots \ldots \ldots$

3.1.2 Low-Mass X-ray Binary Systems . . . . . . . . . . . . . . 919

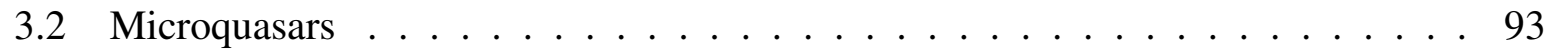

3.2 .1 Accretion disk . . . . . . . . . . . . . . . . 94

3.2 .2 Relativistic radio-jets . . . . . . . . . . . . . . . 96

3.2.3 Black hole microquasars $\ldots \ldots \ldots \ldots \ldots$

3.2.3.1 X-ray states $\ldots \ldots \ldots \ldots \ldots$

3.2.3.2 Disk-jet coupling . . . . . . . . . . . . . . . . 101

3.2.4 VHE radiative processes in microquasars . . . . . . . . . . . 102

3.2.4.1 Leptonic processes . . . . . . . . . . . . . . . . 102

3.2.4.2 Hadronic processes . . . . . . . . . . . . . . 102

3.2.4.3 Radiative processes in a low-mass microquasars . . . . . . 103

3.2.4.4 Photon-photon absorption . . . . . . . . . . . . . 103

3.2.5 VHE observations of microquasars with MAGIC . . . . . . . . 104

4 Cygnus X-1 105

4.1 History . . . . . . . . . . . . . . . . . . . 105

4.2 Fermi-LAT analysis . . . . . . . . . . . . . . . . . . . . . . 108

4.2 .1 Data selection . . . . . . . . . . . . . . . . . . . 108

4.2 .2 Model . . . . . . . . . . . . . . . . . . . . . . . . . . . . 109

4.2 .3 Results . . . . . . . . . . . . . . . . . . 1112

4.2 .4 Discussion . . . . . . . . . . . . . . . . 115

4.3 MAGIC analysis . . . . . . . . . . . . . . . . . . . 1117

4.3.1 Observations and Data analysis . . . . . . . . . . . . 117

4.3.2 Search for steady emission . . . . . . . . . . . . . . . 118

4.3.2.1 Results during Hard State . . . . . . . . . . . . . . . 120 . . . . . . . .

4.3.2.2 Results during Soft State . . . . . . . . . . . . . . 121

4.3.3 Search for variable emission . . . . . . . . . . . . . . . . . . . . 121

4.3 .4 Discussion . . . . . . . . . . . . . . . . . . . . . . 124

4.4 Conclusions . . . . . . . . . . . . . . . . . . . 1125

5 Cygnus X-3 129

5.1 History . . . . . . . . . . . . . . . . . . . . . . 129

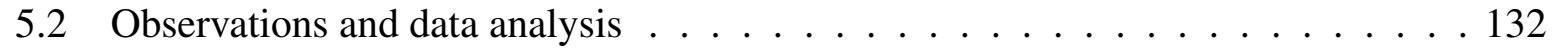

5.2.1 August-September 2016 flare . . . . . . . . . . . . . . 133

5.3 Results before flare $2016 \ldots \ldots$. . . . . . . . . . . . . . . . . . . . 134

5.4 Results of the August-September 2016 flare . . . . . . . . . . . . . . 135

5.5 Discussion and Conclusions . . . . . . . . . . . . . . . . . . . . . . . . 1139

6 V404 Cygni 145

6.1 History . . . . . . . . . . . . . . . . . . . . . . . . . . . 145

6.1.1 June 2015 outburst . . . . . . . . . . . . . . . . . . . . . . 145

6.2 Observations and Data analysis . . . . . . . . . . . . . 147 
6.3 Results . . . . . . . . . . . . . . . . . . . . . . . . . . . 148

6.4 Discussion and conclusions . . . . . . . . . . . . . . . . 150

III PWNe in the very high-energy gamma-ray regime 153

7 Introduction to Pulsar Wind Nebulae 155

7.1 Central object in PWNe . . . . . . . . . . . . . . . . . . 155

7.1 .1 Neutron star . . . . . . . . . . . . . . . . . . . . . . . . . . . . . . . . . .

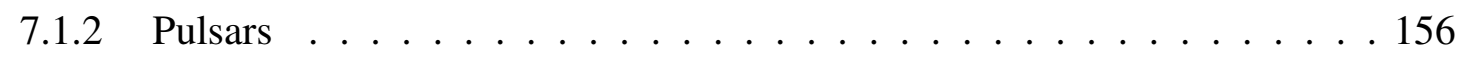

7.1.2.1 Magnetosphere and wind zone . . . . . . . . . . 158

7.1.2.2 Gamma-ray emission models . . . . . . . . . . . . 160

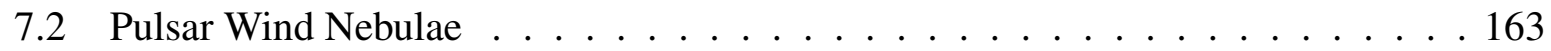

7.2.1 Gamma-ray emission . . . . . . . . . . . . . . . . . . . . . . 164

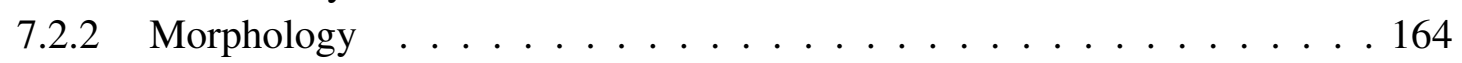

7.2 .3 Evolutionary phases $\ldots \ldots \ldots \ldots$

8 Follow-up studies of HAWC sources 167

8.1 Introduction . . . . . . . . . . . . . . . . . . . . . . . . . . . . . . . . . . . . . .

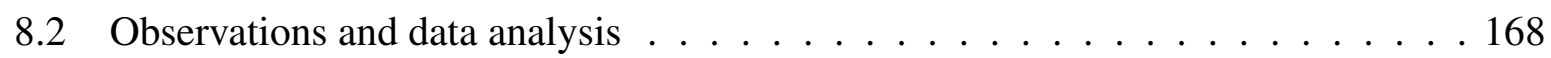

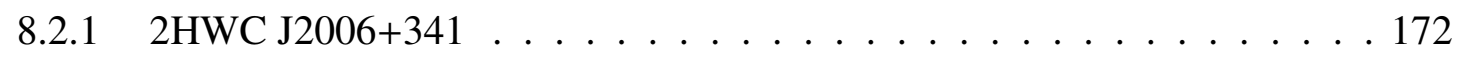

8.2 .2 2HWC J1907+084* . . . . . . . . . . . . . . . . 174

8.3 Results . . . . . . . . . . . . . . . . . 175

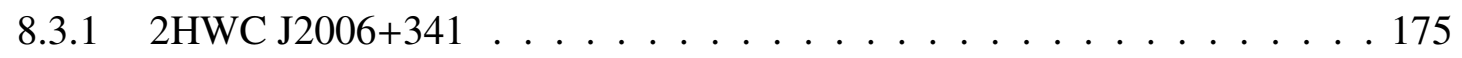

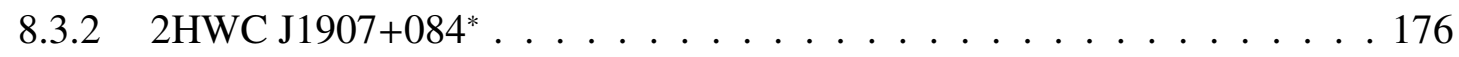

8.4 Discussion . . . . . . . . . . . . . . . . . . . . . . . . . . 176

8.5 Conclusions . . . . . . . . . . . . . . . . . . . . . . . . . .

9 PWN studies around high spin-down power Fermi-LAT pulsars 183

9.1 Introduction . . . . . . . . . . . . . . . . . . . . . . . . 183

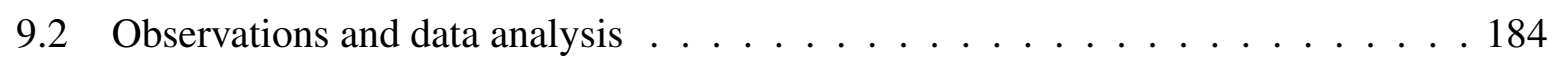

9.2.1 PSR J1954+2838 and PSR J1958+2845 . . . . . . . . . . . . . 185

$9.2 .2 \quad$ PSR J2022+3842 . . . . . . . . . . . . . . . . . . . . 186

9.2 .3 PSR J2111+4606 and PSR J2238+5903 . . . . . . . . . . . . 186

9.3 Results . . . . . . . . . . . . . . . . . . . . . . . . 187

9.3.1 PSR J1954+2838 and PSR J1958+2845 . . . . . . . . . . . . 187

$9.3 .2 \quad$ PSR J2022+3842 . . . . . . . . . . . . . . . . . . . . . . . 188

9.3.3 PSR J2111+4606 and PSR J2238+5903 . . . . . . . . . . . . 189

9.4 Discussion and conclusions . . . . . . . . . . . . . . . . . 190

10 Summary and concluding remarks 
A Very-high-energy gamma-ray observations of SN 2014J

A.1 The death of a star: types of Supernova . . . . . . . . . . . . . 201

A.2 Introduction to SN 2014J . . . . . . . . . . . . . . . . . . . . . . 204

A.3 MAGIC observations and results . . . . . . . . . . . . . . 206

A.4 Discussion . . . . . . . . . . . . . . . . . . . . 207

A.5 Conclusions . . . . . . . . . . . . . . . . . . 211

B F-factor method

C Power supply noise tests with different setup configurations 219

C.0.1 Conducted noise tests . . . . . . . . . . . . . . . . . . . . . 2219

C.0.2 Radiated noise tests . . . . . . . . . . . . . . . . . 221

References

Acronyms 


\section{CONTENTS}




\section{List of Figures}

I Multiwavelength view of the Milky Way. . . . . . . . . . . . . . . . . 1

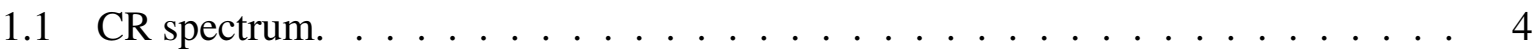

1.2 Cutoff of the CR spectrum. . . . . . . . . . . . . . 6

1.3 Hillas plot. . . . . . . . . . . . . . . . 7

1.4 VHE gamma-ray production processes. . . . . . . . . . . . 10

2.1 EM spectrum. . . . . . . . . . . . . . . . . . . . 18

2.2 Cherenkov radiation effect on the medium. . . . . . . . . . . . . . . 19

2.3 Polarization on a medium when a charged particle travels faster than the speed of light on it $(v>c / n)$. Taken from http://mxp.physics.umn.edu. . . . . . . . . 19

2.4 Spectra of Cherenkov radiation. . . . . . . . . . . . . . . . . . 20

2.5 Cherenkov spectra at different zenith angles. . . . . . . . . . . . . . . 21

2.6 Cherenkov radiation scheme. . . . . . . . . . . . . . . . . . . 21

2.7 Cherenkov light pool scheme. . . . . . . . . . . . . . . . . 22

2.8 Cherenkov photon density VS Energy. . . . . . . . . . . . . . . . 22

2.9 Particle showers sketch. . . . . . . . . . . . . . . . . . . 23

2.10 MC simulation of EM and hadronic cascades. . . . . . . . . . . . . . 25

2.11 Sketch of the imaging atmospheric technique. . . . . . . . . . . . . . 26

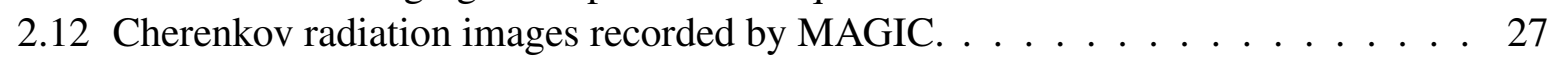

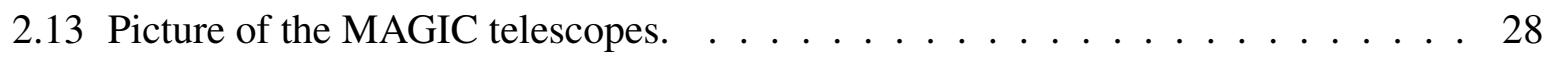

2.14 MAGIC and subsystems. . . . . . . . . . . . . . . . . . . 29

2.15 CCD cameras of MAGIC . . . . . . . . . . . . . . . . . . 30

2.16 MAGIC Starguider camera . . . . . . . . . . . . . . . . . . . 31

2.17 PSF of a bright star. . . . . . . . . . . . . . . . . . . . . . . . . . . . . . . . . .

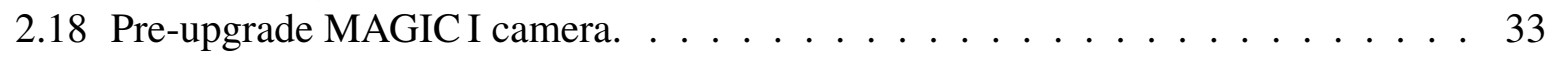

2.19 MAGIC II camera. . . . . . . . . . . . . . . . . . . . . . . 34

2.20 Macrocells in the former MAGIC I camera version. . . . . . . . . . . . . . . 36

2.21 Macrocells in the current MAGIC camera version. . . . . . . . . . . . . . . . . . . . . . . . . . . . . .

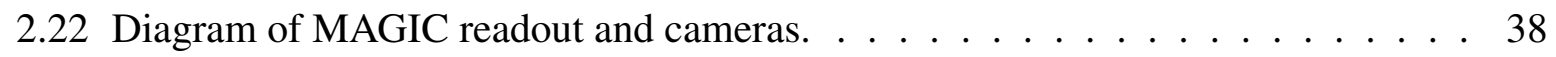

2.23 Scheme of Cherenkov event with MUX andDRS2 . . . . . . . . . . . . . . 40

2.24 MAGIC AllSky-camera image. . . . . . . . . . . . . . . . . 42

2.25 Wobble pointing mode scheme. . . . . . . . . . . . . . . . . 45

2.26 Flowchart of the MAGIC analysis chain. . . . . . . . . . . . . . 47 


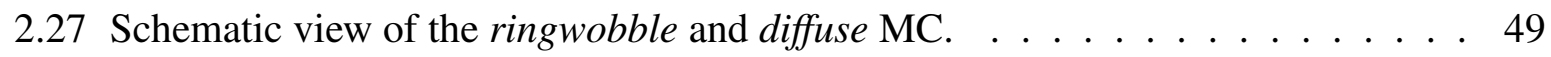

2.28 Data after image cleaning. . . . . . . . . . . . . . . . . . 53

2.29 Hillas parameters scheme. . . . . . . . . . . . . . . . . 55

2.30 Data quality selection plots. . . . . . . . . . . . . . . . . . . . . . . . . .

2.31 Geometry of stereo event. . . . . . . . . . . . . . . . . 57

2.32 Shower maximum distribution height for $\mathrm{EM}$ and hadronic cascades. . . . . . . . 58

2.33 Stereo parameters calculation. . . . . . . . . . . . . . . . 59

2.34 Scheme of the $\mathrm{RF}$ classification. . . . . . . . . . . . . . . . . 60

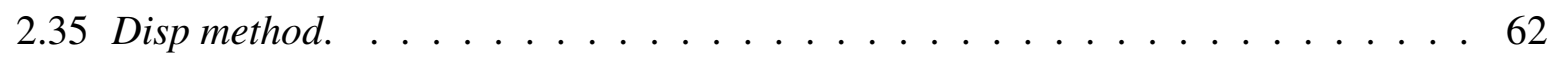

2.36 MAGIC energy resolution, energy bias and energy threshold. . . . . . . . . . 63

2.37 Alpha and $\theta^{2}$ distributions. . . . . . . . . . . . . . . . . . 65

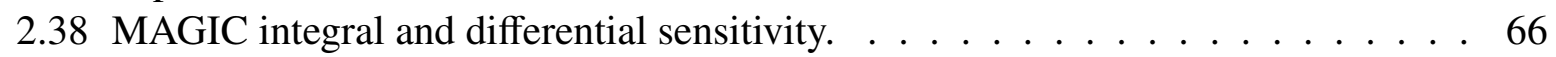

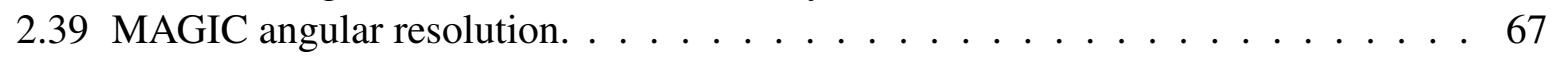

2.40 Example of a MAGIC skymap. . . . . . . . . . . . . . . . . . . 68 . . . . . . . . . . . .

2.41 Scheme of shower development at high Zd angle . . . . . . . . . . . . 70

2.42 MAGIC collection area. . . . . . . . . . . . . . . . . . . . . . 71

2.43 Distribution of time differences between triggered events. . . . . . . . . . . . 72

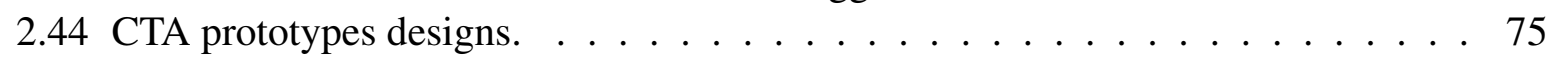

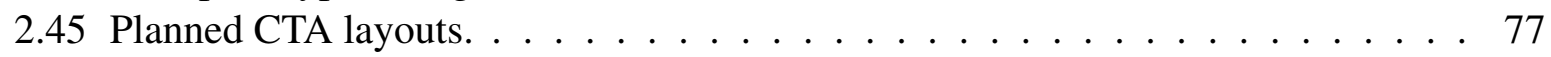

2.46 HAWC Observatory. . . . . . . . . . . . . . . . . . . . . 77

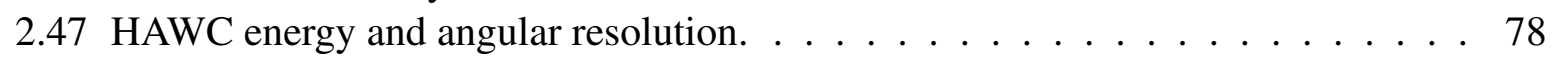

2.48 HAWC sensitivity. . . . . . . . . . . . . . . . . . . . . . . . . . 79

2.49 Fermi-LAT Instrument Response Function (IRF)] and performance. . . . . . . . . 81

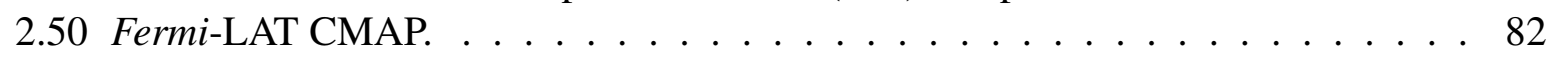

II Artist's impression of a microquasar. . . . . . . . . . . . . . . . . . 85

3.1 Artist's vision of an X-ray binary composed of a $\mathrm{BH}$ and a massive star. . . . . . 89

3.2 Distribution of $\mathrm{HMXBS}$ and LMXBs in galactic coordinates. . . . . . . . . . . . 90

3.3 Sketch of a Be/X-ray binary. . . . . . . . . . . . . . . . . . . 91

3.4 Sketch of different accretion processes. . . . . . . . . . . . . . . . . . . . . . . . . . . . .

3.5 Sketch of a microquasar's anatomy. . . . . . . . . . . . . . . . . . . . . . . . .

3.6 Diagram comparing quasars and microquasars. . . . . . . . . . . . . . . . . . . . . . . . .

3.7 Sketch of a microblazar. . . . . . . . . . . . . . . . . . 97

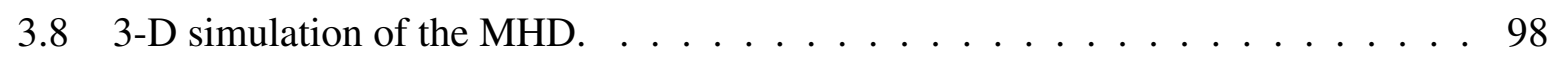

3.9 q-track of the HID . . . . . . . . . . . . . . . . . . . . 99

3.10 Radio/X-ray diagram of $18 \mathrm{BH}$ X-ray binary (Gallo et al.2012). . . . . . . . . . 100

4.1 Representation of the Cygnus region. . . . . . . . . . . . . . . . 106

4.2 Schematic of the Cygnus X-1 orbit. . . . . . . . . . . . . . . . . . . . 107

4.3 TS map centered at Cygnus X-1 including only 3FGL source as background. . . . 110

4.4 TS map centered at Cygnus X-1 for the Hard State (HS) and the Soft State (SS) separately. ................................. 113

4.5 Phase-folded Test Statistics (TS) map centered at Cygnus X-1 for the HS . . . 114 
4.6 SED of Cygnus X-1 during the $\mathrm{HS}$ periods. . . . . . . . . . . . . . . 114

4.7 MAGIC and Fermi-LAT lightcurve of Cygnus X-1 . . . . . . . . . . . . . . 126

4.8 SED of Cygnus X-1 covering from X-ray up to VHE . . . . . . . . . . . . 127

5.1 Cygnus X-3 saxophone plot. . . . . . . . . . . . . . . . . . . . 131

5.2 Cygnus X-3 Fermi-LAT TS evolution. . . . . . . . . . . . . . . . . . . . . 134

5.3 Cygnus X-3 saxophone plot with MAGIC observations. . . . . . . . . . . . . . 135

5.4 Cygnus X-3 MAGIC skymaps for the $29^{\text {th }}$ and the $30^{\text {th }}$ of November 2015 . . . . 137

5.5 Cygnus X-3 Spectral Energy Distribution (SED) for the flaring period AugustSeptember 2016. . . . . . . . . . . . . . . . . . . . . 139

5.6 Cygnus X-3 light curve for the flaring period August-September 2016. . . . . . . 141

5.7 Cygnus X-3 phaseogram. . . . . . . . . . . . . . . . . 142

6.1 V404 Cygni FoV before and after the outburst of June 2015. . . . . . . . . . . 146

6.2 V404 Cygni spectra in the soft gamma-ray regime obtained with INTEGRAL. . . 147

6.3 V404 Cygni light curve obtained with INTEGRAL data. . . . . . . . . . . . . . 149

6.4 Differential MAGIC Upper limits (ULs) for V404 Cygni. . . . . . . . . . . . . . 151

6.1 Crab Nebula in X-rays and optical wavelengths. . . . . . . . . . . . . . . 153

7.1 Artist's view of a $\mathrm{NS}, \ldots \ldots \ldots \ldots \ldots \ldots$

7.2 Internal structure of a $\mathrm{NS} \ldots \ldots \ldots \ldots \ldots \ldots$

7.3 Diagram of a pulsar. . . . . . . . . . . . . . . . . 159

7.4 Sketch of the pulsar magnetosphere with the particle acceleration models highlighted. . . . . . . . . . . . . . . . . . . . 160

7.5 Wind zone model sketch. . . . . . . . . . . . . . . . . . . 161

7.6 Crab pulsar SED obtained by MAGIC up to TeV. . . . . . . . . . . . . . . 162

7.7 Crab pulsar phaseogram obtained with MAGIC . . . . . . . . . . . . . . . . . . . . . . . . . . . . . . . . .

7.8 Acceleration mechanisms inside a $\mathrm{PWN} \ldots \ldots \ldots \ldots$

7.9 Crab Nebula's synchrotron spectrum. . . . . . . . . . . . . . . . . . . 164

7.10 X-ray image of Crab Nebula taken with the Chandra satellite. . . . . . . . . . 165

7.11 Optical image of Crab Nebula taken with the Hubble telescope. . . . . . . . . . 165

8.1 HAWC skymaps. . . . . . . . . . . . . . . . . . . 169

8.2 MAGIC skymap of G70.7+1.2 and 1HWC J1904+080c FoV, . . . . . . . . . 170

8.3 OfWP scheme. . . . . . . . . . . . . . . . . . . 171

8.4 Maximum possible extension for a source in wobble mode. . . . . . . . . . . . 173

$8.5 \mathrm{X}$-ray and NIR image from G70.7+1.2. . . . . . . . . . . . . . . . 174

8.6 Zoom view of the MAGIC G70.7+1.2 skymap. . . . . . . . . . . . . . 175

8.7 2-D speed of pulsars. . . . . . . . . . . . . . . . . . 177

8.8 Simulated electron energy distribution for 2 HWC J1907+084* . . . . . . . . . 179 
8.9 Spectral energy distribution from $10 \mathrm{GeV}$ up to $\sim 90 \mathrm{TeV}$. In all cases, the assumed spectrum for the sources is a power-law function with photon index

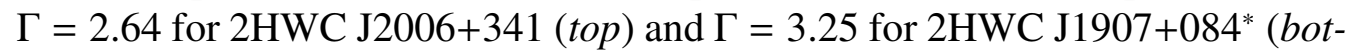
tom), as obtained by HAWC (see Table 8.1). Fermi-LAT UL spectrum is shown in orange assuming a point-like source. MAGIC results for point-like hypothesis (grey) and $0.16 \mathrm{deg}$ radius extension (black) are displayed. The HAWC butterfly is obtained for the parameters given in Table 8.1 . . . . . . . . . . . . . 180

9.1 FoV] of PSR J1954+2838 and PSR J1958+2845 . . . . . . . . . . . . . . . 185

9.2 MAGIC skymap a the position of PSR J1954+2838. . . . . . . . . . . . . . . . . . . . . . . . . . . .

9.3 MAGICISED for PSR J1954+2838 and PSR J1958+2845. . . . . . . . . . . . . 189

9.4 PWN luminosity VS characteristic age and spin-down power of the pulsars. . . . 191

9.5 Milky Way with the spiral arms and MAGICPWNe candidates. . . . . . . . . . . 192

9.6 FIR energy density photon field as a function of the spin-down power of the pulsars 193

A.1 Sketches of different $\mathrm{SNe}$ spectra. . . . . . . . . . . . . . . . . . 202

A.2 MAGIC $\theta^{2}$ distribution of the Type Ia SN SN 2014J. . . . . . . . . . . . . . . 206

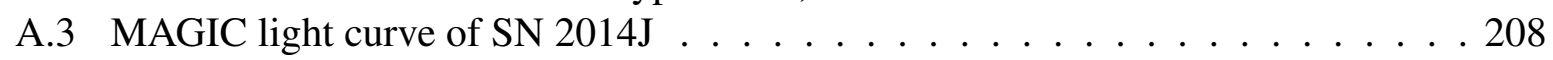

A.4 Full likelihood output for the SN 2014J. . . . . . . . . . . . . . . . . . 208

C.1 Search for conducted noise with linear PSU with $\mathrm{V}_{c c}$ and $\mathrm{HV}$ OFF. . . . . . . 220

C.2 Search for conducted noise with linear $\overline{\mathrm{PSU}}$ with $\mathrm{V}_{c c}=5 \mathrm{~V}$ and $\mathrm{HV}$ OFF. . . . . . 220

C.3 Search for radiated noise with linear PSU with $\mathrm{V}_{c c}, \mathrm{HV}$ and switching PSU OFF. 221

C.4 Search for radiated noise with linear $\overline{\mathrm{PSU}}$ with $\mathrm{V}_{c c}=5 \mathrm{~V}$ and $\mathrm{HV}$ and switching PSU OFF. . . . . . . . . . . . . . . . . . . . . . . 222

C.5 Search for radiated noise with linear $\mathrm{PSU}$ with $\mathrm{V}_{c c}$ and $\mathrm{HV} \mathrm{ON}$ and switching PSUOFF. . . . . . . . . . . . . . . . . . . . 222

C.6 Search for radiated noise with switching PSU with $\mathrm{V}_{c c}, \mathrm{HV}$ OFF and switching PSU ON but unloaded. . . . . . . . . . . . . . . . . . . . . . 223

C.7 Search for radiated noise with switching PSU with $\mathrm{V}_{c c}, \mathrm{HV} \mathrm{ON}$ and switching PSU ON but unloaded. . . . . . . . . . . . . . . . . . . . 224 


\section{List of Tables}

1.1 Classification of the energy domain of the gamma-ray astrophysics. . . . . . . . 8

1.2 Classification of gamma-ray production and absorption mechanisms. . . . . . . 8

1.3 Comparison of gamma-ray detection techniques. . . . . . . . . . . . 15

2.1 Comparison pre- and post-upgrade MAGIC I camera. . . . . . . . . . . . . . . 34

2.2 Image cleaning levels for pre-upgraded cameras. . . . . . . . . . . . . . . 51

2.3 Image cleaning levels for post-upgraded cameras. . . . . . . . . . . . . . . 52

2.4 Sum cleaning image parameters. . . . . . . . . . . . . . . . . . . . 54

2.5 Standard MAGIC analysis cuts. . . . . . . . . . . . . . . . . . 64 . . . . . . . . . . . . . . . . . . . .

2.6 MAGIC size cuts for moon analysis. . . . . . . . . . . . . . . . . . 69

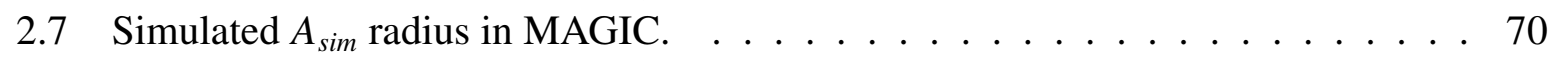

4.1 Intervals in MJD of the $\mathrm{HS}$ and the $\mathrm{SS}$ periods of Cygnus X-1. . . . . . . . 112

4.2 Days with $\mathrm{TS}>9$ in the daily basis analysis of Cygnus $\mathrm{X}-1 . \ldots \ldots$. . . . . . . . . . . . . .

4.3 Details of the MAGIC observations of the microquasar Cygnus X-1 . . . . . . . . 119

4.5 MAGIC integral UL of Cygnus X-1 assuming a power-law spectrum with different photon indices. . . . . . . . . . . . . . . . . . . 120

4.6 MAGIC differential ULs for Cygnus X-1. . . . . . . . . . . . . . . . . . 120

4.7 Cygnus X-1 differential flux ULs at 95\% C.L. for each X-ray spectral state with MAGIC . . . . . . . . . . . . . . . . . . . . . . . . 1121

4.8 Cygnus X-1 orbital phase-folded 95\% C.L. flux ULs with MAGIC. . . . . . . . . 122

4.9 MAGIC daily integral ULs for Cygnus X-1. . . . . . . . . . . . . . . 123

5.1 Cygnus $\mathrm{X}-3 \mathrm{GeV}$ flaring periods. . . . . . . . . . . . . . . . . . 132

5.2 Image cleaning levels for the Cygnus X-3 analysis. . . . . . . . . . . . . . 133

5.3 Cygnus X-3 observations from November 2013 until August 2016. . . . . . . . . 136

5.4 UL to the integral flux above $300 \mathrm{GeV}$ at $95 \% \mathrm{CL}$ assuming a power-law spectrum with different photon indices, $\Gamma$. . . . . . . . . . . . . . . . . 138

5.5 MAGIC differential flux ULs for Cygnus X-3 . . . . . . . . . . . . . . . . . . . 138

5.6 MAGIC daily flux ULs for Cygnus X-3 . . . . . . . . . . . . . . . . . 140

6.1 Time intervals used in the MAGIC analysis based on the Bayesian Block algorithm applied on INTEGRAL data. 
6.2 From left to right: MAGIC observation period in calendar and Modified Julian Day (MJD), effective time, significance and integral ULs for energies between 200 and $1250 \mathrm{GeV}$, assuming a power-law function of index $\Gamma=2.6$. The last two rows report the results for the whole data sample and for the sample obtained based on the Bayesian Blocks algorithm (see Section 6.3). . . . . . . . . . 150

8.1 HAWC parameters for the sources analyzed with MAGIC . . . . . . . . . 168

8.2 Distance between 2HWC candidates and MAGIC wobble positions. . . . . . . . 171

8.3 MAGIC differential flux ULs for 2HWC J2006+341 and 2HWC J1907+084* . . 176

9.1 Characteristic of the six PWNe observed by MAGIC . . . . . . . . . . . . . . 184

9.2 MAGIC differential ULs for PSR J1954+2838 and PSR J1958+2845. . . . . . . 188

9.3 MAGIC differential ULs for PSR J2022+3842 . . . . . . . . . . . . . . . . . . 188

9.4 MAGICISED for PSR J2111+4606 and PSR J2238+5903. . . . . . . . . . . . . 189

9.5 From left to right: Integral UL above $300 \mathrm{GeV}$, UL on the TeV luminosity (1-10

$\mathrm{TeV})$, efficiency converting rotational energy into $\mathrm{TeV}$ gamma rays $\left(L_{\gamma, 1-10 \mathrm{TeV}} / \dot{E}\right) .190$

A.1 Summary of the MAGIC observations of SN 2014J. . . . . . . . . . . . . . 207 


\section{Part I}

\section{Introduction to the non-thermal Universe}

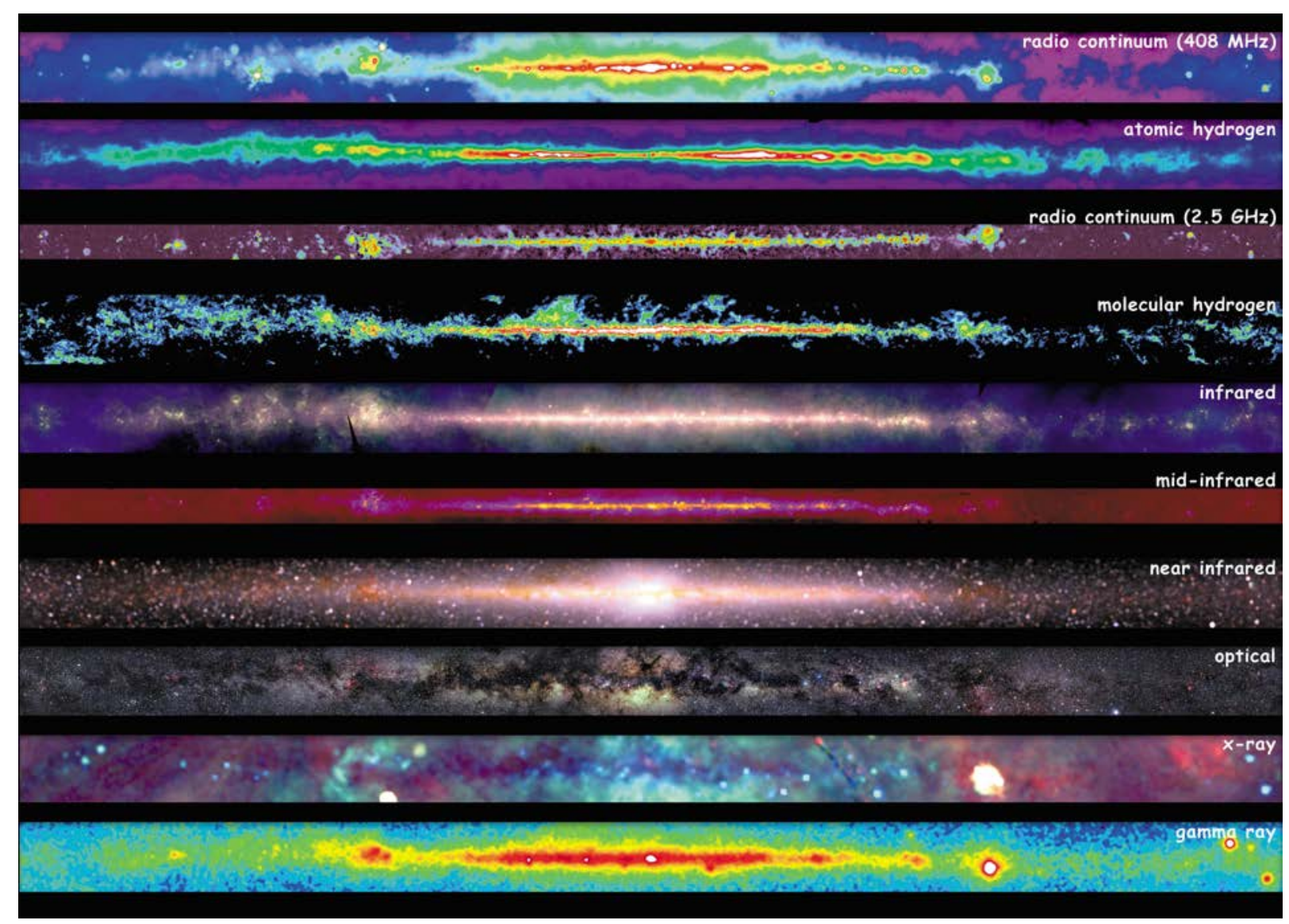

Figure I: Multiwavelength view of the Milky Way. Credit: NASA/Goddard Space Flight Center. 



\section{1 \\ Cosmic rays and gamma-ray astrophysics}

The history of the gamma-ray astronomy, or the non-thermal astrophysics in general, is quite recent. It goes back only to the 20th century, when the physicist Victor Hess discovered what it was thought to be a new kind of radiation that would forever change our conception of the Universe. In 1912, with the aid of balloon flights, Hess measured that the density of ionized particles increased with the altitude (Hess 1912). Some years later, given that its origin was attributed to sources that lay beyond the Earth's atmosphere, Robert Millikan would name this radiation Cosmic Rays (CRs) (Millikan \& Cameron 1926). CRs were not strictly a new type of radiation: they are mostly composed in a $99 \%$ by protons and Helium (He) nuclei, and, in a minor fraction, by heavier nuclei, electrons, positrons and neutrinos. However, CRs present the highest energies observed thus far, reaching the level of $10^{21} \mathrm{eV}$. This highly energetic radiation cannot be thermal in origin, otherwise it would need to have been created during the very early stages of the Big Bang explosion. Therefore, different processes are needed to explain CRs production (Sections 1.1.2 and 1.2.1).

CRs offer us a new window from which to observe the Universe, but they give no clue on the astrophysical sources that produce them. Because CRs are composed of charged particles, they are deflected by the randomly oriented magnetic fields they cross in their travel to Earth, erasing any trace of their origin. This is at least true for CRs with energies below $10^{20} \mathrm{eV}$ : above this threshold, particles can travel several Mpc without being affected by the magnetic fields. Consequently, CRs with energies below $\mathrm{EeV}$ are detected randomly distributed across our atmosphere. By observing them, we can obtain information of their spectrum and composition, but in order to deduce their origin, we need to study neutral particles related to them. To do so, we observe the non-thermal products of the CR acceleration, neutrinos and gamma rays. Their neutral charge allows us to trace back their origin. 
This thesis is focused on VHE gamma-ray astrophysics. The relationship between CRs and gamma rays is presented in this section, and the processes and sources from which these VHE gamma rays originate.

\subsection{Cosmic rays}

The term $\mathrm{CRs}$ is nowadays used to define the energetic particles arriving from outside of our atmosphere. As mentioned before, they are composed mainly by protons and $\mathrm{He}$ nuclei, while only $1 \%$ is formed by electrons, positrons, neutrons and heavier nuclei. Gamma rays are also considered part of the CRs. They can be measured directly with balloon experiments or satellites. Normally these techniques explore CRs up to $10^{14} \mathrm{eV}$. Higher energies can be studied by indirect observations in ground based experiments. These detectors observe the secondary particles produced in the Extended Air Showers (EASs), i.e. cascades of particles originate by the interaction of primary CRs with the nuclei of the Earth's atmosphere. These EASs, first detected by Pierre Auger in 1938, can extend hundred meters on the ground. The study of CRs led to important discoveries, such as the existence of the positron $\left(e^{+}\right)$or pions. However, many question lack for an answer yet, as e.g. which are the sources responsible for the CRs production?.

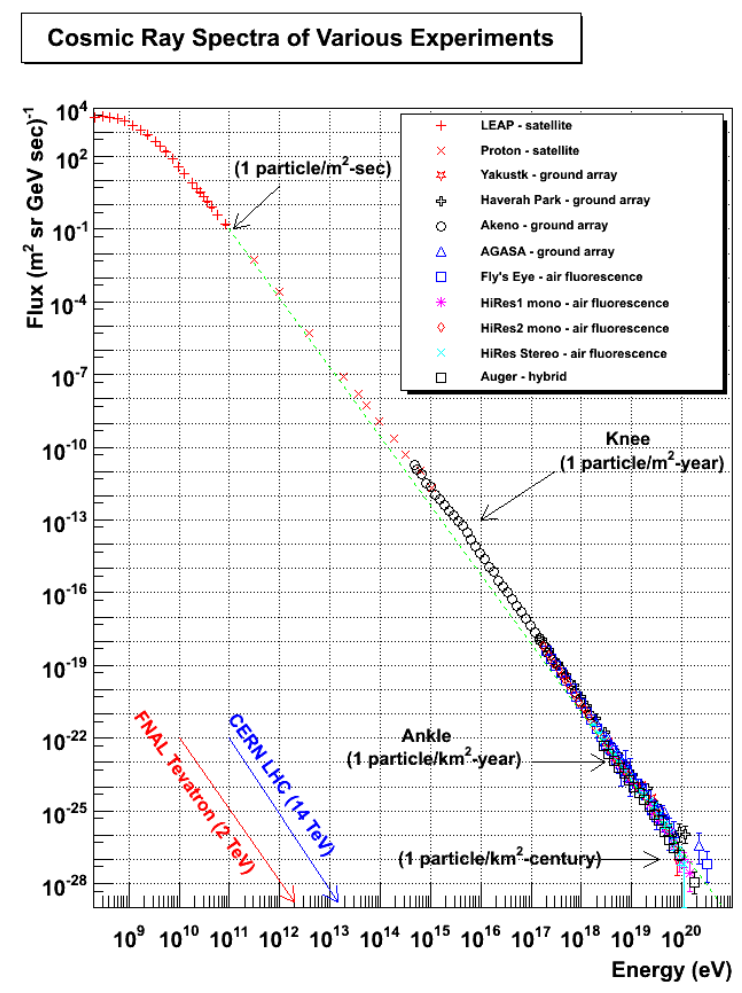

Figure 1.1: $\mathrm{CR}$ spectrum obtained with data from different experiments. Credit: Hanlon 2010). 


\subsubsection{Spectrum}

The CR spectrum extends from $10^{8}$ to $10^{21} \mathrm{eV}$, approximately (see Figure 1.1). Particles with energy below $\sim 1 \mathrm{GeV}$ have solar origin, as the solar magnetic field in the wind blocks particles at those energies arriving from outside from the solar system. The rest of the spectrum is welldefined by a broken power law, $d N / d E \propto E^{-\Gamma}$, with 3 different photon indices $\Gamma$. The first part, from $\sim 100 \mathrm{MeV}$ up to $\sim 5 \mathrm{PeV}$, presents a photon index of $\Gamma \sim 2.7$. Its upper limit is known as knee, which is charge dependent: particles with higher charge will extend the knee to higher energies. The second region covers the range from the knee up to $\sim 3 \mathrm{EeV}$, the so-called ankle, in which the spectrum follows a power law with $\Gamma \sim 3$. Finally, beyond $\sim 3 \mathrm{EeV}$, the spectrum hardens again (with $\Gamma \sim 2.6$ ) up to $\sim 30 \mathrm{EeV}$. The different slopes are thought to be related with the origin of the CRs Particles with energies covering the first range up to the knee are believed to be accelerated inside our Galaxy, whilst particles above the ankle seem to have extragalactic origin. Between the knee and the ankle the origin is not that clear. Several proposals were made to explain the steepening of the spectrum at the knee: changes on the acceleration mechanism, as e.g. two-step acceleration in the Supernova Remnants (SNRs), first at the front shock and re-aceleration in the inner pulsar-driven remnant (Bell 1991 ; see Section ??); leakage of CRs out of the Galaxy by diffuse propagation (Ptuskin et al. 1993) or even a cutoff of light elements (Antoni et al. 2005).

The CR spectrum is affected at the edge by the so-called Greisen-Zatsepin-Kuzmin (GZK) cutoff (Figure 1.2). This cutoff is produced by the interaction of the CRs with energies $\gtrsim 10^{20}$ $\mathrm{eV}$ with the Cosmic Microwave Background (CMB), which gives rise to hadrons as a product of $\Delta$ resonance:

$$
\begin{aligned}
& p+\gamma_{C M B} \rightarrow \Delta^{+} \rightarrow p+\pi^{0} \\
& p+\gamma_{C M B} \rightarrow \Delta^{+} \rightarrow n+\pi^{0}
\end{aligned}
$$

This interaction also limits the maximum distance that the CRs with energies greater than $10^{20} \mathrm{eV}$ can travel to $\sim 50 \mathrm{Mpc}$.

\subsubsection{Cosmic ray acceleration}

CRs, as charged particles, can be accelerated within magnetic and electric fields. Nevertheless, it is thought that the bulk of CRs are accelerated through diffuse shock acceleration mechanisms. These processes can be split into two main mechanisms, proposed by Enrico Fermi (Fermi 1949) whose names arise from: first order and second order Fermi acceleration. They are differentiated attending to the features of the moving plasma.

$\odot$ First order Fermi acceleration: The acceleration takes places in a plasma that presents shock waves (blobs of material moving at supersonic velocities) and magnetic field inhomogeneities. The particles get accelerated every time they cross the shock wave. The energy gained in each reflection is proportional to the relative velocity between the shock and the particle, $\langle\Delta E / E\rangle \propto v_{\text {rel }} / c$, and therefore the larger the difference, the larger the energy gain. The number of times that a particle cross the shock is directly proportional to 


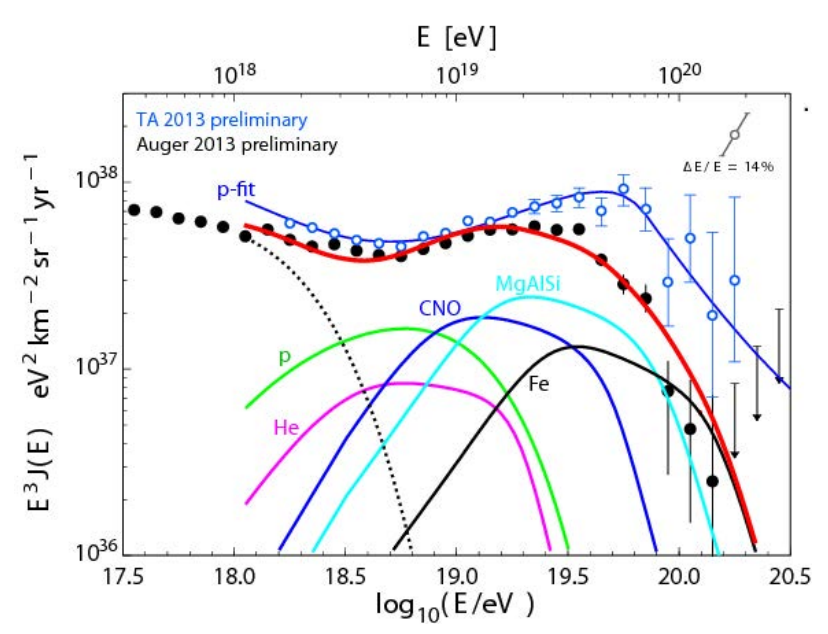

Figure 1.2: CR flux measurements by Auger and Telescope Array (TA) Collaboration, where a cutoff at energies $\sim 10^{21} \mathrm{eV}$ is evidenced. Taken from Kampert \& Tinyakov (2014).

the magnetic field strength. Thus, the crossing frequency is higher as larger the magnetic field strength is. This efficient mechanism is thought to be the responsible of the particle acceleration up to $\mathrm{TeV}$ and $\mathrm{PeV}$ ranges.

$\odot$ Second order Fermi acceleration: This acceleration happens within moving magnetized clouds. The energy gained by the particles in each interaction with the magnetized material is proportional to the square of the speed of the moving cloud, $\langle\Delta E / E\rangle \propto\left(v_{\text {cloud }} / c\right)^{2}$. However, this acceleration is not very efficiency: typical values of $v_{\text {cloud }} / c \sim 10^{-5}$ lead to a gain of $\langle\Delta E / E\rangle \sim 10^{-10}$ per interaction.

If particles escape from the acceleration region, they will not be able to gain more energy. Thus, the maximum energy that the accelerated CRs can reach is limited by the radius of the circular motion they describe under the presence of an uniform magnetic field, the so-called Larmor radius or gyroradius. This gyroradius cannot exceed the size of the acceleration region, otherwise the particle would not be confined on this region anymore. This geometrical constraint is known as Hillas criterion. The maximum energy, $E_{\max }$, can be expressed as follows:

$$
E_{\text {max }}=10^{18} \mathrm{eV} q\left(\frac{R}{\mathrm{kpc}}\right)\left(\frac{B}{\mu \mathrm{G}}\right)
$$

where $q$ is the charge of the particle, $R$ and $B$ are the radius and magnetic field of the acceleration region, respectively.

Figure 1.3, the so-called Hillas plot, shows the relation between the magnetic field strength and the radius of the acceleration region. The diagonal lines confine the allowed region for acceleration of different particles with certain energy.

The possible astrophysical sources responsible for the production of CRs, both galactic and extragalactic, are explained in Section 1.2.3. As mentioned before, in order to study the CRs origin, we need to make use of neutral particles observations, as gamma rays. 


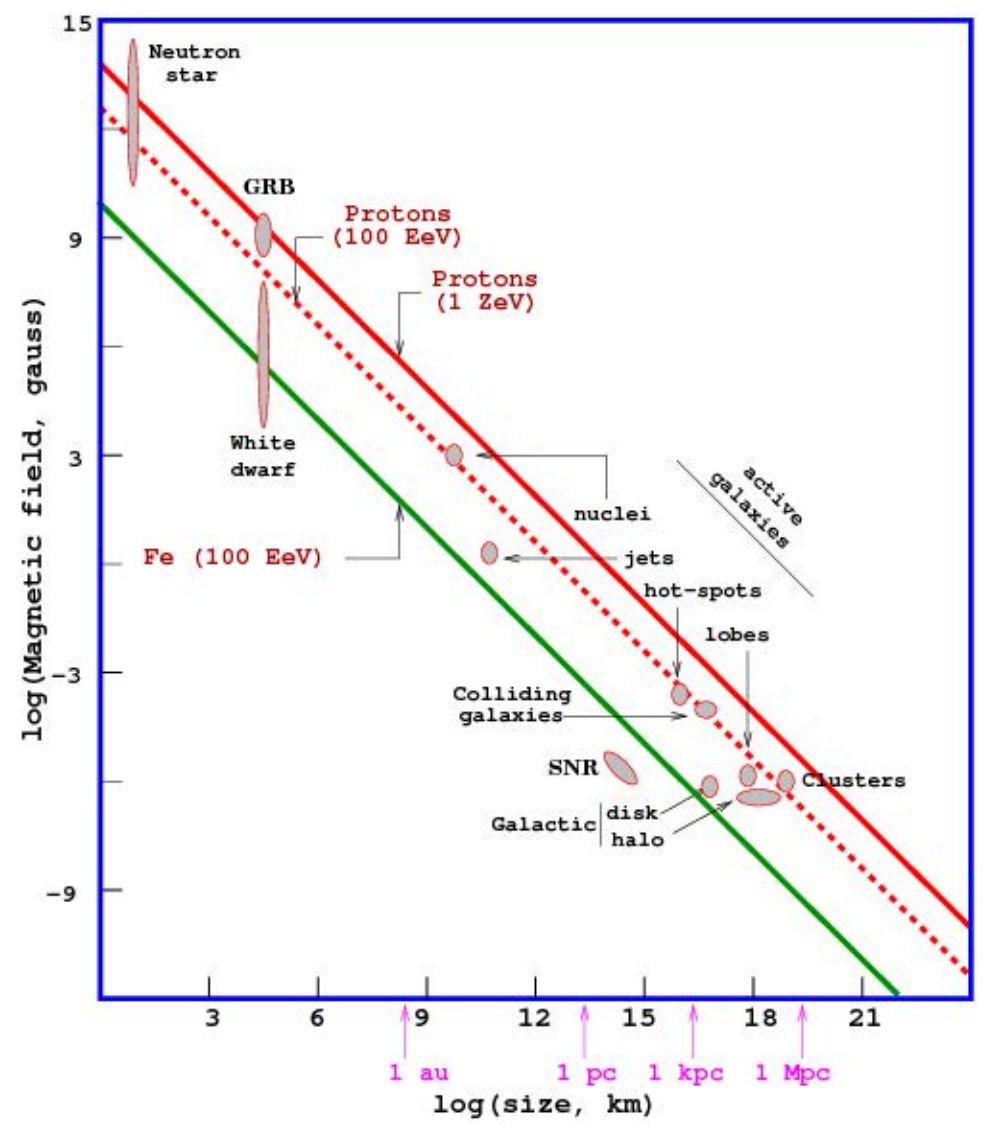

Figure 1.3: Hillas plot that depict the possible $\mathrm{CR}$ sources as a function of their magnetic field strength and size. The lines indicate the allowed acceleration region for different particles at a maximum energy (solid red line for protons with $E_{\max }=1 \mathrm{ZeV}=10^{21} \mathrm{eV}$, dashed red line for protons with $E_{\max }=100$ $\mathrm{EeV}=10^{20} \mathrm{eV}$, and solid green line for Fe nuclei with $E_{\max }=100 \mathrm{EeV}=10^{20} \mathrm{eV}$ ). Objects below each line cannot accelerate those particles up to the indicated energies Hillas (1984).

\subsection{Gamma-ray astrophysics}

Gamma rays, photons with the highest energies, are produced in the acceleration of CRs or their interaction with the environment. The gamma-ray spectrum extend beyond $\sim 1 \mathrm{MeV}$, minimum energy of gamma rays produced in electron/positron annihilation $\left(2 \times m_{e}=2 \times 0.511 \mathrm{MeV}\right.$, where $m_{e}$ is the mass of the electron). The different gamma-ray domains are listed in Table 1.1 .

Up to now, only gamma rays belonging to the first three energy domains quoted in Table 1.1 were detected. However, given that the $\mathrm{CR}$ spectrum extends up to $\mathrm{ZeV}$ energies, gamma rays with energies greater than $100 \mathrm{TeV}$ are expected.

Given the high energies that the gamma rays achieve, thermal mechanism cannot be responsible for their production. We need to evoke non-thermal processes to explain their origin. The interaction of $\mathrm{CRs}$ and gamma rays with their environment can be encompassed into three cat- 


\begin{tabular}{ccc}
\hline \hline Domain & Abbreviation & Energy range \\
\hline Low energy & LE & $1 \mathrm{MeV}-30 \mathrm{MeV}$ \\
\hline High energy & $\mathrm{HE}$ & $30 \mathrm{MeV}-50 \mathrm{GeV}$ \\
\hline Very-high energy & VHE & $50 \mathrm{GeV}-100 \mathrm{TeV}$ \\
\hline Ultra-high energy & UHE & $100 \mathrm{TeV}-100 \mathrm{PeV}$ \\
\hline Extremely-high energy & EHE & $>100 \mathrm{PeV}$ \\
\hline
\end{tabular}

Table 1.1: Classification of the energy domain of the gamma-ray astrophysics.

egories: interaction with matter, interaction with magnetic fields and interaction with photon fields. The gamma-ray production processes (Section 1.2.1), as well as the gamma-ray absorption (Section 1.2.2), will then depend on these targets. Table 1.2 lists the production and absorption mechanisms according to them.

\begin{tabular}{c|c||c|c||c|c}
\hline \hline \multicolumn{2}{c|}{$\begin{array}{c}\text { Interaction with } \\
\text { matter }\end{array}$} & \multicolumn{2}{c||}{$\begin{array}{c}\text { Interaction with } \\
\text { magnetic fields }\end{array}$} & \multicolumn{3}{c}{$\begin{array}{c}\text { Interaction with } \\
\text { photon fields }\end{array}$} \\
\hline $\begin{array}{c}\text { Production } \\
\text { Bremsstrahlung }\end{array}$ & Absorption & Production & Absorption & Production & Absorption \\
\hline $\begin{array}{c}e^{-} / e^{+} \text {annihilation } \\
\pi^{0} \text { decay }\end{array}$ & - & $\begin{array}{c}\text { Synchrotron } \\
\text { Curvature radiation }\end{array}$ & - & & \\
\hline
\end{tabular}

Table 1.2: Classification of gamma-ray production and absorption mechanisms according to the interaction targets of CRs and gamma rays.

\subsubsection{Gamma-ray production}

The main non-thermal processes that give rise to gamma rays are quoted in Table 1.2 and represented in Figure 1.4. In the following, I will summarized the main features of each mechanism. For a comprehensive review of the topic, the reader is referred to Aharonian (2004).

\subsubsection{Bremsstrahlung}

Gamma-ray emission is produced when a charged particle is accelerated in the electric field of a nuclei (Figure 1.4 a). This mechanism is more efficient when the charge particle is an electron. Bremsstrahlung becomes dominant against the ionization above the so-called critical energy, at which the energy loss by both mechanism is equal. The mean bremsstrahlung energy loss as a function of the distance traveled by the electron in the medium is given by:

$$
-\left.\frac{d E}{d x}\right|_{B r e m s}=\frac{1}{\chi_{0}} E
$$


where $\chi_{0}$ is the radiation length, which represents the average distance over which the electron loses all but $1 / e$ of its energy due to bremsstrahlung. The radiation length depends on the material: low/high density materials will present larger/smaller radiation length. This implies that the charged particles penetrate longer without losing energy in mediums with lower density.

Taken into account that the energy loss is directly proportional to the energy of the charged particles, the bremsstrahlung gamma-ray spectrum follows the same distribution as the charged particle. Bremsstrahlung is an important mechanism for the production of $\mathrm{MeV}$ photons. Gamma rays at VHE can be generated through bremsstrahlung of Ultra-High-Energy (UHE) charges.

\subsubsection{Electron/positron annihilation}

When $\mathrm{HE}$ electron and positron collide, two gamma rays are produced $\left(e^{-}+e^{+} \rightarrow 2 \gamma\right.$, see Figure 1.4b). Taken into account the electron and positron mass, this interaction will give rise to a spectral line peak at $511 \mathrm{keV}$. Nevertheless, given the kinetic energy of the particles this peak is normally broad due to the Doppler broadening effect (see e.g. Figure 6.2).

The origin of the positrons is not clear yet, although the simplest possible source is the direct decay of positive pions (Equation 1.6). Another possibility is the creation of electron/positrons through photon-photon annihilation (Section 1.2.2.1), happening e.g. inside of low-mass microquasars (see Section 6.1.1). Finally, positrons could arise from the decay of long-lived radioactive isotopes produced in the Supernova (SN) nucleosynthesis.

\subsubsection{Pion decay}

Pion decay is the dominant hadronic mechanism for gamma-ray production. The interaction takes place between a $\overline{C R}$ proton with ambient protons or nuclei, resulting on emission of charged pions $\left(\pi^{ \pm}\right)$or neutral pions $\left(\pi^{0}\right)$ with the same probability. Whilst positive and negative pions decay into muons and neutrinos, neutral pions decay into two gamma rays $99 \%$ of the time:

$$
\begin{gathered}
\pi^{0} \rightarrow \gamma+\gamma \\
\pi^{+} \rightarrow \mu^{+}+v_{\mu} \text { and } \mu^{+} \rightarrow e^{+}+\overline{v_{\mu}}+v_{e} \\
\pi^{-} \rightarrow \mu^{-}+\overline{v_{\mu}} \text { and } \mu^{-} \rightarrow e^{-}+v_{\mu}+\overline{v_{e}}
\end{gathered}
$$

The rest energy of these gamma rays is half of the neutral pion enegy, i.e. $E_{\gamma}=m_{\pi^{0}} c^{2} / 2 \sim 67.5$ $\mathrm{MeV}$. However, if the neutral pion is moving, then the gamma ray energy increases. The final gamma-ray spectrum can be defined by a power-law spectrum with index $\Gamma \sim 2.5$.

\subsubsection{Synchrotron}

Due to Lorentz force, charged particles in the presence of a magnetic field follow spiral traces around the magnetic field lines, being accelerated and consequently, emitting radiation. This mechanism is more efficient in electrons than in protons. If we assume uniform magnetic field, then the bulk of electrons would be mono-energetic and the synchrotron gamma-ray spectrum would peak at:

$$
E_{\gamma} \sim 1.5 \times 10^{-5}\left(\frac{B}{[G]}\right)\left(\frac{E_{e}}{[T e V]}\right)^{2}
$$



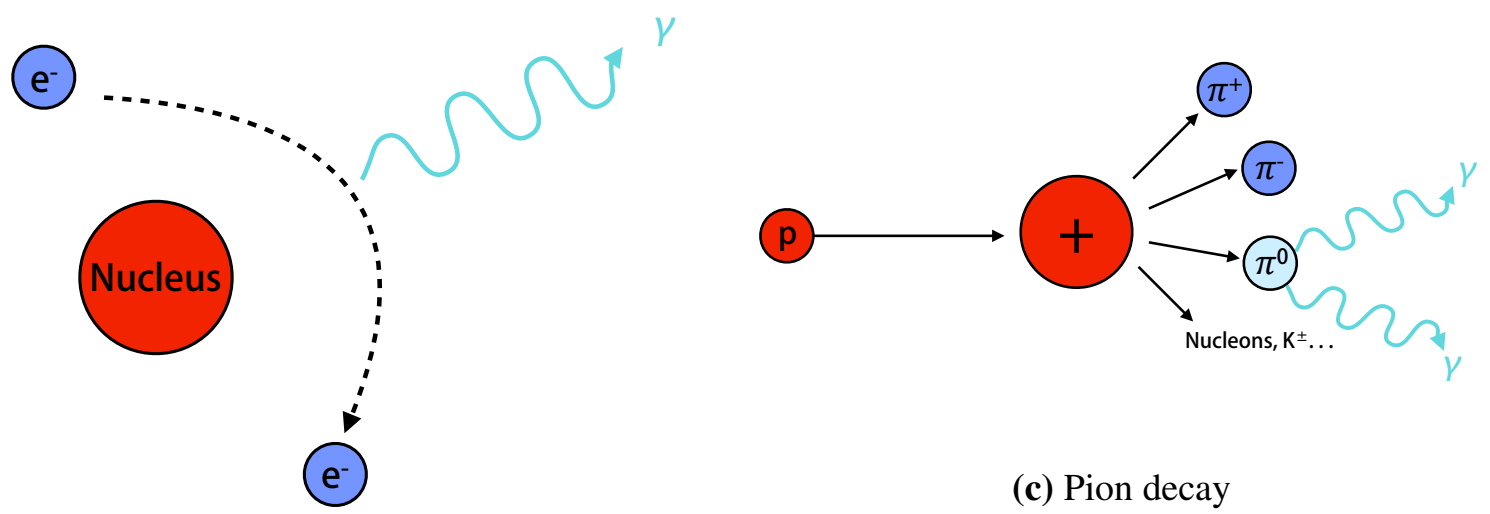

(c) Pion decay

(a) Bremsstrahlung
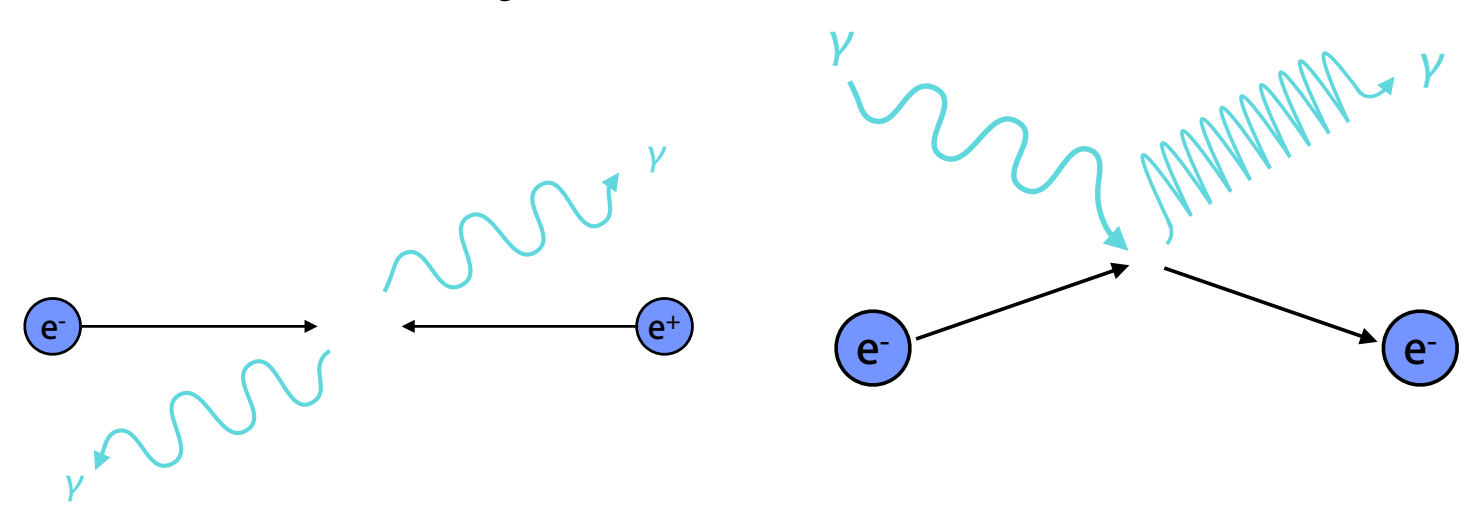

(b) Electron/Positron annihilation.

(d) Inverse Compton

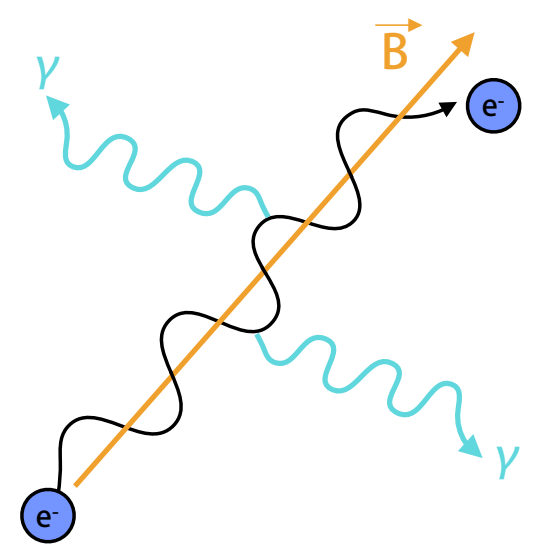

(e) Synchrotron.

Figure 1.4: VHE gamma-ray production processes. From López-Coto 2015. 
where $E_{e}$ is the energy of the seed electrons and $B$ is the magnetic field component perpendicular to the electrons movement. The synchrotron emission does not normally reach VHE.

Curvature on the magnetic field lines can lead to curvature radiation. This process is similar to synchrotron, but the particles follow the curved magnetic field lines instead of describing spiral paths around them.

\subsubsection{Inverse Compton}

This seems to be the most effective mechanism for production of VHE gamma rays. In this mechanism, relativistic electrons transfer most of their energy to the low-energy photons with which interact, turning them into $\mathrm{VHE}$ gamma rays (Figure 1.4). The energy of both, electrons and target photons ( $E_{e}$ and $E_{\gamma}$, respectively), affects the cross-section of the interaction and thus, one can distinguish two regimes:

$\odot$ Thomson regime: This regimes happens when $E_{\gamma} E_{e}<<m_{e}^{2} c^{4}$, leading to a constant cross-section of $\sigma_{T}=\frac{8}{3} \pi r_{e}^{2}$, where $r_{e}$ is the electron radius. For a power-law distribution of electrons with photon index $\Gamma_{e}$, the up-scattered gamma-ray spectrum follows a power-law function as well with index $\Gamma=\left(\Gamma_{e}+1 / 2\right)$ (Ginzburg \& Syrovatskii 1964). The energy loss in this regime is proportional to $E_{e}^{2}\left(d E_{e} / d t=\frac{4}{3} \sigma_{T} c E_{\gamma} n_{\gamma} E_{e}^{2}\right.$, where $n_{\gamma}$ is the density of initial photons).

$\odot$ Klein-Nishima regime: This regime is considered when $E_{\gamma} E_{e} \approx m_{e}^{2} c^{4}$, for which the cross-section is:

$$
\sigma_{K N}=2 \pi r_{e}^{2}\left\{\frac{1+\varepsilon}{\varepsilon}\left[\frac{2+2 \varepsilon}{1+2 \varepsilon}-\frac{\ln (1+2 \varepsilon)}{\varepsilon}\right]+\frac{\ln (1+2 \varepsilon)}{2 \varepsilon}-\frac{1+3 \varepsilon}{(1+3 \varepsilon)^{2}}\right\}
$$

where $\varepsilon=E_{\gamma} / m_{e} c^{2}$. In cases where $E_{\gamma} E_{e}>>m_{e}^{2} c^{4}$, one can follow the Klein-Nishima approximation where $\sigma_{\text {KNapprox }}=\frac{1}{\varepsilon} \pi r_{e}^{2}\left[\ln (2 \varepsilon)+\frac{1}{2}\right]$. In the Klein-Nishima regime, the resulting gamma-ray spectrum, given an electron spectrum again well-fitted by a power-law with $\Gamma_{e}$, is considerably steeper, with a photon index of $\Gamma=\Gamma_{e}+1$. The energy loss is here independent from the electron energy, but proportional to the density of photons.

When the seed photons on the Inverse Compton (IC) scattering are synchrotron gamma rays emitted by the same electron population, then the process is called Synchrotron Self Compton (SSC). This mechanism allows synchrotron radiation to reach the VHE regime.

\subsubsection{Gamma-ray absorption}

\subsubsection{Pair production}

Gamma rays also suffer from annihilation, which decreases their flux and make more difficult their detection in some scenarios. The main gamma-ray absorption mechanism is the so-called 
pair production, in which a $\mathrm{HE}$ photon interact with a lower energy one to give rise to an electron/positron pair. It is therefore the inverse process from the pair annihilation (Section 1.2.1.2). There are two types of pair production:

$\odot$ Classical pair production: This process is the responsible for the cascades produced in our atmosphere (see Chapter 2). It occurs when a $\mathrm{HE}$ photon interacts with a virtual photon of a nucleus' electric field:

$$
\gamma(\gamma) \rightarrow e^{-}+e^{+}
$$

$\odot$ Photon-photon annihilation: Interaction between HE photons with lower-energy photons from the ambient gas. This process is the responsible from the attenuation of extragalactic VHE by the Extragalactic Background Light (EBL) and plays an important role in the absorption of galactic VHE gamma rays inside the binary systems:

$$
\gamma \gamma \rightarrow e^{-}+e^{+}
$$

The cross-section of the pair production presents a peak at:

$$
E_{\gamma 1} E_{\gamma 2}(1-\cos \theta) \sim 2\left(m_{e} c^{2}\right)^{2}
$$

where $E_{\gamma 1}$ and $E_{\gamma 2}$ are the energy of the seed photons, $\theta$ is the collision angle between them and $m e=0.511 \mathrm{MeV}$ is the mass of the electron. This implies that the highest cross-section and hence, the highest probability of absorption for a gamma ray of $\sim 100 \mathrm{GeV}$ takes places with a photon of $\sim 6 \mathrm{eV}$, around Infrared (IR) and Ultraviolet (UV) band. This is the reason why VHE photons created in the microquasars jets can be strongly affected by the stellar wind. On the other hand, $\mathrm{HE}$ photons from these sources are more affected at the base of the jet where the soft $\mathrm{X}$-ray population is higher (see Section 3.2.4).

\subsubsection{Gamma-ray sources}

The processes shown in Section 1.2.1 can occur in different astrophysical objects. Here I provide a brief description of the already established gamma-ray sources, divided according to their galactic or extragalactic nature.

\subsubsection{Galactic sources}

$\odot$ SNR; Leftovers of the $\mathrm{SN}$ explosions. Gamma-ray emission is produced by the interaction between CRs, accelerated through the first order Fermi mechanism in the shock wave from the $\mathrm{SN}$ explosion, and nuclei in the Interstellar Medium (ISM). More information is given in Section ??.

$\odot$ Pulsars: Highly magnetized rotating Neutron Star (NS) (see Section 7.1). Particles can be accelerated in very specific regions along the magnetic field, from which gamma-ray emission is produced in narrow beams. Its emission is characterized by a pulsation: since the magnetic fields lines and the rotation axis of the NS are not usually aligned, the beam 
emission is only detected when crossing our light of sight. The best studied pulsar is the Crab pulsar, first detected by MAGIC (Aliu et al. 2008a) and recently detected up to TeV energies (Ansoldi et al.2016). Along with Crab, only Vela pulsar has been detected in the gamma-ray band (Brun, P. 2014).

$\odot$ PWN: Magnetized cloud of relativistic particles created when the pulsar wind interacts with the ISM. VHE emission originates through IC scattering of ambient photons by accelerated electrons. An extended description is given in Chapter 7 .

$\odot$ Gamma-ray binaries: Binary system composed of a massive star and a compact object, either a $\mathrm{BH}$ or a $\mathrm{NS}$, whose peak of luminosity lays in the gamma-ray regime. Their emission is explained by two models: pulsar wind scenario and microquasars. In the former, the orbiting compact object is a pulsar. The VHE emission arises from the interaction of the pulsar wind with the stellar wind. In the microquasar scenario, the compact object accretes material from the companion. The system presents relativistic jets, whose existence depends on the accretion rate, where particles are accelerated and emit gamma rays in the IC interaction with the stellar wind. Five gamma-ray binaries have been detected so far: PSR B1259-63, LS I +61³03, HESS J0632+057, 1FGL J1018.6-58 and LS 5039. None of them belong to the microquasar scenario. Detailed information is available at Section 3.1 .

\subsubsection{Extragalactic sources}

$\odot$ AGN: Galaxies hosting a super-massive $\mathrm{BH}$ in their center, which accretes material from the surrounding. They present two relativistic jets perpendicular to the accretion disk formed around the compact object. As in the case of microquasars, the gamma-ray emission is produced in the jets where charged particles can get accelerated.

$\odot$ Starburst galaxies: Galaxies in which the star formation rate is high. Consequently, the SN explosion rate is large giving rise to a high CR density. The starburst galaxy M82, detected by Very Energetic Radiation Imaging Telescope Array System (VERITAS) (VERITAS Collaboration et al. 2009), hosted the closest Type Ia in the last decades, SN 2014J, studied in this thesis (Appendix A).

$\odot$ Gamma-Ray Burst (GRB); Sudden and short gamma-ray outburst. They correspond to the most energetic gamma-ray flares. Their nature is still unclear, but two scenario have been proposed: collapse of highly rotating very massive stars $\left(M_{\star}>100 M_{\odot}\right)$, the so-called hypernovae, or merge of two compact objects.

\subsubsection{Detection techniques}

In order to detect the broadband gamma-ray spectrum, different detection techniques have been applied. Focusing on the $\mathrm{HE}$ and $\mathrm{VHE}$ regime, one can divide these techniques into three types: 
satellites, which perform direct observations of the gamma rays above the Earth's atmosphere, and Imaging Atmospheric Cherenkov Telescopes (IACTs) and Water Cherenkov arrays, which perform indirect observations at the ground level. The main characteristic of these techniques are summarized in Table 1.3 .

$\odot$ Satellites: Due to the reduced collection area that detectors on-board satellites have $(\sim 1$ $\mathrm{m}^{2}$ ), this technique provides information from $\mathrm{MeV}$ to a few hundred $\mathrm{GeV}$. Pair production (Section 1.2.2.1) is the dominant mechanism for the detection of gamma rays above $\sim 30$ $\mathrm{MeV}$. These detectors present wide Field of View (FoV) that observe during a 100\% duty cycle. They provide excellent $\gamma /$ hadron separation, but with a poor angular resolution at low energies $\left(>0.5^{\circ}\right.$ below $\left.\sim \mathrm{GeV}\right)$. On the other side, given that satellites are calibrated on ground before launch, their energy resolution is very good, with small systematic errors. The space based gamma-ray detector Fermi-LAT is introduced in Section X.

$\odot$ IACTs: This technique is based on the detection of Cherenkov light produced in the electromagnetic cascades originate by the interaction of a primary gamma ray with the nuclei of our atmosphere. The array of this type of telescopes allows to increase the collection area up to several $\mathrm{km}^{2}$ and therefore, the energy range increases considerably compared to the one covered by satellites. In turn, this technique provides very good $\gamma /$ hadron separation and excellent angular resolution. Nevertheless, the typical detectors used in the cameras, PMTs, do not permit to observed during daytime. Strong bright moonlight can also limit the duty cycle, but hardware modifications in MAGIC have been proved to be fruitful enlarging the observation period (see Section 2.4.2). Detailed description of the MAGIC telescopes is given in Section 2.4.

$\odot$ Water Cherenkov arrays: Particles in the EASs produced by VHE gamma rays (at energies greater than $\sim 100 \mathrm{GeV}$ ) can reach the ground. Gamma rays can be studied by the indirect observation of the Cherenkov light produced when these particles cross water tanks. Their very large collection area allows to detect multi-TeV gamma-rays. However, they present the worst angular resolution among all detection techniques, around $\sim 1^{\circ}$. Their energy resolution is as well lower than the one achieve by IACTs. Nonetheless, they have full duty cycles, since daytime observations are possible, and large FoV. HAWC, a recent Water Cherenkov array, is presented in Section X. 


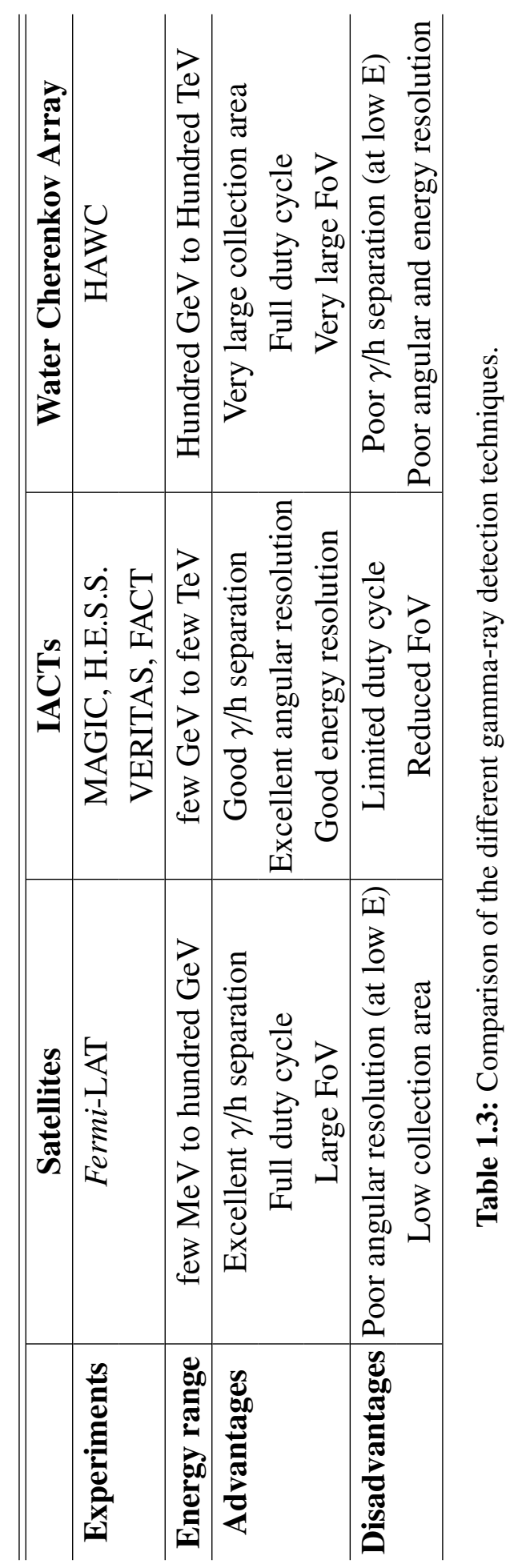




\section{2 \\ Gamma-ray telescopes}

In this chapter, I will describe in detail both hardware of the Major Atmospheric Gamma-ray Imaging Cherenkov (MAGIC) telescopes as well as the software used in the analysis of the data. Moreover, I will give an overview of the planned CTA, future generation of Cherenkov telescopes. Although this thesis focuses on the work performed with these two IACTs, other gamma-ray detection techniques were used in the analysis and discussion of several sources included here. Therefore, I will also describe briefly in this chapter the Fermi-LAT satellite and the wide FoV observatory HAWC, whose results, among with the ones provides by the Fermi Collaboration, are used in the discussion of a joint work between collaborations presented in Chapter X.

Before delving into the MAGIC and CTA characteristics, I will start giving a general view of the imaging atmospheric Cherenkov technique.

\subsection{Cherenkov light}

Gamma rays correspond to the highest radiation in the Electromagnetic (EM) spectrum, which covers 20 energy decades (or orders of magnitude) between radio and the $\mathrm{TeV}$ regime. Most of the lowest energy emission can travel across the atmosphere reaching the ground (see Figure 2.1). However, this is not the case for the gamma rays, whose interaction with the molecules in the atmosphere prevents the most energetic radiation from penetrating and reaching us. In order to detect high-energy photons, detectors on-board satellites can be used. Nevertheless, due to weight limitations, they can only support detectors with small collection area and therefore, they cannot provide results for energies above $100 \mathrm{GeV}$, regime in which the photon fluxes are already low. 


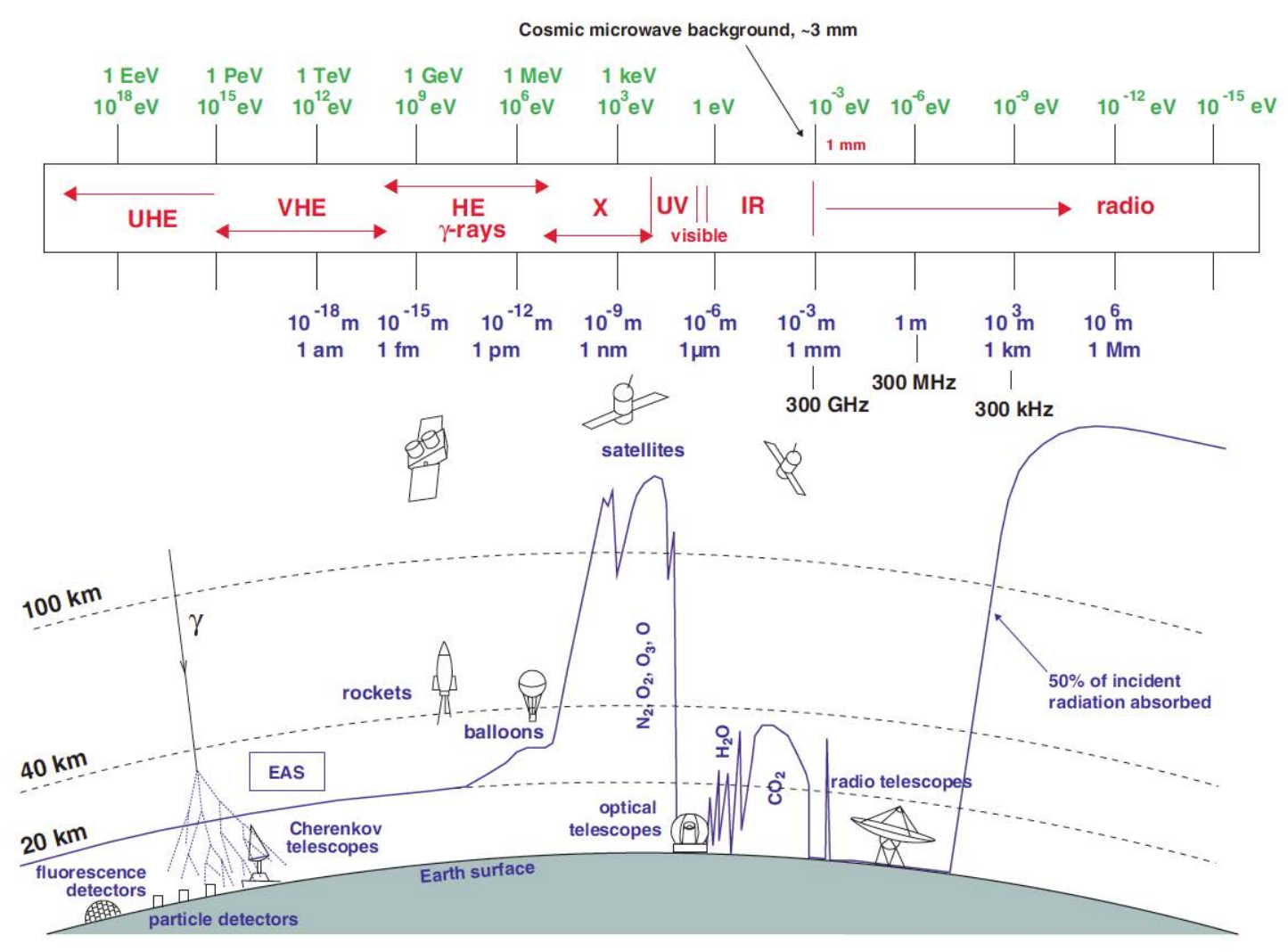

Figure 2.1: $\mathrm{EM}$ spectrum and its corresponding absorption level in the atmosphere. Credit: Wagner (2006), plot adapted from Longair (2011) and Moralejo (2000).

For energies $\geqslant 50 \mathrm{GeV}$, the IACTs, with larger sensitive area, dominates the study of gamma rays. The technique is based on indirect detection. When a VHE gamma ray or CR interacts with the atmospheric nuclei, a particle cascade is initiated, the so-called EAS. If the resulting charged particles of this interaction travel faster than the speed of light in the atmosphere, Cherenkov light is emitted, whose wavelength ranges between 300 and $500 \mathrm{~nm}$. The existence of this type of light was proposed by the Soviet physicist Pavel Alekseyevich Cherenkov (Cherenkov 1934) who, along with Ilya Frank and Igor Tamm, received the Nobel prize in 1958 for the discovery and interpretation of the Cherenkov effect. 


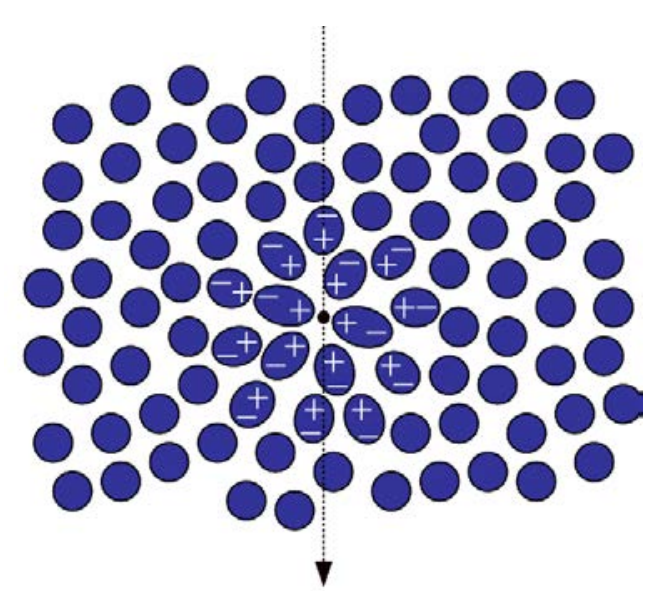

Figure 2.2: Polarization on a medium when a charged particle travels with low velocity $(v<c / n)$. Taken from http://mxp.physics.umn.edu.

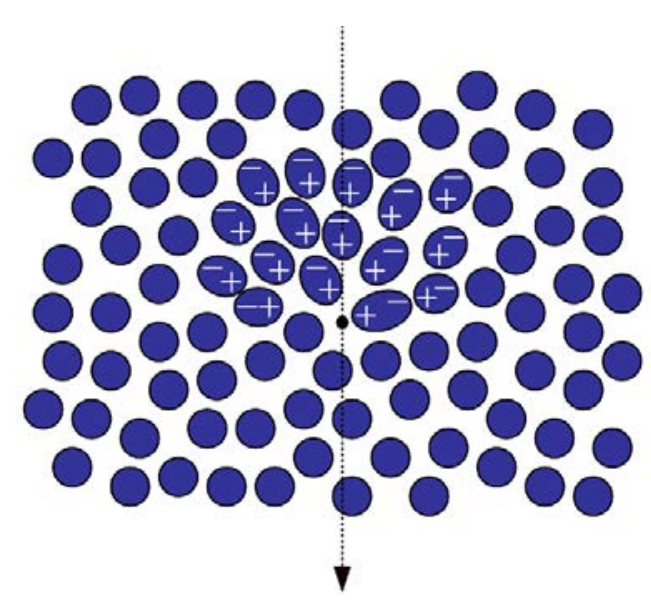

Figure 2.3: Polarization on a medium when a charged particle travels faster than the speed of light on it $(v>c / n)$. Taken from http://mxp.physics.umn.edu.

The atmosphere is a dielectric medium and therefore, the molecules in it are disrupted by the charged particles crossing, changing their polarization. If the particle velocity is low, $v<c / n$ (where $c$ is the speed of light in vacuum and $n$ is the refraction index of the medium), the polarization is symmetrical (see Figure 2.2) and therefore, the electromagnetic field remains null. However, when its velocity is higher than the speed of light in the medium $(v>c / n)$, the particle travels faster than its electric field leaving behind a non-symmetric perturbation (see Figure 2.3). To return to the equilibrium, Cherenkov radiation is emitted in the depolarization of the molecules.

The number of Cherenkov photons can be estimated as a function of unit track length of the charged particle and its own wavelength, following Yao et al. (2006):

$$
\left.\frac{d^{2} N}{d x d \lambda}=\frac{2 \pi \alpha}{\lambda^{2}}\left(1-\frac{c^{2}}{v^{2} n^{2}(\lambda)}\right) \approx 379 \sin ^{2} \theta(\lambda) \mathrm{eV}^{-1} \mathrm{~cm}^{-1}\right]
$$

where $\alpha \approx 1 / 137$ is the known fine structure constant. Therefore, the number of Cherenkov photons is inversely proportional to the squared wavelength, $\mathrm{dN} / \mathrm{d} \lambda \propto \lambda^{-2}$. This is the reason why this radiation peaks at $\sim 320 \mathrm{~nm}$, i.e. in the $\overline{\mathrm{UV}}$ band (see Figure 2.4). However, the emitted and observed Cherenkov radiation spectrum differ due to the transmission losses in the atmosphere. The main sources of this attenuation are:

$\odot$ Rayleigh scattering: Scattering off air molecules, which a wavelength dependency of $\lambda^{-4}$. It affects mostly UV radiation.

$\odot$ Mie scattering: Scattering off aerosols, dust and droplets water. It does not show any strong wavelength dependency. 
$\odot$ Ozone molecules: These molecules are responsible for the strong absorption of hard UV photons $(<300 \mathrm{~nm})$.

$\odot \mathbf{H}_{2} \mathrm{O}$ and $\mathrm{CO}_{2}$ molecules: They produce absorption in the $\mathrm{IR}$ band.

$\odot$ Zenith angle: The larger the zenith angle of the EAS, the higher the attenuation. This is due to the fact that at high zenith angle, the cascades develop in the highest layers of the atmosphere and hence, particles need to travel a larger path. Consequently, the probability of suffering absorption from some of the above mentioned processes increases. Only EASs initiated by particles at the highest energies are significantly detected by the telescopes at high Zenith distance (Zd) range. Thus, at larger zenith ranges the peak of the Cherenkov radiation spectrum shifts to larger wavelengths. Figure 2.5 shows how the density of Cherenkov photons, as well as the peak of their spectrum, vary at different zenith angles.

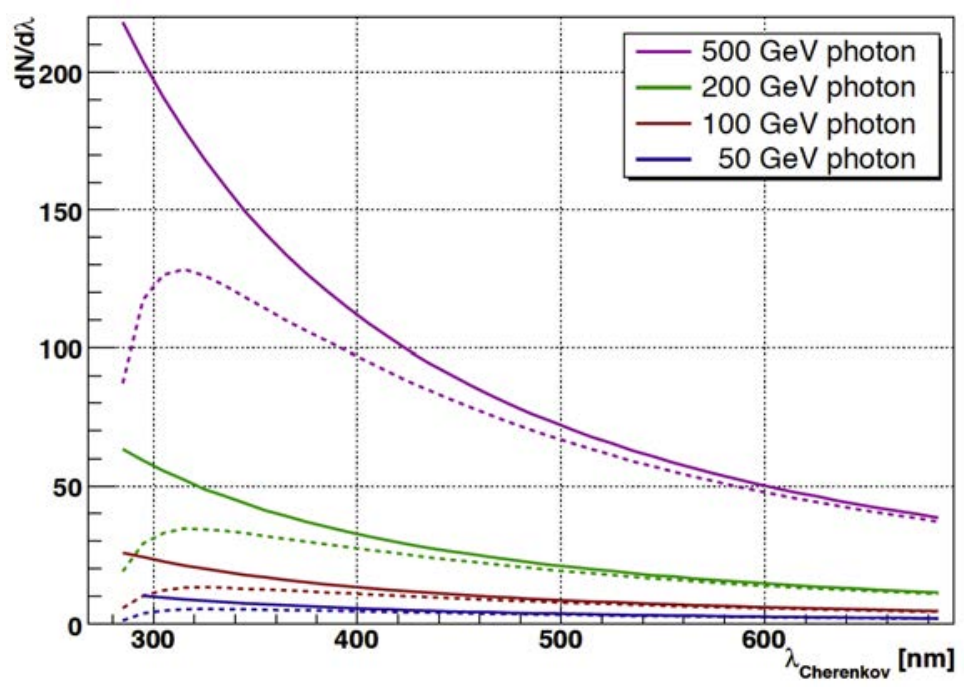

Figure 2.4: Spectra of Cherenkov radiation produced by vertical EAS initiated by gamma rays at different energies. The solid lines corresponds to the unabsorbed spectra at $10 \mathrm{~km}$ altitude, while the dashed line are the observed spectra attenuated by Rayleigh and Mie scattering (see Section 2.1).

The shape of the Cherenkov radiation around the track of the charged particle is a cone with an aperture angle $\theta$, (the so-called Cherenkov angle; see Figure 2.6), given by:

$$
\cos \theta=\frac{c^{\prime}}{v}=\frac{c}{v n(\lambda)}
$$

where $c^{\prime}=c / n$ is the speed of light in the medium and $n(\lambda)$ is the refractive index of the medium, whose value varies with the wavelength $(\lambda)$ of the Cherenkov light. The mean value of $\theta$ in air is $\sim 1^{\circ}$.

An ultrarelativistic particle propagating vertically through the atmosphere creates a doughnut ring of Cherenkov light in the ground. The contribution of all the involving particles in a EAS 

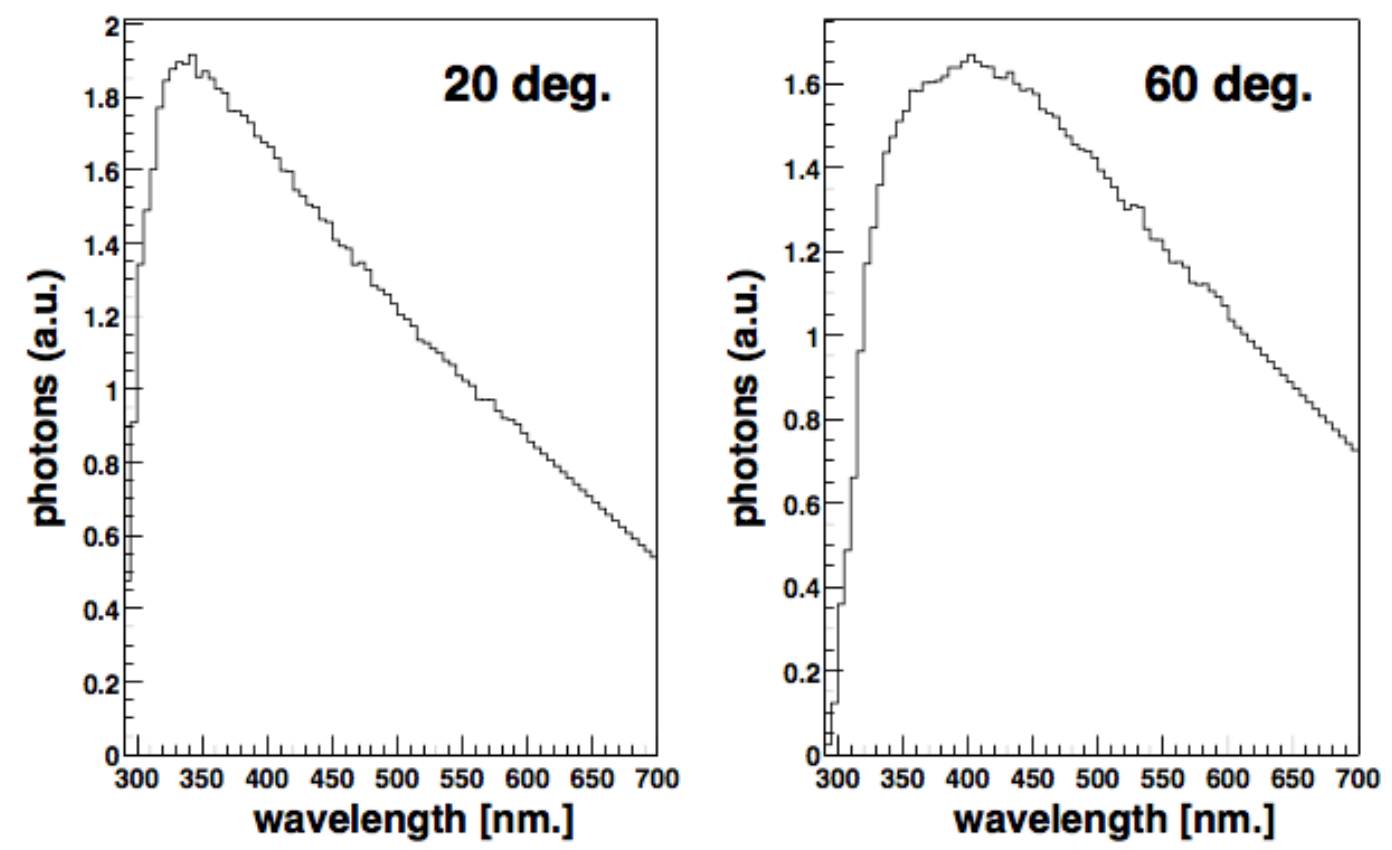

Figure 2.5: Cherenkov spectra at different zenith angles.

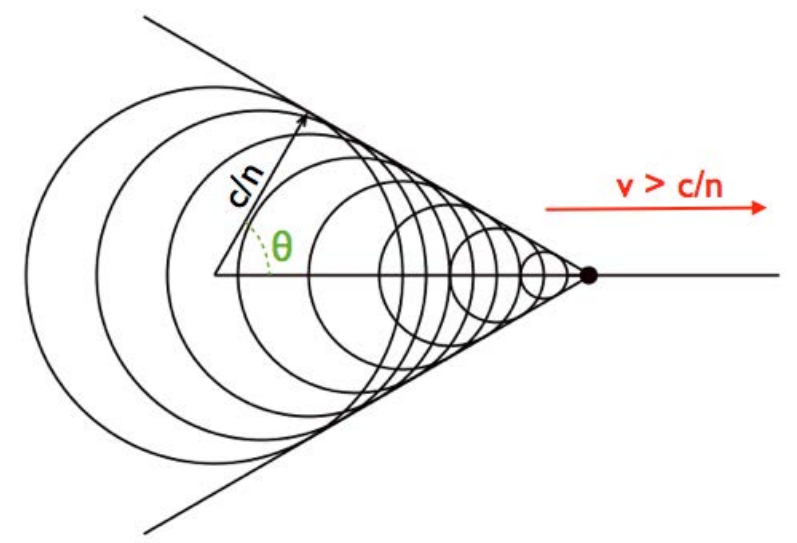

Figure 2.6: Cherenkov radiation scheme.

that emit Cherenkov radiation leads to a full circle on the ground, the so-called Cherenkov light pool (Figure 2.7). If the cascade produced in the atmosphere does not propagates vertically but with some inclination, the superposition of the Cherenkov light rings illuminates an ellipse on the ground.

In the case of a vertical EAS initiated by a gamma ray, the Cherenkov photons density is approximately uniform in a circle from the core of the cascade up to $\lesssim 120 \mathrm{~m}$. There is a slightly increase on the density around this distance, which is known as hump, whose origin arises from an increase in the opening angle, $\theta$, due to the changes in the refraction index as the 


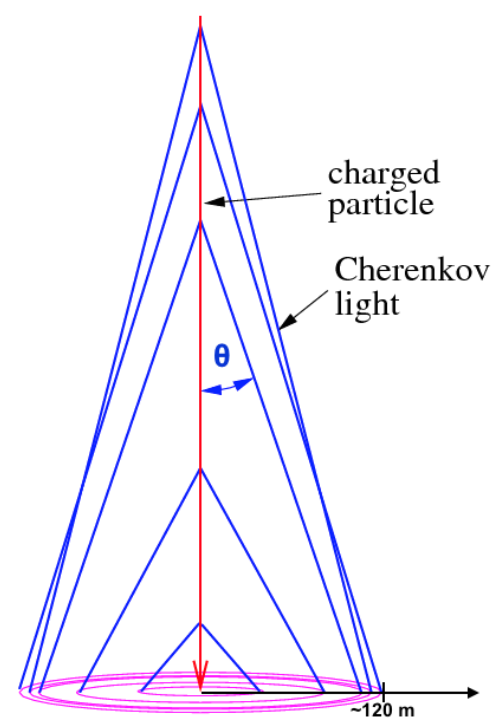

Figure 2.7: The superposition of the Cherenkov light rings produces a circle (or ellipse) in the ground, the so-called Cherenkov light pool.

particle penetrates the atmosphere. Beyond the hump, the density fades rapidly. The density of Cherenkov photons is proportional to the energy of the primary particle when this is a gamma ray, which is not true in case of different incident particle (see Figure 2.8). Therefore, this relation can be used to estimate the energy of the incident gamma ray.

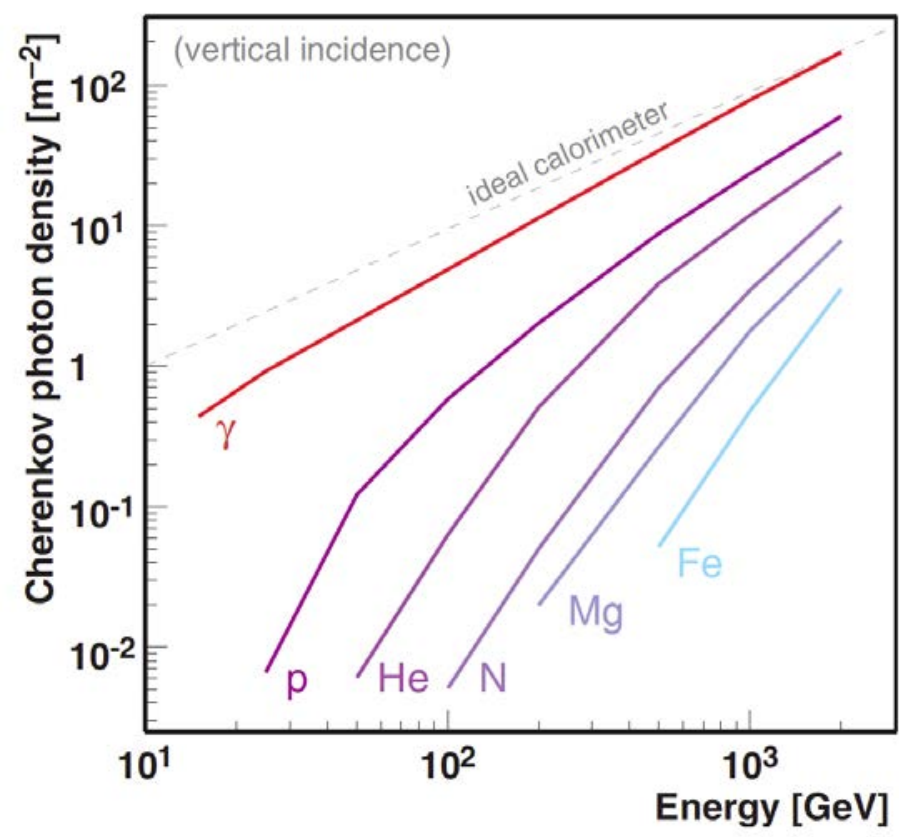

Figure 2.8: Cherenkov photon density within a radius of $125 \mathrm{~m}$ from the core shower as a function of photons energy for different primary particles. Taken from Wagner (2006). 
Although we are interesting in the EASs initiated by gamma rays, cascades induced by hadrons (mainly protons) are much more numerous. Even for strong gamma-ray sources, as it is the case of the Crab Nebula, the ratio between hadron-induced and gamma ray-induced cascades is considerably high, around 1000 hadronic cascades for each electromagnetic shower. Therefore, hadronic cascades represent the major source of background in our observations. The better we understand both types of shower, the better we can get rid of the background that embedded our observations.

\subsection{Types of EAS}

The imaging atmospheric Cherenkov technique differentiates between cascades induced by gamma rays and hadrons based on the images that leave on the telescope cameras. These images are different depending on the particle interaction with the atmospheric nuclei and its development along the air, which gives rise to showers with distinct features. In the following sections, I will give an overview of the difference between gamma ray- and hadron-induced EAS.

\subsubsection{Electromagnetic showers}

Gamma rays can initiate particle cascades through the pair creation process on air nuclei (see Chapter 1.2.2.1) if their energy is $\gtrsim 20 \mathrm{MeV}$. The electron and positron, product of this interaction, emit in turn gamma rays via bremsstrahlung (see Chapter 1.2.1.1). The latter takes place until the electron and positron reach the so-called critical energy, that in air is $E_{C}=86 \mathrm{MeV}$, below which ionization energy loss dominates. If photons emitted through bremsstrahlung present enough energy, they undergo pair creation as well, leading to a EM cascade (see Figure 2.9). The bremsstrahlung radiation length for electrons and positrons in air is $\chi_{0}^{e}=37 \mathrm{~g} \mathrm{~cm}^{-2}$ and

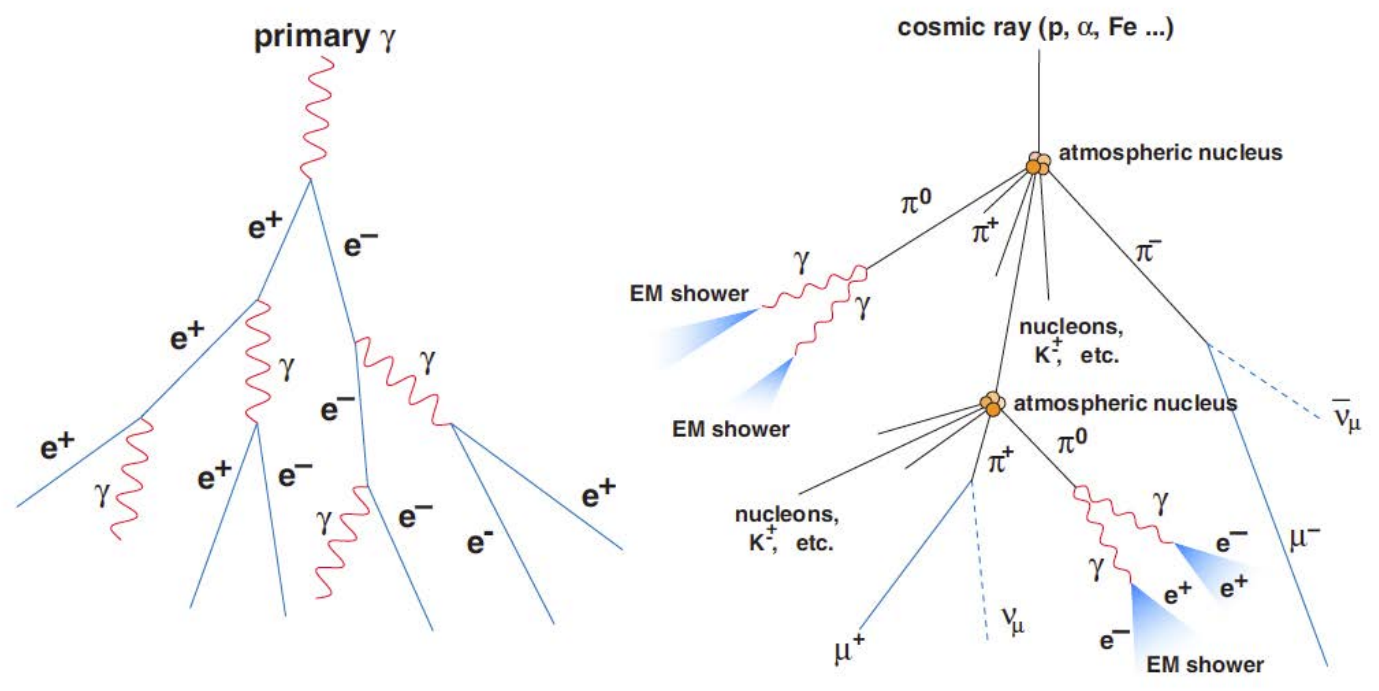

Figure 2.9: Schemes of an EM (left) and hadronic (right) showers. Credit: Wagner (2006). 
the mean free path (average distance traveled between collisions) of gamma rays due to pair creation is $\chi_{0}^{\gamma}=7 / 9 \chi_{0}^{e}$. Consequently, the particles in an EM shower do not scatter too much from the shower axis, leading to a quite symmetric cascade (see left plots on Figure 2.10). The crosssection for the interaction of gamma rays with the atmospheric nuclei is weakly dependent on the photon energy, and therefore the height of the collision of the primary gamma ray is similar for different gamma ray energies, being located at 20-30 km above sea level (a.s.l.). In each step of the shower, the number of particles it is doubled, while the particle energy decreases half, until reaching $E_{C}$, moment at which the shower is disrupted and the number of particles maximum. The altitude at which this condition is fulfilled is called height of the shower maximum and it is inversely proportional to the logarithm of the primary gamma-ray energy, $H_{\max } \propto 1 / \ln (E)$.

\subsubsection{Hadronic showers}

The hadronic cascades are those produced by the interaction between a cosmic and atmospheric nuclei. Normally, the primary particle of these interaction are protons which give rise mostly (in a $90 \%$ ) to pions (approximately in the same proportion $\pi^{+}, \pi^{-}, \pi^{0}$; see Chapter 1.2.1.3). Besides pions these collision produce kaons and nucleus (Figure 2.9p). Both hadrons and pions undergo more collisions or decays that generate the shower. The cascade stops when the energy per nucleon is less than $\sim 1 \mathrm{GeV}$, minimum energy needed for pion production. Different components can be distinguished in the hadronic showers:

$\odot$ Hadronic component: Composed by nuclei and mesons (like pions). Both of them are heavy particles and therefore, the transferred transversal momentum in each collision is high. The following pionic decays take place inside these cascades:

$$
\begin{gathered}
\pi^{+} \rightarrow \mu^{+} v_{\mu} \\
\pi^{-} \rightarrow \mu^{-} \overline{v_{\mu}} \\
\pi^{0} \rightarrow 2 \gamma
\end{gathered}
$$

$\odot$ EM component: Composed by secondary photons, electrons and positrons, mostly arriving from the decay of $\pi^{0}$. If these $\mathrm{EM}$ subcascades are detected, the distinction between them and a gamma ray-induced shower is almost impossible.

$\odot$ Muonic component: Muons and neutrinos are produced by the decay of charged pions. Neutrinos cannot be detected by Cherenkov telescopes as they cannot produce Cherenkov radiation due to the lack of charge. On the other hand, muons can be detected and also decay into:

$$
\begin{aligned}
& \mu^{+} \rightarrow e^{+} v_{e} \overline{v_{\mu}} \\
& \mu^{-} \rightarrow e^{-} \bar{v}_{e} v_{\mu}
\end{aligned}
$$

Hadronic shower are wider than the EM ones, because the transversal momentum that the kaons and pions received are higher than those in electrons and positrons. Furthermore, this type 


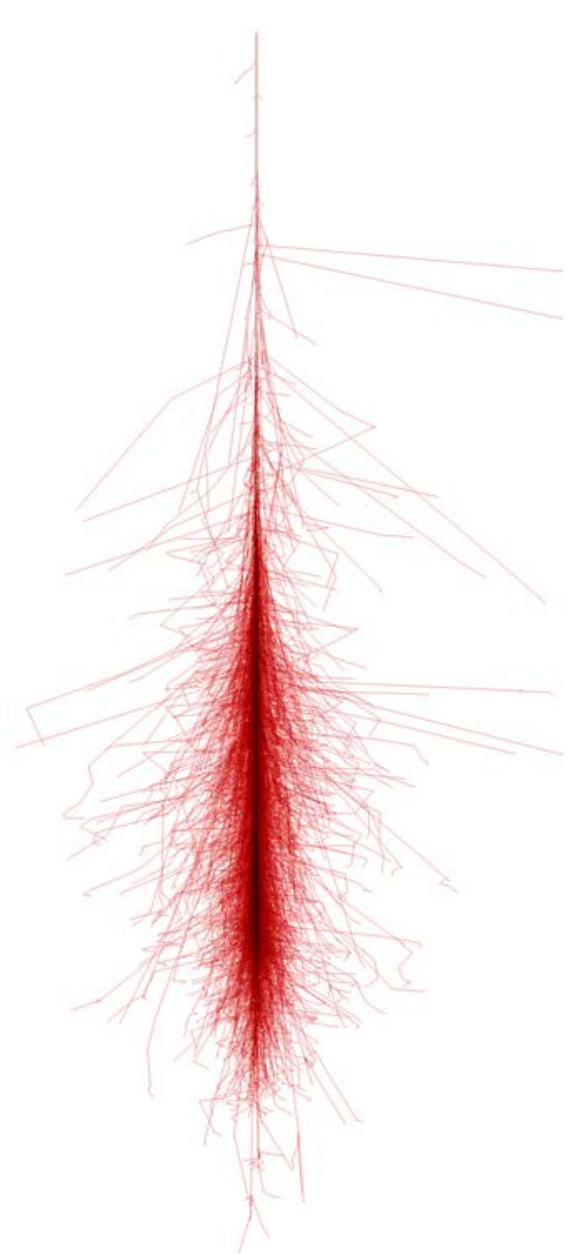

(a)

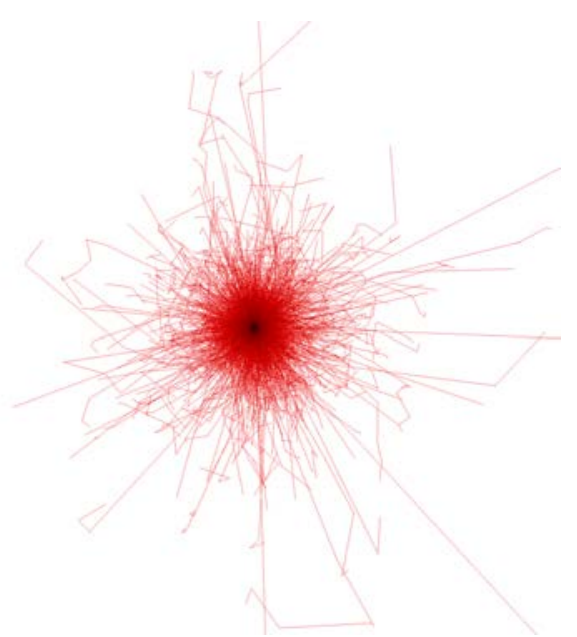

(b)

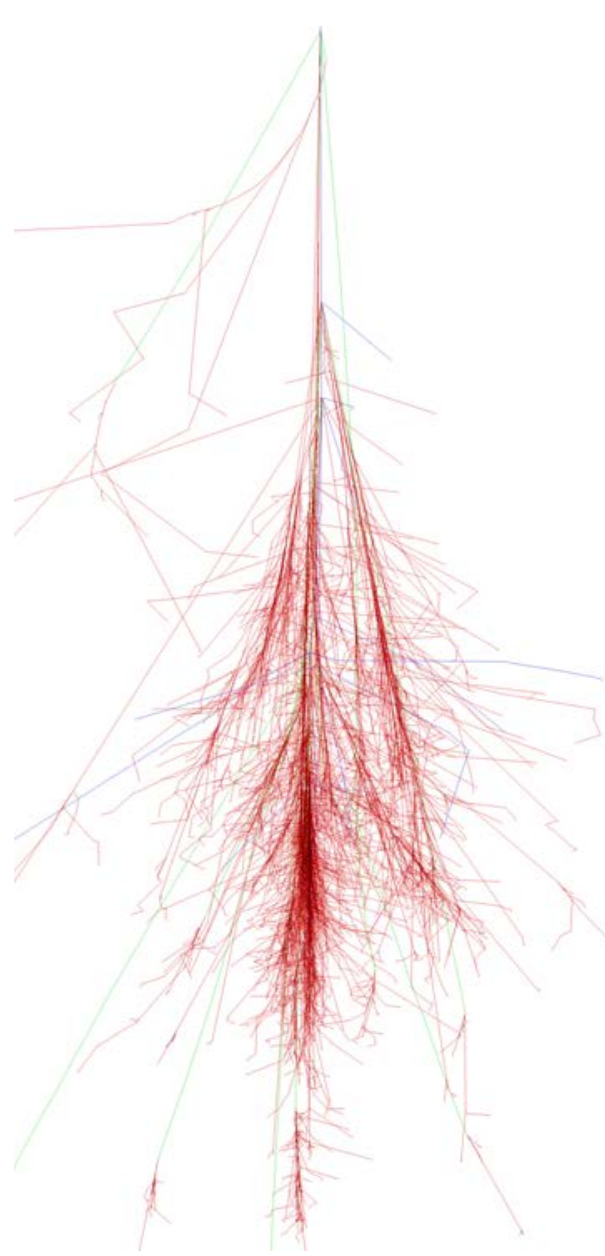

(c)

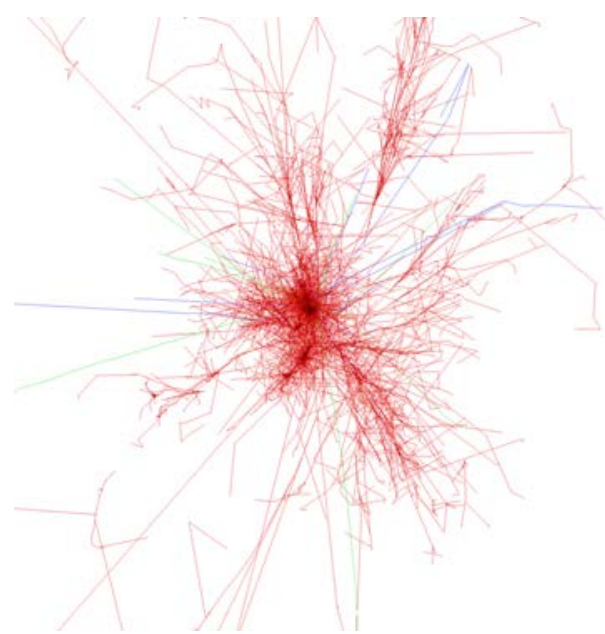

(d)

Figure 2.10: On the left: $\mathrm{MC}$ simulation of an $\mathrm{EM}$ cascade initiated by a $100 \mathrm{GeV}$ gamma ray. On the right: MC simulation of a hadronic cascade initiated by a $100 \mathrm{GeV}$ proton. Red lines show the gammaray, electron and positron tracks, green lines are used for muons and blue ones for hadrons. The upper plots represent the vertical trajectory, while the lower plots represent the transversal planes. 
of cascade undergoes more subshowers, leading to a not only wider but more asymmetric EAS, Figure 2.10 presents MC simulations of gamma ray- (left) and hadron-induced (right) cascades, where the shape difference is evident. On the other hand, EM showers develop faster than the hadronic ones. The former develops in less than $3 \mathrm{~ns}$ compared to the $10 \mathrm{~ns}$ taken in hadronic cascades. Thus, besides their image feature left on the telescopes, the timing parameter can be used to distinguish these type of showers.

\subsection{Imaging atmospheric Cherenkov technique}

As mentioned before, the imaging atmospheric Cherenkov technique bases its study of gamma rays on the indirect observations of the Cherenkov radiation produced in the EAS. In Section 2.2 it was shown the main difference between the possible EM and hadronic cascades. The several particles involved in each type lead to different shower shape and timing features that can be used to distinguish them through the Cherenkov telescopes. In Figure 2.11, we can see the way IACTs work. If the telescopes are inside the Cherenkov light pool, part of the Cherenkov light is reflected in their mirrors and collected in their fast pixelized cameras. The images created in these cameras are projections of the EASs, from which spatial and timing information is obtained. The pixels of the cameras are usually PMTs, although other detector as Silicon PhotoMultipliers (SiPMs) have provided successful results as well.

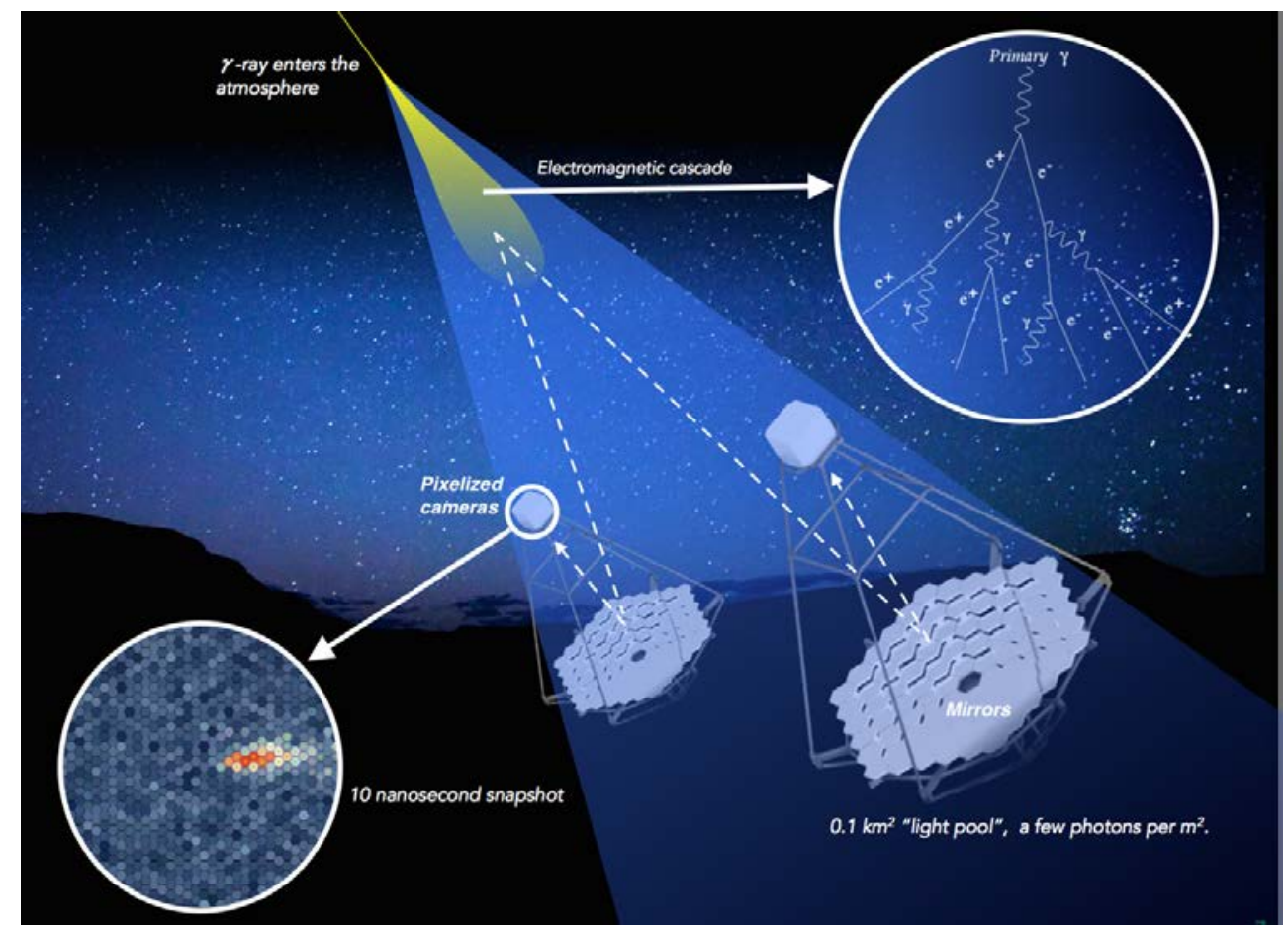

Figure 2.11: Sketch of the imaging atmospheric technique. The Cherenkov light from the cascade is reflected in the mirrors and collected in the camera. Modified image from www.cta-observatory.org. 
The PMTs are very efficient detectors which are able to collect the fast ( $\sim 3 \mathrm{~ns}$ ) Cherenkov radiation emitted in a $\mathrm{EM}$ cascade. A fast response time is also important to avoid collect undesirable photons, product of the background sources. As mentioned before, the main background sources are the hadronic showers, from which the EM subcascades act like an irreducible background for the gamma-ray observations. The other components from the hadronic EASs (see Section 2.2.2 can be discriminated through the different images produced in the camera. Actually, muons leave a canonical ring-like structure clearly differentiated from the ellipse produced in gamma ray-induced cascades (see Figure 2.12). Diffuse gamma rays from the Galactic plane constitute also an important contribution of background when observing galactic source (not that relevant for extragalactic objects). Besides the background from the EASs, photons isotropically distributed on the sky can affect the observations as well. This is the so-called Night Sky Background (NSB) that is formed by the stars' light, airglow, polar and zodiacal light and artificial lights. In La Palma, where the MAGIC telescopes are located, this NSB contribution was measured to be $(1.75 \pm 0.4) \times 10^{12}$ photons $\mathrm{m}^{-2} \mathrm{sr}^{-1} \mathrm{~s}^{-1}$.
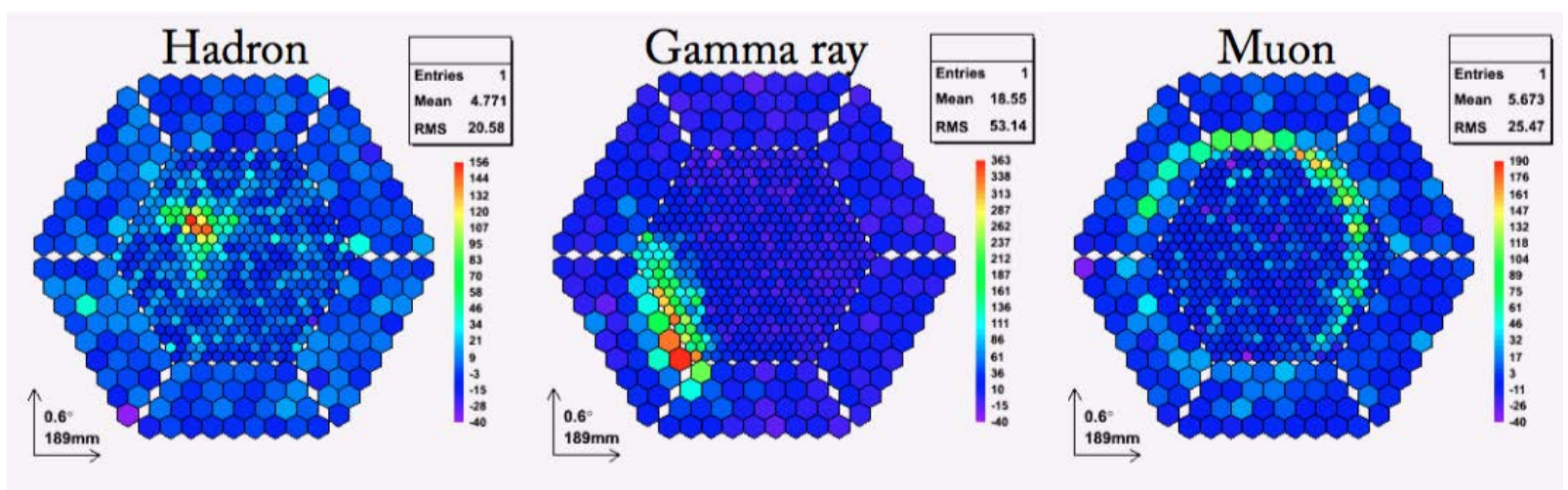

Figure 2.12: Images recorded by the MAGIC telescopes from the Cherenkov radiation induced by hadron (left), gamma ray (middle) and muon (right). Taken from www.magic.mpp.mpg.de.

\subsection{MAGIC}

The MAGIC is a stereoscopic system consisting of two $17 \mathrm{~m}$ diameter imaging atmospheric Cherenkov telescopes (IACTs) located in El Roque de los Muchachos in the Canary island of La Palma, Spain (28.8 N, 17.8 W, 2225 m a.s.1.). Until 2009, MAGIC consisted of just one standalone IACT with an integral flux sensitivity around $1.6 \%$ of the Crab Nebula flux in 50 hours of observation (Aliu et al.2009a). After autumn 2009, the second telescope (MAGIC II) started operation, allowing us to reach in stereo mode an energy threshold as low as $50 \mathrm{GeV}$ at low zenith angles (Aleksić et al. 2012b). In this period the sensitivity improved up to $0.76 \pm 0.03 \%$ of the Crab Nebula flux for energies greater than $290 \mathrm{GeV}$ in 50 hours of observations. Between summer 2011 and 2012 both telescopes underwent a major upgrade that involved the digital trigger, readout systems and the MAGIC I camera (Aleksić et al.2016a). After this upgrade, the system achieves, in stereoscopic observational mode, an integral sensitivity of $0.66 \pm 0.03 \%$ of the Crab 
Nebula flux in 50 hours above $220 \mathrm{GeV}$ (Aleksić et al. 2016b). The data analyzed in this thesis covered both mono (only MAGIC I) and stereoscopic observations and therefore, both will be described in this section.

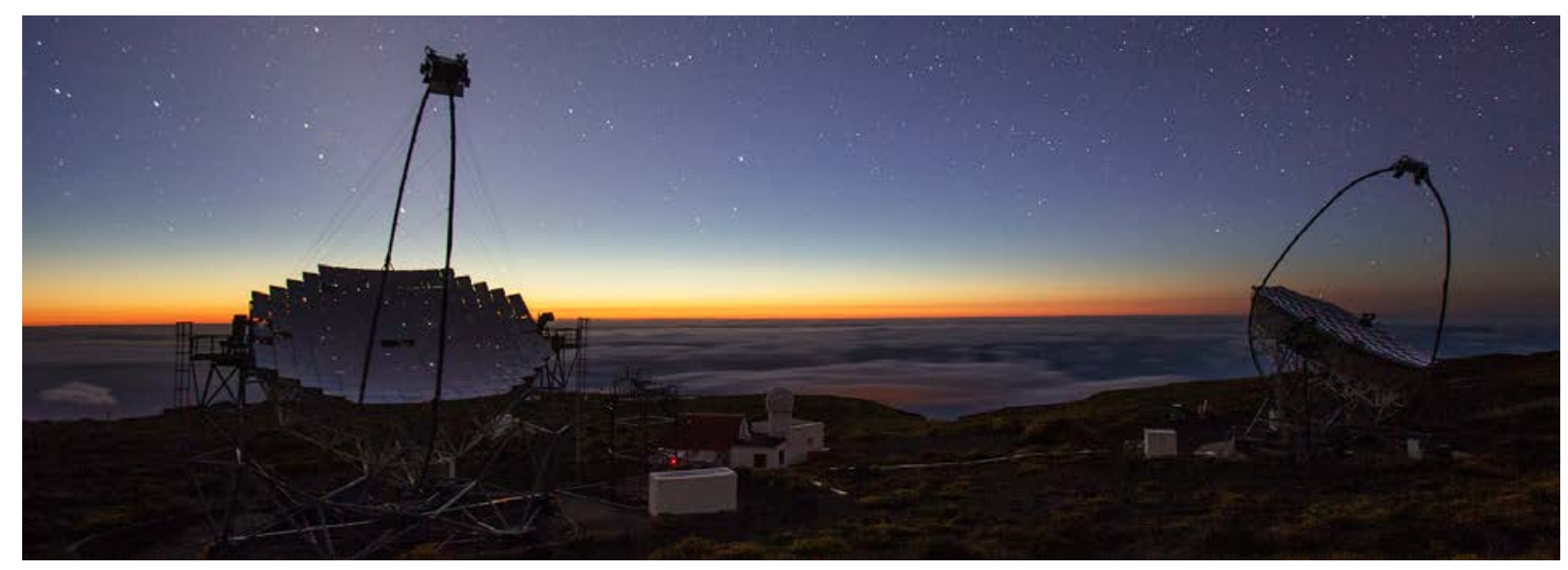

Figure 2.13: Picture of the MAGIC telescopes at El Roque de los Muchachos. Image taken from https://magic.mpp.mpg.de/.

\subsubsection{Hardware}

In this section, I will give a description of the main components of the MAGIC telescopes, depicted in Figure 2.14 and listed below:

$\odot$ Alt-azimuth frame and drive system.

$\odot$ Mirrors and reflector.

$\odot$ Camera.

$\odot$ Receivers.

$\odot$ Trigger system.

$\odot$ Readout system.

$\odot$ Other subsystems.

The Central Control (CC) software of the telescopes is called SuperArehucas, responsible for all the subsystems. It receives and sends reports, monitoring each hardware subsystem, and provides access to most of the funcionalities of the telescopes through a LabView interface. A complete description of SuperArehucas is available in Zanin (2011). 


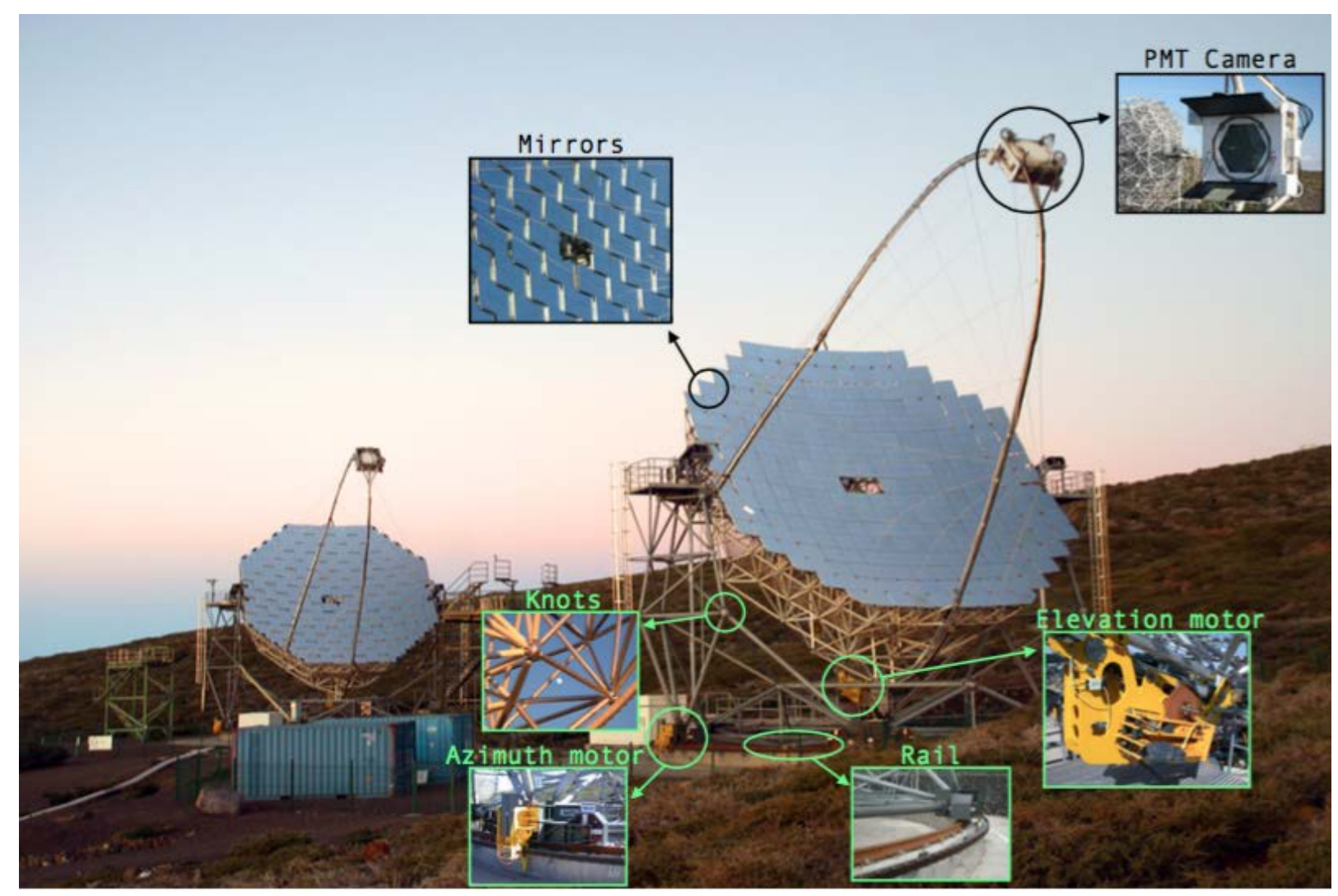

Figure 2.14: Picture of the MAGIC telescopes (MAGIC I behind, MAGIC II on the front) with some of their hardware subsystems. Image taken from https://magic.mpp.mpg.de/.

\subsubsection{Alt-azimuth frame and drive system}

The octogonal telescope structure that supports the $17 \mathrm{~m}$ reflector dish is made of light carbon fibre-epoxy tubes which hold together through aluminum knots (see Figure 2.14). The total weight of the structure, including the reflector and the camera support, is less than 20 tons. This allows a fast repositioning of the telescopes. The camera is held by an aluminum circular tube secured to the main structure by 20 steel cables. The telescopes have an alt-azimuthal mount and therefore, they are moved in two axes, Azimuth (Az) and $\mathrm{Zd}$, when pointing to a source. For any orientation of the telescopes, the deformation of the structure is lower than $3.5 \mathrm{~mm}$ (Bretz et al. 2009). Small bending of the structure during the movement of the telescopes are corrected before starting data taking through the Active Mirror Control (AMC) (see Section 2.4.1.2).

The $\mathrm{Az}$ range covers $-90^{\circ}$ to $318^{\circ}$ and the $\mathrm{Zd}$ does so between $-70^{\circ}$ to $105^{\circ}$. The $\mathrm{Az}$ movement on a $20 \mathrm{~m}$ diameter circular rail is carried out by two $11 \mathrm{~kW}$ motors. The telescopes move on the $\mathrm{Zd}$ or elevation axis thanks to one motor of the same power, located behind the dish structure (see Figure 2.14). For safety reasons, the drive system is automatically stopped to avoid the movement if the fence around the telescopes is open (i.e. somebody is inside the telescopes area).

The position at which the telescopes are pointing is measured by three 14-bit shaft encoders 


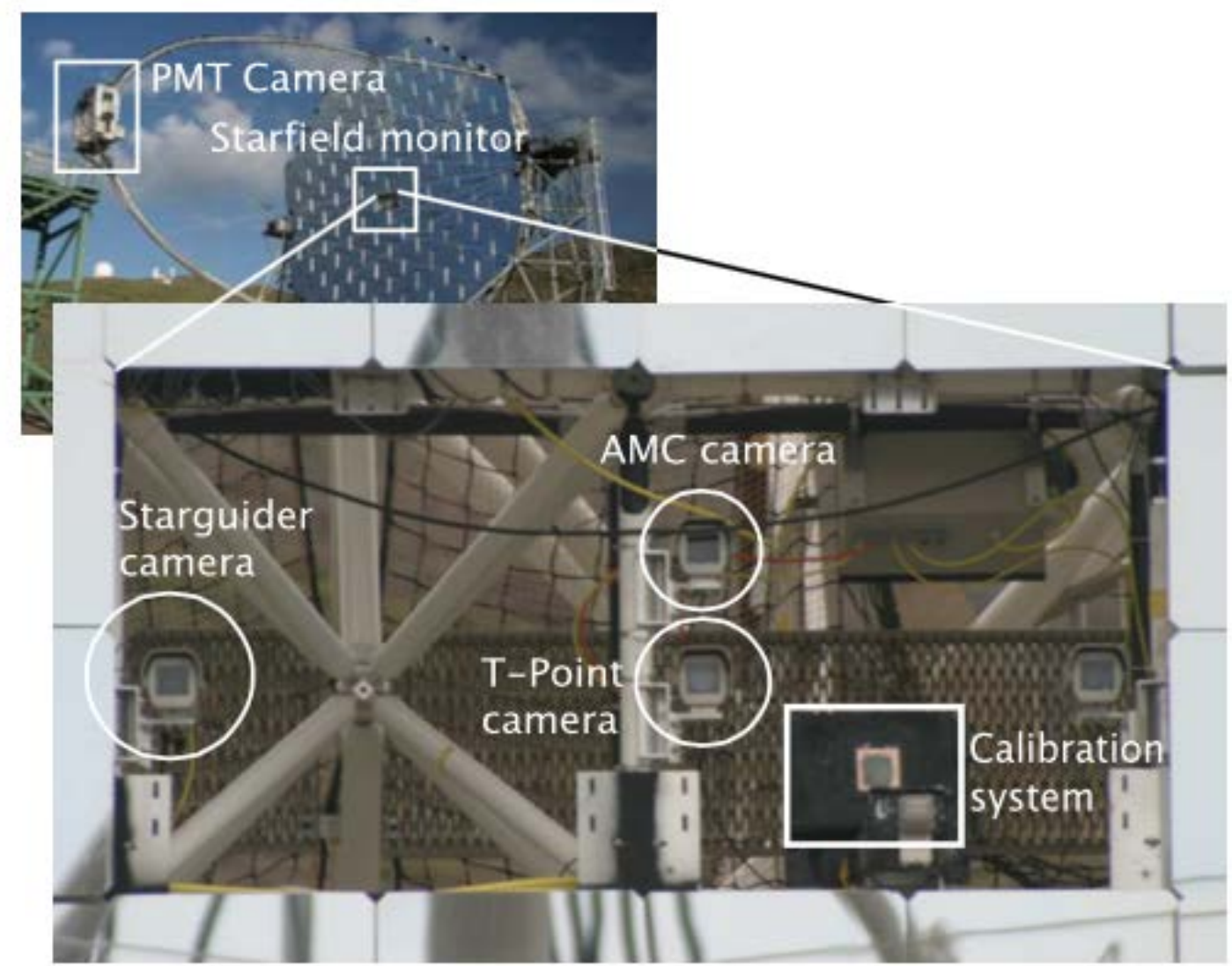

Figure 2.15: $\mathrm{CCD}$ in the middle of the reflector dish. Credit: Wagner 2006, Mazin (2007)

(two in $\mathrm{Az}$, one in $\mathrm{Zd}$. Thus, the telescope position is given with an accuracy of $\sim 0.02^{\circ}$. Along with the shaft encoders, a bending model is used to correct any mispointing (undesirable shift between the camera center and the real coordinates of the source). This bending model is created comparing cataloged and observed coordinates of $\sim 150$ bright stars taken with the so-called T-Points camera (Charge-Coupled Device (CCD) camera located in the middle of the dish, see Figure 2.15) at the end of each night ( 5 stars/night). It is updated every observational period (period between two consecutive full moon breaks). Thus, making use of this model and the shaft encoders, a pointing precision of $0.01^{\circ}$ is achieved. Moreover, the pointing of the telescopes is constantly checked during observations with the Starguider camera, another CCD camera. Located also in the dish (Figure 2.15), it points to the same direction of the telescopes and analyzes the stars in the FoV. This FoV is compared with a star catalog to find any possible offset. The reliability of the Starguider camera is measured by the ratio between the number of observed stars and the number of expected stars in a given FoV, which can be disturbed by a low sky visibility (high cloudiness or humidity). The Starguider camera observes, along with the sky, part of the PMT camera in which a set of Light-Emitting Diodes (LEDs) are installed to provide a reference frame (see Figure 2.16). 


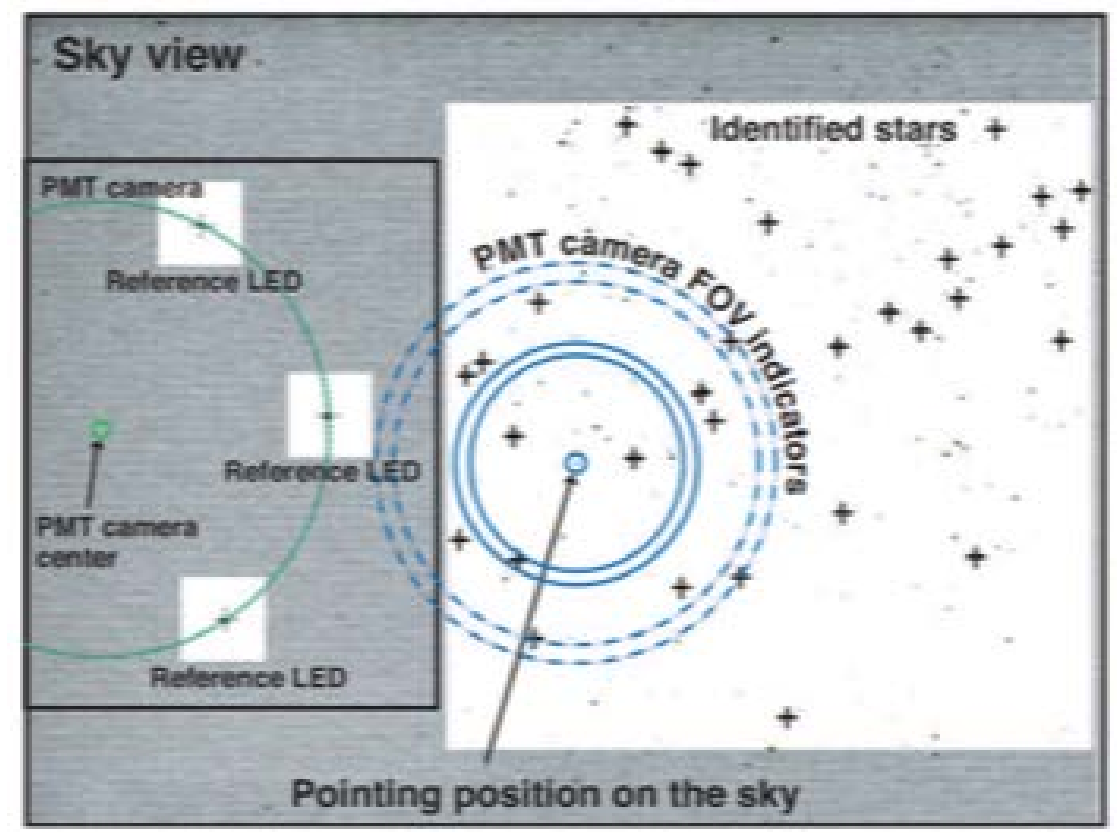

Figure 2.16: MAGIC Starguider camera. Image taken from Wagner $\sqrt{2006) .}$

\subsubsection{Mirrors and reflector}

The $17 \mathrm{~m}$ reflectors used in MAGIC are parabolic and therefore, isochronous. This is important in large size detectors, given that the arrival timing difference of the reflected light from different parts of the dish becomes also larger. The total time spread of the Cherenkov light using parabolic mirrors is $\sim 1-2 \mathrm{~ns}$. Consequently, the signal does not present a significant broadening, allowing us to apply a smaller integration signal window and hence, less background or noise is saved. The focal length of the reflector (distance at which the camera is placed) is also $17 \mathrm{~m}$. The radius of the 247 spherical individual mirrors that composed both telescopes ranges between 34 and $36.7 \mathrm{~m}$ in order to be able to form a $17 \mathrm{~m}$ parabolic reflector. In MAGIC I each mirror is in turn composed by four $0.5 \times 0.5 \mathrm{~m}^{2}$ aluminum honeycomb mirrors, whilst in MAGIC II each mirror is an unique device: 104 are $1 \times 1 \mathrm{~m}^{2}$ glass-honeycomb-glass mirrors and 143 are $1 \times 1 \mathrm{~m}^{2}$ allaluminum mirrors on a honeycomb structure. The Point Spread Function (PSF) in each mirror is defined as the diameter at which $39 \%$ of the light from a point-like source is contained. This value is $\sim 10 \mathrm{~mm}$ at the on-axis of the camera. In order to account for any structure deformation, at the beginning of each operational night images of bright stars (at different $\mathrm{Zd}$ and $\mathrm{Az}$ ) are taken with the T-points camera. Any deformation, and even non-optimal weather conditions as high cloudiness, can be measured quantitatively based on the $\overline{\mathrm{PSF}}$ of the bright stars' images (see Figure 2.17). If needed, the mirrors can be focused with the AMC system. The latter is a system composed by two actuators installed on the back of each mirror. Each actuator moves the mirrors with a precision of less than $20 \mu \mathrm{m}$, providing a very good pointing accuracy. The adjustment is made through Look-Up Tables (LUTs) binned in $\mathrm{Az}$ and Zd. 


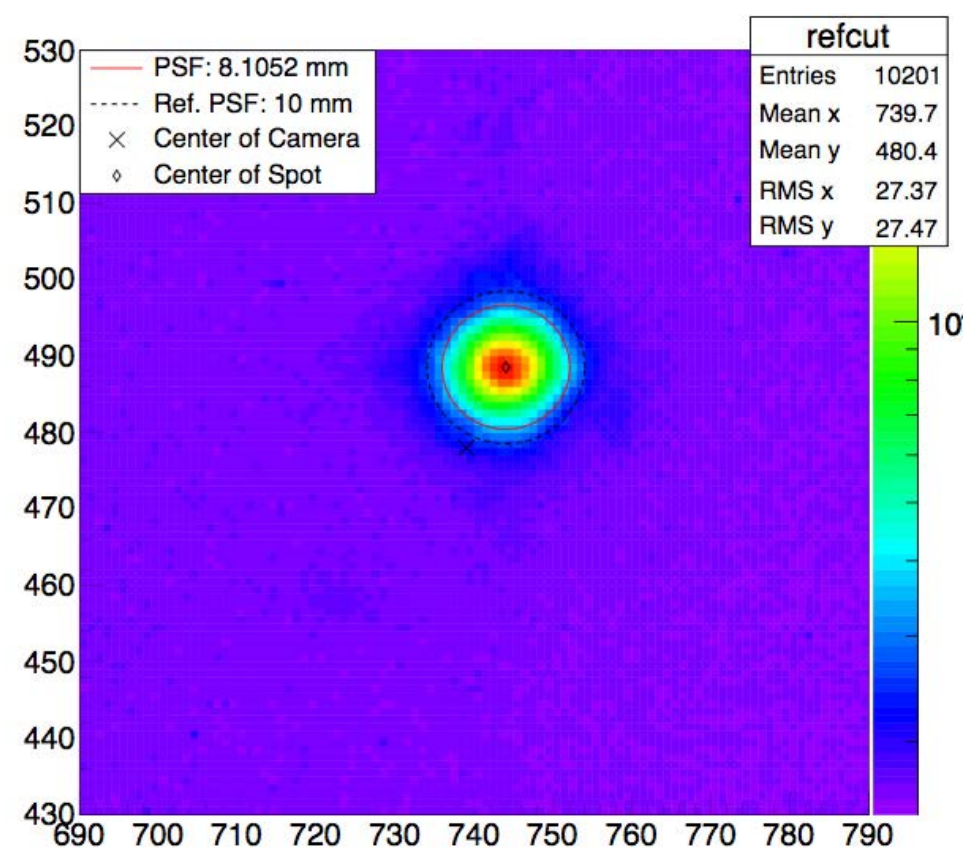

Figure 2.17: $\mathrm{PSF}$ of the bright star Betelgeuse taken at the beginning of the night with MAGIC I in order to check any possible mispointing. The obtained $\mathrm{PSF}(8.1 \mathrm{~mm})$ is inside the reference $(10 \mathrm{~mm})$ and therefore no correction is needed.

\subsubsection{Pre- and post-upgrade camera}

The PMT cameras of the telescopes play a key role in the overall instrument, being responsible for example of the achieved sensitivity. As discussed at the beginning of this chapter, during summers of 2011 and 2012, besides upgrades on the trigger and readout systems that affected both telescopes, the MAGIC I camera suffered a major upgrade to mimic the MAGIC II camera. In this thesis, I analyzed data taken in stand-alone mode (only with MAGIC I, before 2009), in stereoscopic mode before the upgrade period and also post-upgrade data. Therefore, the former MAGIC I camera and the current one, clone of the MAGIC II camera, are described in this section (see Table 2.1).

MAGIC I camera (pre-upgrade) Before 2011, the MAGIC I camera presented an hexagonal shape, covering a FoV of $3.6^{\circ}$ (see Figure 2.18). It was composed by 576 PMTs 396 inner PMTs, located within a $1.2^{\circ}$ radius from the center of the camera, and 180 PMTs in the outer part of the camera. The former, with a diameter of $1 \mathrm{inch}$, covered $0.1^{\circ}$ FoV each, while the outer PMTs covered $0.2^{\circ}$ FoV, The trigger region corresponded to the inner zone covered only by 1 inch PMTs. These two different types of PMTs aim to achieve a good compromise between the cost and performance.

The PMT photocathodes were covered with a diffuse lacquer coating doped with P-Terphenyl which shifted the wavelength of the photons at the lowest regime of the UV]band into the ranges in which the sensitivity was larger. Thus, a 28\% Quantum Efficiency (QE) PMT was achieved. 

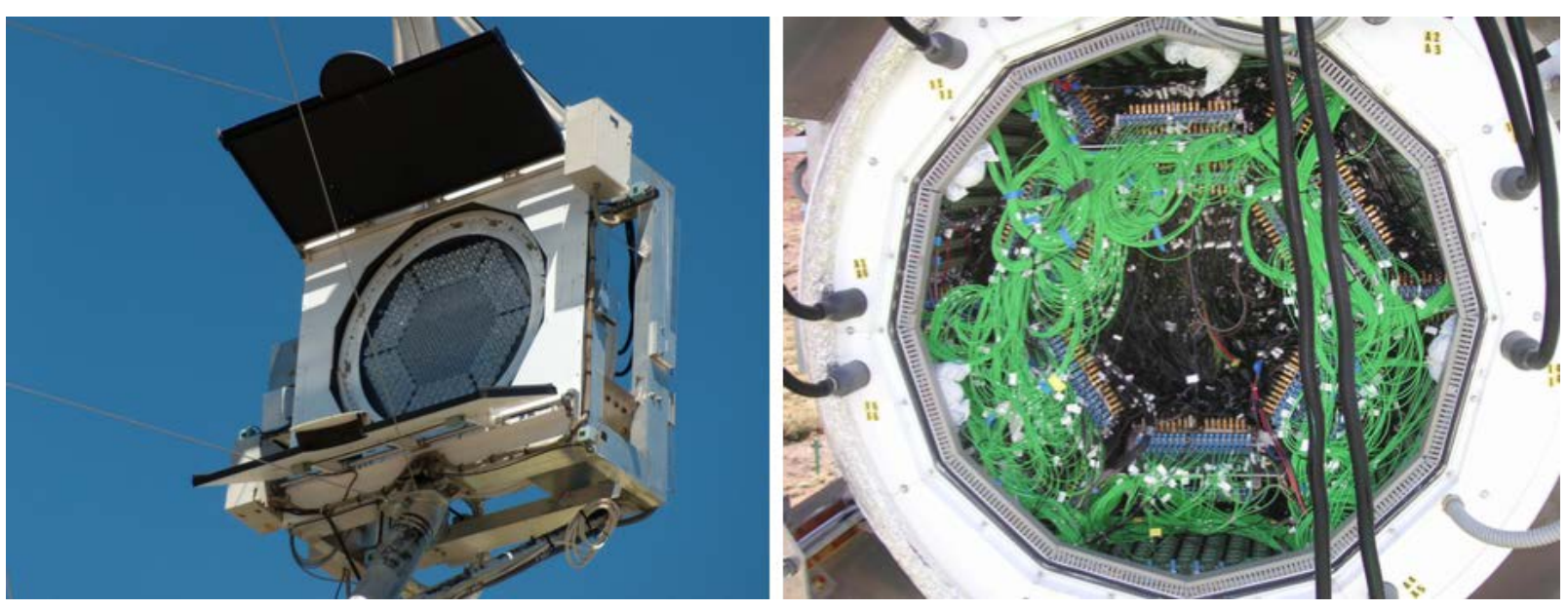

Figure 2.18: Front (left) and back side (right) of the pre-upgrade MAGIC I camera.

Aluminum tubes (modified version of Winton cones) were used as light concentrators to avoid losing photons in the spaces between PMTs, increasing this way the light collection efficiency. On the other hand, light collectors prevent photons coming from large angles to reach the detector, avoiding part of the $\mathrm{NSB}$ radiation and emission reflected by the ground. Light arriving from an incident angle larger than $40^{\circ}$ with respect to the $\mathrm{PMT}$ was not collected. The total $\mathrm{QE}$ of the camera, accounting for PMTs, mirrors and light collectors was $\sim 15 \%$ in a wavelength range between 300-600 $\mathrm{nm}$. Equal to the current design, the PMTs used in the former MAGIC I camera had 6 dynodes and worked at a low gain to be able to observe during moonlight conditions without damaging the detector or accelerating its aging. The gain ranged between $(2-4) \times 10^{4}$. Due to this low gain requirement, the signal was amplified after the PMT by a low-noise pre-amplifier. The High Voltage (HV) applied to each PMT was controlled by a 12-bit Analog-to-Digital Converter (ADC), The communication between the hardware (including $\mathrm{HV}$ monitoring, power and cooling system of the camera) and the Counting House (CH), where signal is digitized, was performed through optical fibers.

MAGIC I and MAGIC II camera (post-upgrade) The current MAGIC I camera, upgraded between 2011-2012, was a clone of the MAGIC II camera installed in 2009 (Figure 2.19). Both present circular shape with $\sim 1.2 \mathrm{~m}$ diameter and a FoV of 3.5. They are composed of 1039 PMTs uniformly distributed with a $0.1^{\circ}$ FoV each one. The PMTs are grouped in 169 clusters of 7 pixels (PMTs), of which 127 clusters are completed whilst 43 are only partially equipped (outer clusters). The trigger region is $5 \%$ larger than the previous MAGIC I camera with $2.5^{\circ}$ diameter. The PMTs are Hamamatsu R10408 with also 6-dynode system, whose QE is around $32-34 \%$ at $350 \mathrm{~nm}$ (Nakajima et al. 2013). A slow control processor installed in each PMT cluster is responsible for both setting and monitoring the $\mathrm{HV}$ and Direct Current (DC) of each PMT. The $\mathrm{HV}$ at $\sim 1.25 \mathrm{kV}$ is produced by a Cockroft-Walton DC-DC converter, leading to a gain of $\sim 3 \times 10^{4}$. In the same way as for the former camera, the low gain of the PMTs is then compensated by a low-noise pre-amplifier. A pulse generator is also installed in the clusters to inject signal directly to the pre-amplifier for testing purposes. After the pre-amplification, elec- 
trical signal is convert into optical by Vertical Cavity Surface Emitting Lasers (VCSELs) to be able to transmit the information through $162 \mathrm{~m}$ long optical fibers to the $\overline{\mathrm{CH}}$. The use of optical fibers minimizes the dispersion during transmission and avoid electromagnetic pickup and most of the attenuation. Post-upgraded cameras present a modular distribution that allows to extract groups of PMTs and makes the access or replacement of them easier. Pre-upgraded camera did not have this modular design.

The cameras have a plexiglass window installed in front of the light collectors to protect it from the environment conditions. There are also movable lids that prevents damage on the camera due to strong light (as Sun light) and external agents. The pre-upgraded camera was also equipped with both the plexiglass panel and lids.
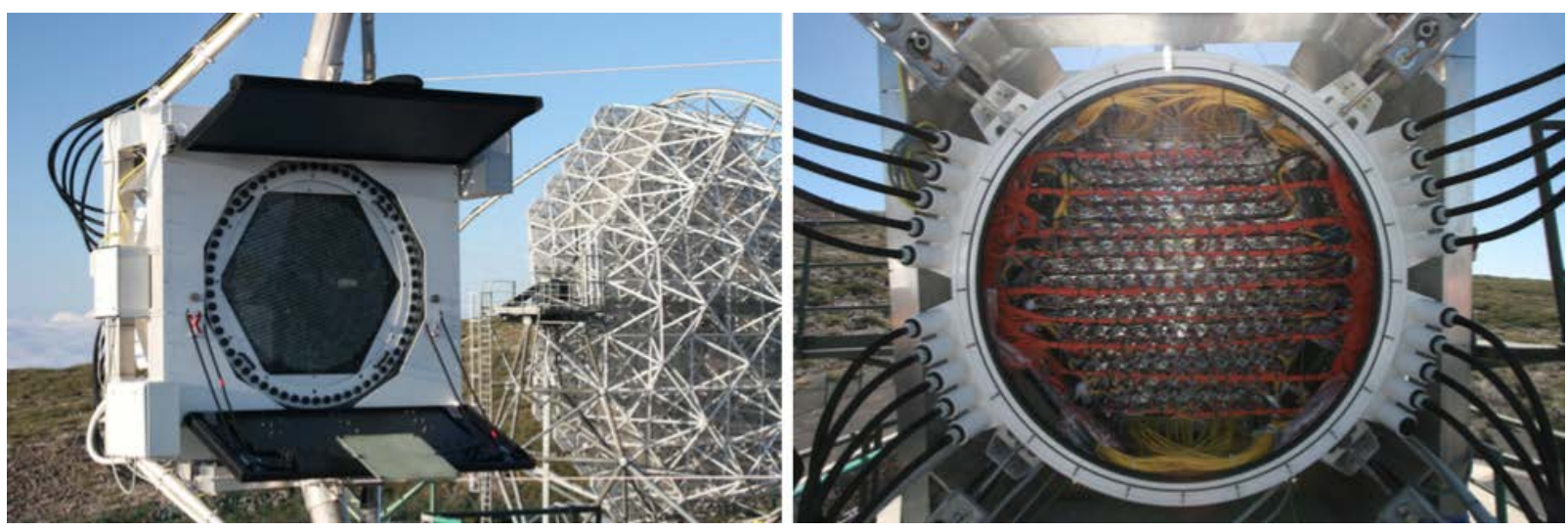

Figure 2.19: Front (left) and back side (right) of the MAGIC II camera. The current MAGIC I camera is a clone of this.

\begin{tabular}{|l|c|c|}
\cline { 2 - 3 } \multicolumn{1}{c|}{} & \multicolumn{2}{c|}{ MAGIC I camera } \\
\cline { 2 - 3 } \multicolumn{1}{c|}{} & Pre-upgrade & Post-upgrade \\
\hline Shape & hexagonal & circular \\
Total FoV & $3.6^{\circ}$ & $3.5^{\circ}$ \\
Back design & Non modular & Modular \\
Number of PMTs & 576 & 1039 \\
\hline PMT FoV & $0.1^{\circ}-0.2^{\circ}$ & $0.1^{\circ}$ \\
\hline PMT $\mathbf{Q E}$ & $28 \%$ & $32 \%$ \\
\hline Trigger region & $2.4^{\circ} \oslash$ & $2.5^{\circ} \oslash$ \\
Light concentrators & Winston cones & Winston cones \\
\hline
\end{tabular}

Table 2.1: Comparison pre- and post-upgrade MAGIC I camera. The MAGIC II camera is identical to the post-upgrade MAGIC I and therefore, the listed features also apply to MAGIC II. 


\subsubsection{Calibration system}

The calibration system has three goals:

$\odot$ Adjust the $\mathrm{HV}$ of all $\mathrm{PMTs}$ in order to obtain an uniform gain in the camera. This is important to cover a broad uniform dynamic range, which is defined as the difference between the highest and lowest signal intensity that the telescopes can detect.

$\odot$ Obtain the conversion factor, $\mathrm{C}$, between the signal amplitude and the corresponding number of photoelectron (phe).

$\odot$ Convert ADC timing into absolute timing.

To perform these tasks the current calibration system consists on a Nd-YAG laser of $355 \mathrm{~nm}$ and $0.7 \mathrm{~ns}$ pulse width, while the former MAGIC I configuration was composed of a box with 64 LEDs emitting at three wavelength of $370 \mathrm{~nm}(\overline{\mathrm{UV}}), 460 \mathrm{~nm}$ (blue) and $520 \mathrm{~nm}$ (green). This system is equipped with two rotating filters that attenuate the laser light and provides a dynamic range betwen 1 and 1000 phe. The system is located in the middle of the reflector (see Figure 2.15). To distribute the light on the camera uniformly, the laser beam is diffused by an integrating (Ulbricht) sphere.

To achieve the first goal (adjusting the $\mathrm{HV}$ ) a special flat-fielding procedure is applied. An uniformly distributed signal from the laser is used to illuminate the entire camera and the $\mathrm{HV}$ of individual pixels is corrected manually until all of them present the same output of integrated signal. This procedure is rarely needed, normally once every few months. The calibration constant to convert ADC counts into the physical quantity of phe (second goal) is obtained through the F-factor method (Mirzoyan 1997; see Appendix B for more information).

\subsubsection{Receivers}

After leaving the camera (through optical fibers), the signal arrives to receiver boars placed in the $\overline{\mathrm{CH}}$, where it is converted to electric signal again via photodiodes. From these receivers the signal is divided and sent to two subsystems simultaneously: the trigger and the readout systems.

\subsubsection{Trigger system}

The function of the trigger system consists on discriminate gamma ray-induced cascades from the hadronic ones and the NSB. The total system is comprised of several steps. The following description of these steps is valid for both old and current MAGIC cameras working in stereoscopic mode. The main difference between the pre- and post- upgraded cameras is the already mentioned 5\% increased in the trigger region in the newest device. Some discrepancies appear for mono observations, i.e. when MAGIC I operated in stand-alone mode (before 2009). Differences on that trigger system with respect to the one used nowadays are highlighted in the following when applies.

$\odot$ Level 0 (L0) trigger: Located already in the receiver boards, this trigger releases a squared signal every time the analog signal from an individual PMT overpasses a certain amplitude 


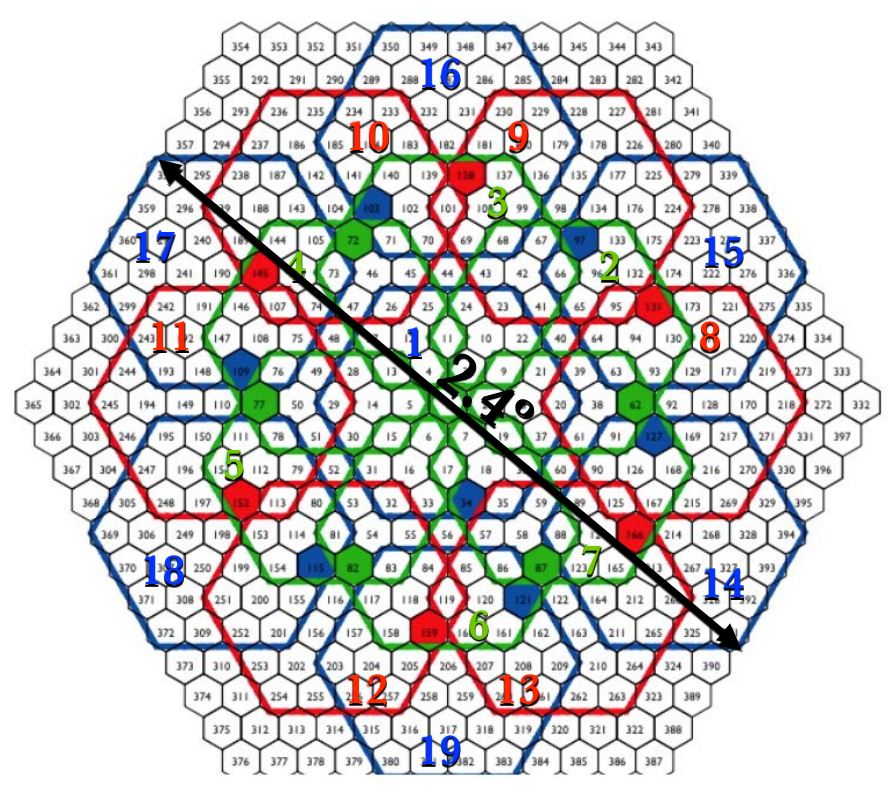

Figure 2.20: Hexagonal L1 macrocells in the former MAGIC I camera version, each of which contains 37 PMTs. The numbers on the macrocells are the internal MAGIC identification. The trigger FoV is $2.4^{\circ}$ diameter. Modified plot from Zanin (2011).

threshold, the so-called Discriminator Threshold (DT), The level of this DT depends on the moonlight, being more relax during dark (no moonlight) observations and more conservative as higher the strength of the Moon is (see Section 2.4.2). A dedicated channel rate counter allows to obtain the individual pixel rate on-line (during data taking). According to this, the DT values are slightly modified automatically to keep a stable rate during variable light conditions.

$\odot$ Level 1 (L1) trigger: The L1 trigger works over 19 overlapping hexagonal cells, the socalled macrocells, composed of 37 PMTs, one of which is blind (see Figures 2.20 and 2.21). Its inputs are the digitized signals given by the L0 trigger from each pixel. Thus, L1 is used to find spatial and timing coincidence between closer pixels. If a number $n$ of neighboring pixels in any macrocell, defined in MAGIC as $n$ Next Neighbour (NN), contains a signal above the DT (i.e. the L0 issued the squared signal), the L1 trigger releases a signal. The possible $n \mid \mathrm{NN}$ are $\mathrm{n}=2,3,4$ and 5, although during mono observation $\mathrm{n}=4$ was usual whilst currently in stereoscopic mode applies $3 \mathrm{NN}$. Therefore, to accept the trigger in a certain macrocell, a pixel that exceeds the $\mathrm{DT}$ must be in contact with at least other two PMTs (three in stand-alone mode observations) which also overpass the amplitude threshold. The L1 signal from each macrocell that fulfills the $3 \mathrm{NN}$ criterion are processed by a Trigger Processing Unit (TPU), This is the last trigger step for mono observations.

$\odot$ Level 3 (L3) trigger: This trigger level only applies for stereo observations. In that case, 
the $\mathrm{L} 3$ receives the output of the TPU, one signal from each telescope that triggered. If only one telescope triggered an event, it is discarded. The signals are artificially stretched to $100 \mathrm{~ns}$ and delayed accounting by the $\mathrm{Az}$ and $\mathrm{Zd}$ of the observations to take into account the different arrival times of the cascade in each telescope. The width of $100 \mathrm{~ns}$ is used to avoid loose events due to some time misalignment of the telescopes. If L1 signals from MAGIC I and MAGIC II are spaced less than $180 \mathrm{ns,} \mathrm{then} \mathrm{the} \mathrm{event} \mathrm{is} \mathrm{accepted} \mathrm{and} \mathrm{the}$ readout starts.

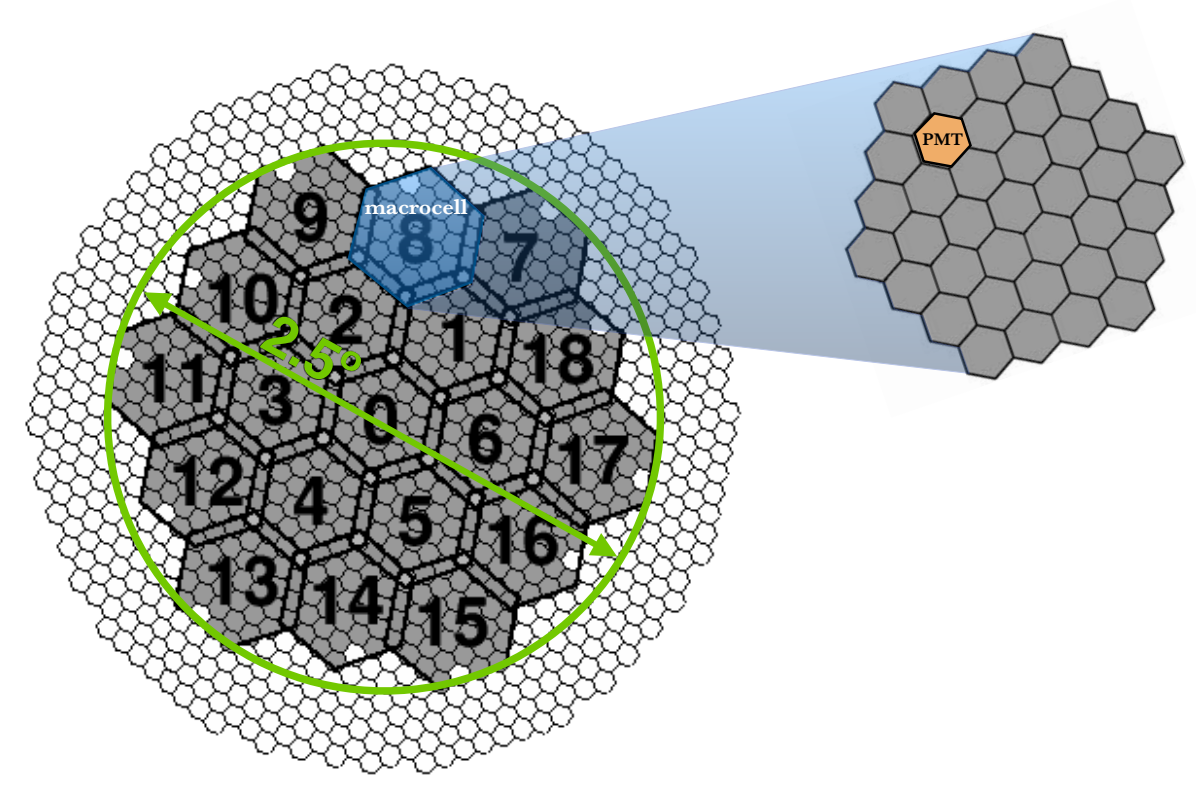

Figure 2.21: Hexagonal L1 macrocells in the current MAGIC camera version, each of which contains 37 PMTs (one blind). The numbers on the macrocells are the internal MAGIC identification. The hexagonal shape of the PMTs is given by the Winston cones. The trigger FoV is $2.5^{\circ}$ diameter. Modified plot from López-Coto (2015).

Different trigger approaches have been tested in MAGIC in order to detect gamma rays at very low energy, below $50 \mathrm{GeV}$. To do so, the trigger procedure needs to be able to discriminate gamma ray-induced events from those produced by $\mathrm{NSB}$, which at the lowest energies are similar. Two trigger systems were and are currently being checked: Sum trigger (García et al. 2014) and Topo trigger (López-Coto et al. 2016). The former was already designed for using in stand-alone mode with MAGIC I (Rissi et al. 2009) with which pulsed emission from the Crab pulsar was detected above $25 \mathrm{GeV}$ (Aliu et al. 2008b). In this case, the L1 level accepts trigger if the sum of the signal of each pixel contained in a macrocell reaches or excesses a certain threshold. The macrocells in the Sum trigger are reduced to be formed by 19 PMTs, In order to avoid contamination and fake triggers by After Pulses (APs) (spurious signal coming from 
the scattering of electrons in the dynode system or ionization of the gas inside the (PMT), before summing, all signals above a determined amplitude are "clipped". Once the signal of the macrocell is summed, it passes through a DT, currently set to several tens phe. On the other hand, the goal of the Topo trigger is as well to reduce the energy threshold. To do so it makes use of both spatial and timing information of the signals at L1 level. Due to geometrical development of the camera, events in both telescopes are expected to trigger the same macrocells. Given that $\mathrm{NSB}$ events are randomly distributed, they are only expected to trigger one macrocell, instead of several like the gamma-ray events. The Topo Trigger sends a veto to the L3 trigger if the L1 trigger macrocell criterion is not fulfilled. With this trigger, $85 \%$ of the accidental stereo trigger are rejected without decreasing the L0DT, which helps to differentiate lower energy cascades.

\subsubsection{Former and current readout systems}

As mentioned in Section 2.4.1.5, one of the two signal branches goes to the readout system, responsible for the $\mathrm{DAQ}$. This signal is delayed a few ns in order to wait for the trigger system response, which will determined if an event is produced by a gamma ray and hence, has to be recorder by DAQ. The DAQ, consisting of a Flash Analog-to-Digital Converter (FADC) in the older versions and a ADC in the current ones, digitizes the incoming electrical signal and saves it in raw files. The former MAGIC I and the current MAGIC I and MAGIC II telescopes present very different readout systems, which have been updated along the years (see Figure 2.22). In total, MAGIC has used four different readout electronics. Data from all, except the first readout system (Siegen), was analyzed for this thesis. I will give a description of all the readouts used in MAGIC

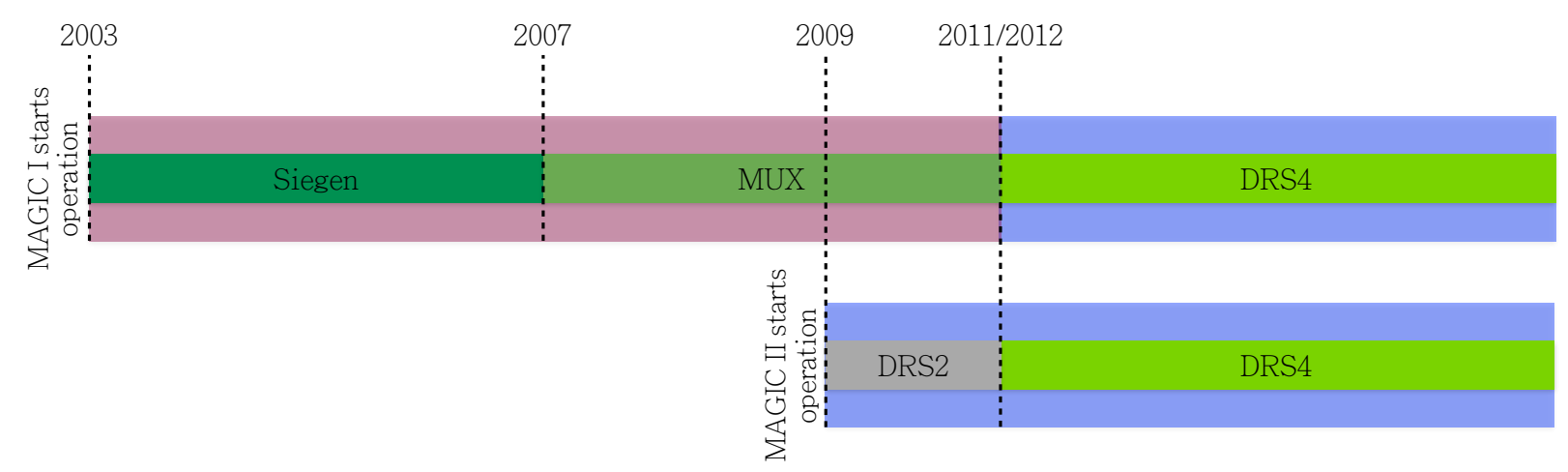

Figure 2.22: Diagram of MAGIC readout systems and cameras along the years. The pink box represents the period in which the old camera was used, while the blue box corresponds to the new and currently used camera. The different readout systems periods are labeled and depicted in different colors.

Former MAGIC readout From the beginning of operation (in 2003) until February 2007, the MAGIC readout system (Siegen) sampled at a frequency of $300 \mathrm{MSample} / \mathrm{s}$ (i.e. $3 \times 10^{8}$ samples per second). Once the signal arrived to the readout, it was split again into two branches. 
In one of them, the signal is amplified by a factor of ten. This is the so-called high-gain path. The other branch, known as low-gain, kept its original amplitude but it is forced to wait $\sim 55$ ns. Normally, high-gain signal is digitized, but if it saturates, then the delayed low-gain signal is digitized. This division into high- and low-gain signal was and is currently still used in the succeeding readout systems that followed Siegen. In the case of the Siegen electronics, before the digitalization (independently from the branch the signal arrived from) the signal was stretched up to 6-7 ns (see Figure 2.23). This was motivated by the fact that FADC were too slow to handle fast $\sim 2$ ns events. While waiting for the trigger decision, the signal was sent to a long ring buffer, in which it was sampled at $300 \mathrm{MSample/s.} \mathrm{If} \mathrm{the} \mathrm{trigger} \mathrm{is} \mathrm{positive,} \mathrm{part} \mathrm{of} \mathrm{the} \mathrm{signal}$ (30 samples or slides) per event and per PMT were recorded in the disk. The deadtime (period in which the system is saving an event and therefore it cannot keep reading further incoming signal) with this configuration was $25 \mu \mathrm{m}$.

In February 2007, the $300 \mathrm{MHz} 8$-bit FADC were updated to a 2 GSample/s one $\left(2 \times 10^{9}\right.$ samples per second). With a faster readout, the integration time was reduced and so did the NSB influence. It used a fiber-optic multiplexing technique, MUX. Multiplexing was possible because the low duration of the signals (around a few ns) and the typical trigger rate of $1 \mathrm{kHz}$ resulted in low duty-cycle for the digitizer. To reduce cost and consumption, instead of using one FADC per channel (i.e. per PMT) as in Siegen, in the MUX readout each FADC could digitize 16 channels. This new readout system led to an improvement in sensitivity of the $\sim 40 \%$. The deadtime remained to be $25 \mu \mathrm{m}$.

MAGIC II started operation in 2009 with a readout system based on a Domino Ring Sampler version 2 (DRS2) analog memory chip (while MAGIC I remained using MUX). These analog memories worked as follow: the signal coming from the receiver boards enters in a 1024 capacitor array. While the signal passes through a capacitor, the device is charged during a certain time. The velocity sample is 2 GSample/s. The switch to the next capacitor is controlled by fast switches synchronized to an external clock. Therefore, the charge in a capacitor is proportional to the time period of the clock (which is known as Domino wave). Once all the capacitors are charged, the system starts to overwrite them. With a sample frequency of 2 GSample/s and a total of 1024 capacitors, the buffer longitude in DRS2 was 512 ns. For the DRS2 readout, if the trigger system accepted an event, the readout stopped the Domino wave and the charge of all the 1024 capacitors were digitized. From these 1024 capacitors, only a small fraction of them were saved by DAQ, the so-called Region of Interest (RoI). The Rol in DRS2 was 80 capacitors. Although only 80 capacitors are recorded, the system needed to read all the buffer, which led to a considerably high deadtime of $500 \mu \mathrm{s}$. The reader is referred to Bitossi (2009) for more information on the DRS2,

Current MAGIC readout During the major upgrade in 2011, both MAGIC I and MAGIC II readout were substituted by Domino Ring Sampler version 4 (DRS4) analog memories (Sitarek et al. 2013a). Besides uniforming the readout system in both telescopes, the DRS4 allows us to reduce the high deadtime. Its functionality is the same as described for DRS2. Nevertheless, instead of digitizing the 1024 capacitors after triggering, the DRS4 only digitizes and records the selected Rol. The Rol is determined attending to two points: first, if the Rol is too large, 


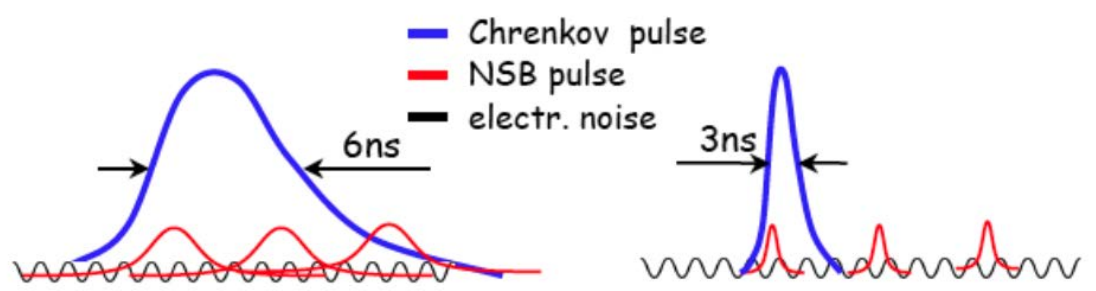

Figure 2.23: Scheme of the PMT pulse from a Cherenkov event, as seen by the 300 MSample/s MUX readout (left) and the 2 GSample/s DRS2 (right).

memory space is being misused with undesirable electronic noise. On the other side, if the RoI is too short, cascades with long timing development could not be entirely recorded, losing information. The RoI was reduced to 60 capacitors in the new system, which has proved to be a good agreement between both conditions. With a $33 \mathrm{MHz}$ ADC, the deadtime is only $26 \mu$ s. Besides an improvement of the deadtime with respect to the DRS2, the DRS4 chips also presented low pedestal noise (0.7 phe per capacitor, instead of the former 1.4 phe). Both Domino Ring Sampler (DRS) versions are temperature-dependent and therefore, observations are performed after reaching a stable electronic temperature.

The DRS4 chips are placed in the PULSer And Recorder (PULSAR) boards. Each PULSAR board contains 4 mezzanines each of them hosting 3 DRS4. Since each DRS4 chip has 8 channels (it can proccess data from 8 PMTs simultaneously), each PULSAR board digitize 96 pixels. A total of 14 PULSAR boards are used to digitize the entire pixels in the camera. The PULSAR boards also host the so-called High Optical Link ATLAS (HOLA) mezzanines, responsible of the transmission from the PULSAR to the Four Input Links for ATLAS Readout (FILAR) cards through optical S-Link. These FILAR cards are Peripheral Component Interconnect (PCI) cards installed in the DAQ computer, where the DAQ program run three tasks in parallel : reading, analyzing and storing the signal from the RoI Apart from the 14 PULSAR boards dedicated to the signal digitalization, another two more PULSAR boards are used with different goals. One of them is the called digital PULSAR, on charge of providing relevant digital information to the stored data, such as trigger number and absolute time. The second is the busy PULSAR, whose task consists on stopping the trigger when an event is being processing or in case an error occurs (Tescaro et al.|2009, 2013).

Along the years and different readout configurations, the DAQ presented certain rate limitations, i.e. a maximum number of events that can process per second. The main limitation is due to the writing speed on the disk. If the rate is too high, instabilities, unnecessary loss of time due to the deadtime and, more important, crashes on the system can happen. The recovery from a DAQ crash takes several minutes, during which data taking is not possible. An increase of rates can arise if the DT of the L0 trigger is too low, allowing $\mathrm{NSB}$ events to fulfill the amplitude threshold condition and increasing this way the probability of accomplishing the $3 \mathrm{NN}$ criterion with just sky background. This very low DT scenario and consequences also apply to 
APs. Even if the DT is set according to the observational conditions, a very bright star in the FoV during the automatic repositioning of the telescopes due to a GRB alert (see Section 2.4.1.8) or artificial lights pointing to the camera (like car flashes) can disturb the DAQ system. To avoid this, besides the automatic tunning of individual pixels DT under bright observational conditions (Section 2.4.1.6), a rate limiter is installed to stop momentarily the trigger system if the stereo rate exceeds $1 \mathrm{kHz}$. During moon observations, the maximum rate that the DAQ could handle was $600 \mathrm{~Hz}$.

\subsubsection{Other subsystems}

$\odot$ Timing system: A Rubidium clock with a precision of $3 \times 10^{-11}$ per second provides the absolute time. It is in turn synchronized with a Global Positioning System (GPS) with a precision of ns. The Rubidium clock proffers the time stamp of the recorded events through the digital PULSAR

$\odot$ Central pixel: The PMT at the center of the camera is not used in the analysis of gamma rays and hence, its information does not go through the standard readout system. Its electronics are changed to use this pixel for regular optical observations.

$\odot$ GRB monitoring alert system: This system monitors the Gamma-ray Coordinate Network (GCN) 1 in order to alert from a possible GRB event. The program evaluates the GCN alert in terms of observability (zenith range, distance to the Moon and Sun, uncertainties on the given GRB coordinates). If the GRB alert accomplishes the observational conditions, the $\mathrm{CC}$ takes control over the telescopes and move them automatically to the GRB position in order to lose the minimum possible observation time. The repositioning of the telescopes for the maximum $\mathrm{Az}$ possible difference of $180^{\circ}$ takes only $20 \mathrm{~s}$, thanks to their light structure.

$\odot$ Weather station: The weather conditions such as humidity, wind speed and direction, temperature and pressure are given by a Reinhardt 5MW weather station, located in the roof of the CH. MAGIC telescopes can operate only if safety conditions are fulfilled (limits are given in Section 2.4.2.3.

$\odot$ Pyrometer: This instrument measures the temperature of the sky and provides an atmospheric transparency estimation. It is only installed in the dish of MAGIC I and points to the same direction than the telescope. It measures IR radiation (in the $8-14 \mu \mathrm{m}$ band) that fits to a blackbody spectrum, obtaining this way the temperature. The measured temperature increases if the sky is cloudy, because it reflects radiation from the ground. Thus, an estimation of the cloudiness (higher cloudiness implies lower transparency) is given by:

$$
\frac{T_{l o w}-T_{m}}{T_{\text {low }}-T_{u p}}
$$

${ }^{1}$ https://gcn.gsfc.nasa.gov/ 
$T_{\text {low }}$ and $T_{u p}$ correspond to the temperature of the sky at its worst and best conditions, respectively, which are set to $T_{\text {low }}=250 \mathrm{~K}$ and $T_{u p}=200 \mathrm{~K} . T_{m}$ is the measured temperature by the pyrometer.

$\odot$ LIght Detection And Ranging (LIDAR); The LIDAR, located in a dome on the $\mathrm{CH}$ roof, is equipped with a $5 \mathrm{~mW}$ Q-switched pulsed laser, a $60 \mathrm{~cm}$ diameter aluminum reflector with $1.5 \mathrm{~m}$ focal length, a Hybrid Photo Detector (HPD), a robotic equatorial mount and a computer that uses FADC cards to digitize the signal from the HPD. The LIDAR shoots the laser at a position shifted by $3^{\circ}$ from the observing source, taking care not to disturb MAGIC data taking, to measure the transparency of the atmosphere. It fires 50000 laser shots with a frequency of $300 \mathrm{~Hz}$ and hence, each data run takes around 3 minutes. A new run is started every 5 minutes. The pulsed light from the laser is backscattered by the clouds and aerosols on the sky. The transparency is measured as a function of the arrival time distribution of the backscattered photons. It can provide transmission estimation at different altitudes of 3, 6, 9 and $12 \mathrm{~km}$ (Fruck et al. 2014).

$\odot$ AllSky camera: Monochrome AllSky-340 SBIG camera, placed in the roof of the CH. It points to the $\mathrm{Zd}$ and provides an image of the sky every 2 minutes. It has complete FoV of $360^{\circ}$ in $\mathrm{Az}$ and almost $90^{\circ}$ in $\mathrm{Zd}$ (see Figure 2.24). These images are monitored online during observations.

$\odot$ MAGIC OnLine Analysis (MOLA); Multithreaded C++ program that gives an on-theflight estimations of the gamma-ray emission when observing a source (Tescaro et al. 2013). It provides $\theta^{2}$ plots, light curves and skymaps (see Section 2.4.3) of the target. This tool is specially interesting when observing a transient system, where flares can occurred.

\subsubsection{Data taking}

The low gain PMTs used in the MAGIC cameras allow us to observe under dark (non Moon presence) and moderate moonlight conditions. The maximum dark duty cycle in a year corresponds to a $18 \%(\sim 1500 \mathrm{hr} /$ year $)$, of which $\sim 65 \%$ is observed and the rest is lost because of technical problems or bad weather conditions. Normally, observation during full Moon nights and around are stopped. Nevertheless, by observing also during decent or strong Moon presence, this duty cycle increases considerably up to $\sim 40 \%$. To do so, small hardware modifications are applied to the standard setup, like decreasing the $\mathrm{HV}$ or using $\mathrm{UV}$-filters, which deal with the higher $\mathrm{NSB}$. Given that the mean $\mathrm{DC}$ is proportional to the $\mathrm{NSB}$ level, the latter can be estimated by comparing the median DC of the observations and the median DC under dark optimal-weather conditions. The latter provides the reference level $\mathrm{NSB}_{\text {lark }}$. Given that the distribution of photons reaching the PMT photocathode follows a poissonian distribution (see Appendix B), an increase of the NSB light will produce a raise of the image noise proportional to $\sqrt{\overline{N S B}}$. A raise of the incoming light will also give rise to higher $\mathrm{AP}$ rate. This situation leads to an increase of the accidental rate in the L0 trigger, i.e. more non gamma ray-induced cascades fulfill the trigger threshold. This has a major effect on the low energy cascades that can be embedded on the high 


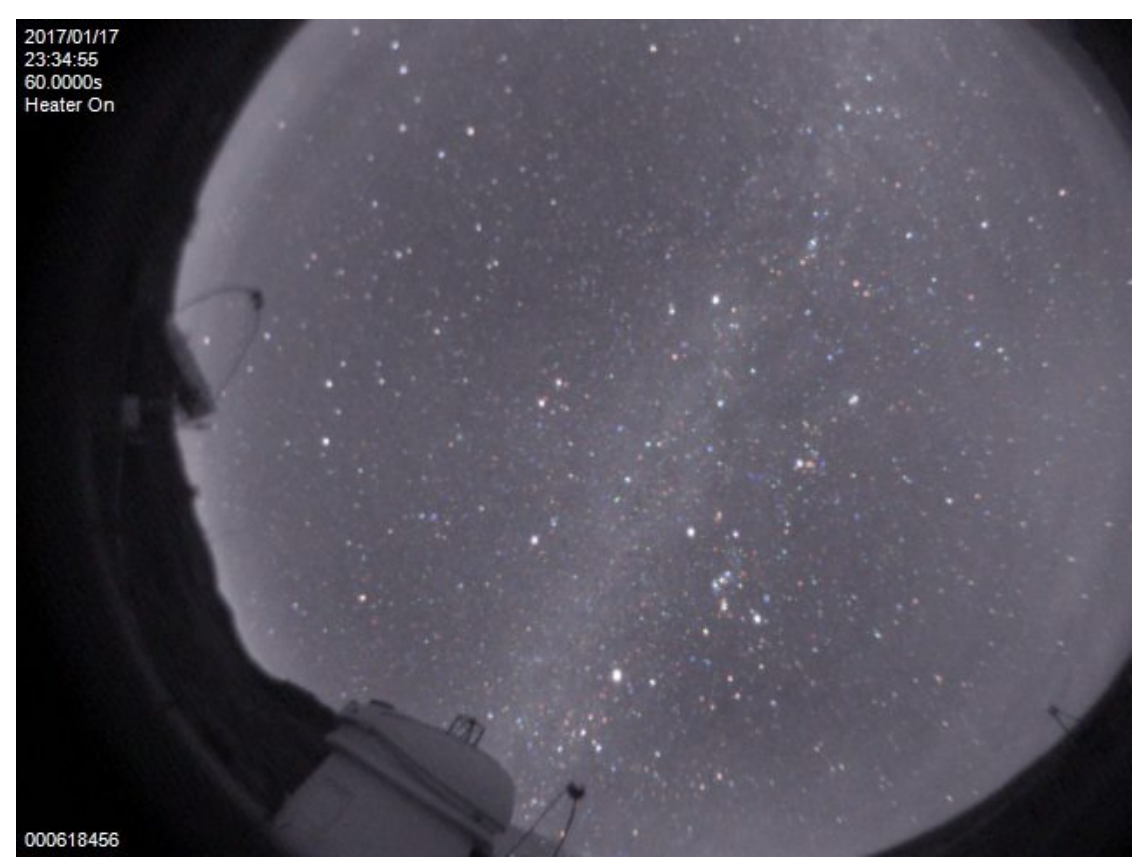

Figure 2.24: MAGICAllSky-camera image.

noise. To suppress these undesirable events, stronger cuts are applied during the data analysis (Section 2.4.3). Attending to safety reasons (see Section 2.4.2.3), with the standard HV of 1.25 $\mathrm{kV}$, the maximum sky brightness under which MAGIC can observed is $12 \times \mathrm{NSB}_{\text {lark }}$. By reducing this $\mathrm{HV}$ value a factor of $\sim 1.7$ (the so-called reduced $\mathrm{HV}$ ), the observations can be extended up to $20 \times \widehat{N S B}_{\text {lark }}$. This reduced $\mathrm{HV}$ performance allows MAGIC to observe up to $90 \%$ of the Moon phase. Observations can be carried out during full Moon if UV -filters are installed. These filters preserve large fraction of the Cherenkov radiation, allowing transmission in the UV band (with a peak at $330 \mathrm{~nm}$ ), while blocking longer wavelength. The MAGIC performance under moonlight is reported in $\mathrm{X}$.

By default, MAGICloperates in stereoscopic mode unless some technical problem prevents it. The L0|DT for stereo observations under dark conditions is set to $1.30 \times \overline{\mathrm{DT}}_{\text {ef }}$, where $\overline{\mathrm{DT}}_{\text {ef }}=4.5$ phe. This value is slightly higher in MAGIC II given the higher reflectivity of the mirrors. Nevertheless, this threshold is not fixed but it is constantly changing for each individual pixel to obtain a L0 and L1 rates stable within the limits, so the accidental L3 rate is low. This task is performed by the Individual Pixel Rate Control (IPRC). If the L0 rate is above $1.2 \mathrm{MHz}$ or below $250 \mathrm{kHz}$, the IPRC decreases or increases the L0 DT, respectively. On average, for dark stereo observations the mean L0 rate is $800 \mathrm{kHz}$. In case stand-alone observations are performed, the usual rate average stays at $100 \mathrm{kHz}$, and the maximum and minimum allowed values are $50 \mathrm{kHz}$ and $150 \mathrm{kHz}$, respectively. Both the L0 DT and rate limits are different in case of Moon presence. The DT level for moonlight conditions during stereoscopic observations is scale a factor 1.70 with respect to the $\overline{\mathrm{DT}}_{\text {ef }}$, and it is changed by the IPRC if the minimum rate is $150 \mathrm{kHz}$ and the maximum $700 \mathrm{kHz}$. Normally this value is around $500 \mathrm{kHz}$. The L1 rate in stereo mode is normally of the order of $10-20 \mathrm{kHz}$, with a maximum constrain at $40 \mathrm{kHz}$. Thus, a L3 rate of 
$\sim 500-600 \mathrm{~Hz}$ is achieved. The accidental L3 rate can be computed as $R_{\text {acc }}=M 1_{L 1} \cdot M 2_{L 1} \cdot 180$ ns, where $R_{a c c}$ is the accidental rate, $M 1_{L 1}$ and $M 2_{L 1}$ are the L1 rate in MAGIC I and MAGIC II respectively and $180 \mathrm{~ns}$ stands for the time difference between telescopes. It has to be lower than $250 \mathrm{~Hz}$ and simultaneously below $60 \%$ of the L3 rate. When observing only with one telescope, the average L1 rate decreases to $400-500 \mathrm{~Hz}$.

\subsubsection{Observational pointing modes}

There are two possible pointing modes with MAGIC, the ON/OFF mode and the so-called wobble mode. Given that both pointing modes were used during observations of sources included in this thesis, I will explain both of them. Nevertheless, the current standard pointing is the wobble mode.

$\odot$ ON/OFF mode: The source is tracked at the center of the camera. The $O N$ region (where the signal from the source is expected) and the OFF region (background signal, used in the significance computation, see Section 2.4.3.9) are observed separately. Here, the background sample is recorded under same conditions (same epoch, zenith angle and atmospheric conditions) as for the $O N$ data but with no candidate source in the field of view.

$\odot$ Wobble mode: In this case, MAGIC points at two or four different positions situated $0.4^{\circ}$ away from the source to evaluate the background simultaneously (Fomin et al.|1994). Each of the positions are usually observed in time slots of 20 or 15 minutes (the so-called runs). With this mode, exactly same conditions of the OFF (background) data are achieved and, in turn, observational time is saved since no dedicated OFF observations are needed. To calculate the significance, one or three OFF regions can be selected (see Figure 2.25). In the former case, the OFF region will be taken at the opposite position of the camera with respect to the source (see Figure 2.25 a). However, three OFF sources are usually encouraged to provide a better background estimation and therefore, most reliable significance. With this pointing mode, there is a reduction of systematic effect produced by different weather or $\mathrm{NSB}$ level conditions with respect to the ON/OFF mode. Nevertheless, there are also some disadvantages. The main one is a decrease on the gamma-ray detection efficiency due to the shift of the source. Because the telescopes is pointing at $0.4^{\circ}$ from the target, some fraction of the EM cascades lay outside of the trigger region (in mono, this fraction reaches 15-20\%). On the other hand, the camera presents inhomogeneities due to the different gain and electrical noise of the PMTs or dead pixels along the trigger region. Therefore, systematic errors in the background estimation arises from the fact that $\mathrm{ON}$ and OFF regions are not taken from the same part of the camera, leading to an overestimation or underestimation of the signal.

\subsubsection{Types of data}

Apart from the source data, MAGIC takes another type of data along the observations for different purposes. Below, I briefly introduce these types and their functionality. 


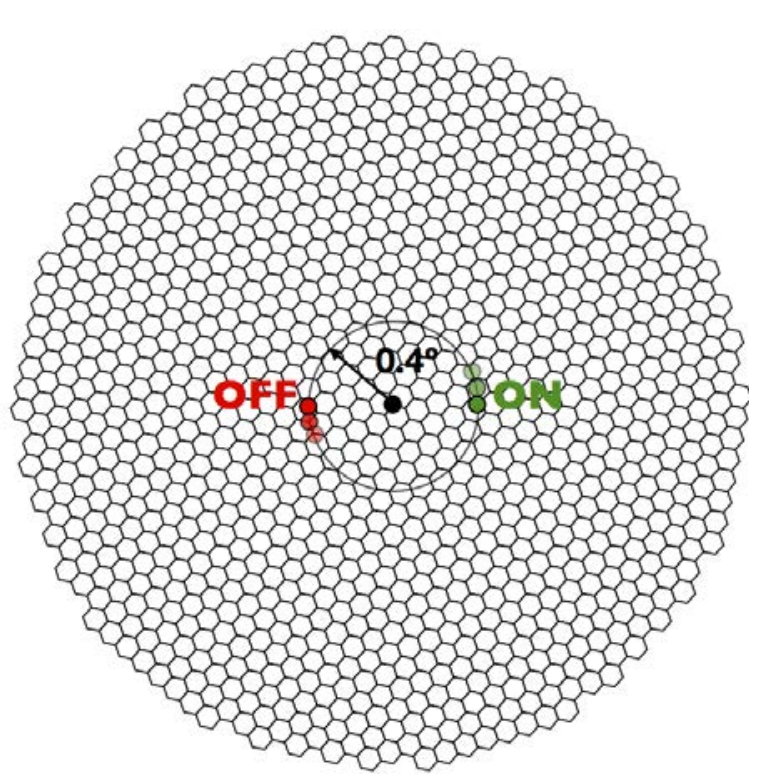

(a) Wobble mode with 1 OFF region.

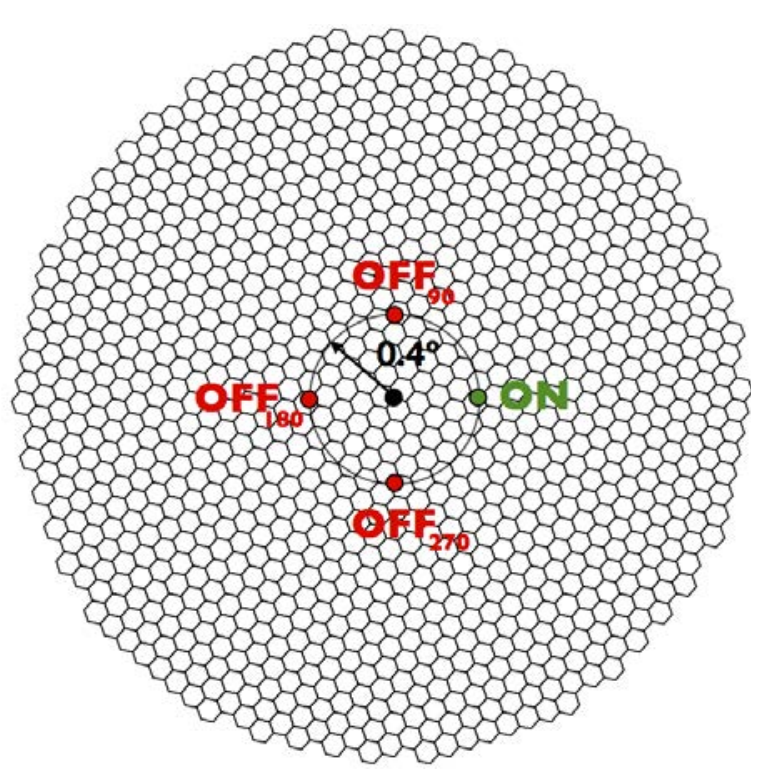

(b) Wobble mode with 3 OFF regions.

Figure 2.25: Scheme of the wobble pointing mode. The black circle corresponds to the center of the camera, located at $0.4^{\circ}$ from the source (green circle). As the source moves in the sky, it remains all the time placed at this distance from the center of the camera, giving rise to a circular movement around the camera center. The background can be simultaneously taken from one region (left plot) or three regions (right plot). In the former, the OFF region (red point) is all the time situated at $0.4^{\circ}$ from the center of the camera, at an opposite direction from the source. If 3 OFF regions are selected, the background is evaluated in regions separated the same distance one from the other, all of them at $0.4^{\circ}$ from the camera center. The subindex of the OFF regions determines the angle at which they lay in the imaginary circle formed by the $\mathrm{ON}$ source movement. These angles $\left(90^{\circ}, 180^{\circ}, 270^{\circ}\right)$ are given counting counterclockwise from the ON region. Plots taken from López-Coto (2015).

$\odot$ Pedestal subtraction run (pedsub): Its goal is to calibrate the pedestal noise (baseline) of each DRS capacitors and set their zero level to 10000 counts, in order to avoid problems at negative values due to fluctuations. With the camera lids closed, DAQ takes $10^{5}$ events at $286 \mathrm{~Hz}$ and hence, this procedure lasts around 6 minutes. It is taken before starting observations at the beginning of the night and it is applied to all the subsequent runs. Given that the DRS chips are temperature dependent, the readout system must be switched on at least 1 hour before taking the pedsub to stabilize temperature. Under usual conditions, this run is only taken once per night. If during observations the electronics are switched off for more than 30 minutes, then pedsub is taken again after another warm-up period of the electronics.

$\odot$ Pedestal run $(\boldsymbol{P}):$ The baseline in the DRS chips remains stable (within 1-2 counts) at a time scale of 1-2 hours. Small drifts of the baseline are corrected with dedicated pedestal runs at the beginning of each source observations and with interleaved pedestal runs at a frequency of $25 \mathrm{~Hz}$ during data taking. This calibration of the baseline is performed online 
by the $\mathrm{DAQ}$ before recording the data runs on the disk.

$\odot$ Calibration run $(\boldsymbol{C})$ : Each calibration run usually contains 4096 events from the calibration system (see Section 2.4.1.4). They are used to update constantly the conversion factor between $\mathrm{ADC}$ counts and number of phe and the arrival times (see Appendix B). They are taken before starting observations of each source and are used before storing data. As for the pedestal runs, there are also interleaved calibration runs.

$\odot$ Data run $(\boldsymbol{D})$ : This run contains all events triggered by the telescopes during observations. Besides cosmic events and accidental triggered events due to the noise, they also contain the interleaved calibration and pedestal runs aforementioned mentioned. These data runs corresponds to 20 or 15 minutes of observations, one run per wobble position. They are split into subruns of 2 minutes.

More information on the different needed runs and performance of the DRS4 can be found in (Sitarek et al.2013b).

\subsubsection{Safety limits}

The telescopes can be only operated under certain safety limits. If these limits are not fulfilled the data taking cannot started or has to be interrupted. Observations under the following listed limits guarantee that none hardware subsystem is damaged:

$\odot$ Wind gust have to be $<40 \mathrm{~km} / \mathrm{h}$ and mean wind speed $<50 \mathrm{~km} / \mathrm{h}$.

$\odot$ Humidity below $90 \%$.

$\odot$ No rain. Sometimes it can happen that it starts raining even though the humidity does not accomplished the aforementioned limit.

$\odot$ Mean DC for the overall camera below $30 \mu \mathrm{A}$.

$\odot$ Individual pixel $\mathrm{DC}<47 \mu \mathrm{A}$. This limit is normally overpasses when a star lays in the FoV of the observation. The IPRC switches off the HV for the PMT that does not fulfill this safety condition.

$\odot$ Humidity inside the camera below $60 \%$. External high humidity conditions do not usually affect the humidity inside the camera because it is sealed. However, under rarely circumstances the humidity can increase inside the camera and observations are stopped until it is dry.

$\odot$ Temperature of the camera $<40^{\circ}$. A higher temperature could suggest a problem in the cooling system.

$\odot$ Zd must be above $1.5^{\circ}$. The drive system prevents movement at lower zenith. 


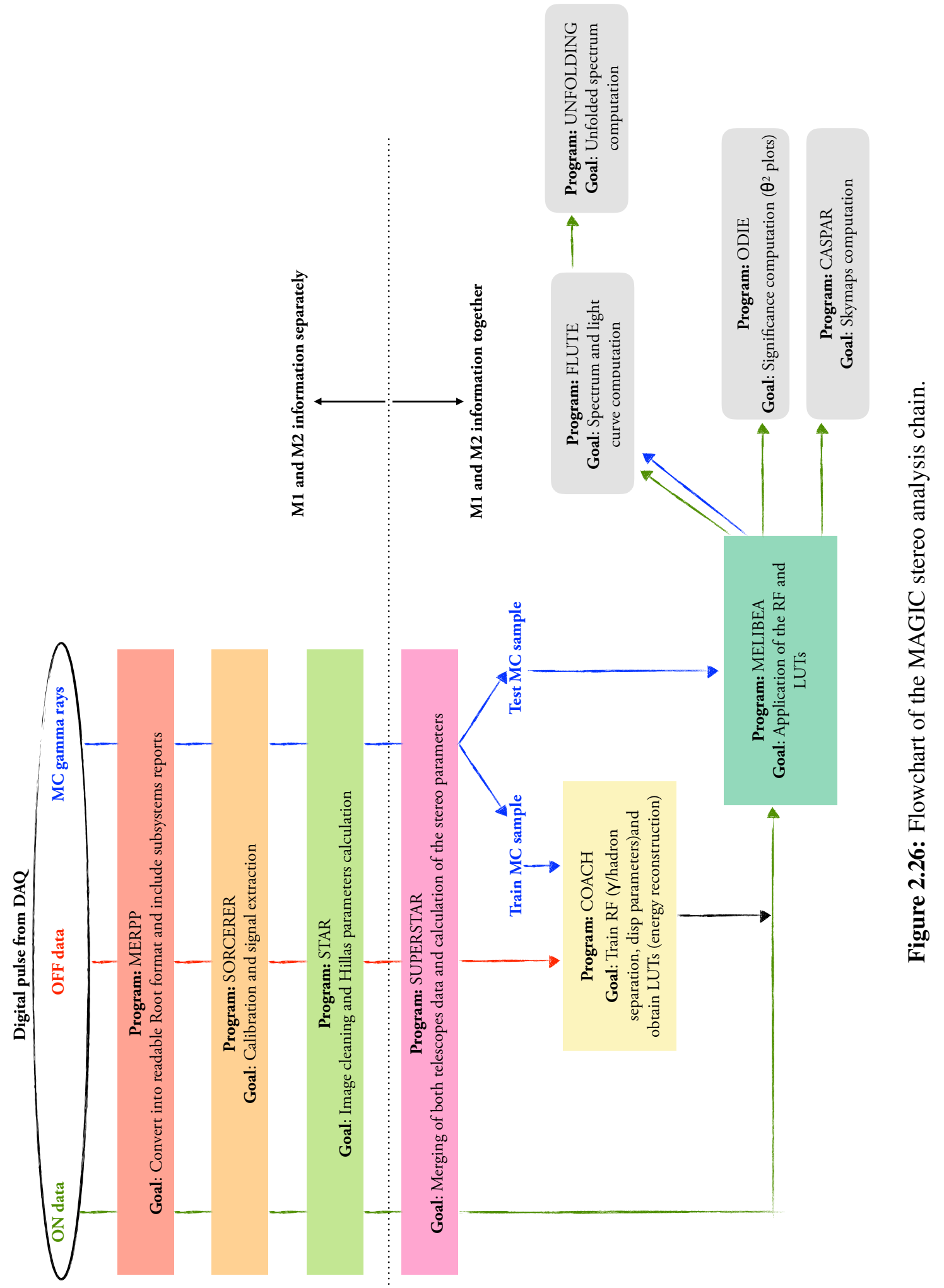




\subsubsection{Data analysis}

Data analysis is performed with the standard MAGIC analysis software called MAGIC Analysis and Reconstruction Software (MARS) (Zanin et al.2013). It makes use of a collection of objectoriented $\mathrm{C}++$ programs and ROOT 1 libraries and classes. MARS converts the raw ADC counts stored by DAQ into processed high-level data. The goal of the analysis is to determine whether an event was generated by a gamma ray or a hadron ( $\gamma /$ hadron separation) as well as to obtain the energy and direction in case of gamma ray-induced showers. In this section, I will give a review of all steps performed by MARS, depicted in Figure 2.26 and listed below:

$\odot$ Calibration of the signal into phe, performed by CALibrate LIght Signals and Time Offsets (CALLIST0) or Simple, Outright Raw Calibration; Easy, Reliable Extraction Routines (SORCERER),

$\odot$ Image cleaning and Hillas parameters calculation computed by the program Star.

$\odot$ Stereo image parameters with the software Superstar.

$\odot$ Train of the Random Forest (RF) for the $\gamma /$ hadron separation, produce the LUTs for the energy reconstruction and compute disp parameters for the arrival direction. This training, carried out by 0steria or Coach, needs simulated Monte Carlo (MC) gamma-ray events and a data sample of real background data (observations with no gamma ray-emitter in the FoV.

$\odot$ Apply RF and LUTs to the real data to obtained hadronness, reconstructed energy and arrival direction. Also applied to the $\mathrm{MC}$ data. The program used for this task is Melibea.

$\odot$ Computation of signal significance with Odie, skymaps with Caspar and spectra and ligth curves with Fluxlc or Flute.

\subsubsection{Monte Carlo simulations}

In order to reconstruct the energy of the primary gamma ray and its arrival direction, IACTs need to use MC simulations. The MC simulation software applied in MAGIC, which does not use the MARS framework, can be divided into three steps:

$\odot$ The development of gamma ray-induced showers are simulated using COsmic Ray SImulations for KAscade (CORSIKA) 6.019 (Heck et al. 1998). An atmospheric model (known as MagicWinter) is used based on measurements at La Palma. This model contains $78.1 \%$ of $\mathrm{N}_{2}, 21.0 \%$ of $\mathrm{O}_{2}$ and $0.9 \%$ of Argon (Ar). High-energy interactions in the atmosphere and hadronic interactions are described with the QGSJet-II model and FLUKA, respectively. The modified version of CORSIKA used in MAGIC allow us to have the direction and position of the photons on the ground.

\footnotetext{
1https://root.cern.ch/
} 


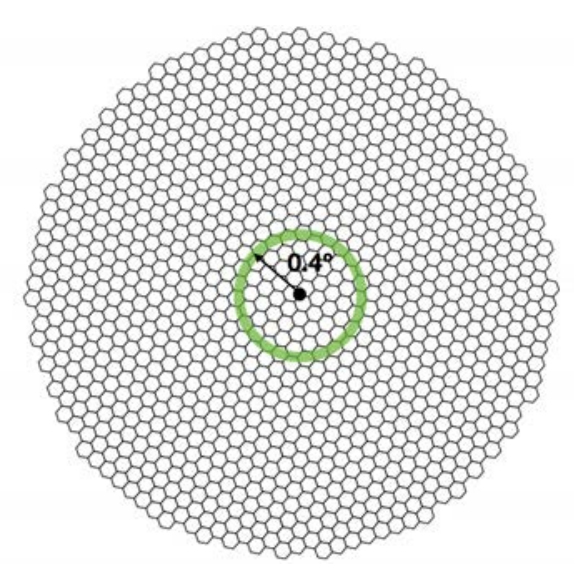

(a)

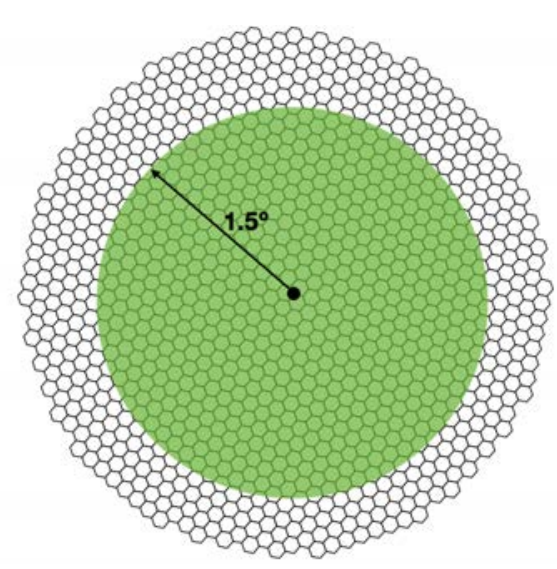

(b)

Figure 2.27: Schematic view of the ringwobble (left) and diffuse (right) $\mathrm{MC}$. The green area corresponds to the region in which gamma rays are simulated. For the ringwobble $\mathrm{MC}$ a width of $0.1^{\circ}$ is used. Credit: López-Coto (2015).

$\odot$ The so-called reflector program is used to take into account the absorption and scattering in the atmosphere (see Section 2.1). Reflector makes use of the Elterman (1964) model for the distribution of aerosols and ozone in the sky. After accounting for the absorption of the Cherenkov photons, the reflector program simulates the reflection of the survival Cherenkov photons in the telescopes' mirror surface, giving the position and arrival time of those photons in the camera plane.

$\odot$ Finally, the camera program simulates the response of the PMTs, the trigger applied $(3 \mathrm{NN}$ or $4 \overline{\mathrm{NN}}$ and readout systems of MAGIC. The QE as well as the measured readout noise are included in the simulation. Camera can also provides the effect of the measured PSF of each mirror and error in their alignment.

After these three steps, data with raw format is obtained, which after converting into ROOT files, can be analyzed with the MARS software. In MAGIC, MC gamma rays are simulated in two ways: as ringwobble $\mathrm{MC}$ and as diffuse $\mathrm{MC}$. The former simulates a ring of $0.4^{\circ}$ radius (with a width of $0.1^{\circ}$ ) from the camera center, accounting for the $0.4^{\circ}$ offset used in the standard wobble mode. It is used for the analysis of point-like sources. The latter is applied for the study of extended sources or sources shifted from the nominal position. In this case, diffuse gamma rays are simulated covering a circle of $1.5^{\circ}$ radius. Figure 2.27 shows a schematic views of both types of MC. In both cases, separated MC data are available for low $\mathrm{Zd}$ range $\left(5^{\circ}-30^{\circ}\right)$, medium $\mathrm{Zd}$ range $\left(35^{\circ}-50^{\circ}\right)$, high $\mathrm{Zd}$ range $\left(50^{\circ}-62^{\circ}\right)$ and very-high $\mathrm{Zd}$ range $\left(62^{\circ}-70^{\circ}\right)$. Although for the source analysis only $\mathrm{MC}$ gamma rays are necessary, $\mathrm{MC}$ of electron, positrons, protons and $\mathrm{He}$ are also computed with CORSIKA for performance studies with MAGIC.

For more information of $\mathrm{MC}$ simulations with MAGIC, the reader is referred to Majumdar et al. (2005). 


\subsubsection{Signal pre-processing}

To be able to analyze the raw data coming from the FADC or ADC (counts as a function of time) with the standard MARS software, one needs to convert it into ROOT format. This conversion is made by MERging and Prepoccesing Program (MERPP), which also attached other subsystems reports to the ROOT files.

Once ROOT files are available, the calibration of the counts into phe is carried out by SORCERER. In the past, another program called CALLISTO was used. As mentioned in Section 2.4.1.7, once the trigger is accepted the DAQ stored the information of a certain Rol per pixel, which was 80 capacitors before 2009 and currently 60 capacitors. Each capacitor contain the number of ADC in a time of $0.5 \mathrm{~ns}$ and hence, a total of $30 \mathrm{~ns}$ are recorded now from the DRS4. The signal pre-processing goal is to achieve the charge (total signal) and arrival time of each event from the pixels. Different algorithms were used along the MAGIC lifetime to obtain this information. For the data presented in this thesis, only two algorithms were applied: the cubic spline extractor for the MUX data and the sliding window used with both DRS2 and DRS4.

$\odot$ Cubic spline extractor: After removing the pedestal, the samples are interpolated with a cubic spline curve. The algorithm then look for the maximum of the interpolation, which corresponds to the signal amplitude. The charge is given as the spline integral of the RoI window around the maximum. The arrival time of the signal is assumed to be the half maximum at the rising edge.

$\odot$ Sliding window:After subtracting the pedestal run (dependent on the capacitor and the last time it was read; Sitarek et al. 2013b), the so-called sliding window algorithm determines the position of the integration window over the whole buffer. The algorithm searches for the maximum integral content in 6 consecutive $\mathrm{ADC}$ slices, which corresponds to the signal charge. The arrival time is defined as the average of the $\mathrm{ADC}$ slices time, weighted with the counts that each slice contains.

After the signal extraction, the measured charge is calibrated to obtain phe through the FFactor method (Mirzoyan 1997) as explained in Appendix B.

\subsubsection{Image cleaning and Hillas parameters calculation}

After the calibration, the images are cleaned and parametrized by the Star program. Although after the signal pre-processing, charge and arrival time for each PMT is available, not all pixel contain useful information. Most of them only contain noise, useless for the signal analysis. Thus, the image cleaning algorithm discards those pixels that, below a certain calibrated signal timing and amplitude thresholds, do not contain useful information of the shower image. In this thesis, data with two different image cleaning methods were used: the absolute image cleaning (Aliu et al. 2009b) and the sum image cleaning (Lombardi 2011). The former is the currently used algorithm.

Absolute image cleaning This method discriminates pixels according to their charge (in phe), dividing between core pixel and boundary pixels. A pixel is considered as the core pixel 
if its charge is above a certain threshold $Q_{c}$. Moreover, this pixel must have at least another neighboring pixel that accomplishes this threshold too, in order to avoid that random high charge pixels unrelated with the cascade are selected as core pixels. The boundary pixels are those with at least one neighboring core pixel and that fulfill an amplitude threshold of $Q_{b}$. The selection of the charge thresholds, $Q_{c}$ and $Q_{b}$, is important for the further analysis. In order to detect the lowest energy gamma-ray cascades, these threshold should not be restrictive. However, very relaxing thresholds can in turn allow noise-induced images to fulfill this condition. To solve this, the absolute image cleaning makes use of the signal arrival time, letting the signal charge thresholds be lower with less risk of accepting non gamma-ray events. The information used is based on the fact that Cherenkov radiation lasts a few ns, much less than the arrival time for $\mathrm{NSB}$. The applied conditions are the following: the core pixel cannot differ from the mean arrival time obtained from all core pixels more than $\Delta t_{c}$, whilst the boundary pixels time cannot differ more than $\Delta t_{b}$ from the core pixel's arrival time. The timing thresholds are, for all periods and performances, $\Delta t_{c}=4.5 \mathrm{~ns}$ and $\Delta t_{b}=1.5 \mathrm{~ns}$. Figure 2.28 shows the effect of the combined charge and timing thresholds.

The threshold values change according to the observational conditions, like the level of moonlight. It also depends on the camera performance and hence, different values were applied for pre- and post-upgraded camera. In this thesis, I analyzed data under dark conditions for preupgrade period and under dark and all moonlight levels (including full Moon with UV-filters) for the post-upgrade period. The corresponding values used in the image cleaning of the data is listed in Tables 2.2 and 2.3. As mentioned in Section 2.4.3, for the complete analysis of the data, $\mathrm{MC}$ data and a real background sample data are needed, both of which need to mimic same observational conditions are those for the source data. This implies that for Moon analysis, one needs appropriated $\mathrm{MC}$ and background data at the same moonlight levels than the observations. MC simulations from Corsika are only computed for dark conditions and therefore, artificial noise is injected in the files before the image cleaning performed at the Star level. In the case of background sample, one can introduce artificial noise as well or use data taken under same moonlight conditions. To calculate the additional noise, we use the interleaved pedestal runs, from which mean pedestal and Root Mean Square (RMS) values are determined in each separate moonlight level. Thus, we can get the scale factor we need to apply to the corresponding mean pedestal and RMS of each pixel of the $\mathrm{MC}$ and background data (see Table 2.3). Arrival time precision is degraded accordingly too.

\begin{tabular}{|l|c|c|c|c|c|}
\cline { 2 - 6 } \multicolumn{1}{c|}{} & $\begin{array}{c}\mathrm{NSB} \\
\left(\times \mathrm{NSB}_{\text {dark }}\right)\end{array}$ & $\begin{array}{c}\text { Equivalent } \\
{[\mu \mathrm{DC}]}\end{array}$ & $\mathrm{HV}$ & \multirow{2}{*}{$Q_{c}$} & $Q_{b}$ \\
\hline MAGIC I & $1-2$ & $<2$ & Standard & 6 & 3 \\
MAGIC II & $1-2$ & $<2$ & Standard & 9 & 4.5 \\
\hline
\end{tabular}

Table 2.2: Image cleaning levels for pre-upgraded cameras. Standard $\mathrm{HV}$ value corresponds to $1.25 \mathrm{kV}$.

Sum image cleaning This algorithm is also based in the previously given definition of core and boundary pixels. However, it uses the compactness of the image to obtain more information. 


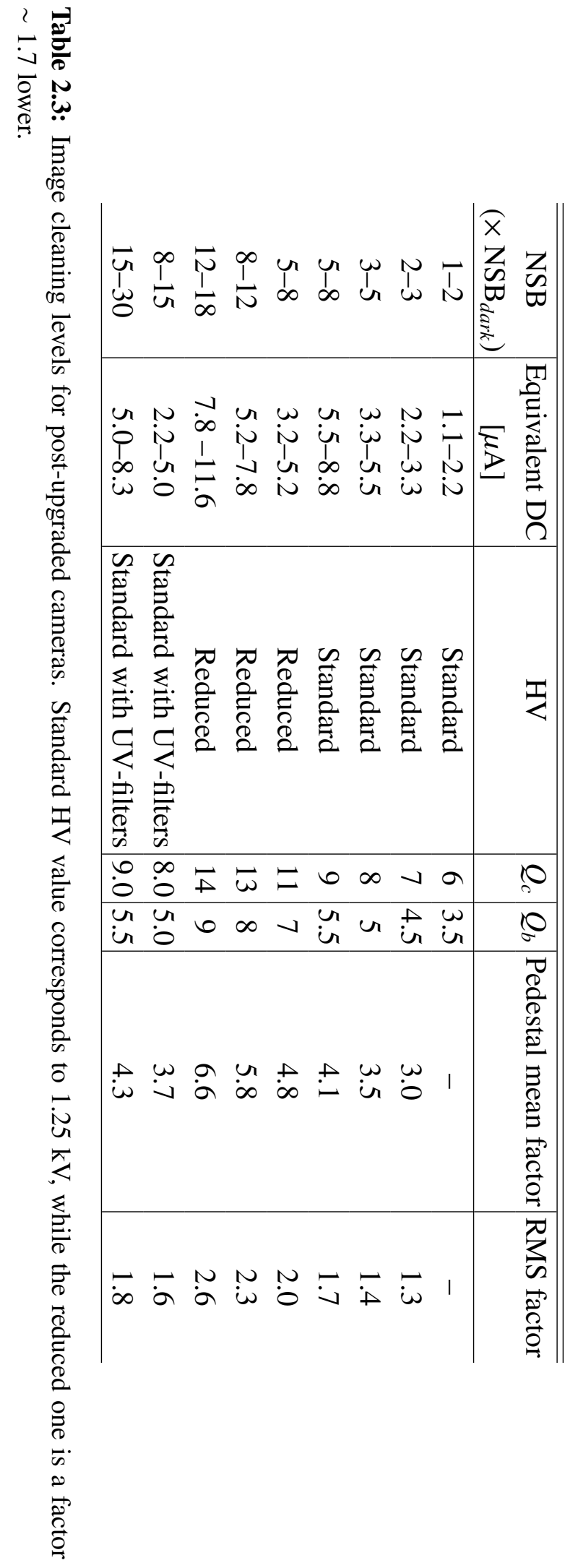




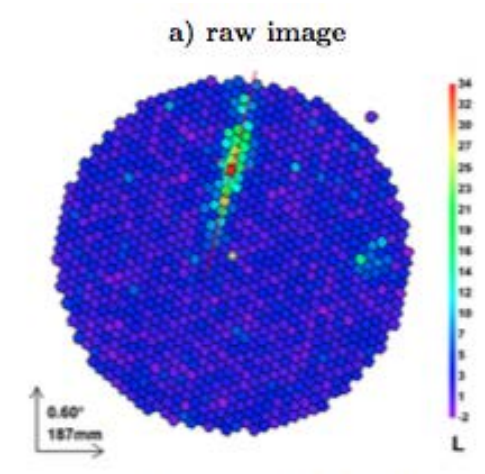

c) cleaned image (15:9 No Timing)

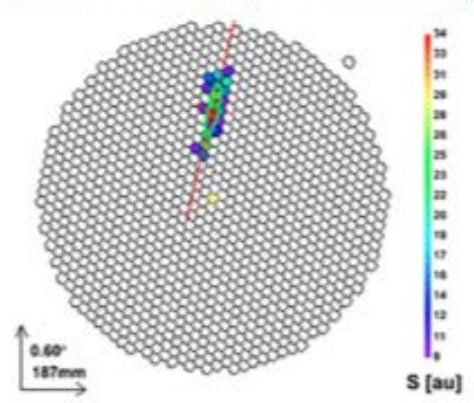

b) arrival time

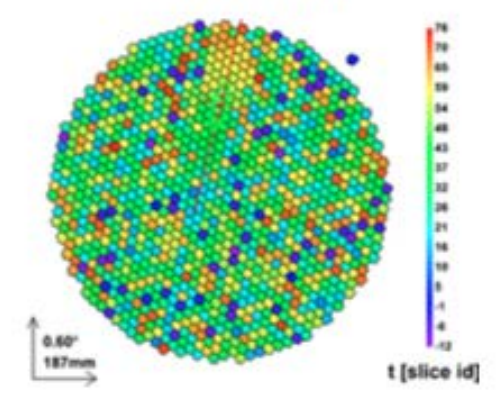

d) cleaned image (9:4.5 No Timing)

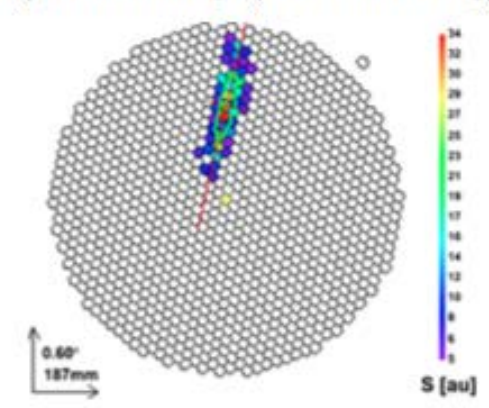

e) cleaned image (9:4.5 Timing)

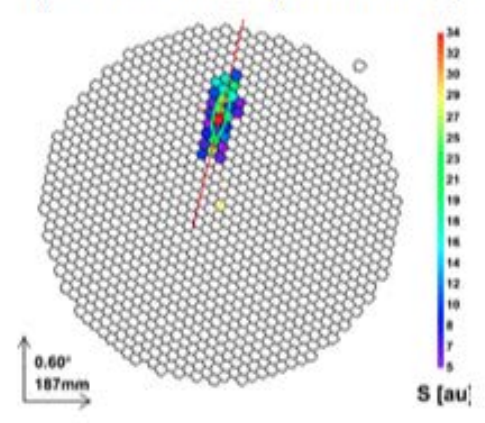

Figure 2.28: Events recorded by MAGIC II at which cleaning levels are applied. The two upper plots (a and $\mathbf{b}$ ) corresponds to the signal charge distribution in the camera and arrival times before image cleaning, respectively. In panel c a strong charge threshold is applied $\left(Q_{c}=15\right.$ phe and $Q_{b}=9$ phe), where part of the cascade image is lost. In panel $\mathbf{d}$, the standard cuts for the DRS2 period are applied $\left(Q_{c}=9\right.$ phe and $Q_{b}=4.5$ phe. This lower values allow more pixels to survive the cuts. Finally, panel e shows the result of the combination between standard charge and timing cuts $\left(\Delta t_{c}=4.5 \mathrm{~ns}\right.$ and $\left.\Delta t_{b}=1.5 \mathrm{~ns}\right)$. The latter can discard more noise than using only charge threshold, while keeping threshold condition sufficiently low. Image taken from Zanin (2011).

With this additional information the charge thresholds can be decreased, which benefits the low energy cascades. The sum image cleaning look for $\mathrm{N}(\mathrm{N}=2,3$ or 4$)$ neighboring pixels whose summed charge is above a threshold $Q_{c}$ within a time window given by $t_{c}$. Before summing, the signals are "clipped" if they are above a certain value to avoid AP (similar to the Sum trigger procedure, see Section 2.4.1.6. The value of $Q_{c}$ and $t_{c}$ depends on the selected $\mathrm{N}$, as presented in 
Table 2.4. The boundary pixels are those adjacent with at least one core pixel, with an amplitude above a fixed value of 3.5 phe and a arrival time difference with respect to their neighboring pixel $<1.5 \mathrm{~ns}$.

\begin{tabular}{|c|c|c|}
\hline Topology & $\begin{array}{r}Q_{c} \\
\text { phe }\end{array}$ & $\begin{array}{c}t_{c} \\
{[\mathrm{~ns}]}\end{array}$ \\
\hline $2 \sqrt{\mathrm{NN}}$ & 10.8 & 0.5 \\
\hline$3 \longdiv { \mathrm { NN } }$ & 7.8 & 0.7 \\
\hline $4 \overline{\mathrm{NN}}$ & 6 & 1.1 \\
\hline
\end{tabular}

Table 2.4: Sum cleaning image parameters.

After the cleaning, the images are ready to be parametrized, from which the Hillas parameters are obtained. To get them an ellipse is fit to the surviving pixels and the momenta of the fit (up to second order), listed below and shown in Figure 2.29, are the parameters used in the analysis of MAGIC. The parametrization information of each event is stored in ROOT files for both telescopes separately.

$\odot$ Size: It corresponds to the sum of the charges in phe of each surviving pixel. The size is proportional to the energy of the primary gamma-ray if the event is contained in the Cherenkov light pool of radius $\sim 120 \mathrm{~m}$.

$\odot$ Length: Longitude of the major semi-axis of the ellipse. It is related with the longitudinal development of the cascade.

$\odot$ Width: Longitude of the minor semi-axis of the ellipse. It is a measurement of the lateral development of the cascade.

$\odot \operatorname{Conc}(\mathbf{N}):$ Fraction of the image charge contained in the $\mathrm{N}$ brightest pixels. It gives the compactness of the image, which for EM cascades is larger than for hadronic showers. The used value is Conc(2).

Some of the Hillas parameters are source-dependent. This means that although they represent the physical features of the showers, these depend on the source position.

$\odot$ Dist: Angular distance between the center of the camera and the center of gravity of the image. The larger the dist value, the larger the impact parameter of the shower in the ground.

$\odot$ Alpha: Angle between the major axis of the ellipse and the imaginary line connecting the camera center and the center of gravity of the image. The camera center is as well the expected source position (in ON observation, for wobble mode this is slightly shifted $0.4^{\circ}$ ) and therefore EM cascade should point to the camera center. Thus, gamma ray-induced showers have smaller alpha angle than the hadronic ones. 


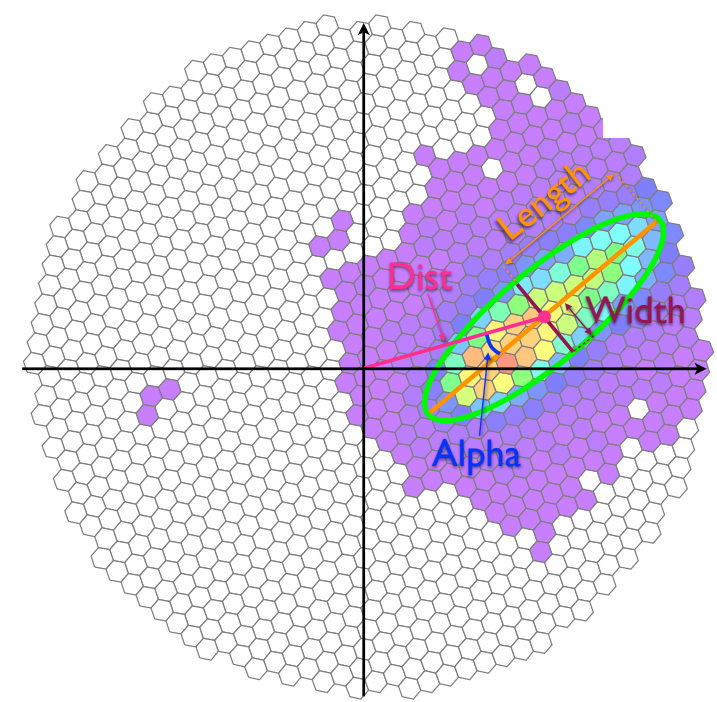

Figure 2.29: Schematic view of the Hillas parameters.

Time-dependent parameters are also useful to discriminate between $\mathrm{EM}$ and hadronic showers, given that the former develop faster $(\sim 3 \mathrm{~ns}$ contrary to the $\sim 10 \mathrm{~ns})$ :

$\odot$ Time RMS: RMS of the arrival time of the surviving pixel, which is smaller for gamma ray-induce cascades.

$\odot$ Time gradient: Slope of the linear fit applied to the arrival time projection along the major axis, which gives the direction of the shower development. [EM] showers are expected to have positive development (from close to the camera center to outside).

Other parameter are used to estimate the image quality. Thus, very noise images or images not well-contained in the camera can be discarded.

$\odot$ LeakageN: Fraction of the shower light contained in the $\mathrm{N}$ outermost rings of PMTs of the camera (usually, $\mathrm{N}=1$ ). This parameter measured how much the image is contained in the camera.

$\odot$ Number of islands: Number of surviving pixels non-related with the image event.

Finally, there are the so-called directional parameters. They are used to differentiate between the head (top of the cascade) and tail (bottom of the cascade). Atmospheric showers presents higher charge in the head part, since particles in the top have higher energies.

$\odot$ Asymmetry: Direction of the line between the center of gravity of the image and the pixel with the highest charge. The EM presents positive asymmetry, i.e. the pixel with the highest charge is located close to the source position.

$\odot$ M3Long: Following the same criterion as the asymmetry parameter, M3Long is the third moment of the image along its major axis. 


\subsubsection{Data quality cuts}
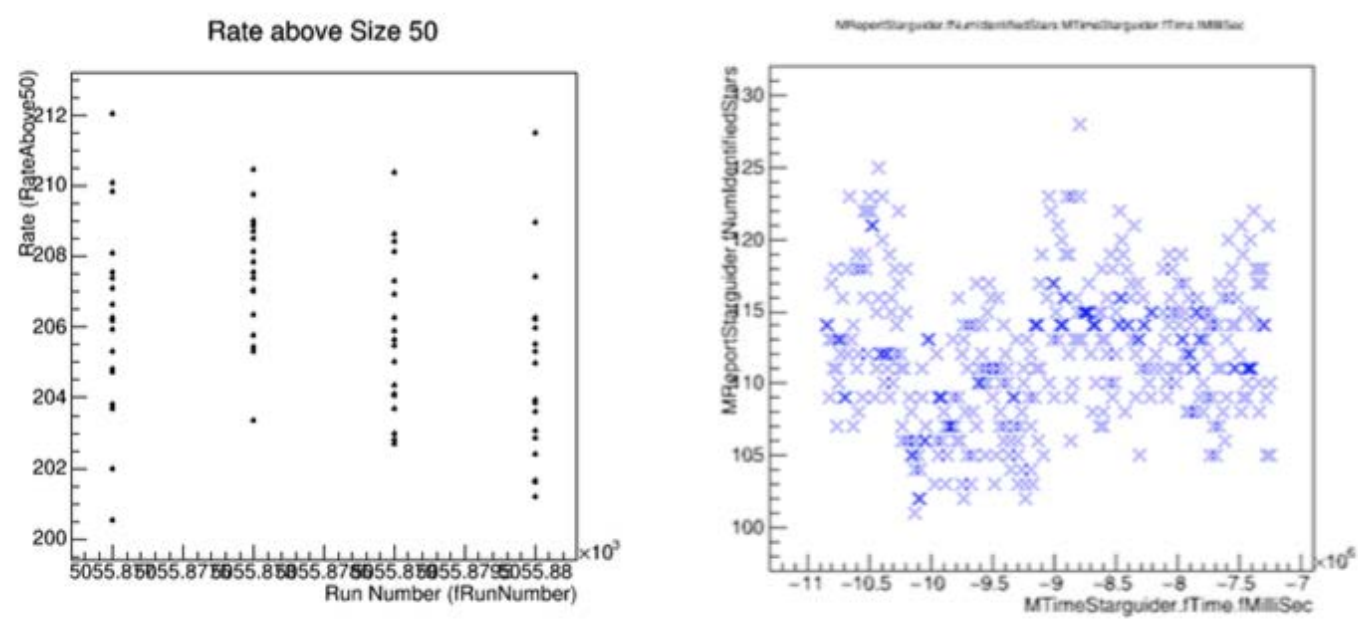

Figure 2.30: Example of two plots used in the data quality selection. On the left: Rate plot as a function of the subrun, taken from Cygnus X-3 observations with MAGIC II applying a size cut of 50 phe. On the right: Number of identified stars by the Starguider camera for the same night. The high detected stars reveal very good quality weather conditions.

After running the program Star, the ROOT data files contain all the information (image parameters) of each event needed for the calculation of the significance or flux. However, before proceeding, data quality cuts are necessary to guarantee reliable results. Data quality of the events is disturbed by technical problems or bad weather conditions and can be estimated by using different indicators. One of them is the Rate of events (in $\mathrm{Hz}$ ) in each subrun for a low size cut of 50 phe (Figure 2.30). Accidental events with unreliable high rate can be discarded. One must take into account that the rate is $\mathrm{Zd}$ dependent, as the absorption is higher at larger $\mathrm{Zd}$ angles, which can be corrected (up to $50^{\circ}$ ) by multiplying the rate by $\cos ^{-1}(\mathrm{Zd}$ ). During a good weather condition night, the rate should stay stable and hence, we normally accept events with rates that differ $\pm 15 \%$ from the mean rate. Because of the higher reflectivity on the MAGIC II mirrors, the rate for this telescope tends to be slightly higher than in MAGIC I. In case the observations were taken under very cloudy night, the rate will be unexpectedly low which helps to reject bad data. A proper estimation of the sky coverage can be made with the cloudiness parameter, given by the pyrometer installed in MAGIC I (see Section 2.4.1.8). For this thesis, and as usually done, I considered bad data those with a cloudiness above 40\%. The information stored from the Starguider camera is also useful to determine if the data should be classified as bad. Given that the Starguider compares the observed[FoV] with a stars catalog, if only a few stars are recognized that would imply high cloudiness (see Figure 2.30). 


\subsubsection{Stereo image parameters calculation}

After the data quality selection, the Superstar program merges the information from both telescopes. The new stereo image parameters obtained are the necessary tools for the energy and direction reconstruction performed in further steps. The most important parameters are listed below. Schemes of some of them are presented in Figure 2.31 and 2.33.

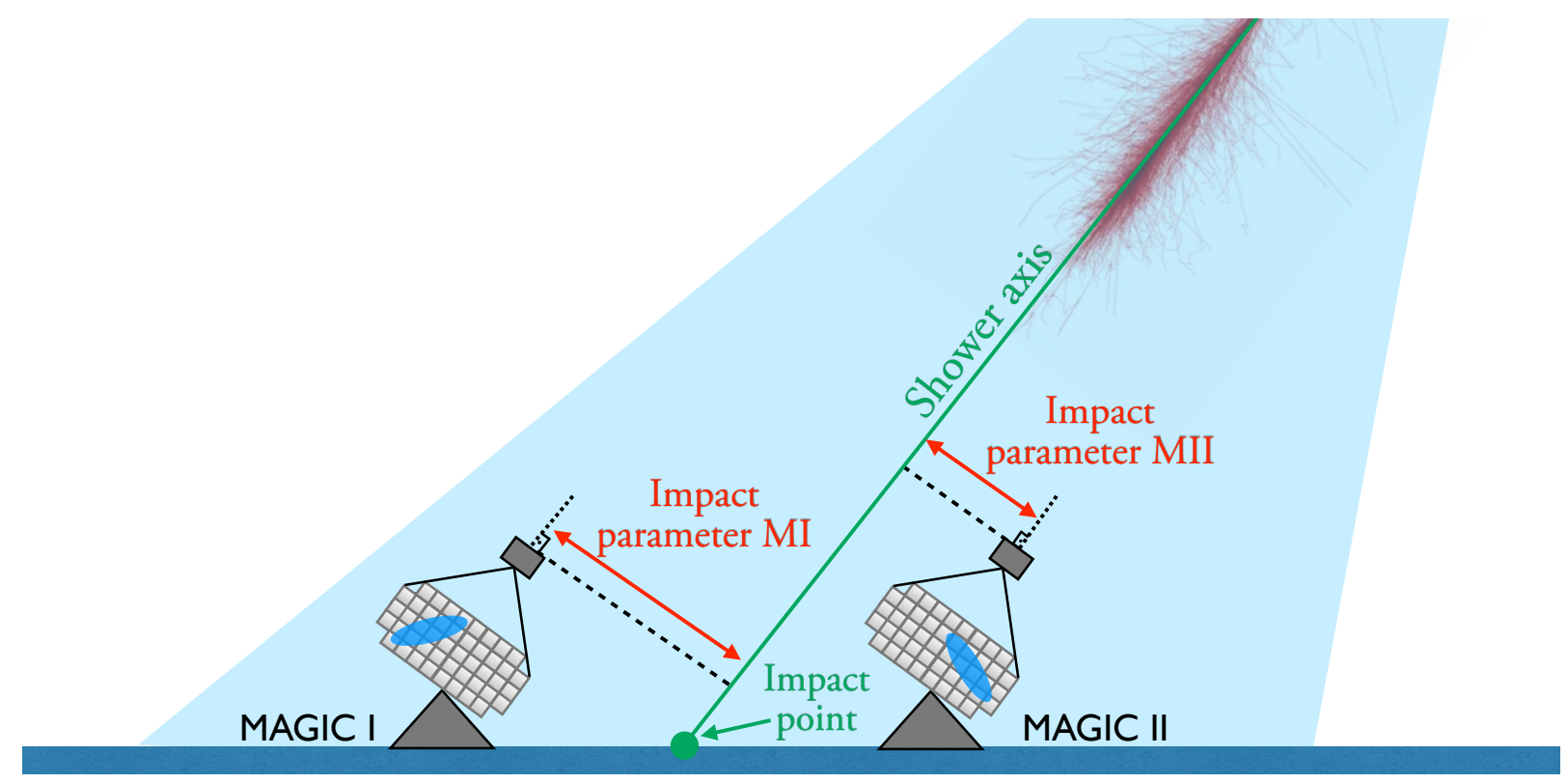

Figure 2.31: Geometry of stereo event, where the impact parameters for both telescopes, impact point and shower axis are highlighted.

$\odot$ Shower axis: This axis can be calculated as the crossing of the enlarged major axes of the two images of the telescopes when they are superimposed in the same camera plane (Figure 2.33b). This is the so-called crossing point (Aharonian et al. 1997; Hofmann et al. 1999). This method cannot be used in mono observations, for which the Disp method is applied. The latter, more robust than the crossing point, is as well used for the current MAGIC stereo observations (see Section 2.4.3.7).

$\odot$ Impact point: The point in the ground that the shower axis reaches. It is determined by the crossing of the enlarged major axes of the image shower in each of the telescopes, taking into account their position (see Figure 2.33a).

$\odot$ Impact parameter: Perpendicular distance in the camera between the pointing direction and the shower axis. There is one impact parameter per telescope. See Figure 2.31.

$\odot$ Shower maximum height $\left(H_{\max }\right):$ The altitude at which the number of particles in the cascade is maximum $\left(H_{\max }\right)$ is determined, once the shower axis is known, with the angle at which the image of the center of gravity is viewed in each telescope. As shown in 
Section 2.2, the $H_{\max }$ depends on the energy of the primary particle: as higher is this energy, closer to the ground the cascade develops and hence, $H_{\max }$ is smaller. It can be used as an indicator for the $\gamma /$ hadron separation, mainly at low energies: at that energy range, the $H_{\max }$ distribution for the gamma ray-induced cascades follows a Gaussian, whilst the $H_{\text {max }}$ distribution for hadrons presents two peaks, the latter produced by muon events. A comparison of $H_{\max }$ distribution for gamma- and hadron-induced showers is presented in Figure 2.32 .
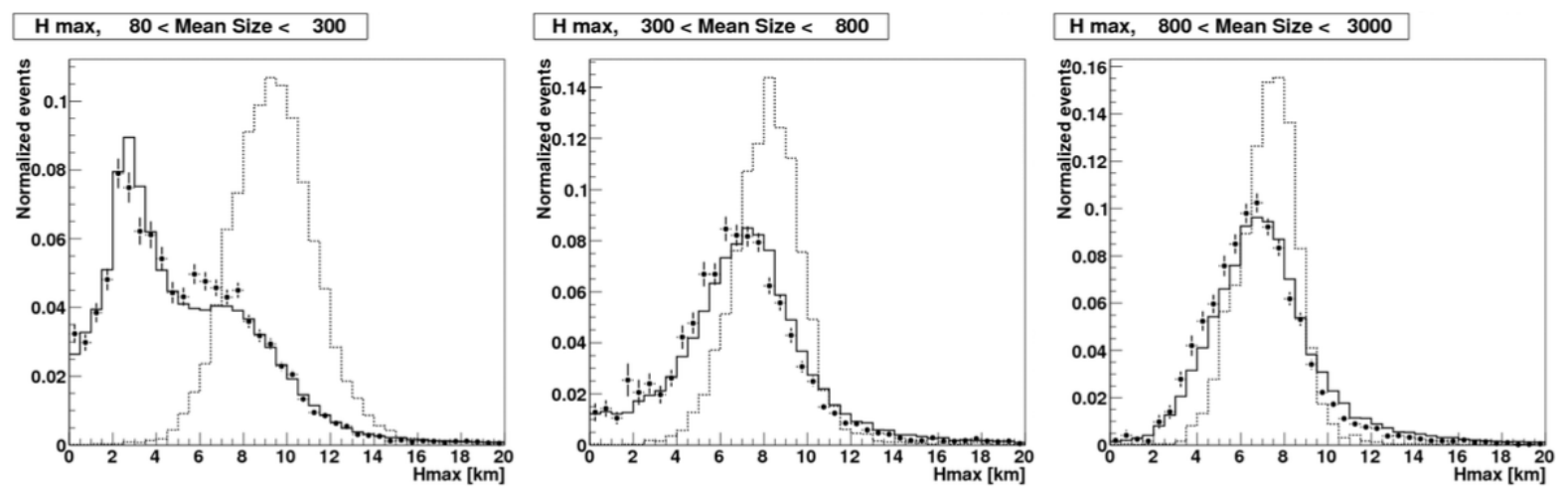

Figure 2.32: Shower maximum distribution height for EM (dotted lines) and hadronic cascades (solid line) for different size cuts (in phe). The MC distribution for hadronic shower is also shown (black points). Images taken from Aleksić et al. (2012b).

$\odot$ Cherenkov radius $\left(R_{C}\right)$ : Radius of the Cherenkov light pool produced by an electron with the bremsstrahlung critical energy $86 \mathrm{MeV}$ at the $H_{\max }$.

$\odot$ Cherenkov photon density: Density of Cherenkov radiation produced by an electron with the bremsstrahnlung critical energy $86 \mathrm{MeV}$ at the $H_{\text {max }}$.

\subsubsection{6 $\gamma /$ hadron separation}

As mentioned before, even for strong sources as the Crab Nebula, the ratio between hadronic and EM] showers is 1000:1. Therefore, the hadronic images surviving the image cleaning is also around 3 orders of magnitude larger than the gamma ray-induced images. This is the reason why an optimal $\gamma /$ hadron separation is key in the analysis. To perform this discrimination, we use RF. a multi-dimensional classification algorithm based on decision trees (Albert et al. 2008). In order to train the $\mathrm{RF}$ on how the gamma-ray events looks like compared to the hadronic ones, the algorithm uses two inputs: $\mathrm{MC}$ simulated gamma rays and a real background data (with no gamma-ray emitter, to avoid misleading the training of the RF algorithm). Both of them need to mimic the observational conditions under which the source data was taken, attending basically to weather conditions, moonlight and zenith range. The $\mathrm{MC}$ set applied here has to be different for the one used later on in the calculation of flux, in order to avoid being biased. Thus, the entire 


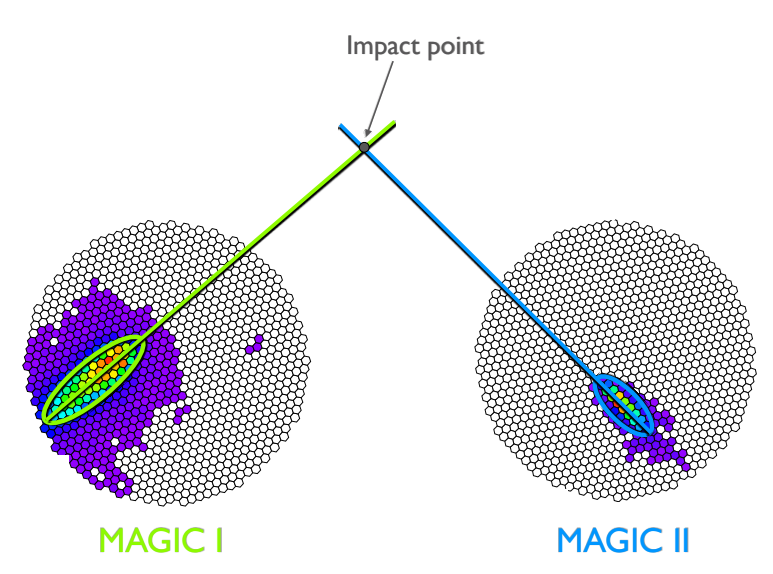

(a) Impact point on the ground.

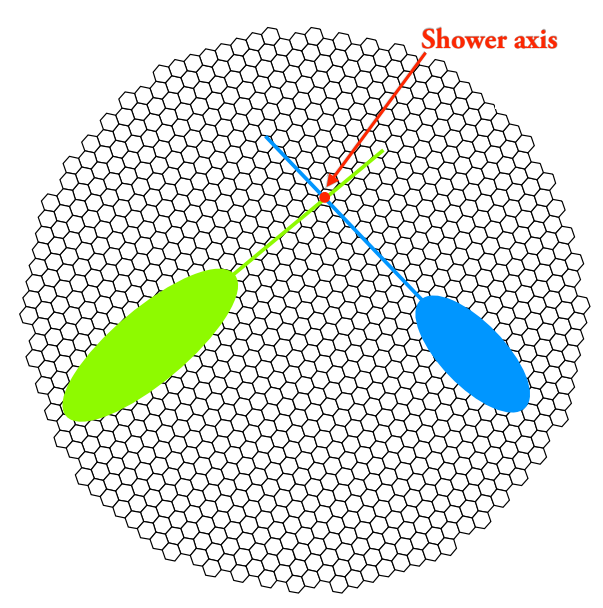

(b) Shower direction calculation.

Figure 2.33: Stereo parameters calculation.

MC sample (simulated with Corsika for different Zdranges, see Section 2.4.3.1) is divided into two sub-sets, a train sample, used for the $\gamma /$ hadron separation, and a test sample, applied for the collection area and migration matrix computation.

The RF tree starts with the whole sample of events (containing both gamma rays from $\mathrm{MC}$ and hadrons from the background data), which provides a reliable image of the real scenario when observing. A randomly selected set of $P$ parameters (such as size, length, arrival time, etc.) are used to discriminate between gamma rays and hadrons. The $\gamma /$ hadron separation is obtained by dividing the initial sample into two subsamples of events, gamma rays and hadrons, based on optimized cuts of one $P$ parameter at a time. The optimization of the cuts is based on the minimization of the Gini coeficient (Gini 1921):

$$
Q_{\text {Gini }}=4 \frac{N_{\gamma}}{N} \frac{N_{h}}{N}
$$

where $N$ is the total number of events, $N_{\gamma}$ is the number of gamma rays and $N_{h}$ is the number of hadrons. The classification selects another parameter randomly and the subsequent division into gammas and hadrons takes place. If one of the subsamples contains only gamma rays or hadrons, the separation process stops in that branch. To evidence the discrimination, if the events from this subsample belongs to the gamma-ray population, they are assigned with a 0 , whilst if they are hadrons the assignation is 1 . The training of the RF grows up to a limit of $n$ trees, which in MAGIC is usually $n=100$. In Figure 2.34 there is a graphical view of the RF classification.

This trained $\mathrm{RF}$ is afterwards applied to the real data from a source with the Melibea software. Each event of the data has to pass through all the trees previously trained, which allows to classify it into gamma ray or hadron. To quantify how likely an event is a gamma ray or hadron, each event is assigned a hadronness value ranging from 0 to 1 (closer to 1 implies hadron-like event). The final hadronness value, $h$, of each event is determined by the mean of the hadronness 


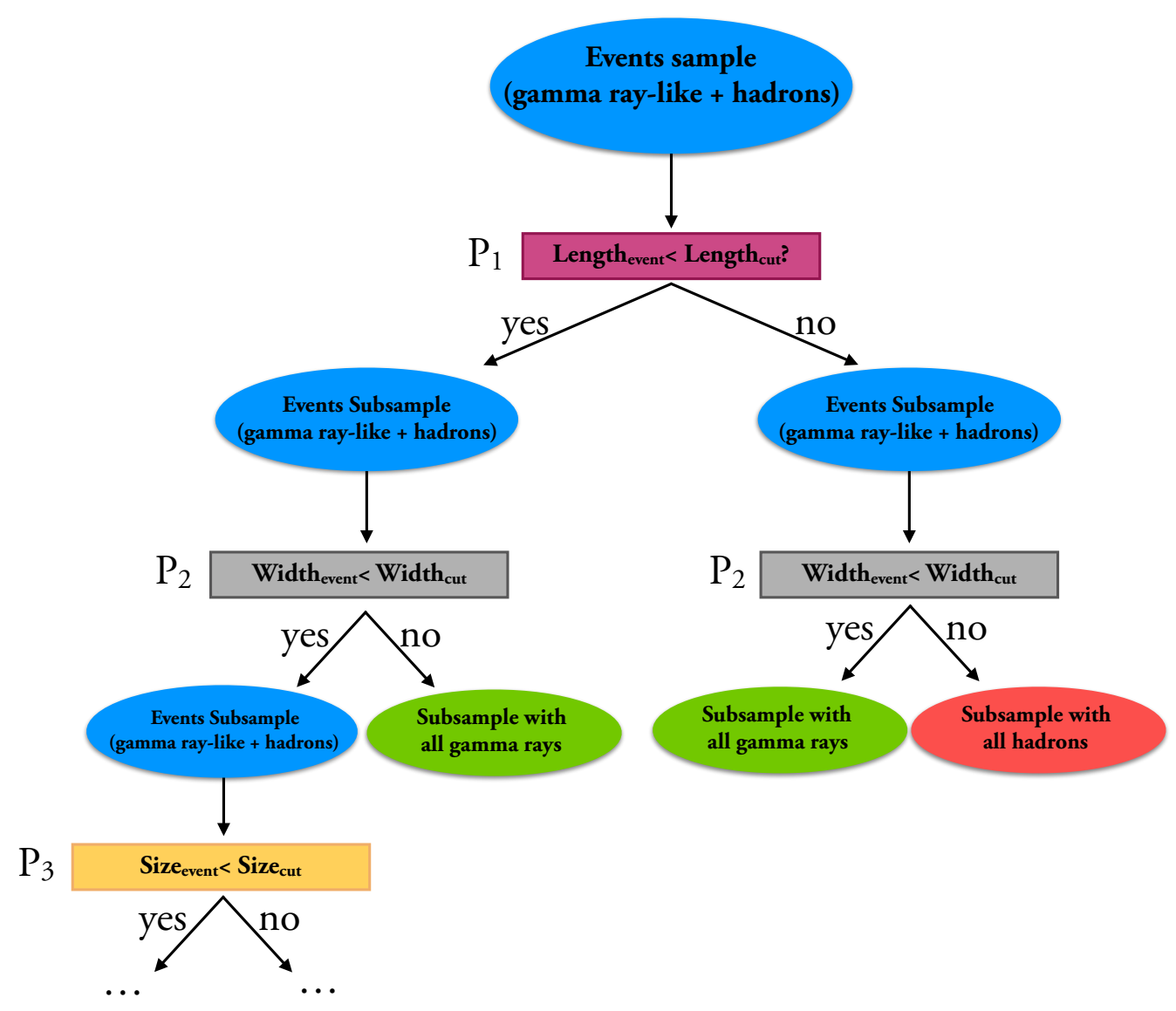

Figure 2.34: Scheme of the RF classification. The $P_{i}$ parameters are selected randomly. Once all events in a subsample belong to the gamma-ray or hadron population, the splitting stops. The cuts applied are optimized with the Gini coefficient.

assigned to all the trees during the training, $h_{i}$ :

$$
h=\sum_{i=1}^{n} \frac{h_{i}}{n}
$$

\subsubsection{Arrival direction reconstruction}

The crossing pointing method (explained in Section 2.4.3.5) can be used in the reconstruction of the arrival direction of the primary gamma ray. However, for stand-alone observations a more sophisticated method, based on MC simulations, is necessary. This method, proposed by Fomin et al.(1994) and revised by Lessard et al.(2001), is the so-called Disp method, which is currently used in stereo observations as well.

The Disp method works as follows: given that the elliptical image obtained in the camera is a projection of the realEM shower, the major axis of it represents the incoming direction of the 
cascade in the camera plane. Therefore, the source position has to be on this axis separated a certain distance, known as disp, from the center of gravity of the image. The disp parameter can be obtained through the relation between the width and the length of the image:

$$
\operatorname{disp}=A(\text { size })+B(\text { size }) \cdot \frac{\text { width }}{\text { length }+\eta(\text { size }) \cdot \text { leakage } 2}
$$

A correction, in terms of leakage2 (see Section 2.4.3.3), is applied to the length to take into account those image that lays at the edge of the camera and hence, are truncated. The coefficients $A($ size $), B($ size $)$ and $\eta($ size $)$ are second order polynomials of $\log ($ size $)$, determined by $\mathrm{MC}$ simulated gamma rays. Once the distance disp is determined, the position of the source is not undoubtedly known, since two possibilities are valid as shown in Figure 2.35a. To disentangle which one is the correct, directional parameters, as Asymmetry or M3Long (Section 2.4.3.3), are used.

However, the correction factor applied on the length is not very efficient at very high energies, where the images in the camera are larger and therefore, the probability of truncation at the edge increases. Thus, a method based on $\mathrm{RF}$ algorithm was implemented to improve and make more robust the Disp method. A $\mathrm{RF}$ is trained in a similar way is done for the $\gamma /$ hadron separation. Given that the disp parameter is known for simulated gamma rays, the $\mathrm{MC}$ events pass through $n$ number of decisional trees to get a correlation between the disp and a set of parameters. In this case, the optimal cuts are those which minimizes the variance of the disp parameter in each division, instead of the Gini coefficient. Among the previously presented image parameters (Section 2.4.3.3), source independent variables are important in the RF training to avoid bias, as for example the time gradient.

In the case of stereoscopic observations, given that we have one image per camera, there are four disp distances or estimations on the source position (Figure 2.35b). To calculate the accurate arrival direction, we compute the distances between these four possible positions and select the smaller one. Between the two closest points, the average of the positions weighted with the number of pixels of each image is computed which corresponds to the reconstructed arrival direction. If none of the distances are smaller than $0.22^{\circ}$, then the algorithm does not accept the reconstruction as valid.

The Disp method brings a new parameter in the analysis, the angular distance $\theta$ (see Figures $2.35 \mathrm{a}$ and 2.35b). It corresponds to the angular distance between the true position of the source and the reconstructed one. Based on this parameter, we obtain the significance plots, the socalled $\theta^{2}$ plots, which will be discussed in Section 2.4.3.9. The introduction of the RF in the determination of the disp distance led to an important improvement in the angular resolution, which is translated into a 20-30\% better sensitivity (Aleksić et al.|2012b).

\subsubsection{Energy reconstruction, energy resolution and energy threshold}

To estimate the energy of a primary gamma ray two methods were applied, accounting whether the observations were performed in stand-alone or stereo mode. For mono observations, the reconstructed energy is obtained in a similar way as the hadronness or arrival direction, by means of a $\mathrm{RF}$. Since with $\mathrm{MC}$ data the true energy of the event $\left(E_{\text {true }}\right)$ is known, the $\mathrm{RF}$ is trained with 


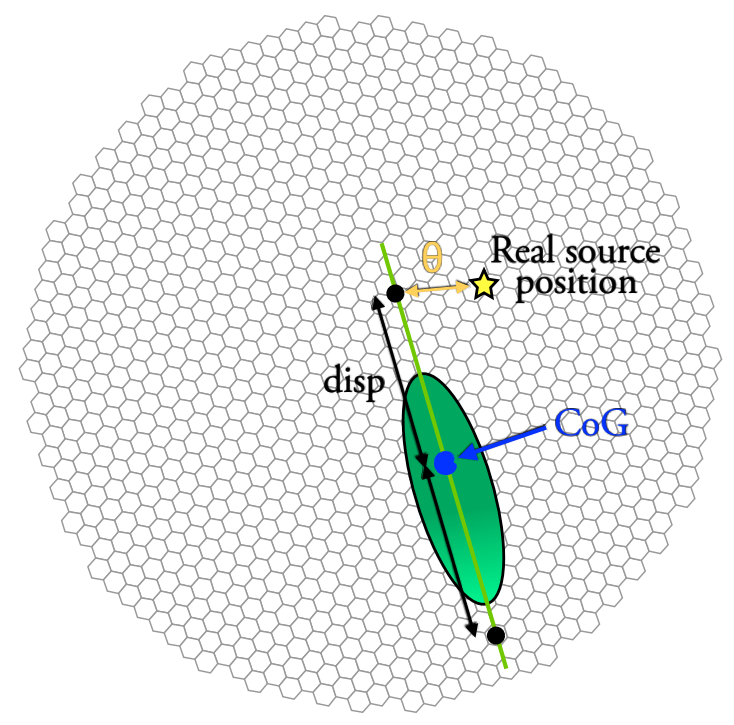

(a)

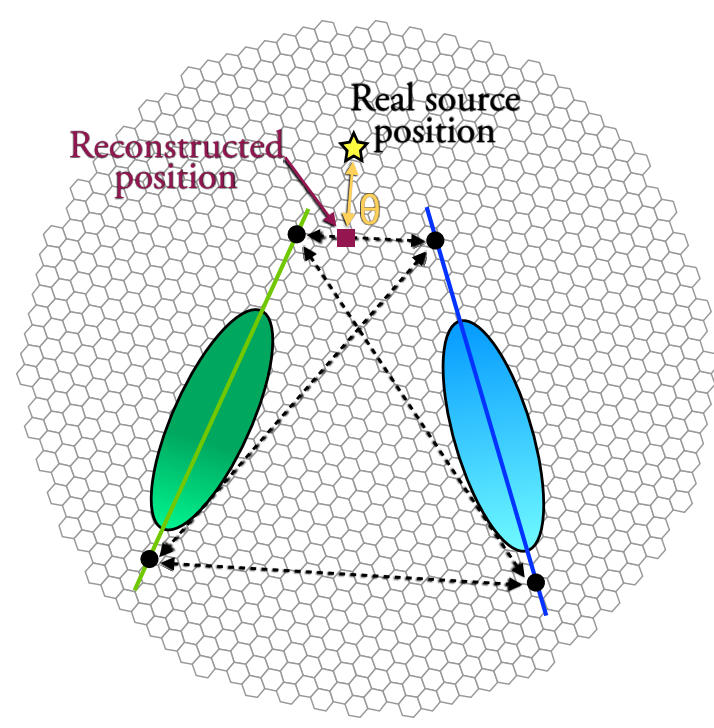

(b)

Figure 2.35: Disp method for stand-alone (left) and stereo observation (right).

a set of randomly selected parameters, whose optimal cut is that which minimizes the variance of $E_{\text {true }}$.

To reconstruct the incident energy in stereoscopic observations, we make use as well of $\mathrm{MC}$ data but through LUTs. In a 2-dimensional histogram, binned in size and impact parameter $/ R_{C}$ (where $R_{C}$ is the Cherenkov radius), each slot is filled with the $E_{\text {true }}$ and its corresponding RMS of each event for MAGIC I and MAGIC II, separately. The estimated energy $\left(E_{\text {est }}\right)$ of an event is obtained with the average of the corresponding $E_{\text {true }}$ for each image of both telescope weighted by the RMS of each bin and corrected by $\cos (\mathrm{Zd})$.

The energy bias, relative error between the $E_{\text {est }}$ and $E_{\text {true }}$, is defines as:

$$
E_{\text {bias }}=\frac{E_{\text {est }}-E_{\text {true }}}{E_{\text {true }}}
$$

The $E_{\text {bias }}$ for an energy bin is obtained by fitting a Gaussian to all $E_{\text {bias }}$ for each individual events included in the bin. The $E_{\text {bias }}$ of the system is the average of all those Gaussians.

The energy resolution, how accurately the instrument can determine the real energy of an event, is defined as the RMS of the Gaussian. Figure 2.36a shows the energy resolution and bias for pre- and post-upgraded periods and different Zd ranges. With the current system, the energy resolution reaches $15 \%$ at a few hundred of $\mathrm{GeV}$, but get worse at higher energies because the higher probability of the image to lay at the edge of the camera (higher impact parameter) and get truncated. At lower energies, the energy resolution is as well worse given the difficulty of reconstruct those images. During mono observations with MAGIC I, the energy resolution was 
$25 \%$ between $200 \mathrm{GeV}$ and $1 \mathrm{TeV}$. On the other hand, the $E_{\text {bias }}$ keeps around zero for energies $>150 \mathrm{GeV}$ at low $\mathrm{Zd}$ range and $>200 \mathrm{GeV}$ at medium range. The relative error between the estimated and true energy increased considerably at lower energies due to the energy threshold.

The energy threshold of the instrument is defined as the peak of the MC simulated energy distribution for a source that follows a power-law function with a standard photon index of $\Gamma=$ 2.6. It is usually evaluated after analysis cuts, such as hadronness, size and $\theta^{2}$ cuts, to obtain the distribution of surviving events. Figure $2.36 \mathrm{~b}$ shows the energy distribution of simulated gamma rays at low and medium $\mathrm{Zd}$ range taken from Aleksić et al. (2016b). The current energy threshold of MAGIC(with a size cut of 50 phe) is $\sim 75 \mathrm{GeV}$. Events with energy below the energy threshold can be detected too, but given the more complicated reconstruction of the image, the spectral points show large errors.

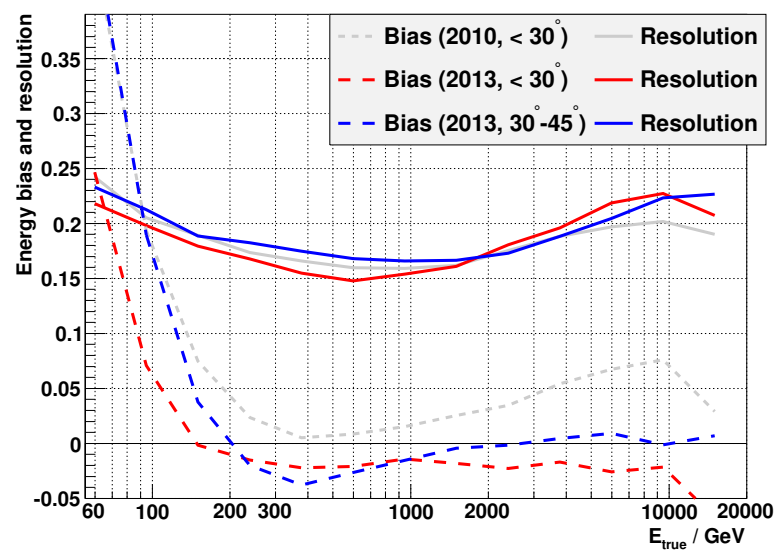

(a)

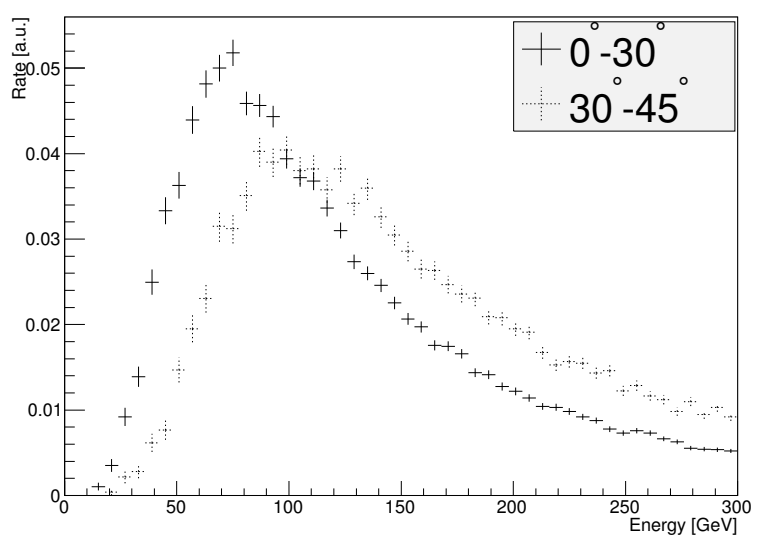

(b)

Figure 2.36: On the left: MAGIC energy bias (dashed lines) and energy resolution (solid lines) for the period before the pre-ugrade of the readout systems (grey lines) and post-upgrade (red and blue lines). On the right: Rate of gamma rays after analysis cuts for low $\mathrm{Zd}$ angle (solid lines) and medium $\mathrm{Zd}$ angle (dashed lines). The peak of the distribution corresponds to the energy threshold, $\sim 75 \mathrm{GeV}$ for Zd between $0-30^{\circ}$ and $\sim 100 \mathrm{GeV}$ between $30-45^{\circ}$. Plots taken from Aleksić et al. (2016b).

\subsubsection{Signal extraction}

To compute the signal from the source, it is used the distribution of events as a function of $\theta^{2}$, angular distance from the real source position and the reconstructed one (obtained through the Disp method, Section 2.4.3.7). The program responsible of calculating the $\theta^{2}$ distance and compute later on the significance is Odie. Assuming that the camera is homogeneous close to the center where we point to the source, the background events $\left(N_{o f f}\right)$ should follow a flat distribution all over the $\theta^{2}$ range, whilst the gamma ray events $\left(N_{o n}\right)$ peak at small $\theta^{2}$ values, i.e. nearby the source. The events used in this distribution are those which survived the analysis cuts, such as size, hadronness or a specified $\mathrm{Zd}$. Although these cuts can be optimized on independent and known sources, e.g. the Crab Nebula, we usually apply standard cuts for the MAGIC analysis. 
The latter are divided into three categories attending to the energy range in which the bulk of events are expected to lay: Low Energy (LE) with $E_{t h r}=100 \mathrm{GeV}$, Full Range Energy (FR) that provides a medium-to-high energy range with $E_{t h r}=250 \mathrm{GeV}$, and $\mathrm{HE}$ with $E_{t h r}=1 \mathrm{TeV}$. The optimized cuts for each range varied from pre- to post-upgraded period. Both set of cuts can be found in Table 2.5 .

\begin{tabular}{|c|c|c|c|c|c|c|}
\cline { 2 - 6 } \multicolumn{1}{c|}{ Energy range } & $\begin{array}{c}E_{t h r} \\
{[\mathrm{GeV}]}\end{array}$ & $\begin{array}{c}\theta^{2} \\
{\left[\mathrm{deg}^{2}\right]}\end{array}$ & Hadronness & $\begin{array}{c}\text { Size in M1 } \\
\text { phe }\end{array}$ & $\begin{array}{c}\text { Size in M2 } \\
\text { phe }\end{array}$ \\
\hline \multirow{4}{*}{ Pre-upgrade } & Low energy & 100 & $<0.026$ & $<0.28$ & $>55$ & $>55$ \\
& Medium-to-high energy & 250 & $<0.01$ & $<0.16$ & $>125$ & $>125$ \\
& High energy & 1000 & $<0.01$ & $<0.17$ & $>300$ & $>300$ \\
\hline \hline \multirow{2}{*}{ Post-upgrade } & Low energy & 100 & $<0.02$ & $<0.28$ & $>60$ & $>60$ \\
& Medium-to-high energy & 250 & $<0.009$ & $<0.16$ & $>300$ & $>300$ \\
& High energy & 1000 & $<0.007$ & $<0.1$ & $>400$ & $>400$ \\
\hline
\end{tabular}

Table 2.5: Standard MAGIC analysis cuts attending to the energy range for periods before (pre-) and after (post-) the upgrade of 2011-2012.

To determine the significance of the signal, we make use of the two distributions separately $N_{o n}$ and $N_{\text {off }}$ (see Figure 2.37a). The later is get from the real data of the target source. Therefore, these events are not purely gamma rays, but includes surviving events from electrons/positrons in hadronic cascades, gamma-like hadrons or diffuse gamma rays (whose contribution is larger in galactic sources). The hadron contribution, given by the $N_{\text {off }}$ distribution, is obtained differently according to the pointing mode: in the case of $O N / O F F$ mode, these events are get from the dedicated OFF observations in a FoV with no gamma-ray candidate and with same observational conditions. For wobble mode, the $N_{o f f}$ is obtained directly from the same source observations, at the same distance from the source but in opposite direction (more information in Section 2.4.2.1). The excess events, $N_{e x c}$, are determined by the difference between $N_{o n}$ and the scaled $N_{o f f}$ :

$$
N_{e x c}=N_{o n}-\alpha \cdot N_{o f f}
$$

where the scale factor $\alpha$ is the normalization between $N_{o n}$ and $N_{o f f}$, given that they are usually not observed the exact amount of time. The value of $\alpha$ is obtained by the fraction of ON events over the OFF events in a region far away from the expected signal region in the $\theta^{2}$ plot, normally between $0.1-0.3 \mathrm{deg}^{2}$. For the wobble mode, taken into account that the observation time is the same for ON and OFF, this value is simply $\alpha=1 /$ (number of OFF regions), where the number of OFF regions can be 1 or 3 for standard observations.

The signal plot can also be given as a function of the alpha angle (see Section 2.4.3.3). Choosing this against $\theta^{2}$ depends basically in the kind of analysis we want. The $\theta^{2}$ plot is obtained in for a source-independent analysis, since the angular distance is obtained with the Disp method, whose $\mathrm{RF}$ is trained with source-independent parameters, in the same way as the hadronness. On the other hand, the alpha plot is given for a source-dependent analysis where the position of the source is assumed, taken into account that the calculation of the alpha angle needs this 


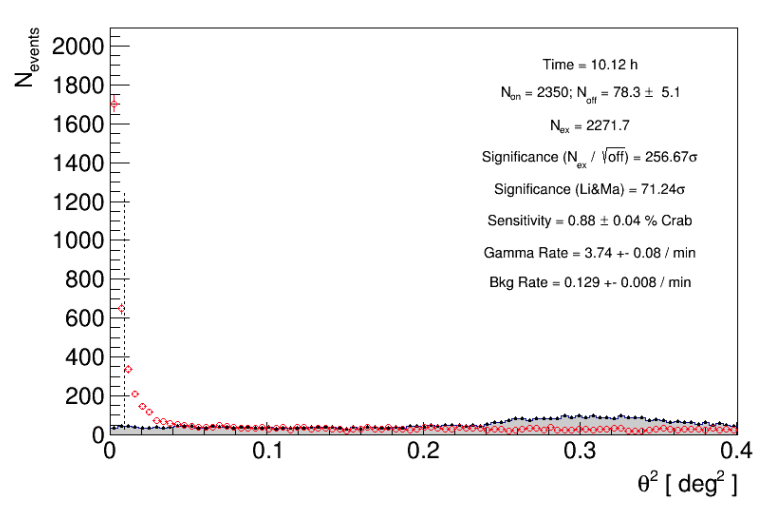

(a)

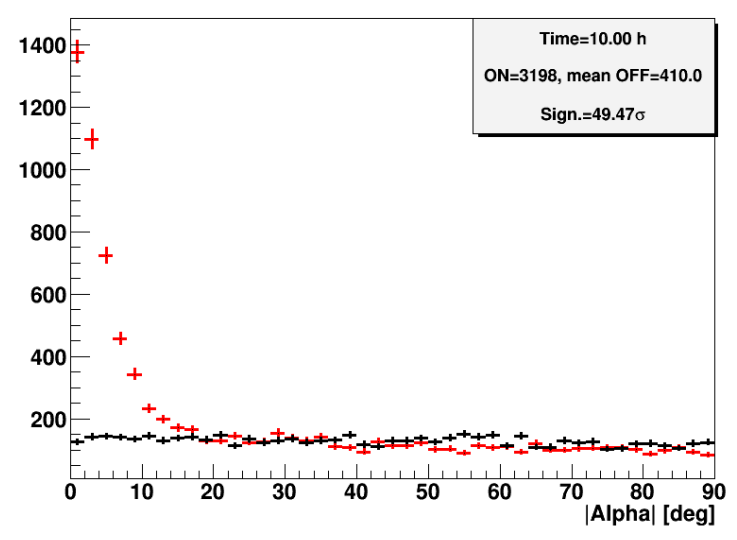

(b)

Figure 2.37: On the left: $\theta^{2}$ distributions for the Crab Nebula data with medium-to-high energy cuts (see Table 2.5). The red points correspond to ON events and the black points to OFF events, taken in wobble mode. On the right: Alpha distribution for a Crab Nebula sample taken with the stand-alone mode with MAGIC I.

information too. The assumption of the position allows to make use of more parameters, like the disp distance, to improve the $\gamma$ /hadron separation. The alpha plots are used for mono data analysis when the source coordinates are known. The standard signal plot for stereoscopic mode is however the $\theta^{2}$ plot, since the source-independent stereoscopic parameters represents a strong tool for the $\gamma /$ hadron separation. For the ON/OFF observations presented in this thesis (mono observations with MAGIC I), the alpha approach was used. Figure $2.37 \mathrm{~b}$ shows an example of the alpha distribution.

Once the number of excess events are known, the significance can be computed roughly by $\sigma=N_{\text {exc }} / \sqrt{N_{\text {off }}}$, a Gaussian approximation of Eq. 17 from Li \& Ma $(1983)$. Nevertheless, to report detection the latter is usually addressed:

$$
\sigma_{\text {LiMa }}=\sqrt{2\left(N_{\text {on }} \ln \left[\frac{1+\alpha}{\alpha}\left(\frac{N_{\text {on }}}{N_{\text {on }}-N_{\text {off }}}\right)\right]+N_{\text {off }} \ln \left[(1+\alpha)\left(\frac{N_{\text {off }}}{N_{\text {on }}-N_{\text {off }}}\right)\right]\right)}
$$

\subsubsection{Sensitivity}

The sensitivity is defined as the minimum flux that the telescopes can detect with $5 \sigma$ using the expression $\sigma=N_{e x c} / \sqrt{N_{o f f}}$ in 50 hours of observation. This flux is normally given in percentage of the Crab Nebula flux (C.U.). For a certain observation with $N_{e x c}$ and $N_{o f f}$ obtained for a time $t$, the significance at a time $t_{0}$ can be estimated as:

$$
\sigma\left(t_{0}\right)=\frac{N_{e x c}}{\sqrt{N_{\text {off }}}} \sqrt{\frac{t_{0}}{t}}
$$


Equation 2.15 evidences the relation between significance and observation time, $\sigma \propto \sqrt{t_{0}}$. One can then compute the sensitivity in terms of Crab Nebula flux by assuming the standard definition where $t_{0}=50$ hours and $5 \sigma$ detection:

$$
\text { Sensitivity }=\frac{5 \sigma}{\sigma(50)}
$$

In the same way as the flux, there are two ways to provide the sensitivity of an instrument: integral and differential sensitivity. The first one is obtained by applying the analysis cuts that gives the best sensitivity above a certain energy threshold. In the case of MAGIC, the best integral sensitivity, $0.66 \pm 0.03 \%$ C.U., is achieved above $220 \mathrm{GeV}$. Figure 2.38a shows the evolution of the MAGIC integral sensitivity as a function of the $E_{t h r}$ for different performances of the system. For the differential sensitivity, the cuts that provides the best sensitivity are searched for each energy bin. The pre- and post-upgraded MAGIC differential sensitivities are displayed in Figure $2.38 \mathrm{~b}$

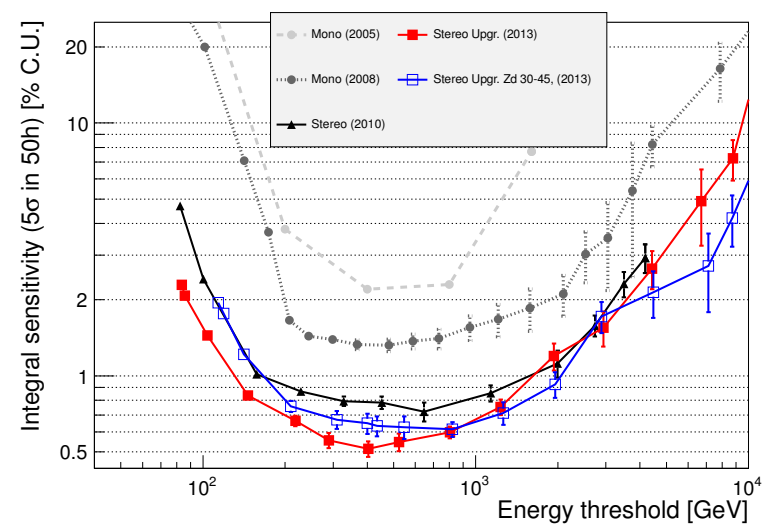

(a)

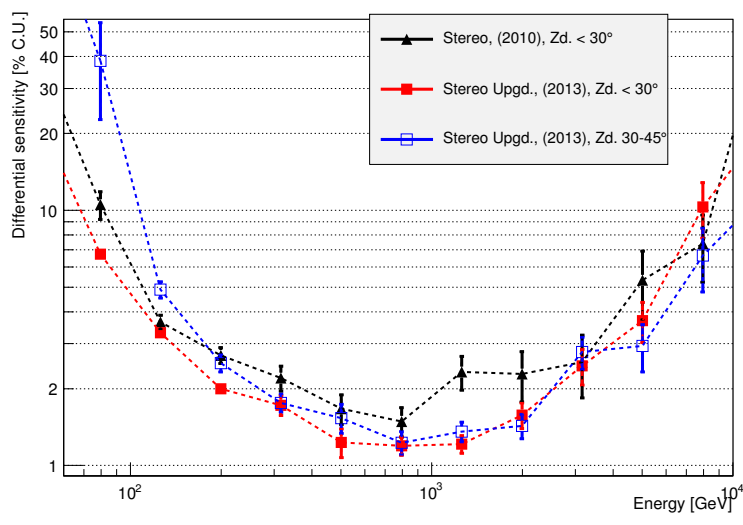

(b)

Figure 2.38: MAGIC integral sensitivity as a function of the energy threshold (left) and differential sensitivity as a function of the energy (right). Integral sensitivity for stand-alone observations with MAGIC I are presented for the Siegen (light grey) and MUX (dark grey) readouts. Black line represents the sensitivity for stereoscopic observations before the upgrade period. The red and blue lines correspond to the current performance of the system for low and medium Zd respectively. Plots from Aleksić et al. (2016b).

\subsubsection{Angular resolution}

Making use the Disp method, the arrival direction of the gamma rays can be determined more accurately. This allows us to improve the angular resolution. If we consider a 2-dimensional distribution of the reconstructed arrival direction, the angular resolution can be determined as the angle that contains $68 \%$ of the events. Usually, the angular resolution is named as $\mathrm{PSF}$, although, if specified, the latter can be considered as the angle that encloses the $39 \%$ of the events. Figure 2.39 presents the MAGIC angular resolution for stereo observations using DRS2 and DRS4. 
Currently, MAGIC obtain a good angular resolution of $\sim 0.10^{\circ}$ at a few hundred $\mathrm{GeV}$, even reaching $0.06^{\circ}$ in the $\mathrm{TeV}$ band.

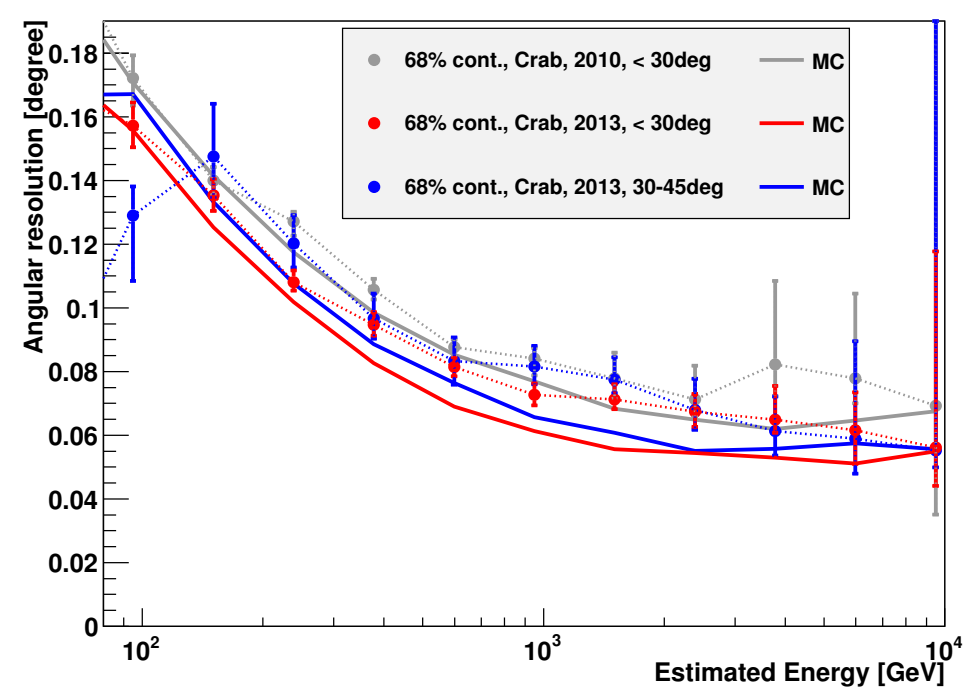

Figure 2.39: MAGIC angular resolution for stereoscopic observations before upgrade (grey line) and after upgrade (red and blue lines). Plot taken from Aleksić et al. (2016b).

\subsubsection{Skymaps}

The skymaps are obtained by reconstructing the arrival direction of each gamma ray-like events, transforming the camera coordinates into sky coordinates (normally into RA and Dec), filling a 2-dimensional histogram with this information and subtracting the background. This task is performed by Caspar in the MARS framework. The arrival direction are obtained by means of the Disp method, which prevent us to use source-dependent parameters and be bias in certain regions of the skymap.

Given the inhomogeneities of the camera, a key point when producing skymaps is to obtain reliable background histograms to subtract. This task becomes easy for the wobble mode observations: the background events are taken from the half of the camera where the source is not present. Thus, for two wobble positions, one would get two background histograms, one for each part of the camera. These two halves are then normalized by the time difference between them. Skymaps are usually given as excess histograms (by subtracting the background histograms to the gamma-like events histograms) or as TS skymaps, where $T S=\sqrt{\sigma_{\mathrm{LiMa}}}$. In both cases, the $\mathrm{ON}$ and OFF events histograms suffer a smoothing for two reasons: first, a density function that describes the events in each part of the camara (number of events in each point) given the camera inhomogeneity. This function in MAGIC is a Gaussian (Gaussian kernel), whose standard deviation can be varied to define the signal region (higher for extended sources). The skymap is also smearing according to the instrument $\mathrm{PSF}$ by a Gaussian distribution with standard deviation equal to the $\mathrm{PSF}, \sigma_{\text {Gauss }}=\mathrm{PSF}=0.1^{\circ}$. Thus, the total smoothing is:

$$
\sigma_{\text {smooth }}=\sqrt{\sigma_{P S F}^{2}+\sigma_{\text {Kernel }}^{2}}
$$


For point-like sources, the $\sigma_{\text {Kernel }}$ is usually equal to the PSF, and hence Equation 2.17kan be simplied as:

$$
\sigma_{\text {smooth }}=\sqrt{2} \cdot \sigma_{P S F}
$$

An example of a skymap after smoothing is provided in Figure 2.40

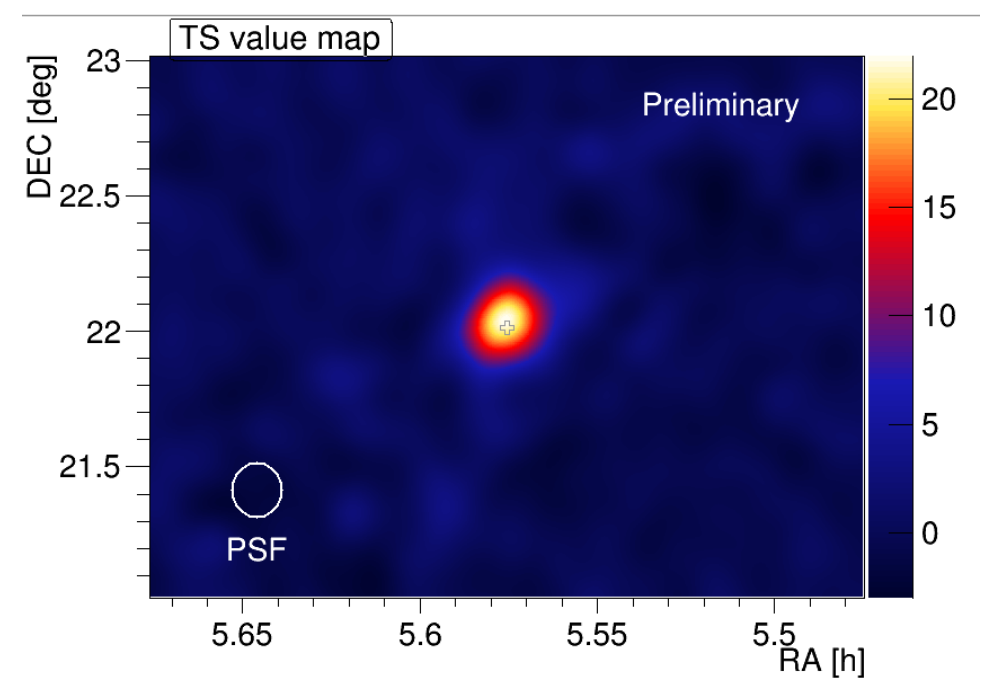

Figure 2.40: $\mathrm{TS}$ skymap of the Crab Nebula.

\subsubsection{Spectrum and Light Curve}

The differential gamma-ray energy spectrum is defined as the number of gamma rays per unit area, time and energy that we observe. Mathematically, it is given by:

$$
\frac{d \phi}{d E}=\frac{d N_{\gamma}(E)}{d E d A_{e f f}(E) d t_{e f f}}\left[\text { photons } \mathrm{TeV}^{-1} \mathrm{~cm}^{-2} \mathrm{~s}^{-1}\right]
$$

where $N_{\gamma}$ is the number of gamma rays in a certain energy range, $A_{\text {eff }}$ is the so-called effective or collection area and $t_{e f f}$ is the effective time. In the MAGIC framework, the computation of differential flux is performed by FLUX and Light Curve (Fluxlc), for mono observations, and by FLUx vs. Time and Energy (Flute), for stereoscopic ones.

The number of gamma rays, $N_{\gamma}$, corresponds to the excess events within an energy range, i.e. $N_{\gamma}=N_{\text {exc }}=N_{o n}-N_{o f f}$. The input to calculate this excess is the Melibea output, after all events passed through a the RF trained branches. Thus, Fluxlc and Flute take the events and perform $\theta^{2}$ (or alpha) and hadronness cuts for each energy bin to get the excess events. These cuts are commonly obtained by efficiency: we select a certain efficiency value for each variable, and the program changes the cut in each energy bin until the number of surviving events reaches the fixed efficiency. Usually, the hadronness efficiency is set to $90 \%$, while the $\theta^{2}$ efficiency is fixed at $75 \%$. These are looser cuts than those applied for the detection of the signal to guarantee 


\begin{tabular}{c|c|c|c}
\hline \hline \begin{tabular}{c|c|}
$\mathrm{NSB}$ \\
$\left(\times \mathrm{NSB}_{\text {dark }}\right)$
\end{tabular} & $\begin{array}{c}\text { Equivalent } \mathrm{DC} \\
{[\mu \mathrm{A}]}\end{array}$ & $\begin{array}{c}\text { Size cut } \\
{[\mathrm{HV}}\end{array}$ \\
\hline $1-2$ & $1.1-2.2$ & Standard & 50 \\
$2-3$ & $2.2-3.3$ & Standard & 80 \\
$3-5$ & $3.3-5.5$ & Standard & 110 \\
$5-8$ & $5.5-8.8$ & Standard & 150 \\
$5-8$ & $3.2-5.2$ & Reduced & 135 \\
$8-12$ & $5.2-7.8$ & Reduced & 170 \\
$12-18$ & $7.8-11.6$ & Reduced & 220 \\
$8-15$ & $2.2-5.0$ & Standard with UV-filters & 100 \\
$15-30$ & $5.0-8.3$ & Standard with UV-filters & 135 \\
\hline
\end{tabular}

Table 2.6: MAGIC size cuts for moon analysis.

a reliable collection area. The default size cut is set to 50 phe Nevertheless, when analyzing data under the effect of the Moon, an increased size cut is used to remove events at the lowest energies, which can be embedded in the NSB. This size cut is dependent on the moonlight level, as shown in Table 2.6 .

The collection area is the geometrical area around the telescopes where the gamma rays are detected. It is calculated with $\mathrm{MC}$ events, applying to the simulated area, $A_{\text {sim }}$, a factor to account for the detection efficiency. $A_{\text {sim }}$ is collection area of an ideal instrument that would detect all simulated events for a given energy and $\overline{\mathrm{Zd}}$ range, whose values according to $\mathrm{Zd}$ are listed in Table 2.7. Thus, $A_{\text {eff }}$ is given as:

$$
A_{e f f}(E)=A_{\text {sim }} \frac{N_{\text {surv }}(E)}{N_{\text {sim }}(E)}
$$

where $N_{\text {sim }}(E)$ are the simulated events for a certain energy range and $N_{\text {surv }}(E)$ are the number of events that survive the analysis cuts (hadronness, $\theta^{2}$, size ) for a given energy range. Usually, $\mathrm{MC}$ gammas rays are simulated with a power-law function, in the case of MAGIC with photon index $\Gamma=1.6$ and in CTA with $\Gamma=2.0$. This spectral function does not need to be the same as the one expected for the source and hence, events need to be weighted according to the source spectrum, as follows:

$$
N_{\text {weighted }}=\sum_{i=0}^{N_{\text {sim }}} \frac{f_{\text {source }}\left(E_{i}\right)}{f_{\text {sim }}\left(E_{i}\right)}
$$

where $N_{\text {weighted }}$ is the total number of events after weighting, $f_{\text {souce }}$ and $f_{\text {sim }}$ are the source and simulated spectra, respectively, and $E_{i}$ is the energy of the event $i$.

As mentioned before, cascades at large $\mathrm{Zd}$ needs to pass through larger atmosphere layer. The distance it follows up to the ground is $D^{\prime} \simeq D / \cos (Z d)$, leading to a illuminated collection 


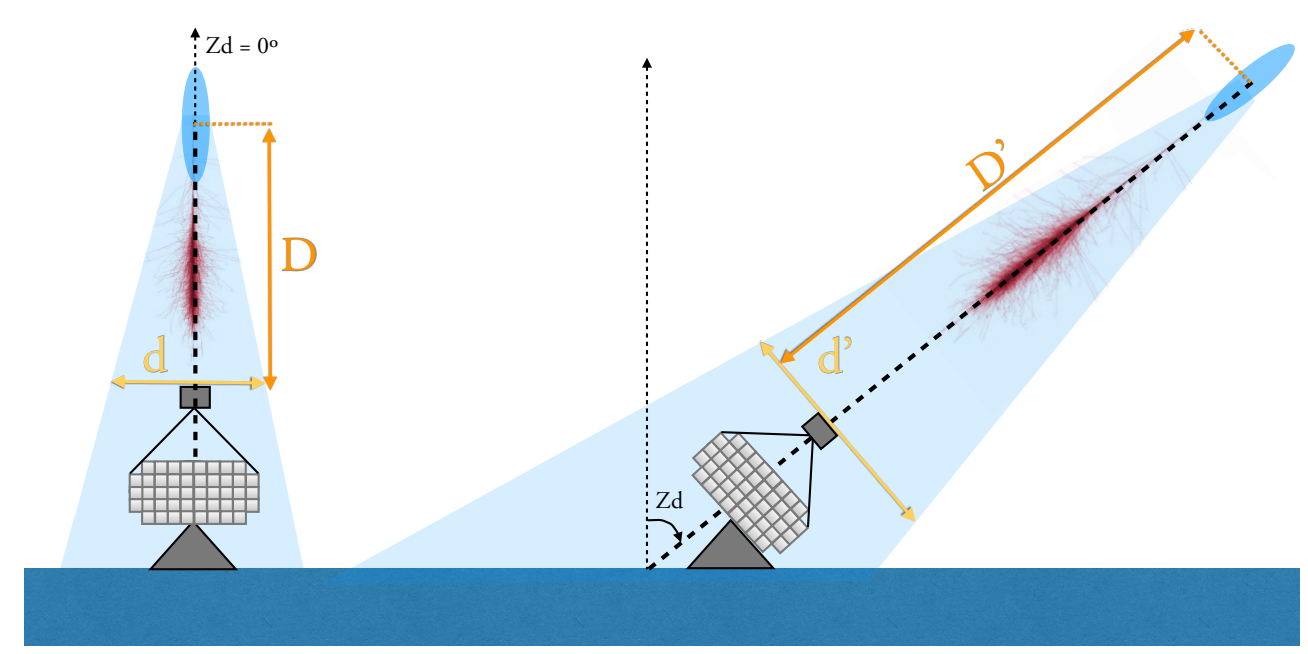

Figure 2.41: Scheme of shower development at low and high $\mathrm{Zd}$ angle, which evidences the larger Cherenkov light pool $\left(d^{\prime} \simeq d / \cos Z d\right)$ in the later.

area perpendicular to the detector of diameter $d^{\prime} \simeq d / \cos (Z d)$. Figure 2.41 shows a schematic view of these distances. Therefore, simulated collection areas are greater as higher the Zd is, as depicted in Table 2.7. The collection area before and after analysis cuts are shown in Figure 2.42a for stereoscopic observations. Figure $2.42 \mathrm{~b}$ gives a typical $A_{\text {eff }}$ for mono observations.

\begin{tabular}{|c|c|}
\hline \multicolumn{2}{|c|}{$\mathrm{Zd}$ range $\left[{ }^{\circ}\right] A_{\text {sim }}$ radius $[\mathrm{m}]$} \\
\hline $5-35$ & 350 \\
\hline $35-50$ & 500 \\
\hline $50-62$ & 700 \\
\hline $62-70$ & 1000 \\
\hline
\end{tabular}

Table 2.7: Radius of the simulated collection area, $A_{\text {sim }}$, in MAGIC for different $\mathrm{Zd}$ ranges.

The last parameter to be calculated in order to compute the spectrum is the effective time. The effective time of a source is not identical to the elapsed time between the beginning and end of observations, given the deadtime after storing each event and some gaps during the data taking (e.g., between runs). The time difference between the arrival time of an event and the next one is $\Delta t$, which follows a Poissonian distribution with stable rate $\lambda$ (without assuming deadtime):

$$
P(n, t)=\frac{(\lambda t)^{n}}{n !} \cdot e^{-\lambda t}
$$

which is the probability of observing $n$ events in a time $t$ with a rate of $\lambda$. By definition, the probability that the consecutive event arrives after a time $t$ is the same as observing 0 events before that time:

$$
P\left(t_{\text {next }}>t\right)=P(0, t)=e^{-\lambda t}
$$




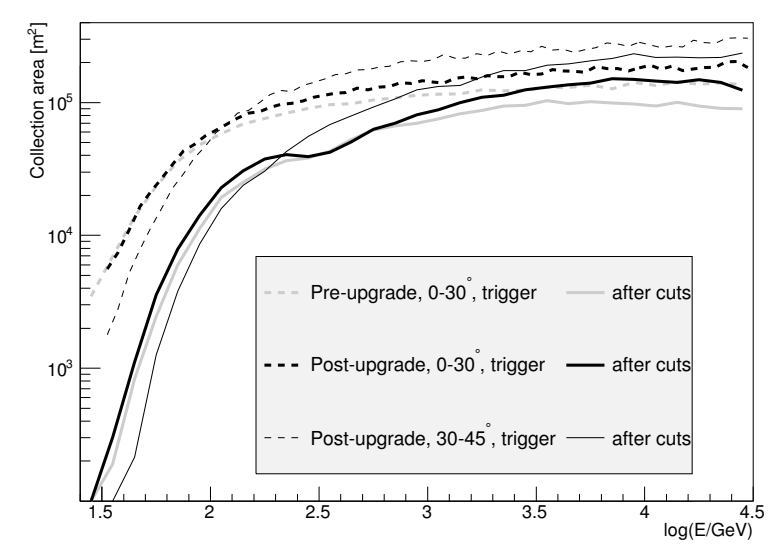

(a)

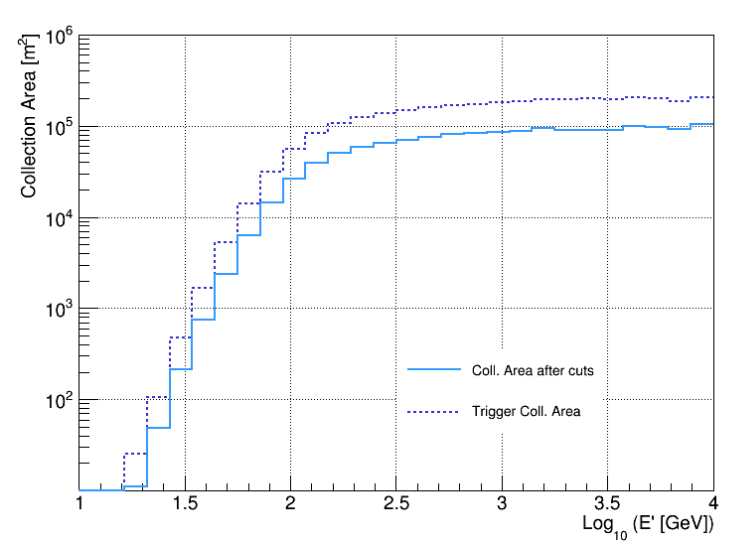

(b)

Figure 2.42: On the left: Collection area before (dashed lines) and after (solid lines) analysis cuts for stereoscopic observations, taken from Aleksić et al. (2016b). On the right: Typical collection area as a function of the energy for mono observations.

The probability $P\left(t_{\text {next }}>t\right)$ is as well defined as:

$$
P\left(t_{\text {next }}>t\right)=\int_{t}^{\infty} \frac{d P\left(t_{\text {next }}=t\right)}{d t} d t
$$

and therefore:

$$
\frac{d P\left(t_{\text {next }}=t\right)}{d t}=\lambda e^{-\lambda t}
$$

Thus, the event rate is the product of the time evolution distribution (Equation 2.25) and the stored events $N_{0}$ :

$$
\frac{d N}{d t}=N_{0} \lambda e^{-\lambda t}
$$

Now if we consider the deadtime $(d)$, the fraction of lost events is $\lambda \cdot d$ and hence, the event rate would be:

$$
\frac{d N}{d t}=N_{d, 0} \lambda e^{-\lambda(t-d)}
$$

Taken into account that the deadtime is fixed for a given observation, the distribution of the time differences of triggered events $\left(N_{d, 0}\right)$ is still exponential with a slope $\lambda$. We can get then the true rate of events, $\lambda$, by fitting an exponential to the distribution. The effective time is easily obtained dividing the number of triggered events by the true rate of events: $t_{\text {eff }}=N_{d, 0} / \lambda$. Figure 2.43a shows a scheme of the time difference distribution for all and triggered events, while Figure $2.43 \mathrm{~b}$ presents a fit on real stereoscopic data with DRS4 (deadtime of $26 \mu \mathrm{s}$ ):

It is as well common to provide SED, defined as:

$$
E^{2} \frac{d \phi}{d E}=\frac{d N_{\gamma}(E)}{d E d A_{e f f}(E) d t_{e f f}} \cdot E^{2}\left[\text { photons } \mathrm{TeVcm}^{-2} \mathrm{~s}^{-1}\right]
$$




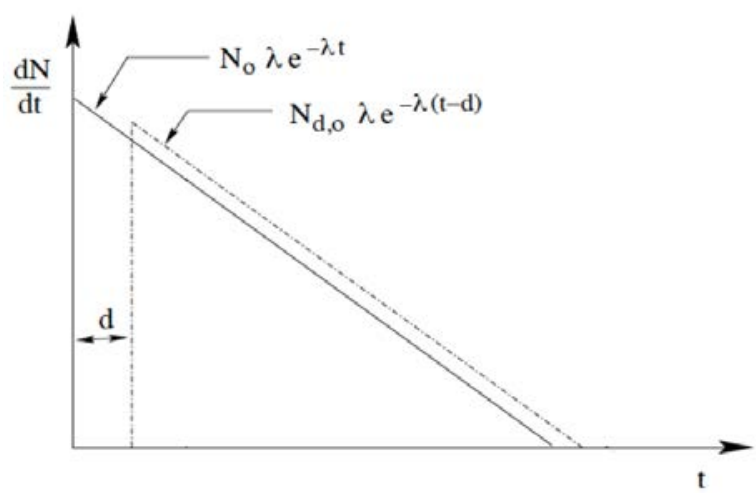

(a)

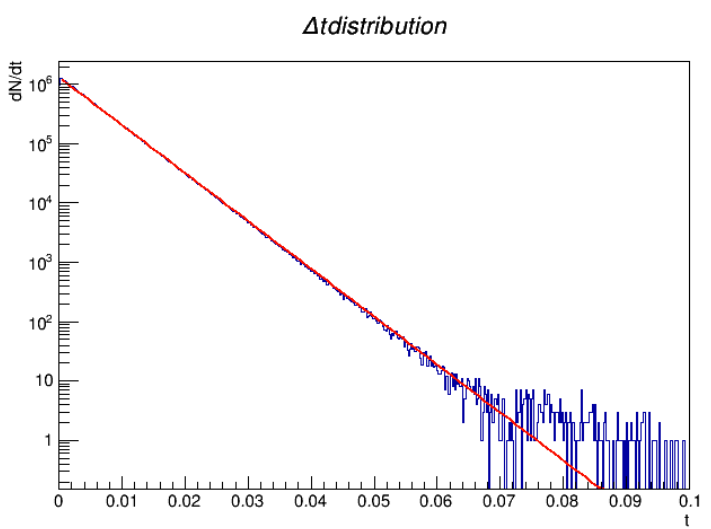

(b)

Figure 2.43: On the left: Scheme of the distribution of time differences for an ideal case in which all arrival events are observed (solid line) and taken into account a deadtime d (dashed line). On the right: Distribution of time differences between triggered events. The fits provides the real rate events with which effective time is calculated.

which is normally used to study broadband spectra, since it shows the relative contribution of each wavelength to the total energy released by the source.

On the other hand, the flux can also be given in time intervals, which are the so-called light curves. In this case, the flux is not integrated within a certain energy range, but given from a threshold $E_{0}$ in each time interval:

$$
\phi_{E>E_{0}}(t)=\int_{E 0}^{\infty} \frac{d N_{\gamma}(t)}{d E d A_{e f f}(t) d t_{e f f}(t)} d E\left[\text { photonscm }^{-2} \mathrm{~s}^{-1}\right]
$$

For the computation of the light curve, the number of excess events is obtained from each time interval separately. In the same way, $A_{e f f}$ is as well get separately taking into account the Zd distribution in each interval.

\subsubsection{Unfolding spectrum}

The detectors are not ideal and hence, physical quantities are systematically distorted. This affects specially the calculation of the spectra, where an estimated energy is measured instead of the true energy, given the finite energy resolution of the instrument (mismatching between $E_{\text {true }}$ and $\left.E_{\text {est }}\right)$. Taking into account the instrument response function, the relation between $E_{\text {true }}$ and $E_{\text {est }}$ can be defined as:

$$
Y\left(E_{\text {est }}\right)=\int M\left(E_{\text {est }}, E_{\text {true }}\right) \cdot X\left(E_{\text {true }}\right) d E_{\text {true }}+B\left(E_{\text {est }}\right)
$$

where $M\left(E_{\text {est }}, E_{\text {true }}\right)$ is the so-called migration matrix which provides the detector response, $Y\left(E_{\text {est }}\right)$ is the measure distribution of energies and $X\left(E_{\text {true }}\right)$ is the true distribution or intrinsic 
distribution from the source. $B\left(E_{\text {est }}\right)$ accounts for possible background that affects the measurements. Equation 2.30 can be also be expressed in bins of $E_{\text {est }}(\mathrm{i})$ and $E_{\text {true }}(\mathrm{j})$ :

$$
Y_{i}=\sum_{j} M_{i, j} \cdot X_{j}+B_{i}
$$

The goal is therefore to determine $X\left(E_{\text {true }}\right)$ knowing $Y\left(E_{\text {est }}\right)$ and $M\left(E_{\text {est }}, E_{\text {true }}\right)$. The problem is that the migration matrix represents the probability that an event with $E_{\text {true }}$ in bin $j$ is classified as an event with $E_{\text {est }}$ in bin $i$, and then we would need an inverted matrix. To solve this we use unfolding methods with regularization, based on the least square minimization method. Thus, the aim is to find the $E_{e s t}$ and $E_{\text {true }}$ that minimize the value $\chi_{0}^{2}$, which represents the degree of agreement between $Y\left(E_{\text {est }}\right)$ and $M\left(E_{\text {est }}, E_{\text {true }}\right) \cdot X\left(E_{\text {true }}\right)$ :

$$
\chi^{2}=\chi_{0}^{2}+\frac{\omega}{2} \chi_{0}^{2}+\operatorname{Reg}
$$

where Reg is a regularization term to avoid unstable results and $\omega$ is the inverse of the Reg strength. MAGIC uses different methods to determine this Reg parameter, described in Schmelling (1994); Bertero (1989); Tikhonov \& Arsenin (1977).

There is another approach, called forward unfolding. In this case, a distribution of $X\left(E_{\text {true }}\right)$ is assumed and a comparison between $Y\left(E_{\text {est }}\right)$ and $M\left(E_{\text {est }}, E_{\text {true }}\right)$ is done. Here, no Reg parameter is needed. The result of the forward unfolding is the best fit given the assumed parameters.

MAGIC normally makes use of the latter approach. However, in case spectral points are wanted to be given, the previous methods are needed.

\subsubsection{Upper limits}

If no signal is found from the source, $\mathrm{UL}$ on the flux are calculated. To compute the UL we make use, as before, of the excess event, $N_{\text {exc }}$, and the number of background events, $N_{\text {backg }}$, and we assume a C.L. and a certain systematic error. Thus, we can calculate the $N_{U L}$, the maximum number of expected gamma rays events, usually with the Rolke et al. (2005) method. In gammaray astronomy, it is common to use a $95 \%$ C.L. and particularly in MAGIC the systematic uncertainty assumed is 30\% (see Section 2.4.3.16). The spectral shape of the source has to be also defined. If no further information is available, a power-law function with photon index $\Gamma=2.6$ (Crab-like spectrum) is usually assumed. The flux of the non-detected source is then defined as:

$$
\phi(E)=K \cdot S(E)=K \cdot\left(\frac{E}{E_{0}}\right)^{-\Gamma}
$$

and therefore, the integral flux above $E_{0}$ would be:

$$
\int_{E_{0}}^{\infty} \phi(E) d E=K \int_{E 0}^{\infty} S(E) d E=\frac{N_{U L}}{\int_{E 0}^{\infty} \int_{0}^{t_{e f f}} A_{e f f}(E) d E d t}
$$

where $t_{\text {eff }}$ is the effective time of the observation. The UL on the integral flux can be obtained easily from the above equation:

$$
K_{U L}<\frac{N_{U L}}{t_{e f f} \int_{E 0}^{\infty} S(E) A_{e f f}(E) d E}\left[\text { photonscm }^{-2} \mathrm{~s}^{-1}\right]
$$




\subsubsection{Systematic uncertainties}

The measurement of the gamma-ray spectra and light curves are affected by systematic uncertainties, summarized below:

1. The following systematic errors affect the estimation of the gamma-ray energy:

$\odot$ Fluctuations in the atmospheric conditions: The atmospheric MagicWinter model used in the MC simulations does not account from nightly basis changes in humidity, temperature, cloudiness or calima (fine sand arriving from the Sahara Desert). There is also a $\sim 15 \%$ variation in the atmospheric transmission from Summer to Winter. In a run-to-run basis the uncertainty was estimated to be $\sim 11 \%$ (Aleksić et al. 2016b).

$\odot$ Light losses: Dust in the mirrors or a wrongly alignment at the beginning of the night (as well as a not accurate enough bending model) lead to light losses. This is estimated to add $\sim 10 \%$ on the systematic uncertainties. Dust can also affect the entrance of the light in the PMTs and Winston cones, whose uncertainty is estimated to be $5 \%$.

$\odot$ Signal extraction: The sliding method or method induce an error on the number of phe. This uncertainty is small, estimated below $3 \%$.

$\odot$ F-factor method: The method used to calibrate the signal produces a systematic uncertainty of $10 \%$.

$\odot$ PMT performance and flat-fielding: The PMTs have temperature-dependent gain, which induces $2 \%$ error. Moreover, there are uncertainties in their QE (around 4\%) and in the collection efficiency of the first dynode $(\sim 5 \%)$. On the other hand, flatfielding is performed with only one wavelength and it is temperature-dependent as well. This produces $6-8 \%$ uncertainty at low energies and less than $2 \%$ at energies greater than $300 \mathrm{GeV}$.

2. Below, there are listed the systematics that affects the flux level.

$\odot \mathrm{MC}$ and data agreement: Difference between the $\mathrm{MC}$ simulated gamma rays and real gamma rays leads to an error of the $\gamma$ /hadron separation efficiency, which in turn, affects the calculation of the collection area.

$\odot$ Background estimation: Dead pixels, dispersion on the PMT response or stars in the FoV can induce an error of $10-15 \%$. This systematic is reduced to $\sim 1 \%$ when using wobble pointing mode for the post-upgrade period and to $\sim 2 \%$ before this. On the side, the background estimation uncertainty is larger as lower the energy is, so it affects mostly weak sources, where the difference between $N_{o n}$ and $N_{o f f}$ (signal-tobackground ratio) is not that accentuated. In the latter case, the systematic can reach $\sim 20 \%$ and, given that is energy-dependent, it can also affects the spectral index.

$\odot$ Telescopes mispoiting: If telescopes point to different position, the computation of stereo parameters used in the analysis worsen. The typical mispointing is less than 
$0.02^{\circ}$ which lead to a systematic uncertainty on the gamma-ray efficiency around $4 \%$ (Aleksić et al. 2016b).

$\odot$ Higher NSB levels: The higher the NSB, more difficult to determine the image parameters due to fluctuations in the images. This can produce $<4 \%$.

To account for all these effects, an average $30 \%$ of systematic uncertainty is included in the Rolke et al. (2005) method to compute flux or UL.

3. Finally, there is uncertainties on the spectral slope:

$\odot$ Analysis cuts and methods: The different approach made in the unfolding methods or efficiencies used in the analysis can induces an error of 0.01 in the photon index.

\subsection{CTA}

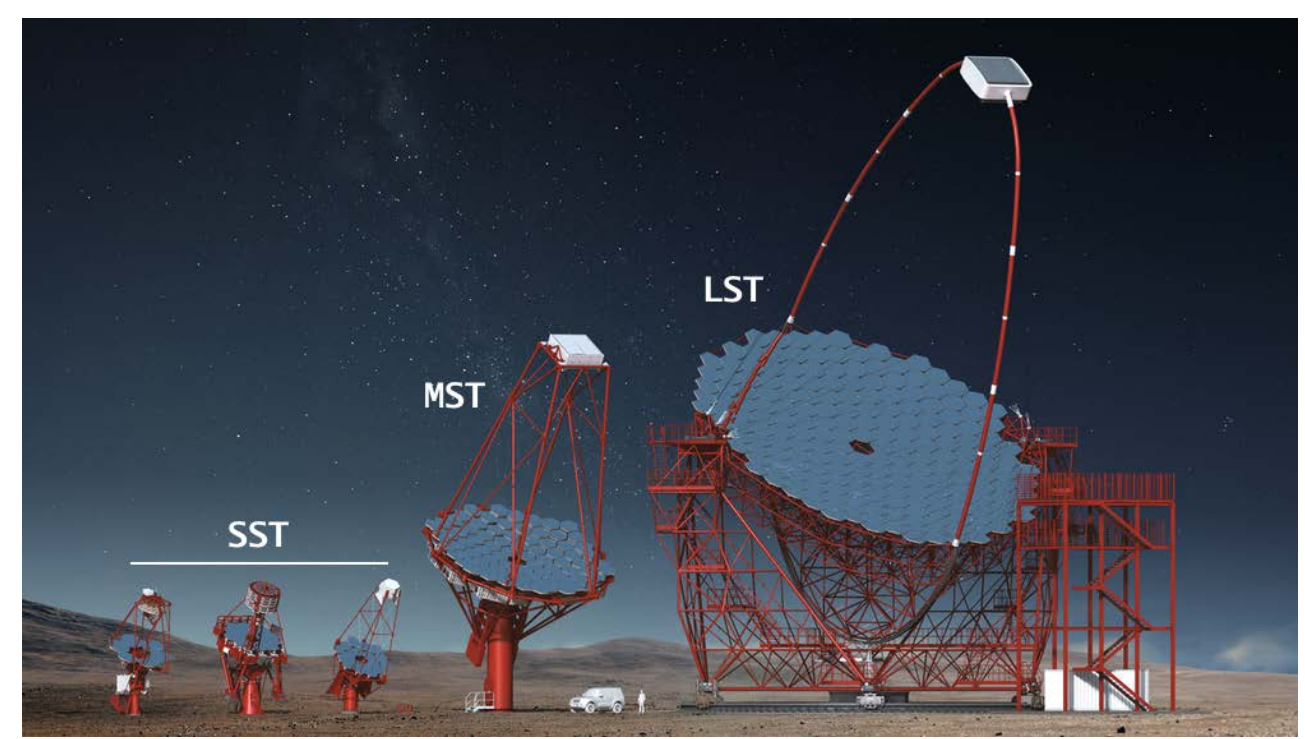

Figure 2.44: CTA prototypes designs. From left to right: Three different Small Size Telescope (SST), Medium Size Telescope (MST) and LST. Picture taken from https://www.cta-observatory.org.

CTA is the future generation of ground-base observatory to study the VHE gamma rays. It is planned to have more than 100 IACTs distributed between the Northern and Southern hemispheres. The Northern array will be located in La Palma, at El Roque de los Muchachos along with the MAGIC telescopes. The Southern one will be placed in the Paranal Observatory (Chile). The proposed array layouts are presented in Figure 2.45. The telescopes are divided into three types (see Figure 2.44), which in turn correspond to different working sub-groups within the Collaboration:

$\odot$ LST: The LST sub-group consists on more than 100 scientist from eight countries (Brazil, France, Germany, India, Italy, Japan, Spain and Sweden). These telescopes are designed 
to achieve the lowest energies, down to $20 \mathrm{GeV}$. The planned baseline for CTA includes 4 LST in each hemisphere. They have a parabolic reflector of $23 \mathrm{~m}$ diameter held by tubular structure made by carbon fiber and steel tubes. The light-structure, despite its $45 \mathrm{~m}$ height, allows the telescope to re-pointing within $20 \mathrm{~s}$. With this fast re-positioning and low energy threshold, LST is thought to delve into galactic transients, GRB, high redshift Active Galactic Nuclei (AGN) studies and, in general, low-energy dominated sources. The LST camera weight less than two tonnes and it is composed by $18550.1^{\circ}$ FoV $\mid$ PMTs, grouped in 265 clusters, that provides a total $\overline{\text { FoV }}$ of $4.5^{\circ}$. The PMTs present $\overline{\mathrm{QE}}$ of $42 \%$ and are equipped with light concentrators. The readout is performed by DRS4 chips. Deeper information regarding the LST prototype camera, its characterization and quality control, can be found in Chapter $\mathrm{X}$ of this thesis. This prototype is expected to be fully installed in La Palma by mid-2017. During commissioning, structure and camera will be evaluated to modify the design of the next LST, if needed. After the commissioning phase, the prototype will be part of the Northern hemisphere array.

$\odot$ MST: The MST collaboration is formed by scientist from six countries: Brazil, France, Germany, Poland, Switzerland and the United States. The best sensitivity is achieved in an energy range of $100 \mathrm{GeV}$ and $10 \mathrm{TeV}$, therefore they are designed to cover mid-energies. In total, CTA will host 40 MST, 25 in the Southern hemisphere and 15 in the Northern. The telescopes have a modified Davies-Cotton reflector of $12 \mathrm{~m}$ diameter with $16 \mathrm{~m}$ focal, held by a polar mount. Two pixelized cameras are designed for the MST NectarCAM and FlashCAM. The former shares many characteristics with the one used in the LST. Currently, a MST prototype (without the final camera and readout) is installed in Berlin for testing purposes.

$\odot$ SST: They are designed to cover an energy range between few TeV and $300 \mathrm{TeV}$, increasing CTA sensitivity at the highest energies. A total of 70 SST will be installed only in the Southern hemisphere. Three different prototypes were tested: one single-mirror design (SST-1M, with the collaboration of Poland and Switzerland) and two dual-mirror designs SST-2M ASTRI, in which Brazil, Italy and South Africa are involved, and SST-2M GCT, with Australia, France, Germany, Japan, the Netherlands, the United Kingdom and the United States). All of them have $4 \mathrm{~m}$ diameter reflector dish and around $9^{\circ} \mathrm{FoV}$.

The scientific goals for CTA can be split into three categories:

1. Study the origin of $\mathrm{CR}$

2. Delve into the understanding of extreme particle acceleration inside pulsars, PWNe, jets, SNRS..

3. Study the nature of dark matter and get deeper into physics beyond the Standard Model.

For specific information on the science planed with CTA, I refer the reader to Acharya et al. (2013). 

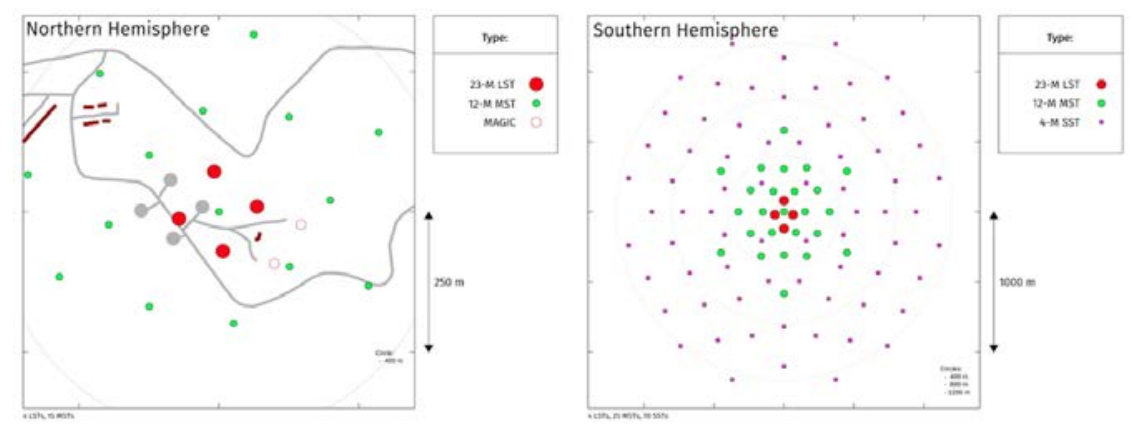

Figure 2.45: Planned CTA layouts, taken from https://www.cta-observatory.org.

\subsection{Other detectors}

\subsubsection{HAWC}

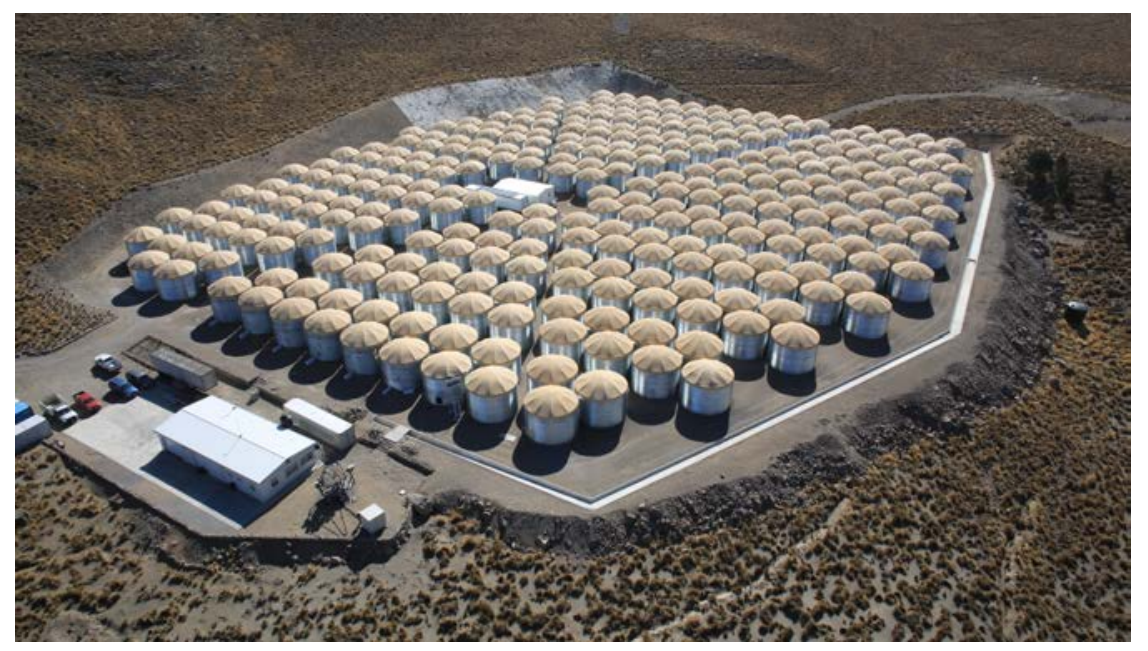

Figure 2.46: HAWC Observatory. Credit: http://www.hawc-observatory.org/.

High Altitude Water Cherenkov (HAWC) Observatory is the second generation of groundbased detectors which applies the Water Cherenkov technique to study the $\mathrm{TeV}$ gamma-ray regime (see Section 1.2.4). It is located in Sierra Negra, Mexico $\left(19.0^{\circ} \mathrm{N}, 97.3^{\circ} \mathrm{W}, 4100 \mathrm{~m}\right.$ a.s.1.), and it is the successor to the Milagro gamma-ray Observatory. The current system, inaugurated in March 2015, is comprised of 300 Water Cherenkov Detector (WCD) over an area of 22,000 $\mathrm{m}^{2}$. Nevertheless, science operation started in August 2013, with a configuration of approximately one-third of the array (111 tanks; HAWC-111). HAWC operates with $>95 \%$ duty cycle with a large instantaneous FoV] of $15 \%$ of the sky, which allows it to scan two-thirds of sky every 24 hours. Large effective area and duty cycle converts HAWC in an optimal instrument to perform survey studies on TeV sources. The dimensions of each tank are $7.3 \mathrm{~m}$ diameter and $5 \mathrm{~m}$ in height, and attached to the bottom four PMTs; one high-QE 10-inch Hamamatsu R7081-MOD 
placed in the center and three 8-inch Hamamatsu R5912 PMTs. Given its high altitude, HAWC can achieve the largest gamma-ray energies, from $\sim 100 \mathrm{GeV}$ to $\sim 100 \mathrm{TeV}$. However, lower energy photons, although they can be detected, are much difficult to discriminate from the background events. In this section, I will briefly comment on the energy and angular resolution and sensitivity of the detector. Nevertheless, the performance of the instrument is deeply presented in Abeysekara et al. (2017a), and the reader is referred to that work for further information.
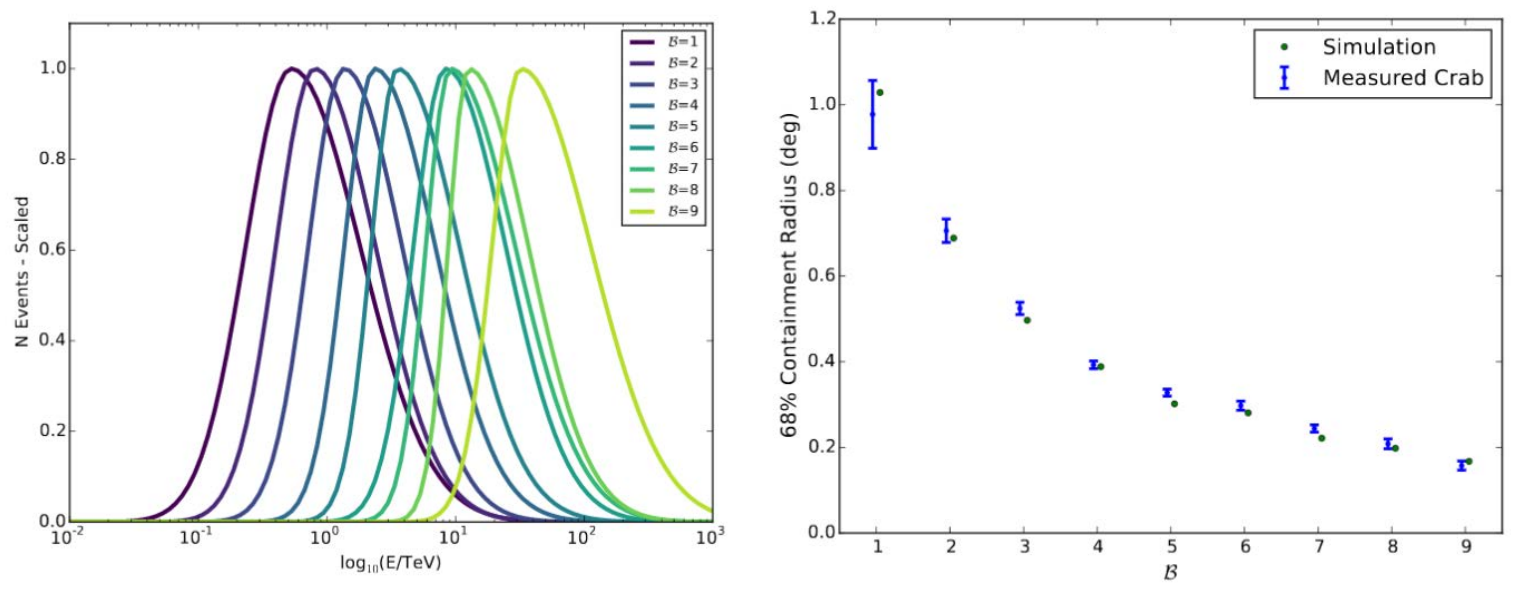

Figure 2.47: On the left: $\mathrm{HAWC}$ true energy distribution, split into 9 fractional hit bins (bin 1 corresponds to the ratio with less PMTs hit and bin 9 with the most). The plot is computed for a source with power-law spectrum of index -2.63 and at a declination angle of $+20^{\circ}$. At high $\mathrm{Zd}$ angles the image reconstruction becomes more difficult and the energy resolution worsen, leading to the broadening of the peaks. On the right: HAWC angular resolution (for the 68\% containment) as a function of each 9 bins. Images taken from Abeysekara et al. (2017a).

The data is divided according to the size of the event (ratio between the number of PMTs hit and the total number of PMTs operating) in 9 bins, where bin 1 implies less PMTs with signal and 9 corresponds to the maximum (see relation between energy bins and true energy in Figure 2.47a). Given the PMT signal and arrival time, the core and direction of the cascade for each event are computed, whose errors increase at lower energies and large impact parameter from the detector. A good core fitter is crucial in HAWC to obtain, not only the reconstructed direction of the cascade, but also a good $\gamma /$ hadron separation. The hadronic cascades produce a signal more randomly distributed all over the detector than the EM showers: while hadronic showers, a part from signal in tanks close to the impact point, leave signal in PMTs located far away, the gamma ray-induced showers are homogeneity and compact around the core. Thus, HAWC makes use of this information to discriminate the nature of the EAS through two parameters, known as compactness and clumpiness of the event. The compactness is the search of high signals outside of an $40 \mathrm{~m}$ radius area centered in the core of the cascade. The presence of such signals far away from the core position reveals the presence of muons and hence, the hadronic origin of the shower. On the other hand, the clumpliness is a type of $\chi^{2}$ fit to the lateral distribution of the cascade. A good fit is expected in case of EM showers, while hadronic, wider cascades, lead to 
failed fits. In Figure 2.48, the sensitivity of HAWC is shown. Finally, a fit to the arrival time of the PMTs is performed in order to get the most probable shower front plane projection, which leads to the direction of the gamma ray and source. The angular resolution is therefore energydependent, as depicted in Figure 2.47b, as more energetic cascades permit to compute a better fit. It ranges from $0.18^{\circ}$ to $1.0^{\circ}$.

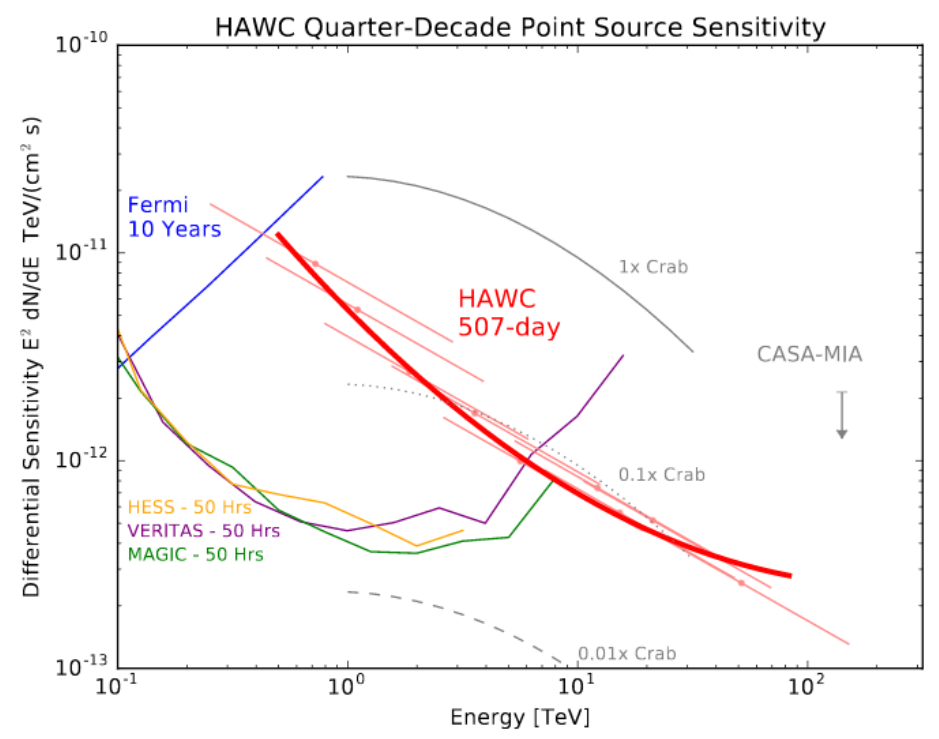

Figure 2.48: $\mathrm{HAWC}$ sensitivity assuming a differential energy spectrum of $E^{-2.63}$. Observations of 507 days corresponds to approximately 3000 hours. Taken from Abeysekara et al. (2017a).

\subsubsection{Fermi-LAT}

\subsubsection{Performance and analysis}

The Fermi Gamma-ray Space Telescope (previously known as GLAST) is a satellite that studies the gamma-ray sky between $8 \mathrm{keV}$ and $\sim 500 \mathrm{GeV}$. The satellite is formed by two instruments: Large Area Telescope (LAT) $(\sim 20 \mathrm{MeV}$ to $\sim 500 \mathrm{GeV}$ ), and GLAST Burst Monitor (GBM) (8 $\mathrm{keV}$ to $\sim 30 \mathrm{MeV}$ ), being the former the one from which I analyzed data in this thesis.

LAT covers a $2 \mathrm{sr}$ FoV, which allows it to observe $20 \%$ of the sky at any moment. It can observe in two modes, the sky-survey mode that covers the entire sky every three hours, and the pointing mode during which the satellite is observing a certain source. It is composed by four subsystems: tracker, calorimeter, anticoincidence detector and data acquisition system. When a particle enters in the LAT, the first interaction occurs in the anticoincidence detector. This device is in charge of rejecting hadronic events. Charge particles produce flashes of light in the anticoincidence detector, which sends a veto signal to the data acquisition system to avoid saving the event. The rejection efficiency reaches $99.97 \%$. The software of data acquisition system in LAT also discriminates events based on the arrival direction to avoid, for example storing gamma rays coming from the Earth's atmosphere. If the primary particle is a gamma ray, it 
passes through the anticoincidence detector without any effect and interacts in one of the 16 thin tungsten sheets placed in the tracker. The interaction gives rise to an electron and positron due to pair production. Given that the energy of the gamma ray is much larger than the rest mass of the electron and positron, they will keep the same track direction as the primary gamma. Thus, the reconstruction arrival of the gamma ray can be computed. Finally, the electron and positron reach the calorimeter that provides their energies, obtaining in this way the energy of the incident gamma ray.

The response of Fermi-LAT to gamma rays of a certain energy and arrival direction (in instrument coordinates) is defined by the IRFs. Here, I will briefly comment on the Pass 8 Release 2 Version 6 (P8R2-V6) data, which was the one analyzed for this thesis. For the analysis, we select events from a certain event class, which includes a set of unique response functions. Formerly, the event class was just divided into two event types: FRONT and BACK events, accounting from the location of the tracker in which the pair production takes place. From the top of the instrument, the tracker device is formed by 12 layers with $3 \%$ radiation length converters (the so-called FRONT or thin section), followed by 4 layers of $18 \%$ radiation length (BACK or thick section). Multiple-scattering is more probable to happen in thicker regions and hence, the angular resolution is better (approximately by a factor of two) on FRONT photons. FRONT and BACK events present different IRF. With the Pass 8 release, two new partitions of the event type were possible:

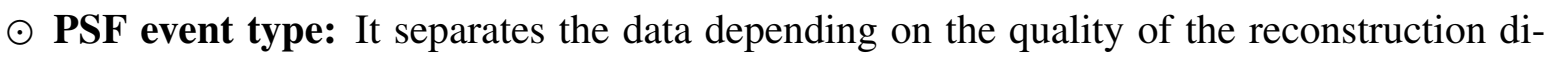
rection (and hence PSF. Data is divided in four quartiles, where $\mathrm{PSF}$, corresponds to the poorest quality and $\mathrm{PSF}_{3}$ to the highest.

$\odot$ EDISP event type: In the same way as for the $\mathrm{PSF}$, the four quartiles in this case represents the quality of the energy reconstruction.

Each quartile has its own IRFs. In Figure 2.49 the effective area, $\mathrm{PSF}$, energy resolution and sensitivity as a function of energy is show for FRONT and BACK events. For comparison, it is also displayed the result from using both FRONT+BACK.

Data format ${ }^{1}$ provided by Fermi-LAT are event lists, to which a selection criteria that best fit to a specific scientific purpose are applied. First of all, cuts based on position, time, energy range, $\mathrm{RoI}$, event class or type or maximum $\mathrm{Zd}$ are computed to set up the data sample to highlevel analysis. Some of these cuts are already recommended by the Fermi-LAT Collaboration, while others are subjective and depend on each analysis. Among the recommended ones, it is worth to highlight the so-called in the Fermi-LAT nomenclature evclass $=128$, which includes only those events with high probability of being photons. There is also a maximum cut in zenith angle, $\mathrm{Zd}=90^{\circ}$, to avoid contamination from atmospheric gammas coming from the Earth's limb (that arrives from angles at $\sim 110^{\circ}$ ). The latter becomes more important for weak sources. These selection of events is performed by the program gtselect. Cuts on time intervals can be also applied. Usually they are computed to remove data from periods in which the spacecraft

${ }^{1}$ Fermi-LAT data is publicly available at the Science Support Center, https://fermi.gsfc.nasa.gov/cgibin/ssc/LAT/LATDataQuery.cgi. 


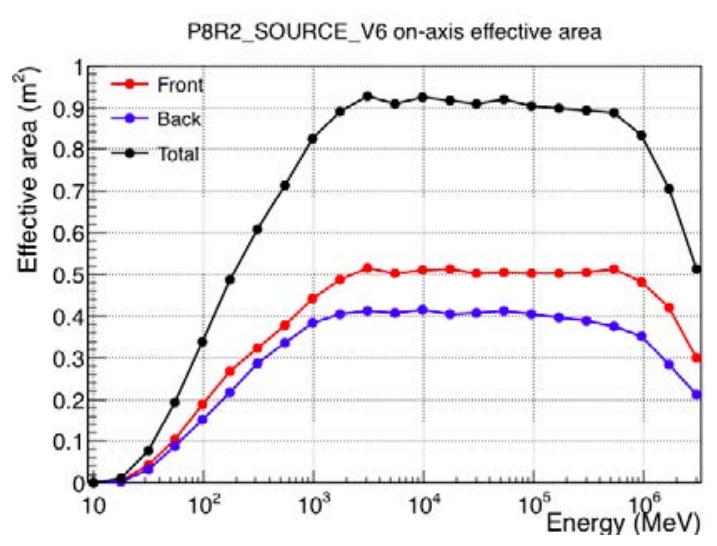

(a) Fermi-LAT collection area.

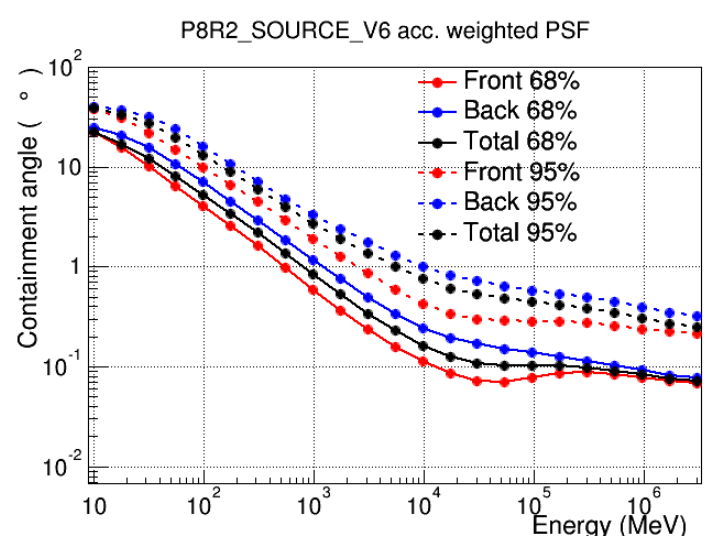

(b) Fermi-LAT PSF

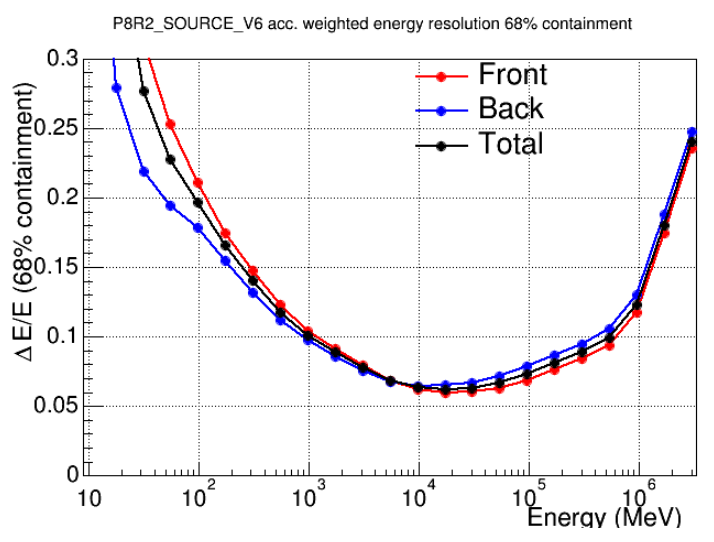

(c) Fermi-LAT energy resolution.

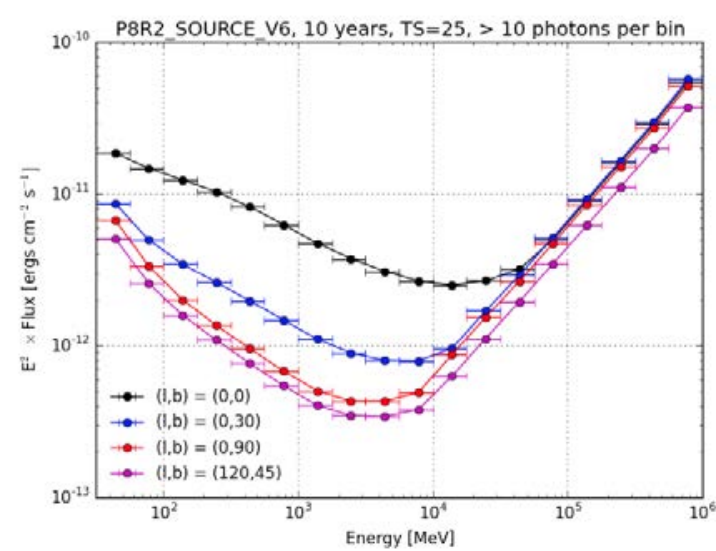

(d) Fermi LAT sensitivity.

Figure 2.49: Fermi-LAT IRF and performance. 
presented problems. The recommended cut to do so is DATA_QUAL $>0 \& \&$ LAT_CONFIG $==1$ and is done by means of gtmktime.

After the data selection, one can obtain the counts map (CMAP) and exposure maps (the latter is needed for further steps of the analysis). The CMAPs represent the number of counts for each spatial bin, whose size (typically $0.2^{\circ}$ /bin) is defined by the analyzer.

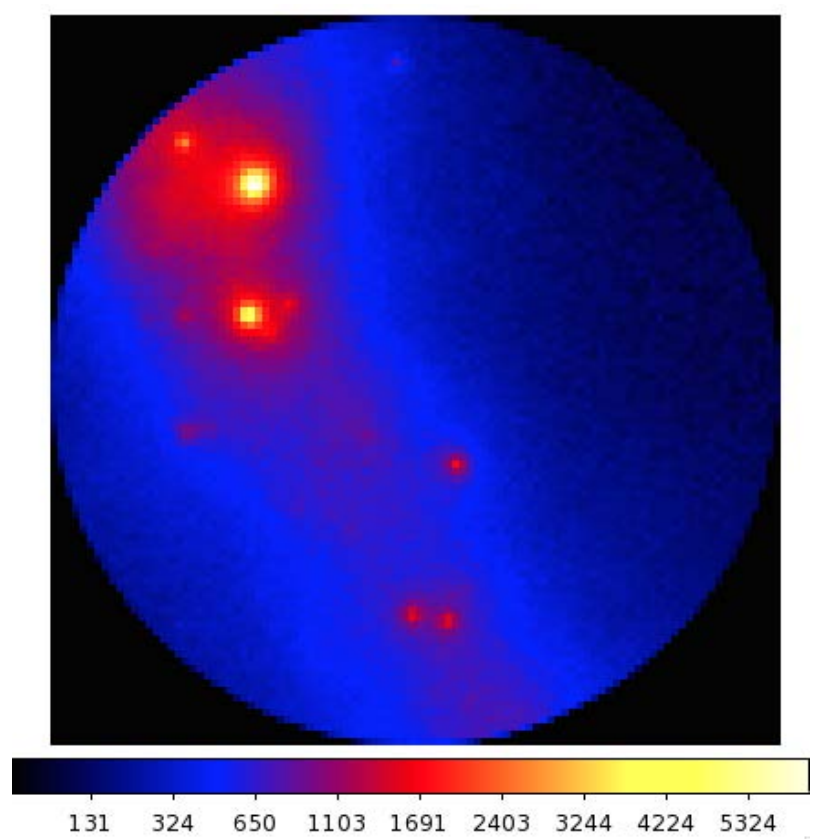

Figure 2.50: Example of a Fermi-LAT CMAP (between $100 \mathrm{MeV}$ and $500 \mathrm{GeV}$ ) centered in Cygnus X-1.
The count map for the entire energy range (see Figure 2.50) is useful to check any failed cut, as inconsistent structures could appear in the FoV in that case. The likelihood method, used later on in the Fermi-LAT analysis, needs a 3-D CMAP. This is simply understood as a CMAP (counts VS space) in each energy range selected. These are obtained with the program gtbin. The energy bins applied here will affect directly the precision of the likelihood: if the bins are too big the IRFs will not be accurately defined in each range (e.g., at lower energies the $\mathrm{PSF}$ is worse than at higher). Normally, 10 bins per decade of energy are recommended.

The exposure maps give the amount of time that the instrument observed the region we aim to analyze. To obtain it, one needs to first compute the so-called livetime cubes, with the gtltcube tool, which are healpix grid 1 that provide the observation time for each source on the sky as a function of the inclination angle (between the normal line of the satellite and the position of the source). According to the livetime calculated, the gtexpcube 2 tool generates a binned exposure map (same spacial and energy bins as before). The exposure map can be done for a certain region around our Rol (always larger to account for the contribution of other sources) or for the entire sky. The consuming time in the latter is just slightly higher.

On the other hand, the likelihood method need a source model (XML file) as an input. The source model is defined by the position of the target source and all sources including in the Rol, as well as their spectral shape and parameters. Usually, point-like background can be defined from the public 4-year point source catalog Third Fermi-LAT catalog (3FGL)|2 (which contains data in the $100 \mathrm{MeV}$ to $300 \mathrm{GeV}$ energy range) to create the model, while additional sources from

${ }^{1}$ http://healpix.jpl.nasa.gov/

${ }^{2}$ https://fermi.gsfc.nasa.gov/ssc/data/access/lat/4yr_catalog/ 
any FHL (above 10 or $50 \mathrm{GeV}$ ) can be included depending on the energy range of the analysis. Taken into account that the available catalogs were published years ago before the release of the improved Pass 8 data, during the current Fermi-[LAT analysis several point-like sources, that work as background for the analysis, arise in the residual maps. These sources have to be added manually to the XML file (see, e.g. Chapter 4.2.2). At low energies, Fermi-LAT presents a PSF as poor as $\sim 3.5^{\circ}$ while at high energies the angular resolution is $\lesssim 0.15^{\circ}$. Therefore, emission from the target source can be affected by that coming from the neighbors. Thus, spectral parameters of sources nearby the source of interest are left free, while the parameters of other objects located further away can be totally fixed (or let free only the normalization parameter) to accelerate the computation. Highly variable or very bright sources can be left free as well. Besides the pointlike background, the model needs to account for the isotropic diffuse background model, i.e. cosmic ray emission. These models are provided by the Fermi-LAT Collaboration, which for the Pass 8 data correspond to the $g l l_{-} i e m_{-} v 06$ and iso_P8R2_SOURCE_V6_v06 files for the galactic and extragalactic contribution, respectively.

At this point of the analysis, one can compute the so-called source map with the gtsrcmaps tools. This is a model counts maps that will be applied in the likelihood method. The gtsrcmaps makes use of the spectrum for each source defined in the XML file, which is multiplied by the exposure of position of the source in the sky (exposure map) and convolved with the effective PSF. Once this is ready, the likelihood method can be computed.

The likelihood is simply defined as the probability of obtaining the observed number of counts given certain model. The model is the one provided as input (XML file). The likelihood tools need to optimize simultaneously the value of those parameters left free inside the model of several sources. The best parameter values and their uncertainties are those that maximize the likelihood expression. First of all, as shown along this section, one needs to take into account that each count is characterized by different observable, e.g. its energy, its inclination angle and its event type. This implies that the Fermi-LAT analysis is a multi-dimensional analysis (multiple number of bins), leading to a small statistic in each bin. Therefore, the distribution in each bin is Poissonian. The likelihood can be then defined as the product of the probability of observing the detected counts in each bin:

$\odot$ Being $m_{i}$ the number of expected counts given a certain model in each bin $i$,

$\odot$ the probability of detecting $n_{i}$ counts in that bin can be defined by $p_{i}=m_{i}^{n_{i}} e^{-m_{i} / n_{i}}$ ! , and therefore

the likelihood, $L$, is the product of all $p_{i}$ in all $i$, which can be expressed as:

$$
L=\exp \left[-N_{\text {exp }}\right] \prod_{i} \frac{m_{i}^{n_{i}}}{n_{i} !}
$$

where $N_{\text {exp }}$ is the total number of expected counts given the assumed model. Therefore, $N_{\text {exp }}$ and $m i$ depend on the model and $n_{i}$ on the data. Equation 2.36 is the expression for the socalled binned Fermi-LAT analysis, and the aforementioned described steps corresponds to this type of analysis, which is applied to large data samples. In this thesis, I analyzed sets of years 
of Fermi-LAT data and hence, this kind of analysis fitted my scientific purposes. The reader is encourage to follow the Fermi-LAT tutorial available in the web page $\left.\right|^{1}$ for deeper information of both binned and unbinned (recommended for small data samples) analysis.

Usually, the maximum likelihood value is mapping out over a grid of coordinates, for which is it is used the TS quantity. This parameter is defined as follows:

$$
T S=-2 \ln \left(\frac{L_{\text {max }, \text { null }}}{L_{\text {max }, \text { source }}}\right)
$$

where $L_{\text {null }}$ and $L_{\text {max,source }}$ are the maximum likelihood values without (null hypothesis) and with an additional target source in the model, respectively.

On the other hand, $S E D$ are produced by performing the maximum likelihood analysis in each energy bin separately, while keeping the background spectral parameters fixed to those values obtained in an overall search. Normally, not all spectral parameters of the target source are left free either: for a power-law distribution, as it is the case of Cygnus X-1 (source discussed in this thesis), the flux normalization is left free to vary in each energy bin, whilst the spectral index is set to that obtained in the overall fit.

Finally, in order to obtain a lightcurve, a set of script and macros are used to download data directly from the Fermi-LAT web page in a daily basis (accounting for those days in which the spacecraft was not in a pointing mode) and analyze based on a data selection criteria and background model created beforehand. Due to the computational consumption of a day-to-day analysis over $\sim 8$ years of Fermi-LAT data, as that performed for this thesis in Cygnus X-1, only spectral parameters of highly variable sources are left free in the background model, besides the target source.

\footnotetext{
${ }_{1}^{1}$ https://fermi.gsfc.nasa.gov/ssc/data/analysis/
} 


\section{Part II}

\section{Microquasars in the very high-energy gamma-ray regime}

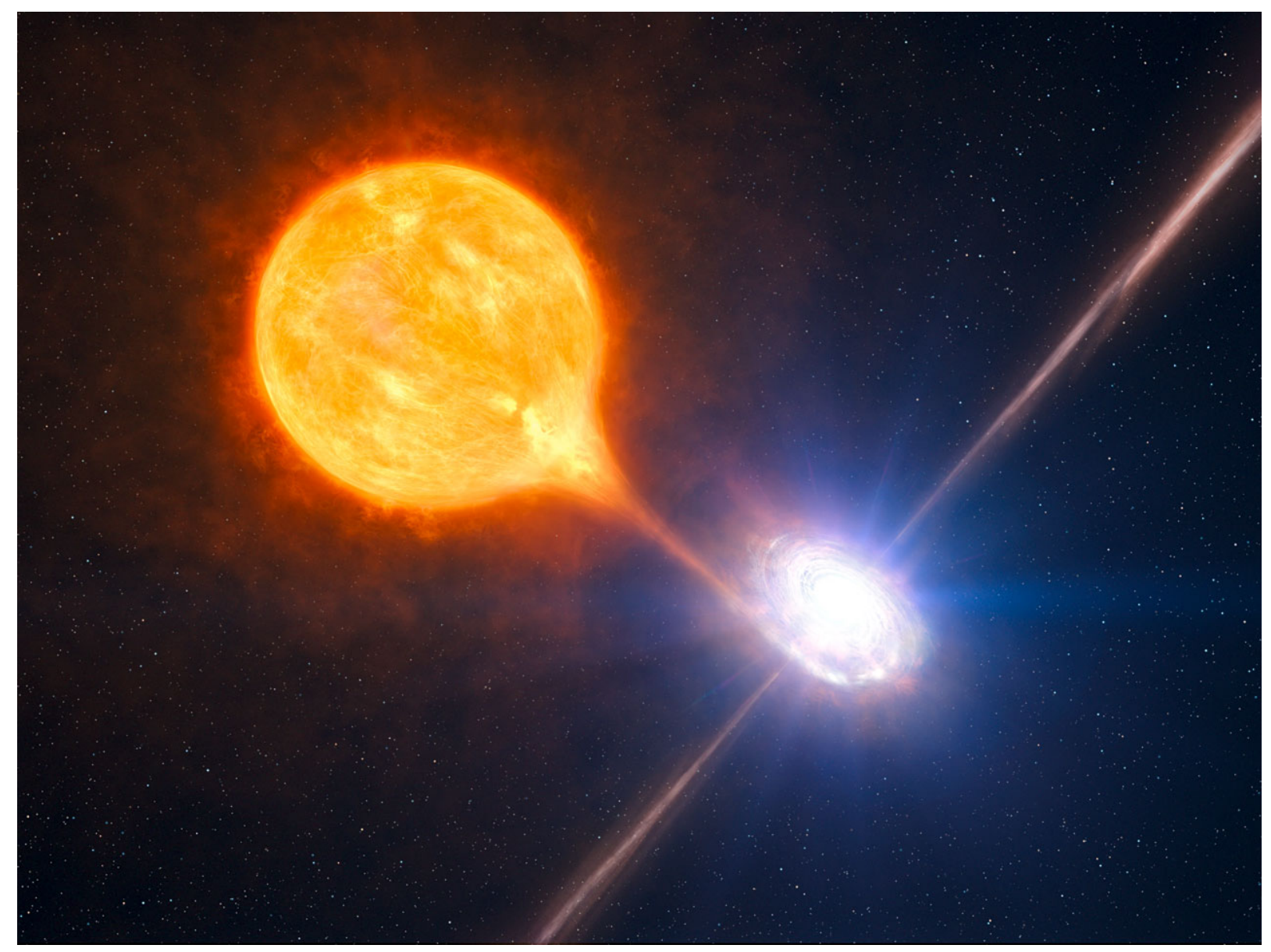

Figure II: Artist's impression of a microquasar. Credit: ESO/L. Calçada. 



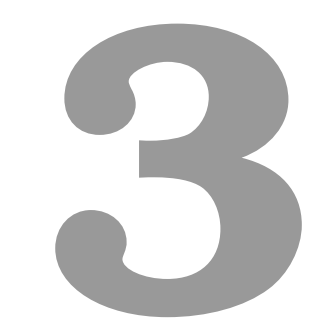

\section{Microquasars, binary systems with powerful jets}

\subsection{X-ray Binaries}

Our Galaxy contains hundred billions of stars with very different features, masses, size and ages. The understanding of their birth, evolution and death was, and still remains to be, a goal to achieve for the astronomers. The life of the stars begins within the molecular clouds, where gravity is responsible of joining the dust and gas of the environment to give rise to the celestial objects. If the created body has enough mass to reach high temperatures and fuse $\operatorname{Hydrogen}(\mathrm{H})$ atoms into $\mathrm{He}$ in its core, then a main sequence star is born. Most of the stars in the Milky Way belong to this type. The main sequence stars are constantly producing energy via nuclear fusion which counteracts the pressure caused by gravity. The fate that a main sequence star follows depends strongly on its initial mass and composition (Heger et al. 2003).

For small stars $\left(M_{\star}<9-10 M_{\odot}\right)$, the mass is not enough to increase the temperature to allow the Carbon (C) fusion point. When this happens, the nuclear fusion ceases and the gravitational pressure starts to dominate. However, as the matter is compressed, the electrons that form this mass get closer, which need to stay in different energy levels given the Pauli exclusion principle. This results into an electron degeneracy that causes internal pressure against the collapse. This degeneracy state is only achieved if the mass of the star, after the fusion processes stop, is lower than the Chandrasekhar limit, $1.4 \mathrm{M}_{\odot}($ Chandrasekhar 1931), generating a remnant known as White Dwarf (WD) In some cases, the core of the star presents layers which reach the necessary temperature to fuse $\mathrm{He}$ atoms into $\mathrm{C}$ in their inward collapse. The pressure originated during this fusion expands the star enlarging its size until forming a red giant. Nevertheless, this is just a 
temporary phase before the inevitable collapse happens.

In the case of more massive stars $\left(9-10 \mathrm{M}_{\odot}<\mathrm{M}_{\star}<40 \mathrm{M}_{\odot}\right)$, the temperature is high enough and fusion of heavy elements like C, Neon (Ne), Oxygen (O), Silicon (Si) or Iron (Fe) takes place. If the mass of the star at the end of its productive phase overpasses the Chandrasekhar mass, the gravitational pressure cannot be halted by the electron degeneracy: the star collapses giving rise to a huge thermonuclear explosion, the so-called $\mathrm{SN}$. The different types of $\mathrm{SNe}$ will be discussed more deeply in Appendix A. The remnant behind such an explosion can be a NS or a $\mathrm{BH}$. The former is created when the mass of the star ranges from 9-10 $\mathrm{M}_{\odot}$ to $25 \mathrm{M}_{\odot}$. Once the electron degeneracy pressure is overcome, the core keeps collapsing while its temperature increases. At this point, electrons and protons combine via electron capture, which produces neutrons and neutrinos. When the core reaches a density of the order of the nuclear one, the neutron degeneracy stops the collapse in the same way electron degeneracy did before, and the infalling outer layers are expelled in the $\mathrm{SN}$, leaving a $\mathrm{NS}$ as remnant. The $\mathrm{BH}$ is created when the mass of the star is higher $\left(25 \mathrm{M}_{\odot}<\mathrm{M}_{\star}<40 \mathrm{M}_{\odot}\right)$ and the gravitational pressure dominates over the neutron degeneracy. At even higher mass ranges $\left(40 \mathrm{M}_{\odot}<\mathrm{M}_{\star}<140 \mathrm{M}_{\odot}\right.$ or $\mathrm{M}_{\star}>260$ $\mathrm{M}_{\odot}$ ), the $\mathrm{BH}$ is formed directly with no visible SN. For a particular case between $140 \mathrm{M}_{\odot}$ and 260 $\mathrm{M}_{\odot}$, a so-called pair-instability $\mathrm{SN}$ takes place without leaving any remnant after the explosion.

When these compact objects (NS) or BH) orbit and accretes material from an optical star (usually a main-sequence one), an X-ray binary system is formed. These kind of sources are extremely luminous in the $\mathrm{X}$-ray band $\left(\mathrm{L}_{X} \sim 10^{35}-10^{38} \mathrm{erg} \mathrm{s}^{-1}\right)$ and the brightest compact sources in the medium X-ray regime, from 2 to $10 \mathrm{keV}$ (Grimm et al. 2002). Given their luminosity, the X-ray binaries were the main subject during the first years of the X-ray astronomy, until the 1980s, when X-ray imaging instruments (first Uhuru, Ariel 5, HEAO-1 and later, EXOSAT, Ginga, RXTE, ROSAT and BeppoSAX, among others) opened the door to fainter sources. Thus, from the first non-solar X-ray source discovered in 1962, Scorpius X-1 (Giacconi et al. 1962) which afterward was confirmed as a binary system (Gursky 1966), up to the present, large number of satellite missions allowed to detect $\sim 200 \mathrm{X}$-ray binaries and to extend our knowledge on the physical properties of these sources.

As explained before, the death of a star can end up in a WD as well. It is, therefore, worth to mention that if the compact object of the binary is a WD, instead of a $\mathrm{BH}$ or $\mathrm{NS}$, then the system is named Cataclysmic Variable (CV). However, although WDs are very dense, the efficiency converting gravitational energy into X-rays is small (around $0.03 \%$ ) compared to systems harboring $\mathrm{NSs}$ or $\mathrm{BHs}(\sim 10 \%$ and $40 \%$, respectively). Therefore, most of the transferred matter is released in the optical or $\mathrm{UV}$ bands, where only a minor fraction is released in X-rays. Consequently, these sources are not usually cataloged as X-ray binaries.

One of the most established ways to classify X-ray binaries is according to the nature of the main sequence or companion star (a.k.a. donor or secondary star). Thus, the X-ray binary systems can be split into two groups: High-Mass X-ray Binaries (HMXBs), if $\mathbf{M}_{\text {donor }} \gtrsim 10 \mathbf{M}_{\odot}$, and Low-Mass X-ray Binaries (LMXBs), if $\mathrm{M}_{\text {donor }} \lesssim 2 \mathrm{M}_{\odot}$. The dominant mass-transfer process in these two types of binaries is different (stellar wind-driven for the HMXBs and Roche-lobe overflow in LMXBs, as explained later), which allows to solve the unclear classification for 
X-ray binaries with 2 to $10 \mathrm{M}_{\odot}$.

\subsubsection{High-Mass X-ray Binary Systems}

In the HMXBs, the primary star, from whose death the compact object arises, was previously forming a star binary system. Since this star was more massive than its companion, evolved faster until it died in a $\mathrm{SN}$. If at the end of its life, its mass is lower than the companion's, the binary system remains together, otherwise, it will be disrupted.

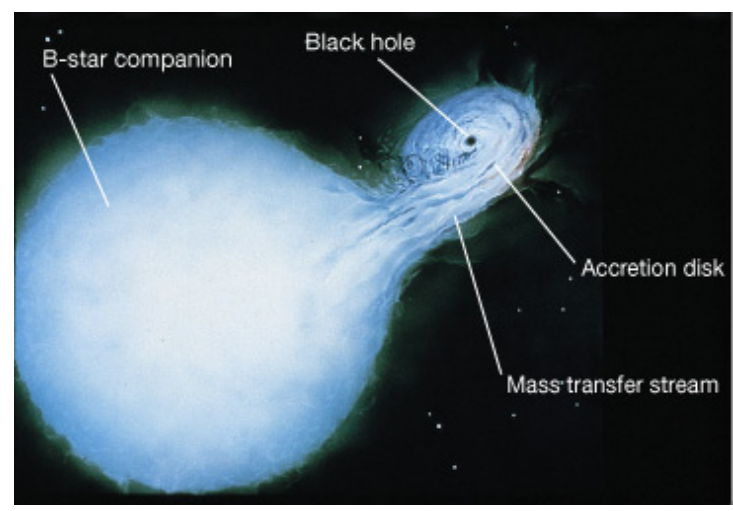

Figure 3.1: Artist's vision of an X-ray binary composed of a $\mathrm{BH}$ and a massive star. Credit: L. Chaisson
These systems are comprised of a massive secondary early $\mathrm{O}$ or B-type companion star, which presents strong stellar winds, with a mass-loss rate around $10^{-6}-10^{-10} \mathrm{M}_{\odot} \mathrm{yr}^{-1}$, and a terminal velocity up to $2000 \mathrm{~km}$ $\mathrm{s}^{-1}$. This type of stars displays high optical, UV and IR luminosity, which dominates the total emission from the system $\left(L_{\text {optical }} / L_{X}>1\right)$. The accretion on these sources is produced in a high-velocity wind-driven form, where the compact object captures a fraction of the $\mathrm{OB}$ star wind that passes within a certain radius, called capture or accretion radius, below which the matter cannot avoid the gravitational attraction of the $\mathrm{BH}$ or NS (see e.g. Bondi 1952).

Additionally to this dominant mass transfer, a secondary transfer through the so-called process Roche-lobe overflow can occur. This type of accretion takes place when the donor star fills its Roche lobe, region in which the material is gravitationally bound to the star. The material that overpasses this lobe will fall onto the compact object via the first Lagrangian point, where the gravitational forces from the $\mathrm{BH}$ or $\mathrm{NS}$ and the donor star are equal (see Figure 3.4). However, if the compact object's mass is greater than the companion's, the Roche-lobe overflow will become unstable after $\sim 10^{5} \mathrm{yr}$ from its beginning (Savonije 1983).

The accreted material presents angular momentum and the conservation of this magnitude prevents the matter from falling directly onto the compact object. Thus, the mass transfer is produced through an accretion disk formed around the compact object (see Figure 3.1), which is the most common mode of accretion in astrophysics. The heating created by friction inside the accretion disk produces the X-rays, which gives the general name to these systems. In the case of $\mathrm{HMXBs}$, the X-ray emission peaks at $k T \geqslant 15 \mathrm{keV}$, characterized normally by regular $X$-ray flux variations (Camenzind 2007). Among other modulations, X-ray binaries normally 
suffer flux variation in an orbital period scale, considered as the time that the compact object needs to complete an orbit around its companion. This modulation can be seen in different wavelengths, from the optical to higher energies, and is produced by the absorption or scattering of the radiation by photons from the companion star or accretion disk. HMXBs present orbital periods from days to hundred of days.

The lifetime of HMXBs is short, around $10^{5}-10^{7} \mathrm{yr}$, due to the high-mass of their secondary stars. This makes the HMXBs distribute along the galactic plane among the young stellar populations (see Figure 3.2).

The HMXBs can be, in turn, classified into two principal sub-groups according to type of the secondary star: Be/X-ray and Supergiant X-ray binary systems. A small fraction of the HMXBs, around the $18 \%$, do not belong to any of these two sub-classes. In this group, it would be fit the X Per like systems (as suggested by Reig \& Roche 1999) or systems harboring Wolf Rayet (WR) stars.

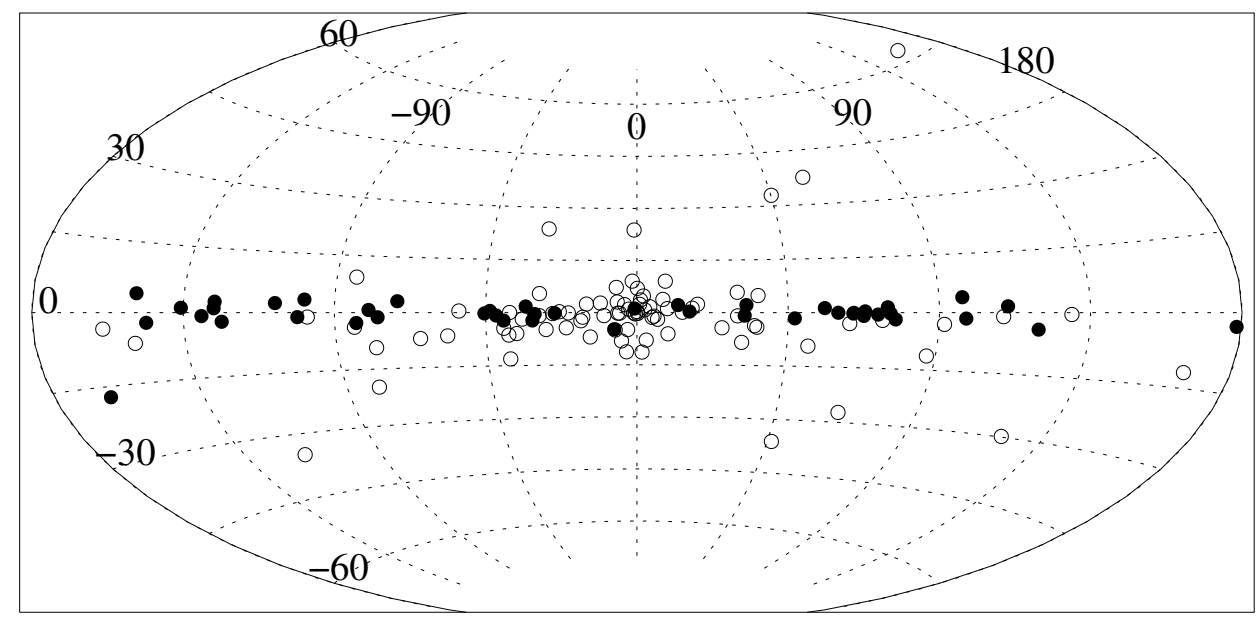

Figure 3.2: Distribution of 52 HMXBs (black circles) and 86 LMXBs (empty circles) in galactic coordinates. HMXBs are distributed along the galactic plane and LMXBs are concentrated in the galactic center and globular clusters (Grimm et al. 2002).

\subsubsection{Be/X-ray binaries}

The Be/X-ray binaries sub-class represent the largest population of HMXBs with a 57\%. These very luminous systems $\left(L_{X} \sim 10^{36}-10^{38} \mathrm{erg} \mathrm{s}^{-1}\right)$ are composed of a Be companion star surrounded by a circumstellar envelope with disk-like geometry (Figure 3.3), which displays Balmer line emission and causes an excess in the IR band. The physical formation of this circumstellar disk, from which the accretion takes place, is not well understood yet, although it is thought to be related with the fast rotation of the star. The compact object is normally a NS (or pulsar in case of rapid rotation and high magnetic field, see Chapter 7.1) and its passage through the circumstellar disk of the donor is the responsible of the X-ray emission. These sources also display 
X-ray outbursts that can be divided into two types: periodic outbursts at the periastron of the orbit (nearest point to the companion star), classified as Type I, and non-regular huge outbursts or Type II outbursts, produced by an expansion of the circumstellar disk. This sub-group of HMXBs usually follows a highly eccentric orbit, which intensifies the Type I outburts. For an extended description of the systems see, e.g., Camenzind (2007).

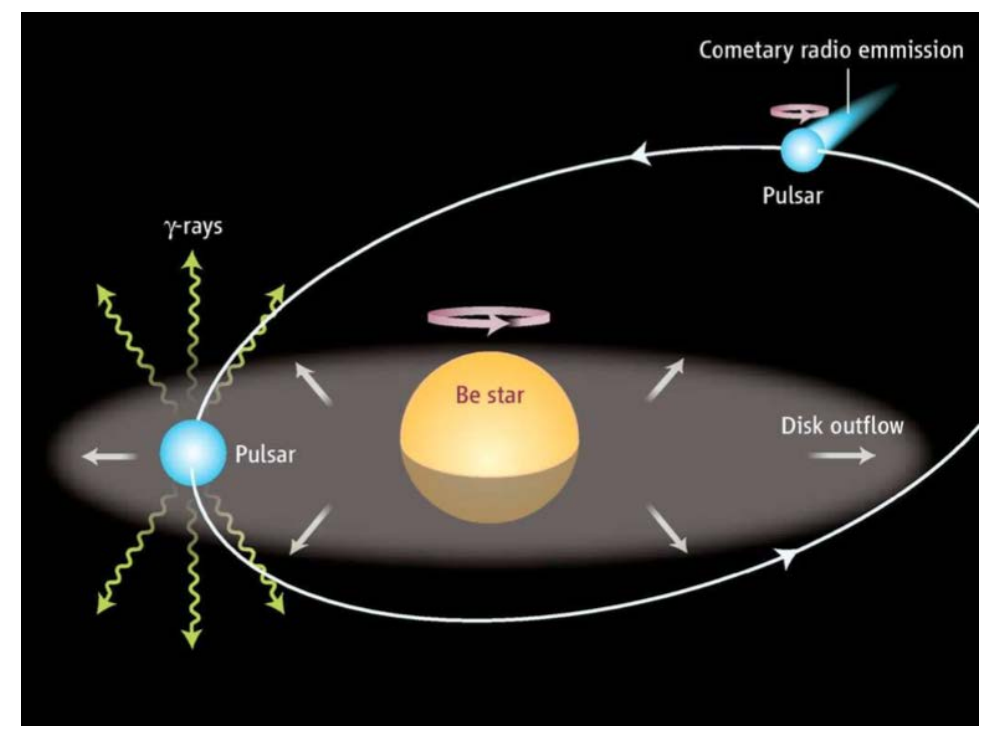

Figure 3.3: Be/X-ray binary composed of a pulsar, whose interaction with the circumstellar disk of the Be star gives rise to X-ray emission and even higher energies as gamma rays (Mirabel 2006).

\subsubsection{Supergiant X-ray binaries}

The supergiant X-ray binary systems are also composed of a secondary OB type star or even type A. The main different with respect to the $\mathrm{Be} / \mathrm{X}$-ray binaries is the mass-transfer process. Due to the lack of a circumstellar disk in these sources, the accretion arises from stellar wind outflow. In this case, the compact object (either a $\mathrm{BH}$ or a $\mathrm{NS}$ ) follows normally a circular orbit around the companion. The steady wind outflow prevents the system to suffer as many Xray outbursts as in Be/X-ray binaries, and produces, in turn, persistent and less luminous X-ray emission $\left(L_{X} \sim 10^{34}-10^{35} \mathrm{erg} \mathrm{s}^{-1}\right)$. The percentage of HMXBs that belong to this type reaches the $25 \%$.

\subsubsection{Low-Mass X-ray Binary Systems}

The origin of the LMXBs is less clear, but it is thought to be created by capture: the compact object, formed after the $\mathrm{SN}$ explosion, interacts with a close cluster and captures a low-mass star due to its strong gravitational force.

These systems are composed of donor stars with type later than A, i.e. low-mass G, K or M companions. Given the faint secondary stars $\left(L_{\text {optical }} / L_{X}<<1\right)$, the optical spectrum is dominated by 
radiation originated at the accretion disk by reprocessed X-ray emission. Actually, the contribution of the donor is only not negligible in cases in which the LMXB arises from an intermediate binary. The late type stars forming the LMXBs do not have strong wind to produce accretion via wind-driven process. This way, the system is powered by the Roche-lobe overflow (Figure 3.4). The X-ray spectrum in these binaries is softer than in their counterparts, peaking at $k T \leq 15 \mathrm{keV}$. The LMXBs also display weaker magnetic fields $\left(\sim 10^{9}-10^{11} \mathrm{G}\right)$ than the HMXBs $\left(\sim 10^{12} \mathrm{G}\right)$, which allow X-ray bursts to happen (produced by thermonuclear fusion of accreted material in the surface of the compact object, Camenzind 2007).

LMXBs are more compact than the HMXBs, i.e. the separation between the star and its accretor is smaller. While the size of the $\overline{\mathrm{LMXBS}}$ is generally around a solar radius, the size of the HMXBs can reach tens of solar radii. Because of this compactness, their orbital period is lower as well, ranging from minutes to hours (see e.g. Table 1.1 of Lewin et al. 1995). On the other side, the lifetime of these systems is higher than the HMXBs', between $10^{7}$ and $10^{9} \mathrm{yr}$, determined mostly by the accretion process. As in the case of old stellar populations, the LMXBs are mostly located close to the galactic center and in globular clusters (see Figure 3.2).
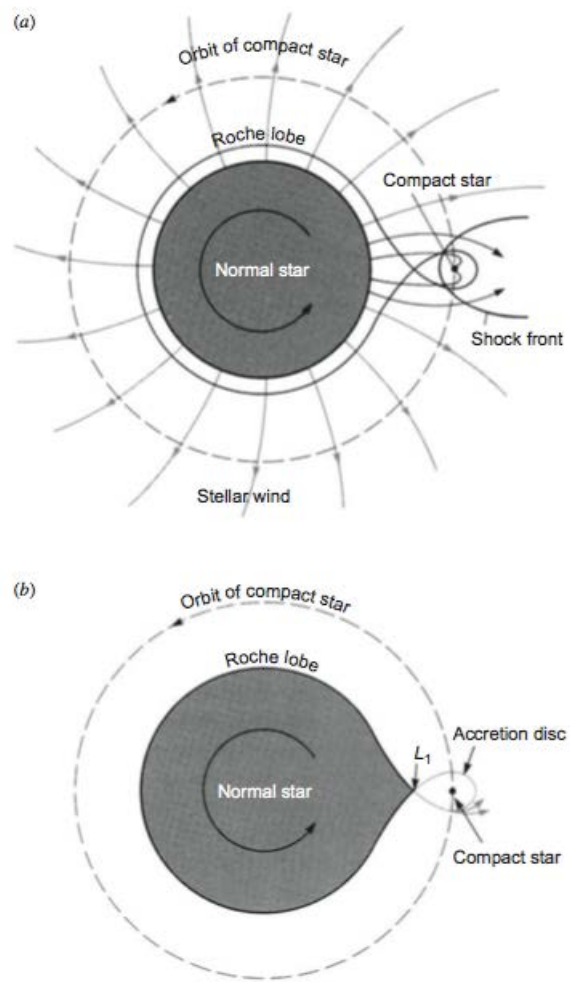

Figure 3.4: Sketches that illustrate the two different accretion processes in binary systems. In $(a)$, the compact object is embedded in the strong stellar wind from its massive companion star, giving rise to the wind-driven mass transfer. In $(b)$, the companion star expands filling its Roche lobe and the material falls onto the compact object through the Lagragian point, $L_{1}$. In both cases, the matter does not reach the compact object directly but through an accretion disk (Longair 2011). 
There is a sub-class of X-ray binaries, in both HMXBs and LMXBs, characterized by the presence of extended radio-emitting jets. These systems are known as Microquasars (Mirabel \& Rodríguez 1999) and constitute one of the main topics of this thesis.

\subsection{Microquasars}

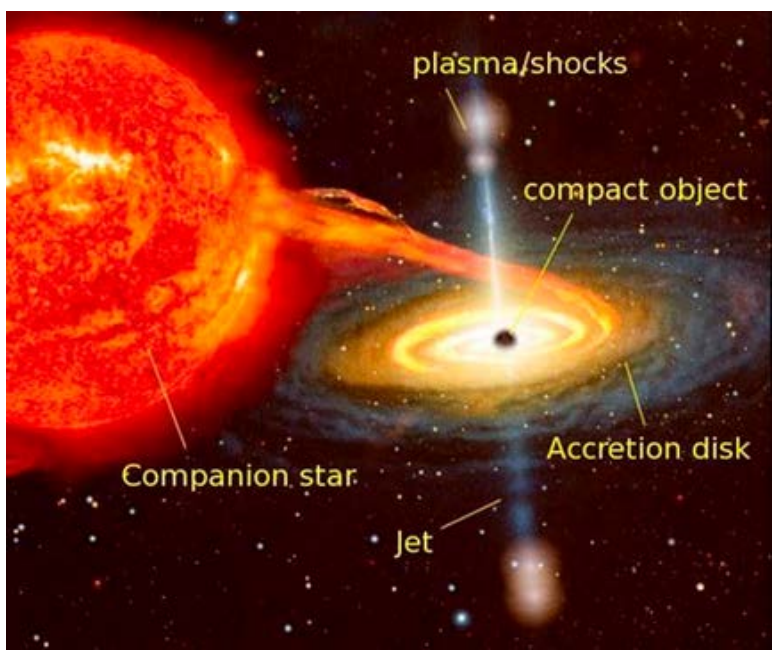

Figure 3.5: Anatomy of a microquasar where the different elements that composed the system are labeled. Credit: Imago Mundi.
The microquasars are a sub-type of X-ray binary systems. Therefore, they are composed of the same elements presented in Chapter 3.1, a high or low-mass companion star and a compact object (either $\mathrm{BH}$ or $\mathrm{NS}$ ) that accretes material through an accretion disk of size $\sim 10^{3} \mathrm{~km}$. However, normally two-sided highly collimated $\left(<15^{\circ}\right.$ opening angle, as defined by Mirabel \& Rodríguez 1999) streams of fluid, gas or plasma, the so-called jets, are launched perpendicularly from the compact object (see Figure 3.5).

These jets, whose speed is usually characterized by the Lorentz factor ${ }^{1}$, are very powerful nonthermal emitters detected at different wavelengths. Nevertheless, they are not steady structures: their presence or absence seems to depend on the accretion rate, which allows to distinguish different X-ray states, as it will be deeply discussed in Section 3.2.3.1.

The name of microquasar, chosen by Mirabel et al. (1992), born from the analogy between these objects and their scaled-up counterparts, the quasars. The latter are distant AGN from which the first evidence of jet-like structures was obtained. These jets were discovered in the optical regime by Curtis (1918), emanating from the galaxy M87. In quasars, this relativistic ejecta can travel several million parsecs, well above the distance reached by microquasars (around few parsecs). The morphological analogy does not only concern the existence of relativistic outflows: quasars also host $\overline{\mathrm{BHs}}$ as central objects which accrete material from the surrounding. The main difference is the scale of the $\mathrm{BH}$ in each case. While the microquasars present stellar-mass $\overline{\mathrm{BHs}}$, the ones forming quasars are super-massive $\mathrm{BHs}$ of the order of several million solar masses (Rees 1998). On the other hand, the accretion disk of the quasars (with size $\sim 10^{9} \mathrm{~km}$ ) is not fed from a companion stellar object but from the ISM] of their galaxies and from disrupted stars due to their strong gravitational force. Nevertheless, the thermal temperatures achieved in the quasars'

\footnotetext{
${ }^{1}$ Lorentz factor described as $\Gamma=\left(1-v^{2} / c^{2}\right)^{-1 / 2}$, where $\mathrm{v}$ is the flow velocity and $c$ the speed of light. In $\mathrm{BH}$ microquasars, the Lorentz factor can reach $\Gamma \sim 2.5(0.92 c)$.
} 
accretion disks by viscous/friction dissipation is of the order of several thousand degrees, instead of several million degrees as in the case of the microquasars. The reason is that as more massive the $\mathrm{BH}$ is, the cooler will be its accretion disk. Given by Rees (1984), the characteristic blackbody temperature at the last stable orbit of the disk for a $\overline{\mathrm{BH}}$ accreting at the Eddington limit, defined in terms of the Eddington luminosity $\left.\left(L_{E d d}\right)\right|^{1}$, is $T \propto 10^{7} M^{-1 / 4}$ (where the temperature, $T$, is in $\mathrm{K}$, and the mass of the $\overline{\mathrm{BH}}, M$, in $\mathrm{M}_{\odot}$ ). Consequently, most of the radiation originated in the accretion disk of quasars is emitted in the UV and optical wavelengths, in contrast with the microquasar scenario, in which the radiation comes out as X-rays (see Chapter 3.1). This is actually the reason why jets in quasars, so far-distant objects, were discovered years before jets in galactic microquasars (first detection in SS 433, Margon 1984): the detection of radio jets was constrained by the development of the X-ray astronomy which would provide new stellar sources.

The main advantage of studying microquasars with respect to quasars is the time scale in which processes happen. The characteristic time of the accretion process is proportional to the mass of the $\mathrm{BH}$. Whilst variations of minutes can take place in microquasars, one would have to wait thousands of years to observe the same effect in a super-massive $\mathrm{BH}$ (Mirabel \& Rodríguez 1999). Thus, observing microquasars brings us the opportunity to study, in a possible human lifescale, the nature and origin of the jets, likely related with the accretion flow. On the other side, the proximity of the galactic microquasars allow us to delve into particle acceleration inside the jets and its expected emission at very-high energies. A schematic view with the aforementioned characteristic of both microquasars and quasars is shown in Figure 3.6.

The main components of the microquasars have been introduced previously, where a detailed discussion of the companion star and the compact object can be found in Chapter 3.1. In the following, I will focus on the accretion disk and the relativistic radio-jet, correlated by the accretion process: the mass of the donor star affects the properties of the transferred material, like its temperature or the accretion rate, giving rise to a clear and direct effect on the accretion disk (influencing e.g. the emitted radiation from the disk or the magnetic field strength on it). Although the mechanism of production and collimation of the jets is still not perfectly understood, it is currently accepted that these streams are also powered by this accretion process, given the correlation evidences of these two mechanism (Fender et al.2004, see Section 3.2.3.1). Thus, an accretion-ejection relation becomes important in these systems.

\subsubsection{Accretion disk}

The movement and energy lost by friction/viscosity of the gas flow inside the accretion disk is normally described by hydrodynamic equations of the Standard Disk Model (SDM), modeled by

\footnotetext{
${ }^{1}$ The Eddington luminosity, $L_{E d d}$, is the maximum luminosity that a body can achieved when the emitted radiation and gravitational force are balance. When the body exceeds this luminosity, the radiation pressure will overcome gravity, and material from the outer layers of the object will be forced away from it rather than falling inwards, giving rise to a very intense radiation-driven wind. This luminosity only depends on the mass of the object, $L_{E d d}=3.2 \times 10^{4}\left(\frac{M}{M_{\odot}}\right) L_{\odot}$. This luminosity is, in turn, associated with the Eddington accretion limit, accretion rate beyond which the $L_{E d d}$ is overpassed
} 


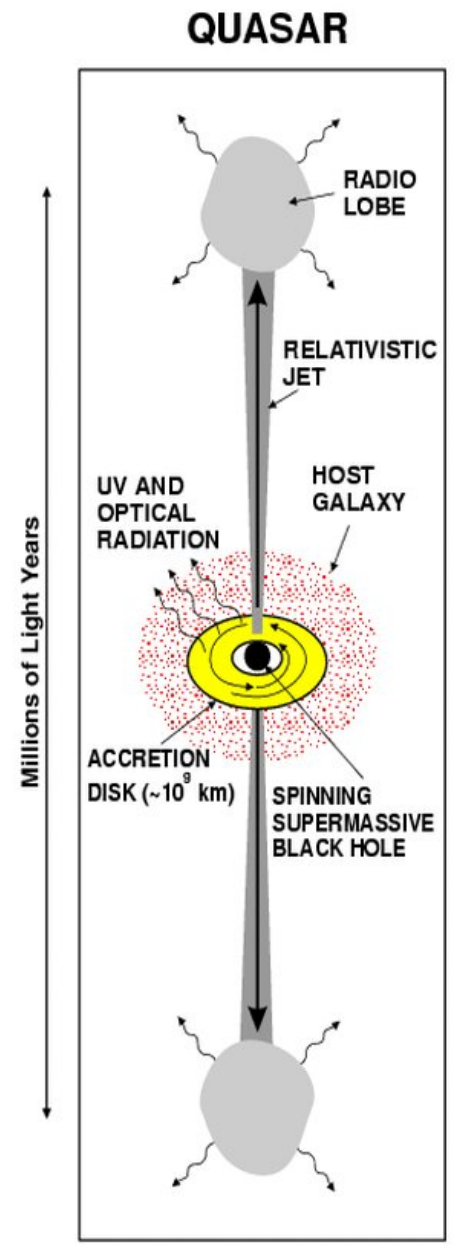

\section{MICROQUASAR}

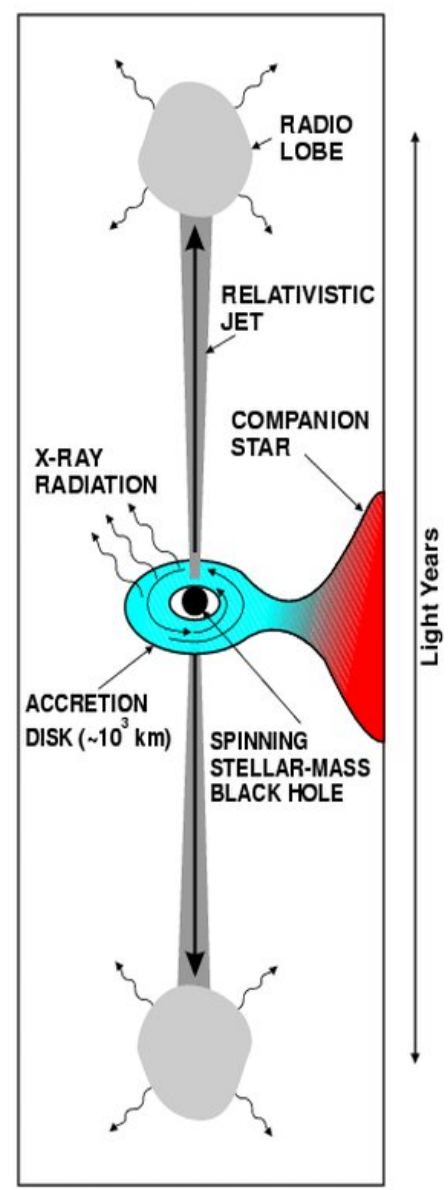

Figure 3.6: Comparison of quasars and microquasars. Three basic components are found in both cases: a central $\mathrm{BH}$ accretion disk and collimated relativistic jets. Differences are also highlighted: mass-transfer from the ISM, accretion disk of $\sim 10^{9} \mathrm{~km}$ emitting mostly in $\mathrm{UV}$ and optical and super-massive $\mathrm{BH}$ in the case of the quasar, and mass-transfer from a stellar companion, accretion disk of $\sim 10^{3} \mathrm{~km}$ emitting in X-rays and stellar-mass $\mathrm{BH}$ in the case of microquasars. The different distance achieved by the jets in each case is also marked (Mirabel \& Rodríguez 1998).

Shakura \& Sunyaev (1973). This energy lost is equivalent to the binding energy at the last stable orbit, whose radio depends on the compact object. In the case of a $\mathrm{BH}$, the innermost stable orbit corresponds to approximately three times the Schwarzschild radius $1, R_{S c h}$, while for the $\mathrm{NS}$, it is its own surface. As mentioned before, the temperature in this orbit is defined by $T \propto 10^{7} M^{-1 / 4}$ and hence, compact objects with tens of solar masses, as in the case of microquasars, will emit

\footnotetext{
${ }^{1}$ The Schwarzschild radius, a.k.a gravitational radius, is the radius of a sphere such that, assuming all its mass inside it, the escape velocity from its surface would be the speed of light. Therefore, all mass falling into the Schwarzschild radius cannot avoid the gravitational attraction of the body inside the sphere. It depends only on the mass of the object, $R_{S c h}=\frac{2 G M}{c^{2}}$, where $G$ is the gravitational constant and $c$ the speed of light.
} 
X-rays.

However, hard X-rays have been detected in several microquasars, e.g. Cygnus X-1, which will be deeply study in the gamma-ray band in this thesis (see Chapter 4). The SDM cannot explain such high emission, unless a new component is introduced. This component is the so-called corona, hot $\left(T \sim 10^{9} \mathrm{~K}\right)$ plasma at the inner region of the accretion flow (Coppi 1999). Close to the compact object (at $\lesssim 100 R_{S c h}$ ), the accretion disk presents low density and hence, the cooling efficiency by viscosity is low as well. This produces an increase of temperature that inflates the gas of the disk forming the corona. The hard X-ray are emitted through the Comptonization of thermal photons from the accretion disk by high-energy electrons in the corona. Hard X-rays were also speculated to be produced inside the jets in terms of Compton scattering of external photons (from the disk or the donor star).

\subsubsection{Relativistic radio-jets}

As seen before, the radio-jets are highly collimated, showing opening angles of less than $15^{\circ}($ Mirabel \& Rodríguez 1999). Normally, the angle between these outflows and the line of sight of the observer is $\gtrsim 30^{\circ}$. However, this feature is purely statistical: the probability of finding jets with smaller angles is low. The microquasars whose axis of ejection form angles $\lesssim 10^{\circ}$ with respect to our line of sight are known as microblazar (Figure 3.7), again because of their scaled-up counterparts, the blazars (Mirabel \& Rodríguez 1999). In this kind of systems, the timescales are shortened by $2 \Gamma$, where $\Gamma$ is the Lorentz factor, and the flux densities are increased by $8 \Gamma^{3}$. Therefore, although the intensity is enhanced with respect to the microquasars, the very fast flux variability, along with the low probability to find one, make the microblazars very difficult sources to detect.

Several models have been proposed along the years to describe the production and collimation of jets and the relation with the accretion process (see e.g., Blandford 1976, Blandford \& Znajek 1977, Blandford \& Payne 1982, Uchida \& Shibata 1985, Uchida \& Shibata 1986 and Meier 1996, among others). Currently, the most accepted one is the Magnetohydrodynamic (MHD) model, which invokes an accretor, a poloidal magnetic field and differential rotation under the assumption of high conductivity (see Figure 3.8). A general review of the model can be found in Meier et al. (2001); here I summarize the main features that describes it:

$\odot$ The plasma expelled through the jets will follow the magnetic field lines in a parallel way (along the rotation axis) without crossing them. In cases where the magnetic field is weak or the density of the plasma is high enough, the magnetic field lines will be bent back. This magnetic pressure is responsible of the outwards particle acceleration.

$\odot$ Magnetic field lines repel to each other, which gives rise to a perpendicular pressure. This characteristic can allow the enhancement of weak magnetic fields by bringing together several parallel lines. 


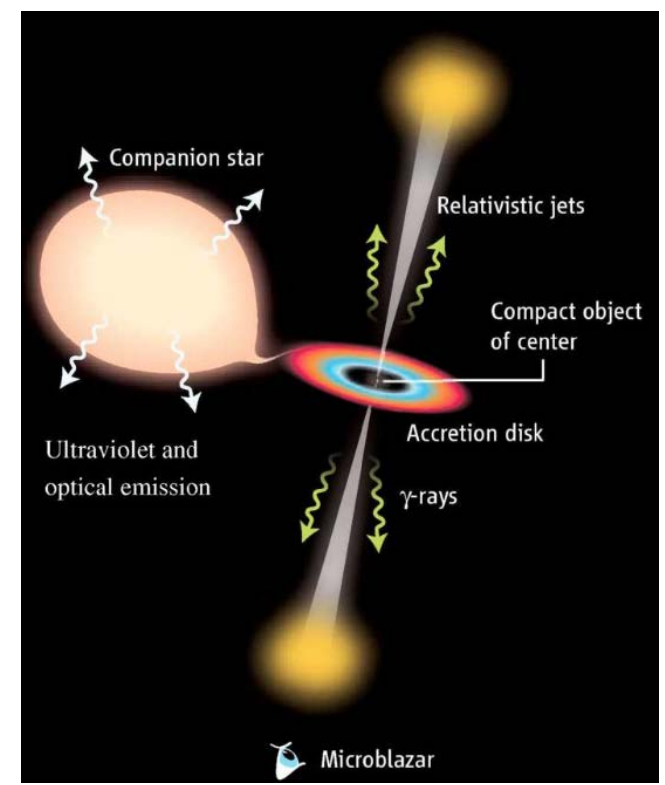

Figure 3.7: Sketch of a microquasar. If the jets are aligned with the line of sight of the observer $\left(\lesssim 10^{\circ}\right)$, the systems are called microblazars (Mirabel 2006).

$\odot$ Magnetic field lines tend to keep straight unless other lines or forces originated in the plasma apply any effect on them. This tension is responsible for the collimation and pinch effect of the plasma.

The creation of the relativistic outflows in microquasars seems to be related with thick accretion disks, since no jets are detected when the system presents optically thin disks, independently of the nature of the compact object. The physical interpretation is still unclear, although one of the reason could be the presence of a not strongly enough magnetic field, during thin accretion disk states, enable to collimate the jets. The different states and their correlation with the radiojets are presented in the following Section 3.2.3.1.

Inside these outflows non-thermal emission has been detected in a broad multiwavelength band, from synchrotron radio, through $[\mathrm{IR}$ and optical (Russell \& Fender 2010), up to X-rays. High-energy gamma rays from microquasars most likely originate inside the jets have been also reported. So far only two microquasars have been detected in the latter regime: the high-mass microquasar Cygnus X-3, at energies greater than $100 \mathrm{MeV}$, by AGILE (Tavani et al.2009a) and Fermi-LAT (Fermi LAT Collaboration et al.2009a), and Cygnus X-1, at energies above $60 \mathrm{MeV}$, using Fermi-LAT data as reported in this thesis in Chapter 4. All these results evidence the existence of a relativistic particle population inside the streams, which are thought to be accelerated via Fermi acceleration (see Chapter 1.1.2 for a detailed description of this process). This mechanism of acceleration evokes shock waves, whose origin in microquasars can be explained by different models:

$\odot$ The jets are discrete ejections of material: in this scenario, the shock waves are formed by the jets itself that are ejected as blobs. These blobs can display different velocities 


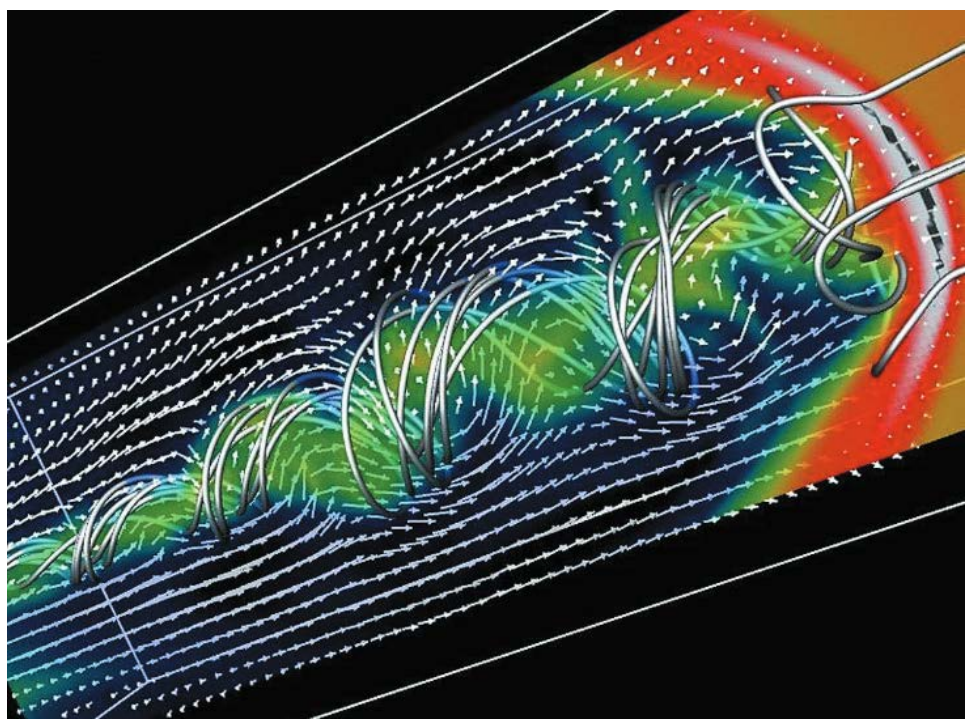

Figure 3.8: In the axisymmetric and rotating jet (due to the conservation of the angular momentum), the plasma moves parallel to the magnetic field lines (metallic tubes). The arrows show the plasma velocity while the colored zones describes the plasma density: white indicates the highest density, blue the lowest. Plasma trapped into the magnetic field suffers Lorentz force, which can be divided into two vectors: magnetic pressure along the rotation axis, which accelerates the plasma outwards, and magnetic tension perpendicular to the former, responsible to the collimation of the streams (Meier et al.2001).

inside the outflow and hence, interact to each other, creating internal shocks. The expected emission would be quasi-steady with a certain variability given by the blob injection van der Laan 1966, Jamil et al. 2010).

$\odot$ The jets are continuous outflows with internal shocks: in this case, the stream is assumed to be continuous and the shocks that produce the particle acceleration originate inside the jet by interactions of regions with different features (like densities or velocities; Kaiser et al. 2000).

\subsubsection{Black hole microquasars}

Large fraction of the microquasars in our Galaxy are composed of a $\mathrm{BH}$, which are normally highly variable X-ray sources. This variability extends to other wavelengths as well. This transient nature is related to a change in the accretion rate: they spend most of their time in low accretion periods (which leads to $L_{X} \lesssim 10^{33} \mathrm{erg} \mathrm{s}^{-1}$ ), with sudden flaring periods that can last from days to months. Only a small number of this type of microquasars are persistent objects, as it is the case of Cygnus X-1 (see Chapter 4), which is always showing high accretion rate and consequently, displaying high luminosity $L_{X}>10^{37} \mathrm{erg} \mathrm{s}^{-1}$. This fast flux modulation complicates the study of the behavior of these systems. However, thanks to deep observations with the $R X T E$ satellite, an overall view of the $\mathrm{BH}$ microquasars was possible, allowing to classify their $\mathrm{X}$-ray states depending on flux and energy spectra changes. The course that these sources follow 
through all the different X-ray states is well described by the so-called $q$-track in the HardnessIntensity Diagram (HID) given by Fender et al. (2004). In the upper plot of Figure 3.9, we have a schematic of the HID the X-axis corresponds to the hardness of the X-ray band, i.e. the ratio between hard and soft X-ray (at higher this value, harder the energy spectrum would be), and the $\mathrm{Y}$-axis represents the intensity of the X-ray flux. The direction of the motion is counterclockwise and give us the opportunity to define the X-ray spectra of these sources mainly by two principal states: the $\mathrm{HS}$ and the $\mathrm{SS}$.

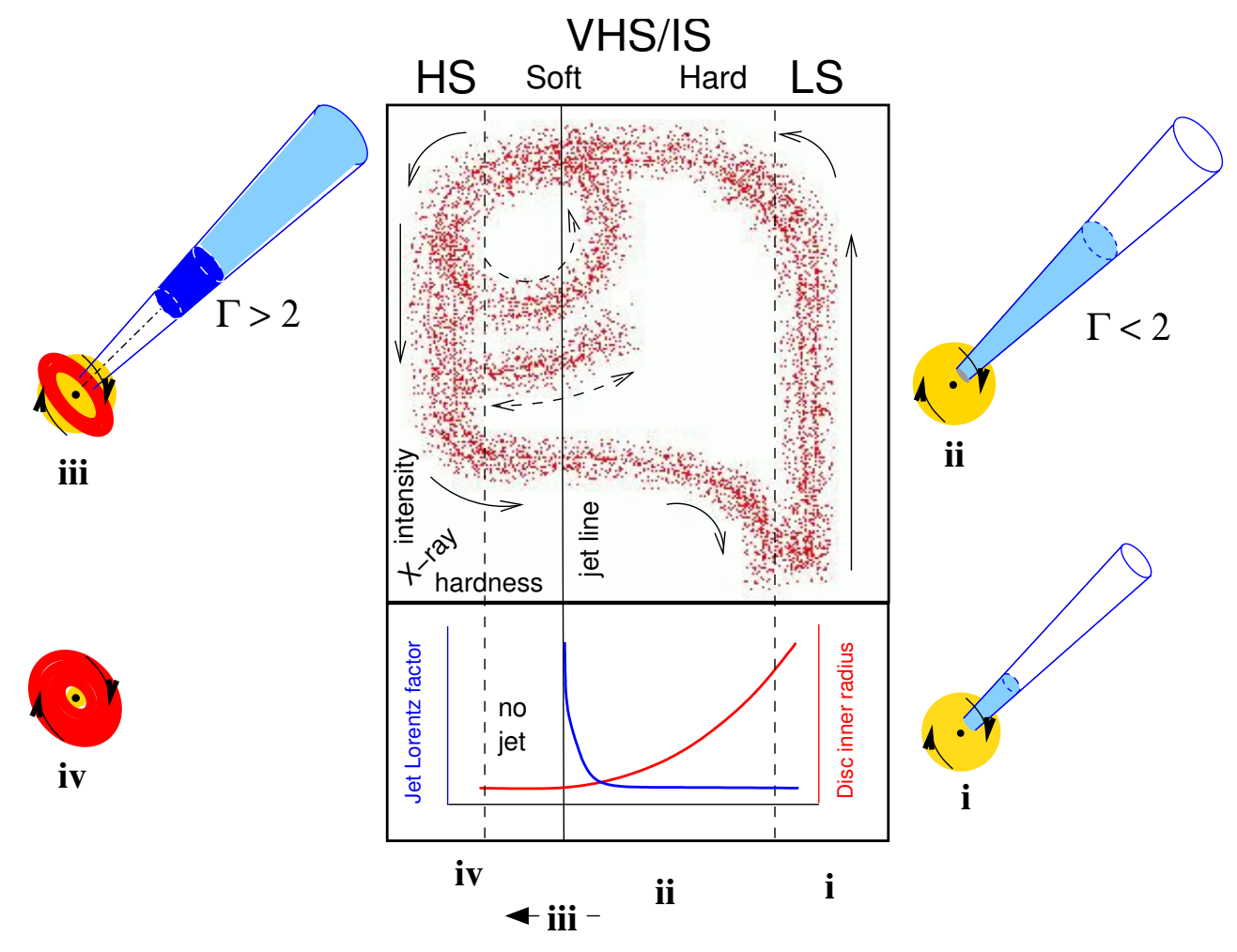

Figure 3.9: Schematic of the jet-disk coupling model presented by Fender et al. (2004).

\subsubsection{X-ray states}

Although it is standardized the use of the two canonical X-ray states on $\mathrm{BH}$ microquasars, the $\mathrm{HS}$ and the $\mathrm{SS}$, a deeper detailed X-ray emission study seems more complicated and more states have been discussed in literature (see, e.g., Belloni et al. 2000, Gilfanov 2010). Nevertheless, these two states give us a well understanding of the systems and will be used in this thesis to discuss the behavior of the microquasars Cygnus X-3 (Chapter X) and Cygnus X-1 (Chapter 4).

Both states are characterized by the sum of a thermal blackbody component that peaks at the $\mathrm{keV}$ energies and a power-law component at higher energies. The origin of the latter seems to happen in the corona, where high-energy electrons scatters (via IC) thermal photons from the accretion disk. The dominance of one or other component will define the state of the system. 
$\odot$ Hard State: It is dominated by a power-law photon distribution $\left(\mathrm{d} \phi / \mathrm{d} E \propto E^{-\Gamma}\right.$, with $\Gamma \sim 1.4-1.9)$ with a high-energy exponential cutoff at hundred $\mathrm{keV}$, whilst the thermal component is very weak, peaking at $k T \sim 0.1 \mathrm{keV}$. The total X-ray luminosity, $L_{X}$, reaches a few $\%$ of the $L_{E d d}$ (Maccarone 2003). During this state, a steady radio-jet is detected, evidencing the correlation between hard X-rays and radio wavelengths. Historically, the relation between the luminosity in these two bands was $L_{\text {radio }} \propto L_{X}^{0.7 \pm 0.1}$ (Gallo et al. 2003). This value was afterwards revised by Gallo et al. (2012) differentiating two population of $\mathrm{BH}$ microquasars probably dependent on the accretion rate. The relation of one of them is still compatible with the former one, $L_{\text {radio }} \propto L_{X}^{0.63 \pm 0.03}$, while a new track slope is obtained, $L_{\text {radio }} \propto L_{X}^{0.98 \pm 0.08}$ (Figure 3.10.

$\odot$ Soft State: Contrary to the HS, this state is dominated by the thermal blackbody component that peaks at $k T \sim 1 \mathrm{keV}$, emitted mainly in the inner region of the accretion disk that extends down to the last stable orbit, and a softer power-law tail (with photon index of $\Gamma \sim 2.2-2.7$ ) that extends beyond $500 \mathrm{keV}$. The X-ray luminosity in this states is $\gtrsim 0.01 L_{E d d}$. During this state, the relativistic outflows are disrupted and radio emission is undetectable.

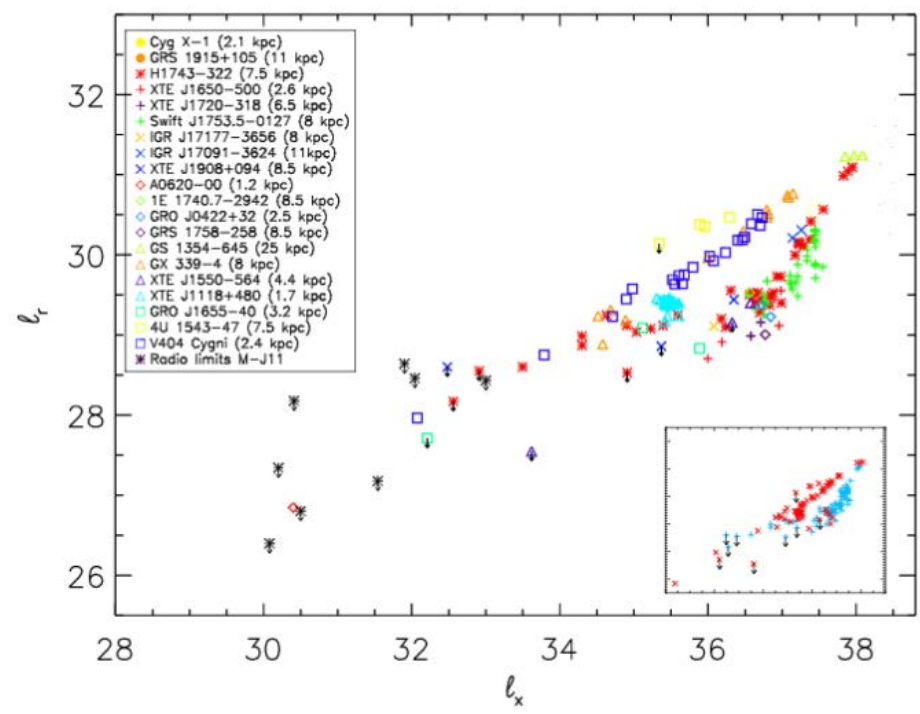

Figure 3.10: Radio/X-ray luminosities $\left(l_{r}\right.$ and $l_{X}$, respectively, in $\mathrm{erg} \mathrm{s}^{-1}$ ) of $18 \mathrm{BH}$ X-ray binary by (Gallo et al. 2012). This diagram evidences that the relation between radio and X-ray during the $\mathrm{HS}$ follows two tendencies (probably related with accretion rate), with slopes $0.63 \pm 0.03$ and $0.98 \pm 0.08$.

The transition between these two states is done through the so-called Intermediate State (IS), which displays spectral properties between both of them and rapid and strong radio variability due to internal shocks inside the jets. 


\subsubsection{Disk-jet coupling}

Instabilities in the accretion disk due to changes of the accretion rate will affect the jets radiation (and consequently, the X-ray and radio emission, as shown previously). This instabilities are accepted to be responsible of the spectral variability in $\mathrm{BH}$ transients. Fender et al. (2004) described these spectral changes within an unified model of coupling between disk and jet in $\overrightarrow{B H}$ binaries. The model is shown conceptually in Figure 3.9. Besides the HID, the figure presents different sketches of the sources at each state to highlight the dominant components. The lower part of the plot shows the variation of the Lorentz factor value, $\Gamma$, and the innermost radius of the accretion disk with respect to the hardness. The different phases according to the model are:

$\odot$ Phase i: The microquasar is in the $\mathrm{HS}$ but in a low-luminosity state, i.e. with low accretion rate. This state can achieve the lowest luminosities, a.k.a. quiescence state. It displays a persistent jet, characterized by synchrotron radio emission, and a hot corona, from where the hard X-rays arise. The last stable orbit of the accretion disk is at its furthest point from the $\mathrm{BH}$. At this point, the radio and $\mathrm{X}$-ray emission is related by the non-linear equation $L_{\text {radio }} \propto L_{X}^{\alpha}$, where $\alpha$ can be $0.63 \pm 0.03$ or $0.98 \pm 0.08$ as given by Gallo et al. (2012) (see Section 3.2.3.1.

$\odot$ Phase ii: The accretion rate starts increasing and the motion through the HID becomes almost vertical, until it enters in the hard/intermediate state (to the left). At this moment, the spectrum starts to suffer softening because of lost in temperature in the corona due to the $\mathrm{IC}$ processes on thermal photons from the disk. Thus, thermal emission is not longer negligible. At the same time, given the increase of accretion rate, the dense of the gas enhances and starts to cool via synchrotron and Bremsstrahlung, helping to move the system to the $\mathrm{SS}$. The corona shrinks and the innermost stable orbit approaches the $\mathrm{BH}$. For angular momentum conservation, as matter gets closer to the compact object, higher the velocity is inside the jets ( still $\Gamma \lesssim 2$ ).

$\odot$ Phase iii: The source keeps moving to a softer state, where thermal emission from the disk becomes dominant with respect to the hard X-ray from the corona. It approaches the jet line, a vertical line in the HID that divides the states where the relativistic outflows are present and where they are not. In this state, the jet becomes unstable giving rise to rapid Lorentz factor increase $(\Gamma \gtrsim 2)$ that originates internal shocks in the outflow before being disrupted once it enters in the $\mathrm{SS}$. Between this phase and the following one, we can see a loop and a path marked with dashed lines and arrows that cross back and forward the jet line. This excursions, that re-activates the jets and produce flaring activity, can happen $\lesssim 10$ times.

$\odot$ Phase iv: The microquasar finally enters the SS, where no radio-jet is detected. Therefore, the X-ray spectrum is dominated by the thermal blackbody component from the accretion disk. In this phase, the accretion rate is the highest, which extends the innermost stable orbit close to the $\mathrm{BH}$. From here on, the accretion rate starts decreasing, the dense of the gas in the inner part of the disk decrease as well creating the corona, and the microquasar 
enters the $\mathrm{HS}$, where relativistic jets, in which particles can be accelerated up to gamma rays, appear again.

\subsubsection{VHE radiative processes in microquasars}

After the discovery of the relativistic jets from the microquasars (Mirabel et al. 1992) and detection of non-thermal processes (from synchrotron radio up to high-energy gamma rays), VHE gamma-ray emission from this kind of systems was proposed in the literature from both leptonic (e.g. Atoyan \& Aharonian 1999, Bosch-Ramon et al.2006) and hadronic processes (e.g. Romero et al. 2003). Bosch-Ramon \& Khangulyan (2009) provides a deep review on the mechanism of particle acceleration and VHE emission of microquasars. Here I just point out the most relevant processes in the $\mathrm{VHE}$ regime.

\subsubsection{Leptonic processes}

The most efficient radiative process at VHE in microquasars seems to be a leptonic one, the IC. There are different possible source photon fields according to the distance of the production site to the compact object: close to the $\mathrm{BH}$, IC of thermal photons (Georganopoulos et al. 2002, Romero et al. 2002), or synchrotron photons (e.g. Bosch-Ramon et al. 2006) may be dominant. When the production region is situated inside the binary but further from the $\mathrm{BH}$, the process can take place on photons from the companion star. In cases with powerful jets, VHE gamma-ray emission may be also produced in the region where the outflows interact with the environment. Other leptonic mechanisms, besides IC, have been proposed in the literature to explain lower energy emission, mainly synchrotron and relativistic Bremsstrahlung. Depending on the conditions, one mechanism would dominate over the other. Both process have been suggested to take place either at the base of the jet (see, e.g. Markoff et al.2001 for sychrotron emission and BoschRamon et al. 2006 for Bremsstrahlung) or at binary scales along the jet (e.g., Yuan et al. 2005 and Bosch-Ramon et al. 2006, respectively). At the termination of the stream, synchrotron and relativistic Bremsstrahlung can also be expected as suggested by Aharonian \& Atoyan (1998) and Bordas et al. (2009).

\subsubsection{Hadronic processes}

In the hadronic scenario, the main processes producing gamma rays are proton-proton and protonphoton interactions. The targets for the proton-proton interaction are the thermal protons or ions in the jets or, more likely, in the stellar wind, whereas the photon sources are either jet synchrotron, accretion disk or stellar photospheric photons. Any of these interactions produce $\pi^{0}$ that, in turn, decay into two gamma rays. This hadronic collision would also produce charge pions $\left(\pi^{ \pm}\right)$that decay into muons and neutrinos. This way, the detection of neutrinos from microquasars would be an irrefutable probe of hadronic processes taking place inside these system. Muons can, in turn, decay into electron-positron pairs and neutrinos, and these secondary pairs could be responsible for the low-energy gamma-ray emission (via synchrotron, IC or Bremsstrahlung; Orellana et al. 2007). 


\subsubsection{Radiative processes in a low-mass microquasars}

As we have seen, the companion star plays a key role on several models, both in leptonic and hadronic mechanisms: the stellar donor is the responsible of providing seed photons for the IC or nuclei and photon targets for the proton-proton and proton-photon interactions. However, lowmass microquasars, with old and cold secondary stars, do not provide a proper environment for these processes to take place and hence, these models are only suitable in cases with high-mass companions.

Nevertheless, several models have been developed to explain gamma-ray emission from this type of systems. Zhang et al. (2015) proposed a pure leptonic model with relativistic electrons along the jet, where the dominant mechanisms ended up to be synchrotron and SSC emissions from an extended dissipation region in the jet. On the other hand, in hadronic scenario, photopion production inside the relativistic outflow by synchrotron jet emission, given the weak wind of the secondary star, could be considered as possible process (Levinson \& Waxman 2001). Moreover, a simple model on proton low-mass microquasars (assuming that a significant part of the jet composition is formed by protons) was developed by Romero \& Vila (2008), where MeV-GeV gamma rays were predicted from these sources. TeV energies are also expected in some cases at detectable levels for the currentIACTs, depending on the ratio protons/leptons. However, this simple model does not take into account rapid variability, one of the outstanding properties of these systems, as we will see with V404 Cygni in Chapter6.

In general, gamma rays in low-mass microquasars, like V404 Cygni, are expected to be produced inside the relativistic jets or the corona by the interaction of their own matter, radiation and magnetic fields, given the lack of targets provided by the low-mass companion star (see, e.g. Bosch-Ramon et al.2006, Vila \& Romero 2008, Vieyro \& Romero 2012).

\subsubsection{Photon-photon absorption}

Close to the corona, at the base of the jet, and at binary scales, the environment not only help to produce gamma ray but extreme conditions can lead to photon-photon absorption as well. Thus, close to the compact object, thermal $[\mathrm{UV}$ and $\mathrm{X}$-ray photons coming from the accretion process produce strong absorption on $\mathrm{GeV}$ gamma rays via pair production. This sub-product could give rise to secondary emission from cascade, however the likely high magnetic field at the base of the jet may suppress this effect.

If $\mathrm{VHE}$ emission is produced at the scale of the binary system $\left(\lesssim R_{o r b} \mathrm{~cm}\right.$ from the compact object, where $R_{\text {orb }}$ is the size of the system) in high-mass microquasars, the VHE photons will suffer also severe absorption because of the Near-infrared (NIR) stellar photon field (Orellana et al. 2007, Bednarek \& Giovannelli 2007). This absorption is orbitally modulated, since it depends in the companion star-emitter-observer relative positions. This means that when the star is between the observer and the compact object (the so-called, superior conjunction of the compact object) the attenuation is at its maximum, while if the compact object is interposed between (inferior conjunction), the absorption is expected to be minimum. 


\subsubsection{VHE observations of microquasars with MAGIC}

Gamma-ray emission from microquasars has been theoretically predicted for several years. However, it was not until less than a decade that gamma rays were detected from this type of systems. In 2009, two satellites AGILE and Fermi-LAT reported, for the first time, excess in energies above $100 \mathrm{MeV}$ from the high-mass microquasar Cygnus X-3 (Tavani et al. 2009b, Fermi LAT Collaboration et al. 2009b). Another firmly established microquasar, composed as well with a high-mass companion, Cygnus X-1, presented a hint of steady emission reported by Malyshev et al.(2013a). Hints of transient radiation from this source was also reported by AGILE (Sabatini et al.2010a, Rushton et al.2012a and Sabatini et al.2013a).

No VHE gamma-ray emission has been detected up to now from microquasars. Although pursued for many IACTs, this complicated task of disentangling the TeV regime in these systems is still not fulfilled, mostly due to the extremely good sensitivity required by the instruments. MAGIC had performed deep observations on microquasars since it starts operation in 2004, looking to Cygnus X-1, Cygnus X-3, SS 433, GRS 1915+105 or Scorpius X-1. In 2006, MAGIC published the detection of LS I +61 303 (Albert et al.2006), however although it was classified as microquasar at the beginning, it is currently accepted to be consistent with a pulsar wind scenario.

In this thesis, I present the latest results of two high-mass and one low-mass microquasars: Cygnus X-1, Cygnus X-3 and V404 Cygni. The former was observed in a long-term campaign from 2007 to 2014 accumulating 100 hours of good quality data. Such a deep campaign was motivated by a hint of signal detected by MAGIC in 2006 at the level of $4.1 \sigma$ in the direction of this source (Albert et al. 2007). Cygnus X-3 was, at the time of starting this thesis, the best candidate for searching $\mathrm{VHE}$ gamma-ray emission given that was at the moment the only microquasar detected in the gamma-ray band. It was observed following a strict follow-up observations campaign: we performed daily analysis of public Fermi-LAT data and according to the results on the MeV-GeV regime, MAGIC observations were triggered. V404 Cygni was observed during an extreme outburst that the system underwent on June 2015 that lasted several days. MAGIC could observed the source at its maximum activity thanks to the automatic Gamma-ray Burst procedure. With all these observations, MAGIC has been able to provide very useful information and shed light on microquasars in the VHE regime. 


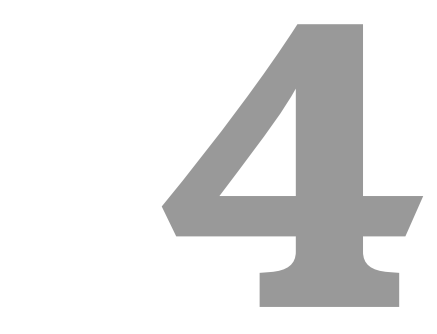

Cygnus X-1

\subsection{History}

Cygnus X-1 is one of the brightest and most studied X-ray sources in our Galaxy and a firmly established stellar-mass $\mathrm{BH}$ X-ray binary system. Discovered in the early stage of the X-ray astronomy (Bolton 1972), the system is located in the Cygnus region $\left(l=71.32^{\circ}\right.$ and $\left.b=+3.09^{\circ}\right)$ at a distance of $1.86_{-0.11}^{+0.12} \mathrm{kpc}$ from the Earth (Reid et al. 2011). It is comprised of a $(14.81 \pm 0.98) M_{\odot}$ $\mathrm{BH}$ and a O9.7 Iab type supergiant companion star with a mass of $(19.16 \pm 1.90) \mathrm{M}_{\odot}$ (Orosz et al. 2011). Nevertheless, the most plausible mass range of the donor star has been recently increased to 25-35 $\mathrm{M}_{\odot}$ by Ziółkowski (2014). This system is the only HMXB for which the compact object has been clearly identified as $\mathrm{BH}$.

The assumption that Cygnus X-1 ranks among the microquasars was accepted after the detection, with the VLBA instrument, of a highly collimated one-sided relativistic radio-jet that extends $\sim 15$ mas from the source (opening angle $<2^{\circ}$ and velocity $\geq 0.6 c$, Stirling et al. 2001). These jets are thought to create a $5 \mathrm{pc}$ diameter ring-like structure observed in radio that extends up to $10^{19} \mathrm{~cm}$ from the BH (Gallo et al. 2005). The total power carried by these relativistic outflows is $10^{36-37} \mathrm{erg} \mathrm{s}^{-1}$ (Gallo et al. 2005; Russell \& Fender 2010).

The binary system moves following a slightly elliptical orbit with eccentricity of 0.018 (Orosz et al. 2011), orbital period of 5.6 days (Brocksopp et al. 1999) and an inclination angle of the orbital plane to our line of sight of $27.1 \pm 0.8^{\circ}$ (Orosz et al. 2011). The superior conjunction phase of the compact object, when the companion star is interposed between the $\mathrm{BH}$ and the observer (see Figure 4.2), corresponds to phase 0, assuming the ephemerides $T_{0}=52872.788$ HJD taken from Gies et al. (2008). As mentioned in Chapter 3.1, the X-ray binaries generally suf- 


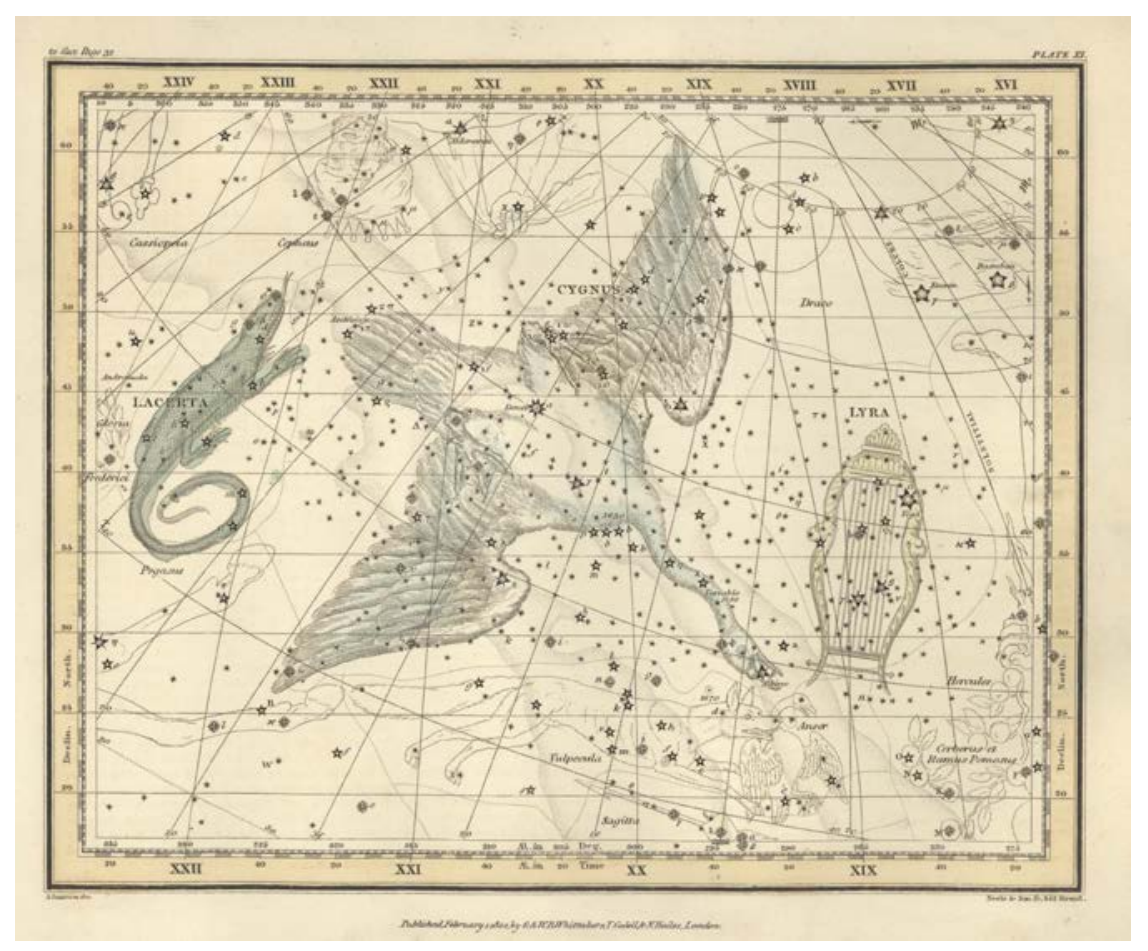

Figure 4.1: Alexander Jamieson's Celestial Atlas representation of the Cygnus Constellation (1822). The location of Cygnus X-1 corresponds to the $\eta$ symbol in the neck of the swan figure.

fer flux periodicity at their own orbital period. Cygnus X-1 shows this kind of modulation both in X-ray and radio wavelengths (Wen et al. 1999. Brocksopp et al. 1999, Szostek \& Zdziarski 2007), which may be caused by absorption or scattering by the wind of the donnor star over the radiation emitted from the compact object. Besides this modulation, several X-ray binary systems also present flux variations at periods much longer than their respective orbital period. This effect is known as superorbital modulation and is thought to be caused by the precession of the accretion disk or jet (Poutanen et al. 2008). The X-ray superorbital period of Cygnus X-1 was under debate for several years. Initally, it was estimated to be $\sim 290 \mathrm{~d}$ by Priedhorsky et al. (1983). Later, a large number of authors claimed a superorbital periodicity of half this value, $\sim 150 \mathrm{~d}$ (e.g., Brocksopp et al. 1999 or more recent Lachowicz et al. 2006). The latest results confirm again a superorbital period of $~ 300 \mathrm{~d}$, as suggested by Rico (2008) and confirmed by Zdziarski et al. (2011).

Given that is composed of a $\mathrm{BH}$. Cygnus X-1 displays the two canonical X-ray spectral states of a $\mathrm{BH}$ transient system (see Chapter 3.2.3), the HS and the SS (Esin et al. 1998), and the course that it follows through all the different states is well defined by the HID (Fender et al. 2004). Therefore, its X-ray spectrum can be described as the sum of two components: a blackbodylike emission coming from the disk and dominant during the SS state, and a power-law tail, most likely originated due to IC of disk photons by hot thermal electrons in the corona, and dominant during the $\mathrm{HS}$ state. As we have seen in Chapter 3.2.3, there is a relation between 


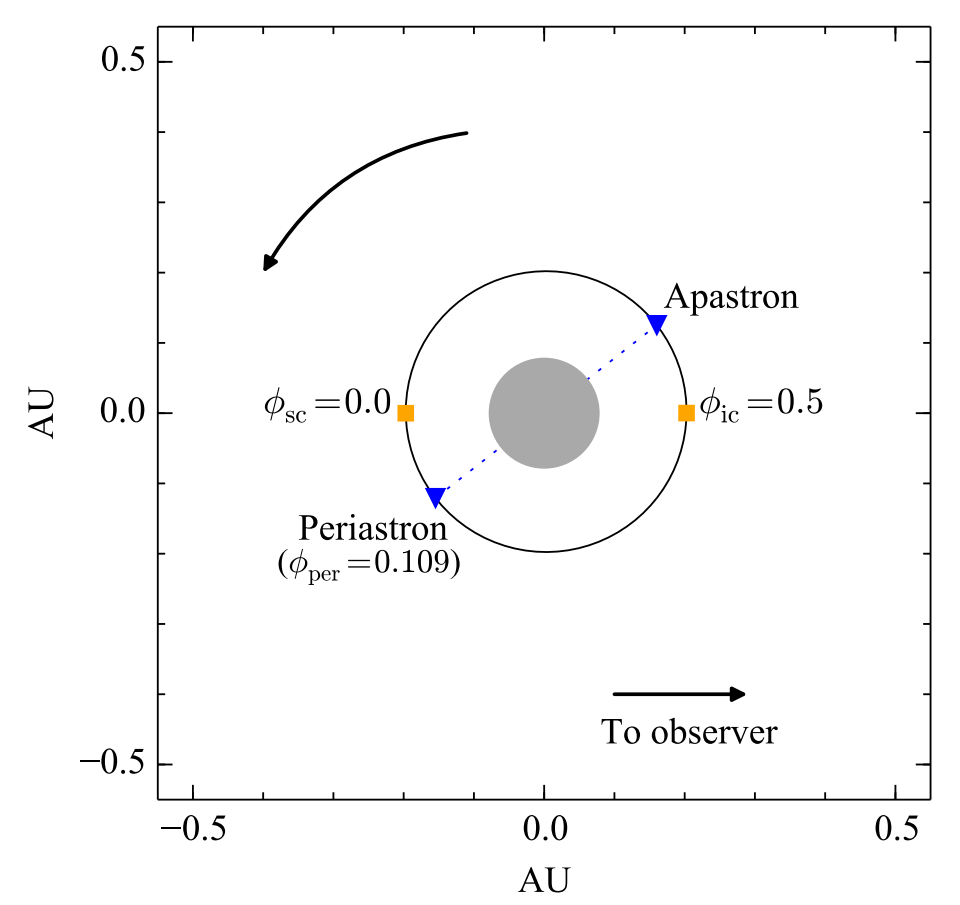

Figure 4.2: Schematic of the Cygnus $X-1$ orbit where the superior $\left(\phi_{S C}=0\right)$ and inferior conjunction $\left(\phi_{I C}=0.5\right)$ are marked. The almost circular orbit (eccentricity 0.018$)$ that follows the $\mathrm{BH}$ is on scale with the companion star (in grey) of $16.4 \mathrm{R}_{\odot}$ (radius taken from Orosz et al.|2011, as well as the periastron and apastron phases). Neither the inclination of the orbit with the line of sight or the longitude of the ascending node were considered here. AU stands for astronomical units.

radio and X-ray: whilst in the $\mathrm{HS}$, microquasars display steady relativistic synchrotron jets at $\mathrm{GHz}$ frequencies, except for some unusual flares in Cygnus X-1 (Fender et al. 2006), during SS that emission is strongly quenched. However, Cygnus X-1 is a persistent X-ray source never fully disk-dominated, i.e. even during its $\mathrm{SS}$ the system presents a strong power-law component and evidences of an unresolved compact jet during this state (Rushton et al. 2012b). Nevertheless, this jet is 3-5 times weaker than the one observed during the $\mathrm{HS}$ that reached $0.6 c$ (Gallo et al. 2005). Thus, there is a constant level of radio emission around $10-15 \mathrm{mJy}$, that extends with no cutoff, up to the IR band, where the contribution from the O9.7 Iab type donor dominates, hindering the measurement of such cutoff.

Observations with COMPTEL when Cygnus X-1 remained in the SS suggested, for the first time, the existence of non-thermal component beyond MeV (McConnell et al. 2002). This result gave rise to an increase of the interest for this source in the gamma-ray regime. Nevertheless, observations with INTEGRAL excluded the existence of this $\mathrm{MeV}$ tail in the SS, but probed, in turn, the presence of such non-thermal hard emission during $\mathrm{HS}$, when the jets were present (Rodriguez et al. 2015b). INTEGRAL-IBIS also reported a hard tail in $\mathrm{HS}$ which was shown to be polarized in the energy range of $0.4-2 \mathrm{MeV}$ at a level of $\sim 70 \%$ with a polarization angle of $(40.0 \pm 14.3)^{\circ}$ (Laurent et al.|2011, Jourdain et al.|2012). The origin site of this polarized MeV tail 
was suggested to be the jets where ultra-relativistic electrons would produce it via synchrotron. The corona was also considered as source of this radiation, where a population of secondary leptons would emit synchrotron soft gamma rays (Romero et al.2014).

Steady high-energy gamma-ray emission during $\mathrm{HS}$ was hinted by Malyshev et al. (2013b) at the level of $4 \sigma$ in the energy range of $0.1-10 \mathrm{GeV}$ by using 3.8 years of Fermi-LAT data. At the time of this thesis, the 7.5 years of Pass 8 Fermi-LAT data was released (see Chapter 2.6.2.1). Given the hint spotted in the past, with more available data and better sensitivity, the analysis of this Cygnus X-1 data could clearly provide new information on accreting X-ray binaries. The data used and the results of such analysis can be found in Section 4.2. Besides this persistent emission, the source underwent 3 preceding episodes of transient activity detected by AGILE. The first two flaring events occurred during $\mathrm{HS}$ on the 16th of October 2009, with an integral flux of $(2.32 \pm 0.66) \times 10^{-6} \mathrm{ph} \mathrm{cm}^{-2} \mathrm{~s}^{-1}$ between 0.1 and $3 \mathrm{GeV}$ (Sabatini et al. 2010b), and on the 24th March 2010, with an integral flux of $2.50 \times 10^{-6} \mathrm{ph} \mathrm{cm}^{-2} \mathrm{~s}^{-1}$ for energies above 100 $\mathrm{MeV}$ (Bulgarelli et al. 2010). The third one, on the 30th of June 2010 with a flux of $(1.45 \pm$ $0.78) \times 10^{-6} \mathrm{ph} \mathrm{cm}^{-2} \mathrm{~s}^{-1}$ also for energies above $100 \mathrm{MeV}$ (Sabatini et al. 2013b), took place during the intermediate state when the source was leaving the $\mathrm{HS}$ but just before an atypical radio flare (Rushton et al. 2012b). All these episodes lasted only 1-2 days. An independent analysis performed by Bodaghee et al. (2013) using 3.6 years of Fermi-LAT data confirmed, at the level of 3-4 $\sigma$, transient emission from Cygnus X-1, although not coincident with the AGILE flares (between one and two days before the event reported by Sabatini et al. 2010b).

MAGIC observed the source in the past for a total of 40 hours, spanning in 26 nights between June and November of 2006. At that period, the observations were carried out with the stand-alone MAGIC telescope, MAGIC I. Although no significant excess for steady gamma-ray emission using the all data sample was found, during the daily basis analysis a hint on the 24th of September 2006 (MJD=54002.96), corresponding to an orbital phase of 0.9 (i.e. close to the superior conjunction of the compact object) was spotted (Albert et al. 2007). This search yielded an evidence of gamma rays at $4.9 \sigma$ ( $4.1 \sigma$ after trials) in an effective time of 79 minutes. This excess took place at the maximum superorbital modulation of the source and simultaneously with the rising edge of a hard X-ray flare detected by INTEGRAL, Swift-BAT and RXTE-ASM (Malzac et al. 2008). The energy spectrum computed for this day is well defined by a simple power-law of $\mathrm{d} \phi / \mathrm{d} E=(2.3 \pm 0.6) \times 10^{-12}(E / 1 \mathrm{TeV})^{-3.2 \pm 0.6} \mathrm{~cm}^{-2} \mathrm{~s}^{-1} \mathrm{TeV}^{-1}$. VERITAS Collaboration also observed Cygnus X-1 on 2007 without any significant detection (Guenette et al. 2009) and therefore, former MAGIC results were the first experimental hint of VHE emission from a stellar $\mathrm{BH}$ binary. Consequently, both $\mathrm{HE}$ and $\mathrm{VHE}$ hints triggered a deep campaign on Cygnus $\mathrm{X}-1$, whose results are shown in this chapter.

\subsection{Fermi-LAT analysis}

\subsubsection{Data selection}

For this analysis, we used 7.5 years of Pass 8 data, from the 4th of August 2008 (MJD 54682) to the 2nd of February 2016 (MJD 57420). As shown in Chapter 2.6.2.1, using Pass 8 data 
allowed us to increase the energy range and cover from $60 \mathrm{MeV}$ up to $500 \mathrm{GeV}$. These results were obtained through two independent methods that worked as cross-check for the results: on one hand, I analyzed the sample following the standard FERMI SCIENCE TOOLS $\square$, and on the other, results were obtained using the Fermipy ${ }^{2}$ package, a set of python tools recently released to automatize the Pass 8 analysis of Fermi-LAT data. In both cases, and in order to properly compare results, the cuts applied to the data were the same. It was selected "P8R2_SOURCE" class photons from the LAT archive, with the aim of looking in the widest possible energy range $(60 \mathrm{MeV}-500 \mathrm{GeV})$, that were located within a $30^{\circ}$ acceptance cone from Cygnus X-1, assuming the coordinates $\mathrm{RA}_{\mathrm{J} 2000}=19 \mathrm{~h}: 58 \mathrm{~m}: 21.676 \mathrm{~s}, \mathrm{Dec}=+35^{\circ}: 12 \mathrm{~m}: 5.78 \mathrm{~s}$ (van Leeuwen 2007). Only "SOURCE" class events were used, which corresponds to those with highly probability of being photons. Therefore, the corresponding "P8R2_SOURCE_v16" IRFs were taken, which define the response of the satellite to gamma rays with a certain energy (defined by the EDISP event-type) and arrival direction (whose quality is divided in PSF event-type) in the coordinates of the instruments for the given period. When performing the analysis with the standard FERMI SCIENCE TOOLS, I used those events with PSF event-type FRONT+BACK and cross-checked results by selecting the events separately from the FRONT and BACK track. However, with Pass 8 the photon class events can be subdivided into quartiles regarding the quality of their PSF and energy reconstruction. Thus, the analysis with the Fermipy tools was computed for the four PSF event-type separately and combining the output results with a joint likelihood fit. Given the possible contamination by the albedo gamma rays from the Earth, those photons whose reconstructed direction angle with respect to the instrument was larger than $90^{\circ}, 85^{\circ}, 75^{\circ}$ and $70^{\circ}$ for each of the four PSF quartiles (the tighter the better the PSF is) were not included in the study. In the case of the standard analysis, I fixed the conservative value of $90^{\circ}$. On the other side, no cut was applied to the rocking angle, since the analysis is not very sensitive to the Earth Limb and data in which the Fermi-LAT satellite was in pointing mode to the Galactic-Center was also included. By analyzing the PSF event-type separately with the Fermipy tools, the best possible angular resolution was achieved, e.g. $\sim 0.5^{\circ}$ at $1 \mathrm{GeV}$. Both analysis, using standard FERMI SCIENCE TOOLS and Fermipy tools were probed to be compatible.

\subsubsection{Model}

To create a model that defines the emission coming from the region around Cygnus X-1, we used $14^{\circ} \times 14^{\circ} \mathrm{RoI}$ in galactic coordinates. The model needs to account for the galactic diffuse emission as well as the isotropic contribution, mainly composed by extragalactic diffuse radiation and cosmic rays. The corresponding background models, utilized in this analysis, were $g l l_{-} i e m_{-} v 06$. fits, for the galactic contribution, and iso_P8R2_SOURCE_V6_PSF F_v06.txt, where $\mathrm{x}=0,1,2$ and 3 , for the isotropic one. As point-like background sources, we first selected all those contained within $22^{\circ}$ radius from the third LAT catalogue (3FGL, Acero et al. 2015), which used $\sim 4$ years of Pass 7 Reprocessed LAT data. All spectral parameters from sources further away more than $14^{\circ}$ from the Cygnus X-1 location were fixed, given the small contribu-

\footnotetext{
${ }^{1}$ http://fermi.gsfc.nasa.gov/ssc/data/analysis/software/

${ }^{2}$ http://fermipy.readthedocs.org/en/latest/
} 
tion to the total amount of photons arriving from the target nominal position. We left free the flux normalization of sources between $7^{\circ}$ and $14^{\circ}$ and that of extremely bright sources (with significance above $100 \sigma$ ), as well as the flux normalization of the diffuse components (both galactic and isotropic). The closest sources, those placed at less than $7^{\circ}$ from Cygnus X-1, preserved all their spectral parameters free to vary in the maximum likelihood fit performed with the gtlike function.

The significance is determined through the TS value, defined as TS $=-2 \ln \left(L_{0} / L_{1}\right)$, where $L_{0}$ is the likelihood value for the model without including Cygnus X-1 (the so-called null hypothesis) and $L_{1}$ is the likelihood including it. This way, the TS will be maximized when the likelihood of the model with our source is maximized as well. For all the TS maps we obtained around the microquasar, we adopted a power-law function to describe the Cygnus X-1 spectrum with a photon index of 2.5 , while all the point-like background sources remained fixed.

The best localization of Cygnus X-1 was search for energies above $1 \mathrm{GeV}$ (assuring thus a good angular resolution), using Fermipy. The method consists in two steps: from a $4^{\circ} \times 4^{\circ} \mathrm{TS}$ map centered around Cygnus X-1, the algorithm looks for the location with the maximum TS value, and afterwards it performs a full likelihood fit around this peak of TS to provide the best position. During this process, the flux normalization of extremely bright source are free to vary!

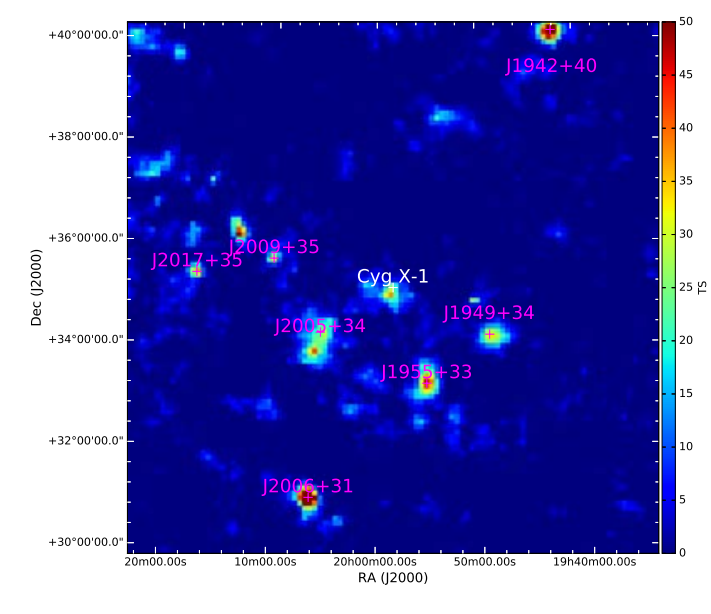

Figure 4.3: $5^{\circ} \times 5^{\circ} \mathrm{TS}$ map centered at Cygnus $\mathrm{X}-$ 1 for energies above $1 \mathrm{GeV}$ in which the point-like background model is only defined by sources from the 3FGL catalogue.
The first TS maps obtained were not flat, i.e. the background emission was not well described including only the 3FGL sources. While the diffuse and isotropic contribution is not expected to change from the period in which the 3FGL catalogue was released and the time of our analysis, the larger ( 7.5 years) Pass 8 data sample allowed to improve the sensitivity and hence new sources arised. We found in this first model 7 new hotspots with TS above 25 (for the full energy range $0.6-500 \mathrm{GeV}$ ), a part from an already clear excess at the position of Cygnus X-1 (see Figure 4.3).

In order to account for the contribution of these candidates, we included them in the model by assuming, in most of the cases, that their spectrum follow a power-law function. General features of these sources are:

$\odot$ J1942+40: This source presented a TS $=55$ between $0.6-500 \mathrm{GeV}$. The excess corresponds

\footnotetext{
${ }^{1}$ http://fermipy.readthedocs.io/en/latest/advanced/localization.html
} 
to the location $\mathrm{RA}_{\mathrm{J} 2000}=19 \mathrm{~h}: 42 \mathrm{~m}: 7 \mathrm{~s}$, Dec $=+40^{\circ}: 14 \mathrm{~m}: 7 \mathrm{~s}$, most probably associated to the open cluster NGC 6819, where Gosnell et al. (2012) detected several X-ray sources with the XMM-Newton Observatory.

$\odot$ J1949+34: This source displayed the lower significance with a TS=35 also in the full energy range. The coordinates associated to it are $R_{\mathrm{J} 2000}=19 \mathrm{~h}: 49 \mathrm{~m}: 7 \mathrm{~s}$, Dec $=+34^{\circ}: 15 \mathrm{~m}: 44 \mathrm{~s}$.

$\odot$ J1955+33: It showed strong excess with $\mathrm{TS}=90$ (between $0.6-500 \mathrm{GeV}$ ) at $\mathrm{RA}_{\mathrm{J} 2000}=19 \mathrm{~h}: 55 \mathrm{~m}: 10 \mathrm{~s}$, Dec $=+33^{\circ}: 18 \mathrm{~m}: 34.8 \mathrm{~s}$.

$\odot \mathbf{J 2 0 0 5 + 3 4 :}$ The LAT excess (centered at $\mathrm{RA}_{\mathrm{J} 2000}=19 \mathrm{~h}: 42 \mathrm{~m}: 7 \mathrm{~s}, \mathrm{Dec}=+40^{\circ}: 14 \mathrm{~m}: 7 \mathrm{~s}$ ), came to a $\mathrm{TS}=49$ in the full energy range.

$\odot$ J2006+31: This is an extremely bright new LAT source with $\mathrm{TS}=115$ at $\mathrm{RA}_{\mathrm{J} 2000}=20 \mathrm{~h}: 06 \mathrm{~m}: 12.8 \mathrm{~s}$, Dec $=+31^{\circ}: 02 \mathrm{~m}: 38.3 \mathrm{~s}$. It is spatially coincident with the $164 \mathrm{~ms}$ radio pulsar PSR J2006 +3102 (Nice et al. 2013). Just for this case, the spectrum was better described (at more than $3 \sigma$ level) by a LogParabola instead of power-law function.

$\odot$ J2009+35: This source presented a $\mathrm{TS}=48$ for energies greater than $60 \mathrm{MeV}$ centered at $\mathrm{RA}_{\mathrm{J} 2000}=20 \mathrm{~h}: 09 \mathrm{~m}: 57.8 \mathrm{~s}$, Dec $=+35^{\circ}: 44 \mathrm{~m}: 48.6 \mathrm{~s}$.

$\odot \mathbf{J 2 0 1 7 + 3 5}$ : Its excess reached $\mathrm{TS}=90$ at $\mathrm{RA}_{\mathrm{J} 2000}=20 \mathrm{~h}: 17 \mathrm{~m}: 25 \mathrm{~s}, \mathrm{Dec}=+35^{\circ}: 26 \mathrm{~m}: 5 \mathrm{~s}$.

The coordinates given for these 7 new sources were estimated for energies above $1 \mathrm{GeV}$ to consider events with better angular resolution, with a statistical uncertainty of $\sim 0.2^{\circ}$. On the other hand, we found that the source 3FGL J2014.4+3606, associated to the SNR G73.9+0.9, presented a mismatching of $0.24^{\circ}$ between the location given in the 3FGL catalogue and the centroid obtained in our TS maps. The new coordinates were set to $\mathrm{RA}_{\mathrm{J} 2000}=20 \mathrm{~h}: 13 \mathrm{~m}: 33.8 \mathrm{~s}$, $\mathrm{Dec}=+36^{\circ}: 11 \mathrm{~m}: 54.0 \mathrm{~s}$. In the model, the spectrum of this source is described by a power-law function, since the more complex LogParabola function, suggested by Zdziarski et al. (2016c) using Pass 8 data, was not favoured. Taken into account all these modifications in our model, the background was then well defined.

The SED was computed for the entire energy range, i.e from $60 \mathrm{MeV}$ up to $500 \mathrm{GeV}$, using 7 logarithmically spaced bins. For this calculation, the photon index of Cygnus X-1 is fixed to the one obtained during the overall fit (full energy range), while the flux normalization is let free to vary in each energy bin. The spectral parameters of the rest of the point-like background source are fixed by the overall fit too. ULs (at the 95\% C.L.) are calculated if the TS in one bin does not reach at least 4 , i.e. if the significance is less than $2 \sigma$. The SED were obtained with the Fermipy software package.

In order to compute a lightcurve of the more than 7 years of data, which is calculated by applying the maximum likelihood fit in a daily basis, we used the FERMI SCIENCE TOOLS for energies above $100 \mathrm{MeV}$. With this energy range, the results reported here can be compared with the previous flaring activities reported from Cygnus X-1 (Sabatini et al.2010b, 2013b; Bodaghee 


\begin{tabular}{cc}
\hline \hline $\mathrm{HS}$ & $\mathrm{SS}$ \\
\hline $54682-55375$ & $55391-55672$ \\
$55672-55790$ & $55797-55889$ \\
$55889-55945$ & $55945-56020$ \\
$56020-56086$ & $56086-56330$ \\
$56718-56753$ & $56338-56718$ \\
$56759-56839$ & $56839-57009$ \\
$56848-56852$ & $57053-57103$ \\
$57009-57053$ & $57265-57325$ \\
$57103-57265$ & \\
$57325-57420$ & \\
\hline
\end{tabular}

Table 4.1: Intervals in MJD of the HS and the SS periods of Cygnus X-1.

et al. 2013). In this case, ULs at the 95\% C.L. are computed when the TS in 1 day-bin was lower than 9 (significance $<3 \sigma$ ). If the TS was higher, an integral flux for energies between 100 $\mathrm{MeV}$ and $20 \mathrm{GeV}$ was calculated. The former maximum value of $20 \mathrm{GeV}$ was constrained by the results from the SED, as shown in the next subsection.

\subsubsection{Results}

The model (including the 3FGL and the new hotspots as point-like background emitters) lead us to claim the first firmly gamma-ray detection at the location of Cygnus X-1 with a TS=53, from $60 \mathrm{MeV}$ to $500 \mathrm{GeV}$. The microquasar is also detected at energies above $1 \mathrm{GeV}$ at the level of $\mathrm{TS}=31$. With the previously mentioned Fermipy algorithm to calculate the position of the source, the best coordinates for Cygnus X-1 were set to $\mathrm{RA}_{\mathrm{J} 2000}=19 \mathrm{~h}: 58 \mathrm{~m}: 56.8 \mathrm{~s}, \mathrm{Dec}=+35^{\circ}: 11 \mathrm{~m}: 4.4 \mathrm{~s}$, which shows an offset of $0.05^{\circ}$ with respect to the van Leeuwen (2007) location, but still compatible with Cygnus X-1 within the statistical uncertainties of $0.2^{\circ}$.

Given the former hinted dependency on the X-ray spectral state, we also performed the analysis of the source dividing the sample according to its $\mathrm{HS}$ and $\mathrm{SS}$ periods. These periods were defined with the public available Swift-BAT (15-50 keV) data, following the criterion given by Grinberg et al. (2013): above 0.09 counts $\mathrm{cm}^{-2}$ Cygnus X-1 stays in the HS +IS state and below in the SS. It is worth to mention that, taken into account the short duration (around days) of the transition periods between these two main states, the inclusion of the IS states into the HSperiod cannot alter the results. The division made into these two main periods is shown in Table 4.1 . The total amount of time in each subsample is very similar, 3.6 years for the $\mathrm{HS}$ and 3.7 years for the SS.

This analysis results on a clear detection of Cygnus X-1 during the HS with a TS $=49$ for the full energy range, which lead to an energy flux of $(7.7 \pm 1.3) \times 10^{-6} \mathrm{MeV} \mathrm{cm}^{-2} \mathrm{~s}^{-1}$. However, no significant gamma-ray emission from the source is detected during its spectrally soft state, which 
showed a very low $\mathrm{TS}=7$. For the latter, integral UL for energies greater than $60 \mathrm{MeV}$ and a $95 \%$ C.L. was set at $5.4 \times 10^{-6} \mathrm{MeV} \mathrm{cm}^{-2} \mathrm{~s}^{-1}$. This result evidences the correlation between the $\mathrm{GeV}$ emission and the hard X-ray, previously hinted by Malyshev et al. (2013a) and confirmed, just after the time of the publication of these results, by an independent analysis done by Zdziarski et al. (2016a). In Figure 4.4, the TS maps for energies above $1 \mathrm{GeV}$ for each of the X-ray states are shown.
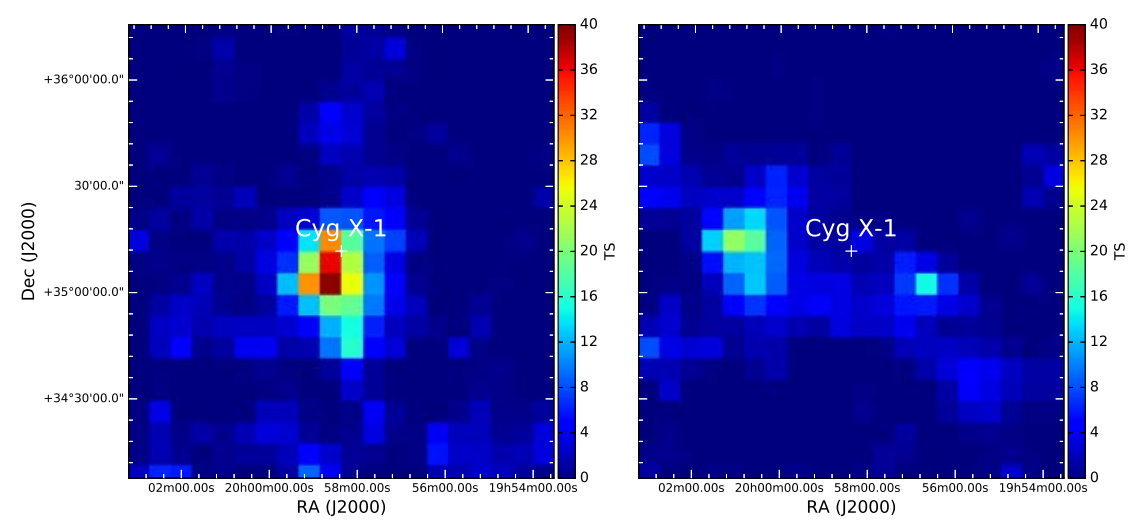

Figure 4.4: $2^{\circ} \times 2^{\circ} \mathrm{TS}$ maps for energies above $1 \mathrm{GeV}$ centered at the position of Cygnus X-1 (white cross). On the left: $\overline{\mathrm{TS}}$ map corresponding to the subsample data during the $\mathrm{HS}$, where the detection (TS=49) is evident. On the right: TS map corresponding to the subsample data during the $\mathrm{SS}$.

Due to the variability shown by Cygnus X-1, like the orbital modulation in X-ray or radio wavelengths, $\mathrm{HS}$ data sample was also analyzed according to the orbital phase. However, due to the low statistics, the division was performed in only two bins, aiming to center the data on the superior conjunction of the compact object (at phases between $\phi>0.75$ and $\phi<0.25$ ) and the inferior conjunction $(\phi>0.25$ and $\phi<0.75)$, which are critical phases given the almost circular orbit of the system. The source is detected around the superior conjunction at TS=31, while it remains undetectable $(\mathrm{TS}=10)$ during the inferior conjunction (Figure 4.5). Even though the statistics are low to study deeper this excess, the strong difference between the two bins (with the same effective time) can be understood as a hint of gamma-ray orbital modulation. The energy flux during the superior conjunction is $(7.6 \pm 1.7) \times 10^{-6} \mathrm{MeV} \mathrm{cm}^{-2} \mathrm{~s}^{-1}$.

The SED obtained for the $\mathrm{HS}$ is well defined by a simple power-law function with a photon index $\Gamma=(2.3 \pm 0.1)$ and a flux normalization of $f_{0}=(5.8 \pm 0.9) \times 10^{-13} \mathrm{MeV}^{-1} \mathrm{~cm}^{-2} \mathrm{~s}^{-1}$ at a pivot energy of $1.3 \mathrm{GeV}$ (see Figure 4.6). It extends from $60 \mathrm{MeV}$ up to $\sim 20 \mathrm{GeV}$, energy from which ULs were set. A broken power-law function was not favoured against a simple power-law: the improvement was not statistically significant, $\Delta \mathrm{TS}<2$. The spectrum computed for the phase-folded analysis yielded photon indices compatible within $1 \sigma$ with the $\Gamma=2.3$, in both superior and inferior conjunctions. The flux normalization for the superior conjunction was 

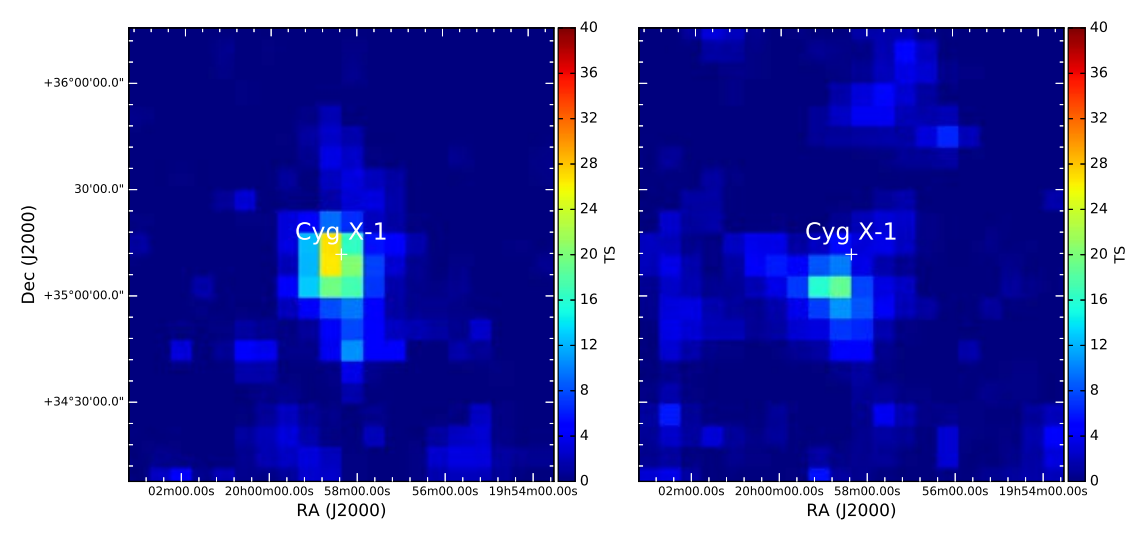

Figure 4.5: Phase-folded $2^{\circ} \times 2^{\circ} \mathrm{TS}$ maps for energies above $1 \mathrm{GeV}$ centered at the position of Cygnus X1 (white cross) when the source was in the HS. On the left: TS map for phases around the superior conjunction of the compact object $(\phi>0.75$ and $\phi<0.25)$. On the right: TS map for phases around the superior conjunction of the compact object $(\phi>0.25$ and $\phi<0.75)$.

$f_{0}=(5.7 \pm 1.3) \times 10^{-13} \mathrm{MeV}^{-1} \mathrm{~cm}^{-2} \mathrm{~s}^{-1}$.

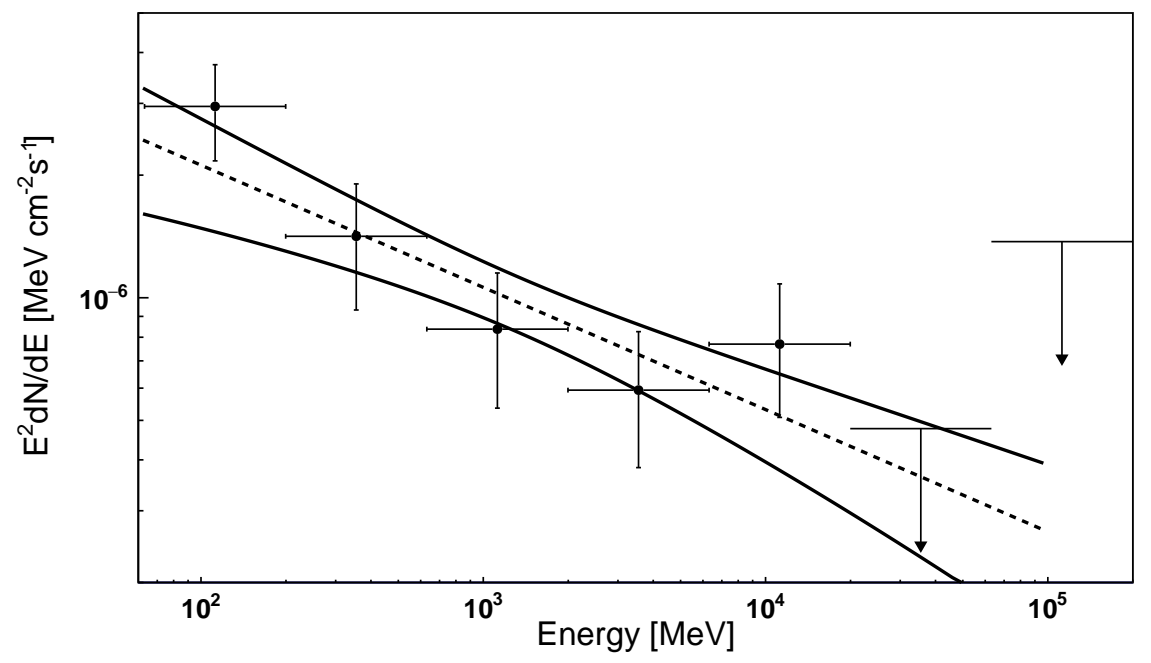

Figure 4.6: $\mathrm{SED}$ of Cygnus X-1 during the $\mathrm{HS}$ periods. It is well described by a power-law function with photon index $\Gamma=(2.3 \pm 0.1)$ and extends up to $\sim 20 \mathrm{GeV}$.

Given the rapid variation of the flux level previously reported by MAGIC on a timescale of hours (Albert et al. 2007), as well as the flaring activity hinted by Sabatini et al. (2010b, 2013b); Bodaghee et al. (2013), we carried out daily basis analysis of the $\sim 7.5$ years of data in an energy range between $100 \mathrm{MeV}$ and $20 \mathrm{GeV}$ (Figure 4.7). This search yielded no gamma-ray excess in any of the nights. All days with TS $>9$ are listed in Table 4.2 and among them, only MJD 55292 


\section{CYGNUS X-1}

is coincident with one transient event formerly reported by Bodaghee et al. (2013). No hint was either highlighted any day around the flaring events reported by AGILE (Sabatini et al. 2010b; Bulgarelli et al. 2010; Sabatini et al. 2013b). This apparent discrepancy can be explained based on the different exposure time and off-axis angle distance both satellites, Fermi-LAT and AGILE, presented during Cygnus X-1 observations, as discussed by Munar-Adrover et al. (2016) for the case of AGL J2241+4454.

\begin{tabular}{|c|c|c|c|c|}
\hline \multicolumn{2}{|l|}{ Date } & \multirow[t]{2}{*}{$\mathrm{TS}$} & \multirow{2}{*}{$\begin{array}{c}\text { Fermi-LAT flux } \\
\left(10^{-7} \text { photons } \mathrm{cm}^{-2} \mathrm{~s}^{-1}\right)\end{array}$} & \multirow[t]{2}{*}{ X-ray State } \\
\hline (уууу mm dd) & (MJD) & & & \\
\hline 2009-03-05 & 54895 & 10.3 & $4.8 \pm 2.0$ & $\mathrm{HS}$ \\
\hline 2010-02-02 & 55229 & 10.5 & $6.2 \pm 2.3$ & $\mathrm{HS}$ \\
\hline 2010-04-06 & 55292 & 12.2 & $3.1 \pm 1.1$ & $\mathrm{HS}$ \\
\hline $2012-12-31$ & 56292 & 9.2 & $5.3 \pm 2.2$ & SS \\
\hline 2014-02-10 & 56698 & 9.7 & $7.7 \pm 3.1$ & SS \\
\hline 2014-03-01 & 56717 & 10.5 & $6.3 \pm 2.5$ & $\mathrm{SS}$ \\
\hline 2014-03-06 & 56722 & 9.4 & $6.0 \pm 2.5$ & $\mathrm{HS}$ \\
\hline 2014-08-08 & 56877 & 10.2 & $6.7 \pm 2.5$ & $\mathrm{SS}$ \\
\hline $2015-05-26$ & 57168 & 9.6 & $4.5 \pm 1.8$ & $\mathrm{HS}$ \\
\hline
\end{tabular}

Table 4.2: Days, also in MJD, with a TS $>9$ (significance $\geq 3 \sigma$ ) obtained during the daily basis analysis in the energy range between $100 \mathrm{MeV}$ and $20 \mathrm{GeV}$. The fourth and fifth columns correspond to the FermiLAT flux and the X-ray state the source stayed for each day, respectively.

\subsubsection{Discussion}

As it was discussed in Chapter 3.2.4.3, several mechanism have been used to explain $\mathrm{HE}$ and VHE gamma-ray emission from microquasars. The IC scattering on seed photons would be dominant if the $\mathrm{HE}$ radiation does not comes from the lower part of the jet. Along the outflow, different photon fields can work as target for this process. At a distance of $\sim 10^{11} \mathrm{~cm}$ from the compact object, the dominant photon field is the stellar radiation one (Romero et al. 2014), defined as $\omega_{\star}=L_{\star} / 4 \pi\left(R_{\text {orb }}^{2}+Z^{2}\right) c$, where $L_{\star}=7 \times 10^{39} \mathrm{erg} \mathrm{s}^{-1}$ is the luminosity of the supergiant star in the Cygnus X-1 system (Orosz et al. 2011), $R_{\text {orb }}=3 \times 10^{12} \mathrm{~cm}$ is the orbital distance and $Z$ is the distance from the compact object along the jet. In comparison, the soft X-rays (1-20 keV) produced in the accretion disk due to viscosity forces, with a density of $\omega_{\text {softXrays }}=L_{\text {softXrays }} / 4 \pi Z^{2} c$, present a luminosity, $L_{\text {softXrays }}$, between $10^{36}$ and $2 \times 10^{37} \mathrm{erg} \mathrm{s}^{-1}$ (depending on the model, Di Salvo et al. 2001). Further away from the BH, the photon density from the companion star diminishes, but gamma rays can be produced in the shocks between the relativist outflow and the surrounding medium. This kind of interaction is believed to take place in Cygnus X-1 due to the inflate ring-like structure detected in radio by Gallo et al. (2005). This structures extends up to $10^{19} \mathrm{~cm}$ from the $\mathrm{BH}$ and hence, this distance is assumed to be the 
maximum extension of the jets in Cygnus X-1.

However, inside the binary, the gamma rays will suffer severe absorption due to photonphoton collision. In the case of $\mathrm{GeV}$ photons, this absorption is heavier close to the base of the jet where the UV] or soft X-rays photons density coming from the disk is high. For TeV photons (in the range of the MAGIC telescopes observations), this effect is greater at distances in which the stellar radiation field is dominant, given the high contribution of IR emission.

Taken this into account and the fact that we do detect $\mathrm{GeV}$ gamma rays from Cygnus X-1, we can calculate the minimum distance from the $\mathrm{BH}$ to avoid pair production between the $\mathrm{GeV}$ photons and the soft X-rays ( $\sim 1 \mathrm{keV})$ from the disk, following Akharonian et al. (1985) approach:

$$
R \geq 6 \times 10^{6}\left(\frac{d}{1 \mathrm{kpc}}\right)^{2}\left(\frac{F_{X-\text { rays }}}{10^{-2} \mathrm{keV}^{-1} \mathrm{~cm}^{-2} \mathrm{~s}^{-1}}\right)
$$

where $d=1.86 \mathrm{kpc}$ is the distance to Cygnus X-1 (Reid et al. 2011 ) and $F_{X-\text { rays }}=1.6 \times 10^{-9}$ erg cm $\mathrm{cm}^{-2} \mathrm{~s}^{-1}$ is the de-absorbed flux at $1 \mathrm{keV}$ given by Di Salvo et al. (2001). Considering this values, the production site of $\mathrm{GeV}$ gamma rays must be larger than $\mathrm{R}>2 \times 10^{9} \mathrm{~cm}$ from the $\mathrm{BH}$ to avoid being absorbed. Given that the radius of the corona is around $20-50 \mathrm{R}_{g}$, where $\mathrm{R}_{g}$ is the gravitational radius, i.e. $\sim 5-10 \times 10^{7} \mathrm{~cm}$, we can exclude the corona as the emission region for the observed gamma rays. Moreover, the GeV should be produced, not only outside of the corona, but most likely inside the jets. This conclusion is based on the exclusive detection of the system during $\mathrm{HS}$, when the relativistic jets are present.

If the hint of orbital modulation reported here is finally confirmed, we could exclude the jetmedium interaction regions, given that it is not affected by orbital variability and hence, the emission should originate from the jets themselves. Thus, we can establish an UL on the maximum distance with respect to the compact object at $Z<10^{13} \mathrm{~cm}$. Additionally, this type of modulation is only expected in case the $\mathrm{GeV}$ emission arises from anisotropic IC]scattering on stellar radiation (Jackson 1972; Aharonian \& Atoyan 1981; Zdziarski \& Pjanka|2013; Khangulyan et al. 2014). IC or SSC mechanism on thermal photons from the accretion disk can be discarded since no orbital modulation is expected (assuming no additional sources of variability). As mentioned before, the stellar photon field becomes dominant at $Z>10^{11} \mathrm{~cm}$ from the $\mathrm{BH}$, which allow us to constrain the GeV gamma-ray emitter location to $10^{11}-10^{13} \mathrm{~cm}$. This conclusion is compatible with the hydrodynamic simulations of the interaction between the stellar wind and Cygnus X-1like jets with power $\sim 10^{36-37} \mathrm{erg} \mathrm{s}^{-1}$ (Perucho \& Bosch-Ramon 2008; Yoon et al. 2016).

To achieve gamma rays between $60 \mathrm{MeV}$ and $20 \mathrm{GeV}$, the energy of the primary electrons needs to be at least of the order of several tens of $\mathrm{GeV}$, which means that the IC]scattering takes place in the Thomson region (where $E_{\gamma} E_{e^{-}}<<m_{e}^{2} c^{4}$, as explained in Chapter 1.2.1.5). In order to accelerate the electrons to those energies within the stellar photon field, a moderate magnetic field would be enough, as suggested by Khangulyan et al. (2008), i.e. $\mathrm{B} \sim 10^{-2} \mathrm{G} \times \eta$, where $\eta$ is the acceleration efficiency. If we now assume that the same population of electrons that produces the $\mathrm{GeV}$ gamma-ray emission is responsible of the synchrotron radiation detected in the jets at lower energies, we can constrain the magnetic field strength by the ratio of the X-ray and $\mathrm{HE}$ 
gamma-ray luminosities observed:

$$
\frac{B^{2}}{8 \pi}=\omega_{\star} \frac{L_{X-r a y}}{L_{G e V}}
$$

The energy flux obtained in this work during the $\mathrm{HS}$ for the full energy range, $(7.7 \pm 1.3) \times 10^{-6}$ $\mathrm{MeV} \mathrm{cm}-2 \mathrm{~s}^{-1}$, corresponds to a luminosity of $L_{\mathrm{GeV}}=5 \times 10^{33} \mathrm{erg} \mathrm{s}^{-1}$, assuming the distance of $1.86 \mathrm{kpc}$ to the system. Note that this value is a few orders of magnitude smaller than the total power emitted by the jets, $10^{36-37} \mathrm{erg} \mathrm{s}^{-1}$ (Gallo et al. 2005; Russell \& Fender 2010). In this case, we considered the $L_{X-\text { ray }}=2.2 \times 10^{37} \mathrm{erg} \mathrm{s}^{-1}$ between 20 and $100 \mathrm{keV}$. Thus, for a distance of $Z=10^{12} \mathrm{~cm}$, we obtain a maximum magnetic field strength of $\sim 2 \mathrm{kG}$, which decreased up to $700 \mathrm{G}$ at the edge of our production region $\left(Z=10^{13} \mathrm{~cm}\right)$.

\subsection{MAGIC analysis}

\subsubsection{Observations and Data analysis}

MAGIC observed Cygnus X-1 from July 2007 to October 2014. Therefore, observations were carried out in stand-alone mode with MAGIC I (Aliu et al. 2009a), with pre-upgrade telescopes (Aleksić et al. 2012b) and with post-upgrade stereo system (Aleksić et al. 2016ab). Further information on the performance of each period can be found in Chapter 2.4.

The analysis was performed using the MARS software described in Chapter 2.4.3. Integral and differential flux ULs were computed making use of the full likelihood analysis developed by Aleksić et al. (2012a), which takes into account the different IRFs of the telescopes along the years, assuming a $30 \%$ systematic uncertainty.

At La Palma, Cygnus X-1 culminates at a zenith angle of $6^{\circ}$. Observations, performed up to $50^{\circ}$, were carried out in a stand-alone mode (with just MAGIC I) from July 2007 to summer 2009, and, in stereoscopic mode, from October 2009 up to October 2014. Two data taking modes were used: the false-source tracking mode, wobble-mode, and the on-off mode (for deeper information see Chapter 2.4.2.1). In the former one, for this analysis, MAGIC pointed at two and four different positions situated $0.4^{\circ}$ away from the source to evaluate the background simultaneously (Fomin et al. 1994). In the latter mode, the background sample, observed separately from the on region, was recorded under same conditions (same epoch, zenith angle and atmospheric conditions) as for the on data but with no candidate source in the field of view. The total Cygnus X-1 data sample recorded by MAGIC amounts to $\sim 97 \mathrm{hr}$ after data quality cuts distributed over 53 nights between July 2007 and October 2014. The whole data sample extends over five yearly campaigns, characterized by different performances of the telescopes. Because of this, each epoch was analyzed separately with appropriate MC-simulated gamma-ray events. The details of the observations for each campaign are summarized in Table 4.3. For convenience, the following code is used in the table to describe the different observational features: STEREO 
stands for stereoscopic mode while MONO is used when only MAGIC I was operating. In the latter, the subscript specifies the observational mode: on-off or wobble mode. In STEREO, only wobble mode was used, so the subscript is used to specify whether the observations were taken before (pre) or after (post) the MAGIC upgrade.

Different criteria to trigger observations were used during the campaign to optimize observations, aimed at observing the system in a state, $\mathrm{HS}$, similar to that in which MAGIC previously reported evidence of emission Albert et al. (2007). The X-ray spectral states were defined by using public Swift-BAT (15-50 keV) and RXTE-ASM (1.5-12 keV) data, except for the data taken in 2014 where only Swift-BAT was considered (since RXTE-ASM was not available any more). Between July and November 2007, the criteria used to prompt the observation was a Swift-BAT flux larger than 0.2 counts $\mathrm{cm}^{-2} \mathrm{~s}^{-1}$ and a ratio between $R X T E$-ASM one-day average (in counts $\mathrm{s}^{-1}$ in a Shadow Scanning Camera (SSC)) and Swift-BAT lower than 200. This criterion is in agreement with the one set by Grinberg et al. (2013) to define the X-ray states of Cygnus X-1 using Swift-BAT data and utilized also in the Fermi-LAT analysis (see Section 4.2.3): above 0.09 counts $\mathrm{cm}^{-2} \mathrm{~s}^{-1}$ the microquasar stays in the $\mathrm{HS}$ - IS and below in the SS. The trigger criterion we selected is higher to achieve a count rate similar to that of the previous MAGIC hint. In July 2008, on top of the HS triggering criteria described above, observations were intensified following the X-ray superorbital modulation. The observations were triggered when the source was on the same superorbital phase as during the hint. Between June and October 2009, a new hardness ratio constraint using RXTE-ASM data of the energy ranges $5-12 \mathrm{keV}$ and $1.3-2 \mathrm{keV}$ was included: the observations were only stopped after 5 consecutive days of this ratio being lower than 1.2, to avoid interrupting the observations during the IS. In May 2011, the source was observed two nights based on internal analysis of public Fermi-LAT data that showed a hint at $\mathrm{HE}$ during a hard X-ray activity period. Since all the above mentioned data were taken during the $\mathrm{HS}$, for completeness, Cygnus X-1 was observed in its SS on September 2014 to search for gamma-ray emission in this state at the same flux level as in the previous one. To define the X-ray state of the source, Swift-BAT public data was again used following Grinberg et al. (2013) criteria.

\subsubsection{Search for steady emission}

We search for steady VHE gamma-ray emission from Cygnus X-1 at energies greater than 200 $\mathrm{GeV}$ making use of the entire data set of almost 100 hours. We did not find any significant excess and hence, we computed ULs assuming a simple power-law function with different photon indices. The lower value, $\Gamma=2$, is consistent with the results obtained in the $\mathrm{HE}$ band (see Section 4.2.3), while the upper one, $\Gamma=3.8$, is constrained by the former MAGIC results $(\Gamma=3.2 \pm 0.6$, Albert et al. 2007). Deviations in the photon index do not critically affect our results, quoted in Table 4.5, so all ULs obtained during this analysis are given at a C.L. of 95\% with $\Gamma=3.2$, which is the photon index obtained for the hint of signal reported by the MAGIC Collaboration (Albert et al. 2007). For steady emission, we obtained an integral flux UL for energies greater than 200 $\mathrm{GeV}$ of $2.6 \times 10^{-12} \mathrm{~cm}^{2} \mathrm{~s}^{-1}$. Differential flux ULs for the entire data sample can be found in Table 
Table 4.3: From left to right: date of the beginning of the observations in calendar and in MJD, effective time after quality cuts, zenith angle range, $X$-ray spectral state and observational conditions of the MAGIC observations. Horizontal lines separate different observational modes along the five yearly campaigns. During MJD 54656, 54657 and 54658, data under different observational modes were taken.

\begin{tabular}{|c|c|c|c|c|c|}
\hline \multicolumn{2}{|l|}{ Date } & \multirow{2}{*}{$\begin{array}{c}\text { Eff. Time } \\
{[\mathrm{hr}]}\end{array}$} & \multirow{2}{*}{$\begin{array}{l}\mathrm{Zd} \\
{\left[{ }^{\circ}\right]}\end{array}$} & \multirow{2}{*}{$\begin{array}{c}\text { Spectral } \\
\text { State }\end{array}$} & \multirow{2}{*}{$\begin{array}{c}\text { Obs. } \\
\text { conditions }\end{array}$} \\
\hline [yyyy mm dd] & {$[\mathrm{MJD}]$} & & & & \\
\hline 20070713 & 54294 & 1.78 & $6.5-17$ & \multirow{11}{*}{ HS } & \multirow{11}{*}{$\mathrm{MONO}_{\text {wobble }}$} \\
\hline 20070919 & 54362 & 0.71 & $25.1-50.8$ & & \\
\hline 20070920 & 54363 & 1.43 & $21.3-40.9$ & & \\
\hline 20071005 & 54378 & 0.85 & $6.5-26.4$ & & \\
\hline 20071006 & 54379 & 1.85 & $6.4-25.8$ & & \\
\hline 20071008 & 54381 & 1.95 & $17.8-43.1$ & & \\
\hline 20071009 & 54382 & 0.77 & $9.6-34.3$ & & \\
\hline 20071010 & 54383 & 2.26 & $6.9-33.3$ & & \\
\hline 20071011 & 54384 & 0.76 & $11.1-33.3$ & & \\
\hline 20071105 & 54409 & 0.58 & $34.2-48.6$ & & \\
\hline 20071106 & 54410 & 0.96 & $20.0-33.2$ & & \\
\hline 20080702 & 54649 & 4.24 & $6.5-30.1$ & \multirow{10}{*}{$\mathrm{HS}$} & \multirow{10}{*}{$\mathrm{MONO}_{\mathrm{on} / \mathrm{off}}$} \\
\hline 20080703 & 54650 & 3.26 & $6.5-30.3$ & & \\
\hline 20080704 & 54651 & 4.27 & $6.5-30.1$ & & \\
\hline 20080705 & 54652 & 4.15 & $6.4-36.1$ & & \\
\hline 20080706 & 54653 & 3.75 & 6.5 .36 .3 & & \\
\hline 20080707 & 54654 & 3.69 & $6.5-37.4$ & & \\
\hline 20080708 & 54655 & 3.94 & $6.5-34.1$ & & \\
\hline 20080709 & 54656 & 3.06 & $6.5-33.8$ & & \\
\hline 20080710 & 54657 & 2.89 & $6.5-36.8$ & & \\
\hline 20080711 & 54658 & 1.18 & $6.5-30.1$ & & \\
\hline 20080709 & 54656 & 0.33 & $28.5-33.5$ & \multirow{8}{*}{$\mathrm{HS}$} & \multirow{8}{*}{$\mathrm{MONO}_{\text {wobbl }}$} \\
\hline 20080710 & 54657 & 0.39 & $21.5-36.5$ & & \\
\hline 20080711 & 54658 & 0.32 & $14.8-19.6$ & & \\
\hline 20080712 & 54659 & 2.51 & $6.5-31$ & & \\
\hline 20080724 & 54671 & 0.62 & $13-19.6$ & & \\
\hline 20080725 & 54672 & 0.63 & $8.4-14.4$ & & \\
\hline 20080726 & 54673 & 0.84 & $6.5-9.1$ & & \\
\hline 20080727 & 54674 & 0.30 & $9.5-12.7$ & & \\
\hline 20090630 & 55012 & 3.50 & $6-30$ & \multirow{4}{*}{$\mathrm{HS}$} & \multirow{4}{*}{$\mathrm{MONO}_{\text {wobbl }}$} \\
\hline 20090701 & 55013 & 2.63 & $6-30$ & & \\
\hline 20090702 & 55014 & 1.83 & $6-30$ & & \\
\hline 20090705 & 55017 & 0.22 & $25-35$ & & \\
\hline 20091008 & 55112 & 0.26 & $610-14.3$ & \multirow{15}{*}{ HS } & \multirow{15}{*}{ STEREO$_{\text {pre }}$} \\
\hline 20091010 & 55114 & 0.67 & $20-32.6$ & & \\
\hline 20091011 & 55115 & 2.03 & 6-40.4 & & \\
\hline 20091012 & 55116 & 2.34 & $6.9-42.4$ & & \\
\hline 20091013 & 55117 & 0.95 & $26-41.2$ & & \\
\hline 20091014 & 55118 & 1.98 & $7.5-40$ & & \\
\hline 20091016 & 55120 & 1.37 & $7.5-40$ & & \\
\hline 20091017 & 55121 & 0.96 & $7.5-40$ & & \\
\hline 20091018 & 55122 & 1.60 & $7.5-40$ & & \\
\hline 20091019 & 55123 & 0.68 & $7.5-40$ & & \\
\hline 20091021 & 55125 & 1.99 & $7.5-40$ & & \\
\hline 20091106 & 55141 & 0.37 & $7.5-40$ & & \\
\hline 20091107 & 55142 & 0.64 & $7.5-40$ & & \\
\hline 20091113 & 55148 & 0.89 & $7.5-40$ & & \\
\hline 20100326 & 55281 & 0.78 & $38.5-50$ & & \\
\hline 20110512 & 55693 & 1.35 & $12.3-42.1$ & \multirow[t]{2}{*}{$\mathrm{HS}$} & \multirow[t]{2}{*}{ STEREO $_{\text {pre }}$} \\
\hline 20110513 & 55694 & 1.20 & $9.1-29.0$ & & \\
\hline 20140917 & 56917 & 2.55 & $6.8-38.4$ & & \\
\hline 20140918 & 56918 & 1.29 & $6.3-26.5$ & & \\
\hline 20140920 & 56920 & 2.38 & 6-38 & ss & STEREO $_{\text {post }}$ \\
\hline 20140923 & 56923 & 3.00 & 6-39 & & \\
\hline 20140924 & 56924 & 3.26 & $6.6-37.5$ & & \\
\hline 20140925 & 56925 & 1.81 & $6.2-39$ & & \\
\hline
\end{tabular}


4.6

\begin{tabular}{cc}
\hline \hline$\Gamma$ & Flux \\
\cline { 2 - 2 } & {$\left[\times 10^{-12} \mathrm{~cm}^{-2} \mathrm{~s}^{-1}\right]$} \\
\hline 2.0 & 2.20 \\
2.6 & 2.44 \\
3.2 & 2.62 \\
3.8 & 2.71 \\
\hline
\end{tabular}

Table 4.5: UL to the integral flux above $200 \mathrm{GeV}$ at $95 \%$ C.L. assuming a power-law spectrum with different photon indices, $\Gamma$.

\subsubsection{Results during Hard State}

Most of the observations ( $~ 83$ hours) of Cygnus X-1 presented were focused on the HS of the source. Observations under this X-ray spectral state were carried out between July 2007 and May 2011. However, no relevant excess can be reported during this spectral state. The integral flux UL for energies greater than $200 \mathrm{GeV}$ is $2.6 \times 10^{-12} \mathrm{~cm}^{-2} \mathrm{~s}^{-1}$. Differential flux ULs listed in the upper part of Table 4.7 .

In order to search for VHE orbital modulation, we carried out an orbital phase-folded analysis. To accomplish a good compromise between orbital phase resolution and significant amount of data, the binning in this analysis was 0.2. Moreover, in order to obtain enough statistics and cover the superior conjunction of the $\mathrm{BH}$ (phases 0.9-0.1), we started to bin the data at phase 0.1 . No VHE orbital modulation is evident either. Integral ULs for this phase-folded analysis are shown in Table 4.8 .

Table 4.6: Differential flux ULs at 95\% CL for the overall data sample assuming a power-law spectrum with photon index of $\Gamma=3.2$.

\begin{tabular}{ccc}
\hline \hline Energy range & Significance & Differential flux UL for $\Gamma=3.2$ \\
\hline$[\mathrm{GeV}]$ & {$[\sigma]$} & {$\left[\times 10^{-13} \mathrm{TeV}^{-1} \mathrm{~cm}^{-2} \mathrm{~s}^{-1}\right]$} \\
\hline $186-332$ & 2.15 & 0.02 \\
$332-589$ & -0.14 & 0.33 \\
$589-1048$ & 0.44 & 0.18 \\
$1048-1864$ & 0.17 & 6.41 \\
$1864-3315$ & 0.03 & 75.64 \\
\hline
\end{tabular}




\begin{tabular}{cccc}
\hline \hline Spectral State & Energy range & Significance & Differential flux UL for $\Gamma=3.2$ \\
\hline \multirow{6}{*}{$\mathrm{HS}$} & {$[\mathrm{GeV}]$} & {$[\sigma]$} & {$\left[\times 10^{-12} \mathrm{TeV}^{-1} \mathrm{~cm}^{-2} \mathrm{~s}^{-1}\right]$} \\
& $186-332$ & -2.57 & 0.20 \\
& $332-589$ & -0.03 & 3.70 \\
& $589-1048$ & 2.09 & 1.31 \\
& $1048-1864$ & 0.02 & 99.22 \\
& $1864-3315$ & 0.51 & 16.34 \\
\hline \multirow{3}{*}{$\mathrm{SS}$} & $186-332$ & 1.14 & 0.49 \\
& $332-589$ & 1.22 & 0.11 \\
& $589-1048$ & 0.06 & 4.71 \\
& $1048-1864$ & -1.23 & 51.62 \\
& $1864-3315$ & -1.34 & 16.37 \\
\hline
\end{tabular}

Table 4.7: Differential flux ULs at 95\% CL for each X-ray spectral state.

\subsubsection{Results during Soft State}

Cygnus X-1 was observed for a total of $\sim 14$ hours in the SS, bringing forth a clear difference on effective time with respect to the $\mathrm{HS}$. Nevertheless, this corresponds to the post-upgrade period, in which MAGIC achieved its best sensitivity, $0.66 \pm 0.03 \%$ of the Crab Nebula flux above 220 $\mathrm{GeV}$ in 50 hours (Aleksić et al. 2016b), implying that the previous observations flux was nearly reached in only about 9 hours. This data set guarantees, in turn, a full coverage of the X-ray spectral states that the source is used to exhibit. Although steady gamma-ray emission in the SS, when no persistent jets are present, is not theoretically predicted, transient jet emission cannot be dismissed during this state, as it happens in the case of Cygnus X-3 (Tavani et al. 2009b, Fermi LAT Collaboration et al. 2009b). Nevertheless, we did not find significant VHE gamma-ray emission from Cygnus X-1 in its spectrally soft state. Integral UL for energies beyond $200 \mathrm{GeV}$ and $\Gamma=3.2$ was set to $1.0 \times 10^{-11} \mathrm{~cm}^{-2} \mathrm{~s}^{-1}$. Differential ULs are quoted in the lower part of Table 4.7. The orbital phase-folded analysis did not yield any significant emission either. The integral ULs for this phase-folded study are also given in Table 4.8 .

\subsubsection{Search for variable emission}

As done for the Fermi-LAT data analysis, because of the rapid flux variation spotted in Cygnus X1 , I carried out daily basis analysis for the 53 nights. This search yielded no significant excess in any of the nights and therefore, integral ULs (95\% C.L.) for energies above $200 \mathrm{GeV}$ were computed for single-night observations (listed in Table 4.9).

MAGIC results are included in the top panel of the multiwavelength lightcurve presented in 


\begin{tabular}{ccccc}
\hline \hline Spectral State & Phase & Eff. Time & Significance & Integral flux \\
\hline & & {$[\mathrm{hr}]$} & {$[\sigma]$} & for $\Gamma=3.2$ \\
\hline \multirow{4}{*}{$\mathrm{HS}$} & $0.1-0.3$ & 15.47 & -0.77 & 7.89 \\
& $0.3-0.5$ & 22.34 & 1.88 & 6.91 \\
& $0.5-0.7$ & 14.08 & 0.00 & 21.32 \\
& $0.7-0.9$ & 14.81 & 0.99 & 6.92 \\
& $0.9-0.1$ & 15.62 & -0.96 & 4.34 \\
\hline \multirow{4}{*}{ SS } & $0.1-0.3$ & 2.58 & 0.45 & 19.32 \\
& $0.3-0.5$ & 4.35 & -1.23 & 7.96 \\
& $0.5-0.7$ & 3.91 & 0.59 & 15.49 \\
& $0.7-0.9$ & 3.64 & 0.23 & 18.23 \\
& $0.9-0.1$ & - & - & -
\end{tabular}

Table 4.8: Orbital phase-wise 95\% C.L. integral flux ULs for energies $>200 \mathrm{GeV}$ for HS and SS observations. The latter did not cover phases from $0.9-0.1$, so no ULs are provided.

Figure 4.7. Along with MAGIC ULs, the figure shows data in the HE gamma-ray regime from Fermi-LAT (0.1-20 GeV) data analysis performed in this thesis, hard X-ray (Swift/BAT in 15$50 \mathrm{keV}$, Krimm et al. 2013), intermediate-soft X-ray (MAXI between 2-20 keV, Matsuoka et al. 2009), soft X-ray (quick-look results provided by the RXTE/ASM team in 3-5 keV) and radio data (AMI at $15 \mathrm{GHz}$ and RATAN-600 at $4.6 \mathrm{GHz}$ ). The three transient episodes observed by $A G I L E$ are also marked. The X-ray states are identified in the lightcurve by the horizontal dashed line drawn in the X-ray pad at the level of 0.09 counts $\mathrm{cm}^{-2} \mathrm{~s}^{-1}$ (Grinberg et al. 2013) and by the colored bands.

During this multi-year campaign, Cygnus X-1 did not display any X-ray flare during this campaign like that in which the previous MAGIC $4.1 \sigma$ result was obtained (Albert et al. 2007). This prevented us to observe the source under strictly the same conditions: the maximum SwiftBAT flux simultaneous to our observations happened on MJD 54379 (1.13 $\sigma$, around superior conjunction of the $\mathrm{BH}$ ) at the level of 0.23 counts $\mathrm{cm}^{-2} \mathrm{~s}^{-1}$, close but still lower than 0.31 counts $\mathrm{cm}^{-2} \mathrm{~s}^{-1}$ peak around the MAGIC hint. However, we observed the microquasar in coincidence with the first AGILE flare. This transient episode (on the 16th of October 2009, MJD 55120) showed a TS=28.09 ( $4 \sigma$ after trials) between $0.1-3 \mathrm{GeV}$ with a gamma-ray flux of $(2.32 \pm$ $0.66) \times 10^{-6}$ photons $\mathrm{cm}^{-2} \mathrm{~s}^{-1}$ (Sabatini et al. 2010b), which took place during the X-ray HS of Cygnus X-1. The corresponding MAGIC integral flux $\mathrm{UL}$ above $200 \mathrm{GeV}$ for this day is $1.3 \times 10^{-11} \mathrm{~cm}^{-2} \mathrm{~s}^{-1}$ (see Table 4.9). It is worth noting that, in accordance with our non-detection, the analysis of Fermi-LAT data did not show any significant signal (between $100 \mathrm{MeV}$ and 20 $\mathrm{GeV}$ ) on or around this date. 
Table 4.9: From left to right: Date of the beginning of the observations in calendar and in MJD, effective time after quality cuts, significance for an energy threshold of $\sim 150 \mathrm{GeV}$ for mono observations (only MAGIC I) and $\sim 100 \mathrm{GeV}$ for stereoscopic observations (separated by the horizontal line) and integral flux ULs at 95\% C.L. for energies above $200 \mathrm{GeV}$ computed on a daily basis. MJD 54656, 54657 and 54658 were analyzed separately according to each observational mode (see Table 4.3). Due to low statistics, neither the integral UL for MJD 55017 nor the significant for MJD 55116 were computed.

\begin{tabular}{|c|c|c|c|c|}
\hline \multicolumn{2}{|l|}{ Date } & \multirow{2}{*}{$\begin{array}{l}\text { Eff. Time } \\
{[\mathrm{hr}]}\end{array}$} & \multirow{2}{*}{$\begin{array}{c}\text { Significance } \\
{[\sigma]}\end{array}$} & \multirow{2}{*}{$\begin{array}{c}\text { Flux UL for } \Gamma=3.2 \\
{\left[\times 10^{-11} \text { photons } \mathrm{cm}^{-2} \mathrm{~s}^{-1}\right]}\end{array}$} \\
\hline [yyyy mm dd] & [MJD] & & & \\
\hline 20070713 & 54294 & 1.78 & -0.67 & 2.19 \\
\hline 20070919 & 54362 & 0.71 & 1.10 & 7.10 \\
\hline 20070920 & 54363 & 1.43 & 1.99 & 4.59 \\
\hline 20071005 & 54378 & 0.85 & -0.84 & 1.84 \\
\hline 20071006 & 54379 & 1.85 & 0.02 & 1.21 \\
\hline 20071008 & 54381 & 1.95 & 0.99 & 2.88 \\
\hline 20071009 & 54382 & 0.77 & -0.57 & 2.38 \\
\hline 20071010 & 54383 & 2.26 & -0.04 & 1.05 \\
\hline 20071011 & 54384 & 0.76 & 1.68 & 2.26 \\
\hline 20071105 & 54409 & 0.58 & 0.31 & 4.38 \\
\hline 20071106 & 54410 & 0.96 & -1.24 & 0.97 \\
\hline 20080702 & 54649 & 4.24 & 2.33 & 0.21 \\
\hline 20080703 & 54650 & 3.26 & 1.53 & 0.15 \\
\hline 20080704 & 54651 & 4.27 & 2.36 & 0.23 \\
\hline 20080705 & 54652 & 4.15 & 2.97 & 0.22 \\
\hline 20080706 & 54653 & 3.75 & 1.75 & 0.39 \\
\hline 20080707 & 54654 & 3.69 & 2.74 & 0.24 \\
\hline 20080708 & 54655 & 3.94 & 2.01 & 0.18 \\
\hline 20080709 & 54656 & 3.06 & 1.66 & 0.49 \\
\hline 20080710 & 54657 & 2.89 & 1.75 & 0.38 \\
\hline 20080711 & 54658 & 1.18 & 0.32 & 0.93 \\
\hline 20080709 & 54656 & 0.33 & 0.06 & 4.84 \\
\hline 20080710 & 54657 & 0.39 & -1.22 & 3.11 \\
\hline 20080711 & 54658 & 0.32 & 1.83 & 8.81 \\
\hline 20080712 & 54659 & 2.51 & 0.11 & 1.16 \\
\hline 20080724 & 54671 & 0.62 & -1.45 & 1.90 \\
\hline 20080725 & 54672 & 0.63 & -0.15 & 2.30 \\
\hline 20080726 & 54673 & 0.84 & -1.33 & 2.40 \\
\hline 20080727 & 54674 & 0.30 & 2.09 & 2.44 \\
\hline 20090630 & 55012 & 3.50 & 0.76 & 3.46 \\
\hline 20090701 & 55013 & 2.63 & 0.73 & 2.50 \\
\hline 20090702 & 55014 & 1.83 & 0.14 & 1.36 \\
\hline 20090705 & 55017 & 0.22 & 0.37 & - \\
\hline 20091008 & 55112 & 0.26 & -1.85 & 1.11 \\
\hline 20091010 & 55114 & 0.67 & 0.19 & 1.50 \\
\hline 20091011 & 55115 & 2.03 & 0.32 & 3.10 \\
\hline 20091012 & 55116 & 2.34 & - & 2.19 \\
\hline 20091013 & 55117 & 0.95 & 1.53 & 3.87 \\
\hline 20091014 & 55118 & 1.98 & -0.30 & 2.44 \\
\hline 20091016 & 55120 & 1.37 & -2.99 & 1.30 \\
\hline 20091017 & 55121 & 0.96 & -0.77 & 4.25 \\
\hline 20091018 & 55122 & 1.60 & -0.27 & 3.05 \\
\hline 20091019 & 55123 & 0.68 & -0.44 & 3.42 \\
\hline 20091021 & 55125 & 1.99 & -1.90 & 1.09 \\
\hline 20091106 & 55141 & 0.37 & -3.04 & 2.23 \\
\hline 20091107 & 55142 & 0.64 & 0.13 & $2 . .35$ \\
\hline 20091113 & 55148 & 0.89 & -1.23 & 3.06 \\
\hline 20100326 & 55281 & 0.78 & 1.75 & 10.92 \\
\hline 20110512 & 55693 & 1.35 & 0.09 & 1.38 \\
\hline 20110513 & 55694 & 1.20 & -1.54 & 0.53 \\
\hline 20140917 & 56917 & 2.55 & 0.32 & 2.56 \\
\hline 20140918 & 56918 & 1.29 & -0.99 & 1.25 \\
\hline 20140920 & 56920 & 2.38 & 0.08 & 2.13 \\
\hline 20140923 & 56923 & 3.00 & 0.85 & 2.85 \\
\hline 20140924 & 56924 & 3.26 & -0.61 & 2.73 \\
\hline 20140925 & 56925 & 1.81 & 0.28 & 2.26 \\
\hline
\end{tabular}




\subsubsection{Discussion}

MAGIC observations carried out between July 2007 and September 2014 for a total of 100 hours covered the two principal X-ray states of Cygnus X-1 with the main focus on the HS. We did not detect any significant excess from either all the data or any of the samples, including orbital phase-folded and daily analysis. This long-term campaign provided, for the first time, constraining ULs on the VHE emission of Cygnus X-1 at the two main X-ray states, the HS and the SS, separately as well as in an orbital binning base, which showed no hint of gamma-ray orbital modulation. This was possible thanks to a comprehensive trigger strategy that allowed to observe the source under flaring activity. The chosen photon index $(\Gamma=3.2$ in this thesis, Crablike in the previous MAGIC observations, Albert et al.2007) and the addition of $30 \%$ systematic uncertainties contributed to obtain more robust ULs compared to the formerly ones reported by MAGIC,

The total power emitted by the jets during the HS in Cygnus X-1 is expected to be $10^{36}-10^{37}$ $\mathrm{erg} \mathrm{s}^{-1}$ (Gallo et al. 2005). The integral UL $2.6 \times 10^{-12}$ photons $\mathrm{cm}^{-2} \mathrm{~s}^{-1}$, for energies greater than $200 \mathrm{GeV}$, obtained in this thesis with MAGIC corresponds to a luminosity of $6.4 \times 10^{32}$ $\mathrm{erg} \mathrm{s}^{-1}$ assuming a distance of $1.86 \mathrm{kpc}$ (Reid et al. 2011). Therefore, the UL on the conversion efficiency of jet power to VHE gamma ray luminosity is $0.006-0.06 \%$, similar to the one obtained previously for Cygnus X-3 (Aleksić et al. 2010a). Note that gamma-ray opacity in Cygnus X-3 is nevertheless about two orders of magnitude higher than in Cygnus X-1.

VHE emission from the jet large scale or jet-medium interaction regions above the sensitivity level of MAGIC can be ruled out, as these regions are not affected by gamma-ray absorption. On the binary scales, however, the non-detection is less conclusive because of pair creation in the stellar photon field. Models do predict $\mathrm{VHE}$ radiation as long as particle acceleration is efficient (e.g. Pepe et al. 2015). Formally, particle acceleration up to $\sim \mathrm{TeV}$ energies can be reached in the jet on the binary region (Khangulyan et al. 2008), and thus $100 \mathrm{GeV}$ IC photons should be produced, but this emission may be right below the detection level of MAGIC (as in Zdziarski et al. 2016b, Fig. 6) even under negligible gamma-ray absorption. It could otherwise be that non-thermal particles cannot reach VHE IC emitting energies in the jet of Cygnus X-1. Besides inefficient acceleration, a very high magnetic field could also prevent particles to reach $\mathrm{VHE}$, and even if these particles were present, a strong magnetic field can suppress intensely VHE photon production.

Nevertheless, one cannot dismiss the possibility of a transient emission as the one hinted by MAGIC in 2006. This flare took place during an orbital phase around the superior conjunction of the $\overline{\mathrm{BH}}$, where the gamma-ray absorption is expected to be the highest. The attenuation constraint may have been relaxed by an emitter at some distance from the BH (Albert et al. 2007), with its intrinsic variability possibly related for instance to jet-stellar wind interaction (Perucho \& BoschRamon 2008, Owocki et al. 2009). On the other hand, even considering absorption by stellar photons, emission closer to the $\overrightarrow{\mathrm{BH}}$ would be possible accounting for extended pair cascades under a reasonable intrinsic gamma-ray luminosity, although rather low magnetic fields in the stellar wind would be required (?; see also Bosch-Ramon et al. 2008). Cygnus X-3, the other microquasar firmly established as a GeV emitter (Tavani et al. 2009b, Fermi LAT Collaboration et al. 2009b), displays a very different behavior from that of Cygnus X-1. The HE gamma- 
ray emission from Cygnus X-3 is transient, occurring sometimes during flaring activity of nonthermal radio emission from the jets (Corbel et al.|2012). If VHE|radiation in microquasars were related to discrete radio-emitting-blobs with high Lorentz factor $(\Gamma \geq 2)$, this may also happen in Cygnus X-1 during hard-to-soft transitions.

The multiwavelength emission from X-rays up to VHE gamma rays in Cygnus X-1 is shown in Figure 4.8. The data used in this spectral energy distribution (SED) corresponds to the HS. The sensitivity curve for 50 and scaled to $200 \mathrm{hr}$ of observations with the future Cherenkov Telescope Array, CTA 1 , on the Northern hemisphere is showed along with the data. The spectral cutoff of the $\mathrm{HE}$ radiation from Cygnus X-1 is still unknown, although if the gamma-ray emission in the $\mathrm{HS}$ reaches $\sim \mathrm{TeV}$ energies, the next generation of IACTs may be able to detect the system for long enough exposure times. Thus, to detect steady VHE emission from the jets, future more sensitive instruments, as CTA, would be needed. This instrument could provide valuable information of the VHE gamma-ray production in Cygnus X-1 $\mathrm{HE}$ spectral cutoff, energetics, impact of gamma-ray absorption/IC cascades), as well as allow the study of possible short-term flux variability.

\subsection{Conclusions}

We obtained, for the first time, a high-significance detection of a $\mathrm{BH}$ binary system in the $\mathrm{HE}$ regime. We established the detection of a point-like LAT source spatially coincident with the microquasar Cygnus X-1 at the level of TS=53 for energies above $60 \mathrm{MeV}$, using 7.5 years of Pass 8 Fermi-LAT data. By analyzing the sample at the two different main X-ray states, $\mathrm{HS}$ and SS, we could confirm a correlation between $\mathrm{GeV}$ emission and hard X-rays: the source is only detected during the former state, i.e. when steady relativistic radio-emitting jets are displayed (TS=49). During this spectrally hard states, the emission was detected around the superior conjunction of the compact object $(\phi>0.75$ and $\phi<0.25$, at TS $=31)$, while it becomes undetectable at the inferior conjunction, evidencing a hint of orbital modulation in gamma rays.

We could constrain the production site of $\mathrm{HE}$ photons at distances $Z>10^{11} \mathrm{~cm}$ from the $\mathrm{BH}$ along the jet, to prevent pair production mechanism faints the $\mathrm{GeV}$ photons and account for the dominant stellar photon radiation, and $Z<10^{13} \mathrm{~cm}$, to confined the $\mathrm{HE}$ emission inside the binary system given the presumable orbital variability.

On the other hand, observations with the MAGIC telescopes allowed us to discarded the interaction between the relativistic jets and the surrounding medium as production site for $\mathrm{VHE}$ gamma rays at the level of the MAGIC sensitivity, taken into account that this region is not affected by photon-photon absorption. Nevertheless, $\mathrm{TeV}$ gamma rays produced inside the binary still remains a valid possibility.

\footnotetext{
${ }^{1}$ Taken from https://www.cta-observatory.org/science/cta-performance/
} 


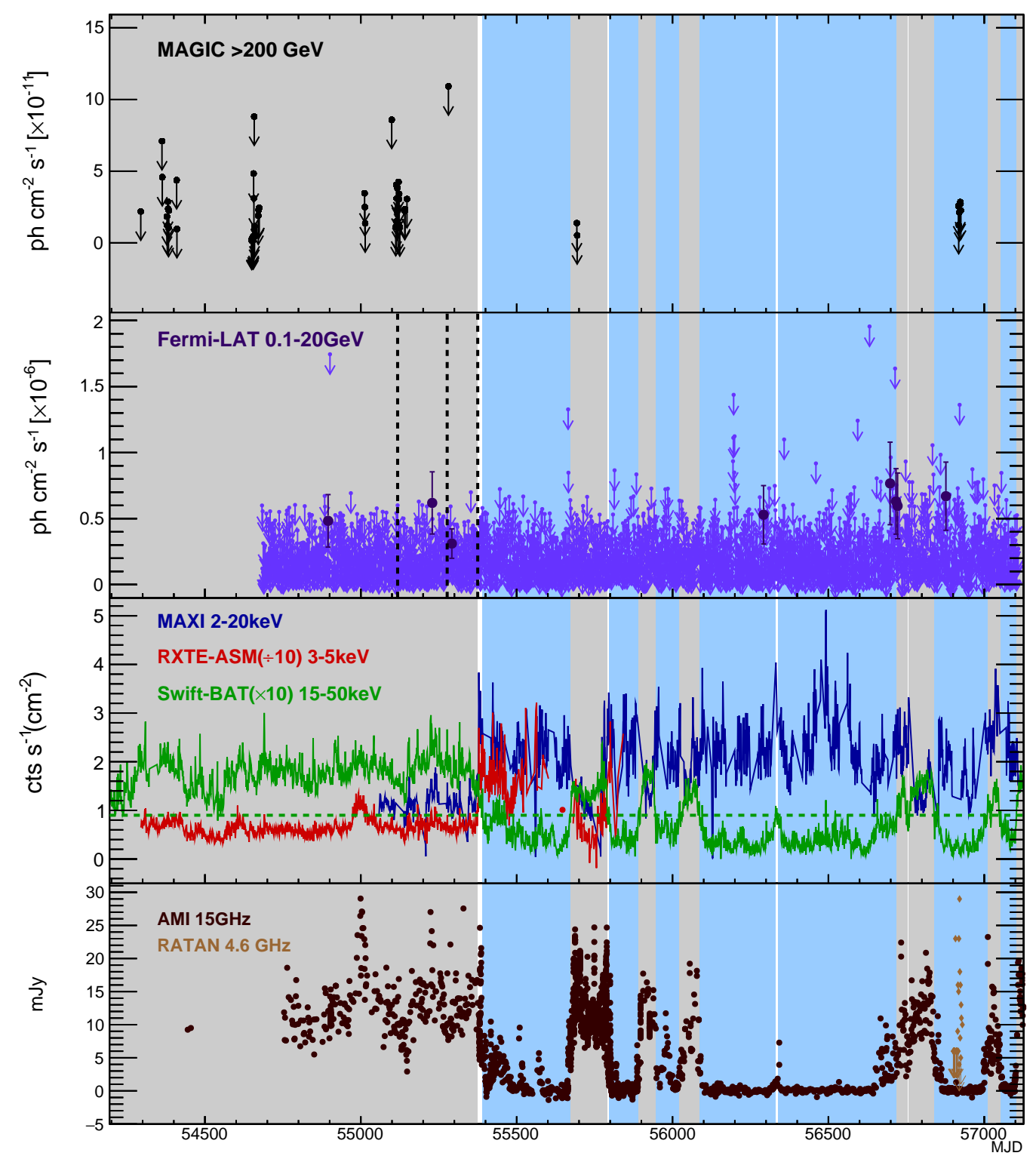

Figure 4.7: From top to bottom: daily MAGIC integral ULs for $E>200 \mathrm{GeV}$ assuming a power-law function with photon index $\Gamma=3.2$, $\mathrm{HE}$ gamma rays from Fermi-LAT, hard X-ray (Swift-BAT, $\times 10$ counts $\mathrm{s}^{-1} \mathrm{~cm}^{-2}$ in the $15-50 \mathrm{keV}$ range), intermediate-soft X-ray (MAXI, in counts $\mathrm{s}^{-1}$ in the $2-20 \mathrm{keV}$ range), soft X-ray (RXTE-ASM, counts $\mathrm{s}^{-1}$ divided by 10 in the 3-5 keV range), and finally, radio integral fluxes from AMI at $15 \mathrm{GHz}$ and RATAN-600 at $4.6 \mathrm{GHz}$. In the $\mathrm{HE}$ pad, daily fluxes with $T S>9$ are displayed as filled black points while days with $T S<9$ are given as 95\% Confidence Level (C.L.) ULS. Dashed lines, in the same pad, correspond to AGILE alerts. For convenience, an horizontal green dashed line in Swift-BAT plot is displayed at the limit of 0.09 counts $\mathrm{cm}^{-2} \mathrm{~s}^{-1}$, above which the source can be considered to be in the HS and below which it is in the SS (Grinberg et al. 2013). This distinction between $\mathrm{X}$-ray states is also highlighted by the color bands: gray bands correspond to the HS+IS and blue ones to the SS periods. White bands correspond to transitions between these two main X-ray spectral states which cannot be included within the $\mathrm{HS}$ periods. 


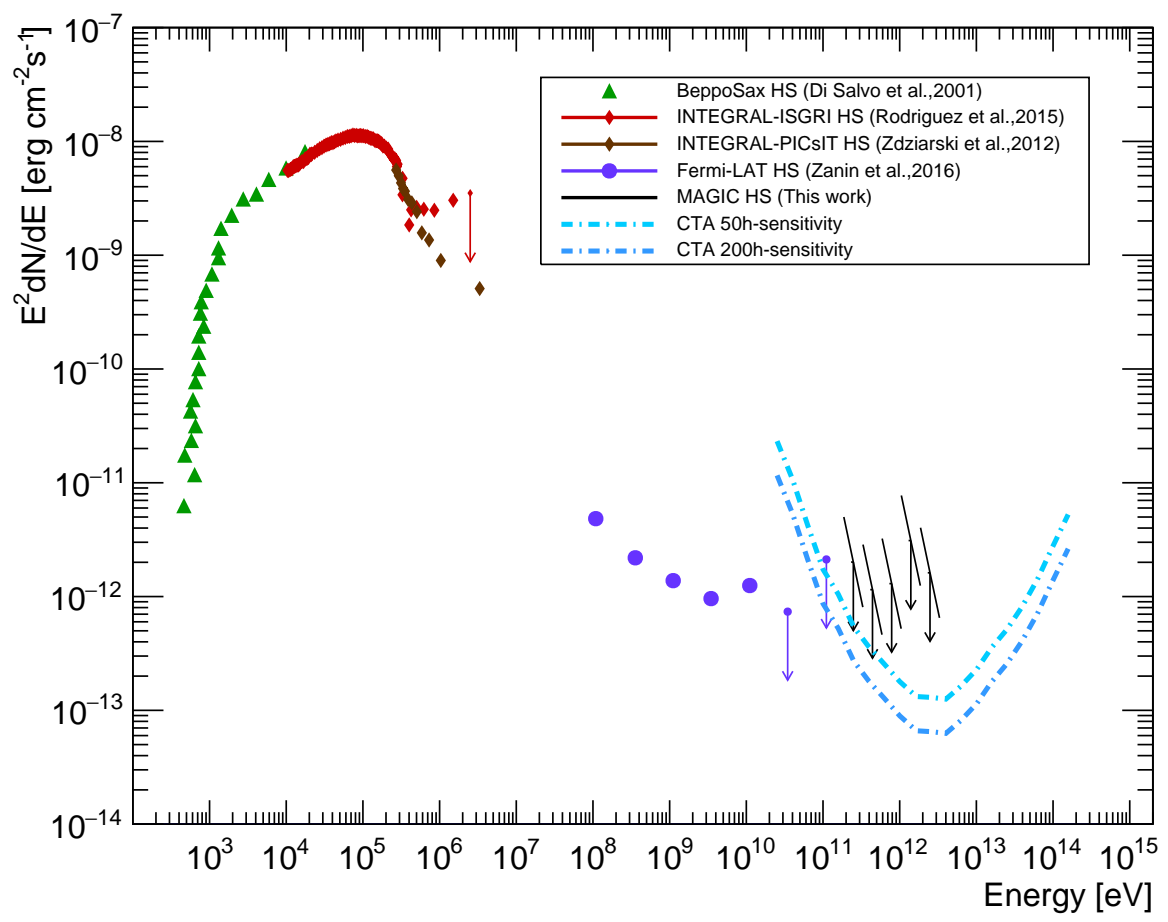

Figure 4.8: Spectral energy distribution (SED) of Cyg X-1 covering X-ray, HE and VHE gamma-ray regimes during the HS. BeppoSAX soft X-ray data (in the $\mathrm{keV}$ band, green triangles) is taken from Di Salvo et al. (2001), while for the hard X-ray band data from both INTEGRAL-ISGRI (10 keV-2 MeV, red diamond and UL; Rodriguez et al. 2015b) and INTEGRAL-PICsIT (150 keV-10 MeV, brown diamond; Zdziarski et al. 2012) are displayed, given their incompatibility spectral results above $1 \mathrm{MeV}$. In the HE gamma-ray band (60 MeV-few hundred GeV, violet circles and ULs), results from Zanin et al. (2016) obtained with Fermi-LAT data are shown. At VHE, results from this work during the HS are plotted (black) assuming a power-law function of $\Gamma=3.2$. The dashed blue lines correspond to the 50 and scaled to $200 \mathrm{hr}$ sensitivity curves for CTA North. No statistical errors are drawn. 
4. CYGNUS X-1 


\section{5 \\ Cygnus X-3}

\subsection{History}

Cygnus X-3, discovered 50 years ago (Giacconi et al.|1967), is one of the brightest X-ray sources in our Galaxy and the strongest radio source among the X-ray binaries, displaying two-sided powerful relativistic radio jets (Martí et al. 2001; Miller-Jones et al.2004). Located in the Galactic plane $\left(l=71.32^{\circ}\right.$ and $\left.b=+3.09^{\circ}\right)$, the system lies at a most likely distance of $7.4 \pm 1.1 \mathrm{kpc}$ from Earth, based on a Bok globule study situated along the light of sight of Cygnus X-3 (McCollough et al. 2016). The features of this microquasar is not representative of the typical X-ray binary. First of all, despite hosting a WR star (van Kerkwijk et al. 1992), it shows an orbital period of 4.8 hours, more common of low-mass binaries. This value was ascertained by X-ray and IR flux orbital modulation detected during flaring activity of the source (Parsignault et al. 1972; Becklin et al. (1973). This periodic intensity variations may arise from the scattering or absorption of the emission from the stellar wind, as in other binary systems. On the other hand, the proximity between the compact object and the companion star gives rise to an unusual strong absorption, as a consequence of the embed of the system within the companion stellar wind. This complicates the understanding of the system, from which the nature of the compact object is unclear so far: it can be composed by a $1.4 \mathrm{M}_{\odot} \mathrm{NS}\left(\right.$ Stark \& Saia 2003) or a $<10 \mathrm{M}_{\odot} \mathrm{BH}($ Hanson et al. 2000). Nevertheless, the latter scenario involving a $\overline{\mathrm{BH}}$ is reinforced given that the only other known binaries composed by WR stars host this type of compact object (see Prestwich et al. 2007 for IC 10 X-1 and Carpano et al.2007 for NGC 300 X-1) and the resemblances that the Cygnus X-3 spectral features present with respect to the spectra of $\mathrm{BH}$ transients.

Actually, despite the difficult task that the study of the Cygnus X-3 spectrum implies, given the strong X-ray absorption, the two main X-ray spectral states typical from $\mathrm{BH}$ transients are 
observed (the $\mathrm{HS}$ and the $\mathrm{SS}$ ). The $\mathrm{HS}$ was studied by Hjalmarsdotter et al. (2008), which determined an unusually low energy cutoff of $\sim 20 \mathrm{keV}$ (compared to the typical cutoff at hundred $\mathrm{keV}$ displayed by the counterparts). A possible explanation for this additional peculiarity could be the presence of a very massive $\mathrm{BH}$ with $>20 \mathrm{M}_{\odot}$ forming the system. With such mass, the last stable orbit would be further away from the compact object than in other sources and hence, Cygnus X-3 could not reach temperatures as high as other binaries. Thus, $\mathrm{HS}$ needs to be modeled by the Comptonization of hybrid (thermal and non-thermal) electron population along with a Compton reflection component, instead of only thermal one as expected in the standard scenario.

Although Cygnus X-3 is always detected in the radio band, its flux level in this wavelength can vary several orders of magnitude during its frequent radio outbursts, the first of which was reported at the beginning of the 70's by Gregory et al. (1972). Waltman et al. (1994, 1995, 1996) discussed the existence of four radio states: quiescent state, with fluxes around $\sim 50-200 \mathrm{mJy}$ that lasts months; minor flaring episodes with $\lesssim 300 \mathrm{mJy}$; quenched periods in which the radio flux is as low as $\lesssim 30 \mathrm{mJy}$; and major flaring periods, during which the flux level increases large fraction up to $\sim 1-20 \mathrm{Jy}$. These strong radio flares happen during the SS. The correlation between the radio and the soft X-rays was deeply studied afterwards by Szostek et al. (2008). This connection can be split into six states, following the previous division, represented in the so-called saxophone plot (see Figure 5.1), which I summarize below:

$\odot$ Quiescence state: Low flux in both energy bands, radio and soft X-rays. Variation is positively correlated.

$\odot$ Minor Flaring state: State in which small oscillations can happen as the source is overpassing the X-ray transitional level (set to 3 counts/s using RXTE-ASM data in the 3-5 $\mathrm{keV}$ band). To the left of this level, the source stays in the $\mathrm{HS}$, at the right it is in the SS. The radio level remains below its transitional level of $300 \mathrm{mJy}$.

$\odot$ Suppressed state: There is anti-correlation between fluxes. While the source is finally entering in the SS in which flux of soft X-rays increases, the radio flux keeps decreasing. It is not always followed by a major flare.

$\odot$ Quenched state: The radio flux is at its lowest level, while the soft X-rays show one of the highest fluxes. This state is directly followed by a radio outburst.

$\odot$ Major Flaring state: The soft X-rays keep roughly constant, whilst radio suffers huge variation of several factors of magnitude.

$\odot$ Post Flaring state: It corresponds to a returning state to either the minor flaring or the suppressed state.

Cygnus X-3 was detected in the HEregime (above $100 \mathrm{MeV}$ ) by both Fermi-LAT and Astrorivelatore Gamma ad Imagini LEggero (AGILE) (Fermi LAT Collaboration et al. 2009b; Tavani et al. 2009b). AGILE detected four gamma-ray flares of 1-2 days each one between mid-2007 and mid-2009, leading to an average spectrum between $100 \mathrm{MeV}$ and $3 \mathrm{GeV}$ described by a 


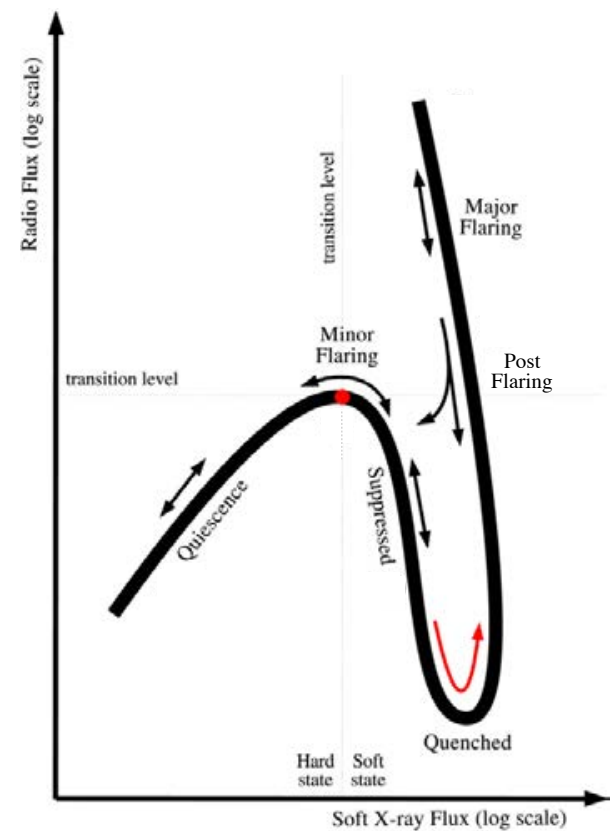

Figure 5.1: Cygnus $X-3$ saxophone plot that represents the evolution of the source through the different radio and X-ray states. The dotted lines correspond to the transition level (determined at $0.3 \mathrm{Jy}$ and 3 counts/s with GBI radio and RXTE-ASM soft X-rays data, respectively). The six different defined states are labeled along the path in which arrows show the possible direction that the system can follow. Modified plot from Szostek et al. (2008).

power-law function with photon index $1.8 \pm 0.2$. On the other hand, Fermi-LAT detection took place mainly during two activity periods of around two months MJD 54750-54820 and MJD 54990-55045), an the estimated spectrum was as well defined by a power-law distribution but with a softer index of $2.70 \pm 0.05_{\text {stat }} \pm 0.20_{\text {syst }}$. All GeV detections happened during the $\mathrm{SS}$ of the source and before a radio major flare. Fermi-LAT estimated this gap between HE and radio photons on $(5 \pm 7)$ days. These results evidence the episodic nature of the HE emission, opposite to the steady radiation detected in Cygnus X-1 (see Chapter 4). After these two flaring activity periods that yielded to its detection in the $\mathrm{HE}$ regime, Cygnus X-3 underwent another five outburst (see Table 5.1).

This microquasar was also claimed to be a TeV emitter (see e.g. Vladimirsky et al. 1973) and even a PeV emitter (see Bhat et al. 1986). Nevertheless, this presumable detection in the $\mathrm{TeV}$ regime was not confirmed by more recent and sensitive instruments, as MAGIC (Aleksić et al. 2010b) or VERITAS (Archambault et al. 2013). Former MAGIC observations amounted a total of $\sim 60$ hours of good quality data, between 2006 and 2009 in stand-alone mode with MAGIC I. Observations during this previous campaign were triggered by either radio or gamma-rays alerts sent by RATAN-600 and AGILE, respectively. These MAGIC observations yielded to an integral $\mathrm{UL}$ at $95 \% \mathrm{CL}$ of $2.2 \times 10^{-12}$ photons $\mathrm{cm}^{-2} \mathrm{~s}^{-1}$ at energies greater than $250 \mathrm{GeV}$. 
Table 5.1: Cygnus X-3 GeV flaring periods as seen by Fermi-LAT.

\begin{tabular}{l|c|c}
\hline \hline Name & $\begin{array}{c}\text { Period } \\
\| \text { MJD }\end{array}$ & \multicolumn{1}{|c}{ Status } \\
\hline Flare 1 & $54750-54820$ & Reported in \\
Flare 2 & $54990-55045$ & Fermi LAT Collaboration et al. (2009b) \\
\hline Flare 3 & $55585-55610$ & Reported in Corbel et al. (2012) \\
Flare 4 & $55640.5-55643.5$ & Unpublished \\
\hline Flare 5 & $57398-57415$ & Reported in this thesis at VHE \\
\hline Flare 6 & $57622-57653$ & Unpublished \\
\hline Flare 7 & $57799-57873$ & \\
\hline
\end{tabular}

\subsection{Observations and data analysis}

Cygnus X-3 observations belong to a long-term observational campaign within the MAGICinternally so-called Key Observation Program (KoP), These projects comprehend those sources that, due to the high scientific interest and impact, have observational priority. Sources under KoP are not usually scheduled commonly, but their observations are triggered based on multiwavelength information in order to observe them at the most appropriate period. In the case of Cygnus X-3, the trigger criterion is based on a Fermi-LAT pipeline performed at the Universitat de Barcelona (UB). Currently, we observe the system when the TS on their daily analysis of public FermiLAT data (using photon-like events between 00:00:01 and 00:00:01 of two consecutive days, approximately) is higher than 13. In order to decide to keep or interrupt observations, I analyze the MAGIC data as soon as it is available the following day: if a significant hint is obtained in this analysis or the Fermi-LAT pipeline fulfills the aforementioned criterion again, observations are scheduled for the next day, otherwise they are interrupted. The Cygnus X-3 KoP started on November 2013 and keeps active up to now. The source is observable from November to April, due to its position in the Cygnus Constellation, for a broad $\mathrm{Zd}$ range of (12-50) ${ }^{\circ}$.

Cygnus X-3 is observed making use of the wobble tracking mode (see Chapter 2.4.2.1) but with non-standard positions in order to avoid the speculative pulsar binary TeV J2032+4130 and the binary star systems $\mathrm{WR} 146$ and $\mathrm{WR}$ 147, situated in the FoV of the target microquasar. Thus, the wobble positions for Cygnus X-3 observations are placed at a RA angles of $51^{\circ}, 141^{\circ}$, $231^{\circ}$ and $321^{\circ}$, at a nominal offset distance of $0.4^{\circ}$ (see Figure 5.4).

For the latest observational period (August-September 2016 campaign), although most of the data were taken under dark conditions, MAGIC pointed to Cygnus X-3 under different moonlight levels in order to extend observations as much as possible. Thus, a proper analysis with dedicated $\mathrm{MC}$ and OFF data was applied in each case. The data was split into six categories according to the $\mathrm{NSB}$ background level, which are: $1 \times \mathrm{NSB}_{\text {lark }}$ (corresponding to dark conditions), $2-3 \times$ $\mathrm{NSB}_{\text {lark }}, 3-5 \times \mathrm{NSB}_{\text {lark }}, 5-8 \times \mathrm{NSB}_{\text {lark }}, 8-12 \times \mathrm{NSB}_{\text {lark }}$ and $12-18 \times \mathrm{NSB}_{\text {lark }}$. It is worth to mention that all moon data was taken using nominal $[\mathrm{HV}](\sim 1.25 \mathrm{kV})$, including the highest levels, without resorting to the reduced HV. Therefore, I summarize in Table 5.2 the cleaning 
levels and size cuts applied for the Cygnus X-3 analysis as well as the pedestal mean and RMS distribution values used in the additional noise included in the $\mathrm{MC}$ and OFF data.

Table 5.2: Image cleaning levels for the Cygnus X-3 analysis.

\begin{tabular}{|c|c|c|c|c|}
\hline NSB & Cleaning Levels & Pedestal & RMS & Size cut \\
\hline$\left[\times \mathrm{NSB}_{\text {dark }}\right]$ & $\begin{array}{c}\mathrm{Lvl}_{1}: \mathrm{Lvl}_{2} \\
\text { [phe] }\end{array}$ & $\begin{array}{c}\text { distributior } \\
\text { [phe] }\end{array}$ & $\begin{array}{l}\text { stribution } \\
\text { [phe] }\end{array}$ & [phe] \\
\hline $1-2$ & $6: 3.5$ & - & - & 50 \\
\hline $2-3$ & $7: 4.5$ & 3.0 & 1.3 & 80 \\
\hline $3-5$ & $8: 5$ & 3.6 & 1.5 & 110 \\
\hline $5-8$ & $9: 5.5$ & 4.2 & 1.7 & 150 \\
\hline $8-12$ & $10: 6$ & 4.8 & 2.0 & 210 \\
\hline $12-18$ & $13: 8$ & 5.8 & 2.3 & 250 \\
\hline
\end{tabular}

\subsubsection{August-September 2016 flare}

During summer 2016, Cygnus X-3 underwent a flaring activity in both radio and HE gammaray regimes. The outburst period was initiated the $21^{\text {st }}$ of September, reference based on the UB Fermi-LAT pipeline, which showed a TS=20.5 $(\sim 4.5 \sigma)$ that day. This enhanced activity continued almost a month, during which Cygnus X-3 was firmly detected in three occasions by this Fermi-LAT pipeline: on MJD 57631 with TS $28(\sim 5.3 \sigma)$, on MJD 57647 with TS 33 $(\sim 5.7 \sigma)$ and on MJD 57649, when a major flare with TS $73(\sim 8.5 \sigma)$ happened. Although no other days showed significance well above $5 \sigma$, several intervening days presented TS higher than the internal criterion of $\mathrm{TS}=13$. The TS light curve obtained from the daily Fermi-LAT analysis performed at $\mathrm{UB}$ since August 2008 is depicted in Figure 5.2. This HE emission during AugustSeptember 2016 was confirmed by AGILE (Piano et al. 2016) and the Fermi-LAT Collaboration (Cheung \& Loh 2016) as well.

On the other hand, this $\mathrm{HE}$ activity period started while the radio emission began to decrease, revealing that Cygnus X-3 was entering in the SS (Trushkin et al. 2016a). Actually, radio flux at 4.6, 8.2 and $11.2 \mathrm{GHz}$ from RATAN-600 reached quenched level (around 10-30 mJy) during MJD 57622-57625, foreshadowing a major flare. Thus, on the $13^{\text {th }}$ of September (MJD 57643.8) a giant flare of $15 \mathrm{Jy}$ at $4.6 \mathrm{GHz}$ was detected by RATAN-600 (Trushkin et al. 2016b). The combination of all these conditions provided an excellent opportunity to observe the source under the state in which VHE gamma rays are expected.

MAGIC follow-up observations started on the $23^{\text {rd }}$ of August, triggered by the internal UB Fermi-LAT pipeline. The campaign was extended up to the $22^{\text {nd }}$ of September, point at which MAGIC had observed the system for 18 nights (among which only one was discarded afterwards during the analysis for presenting cloudiness above 40\%). From the $27^{\text {th }}$ of August to the $7^{\text {th }}$ of September, observations were carried out uninterruptedly every night aiming to observe the source the greatest possible time. This deep MAGIC campaign allowed us to observed the source 
for a total of $\sim 70$ hours of good quality data and, most important, from the very beginning of the $\mathrm{HE}$ emission until it ceased. Therefore, for the first time, we were able to obtain a great amount of data from Cygnus X-3 during an entire outburst that behaved as the one that led to its detection in the $\mathrm{HE}$ gamma-ray regime in 2009 (Fermi LAT Collaboration et al. 2009b).

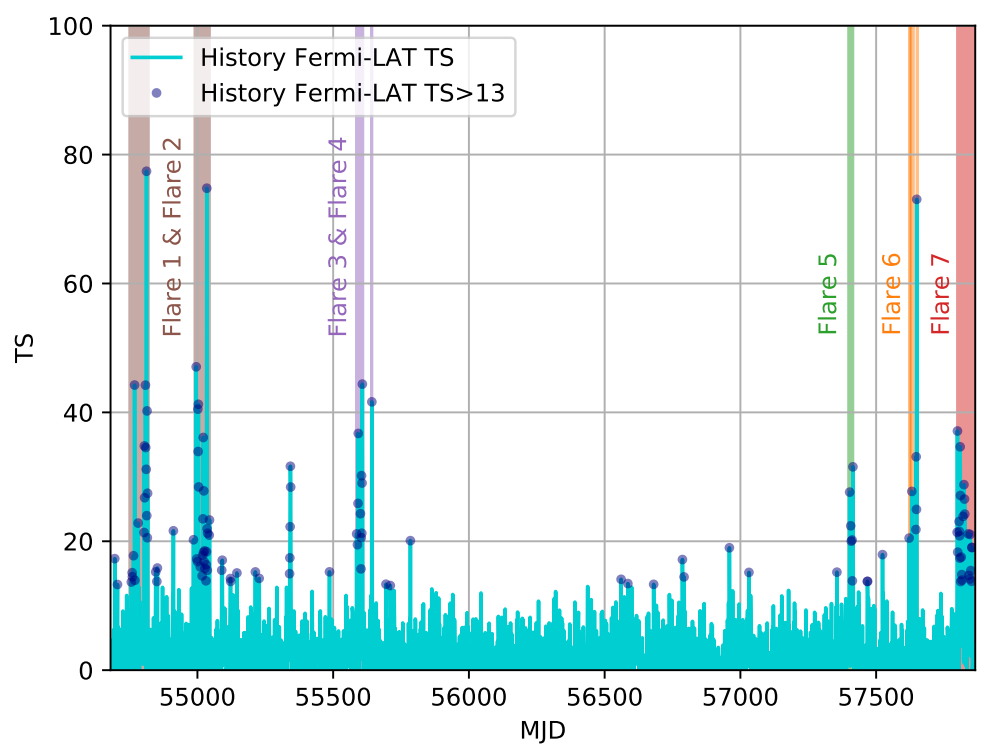

Figure 5.2: Cygnus X-3 TS evolution taken from the UB Fermi-LAT pipeline. Days with TS higher than 13 are marked with a dark blue dot. All $\mathrm{HE}$ flaring periods of the source are highlighted and labeled according to Table 5.1. A zoom view of Flare 6 reported in this thesis can be found in Figure 5.6

Figure 5.3 shows a schematic view of the saxophone plot given by Szostek et al. (2008), in which MAGIC observations are set in context. Given the lack of simultaneous soft X-ray data that covers the entire observational period, I defined two periods based on $15 \mathrm{GHz}$ Owens Valley Radio Observatory (OVRO) radio information: Period 1, from MJD 57623 to 57638, in which Cygnus X-3 was most of the time below the transition level (with an upper value of $\sim 500 \mathrm{mJy}$ ); and Period 2, from MJD 57650 to 57653, during which the source was entirely at a major flaring state (lower value at $\sim 3 \mathrm{Jy}$ and reaching around $9.5 \mathrm{Jy}$ ).

\subsection{Results before flare 2016}

From the beginning of the project until August 2016, we observed Cygnus X-3 for 16 nights, all of them under dark conditions. The dates and the corresponding significance (for FR and LE cuts, see Chapter 2.4.3.9p are listed in Table 5.3. No significant excess was found in any of these nights. The only remarkable episode happened on the $29^{\text {th }}$ of November 2015, when a hotspot at the level of $5 \sigma$ appeared close to the nominal position at a distance of $\sim 0.23^{\circ}$ (approximated coordinates $\mathrm{RA}=308.03^{\circ}, \mathrm{Dec}=41.18^{\circ}$ ). This excess was not coincident with the location of the 


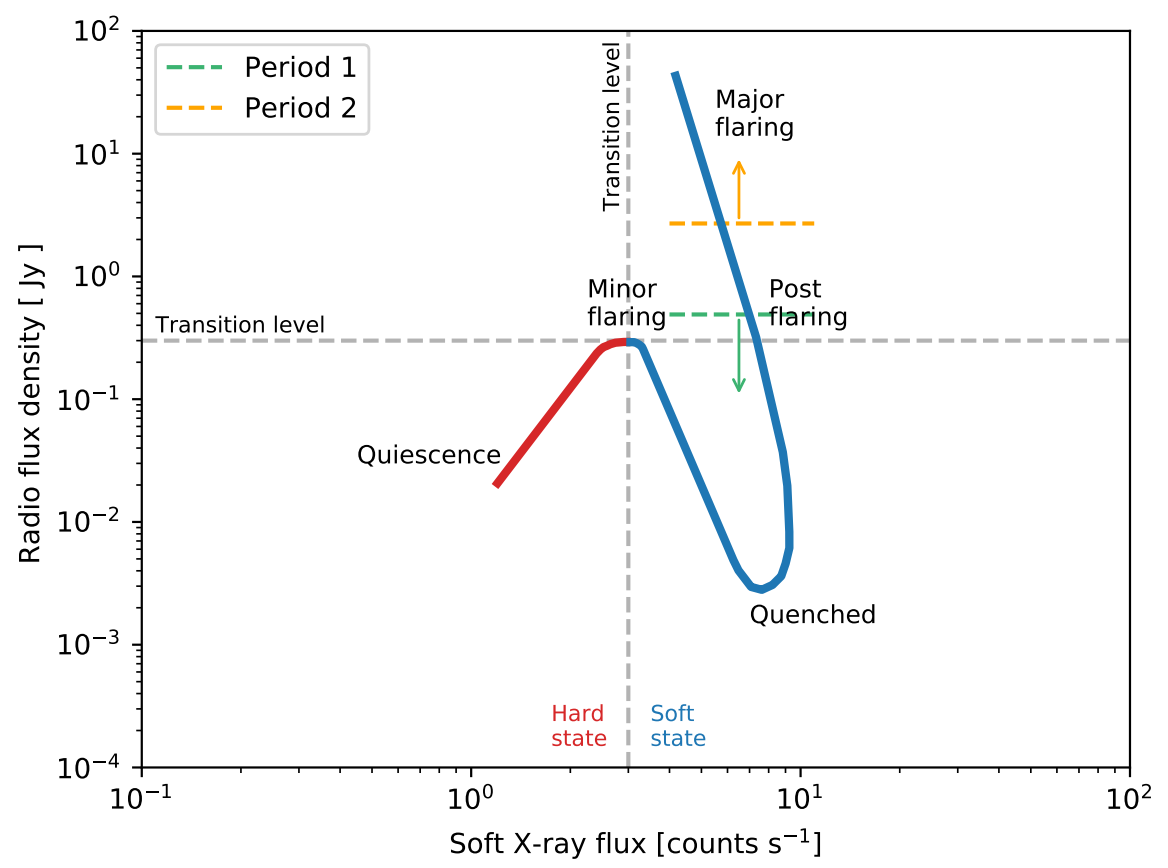

Figure 5.3: Modified saxophone plot from Szostek et al. (2008). Due to the lack of soft X-ray information, MAGIC observations periods are marked with two arrows based on OVRO data: the green one corresponds to Period 1 (MJD 57623-57638) and the orange one to Period 2 (MJD 57650-57653).

source TeV J2032+4130 either (see Figure 5.4). The significance, computed with Odie following the Off from Wobble Partner (OfWP) (see detailed information of this background estimation in Chapter 8.2 , was however $\sim 2.5 \sigma$. Nevertheless, and even though the signal from Cygnus X-3 was compatible with background from both $\theta^{2}$ plots and skymaps, observations were extended the $30^{\text {th }}$ triggered by this interesting hotspot in the FoV. On the $30^{\text {th }}$ of November, the skymap did not present any highlighted region and observations were stopped.

\subsection{Results of the August-September 2016 flare}

I searched for VHE gamma-ray emission in the entire period of activity, i.e. making use of the whole available data sample of $\sim 70$ hours. However, no excess was found, being the signal compatible with background with a significance of $-1.27 \sigma$ applying Equation 2.14 with FR cuts. Thus, integral UL at 95\% C.L. assuming a power-law distribution with photon index $\Gamma=2.6$ for energies above $300 \mathrm{GeV}$ was computed. This calculation yielded an UL of $2.6 \times 10^{-13}$ photons $\mathrm{cm}^{-2} \mathrm{~s}^{-1}$. Note that the minimum energy is constrained by the highest moonlight level data, to which a size cut of 250 phe is applied (see Table 5.2), implying an energy threshold of $\gtrsim 250 \mathrm{GeV}$. On the other side, variations of $\pm 30 \%$ on the photon index were probed and gave rise to less than $10 \%$ difference (see Table 5.4). Therefore, for the rest of the discussion, 
Table 5.3: Cygnus X-3 observations from November 2013 until August 2016. From left to right: Calendar date, effective time after quality cuts, significance with $\mathrm{FR}$ cuts and LE cuts at the nominal position.

\begin{tabular}{cccc}
\hline \hline Date & Effective time & \multicolumn{3}{c}{ Significance } \\
\cline { 3 - 4 } & & {$[\mathrm{FR}$} & $\overline{\mathrm{LE}}$ \\
[yyyy mm dd] & {$[\mathrm{hr}]$} & {$[\sigma]$} & {$[\sigma]$} \\
\hline 20131126 & 1.30 & 1.18 & -0.48 \\
20140505 & 0.86 & 0.32 & -1.24 \\
20140506 & 2.10 & 0.32 & 0.24 \\
20140525 & 2.10 & -1.67 & -0.15 \\
20140526 & 2.15 & 1.76 & 0.43 \\
20140609 & 1.32 & 0.05 & 0.76 \\
20141011 & 0.98 & 1.68 & 2.33 \\
20141012 & 2.62 & 1.60 & -0.50 \\
20141014 & 2.29 & -0.09 & -1.50 \\
20141016 & 0.53 & -0.97 & -1.46 \\
20141028 & 0.16 & 0.76 & -1.06 \\
20150729 & 5.79 & -3.10 & -0.96 \\
20151111 & 0.65 & 0.51 & -0.56 \\
20151129 & 0.88 & -1.56 & 0.55 \\
20151130 & 0.56 & 0.41 & -0.14 \\
20160517 & 0.96 & 0.0 & 1.07 \\
\hline
\end{tabular}

I decided to use the standard $\Gamma=2.6$, which allowed me to compare the results obtained in this thesis with the previous MAGIC/ULs (Aleksić et al. 2010b), for which computation a Crablike spectrum was also assumed. In order to decrease the energy threshold up to $100 \mathrm{GeV}$ and be able to extend results at lower energies, I also made use of the data sample taken under dark conditions only ( $\sim 52$ hours) to compute ULs. The integral UL under dark conditions, assuming the above mentioned spectral shape, is $5.1 \times 10^{-12}$ photons $\mathrm{cm}^{-2} \mathrm{~s}^{-1}$. Differential ULs, for the overall data set and only dark sample, are listed in Table 5.5 and presented in Figure 5.5 along with former MAGIC results as well as with the Fermi-LAT spectrum obtained by an independent analysis for the flaring period of 2016. Fermi-LAT spectrum was computed using FRONT+BACK photons from the Pass 8 data between MJD 57642-57652 that encompasses Period 2, in which the peak at $\mathrm{TS}=73$ was observed in the UB pipeline. In this analysis, the background model was created from 3 FGL sources, including the pulsation emission from $\mathrm{TeV}$ $\mathrm{J} 2032+4130$ (for which radio ephemeris from a private communication were used), as well as the corresponding diffuse emission ( $g l l_{-} i e m_{-} \_06$ and iso_P8R2_SOURCE_V6_v06). The off-pulse emission from $\mathrm{TeV} \mathrm{J} 2032+4130$ was described as a power-law with index $\Gamma=2.54$ (value obtained for the 8-years analysis data) and the Cygnus Cocoon contribution was fixed by the 


\section{CYGNUS X-3}

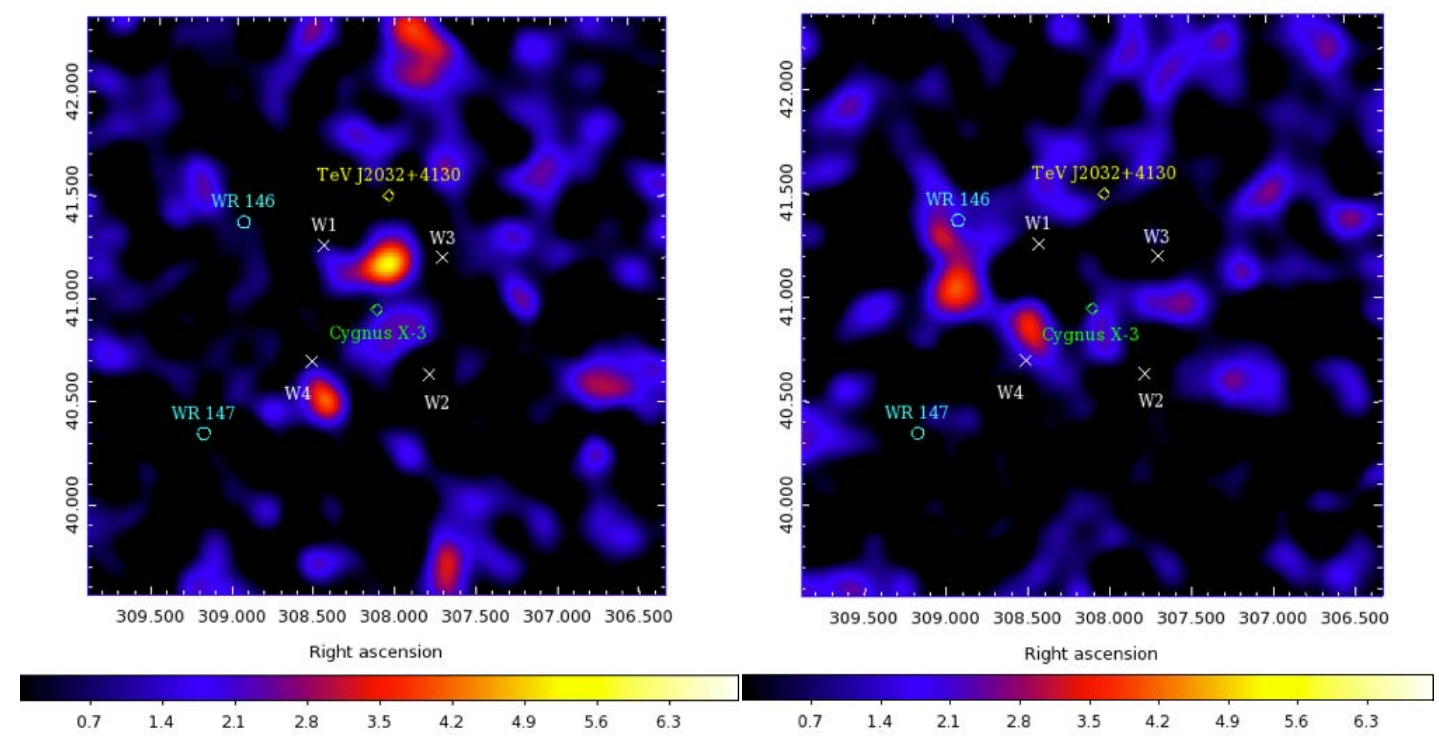

Figure 5.4: Cygnus X-3 LE skymaps for the $29^{\text {th }}$ (left) and the $30^{\text {th }}$ (right) of November 2015. The first night a hotspot at a distance of $\sim 0.23^{\circ}$ from Cygnus X-3 (green diamond) pop-up at the level of $5 \sigma$, not coincident with any known $\mathrm{TeV}$ source or candidate in the FoV This putative excess disappeared the following night. The source TeV J2032+4130 (yellow diamond) and the binary star systems WR 146 and WR 147 (cyan circles), as well as the wobble positions from the Cygnus X-3 observations (white crosses), are highlighted and labeled.

value given in Ackermann et al. (2011). To avoid contamination from another nearby pulsar, PSR $\mathrm{J} 2021+40$ located at $2.3^{\circ}$, the analysis was performed above $300 \mathrm{MeV}$ (energy at which FermiLAT PSF is less than $2^{\circ}$ ). Thus, during this Period 2, GeV emission was detected at TS=27 up to $\sim 20 \mathrm{GeV}$ and it is well defined by $d N / d E=(1.05 \pm 0.59) \times 10^{-11}(E / 1.3 \mathrm{GeV})^{3.23 \pm 0.56} \mathrm{GeV}^{-1}$ $\mathrm{cm}^{-2} \mathrm{~s}^{-1}$. It is worth to mention that no detection was achieved for the Period 1 (TS $\left.=13\right)$. The implications of the new results are discussed in the next section.

On the other hand, as previously introduced, Cygnus X-3 is a highly variable source in Xrays and $\mathrm{HE}$ gamma rays, and hence, one cannot discard this type of variation in the VHE band. Therefore, I performed a daily basis analysis. Nevertheless, no significant excess or hint was found during this search. Integral ULs above 100 and $300 \mathrm{GeV}$ (depending on the observational conditions) for each night are quoted in Table 5.6, with the respective significance and effective time. These results are set in context in Figure 5.6 together with the TS from Fermi-LAT data, hard X-ray data from Swift-BAT (15-50 keV, Krimm et al.2013) and soft X-ray data from MAXI (2-4 keV, Matsuoka et al. 2009). The low Swift-BAT flux during the entire observational campaign, along with the decrease in radio flux (which reached quenched state, Trushkin et al. 
Table 5.4: UL to the integral flux above $300 \mathrm{GeV}$ at $95 \% \mathrm{CL}$ assuming a power-law spectrum with different photon indices, $\Gamma$.

\begin{tabular}{|c|c|}
\hline \multirow[t]{2}{*}{$\Gamma$} & Flux UL at $95 \% \mathrm{CL}$ \\
\hline & {$\left[\times 10^{-13}\right.$ photons $\left.\mathrm{cm}^{-2} \mathrm{~s}^{-1}\right]$} \\
\hline 2.0 & 2.4 \\
\hline 2.6 & 2.6 \\
\hline 3.2 & 2.7 \\
\hline
\end{tabular}

Table 5.5: MAGIC differential flux ULs for Cygnus X-3 during the August-September 2016 flare, assuming a power-law spectrum with spectral index of $\Gamma=2.6$

\begin{tabular}{lcc}
\hline $\begin{array}{c}\text { Energy range } \\
{[\mathrm{GeV}]}\end{array}$ & $\begin{array}{c}\text { Differential flux } \\
{\left[\mathrm{TeV}^{-1} \mathrm{~cm}^{-2} \mathrm{~s}^{-1}\right]}\end{array}$ & $\begin{array}{c}\text { Observational } \\
\text { conditions }\end{array}$ \\
\hline $75.4-119.4$ & $2.7 \times 10^{-10}$ & dark \\
$119.4-189.3$ & $6.8 \times 10^{-11}$ & dark \\
$189.3-300.0$ & $1.1 \times 10^{-11}$ & dark \\
$300.0-475.5$ & $1.1 \times 10^{-12}$ & dark+moon \\
$475.5-753.6$ & $5.0 \times 10^{-13}$ & dark +moon \\
$753.6-1194.3$ & $2.6 \times 10^{-13}$ & dark +moon \\
$1194.3-1892.9$ & $1.5 \times 10^{-13}$ & dark +moon \\
$1892.9-3000$ & $2.9 \times 10^{-14}$ & dark + moon \\
$3000.0-4754.7$ & $9.7 \times 10^{-15}$ & dark + moon \\
\hline
\end{tabular}

2016b) before major outbursts, evidences the SS in which Cygnus X-3 stayed during the flaring activity. $\mathrm{HE}$ and radio outbursts happened almost simultaneously, separated approximately 2 days, shorter period that the one obtained by the Fermi-LAT Collaboration during the 2009 flare ( $5 \pm 7$ days, Fermi LAT Collaboration et al. 2009b). This gap is assumed by comparing the highest flux level in each regime: two days before the major Fermi-LAT flare with $\overline{\mathrm{TS}}=73$ on MJD 57649, radio flux increased to its maximum (see e.g. ??). Nevertheless, note that this presumable separation is get based on the results from the UB Fermi-LAT pipeline. For a more precise gap calculation between Fermi-LAT and radio flares, a dedicated Fermi-LAT flux light curve is needed.

The HE gamma-ray emission detected by Fermi-LAT was found to be orbitally modulated, with its maximum coincident with the superior conjunction of the compact object, which corresponds to phase 0 (Fermi LAT Collaboration et al. 2009b). I computed phase-folded analysis following the ephemeris of $T_{0}=2440949.892 \pm 0.001$ given by Singh et al. (2002). Owing to the great amount of data and the short orbital period of 4.8 hours, binning of 0.1 was possible. No hint was seen at any phase, including at the superior conjunction. The integral UL for each phase, with the corresponding FR significance and the effective time, is illustrated in Figure 5.7. 


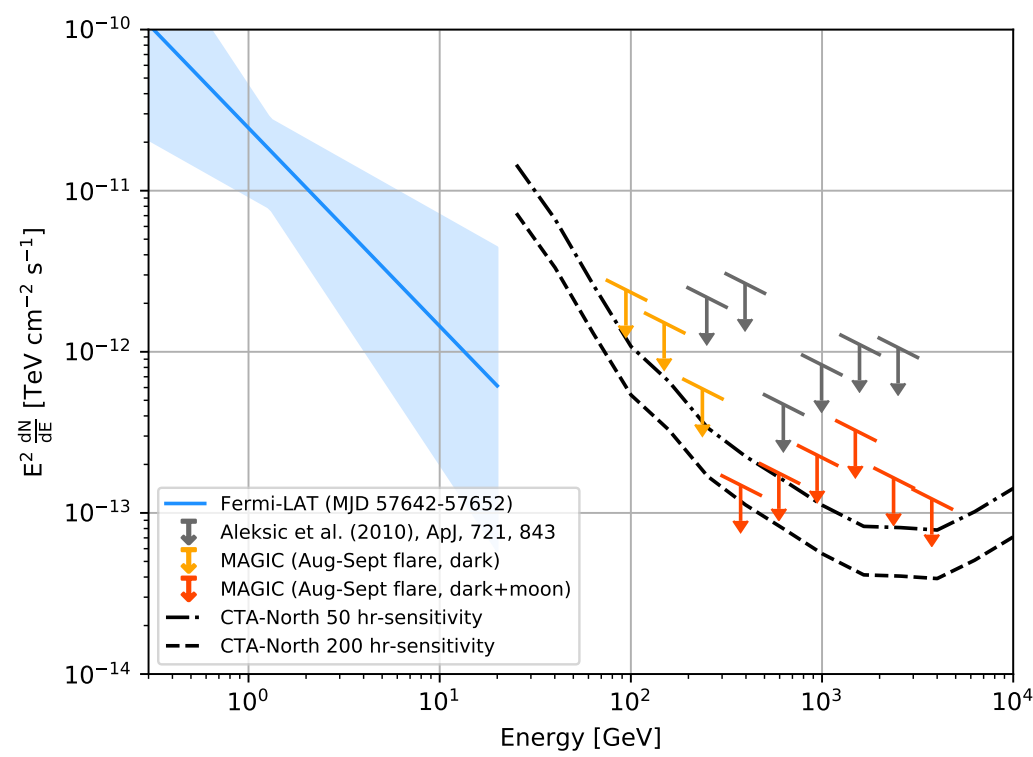

Figure 5.5: Cygnus X-3 SED for the flaring period August-September 2016. The blue butterfly corresponds to the Fermi-LAT results obtained by an independent analysis of the HE data MJD 57642-57652. Former MAGIC results given by Aleksić et al. (2010b) are depicted in grey, while ULs obtained in this thesis are shown in orange. At lower energies, only dark data was used ( $\sim 52$ hours, light orange) while the whole data sample was taken ( 70 hours, dark orange) above $300 \mathrm{GeV}$. Sensitivity curves of CTA-North for 50 hours (dot-dashed line) and 200 hours (dashed lines) of observations are shown too.

\subsection{Discussion and Conclusions}

As discussed in Chapter 3.2.4, VHE emission is postulated to happen inside the jets via both leptonic and hadronic mechanisms. This emission can occur steadily, as suggested for Cygnus X1 in which gamma-ray emission originates in the persistent jet during $\mathrm{HS}$ (see Chapter 4); or it could present transitional behavior, as expected in Cygnus X-3 from powerful radio-emitting blobs during $\mathrm{SS}$.

By the time of the beginning of this thesis, Cygnus X-3 was the best candidate among the microquasars to emit $\mathrm{VHE}$, as it was the only source of this type from which $\mathrm{HE}$ gamma rays had been firmly detected (Fermi LAT Collaboration et al. 2009b; Tavani et al. 2009b). Therefore, MAGIC focused the efforts on observing this source during $\mathrm{HE}$ flares by a dedicated trigger system. Eventually, we were able to observe the source during an entire strong radio and $\mathrm{HE}$ outburst in 2016, from the very beginning of the flare activity until the peak of emission ceased a month later. Nevertheless, no detection was achieved at VHE for any of the analyzed data samples. The obtained integral UL for energies greater than $300 \mathrm{GeV}$ was $2.6 \times 10^{-13}$ pho- 
Table 5.6: Daily basis analysis for the flaring period between August-September 2016. From left to right: Date, in calendar and $\mathrm{MJD}, \mathrm{Zd}$ range, effective time, significance applying $\mathrm{FR}$ and LE cuts, and integral ULs above $150 \mathrm{GeV}$ (dark data only) and $300 \mathrm{GeV}$ (dark+moon data). MJD 57623, 57650, 57651, 57652 and 57653 were observed under all aforementioned moonlight levels and hence, significance with LE cuts is not computed. MJD 57650 shows no integral $\mathrm{UL}$ above $150 \mathrm{GeV}$ due to low statistics.

\begin{tabular}{|c|c|c|c|c|c|c|c|}
\hline \multicolumn{2}{|l|}{ Date } & \multirow[t]{2}{*}{$\mathrm{Zd}$} & \multirow[t]{2}{*}{ Eff. Time } & \multicolumn{2}{|c|}{ Significance } & \multirow{2}{*}{$\begin{array}{l}\text { Integral ULs } \\
(\mathrm{E}>150 \mathrm{GeV})\end{array}$} & \multirow{2}{*}{$\begin{array}{l}\text { Integral ULs } \\
(\mathrm{E}>300 \mathrm{GeV})\end{array}$} \\
\hline & & & & FR & $\mathrm{LE}$ & & \\
\hline [уyуy mm dd] & [MJD] & {$\left[{ }^{\circ}\right]$} & [hr] & {$[\sigma]$} & {$[\sigma]$} & {$\left[\times 10^{-12}\right.$ phot } & ons $\mathrm{cm}^{-2} \mathrm{~s}^{-1}$ ] \\
\hline 20160823 & 57623 & $12-46$ & 5.9 & 1.03 & - & 8.9 & 2.2 \\
\hline 20160824 & 57624 & $12-27$ & 1.8 & -1.63 & 0.08 & 3.9 & \\
\hline 20160827 & 57627 & $22-32$ & 1.0 & 1.25 & 0.83 & 9.0 & \\
\hline 20160829 & 57629 & $20-30$ & 1.0 & -0.66 & -0.35 & 10.8 & \\
\hline 20160830 & 57630 & $20-30$ & 1.0 & 0.21 & -0.29 & 12.8 & \\
\hline 20160831 & 57631 & $12-30$ & 3.0 & -0.35 & -0.47 & 5.4 & \\
\hline 20160901 & 57632 & $12-28$ & 2.8 & 0.61 & -0.97 & 7.4 & \\
\hline 20160902 & 57633 & $10-50$ & 5.7 & 0.12 & -0.35 & 3.4 & \\
\hline 20160903 & 57634 & $10-50$ & 5.8 & -2.95 & 1.20 & 2.8 & \\
\hline 20160904 & 57635 & $10-50$ & 5.8 & -0.26 & -1.30 & 8.9 & \\
\hline 20160905 & 57636 & $10-50$ & 5.8 & -1.53 & -0.02 & 7.2 & \\
\hline 20160906 & 57637 & $10-50$ & 5.2 & 0.26 & 0.30 & 5.8 & \\
\hline 20160907 & 57638 & $10-50$ & 5.5 & -0.54 & -1.16 & 4.8 & \\
\hline 20160919 & 57650 & $12-50$ & 4.7 & 0.05 & - & - & 2.1 \\
\hline 20160920 & 57651 & $12-49$ & 4.8 & -0.68 & - & 10.1 & 1.0 \\
\hline 20160921 & 57652 & $12-50$ & 5.0 & 0.88 & - & 5.6 & 2.7 \\
\hline 20160922 & 57653 & $10-50$ & 3.7 & -0.48 & - & 7.7 & 1.5 \\
\hline
\end{tabular}

tons $\mathrm{cm}^{-2} \mathrm{~s}^{-1}$ and $5.1 \times 10^{-12}$ photons $\mathrm{cm}^{-2} \mathrm{~s}^{-1}$ above $100 \mathrm{GeV}$, correspond to a luminosity UL of $L_{\gamma}(>300 \mathrm{GeV})=2.0 \times 10^{33} \mathrm{erg} \mathrm{s}^{-1}$ and $L_{\gamma}(>100 \mathrm{GeV})=1.3 \times 10^{34} \mathrm{erg} \mathrm{s}^{-1}$, respectively, assuming a distance of $\sim 7 \mathrm{kpc}$. Given that the expected luminosity inside the relativistic jets is around $10^{37} \mathrm{erg} \mathrm{s}^{-1}$ (Martí et al. 2001), the UL on the conversion efficiency of the jet power to the VHE gamma-ray luminosity is about $0.02 \%$ (> $300 \mathrm{GeV})$ and $0.13 \%(>100 \mathrm{GeV})$.

One has to consider the extremely high absorption seen in X-rays by the wind of the $\mathrm{WR}$ companion, which might be not negligible for VHE gamma rays either. In the case of Cygnus X3 , the WR star has a temperature of $T_{*} \sim 10^{5} \mathrm{~K}$, with a bolometric luminosity of $L_{*} \sim 10^{38} \mathrm{erg}$ $\mathrm{s}^{-1}$. For energies above $300 \mathrm{GeV}$, the highest absorption is produced with photons around 1.7 $\mathrm{eV}$, i.e. in the NIR band, emitted by both companion and jet. Following Aharonian (2004), the cross-section of this interaction would be $\sigma_{\gamma \gamma} \sim \frac{2}{3} \sigma_{\text {Thomson }} \omega_{0}^{-1} \ln \left(\omega_{0}\right) \sim 1 \times 10^{-25} \mathrm{~cm}^{-2}$, where $\sigma_{\text {Thomson }}$ is the cross-section of the IC process in the Thomson regime (see Chapter 1.2.1.5) and $\omega_{0}=E_{\gamma 1} \cdot E_{\gamma 2}$ the product of the energies of the two photons colliding ( $300 \mathrm{GeV}$ and $1.7 \mathrm{eV}$, in 


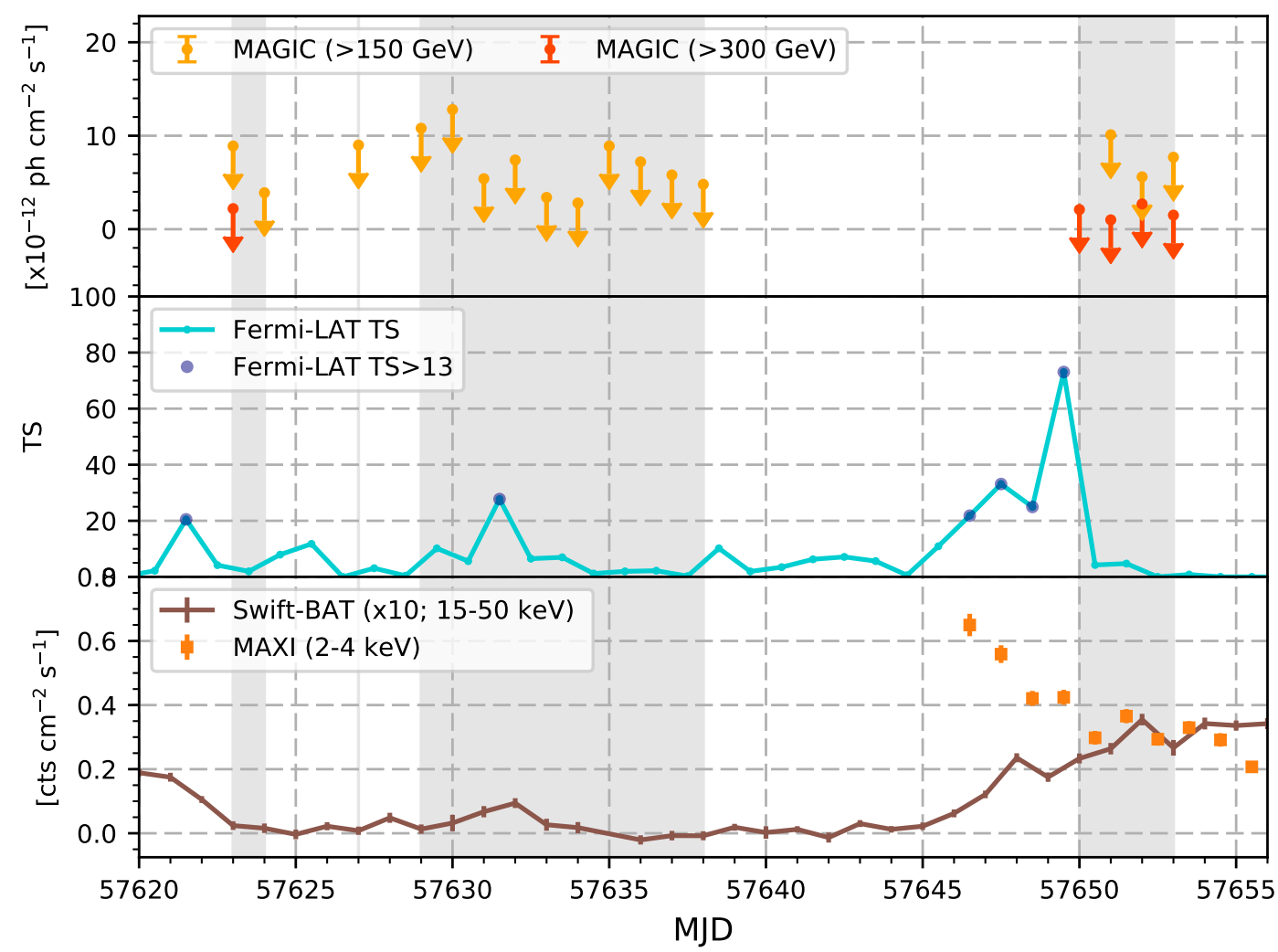

Figure 5.6: Cygnus $X-3$ light curve for the flaring period August-September 2016. From the top to the bottom: MAGIC ULs obtained in this thesis for a minimum energy of $150 \mathrm{GeV}$ (dark data only) and $300 \mathrm{GeV}$ (dark+moon), TS evolution from the UB pipeline where days with TSP 13 are highlighted in dark blue and public available hard and soft X-rays from Swift-BAT and MAXI, respectively. Gray bands corresponds to the MAGIC observations. No integral UL above $150 \mathrm{GeV}$ is shown for MJD 57650 because the total amount of dark data for this day corresponds to less than 5 minutes.

units of $\mathrm{m}_{e} c^{2}$. This way, absorption can be estimated as $\tau \sim \sigma_{\gamma \gamma} \cdot n_{N I R} \cdot r$ (Aharonian et al. 2005), where $r$ is the size of the emitting region and $n_{N I R} \sim L_{N I R} /\left(4 \pi r^{2} c E_{\gamma 2}\right)$, with $c$ the speed of light and $E_{\gamma 2}$ the target photons energy. For the calculation of $n_{N I R}$ I assume $L_{N I R}=L_{*}=10^{38} \mathrm{erg} \mathrm{s}^{-1}$ (i.e. most of the NIR contribution comes from the donor star) and $E_{\gamma 2}=2.7 \times 10^{-12} \mathrm{erg}$. Thus, it would not be until a radius of $\sim 10^{13} \mathrm{~cm}$, i.e. outside of the binary scale $\left(R_{\text {orb, } C y g X-3} \sim 2.5 \times 10^{11}\right.$ $\mathrm{cm}$ ) that the absorption does not affect VHE emission anymore. The independent Fermi-LAT analysis performed for the 2016 flare yielded with a detection between $300 \mathrm{MeV}$ and $20 \mathrm{GeV}$, approximately. To avoid absorption on $\overline{\mathrm{UV}}$ and X-rays from the disk, $\mathrm{HE}$ gamma-ray photons in Cygnus X-3 had to be produced at distances above $10^{10}-10^{11} \mathrm{~cm}$ from the compact object, still inside the binary scales where the interaction with the stellar photon field gives rise to orbital modulated emission. Thus, given the MAGIC non-detection, VHE emission, if produced, could 


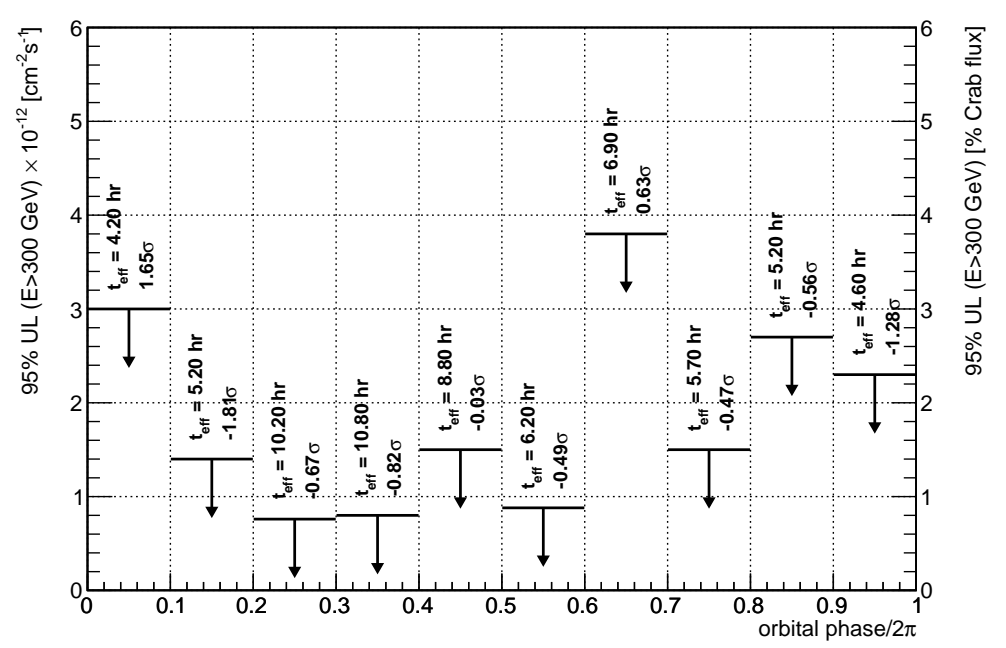

Figure 5.7: Cygnus X-3 phaseogram assuming the ephemeris of $T_{0}=2440949.892 \pm 0.001$ (Singh et al. 2002). The corresponding significance and effective time for each bin are labeled.

be expected inside the jets at a distance $<10^{13} \mathrm{~cm}$, maybe related to the $\mathrm{HE}$ emission site. On the other hand, during the August-September campaign, MAGIC observed Cygnus X-3 during the highest radio flux ( $\sim 9.5 \mathrm{Jy})$ on MJD 57651 for 4.8 hours. This day corresponds to moon observations, in which the $\mathrm{NSB}$ reached the level of $12-18 \times \mathrm{NSB}_{\text {lark }}$. No significant signal or emission was found during this day, which could reinforce the idea that VHE gamma rays are originated inside the binary scale and not at the radio-emitting regions of the jets far from the compact object. Note, however, that with the mentioned effective time, the sensitivity of the current MAGIC telescopes would be around 2.1\% Crab Units (C.U.), to which an additional $\gtrsim 10 \%$ degradation has to be considered given the moonlight (MAGIC Collaboration et al. 2017). Therefore, it would be necessary to observe the source during longer periods simultaneously with radio outbursts to provide strong conclusions regarding the VHE production region.

In Figure 5.5, the SED combining both $\mathrm{HE}$ and VHE results for the 2016 flare is shown. Fermi-LAT butterfly corresponds to the spectrum obtained by the independent analysis for $\mathrm{Pe}$ riod 2 , in which emission above $\sim 20 \mathrm{GeV}$ is not detected. No $\mathrm{HE}$ cutoff is obtained in this Fermi-LAT analysis. There are two facts that are worth to be highlighted: first of all, this SED allows us to probe the improvement achieved on the MAGIC performance after the upgrade of 2011-2012 (see Chapter 2.4). Former and current ULs were obtained assuming the same spectral shape and with very similar effective time $(\sim 60$ hours in Aleksić et al. $2010 \mathrm{~b}$ and $\sim 70$ hours in this campaign). The main difference is that the pre-upgrade stand-alone MAGIC I telescope was used for observations shown in Aleksić et al. (2010b), while here results in post-upgrade stereoscopic mode were presented. Consequently, there is a large difference in sensitivities between both campaigns. While during mono observations, the sensitivity was roughly $\sim 1.6 \%$ C.U. above $270 \mathrm{GeV}$ in 50 hours, currently the sensitivity is $\sim 0.66 \%$ C.U. above $220 \mathrm{GeV}$ for the same time. The latter is just marginally affected by moon observations, around $\gtrsim 10 \%$ with nominal HV (MAGIC Collaboration et al. 2017), given that more than 50 hours were observed 
in dark conditions. The sensitivity difference between both campaigns is remarkable at low energies, where $\mathrm{NSB}$ affects the most our observations. Spurious triggered signals by the $\mathrm{NSB}$ are highly reduced in stereoscopic mode at the L3 trigger, which implies a better sensitivity as evidenced in Figure 5.5. Moreover, results from Aleksić et al. (2010b) were obtained with the two oldest readout systems, Siegen and MUX. The former presented a very slow readout speed (300 MSamples/s) compared to that of MUX and the currently one used nowadays (2GSamples/s and 1.64 GSamples/s, respectively). This slow integration time increased the integrated NSB, worsening the sensitivity up to $40 \%$ (see Chapter 2.4.1.7).

Secondly, the deep campaign performed during August-September 2016 with almost 70 hours of good quality data allowed us to obtain very constraining ULs at 95\% CL. Such low ULs are already at the level or below the 50 hours-sensitivity curve from CTA-North 1 at energies $<1$ $\mathrm{TeV}$. Therefore, large observation time would be needed to detect Cygnus X-3 a low energies even with the next, more sensitive, generation of IACTs. This can be understood in two ways: one possibility is that the VHE flux from Cygnus X-3 is extremely low given the unusually high absorption and consequently, great amount of time would be necessary with CTA under flaring activity to shed light on this regime. In this case, other microquasar with smaller opacity to gamma rays, as Cygnus X-1, are favored to be detected by CTA. Another possible scenario is the existence of a cutoff between $\mathrm{HE}$ (whose emission was detected up to $\sim 20 \mathrm{GeV}$ ) and VHE. The nature of this speculative cutoff could be either the absorption itself or a possible inefficiency inside the Cygnus X-3 jets. Nevertheless, the extrapolation of the Fermi-LAT spectrum, consistent with the ULs obtained in this thesis, seems to make unlikely the second scenario, although a more dedicated Fermi-LAT analysis could bring more information.

\footnotetext{
${ }^{1}$ Taken from https://www.cta-observatory.org/science/cta-performance/
} 
5. CYGNUS X-3 


\section{6 \\ V404 Cygni}

\subsection{History}

V404 Cygni is a low-mass microquasar composed of a 8-15 $\mathrm{M}_{\odot} / \mathrm{BH} /($ Shahbaz et al. 1994) and a $0.7_{-0.2}^{+0.3} \mathrm{M}_{\odot}$ K3 III companion star (Casares \& Charles 1994, Khargharia et al. 2010). The system is located at a distance of $2.39 \pm 0.14 \mathrm{kpc}$ (Miller-Jones et al.2009) in the Cygnus Constellation. It displays an orbital period of 6.5 days (Casares et al. 1992) and a jet inclination angle with respect to our line of sight of $\sim 67_{-1}^{\circ+3}$ (Khargharia et al. 2010).

There are reports from the 18th century in which V404 Cygni was considered by astronomers as a variable star. It was firmly detected in optical in1938, and afterwards in 1956, although misclassified as a nova event. It would not be until 1989, when the source entered in an outburst activity period (releasing great amount of energy in radio, optical and X-rays, as seen by Ginga satellite, Makino et al. (1989), when it was finally classified as a LMXB. This way, the source seems to undergo extreme outbursts every two or three decades. Since 1989, it had remained in a quiescence state until June 2015.

\subsubsection{June 2015 outburst}

On the 15th of June 2015, the hard X-ray satellite Swift-BAT detected a huge outburst from the direction of V404 Cygni, which sent a worldwide alert via the GCN (Barthelmy et al. 2015). This burst-like activity period was similar to that on 1989 but shorter, with a duration of $\sim 11$ days. During these days, the microquasar displayed multiple flares in a time-scale of hours and it became the brightest X-ray source in the sky, reaching fluxes larger than 30 C.U. between 20-40 keV (see Figure 6.1). This exceptional behaviour led to a multiwavelength observational 
campaign from radio (Mooley et al. 2015), through hard X-rays with INTEGRAL (Ferrigno et al. 2015) and HE gamma-ray with Fermi-GBM (Younes 2015), up to VHE, observed by VERITAS (Archer et al.2016) and MAGIC.
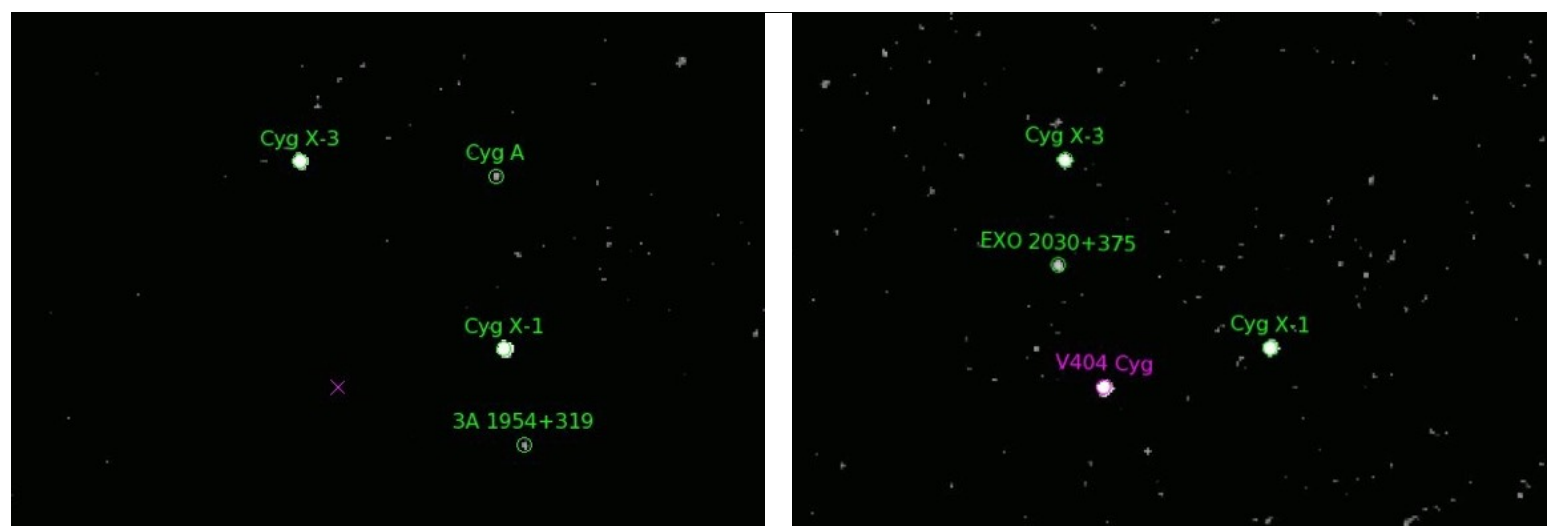

Figure 6.1: V404 Cygni FoV as seen by INTEGRAL-IBIS ( $15 \mathrm{keV}-10 \mathrm{MeV})$ before (left) and after (right) the outburst on the 19th of May 2015. The position of the source is marked with a magenta cross and magenta circle in the left and right panels, respectively. Credit: C. Ferrigno, INTEGRAL Science Data Center.

Specially interesting were the results obtained from the observations performed by INTEGRAL (Siegert et al. 2016) between the 17th to the 30th of June. Data from INTEGRAL-SPI were analyzed in three periods of three days each one (during flaring activity), in which an excess around $511 \mathrm{keV}$ was detected. This excess was compatible with the electron/positron annihilation. Siegert et al. (2016) showed that for energies below $200 \mathrm{keV}$, the hard X-ray spectrum could be described with the common thermal Comptonization, however above this energy an excess stood out. By adding a model spectrum of $e^{ \pm}$plasma with temperature T to the Comptonization model, the overall model improved in a $5 \sigma$. The value of $\mathrm{T}$ (which describes the width of the annihilation line) differed considerably in each epoch, from $\mathrm{T} \sim \mathrm{keV}$ to $\sim 200 \mathrm{keV}$, as seen in Figure 6.2. This $e^{ \pm}$pairs production is a result of the interaction between $\mathrm{MeV}$ gamma rays with an average cross-section of $\sim 10^{-25} \mathrm{~cm}^{2}$. This process becomes efficient given the small size of the V404 Cygni system (radius $3-10 R_{S c h}$, where $R_{S c h}=10 \mathrm{~km}$ is the Schwarzschild or gravitational radius of the $\mathrm{BH}$. The annihilation line emission is expected close by luminous accreting $\overline{\mathrm{BH}}$ when the spectra of the $e^{ \pm}$extend above $511 \mathrm{keV}$.

This observed annihilation line corresponded to a positron production rate of $\sim 10^{42} \mathrm{~s}^{-1}$. Such a positron production rate found in V404 Cygni could support two interesting theories: first of all, that the microquasars could be the main producers of the $e^{ \pm}$plasma responsible for the diffuse annihilation radiation in the bulge region of the Galaxy and, secondly, that they could also be the main origin of the observed $\mathrm{MeV}$ continuum excess present in the inner parts of our Galaxy.

Along with this annihilation line emissions, Loh et al. (2016) found an excess of $4 \sigma$ using 


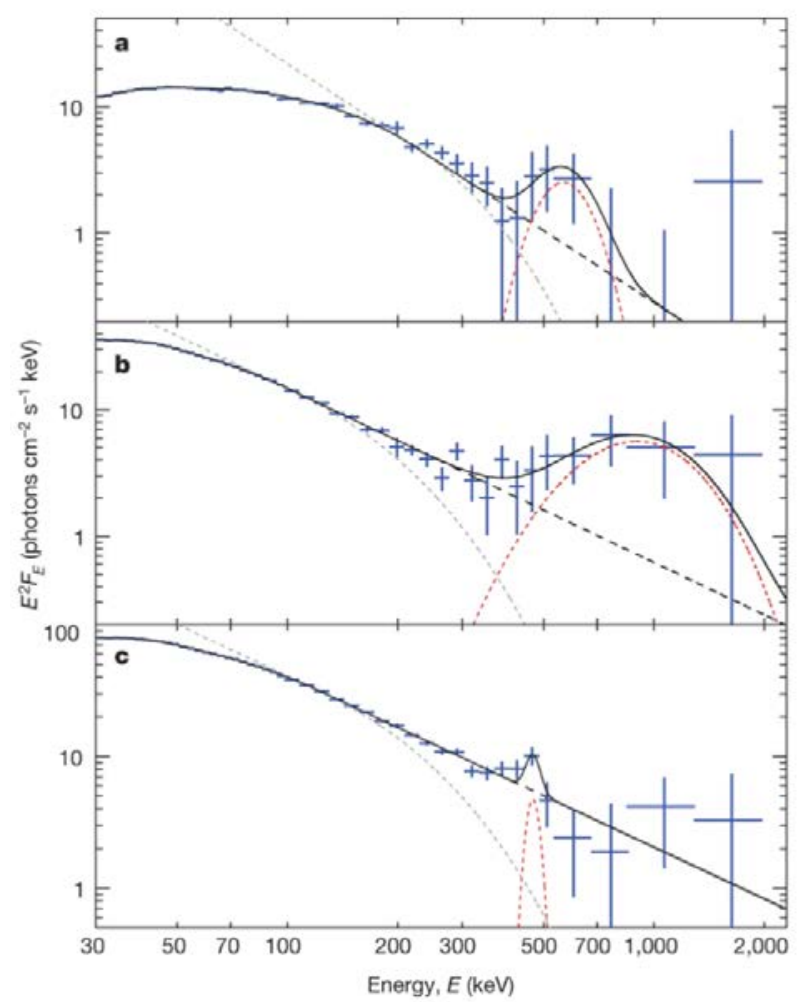

Figure 6.2: Spectra of V404 Cygni in the soft gamma-ray regime obtained with INTEGRAL in three flaring periods between 17th and 30th of June 2015. Blue points (with $1 \sigma$ error bars) correspond to the data, fitted to the Comptonization model (black dashed curve) and annihilation radiation (red dashed curve).

Fermi-LAT data, coincident with a giant radio flare (Trushkin et al. 2015a). Given the simultaneity of the $511 \mathrm{keV}$ excess and the hint in the $\mathrm{HE}$ gamma-ray regime, the production site of latter is expected to be outside of the corona, most likely in the jets: the plasma is continuously emitting annihilation radiation (given rise to broad annihilation lines, see Figure 6.2) increasing the opacity for photons with energies above $100 \mathrm{MeV}$ which would be absorbed by the X-rays in the coronal region.

\subsection{Observations and Data analysis}

MAGIC observed V404 Cygni for 8 non-consecutive nights under dark conditions $\left(D C_{M I}<\right.$ $2 \mu \mathrm{A}$ ) from the 18th to the 27th of June 2015, reaching a total of $\sim 10$ hours. Most of our observations were based on INTEGRAL alerts arrived from the GCN. The first alert, received on the 18th at 00:08:39 UT, presented such a high level flux that the source was believed to be a GRB. Therefore, the MAGIC telescopes pointed automatically to the coordinates of the source, following the GRB procedure (explained in Chapter 2.4.1.8). This scenario would be repeated along the entire period (with several alerts per night). Only on the night of the 23rd, observations 
of V404 Cygni were scheduled in the context of a campaign. Therefore, most of our observations were performed during high hard X-ray activity.

The analysis was performed using the MARS software (see Chapter 2.4.3). First subrun $(\sim 2$ minutes) during observations triggered by INTEGRAL alerts were discarded as they can be affected by a slightly wrong pointing position due to the automatic movement of the telescopes. The source was observed for a full zenith range of $5-50^{\circ}$ in the wobble-mode (Fomin et al. 1994), pointing at 4 position situated $0.4^{\circ}$ away from the V404 Cygni coordinates to evaluate the background simultaneously.

\subsection{Results}

In order to avoid spoil a putative excess due to trials, we searched for time intervals with the highest hard X-ray activity, related with the presence of relativistic jets. To do so, publicly available INTEGRAL-IBIS data $(20-40 \mathrm{keV})$ was analyzed with the OSA software 1 for which the light curve depicted in Figure 6.3 was obtained. Onto this light curve, we applied a Bayesian block analysis (Scargle et al.2013). This method is used to detect signal structures over the time with the aim of separating significant local $\left.\right|^{2}$ features from random errors. By applying the least possible assumptions (e.g. the shape of the signal) to avoid limiting the model, the division is achieved by fitting a piecewise constant model (a step function) to the data. Consequently, the range of the independent variable (time) will be split into bins in which the dependent variable (the intensity of the signal) remains constant within the errors. In our case, these local features could lead to periods in which particle acceleration was specially efficient in V404 Cygni. Nevertheless, none of the INTEGRAL flares presented exceptional intensity compared to the others (see Figure 6.3) and hence, five time intervals, covering the highest flaring activity, were used for the MAGIC analysis. These time intervals are listed in Table 6.1. The total MAGIC data covered in these 5 periods defined with the Bayesian Block algorithm reach $\sim 7$ hours. However, we found no detection, with a significance of $0.08 \sigma$.

For completeness, we searched for steady emission in the whole data sample and, given the high variability shown by the source, we also looked for emission in a daily basis analysis. None of these subsample yielded to a significant excess (see Table 6.2). We therefore computed integral and differential ULs using Rolke et al. (2005) method, assuming a power-law spectrum of photon index $\Gamma=2.6$ and $30 \%$ systematic uncertainty in the effective area of the gamma rays. The results, given for a C.L. of 95\%, are quoted in Table 6.2. Differential ULs for the data within the Bayesian Blocks periods are shown in Figure 6.4.

\footnotetext{
${ }^{1}$ http://www.isdc.unige.ch/integral/analysis

${ }^{2}$ The local features are those located in a sub-range of time which do not repeat continuously, contrary to the global ones, as e.g. the periodicity.
} 


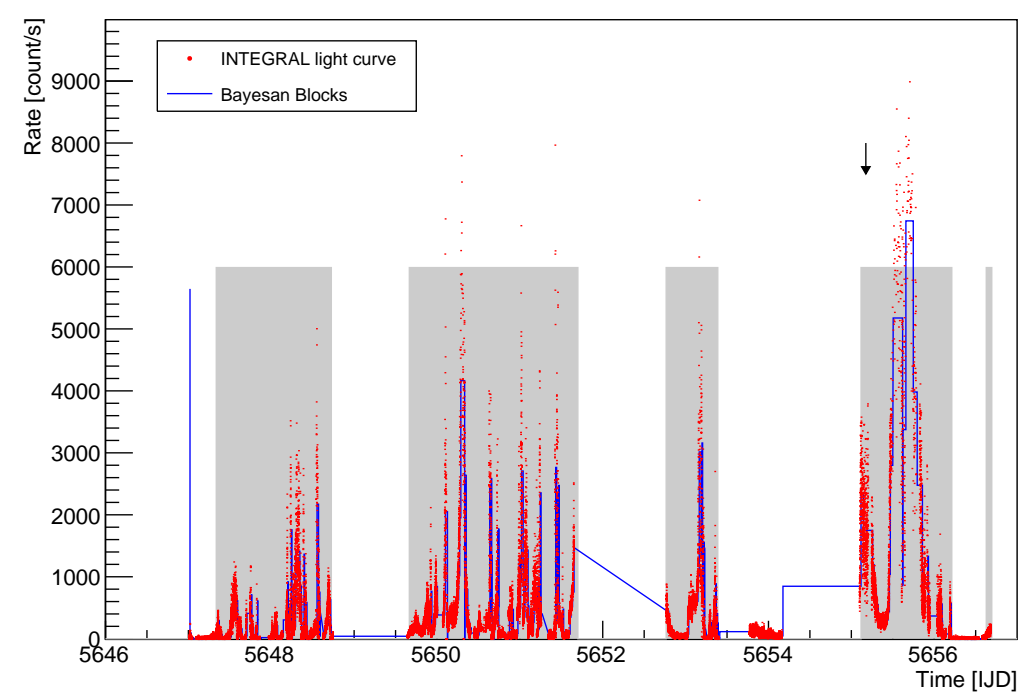

Figure 6.3: Light curve of INTEGRAL-IBIS data (red points, $20-40 \mathrm{keV}$ ) onto which Bayesian Blocks method (Scargle et al. 2013) was applied (blue lines). The intervals with highest flaring activity used in the analysis of MAGIC data are highlighted by grey bands. The time is given in INTEGRAL Julian Date, defined as IJD=MJD-51544.0. The black arrow corresponds to the observation time at which the $4 \sigma$ hint with Fermi-LAT data was obtained (Loh et al. 2016).

The hint at the level of $4 \sigma$ seen in the $\mathrm{HE}$ regime by Loh et al. (2016) with the analysis of Fermi-LAT data, temporally coincident with the brightest radio flare, presented a peak on the $26^{\text {th }}$ of June, at MJD $57199.2 \pm 0.1$ (black arrow in Figure 6.3. MAGIC observed V404 Cygni for around 1 hour simultaneously to this Fermi-LAT excess (during observations between 57199.158-57199.204, see Table 6.2). Nevertheless, the extrapolation of the FermiLAT spectrum, given between $100 \mathrm{MeV}$ and $100 \mathrm{GeV}$ and described by a power-law function of index 3.5, is two orders of magnitude lower than our ULs computed with the same photon index (see Figure 6.4).

Table 6.1: Time intervals used in the MAGIC analysis based on the Bayesian Block algorithm applied on INTEGRAL data.

\begin{tabular}{cc}
\hline $\begin{array}{c}\text { Start } \\
\text { (MJD })\end{array}$ & $\begin{array}{c}\text { Stop } \\
\text { (MJD }\end{array}$ \\
\hline 57191.337 & 57192.725 \\
57193.665 & 57195.700 \\
57196.765 & 57197.389 \\
57199.116 & 57200.212 \\
57200.628 & 57200.695 \\
\hline
\end{tabular}


Table 6.2: From left to right: MAGIC observation period in calendar and MJD, effective time, significance and integral ULs for energies between 200 and $1250 \mathrm{GeV}$, assuming a power-law function of index $\Gamma=2.6$. The last two rows report the results for the whole data sample and for the sample obtained based on the Bayesian Blocks algorithm (see Section 6.3).

\begin{tabular}{|c|c|c|c|c|}
\hline \multicolumn{2}{|c|}{ Observation Period } & \multirow{2}{*}{$\begin{array}{l}\text { Eff. Time } \\
\text { [hr] }\end{array}$} & \multirow{2}{*}{$\begin{array}{c}\text { Significance } \\
{[\sigma]}\end{array}$} & \multirow{2}{*}{$\begin{array}{l}\text { Integral } \mathrm{UL} \\
{\left[\times 10^{-11} \text { photons cm } \mathrm{cm}^{-2} \mathrm{~s}^{-1}\right]}\end{array}$} \\
\hline [yyyy mm dd] & MJD & & & \\
\hline 20150618 & $57191.006-57191.146$ & 2.99 & -0.43 & 0.51 \\
\hline 20150619 & $57191.960-57192.055$ & 1.9 & -0.6 & 1.0 \\
\hline 20150621 & 57193.997-57194.025 & 0.66 & 1.57 & 4.4 \\
\hline \multirow[t]{2}{*}{20150622} & $57195.021-57195.049$ & 133 & 0 & 17 \\
\hline & $57195.103-57195.134$ & 1.53 & 0.09 & 1.1 \\
\hline 20150623 & $57196.003-57196.124$ & 2.74 & -0.45 & 0.37 \\
\hline 20150626 & $57199.158-57199.204$ & 1.03 & -1.41 & 0.66 \\
\hline \multirow[t]{2}{*}{20150627} & $57200.085-57200.115$ & 1.97 & -0.57 & 12 \\
\hline & $57200.144-57200.202$ & & & \\
\hline Full data sample & - & 10.65 & -0.88 & 0.22 \\
\hline Bayesian Block selection & - & 6.88 & -0.42 & 0.48 \\
\hline
\end{tabular}

\subsection{Discussion and conclusions}

MAGIC observed the low-mass microquasar V404 Cygni during a major burst-like period, the last one since 25 years ago. These observations represented a good opportunity to study accretionejection processes given the proximity of the $\mathrm{BH}$ and the well-determined parameters of the binary (as its distance and the masses of the compact object and donor star). Mostly triggered by hard X-ray alerts from INTEGRAL, we obtained a total of $\sim 10$ hours of good quality data. By applying a Bayesian Block model to search for local variability, we reduced this sample to $\sim 7$ hours. However, nor steady emission (at any subsample) or variable emission in a night-wise basis was found.

Given the integral UL (for energies between 200-1250 GeV) obtained for the Bayesian Block selection, $4.8 \times 10^{12} \mathrm{~cm}^{-2} \mathrm{~s}^{-1}$, and a distance of $2.4 \mathrm{kpc}$ (Miller-Jones et al. 2009), the gamma-ray luminosity UL is $\sim 2 \times 10^{33} \mathrm{erg} \mathrm{s}^{-1}$, much lower than the extreme energy flux emitted in the hard X-ray band (20-400 keV) of $\sim 2 \times 10^{38} \mathrm{erg} \mathrm{s}^{-1}\left(\sim 20 \% L_{E d d}\right.$ of a $9 \mathrm{M}_{\odot} \mathrm{BH}$, Rodriguez et al. 2015a). This luminosity UL is, in turn, two orders of magnitude lower than the one obtained in the HE gamma-ray regime by Loh et al. (2016). On the other side, Tanaka et al. (2016) developed a model for the jet emission in V404 Cygni, following a blazar approach (assuming onezone synchrotron plus SSC model), in which the total power carried by the relativistic outflows reach $7.0 \times 10^{37} \mathrm{erg} \mathrm{s}^{-1}$. Therefore, the conversion efficiency of jet power to VHE gamma-ray luminosity in this low-mass microquasar is below $0.003 \%$.

I compare this luminosity UL with the one obtained for the high-mass microquasar Cygnus X1 (Chapter 4.3), given certain similarities shown by both microquasars. On one hand, both 


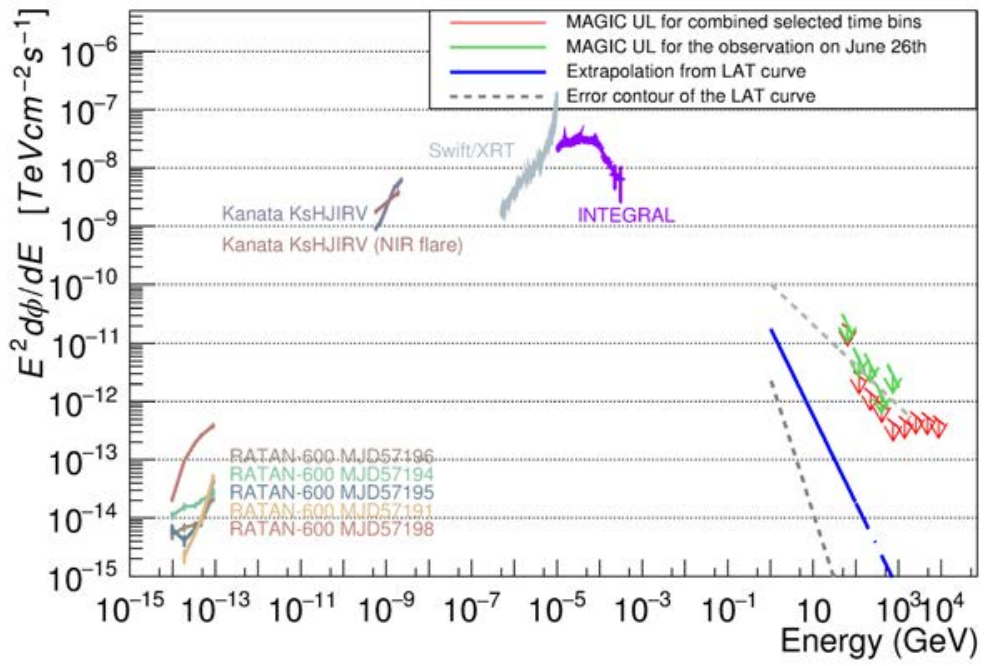

Figure 6.4: Multiwavelength SED of V404 Cygni during the June 2015 flaring period. In red, MAGIC ULs are given for the combined Bayesian block time bins ( 7 hours) for which a power-law function with photon index 2.6 was assumed. In green, MAGIC ULs for observations on June 26th, simultaneously taken with the Fermi-LAThint (Loh et al. 2016). In this case, a photon index of 3.5 was applied following Fermi-LAT results. The extrapolation of the Fermi-[LAT spectrum is shown in blue with $1 \sigma$ contour (gray dashed lines). In the X-ray regime, INTEGRAL (20-40 keV, Rodriguez et al. 2015a) and Swift-XRT (0.2$10 \mathrm{keV}$, Tanaka et al. 2016) data are depicted. At lower energies, Kanata-HONIR optical and NIR data are shown, taken from Tanaka et al. (2016). Finally, RATAN-600 radio data, from/Trushkin et al. (2015b), are presented for different days along to the flaring activity period.

sources are located at similar distances $(2.4 \mathrm{kpc}$ in the case of V404 Cygni and $1.86 \mathrm{kpc}$ for Cygnus X-1, Reid et al. 2011). Secondly, the total power emitted by their relativistic outflows are of the same order (for V404 Cygni, the power jet reaches $7.0 \times 10^{37} \mathrm{erg} \mathrm{s}^{-1}$, as mentioned above, taken from Tanaka et al. (2016), whilst for Cygnus X-1 this is expected to be $10^{36}-10^{37} \mathrm{erg} \mathrm{s}^{-1}$, Gallo et al. 2005). Finally, during the flare that gave rise to the hint in the $\mathrm{HE}$ gamma-ray band (reported by Loh et al. 2016), V404 Cygni appeared to stay in a HS or IS, states during which $\mathrm{GeV}$ emission was detected in Cygnus X-1 (see Chapter 4.2). Despite all the resemblances, the conversion efficiency into gamma-ray luminosity from the jet power is one order of magnitude smaller in the case of V404 Cygni ( $0.003 \%$ compared to the $0.06 \%$ obtained for Cygnus X-1).

The fact that the only two detected microquasars in the gamma-ray regime, Cygnus X-1 (Chapter 4.2) and Cygnus X-3 (Tavani et al. 2009a; Fermi LAT Collaboration et al. 2009a), host hot and massive companion stars suggests that the most efficient mechanism, to emit in this energy band, is the IC on stellar photons. Low-mass microquasars are not suitable to fit in this scenario because of the cold and old companions (see Chapter 3.2.4.3), but they cannot be discarded as VHE emitters. Models predict TeV emission from this type of systems under 
efficient particle acceleration on the jets (Atoyan \& Aharonian 1999) or strong hadronic jet component (Romero \& Vila 2008). If produced, VHE gamma rays may annihilate via pair creation in the vicinity of the emitting region. For gamma rays in an energy range between $200 \mathrm{GeV}-$ 1.25 TeV, the largest cross-section occurs with NIR photons. For a low-mass microquasar, like V404 Cygni, the contribution of the NIR photon field from the companion star (with a bolometric luminosity of $\sim 10^{32} \mathrm{erg} \mathrm{s}^{-1}$ ) is very low. During the period of flaring activity, disk and jet contributions are expected to dominate. During the outburst activity of June 2015, the magnitude of the K-band reached $\mathrm{m}=10.4$ (Shaw et al. 2015), leading to a luminosity on the NIR regime of $L_{N I R}=v \phi_{m=0} 4 \pi d^{2} 10^{-m / 2.5}=4.1 \times 10^{34} \mathrm{erg} \mathrm{s}^{-1}$, where $v$ is the frequency for the $2.2 \mu \mathrm{m} \mathrm{K}$-band, $\phi_{m=0}=670 \mathrm{Jy}$ is the K-band reference flux and $d=2.4 \mathrm{kpc}$ is the distance to the source. The detected NIR radiation from V404 Cygni during this flaring period, was expected to be dominated by optically-thick synchrotron emission from the jet or to be originated inside the accretion flow, given the lack of evidence of polarization (Tanaka et al. 2016). Consequently, stronger gammaray absorption is expected at the base of the jets. The gamma-ray opacity due to NIR radiation inside V404 Cygni can be estimated as $\tau_{\gamma \gamma} \sim \sigma_{\gamma \gamma} \cdot n_{N I R} \cdot r$, given by Aharonian et al. (2005). The cross-section of the interaction is defined by $\sigma_{\gamma \gamma}$, whose value is $\sim 1 \times 10^{-25} \mathrm{~cm}^{2}$. The NIR photon density is calculated as $n_{N I R}=L_{N I R} / \pi r^{2} c \epsilon$, where $\mathrm{r}$ is the radius of the jet where NIR photons are expected to be emitted; $c$ is the speed of light and $\epsilon \sim 1 \times 10^{-12}$ erg is the energy of the target photon field. Assuming the aforementioned luminosity of $L_{N I R}=4.1 \times 10^{34} \mathrm{erg} \mathrm{s}^{-1}$, the gammaray opacity at a typical radius $r \sim 1 \times 10^{10} \mathrm{~cm}$ may be relevant enough to avoid VHE emission above $200 \mathrm{GeV}$. Moreover, if $\mathrm{IC}$ on X-rays at the base of the jets $\left(r \lesssim 1 \times 10^{10} \mathrm{~cm}\right)$ is produced, this could already prevent electrons to reach the $\mathrm{TeV}$ regime, unless the particle acceleration rate in V404 Cygni is close to the maximum achievable including specific magnetic field conditions (see e.g. ?). On the other hand, absorption becomes negligible for $r>1 \times 10^{10} \mathrm{~cm}$. Thus, if the VHE emission is produced in the same region as $\mathrm{HE}$ radiation $\left(r \gtrsim 1 \times 10^{11} \mathrm{~cm}\right.$, to avoid X-ray absorption), then it would not be significantly affected by pair production attenuation $\left(\sigma_{\gamma \gamma}<1\right)$. Therefore a VHE emitter at $r \gtrsim 1 \times 10^{10} \mathrm{~cm}$, along with the non-detection by MAGIC suggests either inefficient particle acceleration inside the V404 Cygni jets or not enough energetics of the VHElemitter. 


\section{Part III}

\section{Pulsar Wind Nebulae in the very high-energy gamma-ray regime}

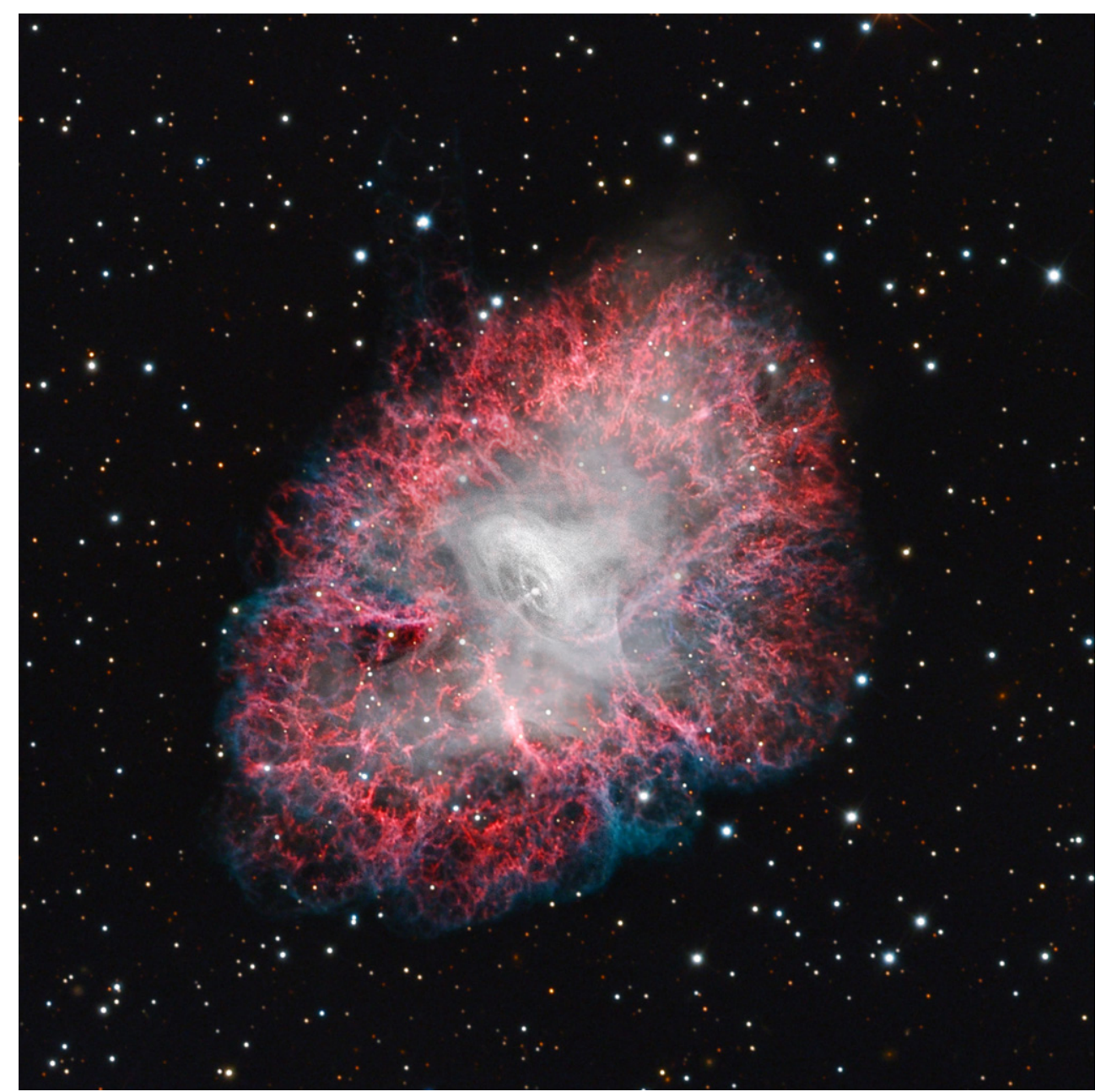

Figure 6.1: Superimposed image of the $\mathrm{X}$-ray emission from the Crab pulsar (white) with the visible Crab Nebular (red). Credit: (Crab Pulsar image) NASA/CXC/SAO/F. D. Seward, W. H. Tucker and R. A. Fesen; (Crab Nebula image) Adam Block/Mount Lemmon SkyCenter/University of Arizona. 



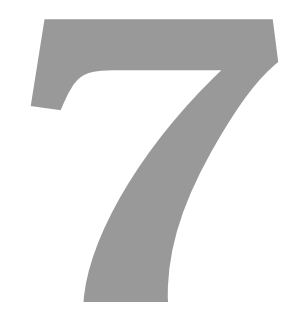

\section{Introduction to Pulsar Wind Nebulae}

All the ejected material by a $[\mathrm{SN}]$ explosion interacts with the surrounding environment, giving rise to a new astrophysical object known as $\mathrm{SNR}$ (for a review of this matter, I refer the reader to Reynolds 2008 and to Appendix A for more information on the first evolutionary phase). In turn, the possible leftovers product of this $\mathrm{SN}$ are very different depending on its initial mass, as described in Chapter 3.1. In this chapter, I will focus on the remnants produced by the interaction of a pulsar created after the explosion and the surrounding medium, the so-called PWNe.

SNRs can be classified into three types: shell-like SNRs, whose name arises from their shelllike structure (as an example, Cassiopeia A); PWNe or plerions, if the central object is a pulsar that constantly powers and injects particles into the remnant (as in the case of the famous Crab Nebula); and finally, composite remnant, produced when the $\overline{\mathrm{PWN}}$ is surrounded in turn by a shell-like SNR (e.g., HESS J1818-154). In the following, I will focus on the components and expected emission from the second type of SNR, the PWNe.

\subsection{Central object in PWNe}

\subsubsection{Neutron star}

$\mathrm{NSs}$ are the most dense objects known in our Universe (Figure 7.1). Born from the explosion of massive stars, these objects normally achieve diameters of $\sim 20 \mathrm{~km}$ and masses around 1.4-3 $\mathrm{M}_{\odot}$, which leads to densities of $\rho \sim 10^{17} \mathrm{~kg} / \mathrm{m}^{3}$. Two properties are specially relevant in these objects: the angular momentum and the magnetic flux, both of them conserved from the initial massive star. As the massive star collapses into a much smaller object, the rotation rate needs to increase as a result of conservation of angular momentum. Therefore, $\mathrm{NSs}$ can experiment 
rotation rates or periods from $1 \mathrm{~ms}$ to $10 \mathrm{~s}$. On the other hand, as the star's surface is smaller, due to the magnetic flux conservation, the magnetic field will be stronger. As a result, a highly magnetized rapidly-spinning $\mathrm{NS}$ is born. NSs are usually modeled as magnetic dipoles.

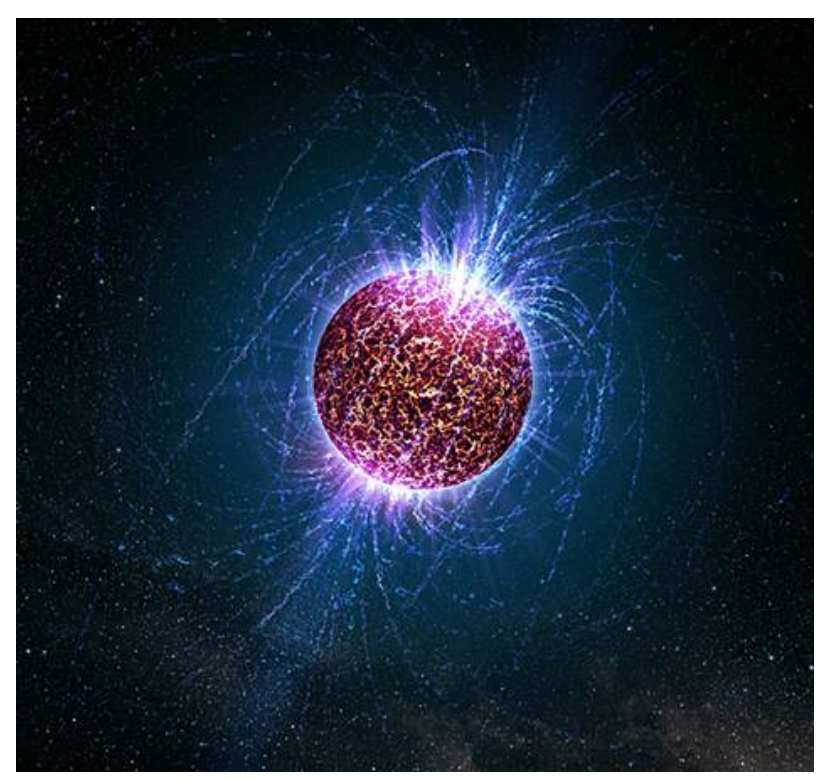

Figure 7.1: Artist's view of a NS. Credit: Casey Reed/Penn State University.

$\mathrm{NSs}$ are mainly composed of neutrons, although their internal structure is more complicated (Figure 7.2). Below a very thin atmosphere, $\mathrm{NSs}$ present an outer crust of $\sim 300 \mathrm{~m}$ deep formed by ions and electrons. Deeper in the star, an inner crust of $\sim 600 \mathrm{~m}$ deep is developed, which mixed neutrons and electrons. The bulk of the NS remains in the so-called outer core, a $\sim 9 \mathrm{~km}$ deep layer, composed of a superfluid neutrons, as well as an small number of superconducting protons. The composition of the innermost layer, the core of the NS, is still unclear, but some theories suggest exotic solid matter consisting on elementary particles, such as quarks and gluons.

There are different types of $\mathrm{NSs}$ according to the primary source of their emission. In this thesis, we are interested in a sub-class known as Rotation-Powered Pulsars (RPPS) or just pulsars, in which the emission arises from the rotational energy of the $\mathrm{NS}$. For an extended review on NSs, see Harding (2013).

\subsubsection{Pulsars}

Pulsars are a type of NS that emit beams of electromagnetic radiation powered by rotational energy. This emission is detected when crossing our light of sight, producing this way the pulsed appearance (the so-called lighthouse effect). Their existence was postulated by Pacini (1967) to 


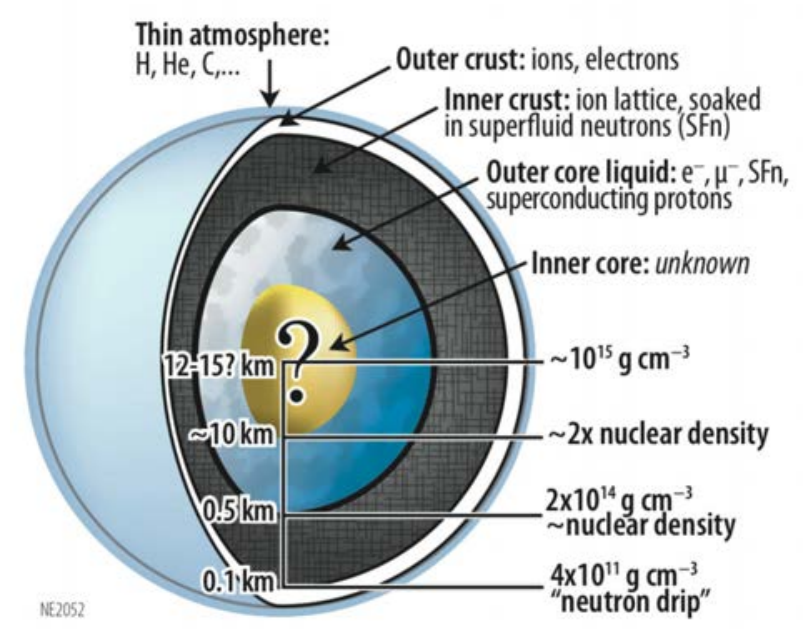

Figure 7.2: Internal structure of a NS. Credit: NASA/GSFC.

explain the Crab Nebula system, one year before their discovery in the radio band by Jocelyn Bell (Hewish et al. 1968).

Given that the primary source of the radiation in these systems is their rotational energy, $E_{\text {rot }}$, in the following we will see the relation of their principal properties and the energy loss. For a comprehensive review on PWNe, the reader is referred to Gaensler \& Slane (2006).

The main feature of the pulsars is the so-called spin-down power, $\dot{E}=-d E_{\text {rot }} / d t$, i.e. the rate at which the rotational energy is released. It is defined as:

$$
\dot{E}=4 \pi^{2} I \frac{\dot{P}}{P^{3}}=I \omega \dot{\omega}
$$

where $P$ is the rotational or spin period and $\omega=2 \pi / P$ is the angular frequency of the $\mathrm{NS}(\dot{P}$ and $\dot{\omega}$ correspond with their derivative with respect to time, respectively), and $I \sim 10^{38} \mathrm{~kg} \mathrm{~m}^{2}$ is the moment of inertia of the star. This leads to $\dot{E}$ values ranging between $10^{28}-10^{38} \mathrm{erg} / \mathrm{s}$.

The rotational period decreases with time with respect to its initial spin period $P_{0}$. This spin down can be defined as $\dot{\omega}=-\kappa \omega^{n}$, where $\kappa$ is a constant and $n$ is the so-called braking index. The value of $n$ has only been firmly measured for four pulsars, ranging between 1.4 and 2.9. It is usually considered $n=3$ for the rest of pulsars, which corresponds to the spin down through magnetic dipole radiation. If we take this spin down as a function of the period, following Manchester \& Taylor (1977), then we get an expression for the age of the pulsar, $\tau$ :

$$
\tau=\frac{P}{(n-1) \dot{P}}\left[1-\left(\frac{P_{0}}{P}\right)^{n-1}\right]
$$


Equation 7.2, for the case of $n=3$ and $P_{0}<<P$, can be simplified as:

$$
\tau_{c}=\frac{P}{2 \dot{P}}
$$

This $\tau_{c}$ is the so-called characteristic age of the pulsar. Normally, the characteristic age obtained through equation 7.3 overestimates the real age of the pulsar.

On the other hand, by following the magnetic dipole assumption (Ostriker \& Gunn 1969), one can obtain the magnetic field on the surface of the $\overline{N S}, B$ in $\mathrm{G}$, as a function of the period (in seconds):

$$
B \sim \mu R \bumpeq 3.2 \times 10^{19}(P \dot{P})^{1 / 2}
$$

where $\mu$ is the magnetic dipole moment and $R$ is the radius of the NS. Common values for the surface magnetic field range between $10^{12}-10^{13} \mathrm{G}$.

All spin-down power, spin period and surface magnetic field will evolve with time. Assuming that the braking index, $n$, is constant, we can defined the evolution as:

$$
\begin{gathered}
\dot{E(t)}=\dot{E}_{0}\left(1+\frac{t}{\tau_{0}}\right)^{-\frac{n+1}{n-1}} \\
P(t)=P_{0}\left(1+\frac{t}{\tau_{0}}\right)^{\frac{1}{n-1}} \\
B(t)=B_{0}\left(1+\frac{t}{\tau_{0}}\right)^{\frac{n-1}{2 n-2}}
\end{gathered}
$$

where $\dot{E}_{0}$ and $B_{0}$ corresponds to the initial values of the spin-down power and magnetic field, and $\tau_{0}=\frac{2 \tau_{c}}{n-1}-t$ is the spin-down time scale.

\subsubsection{Magnetosphere and wind zone}

The charge (due to ions and electrons) in the surface of the NS induce an electric field parallel to the magnetic field, i.e. perpendicular to the surface. This electric field is so strong that pulls the charges out of the surface against the gravitational force. These particles follow the magnetic field lines populating the surrounding of the $\overline{\mathrm{NS}}$, the so-called $\mathbf{N S}$ magnetosphere. In this magnetosphere, the charges co-rotate with the $\mathrm{NS}$ and their charge density is approximately the Goldreich-Julian one, $\rho_{G J} \sim \Omega \cdot B / 2 \pi c$ (Goldreich \& Julian 1969), where $\Omega$ is the angular speed. The $\mathrm{NS}$ magnetosphere is delimited by the so-called light cylinder (see Figure 7.3), whose radius, $R_{L C} \sim 100 \mathrm{~km}$, is the distance at which the co-rotating plasma and magnetic field speed equals the speed of light $\left(R_{L C}=c / \Omega\right.$, where $c$ is the speed of light. Therefore, the open magnetic field lines, through which the charge particles leave the light cylinder, do not co-rotate with the $\mathrm{NS}$. This flowing of particles outside of the magnetosphere creates zones with reduced charge density and hence, contrary to what happens under Goldreich-Julian charge density, an 
electric field (parallel to the magnetic field) can develop. This way, particles following the open magnetic field lines can be accelerated in certain regions and, due to the strong magnetic field, they will be able to emit synchrotron radiation (see models in Section 7.1.2.2). This radiation will, in turn, produce pairs of $e^{ \pm}$, giving rise to electromagnetic cascades. However, these secondary pairs short out the electric field and consequently, constrain their own acceleration. This pulsar-driven wind, during its expansion outwards from the light cylinder, decelerates and the pressure and interaction produced by ISM give rise to the so-called termination shock, in which $e^{ \pm}$get accelerated up to $\mathrm{VHE}$. The region between the light cylinder and the termination shock is called wind zone.

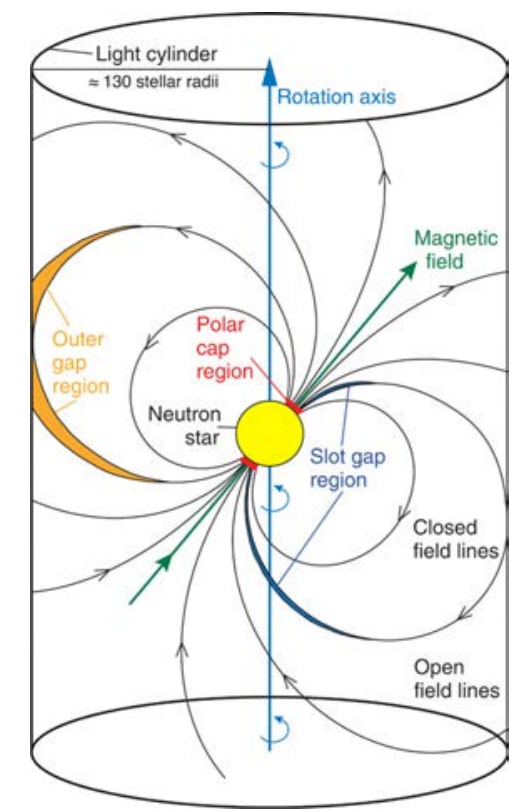

Figure 7.3: Diagram of the canonical magnetic dipole model applied to pulsars. The magnetosphere of the $\mathrm{NS}$ is delimited by the light cylinder. The location for gamma-ray emission on the proposed $\overline{\mathrm{PC}}, \mathrm{OG}$ and $\overline{\mathrm{SG}}$ scenarios are highlighted. Credit: Aliu et al. (2008a).

The magnetization of the pulsar wind is given by $\sigma$ :

$$
\sigma \equiv \frac{F_{E \times B}}{F_{\text {particles }}}=\frac{B^{2}}{4 \pi \rho \gamma c^{2}}
$$

where $F_{E \times B}$ and $F_{\text {particles }}$ are the Poynting and particles fluxes, respectively, and $B, \rho$ and $\gamma$ are the magnetic field, mass density of particles and the Lorentz factor, again respectively. The pulsar wind is assumed to be Poynting flux dominated when it leaves the magnetosphere. Therefore, this magnetization parameter presents values of above $10^{4}$ once the pulsar wind flows out from the light cylinder. Nevertheless, observational conditions require a dramatic decrease in this value close to the termination shock. Thus, values of $\sigma \lesssim 0.01$ are needed to explain e.g. the ratio between the synchrotron luminosity and the total spin-down power of the Crab pulsar ( $\overline{\mathrm{Ken}}-$ nel \& Coroniti 1984), with Lorentz factor of $\gamma \sim 10^{6}$, much higher than values expected from 
free expanding winds (Arons 2002). The reason that sets off this change in the nature of the pulsar wind, from Poynting to particle dominated wind, is not well understood yet (Arons 2009), although magnetic reconnection seems to solve the problem (Porth et al. 2013).

\subsubsection{Gamma-ray emission models}

Pulsars at high and very-high energy have been detected and therefore, it is assumed that particle acceleration is efficiently produced in these systems. However, as mentioned before, the charge density inside the $\mathrm{NS}$ magnetosphere is close to the Goldreich-Julian one which, taken into account that $\mathbf{E}$ is parallel to $\mathbf{B}$, would disrupt particle acceleration inside this region $(\mathbf{E} \cdot \mathbf{B}=0)$. Nevertheless, there are some locations where this condition is not fulfill and therefore, acceleration is possible. The scenarios for the particle acceleration can be split into three models, explained below and depicted in Figure 7.4 .

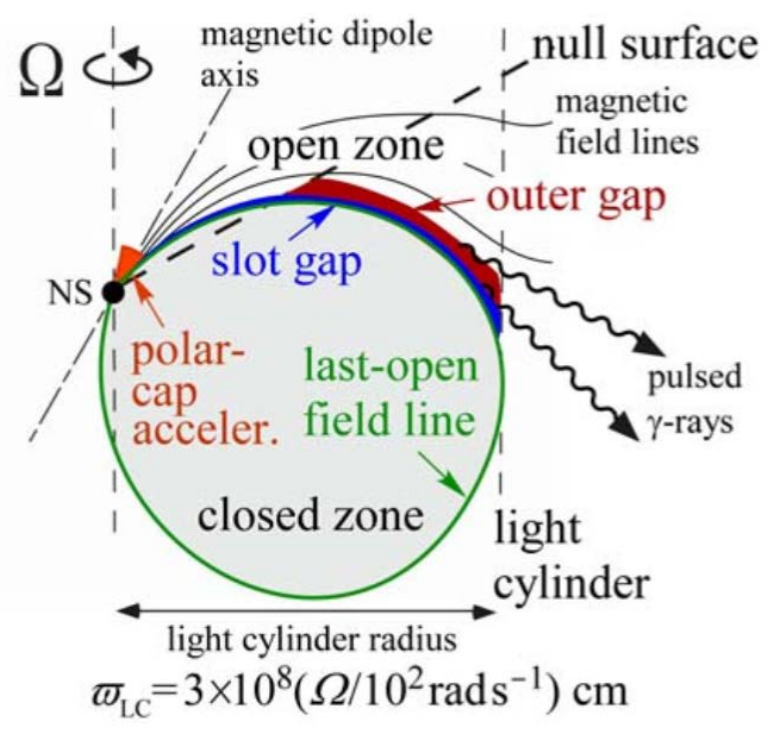

Figure 7.4: Sketch of the pulsar magnetosphere taken from Hirotani (2008) with the particle acceleration models, $\mathrm{PC}, \mathrm{OG}$ and $\mathrm{SG}$, highlighted.

$\odot$ Polar Cap (PC) model: Proposed by Sturrock (1971) and Ruderman \& Sutherland (1975), this model suggests particle acceleration close to the surface of the NS, on the magnetic poles. This particles can emit gamma rays through $[\mathrm{IC}$ (on thermal $\mathrm{X}$-rays from the surface of the star) or via curvature radiation (accelerated in the curved magnetic fields). In turn, these gamma rays produce $e^{ \pm}$pairs that develop in electromagnetic cascades. This model predicts a very sharp energy cutoff at several GeV in the gamma-ray spectrum.

$\odot$ Slot Gap (SG) model: This model was proposed by Arons \& Scharlemann (1979) as an extension of the $\mathrm{PC}$ model at higher altitudes. The acceleration region is placed near to the last-close magnetic field line and far away from the NS surface, allowing particles to 
reach higher energies due to the larger distance they travel. The cone of emission is wider with respect to the $\mathrm{PC}$ one, which could explain the typical two peaks of emission detected from pulsars in the gamma-ray regime.

$\odot$ Outer Gap (OG) model: This is a high-altitude emission model, first proposed by Cheng et al. (1986). The gap in which emission is released is well limited by the null surface (in which $\Omega \cdot \mathbf{B}=0$ ), the last-close magnetic field line, an open magnetic field line and the light cylinder, see Figure 7.4). This model is normally used to explain the highest energies via IC of secondary or tertiary $e^{ \pm}$pairs (produced by the photon-photon interaction of gamma rays originated by curvature radiation) on $[\mathbb{R}]$ or $[\mathrm{UV}]$ photons.

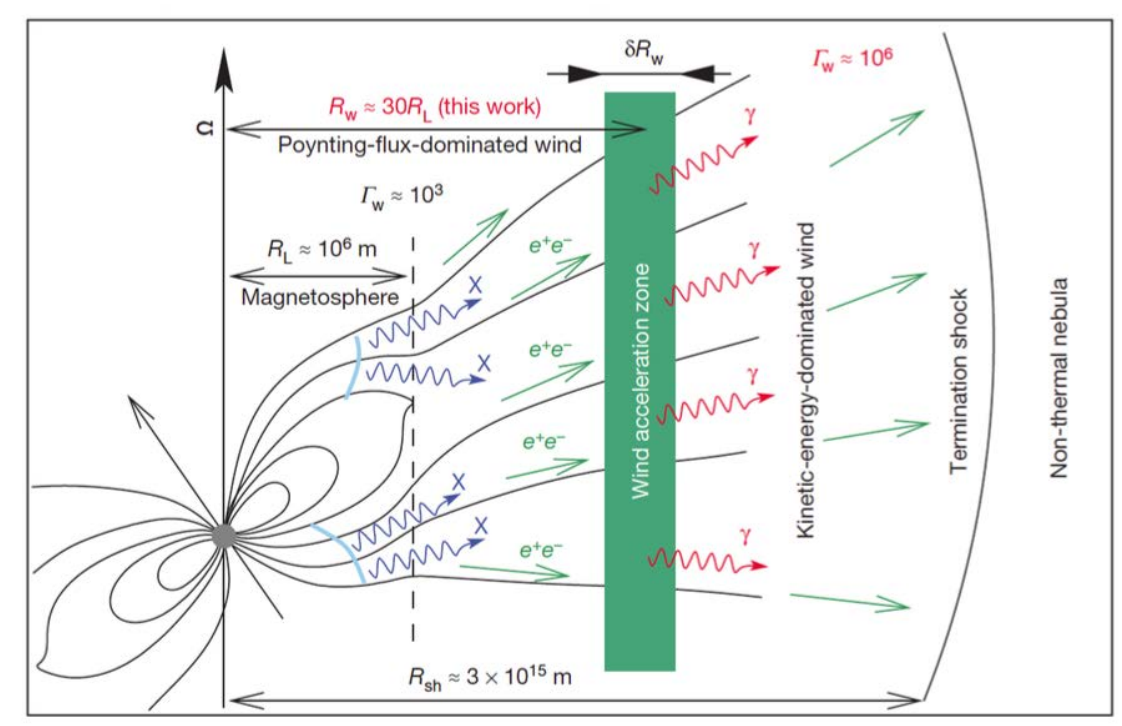

Figure 7.5: Sketch of the wind zone model in which VHE gamma rays are expected from the IC interaction between $e^{ \pm}$(accelerated in the wind acceleration zone) and X-rays coming from the NS magnetosphere. Credit: Aharonian et al. (2012)

It is worth to mention another model where the predicted emission region takes place outside of the magnetosphere, in the wind zone. First proposed by Lyubarskii (1996), this model has been used by Aharonian et al. (2012) to explain VHE gamma-ray emission. This radiation arises from the IC process of X-rays produced inside the magnetosphere by $e^{ \pm}$accelerated outside of it in the known as wind acceleration zone (see Figure 7.5). Nevertheless, this model suggested that the interaction distance lay out between 20 and $50 R_{L C}$, which would lead to a cutoff around $500 \mathrm{GeV}$ in the gamma-ray spectrum. Observations performed by MAGIC on the Crab pulsar, detected up to TeV energies (Ansoldi et al.|2016; see Figure 7.6, suggested a larger distance up to $70 R_{L C}$, if the wind zone works as production site.

The Crab pulsar actually belongs to the small group of pulsars detected in the VHE gammaray regime, along only with Vela pulsar. As many pulsars in the $\mathrm{GeV}$ regime, the Crab displays two peaks of significant emission (P1 and P2, see Figure 7.7), while from Vela only one was detected. Besides the two distinguished peaks, detection of gamma rays up to $400 \mathrm{GeV}$ in the 


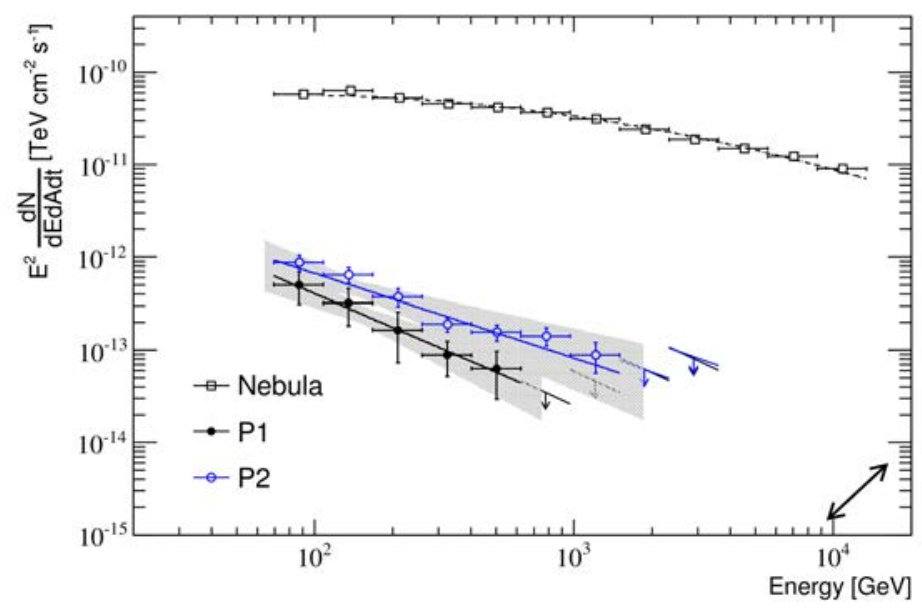

Figure 7.6: Phase-folded SED of the two peaks of emission separately as seen by MAGIC (Ansoldi et al. 2016), between $70 \mathrm{GeV}$ and $1.5 \mathrm{TeV}$.

region between $\mathrm{P} 1$ and $\mathrm{P} 2$, the so-called bridge, was as well reported by MAGIC in the case of Crab between 50 and $400 \mathrm{GeV}$ (Aleksić et al.2014a).

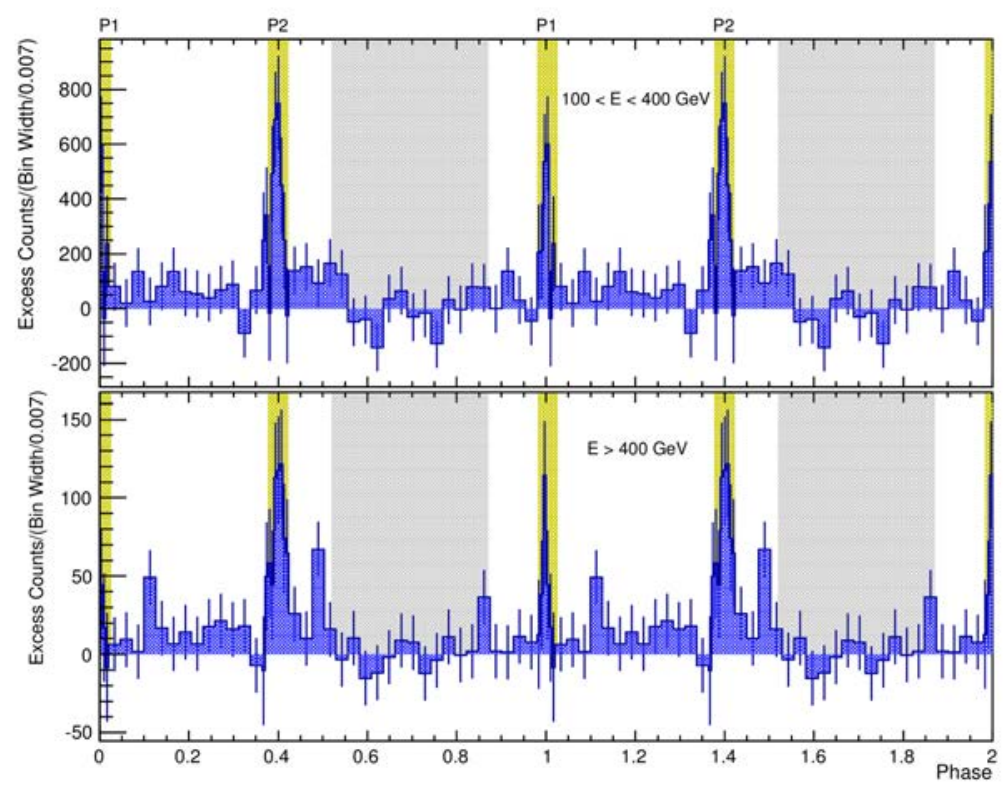

Figure 7.7: Phaseogram of the Crab pulsar emission between 100-400 GeV (upper plot) and above 400 $\mathrm{GeV}$ (bottom plot) taken from Ansoldi et al. (2016). The yellow areas identify the phase intervals of the two peaks of emission. 


\subsection{Pulsar Wind Nebulae}

In the termination shock, the particles are accelerated with a power-law distribution up to multi$\mathrm{TeV}$ energies. When these particles flow out from the termination shock, they interact with the ISM, giving rise to a PWN. These particles emit synchrotron radiation (due to the existing magnetic field in the $\mathrm{PWN}$, creating a synchrotron nebula right after the termination shock, which is detected from radio to X-rays (see Figure 7.8). The size of this synchrotron nebula is inversely proportional to the energy, as more energetic particles loss their energy before traveling long distances. This effect is significant under high magnetic fields, as it is the case for Crab Nebula. In cases with low magnetic field, as e.g. 3C 58, radio and X-ray nebulae are indistinguishable.

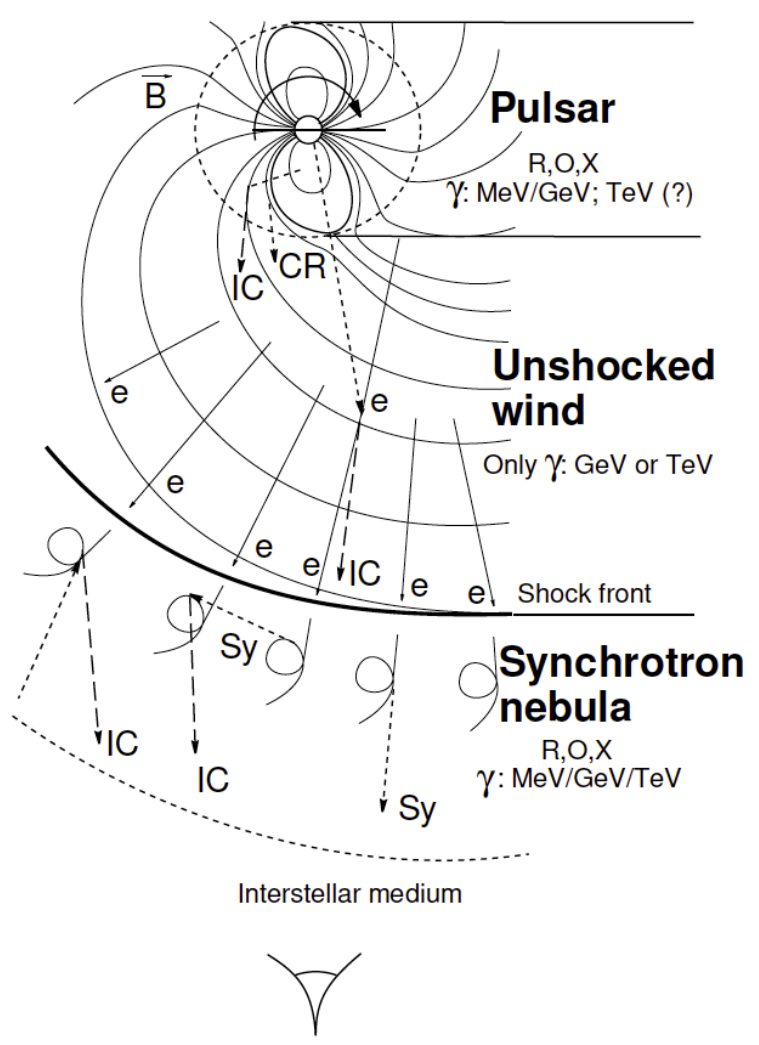

Figure 7.8: Acceleration mechanisms and production sites inside a PWN Credit: Aharonian (2004)

The synchrotron radio emission is characterized by a power-law function defined as $S_{v} \propto v^{\alpha}$, where $S_{v}$ is the observed flux density at a frequency $v$ and $\alpha$ is the spectral index, which ranges from -0.3 to 0 . The radio lumionsity is $\sim 10^{34} \mathrm{erg} \mathrm{s}^{-1}$ (between $100 \mathrm{MHz}$ and $100 \mathrm{GHz}$ ). The $\mathrm{X}$-ray emission is as well defined by a power-law function, $F \propto E^{-\Gamma}$, with a photon index $\Gamma \sim 2$, reaching a luminosity of $\sim 10^{35} \mathrm{erg} \mathrm{s}^{-1}$ (between $0.5-10 \mathrm{keV}$ ). In between, at IR energies, the luminosity is dominated by a component originated by the dust emission that embedded the contribution of the synchrotron IR, as shown in Figure 7.9 for Crab Nebula. 


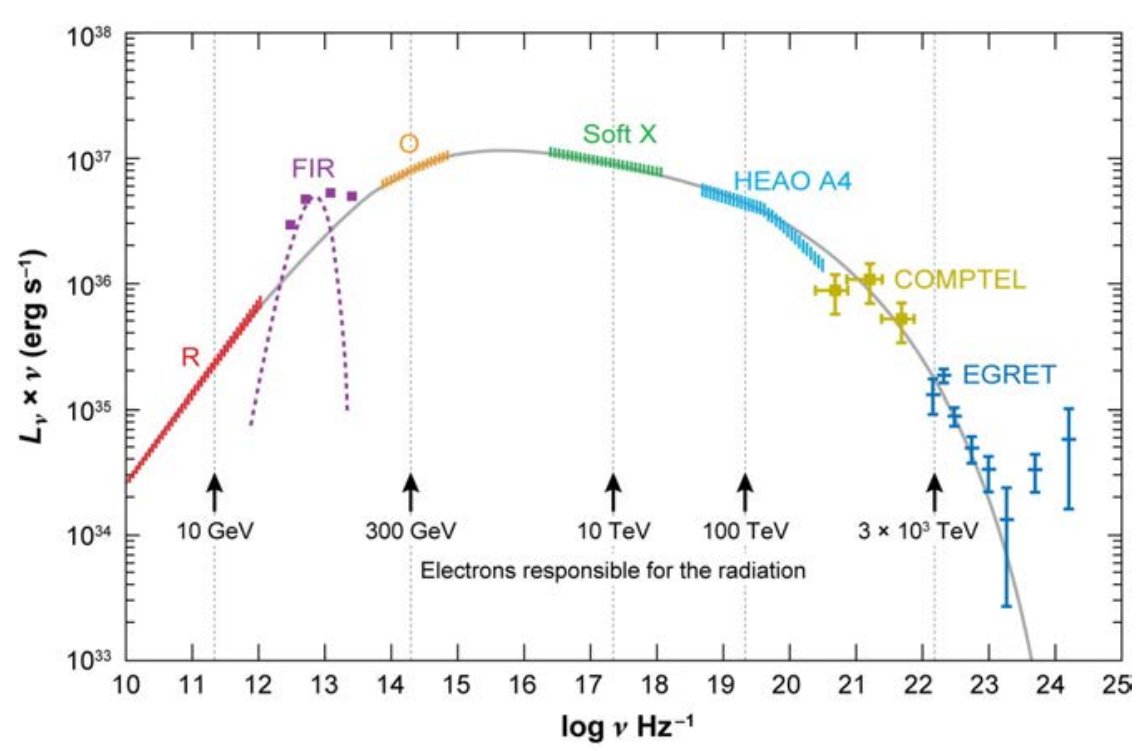

Figure 7.9: Overall Crab Nebula's synchrotron spectrum from radio to soft gamma-rays. The grey solid line represents the fit to the synchrotron emission, whilst the magenta dotted line corresponds to the Far Infrared (FIR) excess produced by the galactic dust. Credit: Gaensler \& Slane (2006).

\subsubsection{Gamma-ray emission}

The synchrotron radiation extends up to hard X-rays or even soft gamma-rays. At higher energies, this process is not efficient anymore and $\mathrm{HE}$ and $\mathrm{VHE}$ gamma rays are produced instead by UHE electrons via IC scattering on different photon fields. These targets are:

$\odot$ CMB: This photon field is uniformly distributed in the Galaxy and does it all over the entire nebula as well.

$\odot$ FIR: Target provided by the excess of photon coming from the galactic dust. It could explain $\sim 50 \%$ of the gamma-ray flux (de Jager \& Harding 1992).

$\odot$ Synchrotron X-rays: This photon field gives rise to gamma rays through $\mathrm{SSC}$ by the same electrons that produced them. Its contribution is important in young PWNe and dominant in the case of the Crab Nebula.

This VHE emission is normally well-defined by a power-law spectrum with photon indices between 1.3 and 2.8, given that detections normally happen at the falling edge of the IC peak. However, in the case of the Crab Nebula, detected down to $\sim 50 \mathrm{GeV}$ by MAGIC, its spectrum is described by a log parabola (Aleksić et al. 2016b).

\subsubsection{Morphology}

PWNe usually present a jet-torus structure: the torus-like structure arises from the emission at the equatorial plane of the pulsar (Bucciantini et al.2006) and two jets expand along the toroidal 
axis (see Figure 7.10). One of the two jets is brighter than the other due to the Doppler beaming effect, which affects the intensity of the emission according to the angle with respect to our line of sight (Pelling et al. 1987). The synchrotron nebula also presents the so-called wisps. Their nature is unclear but it could arise from synchrotron instabilities (Hester et al. 2002) or places where the $e^{ \pm}$plasma gets compressed (Spitkovsky \& Arons 2004). They can vary in timescales of days.

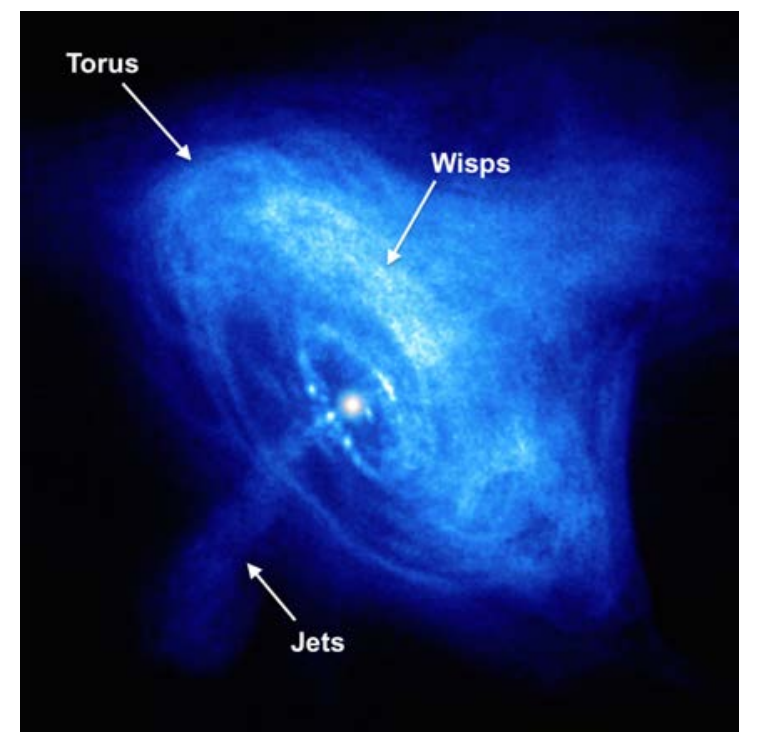

Figure 7.10: $\mathrm{X}$-ray image of Crab Nebula taken with Chandra. The internal structures of jets, torus and wisps are depicted. Credit: $\mathrm{NASA} / \mathrm{CXC} / \mathrm{SAO}$

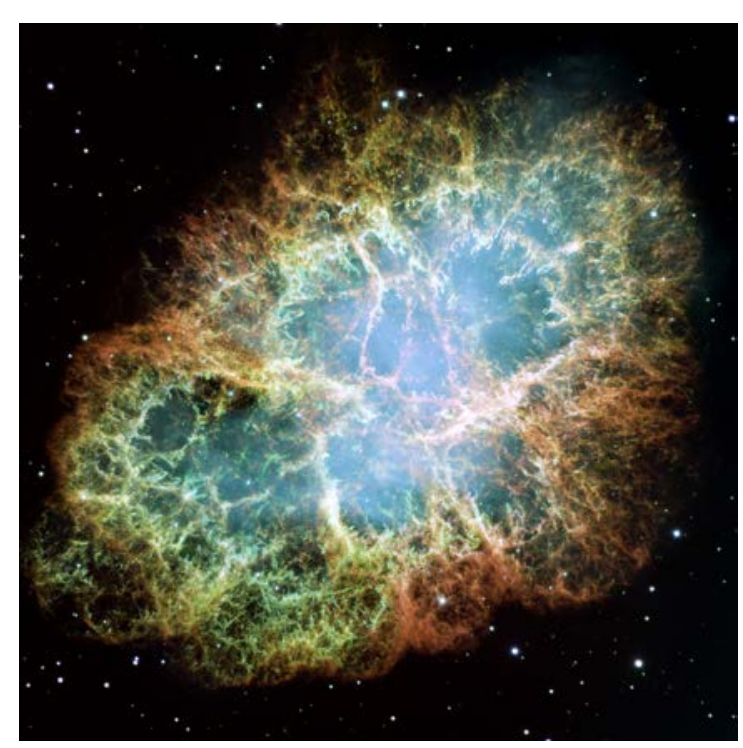

Figure 7.11: Optical image of Crab Nebula taken with the Hubble telescope, where the filamentary structure is observed. Image taken from Hester (2008).

In certain cases, e.g. the Crab Nebula, there are filamentary structures around the non-thermal optical production sites (see Figure 7.11). Its origin was speculated to be Rayleigh-Taylor instabilities ${ }^{1}$ (Hester et al. 1996).

\subsubsection{Evolutionary phases}

As mentioned before, $\mathrm{PWNe}$ can be embedded into a SNR, forming a composite system, in some cases indistinguishable. The evolution of the SNR can affect the development of the PWN Thus, we can separate different phases of the PWN as we did for the SNR:

$\odot$ Free expansion: It happens simultaneously with the first evolutionary phase of the SNR. This phase lasts around thousand years (typically $<6 \mathrm{kyr}$ ), during which the luminosity of the pulsar is the highest. The escape velocity of the pulsar $(<500 \mathrm{~km} / \mathrm{s}$, Arzoumanian et al. 2002) is much lower than the expansion velocity of the $\mathrm{PWN}\left(\sim 10^{3} \mathrm{~km} / \mathrm{s}\right)$ and the SNR s, remaining at the center of the nebula.

\footnotetext{
${ }^{1}$ The Rayleigh-Taylor instabilities are those produced between two fluids of different densities in which the lighter pushes the heavier fluid.
} 
$\odot$ Reverse shock collision: During the Sedov-Taylor phase (second phase, see Reynolds 2008) of the SNR, a reverse shock is formed and starts moving inwards in the direction of the pulsar. At some point, it collides with the expanding PWN The reverse shock compresses the PWN considerably, increasing this way its pressure. This results in a sudden expansion of the PWN. This process is recurrent, causing an oscillation of the nebula that lasts some tens of thousand years. These collisions can produce Rayleigh-Taylor instabilities at the edge of the PWN Moreover, it can affect the overall morphology of the PWN and even displace it from the initial pulsar position.

$\odot$ Relic phase: At this last phase, the pulsar has escaped a distance similar or larger than the PWN radius from the initial position and wind bubble. Thus, it leaves a relic $P W N$ behind, whilst a new smaller $\overline{\mathrm{PWN}}$ is created at the new position. Without compact object that powers it, the relic $P W N$ is usually dominated by $I \mathrm{C}$ emission. 


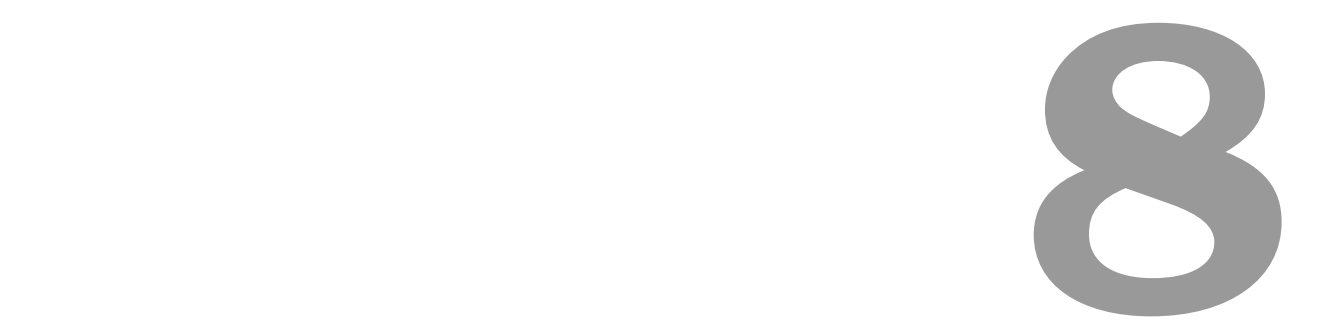

\section{Follow-up studies of HAWC sources}

\subsection{Introduction}

On the $15^{\text {th }}$ of April 2016, the HAWC Collaboration released a preliminary second catalog (2HWC) with $39 \mathrm{TeV}$ sources, from which 16 of them have no association with any known $\mathrm{HE}$ or VHE source. The data used for the analysis comprises 340 days of observation, between the $26^{\text {th }}$ of November 2014 to the $9^{\text {th }}$ of September 2015. The list of sources was sent under a private Memorandum of Understanding (MoU) between HAWC and several collaborations, including MAGIC and Fermi-LAT. A previous catalog (1HWC) had been reported, under the same $\mathrm{MoU}$, in April 2015 for the HAWC-111 configuration. In this first survey, only three sources were detected after trials, all associated with known TeV sources (Abeysekara et al. 2016). Not only the increased number of tanks and observation time, but also the improvements in calibration, event reconstruction and likelihood method to obtain the TS maps, gave rise to an increase of the number of detected sources with HAWC.

HAWC analysis was performed by means of a likelihood method, described in Younk et al. (2015). In this method, a source model needs to be assumed, which is applied to all sources in the FoV. The model is characterized by the source geometry and the energy spectrum. For the $2 \mathrm{HWC}$ catalog, HAWC performed two approaches: a point-like hypothesis by adopting a spectrum defined with a power-law function of spectral index $\Gamma=2.7$, and extended source searches assuming the source geometry as an uniform disk of $0.5^{\circ}, 1^{\circ}$ and $2^{\circ}$ radius, with a spectrum of index $\Gamma=2.0$. All $2 \mathrm{HWC}$ sources presented a pre-trial significance above $5 \sigma$, i.e. $\mathrm{TS}>25$. The systematic uncertainties for the $2 \mathrm{HWC}$ analysis were $0.1^{\circ}$ for the sources' position and $50 \%$ and 0.2 for the reported flux and photon index, respectively. Detailed information of the published catalog can be found in Abeysekara et al. (2017b). 
The 2HWC catalog motivated follow-up studies with the MAGIC telescopes as well as FermiLAT, whose joint work, along with HAWC, allowed us to obtain a multiwavelength view of interesting candidates, presented in this chapter. The main goal was to focus efforts on the 16 sources with no $\mathrm{HE}$ and $\mathrm{VHE}$ association, to provide new information of unknown candidates. Thus, after evaluating those new TeV emitters, we selected a short list of three candidates (see Figure 8.1): $2 \mathrm{HWC} \mathrm{J2006+341}\left(\mathrm{RA}=301.55^{\circ}\right.$, Dec $\left.=34.18^{\circ}\right), 2 \mathrm{HWC} \mathrm{J1907+084^{* }}\left(\mathrm{RA}=286.79^{\circ}\right.$, $\left.\mathrm{Dec}=8.50^{\circ}\right)$ and $2 \mathrm{HWC} \mathrm{J1852+013^{* }}\left(\mathrm{RA}=283.01^{\circ}\right.$, Dec $\left.=1.38^{\circ}\right)$, from which I analyzed the two first sources. The three of them were already located in the FoV of other MAGIC observations, allowing us to study the sources without performing new dedicated observations. In turn, the selected candidates seemed to provide suitable scientific interest for all the instruments involved in the project, HAWC, MAGIC and Fermi-LAT. All of them were detected during the HAWC point-like search. The corresponding photon index and flux normalization obtained during the $\mathrm{HAWC}$ analysis for each case are listed in Table 8.1. An afterward dedicated analysis on these three source, computed by HAWC, provided a specific energy range at which they are detected. The corresponding energy range is as well quoted in Table 8.1 .

It is worth to mention that the likelihood analysis performed by $\mathrm{HAWC}$ was not optimized for disentangling regions with multiple sources. Therefore, HAWC skymaps show crowded regions. In order to distinguish possible sources, HAWC classified the candidates as primary and secondary. The former are local maxima (with $[\mathrm{TS}>25$ ) separated from the closest local maxima at least $\Delta(\sqrt{\overline{T S}})>2 \sigma$. In the latter, this distance is narrower, $1 \sigma<\Delta(\sqrt{\overline{T S}})<2 \sigma$, and therefore they could be non-independent sources but part of primary ones. The secondary sources are labeled with an asterisk at the end of the name, as it is actually the case for $2 \mathrm{HWC}$ J1907+084* and 2HWC J1852+013*.

Table 8.1: Photon index, flux normalization at $7 \mathrm{TeV}$ and energy range for each of the selected sources. Values taken from Abeysekara et al. (2017b) (from which only statistical errors are reported), with the exception of the energy range, which was obtained in an afterward dedicated HAWC analysis.

\begin{tabular}{|c|ccc|}
\cline { 2 - 4 } \multicolumn{1}{c|}{} & Photon index & $\begin{array}{c}\text { Flux normalization } \\
{\left[\times 10^{-15} \mathrm{TeV}^{-1} \mathrm{~cm}^{-2} \mathrm{~s}^{-1}\right]}\end{array}$ & $\begin{array}{c}\text { Energy range } \\
{[\mathrm{TeV}]}\end{array}$ \\
\hline 2HWC J2006+341 & $2.64 \pm 0.15$ & $9.6 \pm 1.9$ & $1-86$ \\
\hline 2HWC J1907+084* & $3.25 \pm 0.18$ & $7.3 \pm 2.5$ & $0.18-10$ \\
\hline 2HWC J1852+013* & $2.90 \pm 0.10$ & $18 \pm 2.3$ & $0.4-50$ \\
\hline
\end{tabular}

\subsection{Observations and data analysis}

As mentioned above, the three sources were situated in the FoV of previous MAGIC observations and hence, archival data was used for this study. The archival data was taken making used of the 

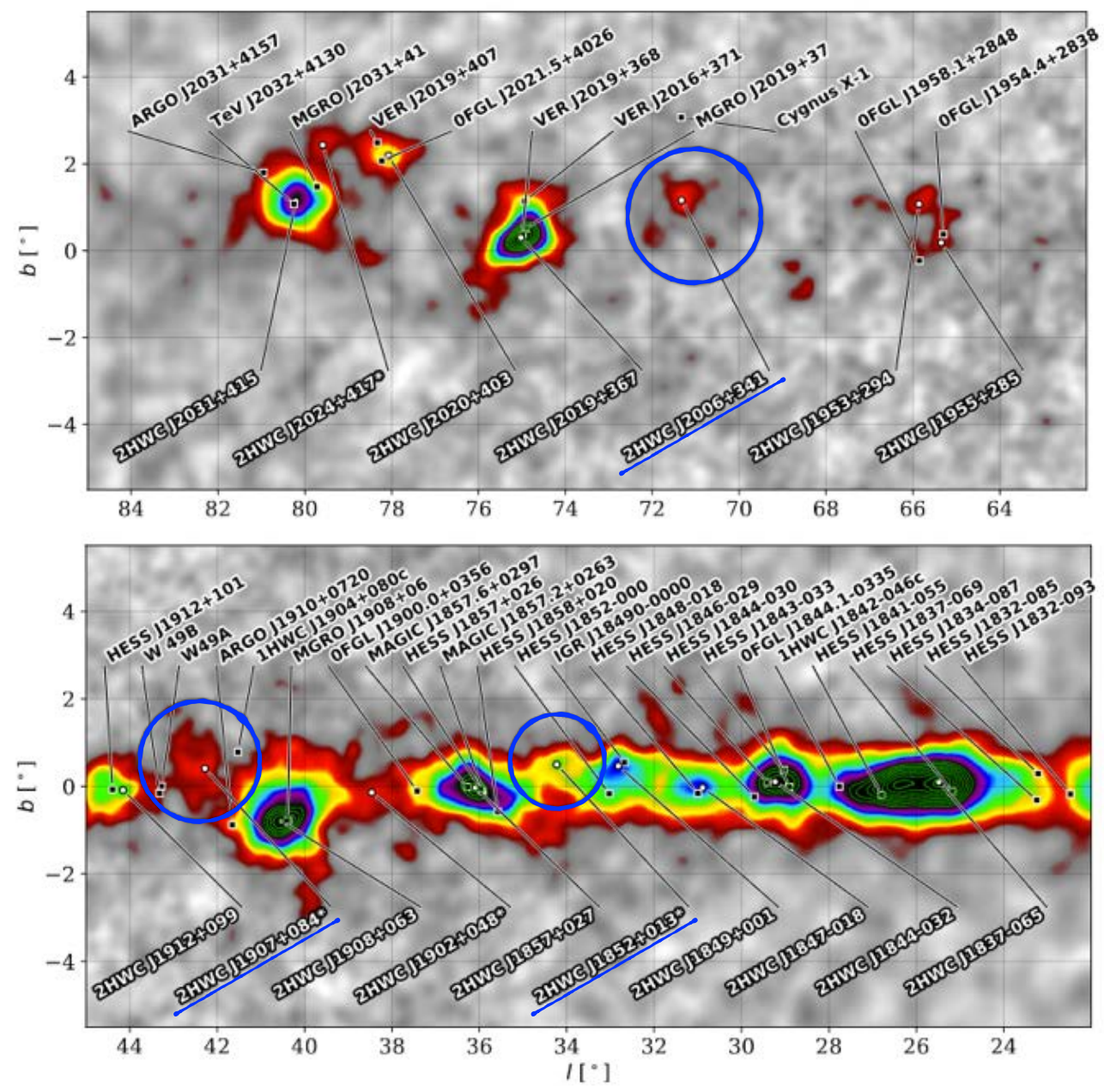

Figure 8.1: HAWC skymaps of the interesting FoV for the joint project with MAGIC and Fermi-LAT,

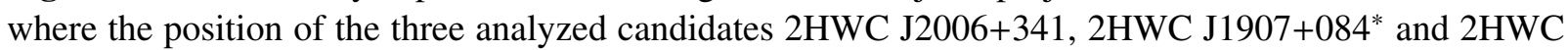
J1852+013* is highlighted with blue circles and lines. Skymaps taken from Abeysekara et al. (2017b).

wobble pointing mode (Section 2.4.2.1). This implies that the coordinates of the HAWC sources were shifted from the camera center more than the standard offset of $0.4^{\circ}$ (see Figure 8.2). To account for their location in the camera, the background is evaluated following the OfWP method. Let's assume two wobble positions, $\mathrm{W} 1$ and $\mathrm{W} 2$, both at $0.4^{\circ}$ from the nominal source at opposite directions. For the $\mathrm{W} 1$ observations, the OFF is obtained from $\mathrm{W} 2$ runs in same region of the camera in which the source lays in W1. If we have now $\mathrm{N}$ wobble pointing (W1, W2, $\mathrm{W} 3 \ldots \mathrm{WN}$ ), we can obtain the $\mathrm{ON}$ region for one of them and $\mathrm{N}-1$ OFF regions from the counterparts. The limitations on the used number of OFF regions, depends on possible sources in the FoV and the extension of the target source. For this study, only one OFF region was used for the analysis of the three sources, taking $\mathrm{W} 1-\mathrm{W} 2$ and $\mathrm{W} 3-\mathrm{W} 4$ pairs. Thus, ON and OFF regions are obtained from the same part of the camera, accounting this way for the same acceptance and 

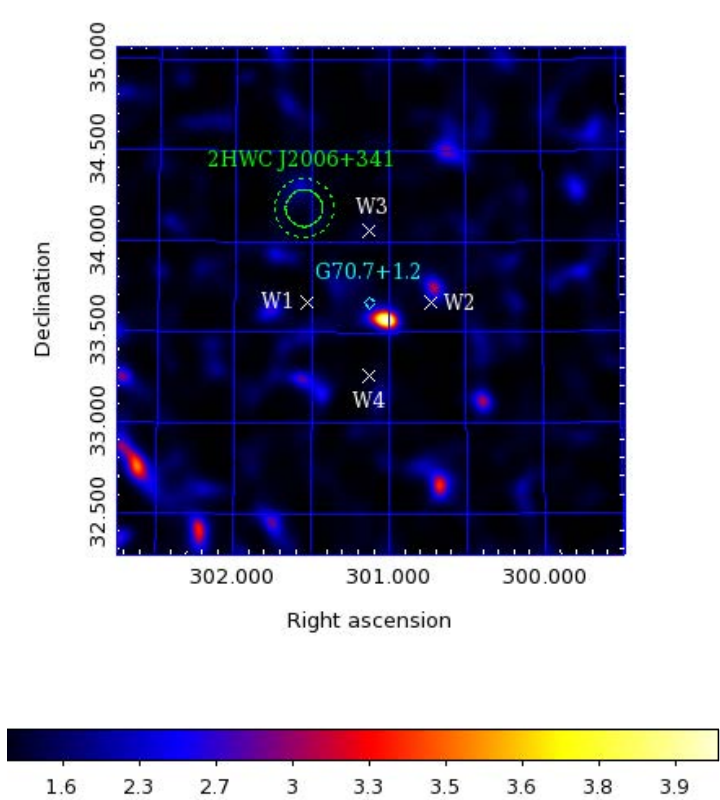
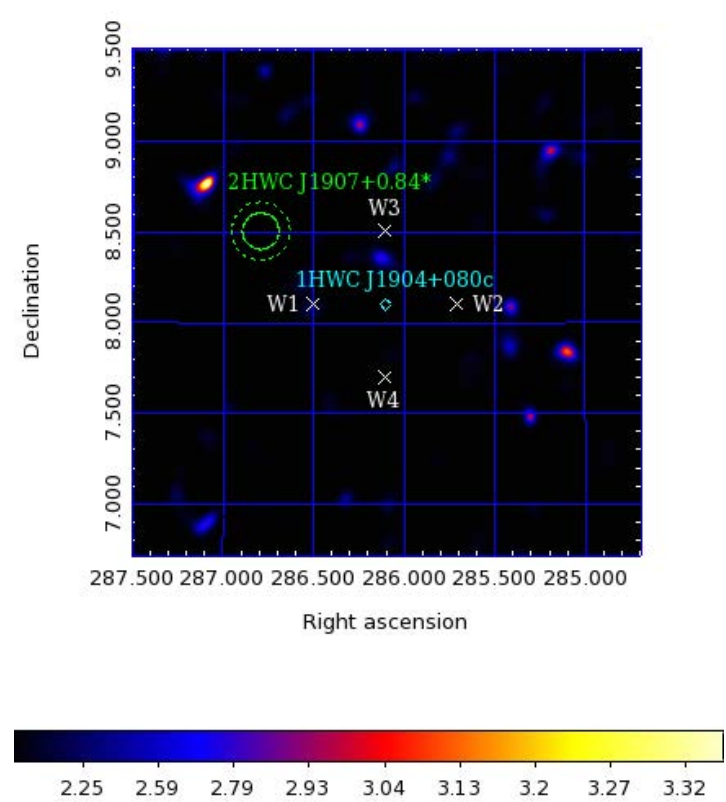

Figure 8.2: On the left: MAGIC significance skymap for the observations of G70.7+1.2 (cyan diamond).

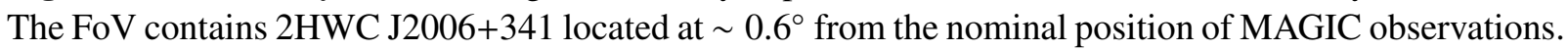
On the right: MAGIC significance skymap for the observations of 1HWC J1904+080c (cyan diamond), FoV in which $2 \mathrm{HWC}$ J1907+084* is located at $0.79^{\circ}$. The green solid and dashed circles in both panels correspond to the MAGIC point-like $\left(\mathrm{PSF}_{8}=0.10^{\circ}\right)$ and extended assumptions (radius of $\left.0.16^{\circ}\right)$, respectively, centered at the HAWC sources positions. The four different wobble positions, to which MAGIC pointed during the G70.7+1.2 and 1HWC J1904+080c observations, are tagged with W1, W2, W3 and W4 in white color.

reducing systematic errors in the background estimation. A schematic view of the $\mathrm{OfWP}$ method is shown in Figure 8.3. MAGIC sensitivity depends on the angular offset from the pointing position. Nevertheless, after the upgrade in 2011-2012, the sensitivity at offset angles larger than $0.4^{\circ}$ improved considerably as shown by Aleksić et al. (2016b). The distances between the camera center and the HAWC sources at the four different wobble positions are summarized in Table 8.2.

The FoV of the two sources I analyzed, 2HWC J2006+341 and 2HWC J1907+084*, were observed under dark and moonlight conditions. The data was divided based on the $\mathrm{DC}$ in the MAGIC I telescope, assuming dark if $D C_{\mathrm{MI}}<2 \mu \mathrm{A}$, moderate moon if $2 \mu \mathrm{A}<D C_{\mathrm{MI}}<4 \mu \mathrm{A}$ and 


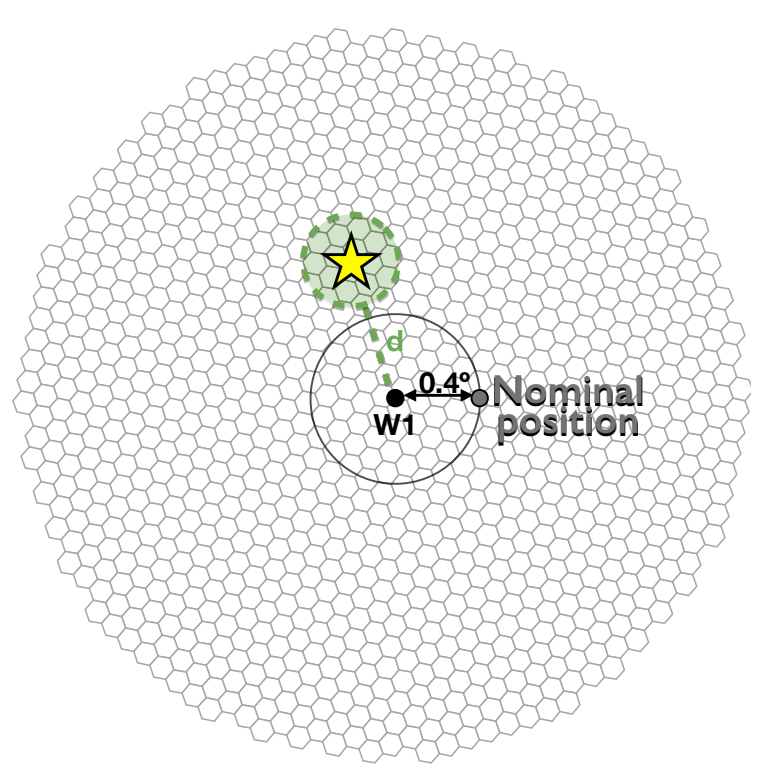

(a) $\mathrm{ON}$ region from the wobble $\mathrm{W} 1$

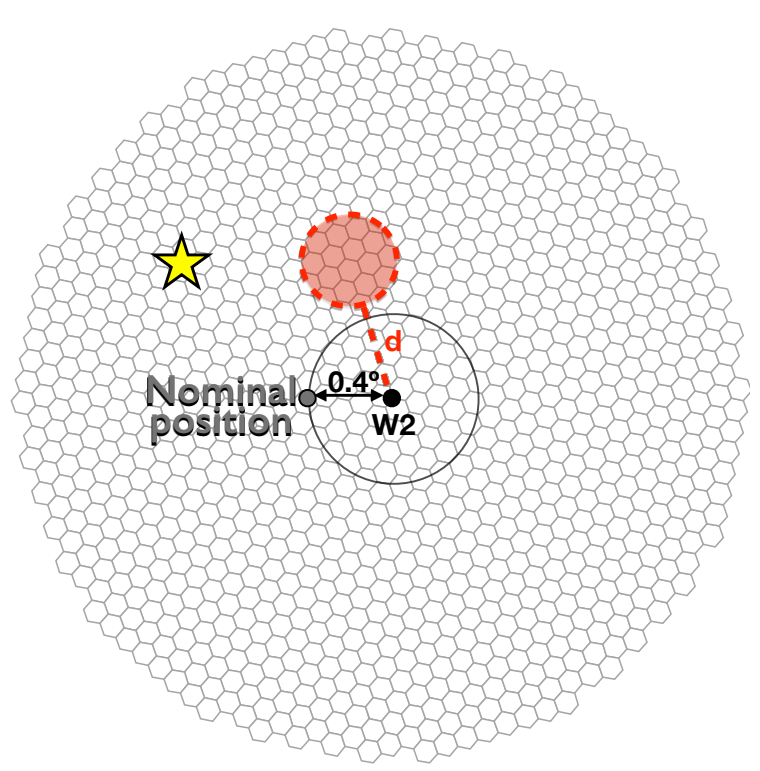

(b) OFF region from the wobble partner, W2.

Figure 8.3: Scheme of the OfWP evaluation background method used in wobble pointing mode. The black circle corresponds to the center of the camera, located at $0.4^{\circ}$ from the nominal source (gray circle), i.e. target of the observations. The yellow star represents a source in the FoV that we aim to analyze. While in W1 (left) the ON region of the interesting source is selected, W2 (right, the wobble partner) is used to get the OFF region at the same position in the camera in which the source stays in W1. The procedure is afterwards performed vice versa, taking ON from W2 and OFF from W1.

decent moon if $4 \mu \mathrm{A}<D C_{\mathrm{MI}}<8 \mu \mathrm{A}$. In the case of moderate and decent moon samples, artificial noise was added to the $\mathrm{MC}$ and background data (used in the $\mathrm{RF}$ training) according to the moonlight levels, as described in Chapter 2.4.3.3. The image cleaning values I used correspond

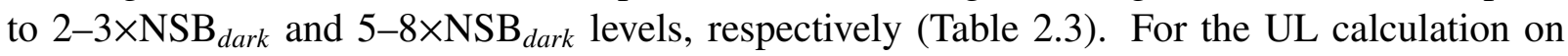
moon data, an increased size cut according to the strength of the Moon was applied, as shown in Table 2.6

Table 8.2: Distance in degrees between the four wobble pointing positions (W1, W2, W3 and W4) and the candidates. The observation time, in hours, achieved in each case is also shown.

\begin{tabular}{|c|c|c|c|c|c|c|c|c|}
\hline & \multicolumn{2}{|c|}{ W1 } & \multicolumn{2}{|c|}{ W2 } & \multicolumn{2}{|c|}{ W3 } & \multicolumn{2}{|c|}{ W4 } \\
\hline & $\begin{array}{c}\text { Distance } \\
{\left[^{\circ}\right]}\end{array}$ & $\begin{array}{l}\mathrm{t}_{o b s} \\
{[\mathrm{hr}]}\end{array}$ & $\begin{array}{c}\text { Distance } \\
{\left[^{\circ}\right]}\end{array}$ & $\begin{array}{l}\mathrm{t}_{o b s} \\
{[\mathrm{hr}]}\end{array}$ & $\begin{array}{c}\text { Distance } \\
{\left[^{\circ}\right]}\end{array}$ & $\begin{array}{l}\mathrm{t}_{\text {obs }} \\
{[\mathrm{hr}]}\end{array}$ & $\begin{array}{c}\text { Distance } \\
{\left[^{\circ}\right]}\end{array}$ & $\begin{array}{l}\mathrm{t}_{o b s} \\
{[\mathrm{hr}]}\end{array}$ \\
\hline 2HWC J2006+341 & 0.5 & 16.0 & 0.9 & 14.0 & 0.4 & 16.3 & 1.0 & 14.8 \\
\hline 2HWC J1907+084* & 0.5 & 1.0 & 1.2 & 1.0 & 0.7 & 1.3 & 1.1 & 0.9 \\
\hline $2 \mathrm{HWC}$ J1852+013* & 1.1 & 30.8 & 0.7 & 28.8 & 1.2 & 29.6 & 0.6 & 27.5 \\
\hline
\end{tabular}

Taken into account that the three selected candidates for this joint project were detected dur- 
ing the HAWC point-like search, one cannot discard them to be point-like for MAGIC as well. Therefore, for the analysis of 2HWC J2006+341 and 2HWC J1907+084*, I performed two approaches: first, I assumed the sources to be point-like for $\mathrm{MAGIC}_{\mathrm{PSF}_{88}} \lesssim 0.10^{\circ}$ beyond a few hundred $\mathrm{GeV}$ ), and second, I computed the analysis under the hypothesis of extension with radius of $\sim 0.16^{\circ}$. The maximum extension possible is strongly constrained by the standard offset of $0.4^{\circ}$ used during wobble pointing mode. Taking only one OFF region, this implies a distance between the center of the position of the source and the background region of $0.8^{\circ}$. The ON region, from which we expect gamma rays, depends, not only on the intrinsic radius of the source, but also on the MAGIC|PSF. The latter changes according to the energy, and given that the observations were performed under different moonlight conditions (to which different analysis cuts are applied, see Table X), the energy threshold is not the same for the entire data sample. This ON region is then defined by the $\theta^{2}$ cut applied to compute the flux. It can be described by the equation $\theta^{2} \sim(2 \cdot \text { Radius })^{2}+\left(2 \cdot P S F_{40}\right)^{2}$, where $P S F_{40}$ is the PSF at a $40 \%$ containment calculated through one 2-D Gaussian fit on a Crab Nebula sample at each of the moonlight conditions. To be conservative, the selected $P S F_{40}$ was the largest one, obtained for the dark sample where lower energies are achieved. This value, $P S F_{40}=0.07^{\circ}$, along with the $0.8^{\circ}$ distance between $\mathrm{ON}$ and $\mathrm{OFF}$ regions, limited the maximum radius to $0.158^{\circ}$, i.e. $\theta^{2}=0.12 \operatorname{deg}^{2}\left(\theta=0.35^{\circ}\right)$. This way, ON and OFF regions do not overlap, while in turn there is distance enough between them to perform the normalization between gamma rays and background events (for a schematic view, see Figure 8.4.

\subsubsection{HWC J2006+341}

$2 \mathrm{HWC} \mathrm{J} 2006+341$ is located at an angular distance of $0.63^{\circ}$ from the compact (20") radio/optical nebula G70.7+1.2, which is embedded in a dense molecular cloud. G70.7+1.2, at a distance of $\sim 4.5 \mathrm{kpc}$ from Earth and situated in the Cygnus constellation, is a very interesting and unique source due to all the features that presents:

$\odot$ It shows a shell-like radio nebula with a broad and blueshifted OI and SII emission lines, which indicate shock with the ISM

$\odot$ Millimeter CO emission suggests shock with the molecular material as well.

$\odot$ A bright $\mathrm{NIR}$ Be star is embedded in the nebula.

Given all the aforementioned characteristics, several explanations were proposed to describe its nature: young SNR, nova shell, HII region, Herbig-Haro-like outflows or stellar wind (see e.g., Reich et al. 1985; Green 1986; Jourdain de Muizon et al. 1988; Bally et al. 1989). However, none of these scenarios could explain the low expansion velocities and the non-thermal radio emission detected from its direction. In 1992, Kulkarni et al. (1992) suggested that the nebula was created by the interaction between the molecular cloud and an hypothetical Be/pulsar binary. This was a speculative theory, whose confirmation depended on the detection of the binary system. With this goal, observations in the X-ray and NIR regimes were carried out. Results with the Chandra 


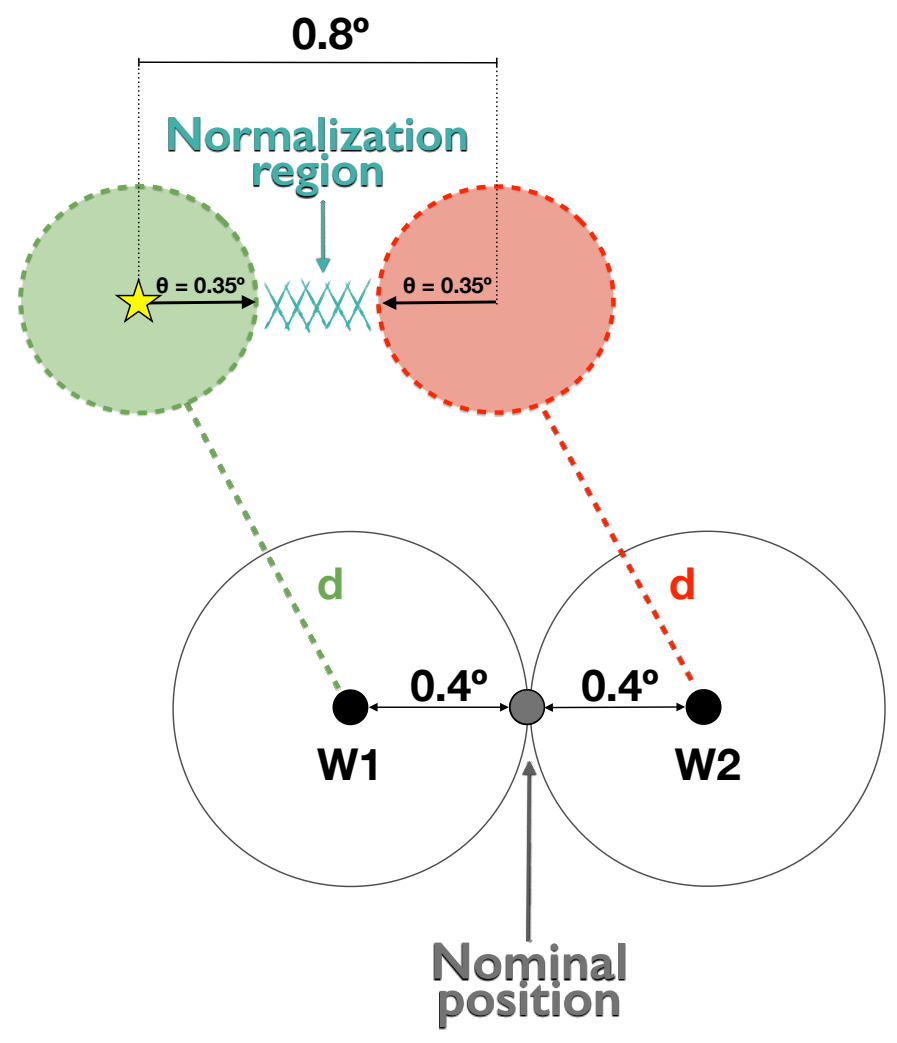

Figure 8.4: Schematic view of the maximum possible extension that MAGIC can assumed for a source taken in wobble mode. Both $\mathrm{W} 1$ and $\mathrm{W} 2$ are depicted simultaneously. From $\mathrm{W} 1$, the ON region (green) is taken while $\mathrm{W} 2$ provides the OFF region (red) at the same distance, $\mathrm{d}$, from the camera center. An intrinsic radius of the source of $\sim 0.16^{\circ}$ leads to a $\theta^{2}$ cut of $0.12 \mathrm{deg}^{2}$, i.e. $\theta=0.35^{\circ}$, taken into account a $\mathrm{PSF}$ of $0.07^{\circ}$. The normalization region is shown in blue between the ON and OFF regions.

X-ray Observatory revealed the existence of a point-like source, CXO J200423.4+333907 (see Figure 8.5), reported by Cameron \& Kulkarni (2007). This source was at 3.6" from the previously detected Be star, turning a binary system composed by both of them impossible. However, NIR observations with the Keck Observatory's Laser Guide Star Adaptive Optics (LGS-AO) system showed in turn a relatively bright counterpart around the X-ray source, which was consistent with a highly obscured B-star. This way, Cameron \& Kulkarni (2007) suggested that the X-ray binary (comprised by CXO J200423.4+333907 and a B-star) was the dominant source that powers the radio/optical nebula G70.7+1.2, moving into the molecular cloud from its far side. On the other hand, the Be star could create a reflection nebula on the near side. An unidentified Fermi-LAT source included in the Second Fermi-LAT catalog (2FGL) catalog, 2FGL J2004.3+3339, was detected at the position of the X-ray source. This discovery reinforced the idea of the binary scenario, although not pulsation was reported so far. In turn, coincident with the Fermi-LAT source, a hotspot at the level of 3-4 $\sigma$ was detected on the re-analysis of the 8-years data sample 
from the Milagro gamma-ray Observatory, whose position was not confirmed by the $2 \mathrm{HWC}$ catalog.

MAGIC pointed to the Fermi-LAT source and amounted a total of $\sim 55$ hours (after quality cuts). The data was recorded between April 2015 and August 2016 within an extended zenith range of $(5,50)^{\circ}$.

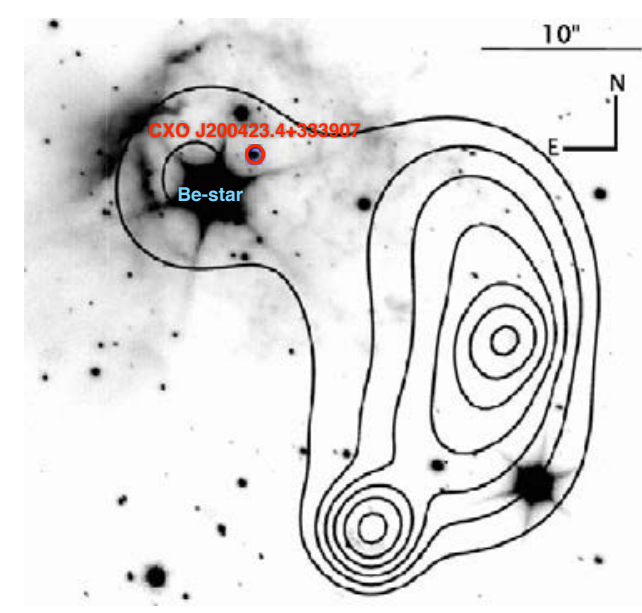

Figure 8.5: LGS-AO NIR image from G70.7+1.2 with the diffuse Chandra X-ray counterparts, taken from Cameron \& Kulkarni (2007). The red circle corresponds to the X-ray source CXO J200423.4+333907. The Be-star, at 3.6" from the X-ray point-like source, is highlighted in blue. The distance between the expected binary system and the center of the diffuse X-ray emission is around 20".

\subsubsection{HWC J1907+084*}

$2 \mathrm{HWC} \mathrm{J} 1907+084^{*}$ is located at $0.79^{\circ}$ from 1HWC J1904+080c $\left(\mathrm{RA}=286.1^{\circ}\right.$, Dec $\left.=8.1^{\circ}\right)$. The latter was included in the first HAWC survey (Abeysekara et al. 2016) and previously reported within a list of interesting candidates via private communication to all collaboration HAWC had a MoU with, including MAGIC. The list was composed of 6 new TeV candidates (1 extragalactic and 5 galactic sources) with significance above $4.2 \sigma$ obtained after 9 months of observations with the HAWC-111 configuration. The PSF at a $68 \%$ radius containment for this HAWC analysis was $0.8^{\circ}$ and the systematic uncertainty on the location was expected to be $<0.2^{\circ}$. The flux for all candidates above $1 \mathrm{TeV}$ was $\sim 20 \%$ C.U. (with a systematic uncertainty on the flux of $\sim 40 \%$ ). The chosen candidate by MAGIC, 1HWC J1904+080c, presented 5.2 $\sigma$ pre-trials, which decreased up to $3.9 \sigma$ after trials, as shown later on in Abeysekara et al. $(2016)$. The coordinates were not coincident with any known $\mathrm{TeV}$ source, but it was close (at $0.3^{\circ}$, coincident within the errors) to a Fermi-LAT hotspot $(<5 \sigma)$ ), 3FGL J1904.9+0818 (Acero et al. 2015).

A point-like source with a flux of $\sim 20 \%$ C.U. in the $\mathrm{TeV}$ regime is detectable by MAGIC in less than an hour. However, given the poor HAWC $\mid \overrightarrow{\text { PSF }}$ in this analysis, the candidate could be extended and, in turn, presented a high systematic uncertainty on the flux. Thus, MAGIC asked 
for observing 1HWC J1904+080c 5 hours, extendable another 5 hours if a significance of $3 \sigma$ was reached. Finally, MAGIC performed follow-up observations of the 1HWC candidate from $10^{\text {th }}$ May of 2015 to $19^{\text {th }}$ May 2015, for 6 non-consecutive nights at a medium Zd range of (30, $50)^{\circ}$. After disposing of the data affected by non-optimal weather conditions, the total amount of time reached 4.2 hours.

\subsection{Results}

\subsubsection{HWC J2006+341}

No significant detection was found in the direction of 2HWC J2006+341 under any of the assumptions, point-like or extended source. Integral ULs at a 95\% C.L. were computed adopting a power-law distribution with photon index $\Gamma=2.64$, as suggested by HAWC analysis. Accounting for the energy threshold at the maximum moonlight level (decent moon, $E_{t h} \sim 220 \pm 10 \mathrm{GeV}$ ) and the fact that the sources were detected by HAWC in the TeV regime, integral ULs are given for energies above $300 \mathrm{GeV}$. For the point-like analysis, this $\mathrm{UL}$ was set to $1.8 \times 10^{-12}$ photons $\mathrm{cm}^{-2} \mathrm{~s}^{-1}$, while for the extended hypothesis, it increases slightly to $3.8 \times 10^{-12}$ photons $\mathrm{cm}^{-2} \mathrm{~s}^{-1}$. Differential ULs for 2HWC J2006+341 were computed as well and can be found in Table 8.3 . The integral UL for a point-like source at the position of G70.7+1.2, for energies above $300 \mathrm{GeV}$ and assuming a power-law distribution with $\Gamma=2.6$, is $1.0 \times 10^{-12}$ photons $\mathrm{cm}^{-2} \mathrm{~s}^{-1}$. Left panel on Figure 8.2 shows the skymap in the FoV of G70.7+1.2, where 2HWC J2006+341 is tagged in green. The skymap is computed assuming point-like source for a blind scan (without assuming the position of any source). No hotspot arises at the position or nearby 2 HWC J2006 +341 .

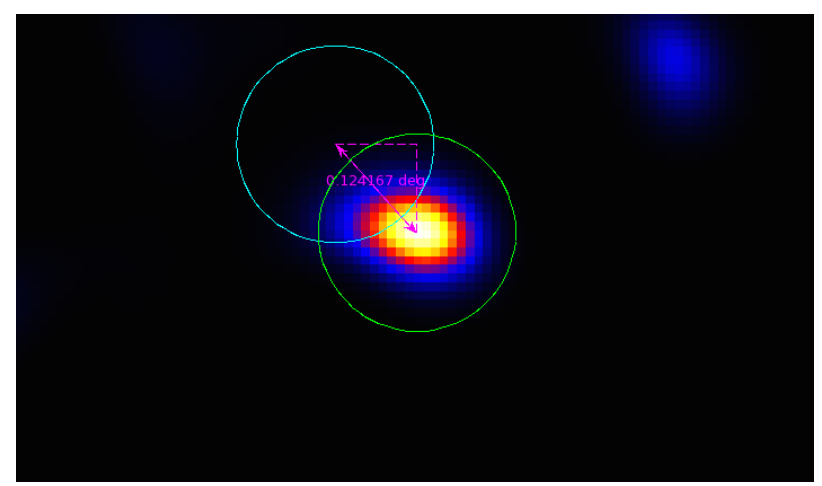

Figure 8.6: Zoom view of the skymap centered in G70.7+1.2 (cyan). The hotspot at the level of $4 \sigma$ (green) is located at $\sim 0.12^{\circ}$ from the nominal position. The radius of the circles indicates the MAGIC PSF of $0.10^{\circ}$.
However, a hotspot appears close to the nominal position of $\mathrm{G} 70.7+1.2$. With approximately $\mathrm{RA}=301.02^{\circ}$ and $\operatorname{Dec}=33.57^{\circ}$, this hotspot is located at $\sim 0.12^{\circ}$ from 2FGL J2004.3+3339. It corresponds to point-like emission since it is contained in the PSF of MAGIC (see zoom view in Figure 8.6). To get an estimation of the significance at this position, again OfWP was used in Odie. In this case, the standard FR cuts were applied, which led to a significance of around $3 \sigma$. 
Table 8.3: MAGIC 95\% CL differential flux ULs for 2 HWC J2006+341 and 2 HWC J1907+084* for both point-like $\left(\lesssim 0.10^{\circ}\right)$ and extended $\left(\sim 0.16^{\circ}\right)$ radius assumptions, considering a power-law spectrum with spectral index of $\Gamma=2.64$ and $\Gamma=3.25$, respectively. $\mathrm{ULs}$ beyond $\sim 4.7 \mathrm{TeV}$ are not computed for J1907+084* due to low statistics.

\begin{tabular}{l|cc|cc}
\hline \hline \multirow{2}{*}{$\begin{array}{c}\text { Energy range } \\
{[\mathrm{GeV}]}\end{array}$} & $\begin{array}{r}\text { Differential flux UL for J2006+341 } \\
{\left[\times 10^{-13} \mathrm{TeV}^{-1} \mathrm{~cm}^{-2} \mathrm{~s}^{-1}\right]}\end{array}$ & $\begin{array}{r}\text { Differential flux UL for J1907+084* } \\
{\left[\times 10^{-13} \mathrm{TeV}^{-1} \mathrm{~cm}^{-2} \mathrm{~s}^{-1}\right]}\end{array}$ \\
\cline { 2 - 5 } & Point-like & Extended & Point-like & Extended \\
\hline $300-475.5$ & 25.7 & 45.7 & 12.1 & 41.2 \\
$475.5-753.6$ & 9.1 & 61.9 & 4.8 & 5.9 \\
$753.6-1194.3$ & 2.9 & 15.0 & 3.2 & 3.0 \\
$1194.3-1892.9$ & 2.2 & 7.1 & 0.6 & 2.1 \\
$1892.9-3000$ & 0.4 & 1.9 & 0.2 & 0.7 \\
$3000-4754.7$ & 0.3 & 0.8 & 0.04 & 0.3 \\
$4754.7-7535.7$ & 0.1 & 0.3 & - & - \\
\hline
\end{tabular}

\subsubsection{HWC J1907+084*}

No excess was found in the direction of 2HWC J1907+084*. The 95\% C.L. integral ULs were again computed for energies greater than $300 \mathrm{GeV}$, but assuming a photon index of 3.25 , following HAWC results (see Table 8.1). The integral ULs are $9.7 \times 10^{-13}$ photons $\mathrm{cm}^{-2} \mathrm{~s}^{-1}$ and $1.4 \times 10^{-12}$ photons $\mathrm{cm}^{-2} \mathrm{~s}^{-1}$, for the point-like and radius $0.16^{\circ}$ hypothesis, respectively. Under the same conditions, differential ULs were calculated, which are listed in Table 8.3 .

1HWC J1904+080c was not detected either. The corresponding integral UL at $E>300$ $\mathrm{GeV}$, assuming point-like source defined by a power-law distribution with $\Gamma=2.6$, is $4.1 \times 10^{-12}$ photons $\mathrm{cm}^{-2}$. The skymap of the FoV shown in Figure 8.2 does not reveal any significant area around the $1 \mathrm{HWC}$ or the $2 \mathrm{HWC}$ candidates.

\subsection{Discussion}

Given that the largest population of TeV emitter in our Galaxy are PWNe, it would not be improbable that our selected candidates belong to this type of sources. However, the non-detection at lower energies neither with MAGIC or Fermi-LAT (see Ahnen et al.2017 for more information on the Fermi-LAT analysis and results), complicates disentangling the nature of these sources. To delve more into the possible $\overline{P W N}$ nature, I looked for detected pulsars nearby the position of the selected candidates using the Australia Telescope National Facility (ATNF) catalog 1 (Manchester et al.2005).

For $2 \mathrm{HWC} \mathbf{\mathrm { J }} 2006+341$, the closest pulsar (and the only one within a $1^{\circ}$ radius) is PSR $\mathrm{J} 2004+3429$, which lays at $\sim 0.4^{\circ}$ from the $2 \mathrm{HWC}$ position. This pulsar is located at a dis-

\footnotetext{
${ }^{1}$ http://www.atnf.csiro.au/people/pulsar/psrcat/
} 
tance of $10 \mathrm{kpc}$ from Earth, with a spin-down power and characteristic age of $\dot{E}=5.8 \times 10^{35} \mathrm{erg}$ $\mathrm{s}^{-1}$ and $\tau=18 \mathrm{kyr}$, respectively. Although the spin-down power of the pulsar seems energetic enough to power a TeV $\overline{P W N}$ (based on observational criterion, see e.g. H. E. S. S. Collaboration et al. 2017), the distance between PSR J2004+3429 and 2HWC J2006+341 allowed to discard connection between both objects: with an offset of $0.4^{\circ}$, i.e. $\sim 70 \mathrm{pc}$, the escape velocity of the pulsar would need to reach $\sim 4000 \mathrm{~km} / \mathrm{s}$. The mean 2D speed for young and old $(<3 \mathrm{Myr})$ pulsars was determined to be $307 \pm 47 \mathrm{~km} / \mathrm{s}$ by Hobbs et al. (2005) in a study involving 233 pulsars. From this sample, the highest 2D speed reached $1624 \mathrm{~km} / \mathrm{s}$. Therefore, a pulsar velocity of $\sim 4000 \mathrm{~km} / \mathrm{s}$ is unreliably high.

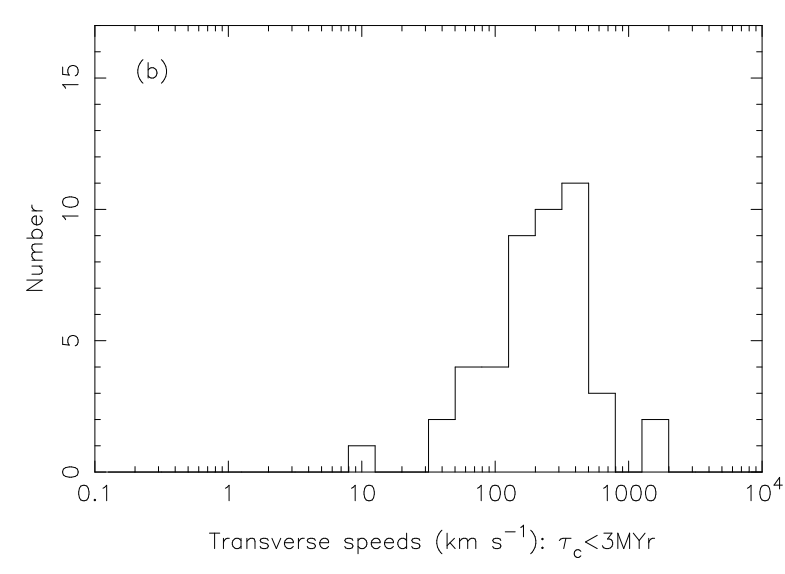

Figure 8.7: 2-D speed of pulsars with a characteristic age of less than 3 Myr. Plot taken from Hobbs et al. (2005).

On the other hand, if $2 \mathrm{HWC} \mathrm{J} 2006+341$ is a point-like source, the distance of $0.63^{\circ}$ between this and G70.7+1.2 makes a direct connection between both very unlikely. However, although $2 \mathrm{HWC}$ J2006+341 was detected during the point-like search with [HAWC, an estimated radius of $0.9^{\circ}$ was given in the $2 \mathrm{HWC}$ catalog. This value is assumed to be the extension from the source after assigning to it the halo-like structures visible in the residual skymaps. With such extension, $2 \mathrm{HWC}$ J2006 +341 could interact or be related with G70.7+1.2 vicinity. This way, although the position of the $2 \mathrm{HWC}$ source is not coincident with the one reported for the hotspot of Milagro, TeV emission would be confirmed to take place on this crowded region. Nevertheless, as mentioned in Abeysekara et al. (2017b), this radius is just a preliminary result which should not be taken as the definite extension. Dedicated morphology studies with HAWC or CTA would be needed to shed light on this matter.

$2 \mathrm{HWC}$ J1907+084* presents two pulsars nearby: PSR J1908 +0833 at $0.30^{\circ}$ from the $2 \mathrm{HWC}$ source's coordinates, and PSR J1908+0839, at $0.33^{\circ}$. The former lays at $\sim 11 \mathrm{kpc}$ from the Earth, with a spin-down power of $\dot{E}=5.8 \times 10^{32} \mathrm{erg} \mathrm{s}^{-1}$ and a characteristic age of $\tau=4.1 \mathrm{Myr}$. The poor spin-down power that presents dismisses the probability of connection with a $\mathrm{TeV}$ PSR J1908+0839, at $8.3 \mathrm{kpc}$ and with $\tau=1.2 \mathrm{Myr}$, is more energetic with a spin-down power 
of $\dot{E}=1.5 \times 10^{34} \mathrm{erg} \mathrm{s}^{-1}$, and therefore a relation between this pulsar and $2 \mathrm{HWC} \mathrm{J1907+084^{* }}$ cannot be directly excluded. Given the characteristic age of the pulsar, the corresponding PWN would remain in the so-called relic phase. This is the last stage of a PWN during which the pulsar escaped from its initial position leaving an old PWN behind with no compact object that injects magnetic or particle flux. Thus, the emission is typically IC-dominated. Therefore, at this stage it is expected to see the pulsar shifted from the PWN position. Following the same exercise as that

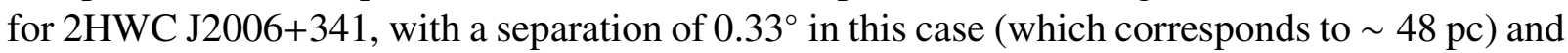
a characteristic age of $1.2 \mathrm{Myr}$, the obtained escape velocity of the pulsar is $\sim 40 \mathrm{~km} / \mathrm{s}$, which is low but plausible (see Figure 8.7). Let's assume that PSR J1908+0839 is indeed the pulsar that powers $2 \mathrm{HWC} \mathrm{J} 1907+084^{*}$. This way, the gamma-ray emission detected by HAWC would be produced by the IC scattering between accelerated electrons, injected by the pulsar, and a low-energy photon field. As shown in Chapter 7.2.1, the target photon field is usually composed by $\mathrm{CMB}$ and IR photons. In order to emit gamma rays above $E_{\gamma}=200 \mathrm{GeV}$ (approximately the minimum energy detected by HAWC in this source), the energy of the electron has to be, at least, $E_{e} \sim 7.5 \mathrm{TeV}$, given by $E_{e}=17 \cdot E_{\gamma}^{0.54+0.046 \cdot \log \left(E_{\gamma}\right)}$ (Aharonian 2004).

Making use of the Naima ${ }^{1}$ software (Zabalza 2015), one can obtain the electron energy distri-

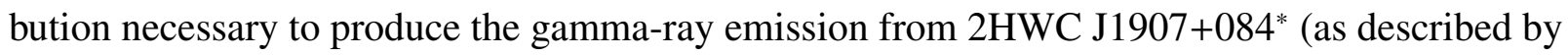
HAWC, see Table 8.1), under certain assumptions. For this calculation, I considered the energy spectrum of the electron population defined by a power law, and IC on CMB and IR photons as the radiative mechanism responsible for the gamma rays. The energy density of the target photon field was set to standard values of $u_{C M B}=0.25 \mathrm{eV} / \mathrm{cm}^{3}$ and $u_{I R}=0.30 \mathrm{eV} / \mathrm{cm}^{3}$, for the CMB and IR radiation, respectively. The obtained electron energy distribution that would be necessary to explain HAWC results on $2 \mathrm{HWC} \mathrm{J} 1907+084^{*}$, under the aforementioned considerations, is defined by $d N / d E=\left(1.0_{-0.2}^{+0.12}\right) \times 10^{44}(E / 7.5)^{-4.4 \pm 0.3} \mathrm{TeV}^{-1} \mathrm{~cm}^{-2} \mathrm{~s}^{-1}$. The total energy carried by these electrons for energies above $7.5 \mathrm{TeV}$ is $W_{e}=\left(1.3_{-0.2}^{+0.17}\right) \times 10^{47} \mathrm{erg}$.

On the other hand, the cooling time of electrons in the Klein-Nishima regime can be computed by:

$$
t_{\text {cool }}=3 \cdot 10^{8}\left(\frac{E_{e}}{\mathrm{GeV}}\right)^{-1}\left(\frac{u}{\mathrm{eV} / \mathrm{cm}^{3}}\right)^{-1}[\mathrm{yr}]
$$

where $u$ is the total energy density of the environment (Aharonian 2004). Considering both synchrotron and IClosses, $u$ can be approximated by the following expression as given by Moderski et al. (2005):

$$
u=\frac{B^{2}}{8 \pi}+u_{C M B}\left(1+0.01 \cdot \frac{E_{e}}{T e V}\right)+u_{I R, T h o m s o n}\left(1+0.1 \cdot \frac{E_{e}}{T e V}\right)
$$

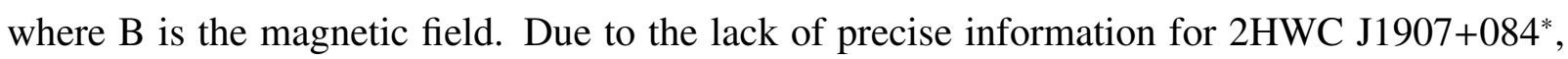
I assumed the minimum possible value of $\mathrm{B}=3 \mu \mathrm{G}$, i.e. the interstellar magnetic field. Again, I assume $u_{C M B}=0.25 \mathrm{eV} / \mathrm{cm}^{3}$ and $u_{I R, \text { Thomson }}=0.30 \mathrm{eV} / \mathrm{cm}^{3}$, as done for the electron energy spectrum computation. These assumptions lead to a $t_{\text {cool }}=1.3 \times 10^{4}$ years. Given the spin-down power of PSR J1908+0839, $\dot{E}=1.5 \times 10^{34} \mathrm{erg} \mathrm{s}^{-1}$, during a time period equal to $t_{\text {cool }}$, the energy released by the pulsar was $W_{e}^{\prime}=\dot{E} \cdot t_{\text {cool }}=6.1 \times 10^{45} \mathrm{erg}$. Therefore, even assuming that all

\footnotetext{
${ }^{1}$ http://naima.readthedocs.org
} 


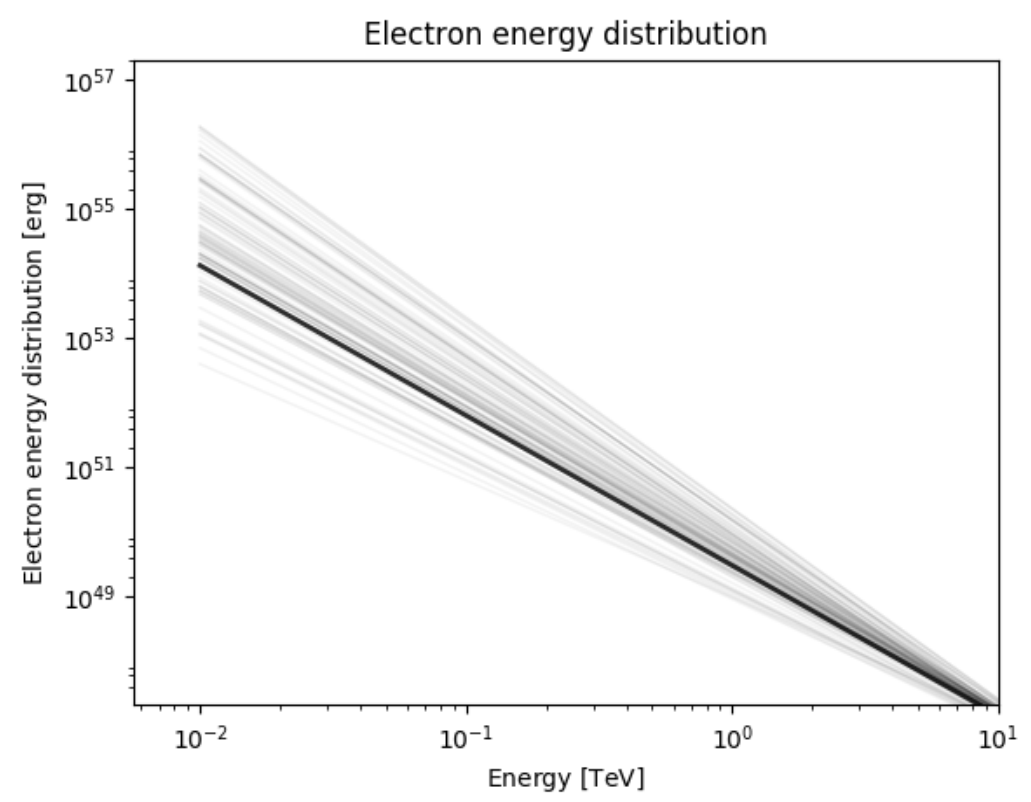

Figure 8.8: Simulated electron energy distribution for $2 \mathrm{HWC} \mathrm{J} 1907+084^{*}$, assuming it is powered by the pulsar PSR J1908+0839.

the energy injected by the pulsar, $W_{e}^{\prime}$, were applied on accelerating electrons above $7.5 \mathrm{TeV}$, the system would not be energetic enough to power a $\overline{P W N}$ with the gamma-ray emission as that detected by HAWC in $2 \mathrm{HWC} \mathrm{J} 1907+084^{*}$, which requires $W_{e}>\left(1.3_{-0.2}^{+0.17}\right) \times 10^{47} \mathrm{erg}$. Consequently, connection between the 2HWC source and PSR J1908+0839 can be discarded. Note that the assumption of the low magnetic field and $u_{I R}$ provides an upper limit on the $t_{\text {cool }}$ and so does in the released pulsar energy. Therefore, higher more reliable magnetic field inside the PWN would still lead to the non-connection between objects.

The SED for the two candidates I analyzed are depicted in Figure 8.9. MAGIC and FermiLAT analysis were computed with the photon index provided by HAWC in each case (see Table 8.1). The multiwavelength context allow us to obtain information on the candidates' extension.

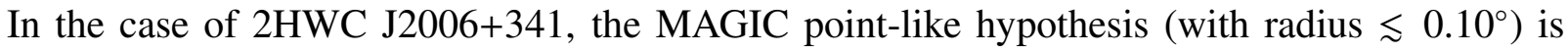
completed discarded, whilst the extended assumption (radius of $\sim 0.16^{\circ}$ ) is in agreement with the HAWC spectrum within errors, and therefore it could be plausible. For 2HWC J1907+084*, both MAGIChypothesis (point-like and extended) are compatible with HAWCresults at energies above $\sim 900 \mathrm{GeV}$. However, in the sub-TeV regime, neither MAGIC nor Fermi-LAT ULs agree with HAWC spectrum. This could be understood in two ways: on one hand, the source could be very extended, well above $0.16 \mathrm{deg}$, which would increase the MAGIC and Fermi-LAT ULs considerable above HAWClevel. On the other side, this mismatching could be due to the fact that this candidate do not emit in the energy range covered by MAGIC and Fermi-LAT (or presents different spectrum), which is supported by the already very restricting ULs obtained by both instruments. 

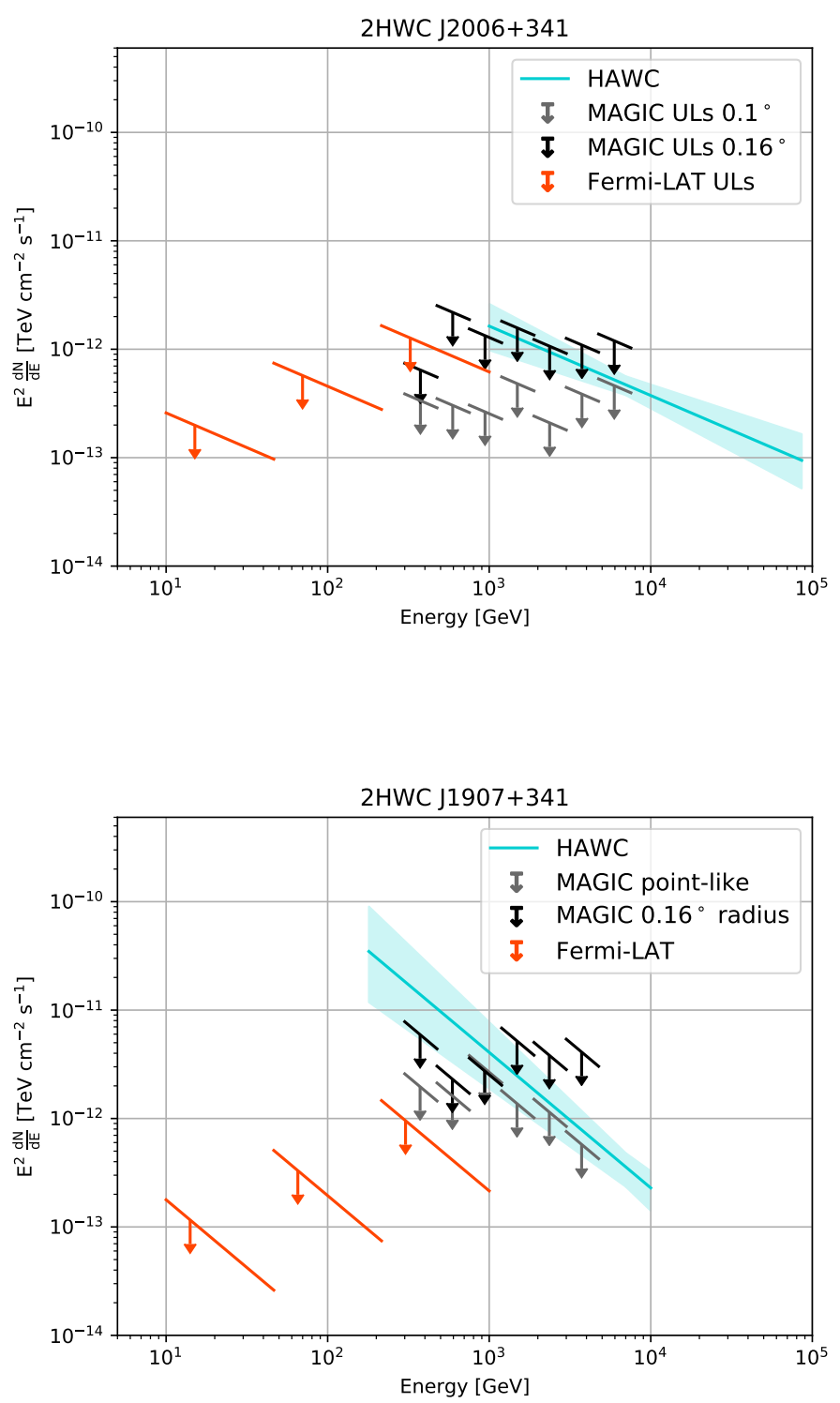

Figure 8.9: Spectral energy distribution from $10 \mathrm{GeV}$ up to $\sim 90 \mathrm{TeV}$. In all cases, the assumed spectrum

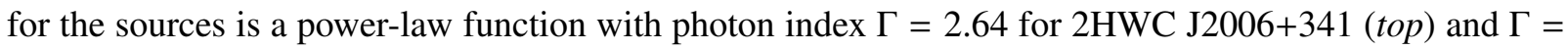
3.25 for 2 HWC J1907+084* (bottom), as obtained by HAWC (see Table 8.1). Fermi-LAT UL spectrum is shown in orange assuming a point-like source. MAGIC results for point-like hypothesis (grey) and $0.16 \mathrm{deg}$ radius extension (black) are displayed. The HAWC butterfly is obtained for the parameters given in Table 8.1 


\subsection{Conclusions}

MAGIC performed a dedicated analysis on 3 new $\mathrm{TeV}$ sources detected on the second survey by the wide FoV] observatory HAWC, of which the results from 2 HWC J2006+341 and 2HWC $\mathrm{J} 1907+084^{*}$ were presented in this chapter. None of them were detected at lower energies and no hotspot was found nearby them. Nevertheless, MAGIC]ULs, computed for two hypothesis based on the radius of the source $\left(\lesssim 0.10^{\circ}\right.$ and $\left.0.16^{\circ}\right)$ allowed to constrain the extension of the candidates. The crowded region in which 2 HWC J2006 +341 is located makes difficult disentangling the extension from the HAWC analysis. However, the assumption of $0.16^{\circ}$ radius made with MAGIC data seems to be already compatible with HAWC results. In the case of $2 \mathrm{HWC}$ $\mathrm{J} 1907+084^{*}$, MAGIC and HAWC results are in agreement in the TeV regime. Below $900 \mathrm{GeV}$, the consistency is not fulfilled anymore, suggesting a larger radius than $0.16^{\circ}$ or different spectrum in the $\mathrm{GeV}$ band. It is worth to mention that the third candidate included in this project, 2HWC J1908+013*, was not detected either. Integral ULs for a power-law distribution with $\Gamma=2.90$ were established at $3.8 \times 10^{-13}$ photons $\mathrm{cm}^{-2} \mathrm{~s}^{-1}$ and $1.7 \times 10^{-12}$ photons $\mathrm{cm}^{-2} \mathrm{~s}^{-1}$ for the point-like and extended hypothesis, respectively. Finally, it was probed that none of the nearby detected pulsars can be related to the HAWC sources, questioning the possible $\mathrm{PWN}$ nature. In order to reveal more information about the nature of these new $\mathrm{TeV}$ emitters, further multiwavelength studies are necessary. 
8. FOLLOW-UP STUDIES OF HAWC SOURCES 


\section{9 \\ PWN studies around high spin-down power Fermi-LAT pulsars}

\subsection{Introduction}

As shown in Chapter 7, pulsars are highly magnetized rotating neutron stars which are constantly releasing their rotational energy in the form of relativistic Poynting and particles flux, the so-called pulsar wind. This wind interacts with the ISM, giving rise to a termination shock in which particles are accelerated. When flowing out, the relativistic particles can, in turn, interact with the surrounding medium generating a magnetized bubble known as PWN. This nebula radiates synchrotron emission, from radio to X-rays, which is dominant the first thousand years. At higher energies, this mechanism is not efficient anymore and gamma rays are instead produced through IC] up-scattering of low-energy photons by ultra-high-energy electrons (see Gaensler \& Slane 2006). The homogeneous target fields are comprised by CMB, FIR and optical photons. Typically, only pulsars with high spin-down power $\left(\gtrsim 10^{34} \mathrm{erg} \mathrm{s}^{-1}\right.$, based on observational criterion, see e.g. H. E. S. S. Collaboration et al. 2017) are able to induce prominent TeV PWNe, but the order parameters of the population are not currently understood.

PWNe represent the most numerous population of TeV galactic VHE gamma-ray sources. MAGIC observed and deeply studied the most luminous galactic gamma-ray PWN, the Crab Nebula (Aleksić et al. 2015), and discovered the least luminous one up to now, 3C 58 (Aleksić et al. 2014b). These objects were also extensively studied by the southern hemisphere IACT H.E.S.S., during their H.E.S.S. Galactic Plane Survey (HGPS) (H. E. S. S. Collaboration et al. 2017). The HGPS revealed 14 firmly identified PWNe at the inner part of our Galaxy. The goal of the MAGIC project is to prove particle acceleration at the outer side of the Galaxy. With this 
aim, six PWN candidates that host known high spin-down power Fermi-LAT pulsars (between $\sim 10^{35}-10^{37} \mathrm{erg} \mathrm{s}^{-1}$ ) were selected: PSR J0631+1036, PSR J1954+2838, PSR J1958+2845, PSR J2022+3842, PSR J2111+4606 and PSR J2238+5903. In turn, all of them present characteristic age around a few tens of kyr, similar to that displayed by detected PWNe Basic information from these pulsars, taken from the ATNF pulsar catalogue ${ }^{1}$ (Manchester et al. 2005), is summarized in Table 9.1. In cases of radio quiet pulsars, in which distance information is missing, this parameter is taken from the literature. When available, pseudo-distance is also provided, which is estimated making used of the spin-down energy loss rate and the gamma-ray luminosity ( $\mathrm{Saz}$ Parkinson et al.2010).

Table 9.1: Characteristics of the six selected PWN candidates for the study. From left to right: Spin-down power, characteristic age, distance and pseudo-distance. Information taken from the ATNF catalog if not specified otherwise. $\dot{E} /$ Distance is computed using the values from the Distance column, except for PSR $\mathrm{J} 2111+4606$, in which pseudo-distance was applied. ${ }^{a}$ Tian \& Leahy (2006), ${ }^{b}$ Saz Parkinson et al. (2010), ${ }^{c}$ Abdo et al. (2013).

\begin{tabular}{|c|c|c|c|c|c|}
\hline Name & $\begin{array}{c}\dot{E} \\
{\left[\mathrm{erg} \mathrm{s}^{-1}\right]}\end{array}$ & $\begin{array}{c}\text { Characteristic Age } \\
{[\mathrm{kyr}]}\end{array}$ & $\begin{array}{c}\text { Distance } \\
{[\mathrm{kpc}]}\end{array}$ & $\begin{array}{c}\text { Pseudo-distance } \\
{[\mathrm{kpc}]}\end{array}$ & $\begin{array}{c}\dot{E} / \mathrm{d}^{2} \\
{\left[\mathrm{erg} \mathrm{kpc}^{-2} \mathrm{~s}^{-1}\right]}\end{array}$ \\
\hline PSR J0631+1036 & $1.7 \times 10^{35}$ & 43.6 & 2.10 & - & $3.9 \times 10^{34}$ \\
\hline PSR J1954+2838 & $1.0 \times 10^{36}$ & 69.4 & $9.2^{a}$ & $1.6^{b}$ & $1.18 \times 10^{34}$ \\
\hline PSR J1958+2845 & $3.4 \times 10^{35}$ & 21.7 & $9.2^{a}$ & - & $4.0 \times 10^{33}$ \\
\hline PSR J2022+3842 & $3.0 \times 10^{37}$ & 8.9 & 10 & - & $3.0 \times 10^{35}$ \\
\hline PSR J2111+4606 & $1.4 \times 10^{36}$ & 17.5 & $<14.8^{c}$ & $2.7^{b}$ & $1.9 \times 10^{35}$ \\
\hline PSR J2238+5903 & $8.9 \times 10^{35}$ & 26.6 & $<12.4^{c}$ & - & $5.8 \times 10^{33}$ \\
\hline
\end{tabular}

\subsection{Observations and data analysis}

From the above mentioned sources, I analyzed all data from PSR J1954+2838, PSR J1958+2845 and PSR J2022+3842 as well as data under dark condition from PSR J2111+4606 and PSR $\mathrm{J} 2238+5903$, as presented in the following sections.

The analysis of the data was performed by means of the standard MAGIC analysis software (Chapter 2.4.3). Flux integral and differential ULs were computed following the Rolke method for a 95\% C.L. assuming a Gaussian background and a systematic uncertainty of $30 \%$ on the effective area. Significance was calculated applying equation 2.14 .

In all cases, observations were carried out under different moonlight conditions. To perform a proper analysis, the data was divided into three groups according to the pixel $D C$ in the camera of MAGIC I during data taking: dark (absence of Moon, $D C_{M 1}<2.0 \mu \mathrm{A}$ ), moderate moonlight $\left(2.0 \mu \mathrm{A}<D C_{M 1}<4.0 \mu \mathrm{A}\right)$ and decent moonlight $\left(4.0 \mu \mathrm{A}<D C_{M 1}<8.0 \mu \mathrm{A}\right)$. The analysis cuts

\footnotetext{
${ }^{1}$ http://www.atnf.csiro.au/research/pulsar/psrcat/
} 


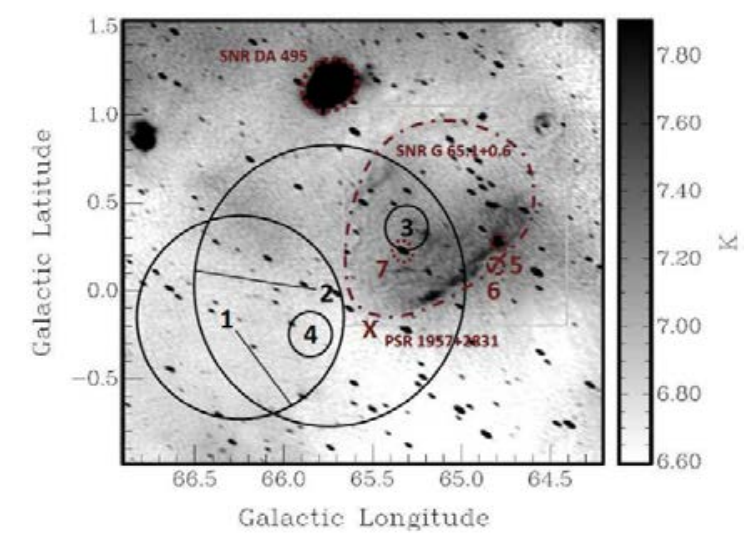

Figure 9.1: Radio image from the FoV of PSR J1954+2838 and PSR J1958+2845. The numbered objects seen in this skymap are: (1) 3EG J1958+2909; (2) 2CG 065+00; (3) PSR J1954+2838; (4) PSR $\mathrm{J} 1958+2845$, (5) region of differing spectral index; (6) IRAS 19520+2759; (7) compact radio object. The errors on the position for (1) and (2) are shown with black circles. SNR G65.1+06 is highlighted with maroon dash-dot line. Skymap taken from Tian \& Leahy (2006).

used correspond to those of $1 \times N S B_{\text {dark }}, 2-3 \times N S B_{\text {dark }}$ and 5-8 $\times N S B_{\text {dark }}$ (with nominal HV, see Table 2.3, respectively, with the latter slightly modified in terms of pedestal and RMS mean factor to 3.8:1.6 in order to mimic more properly the background of the observations. Moreover, appropriated MC-simulated gamma rays and background data (used for the computation of the $\gamma /$ hadron separation) were necessary for each of the moonlight levels.

\subsubsection{PSR J1954+2838 and PSR J1958+2845}

These two pulsars, reported in the First Fermi-LAT |catalog (1FGL) (Abdo et al. 2010), are located in a very dense and crowded region, in which structure associations are still under debate (see Figure 9.1). PSR J1954+2838 is positionally coincident with SNR G65.1+06. The latter corresponds to a very faint $\mathrm{SNR}$ at a distance of $9.2 \mathrm{kpc}$ from Earth with an estimated age between 40-140 kyr (Tian \& Leahy 2006). This SNR seems to be associated with another pulsar in the FoV, PSR J1957+2831, given the compatible distance and kinematic age estimation. In turn, an IR source, IRAS 19520+2759, is detected at the south of the remnant, which was found to be related to $\mathrm{CO}, \mathrm{H}_{2} \mathrm{O}$ and $\mathrm{OH}$ emission lines at a distance similar to SNR G65.1+06, which would suggest interaction with molecular clouds. The re-analysis of 8 years of Milagro data at the position of the Fermi Bright Sources revealed hints of $4.3 \sigma$ and $4.0 \sigma$ in the direction of PSR J1954+2838 and PSR J1958+2845, respectively (Abdo et al.2009b). This emission may be originated from the corresponding $\mathrm{PWN}$ or interaction of the SNR and molecular cloud, in the case of PSR J1954+2838. In 2010, MAGIC observed these two pulsars in the stand-alone mode with MAGIC I for $~ 25$ hours, resulting in a non-detection (Aleksić et al. 2010c). Nevertheless, the major upgrade between 2011-2012 that both telescopes underwent allowed to improve MAGIC sensitivity with respect to former observations. 
In the new campaign, MAGIC observed PSR J1954+2838 from April to November 2015 for a total of $\sim 16$ hours of good quality data. This data, that spans in a broad zenith range from $5^{\circ}$ to $50^{\circ}$, was taken under dark, moderate and decent moonlight levels. In the case of PSR J1958+2845, only moon data were available. After removing data affected by non-optimal weather conditions, the total amount of effective time reached for the latter was $\sim 4$ hours, in a zenith range of $10^{\circ}$ to $40^{\circ}$.

\subsubsection{PSR J2022+3842}

Motivated by Chandra discovery of a point-like source and a faint but distinct surrounding Xray nebula inside the SNR G76.9+1.0 at $10 \mathrm{kpc}$, the pulsar PSR J2022+3842 was detected in radio and X-ray (Arzoumanian et al. 2011). Its spin-down power was revised afterwards by Arumugasamy et al. (2014), establishing a value of $\dot{E}=3 \times 10^{37} \mathrm{erg} \mathrm{s}^{-1}$. Therefore, PSR $\mathbf{J} 2022+3842$ is one of the highest spin-down power pulsars in the sky. Nevertheless, even though it is extraordinarily powerful, it does not show a bright X-ray nebula and presents, in turn, an unusually low conversion efficiency of spin-down power to X-ray luminosity, as reported by Arzoumanian et al. (2011). These features would imply low magnetization in the medium, that favors the scenario in which most of the rotational energy is converted into IC emission, turning PSR J2022+3842 into one of the best candidates for VHE gamma-ray searches. The source was observed by VERITAS during the Cygnus Survey for 10 hours, which ended up in a $3 \sigma$ UL for energies greater than $200 \mathrm{GeV}$ and a flux at $\sim 3 \%$ C.U. The 8-years Milagro skymaps reported a hint at the level $3.5 \sigma$ in the region at multi-TeV energies, although no precise flux or energy information on this source is available (Abdo et al. 2009b). MAGIC observed PSR $\mathrm{J} 2022+3842$ for $\sim 44$ hours, covering a large zenith range from $10^{\circ}$ to $50^{\circ}$. Given the Milagro hotspot, this source is expected to emit at $\mathrm{TeV}$ energies which allowed us to observe it under all aforementioned moonlight levels.

\subsubsection{PSR J2111+4606 and PSR J2238+5903}

PSR J2111+4606 and PSR J2238+5903, which display similar high spin-down power around $10^{36} \mathrm{erg} \mathrm{s}^{-1}$, were both detected during blind Fermi-LAT pulsar searches (Pletsch et al. 2012; Abdo et al. 2009a, respectively). The former is located at a distance of $2.7 \mathrm{kpc}$ near the Galactic plane in a low radio emission area, with faint $21 \mathrm{~cm}$ structures as shown by The EffelsbergBonn $\mathrm{H}_{\mathrm{I}}$ Survey. On the other hand, at the position of PSR J2238+5903, also located in the Galactic plane although its distance has not been yet constrained, Milagro significance maps reached $4.7 \sigma$ (Abdo et al.2009b). The PWNe associated to these Fermi-LAT pulsars are the only ones assumed to be extended included in this study. The nebula hosting PSR J2111+4606 is expected to have a radius of $0.15^{\circ}$, while that surrounding PSR J2238+5903 would display a slightly smaller one of $0.10^{\circ}$. MAGIC performed a deep campaign on these sources, accumulating $\sim 55$ and $\sim 44$ hours of optimal quality data on PSR J2111+4606 and PSR J2238+5903, respectively. Under all moonlight levels as well, PSR J2111+4606 was observed for a full zenith range of $(5,50)^{\circ}$, whilst PSR J2238+5903 data was taken at medium zenith angle, between $30^{\circ}$ and $50^{\circ}$. 


\subsection{Results}

In this section, I present the integral and differential ULs for each source as well as the skymaps for interesting FoV

\subsubsection{PSR J1954+2838 and PSR J1958+2845}

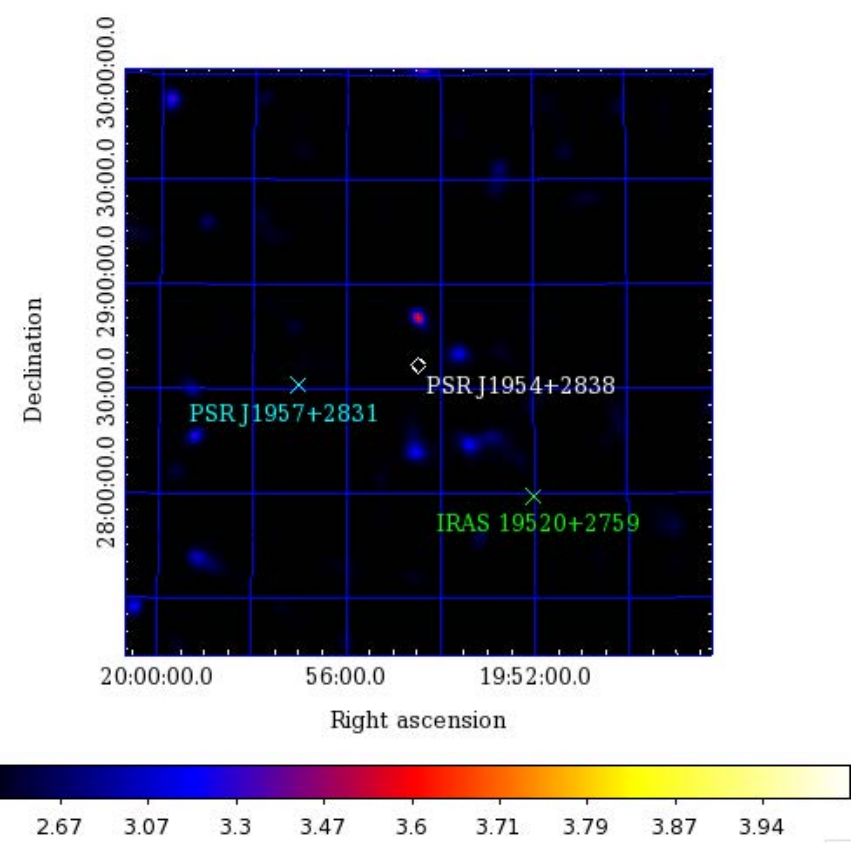

Figure 9.2: MAGIC significance skymap for the observations of PSR J1954+2838 (white diamond). The pulsar PSR J1957+2831 associated to the SNR G65.1+06 is marked in blue, while the IR source, IRAS $19520+2759$, located at the south of the remnant, is shown in green.

No gamma-ray excess was found in the direction of either PSR J1954+2838 or PSR J1958+2845. The measured signal is compatible with background at energies greater than $300 \mathrm{GeV}$ and 1 $\mathrm{TeV}$ (the latter motivated by Milagro hotspots). Nevertheless, it is worth to stress that PSR $\mathrm{J} 1958+2845$ showed a significance of $\sim 2.30 \sigma$ (for $E>1 \mathrm{TeV}$ ) after only $\sim 4$ hours of observations. No hotspots are highlighted in the significance skymaps from PSR J1958+2845. In the case of PSR J1954+2838, a small hotspot situated at an offset of $\sim 0.23^{\circ}$ from the nominal source appears at the level of $\sim 3.5 \sigma$, although its position is not coincident with any known system (see Figure 9.2).

The corresponding integral ULs for energies above $300 \mathrm{GeV}$ assuming a power-law distribution with photon index $\Gamma=2.6$ are $1.1 \times 10^{-12} \mathrm{ph} \mathrm{cm}^{-2} \mathrm{~s}^{-1}(\sim 0.8 \%$ C.U. $)$ and $2.5 \times 10^{-12} \mathrm{ph}$ 
Table 9.2: MAGIC 95\% C.L. differential flux ULs for PSR J1954+2838 and PSR J1958+2845 assuming a power-law spectrum with spectral index of $\Gamma=2.6$.

\begin{tabular}{lcc}
\hline \hline Energy range & $\begin{array}{c}\text { Differential flux ULs } \\
\text { for PSR J1954+2838 } \\
{[\mathrm{GeV}]}\end{array}$ & $\begin{array}{c}\text { Differential flux ULs } \\
\text { for PSR J1958+2845 } \\
{\left[\times 10^{-13} \mathrm{TeV}^{-1} \mathrm{~cm}^{-2} \mathrm{~s}^{-1}\right]}\end{array}$ \\
\hline $\left.3010^{-13} \mathrm{TeV}^{-1} \mathrm{~cm}^{-2} \mathrm{~s}^{-1}\right]$ \\
$475.5-753.5$ & 51.2 & 134.7 \\
$753.6-1194.3$ & 15.3 & 26.9 \\
$1194.3-1892.9$ & 5.7 & 5.7 \\
$1892.9-3000$ & 2.8 & 5.6 \\
$3000-4754.7$ & 4.5 & 2.6 \\
\hline
\end{tabular}

$\mathrm{cm}^{-2} \mathrm{~s}^{-1}(\sim 1.9 \%$ C.U.) for PSR J1954+2838 and PSR J1958+2845, respectively. Differential ULs are also listed in Table 9.2, under same conditions. The SED is depicted in Figure 9.3. including Fermi-LAT and Milagro results as well as former MAGIC spectra.

\subsubsection{PSR J2022+3842}

After $\sim 44$ hours of observation, no significant excess was found in the direction of PSR J2022+3842. The FoV in the skymap is compatible with background with no structure or hotspot popping-up nearby the most energetic pulsar of this study. Integral and differential ULs were therefore computed assuming power-law function of $\Gamma=2.6$ at 95\% C.L. Integral UL for energies greater than $300 \mathrm{GeV}$ is set to $1.5 \times 10^{-12} \mathrm{ph} \mathrm{cm}^{-2} \mathrm{~s}^{-1}$, which corresponds to $\sim 1.2 \%$ C.U. Differential ULs are quoted in Table 9.3 .

Table 9.3: MAGIC 95\% C.L. differential flux ULs for PSR J2022+3842 assuming a power-law spectrum with spectral index of $\Gamma=2.6$.

\begin{tabular}{lc}
\hline $\begin{array}{c}\text { Energy range } \\
{[\mathrm{GeV}]}\end{array}$ & $\begin{array}{c}\text { Differential flux ULs for PSR J2022+3842 } \\
{\left[\times 10^{-13} \mathrm{TeV}^{-1} \mathrm{~cm}^{-2} \mathrm{~s}^{-1}\right]}\end{array}$ \\
\hline $300-475.5$ & 70.8 \\
$475.5-753.6$ & 15.5 \\
$753.6-1194.3$ & 2.9 \\
$1194.3-1892.9$ & 1.3 \\
$1892.9-3000$ & 3.6 \\
$3000-4754.7$ & 0.6 \\
\hline
\end{tabular}



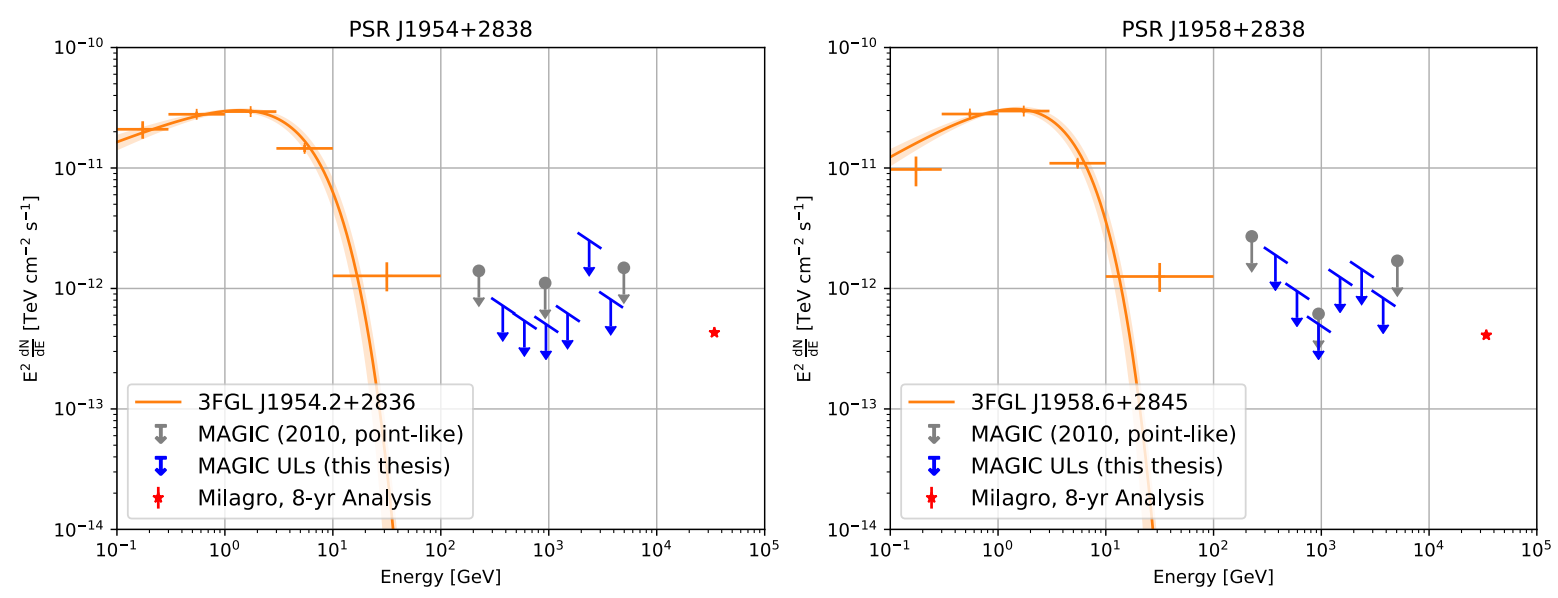

Figure 9.3: SED for the observation on PSR J1954+2838 and PSR J1958+2845, including former MAGIC ULs obtained in 2010 (Aleksić et al. 2010c). Results on Fermi-LAT pulsar as well as Milagro re-analysis are shown, taken from Abdo et al. (2010) and Abdo et al. (2009b), respectively.

Table 9.4: MAGIC 95\% C.L. differential flux ULs for PSR J2111+4606 and PSR J2238+5903 assuming a power-law spectrum with spectral index of $\Gamma=2.6$.

\begin{tabular}{|c|c|c|}
\hline $\begin{array}{c}\text { Energy range } \\
\qquad[\mathrm{GeV}]\end{array}$ & $\begin{array}{l}\text { Differential flux ULs } \\
\text { for PSR J2111+4606 } \\
{\left[\times 10^{-13} \mathrm{TeV}^{-1} \mathrm{~cm}^{-2} \mathrm{~s}^{-1}\right]}\end{array}$ & $\begin{array}{l}\text { Differential flux ULs } \\
\text { for PSR J2238+5903 } \\
{\left[\times 10^{-13} \mathrm{TeV}^{-1} \mathrm{~cm}^{-2} \mathrm{~s}^{-1}\right]}\end{array}$ \\
\hline $300-475.5$ & 106.8 & 50.2 \\
\hline $475.5-753.6$ & 12.5 & 9.4 \\
\hline $753.6-1194.3$ & 2.9 & 2.9 \\
\hline $1194.3-1892.9$ & 1.6 & 1.5 \\
\hline $1892.9-3000$ & 0.6 & 0.4 \\
\hline $3000-4754.7$ & 0.2 & 0.3 \\
\hline
\end{tabular}

\subsubsection{PSR J2111+4606 and PSR J2238+5903}

The MAGIC analysis performed, assuming radius of $0.15^{\circ}$ and $0.10^{\circ}$ for PSR $\mathrm{J} 2111+4606$ and PSR J2238+5903, respectively, yielded no detection. As done for previous sources, we computed integral ULs above $300 \mathrm{GeV}$, considering a standard power-law distribution with photon index 2.6. The constraining ULs are $1.4 \times 10^{-12} \mathrm{ph} \mathrm{cm}^{-2} \mathrm{~s}^{-1}$, i.e. $1.1 \%$ C.U. in the case of PSR 
$\mathrm{J} 2111+4606$, and $8.9 \times 10^{-13} \mathrm{ph} \mathrm{cm}^{-2} \mathrm{~s}^{-1}$, which corresponds to $0.7 \%$ for PSR J2238+5903.

\subsection{Discussion and conclusions}

Despite observing PWNe hosting pulsars with spin-down power as large as the one for PWNe detected at $\mathrm{VHE}$, no detection was achieved for any PWN. Most of them, except for PSR $\mathrm{J} 1958+2845$, were observed for large amount of hours, allowing to explore flux levels below what has been detected from other PWNe. Given that our observations were performed in the outer parts of the Galaxy, a non-detection could be explained if different behaviors are found in the MAGIC candidates with respect to those shown by detected PWNe located in the inner regions. To highlight any possible difference, I compare in Figure 9.4 the PWN luminosity between 1-10 TeV of the five PWNe shown in this chapter with respect to the characteristic age and spin-down power of the hosted pulsars. In turn, I included all detectedPWNe(inside and outside of the HGPS), along with the HGPS candidates and the ULs obtained for the undetected HGPS PWNe (see H. E. S. S. Collaboration et al. 2017). The luminosity of the five above mentioned PWNe is also quoted in Table 9.5 .

Table 9.5: From left to right: Integral UL above $300 \mathrm{GeV}$, UL on the TeV luminosity (1-10 TeV), efficiency converting rotational energy into $\mathrm{TeV}$ gamma rays $\left(L_{\gamma, 1-10 \mathrm{TeV}} / \dot{E}\right)$.

\begin{tabular}{cccc}
\hline \hline Name & $\begin{array}{c}\text { Integral UL } \\
{\left[\times 10^{-12} \mathrm{ph} \mathrm{cm}^{-2} \mathrm{~s}^{-1}\right]}\end{array}$ & $\begin{array}{c}L_{\gamma, 1-10 \mathrm{TeV}} \\
{\left[\mathrm{erg} \mathrm{s}^{-1}\right]}\end{array}$ & $\xi$ \\
\hline PSR J1954+2838 & 1.1 & $5.2 \times 10^{33}$ & $5.2 \times 10^{-3}$ \\
PSR J1958+2845 & 2.5 & $1.2 \times 10^{34}$ & $3.5 \times 10^{-2}$ \\
PSR J2022+3842 & 1.5 & $8.4 \times 10^{34}$ & $2.8 \times 10^{-4}$ \\
PSR J2111+4606 & 1.4 & $5.7 \times 10^{32}$ & $4.1 \times 10^{-4}$ \\
PSR J2238+5903 & 0.89 & $7.7 \times 10^{33}$ & $8.7 \times 10^{-3}$ \\
\hline
\end{tabular}

MAGIC results are in agreement with the fit obtained by H. E. S. S. Collaboration et al. (2017) using detected TeV PWNe and ULs. There is only a small deviation in the case of PSR $\mathrm{J} 2111+4606$ when comparing the luminosity versus the spin-down power. Nevertheless, this mismatching is not very pronounced and, in turn, this source follows, within the errors, the fit obtained for the luminosity against the characteristic age. Therefore, one can conclude that the PWNe included in this thesis are not outliers of the TeV PWN population and so, gamma-ray emission should be expected given their features.

Thus, the possible reasons for a non-detection can be basically encompass into two: larger extension of source than expected or low target photon field. The former would applied PSR J2111+4606, given its most likely distance. For the analysis presented in this thesis, PSR 

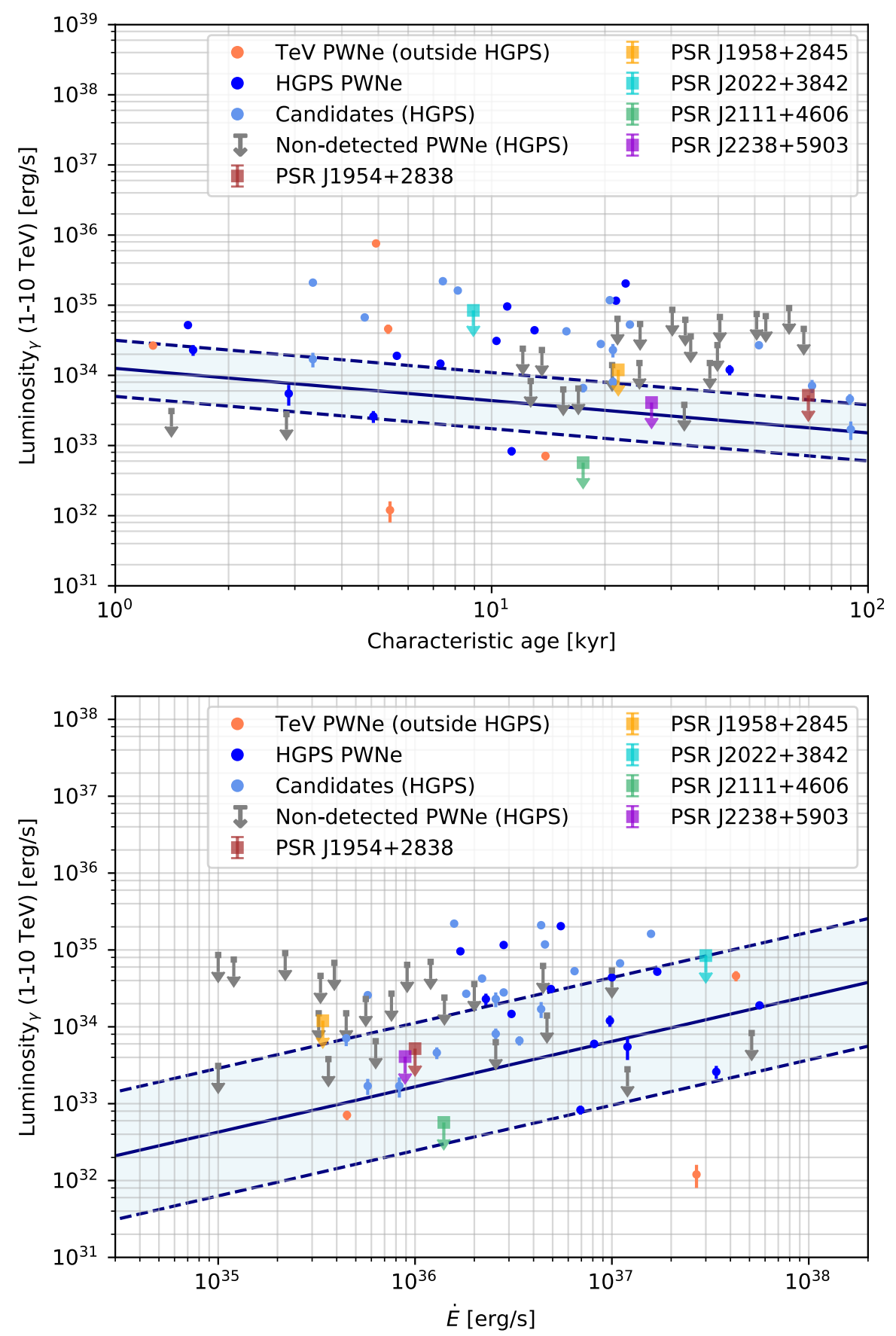

Figure 9.4: $\mathrm{TeV}$ luminosity $(1-10 \mathrm{TeV}$ ) with respect to the characteristic age (top) and the spin-down power of the pulsar (bottom). The five candidates analyzed in this thesis are marked with squares, while external PWNe are shown with circles. In the latter, detectedPWNe from inside and outside of the HGPS, candidates and non-detected nebulae from it are included. The fit obtained in the study of PWNe by H. E. S. S. Collaboration et al. (2017) is depicted as a blue band. 


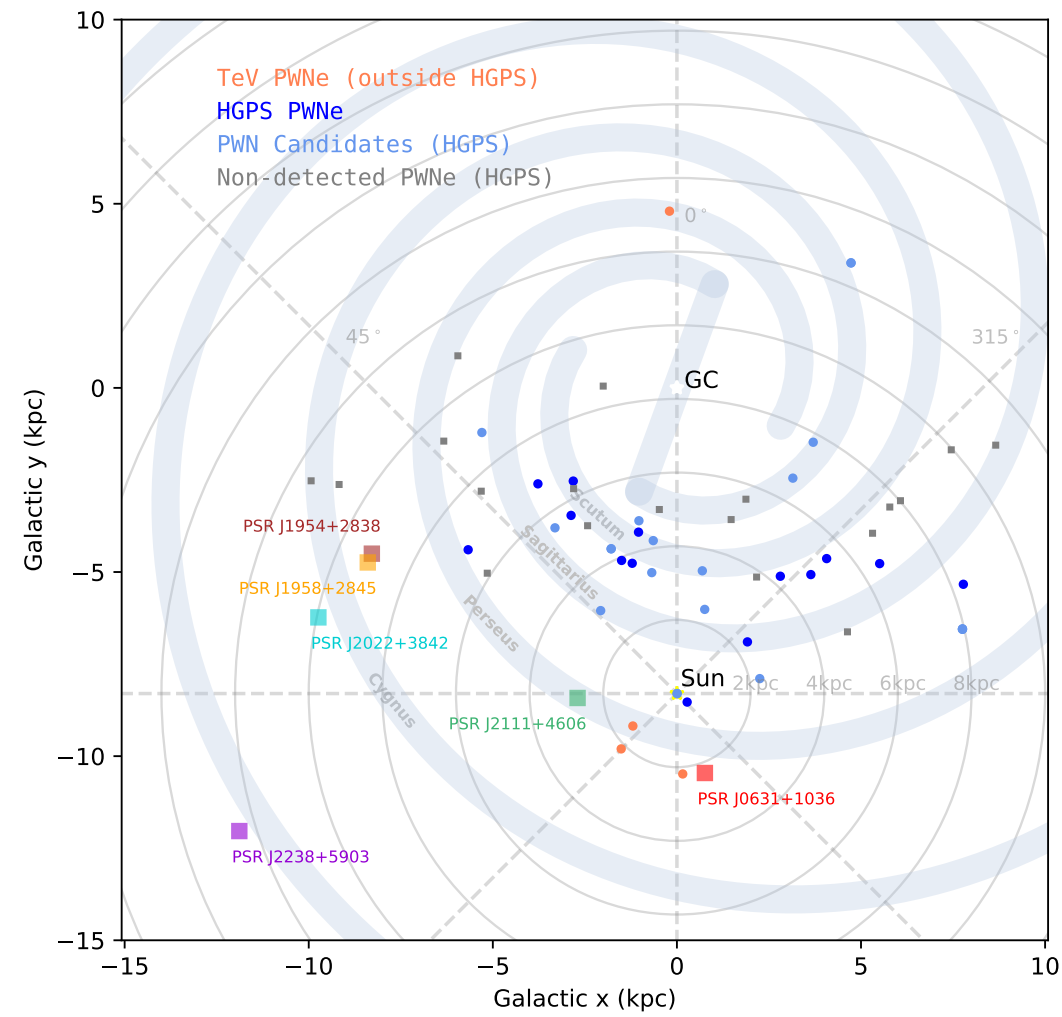

Figure 9.5: Schematic view of the Galaxy with the spiral arms in which sources included in this study are marked. PWNe detected (inside and outside the HGPS are depicted as orange and dark blue dots, while candidates included in the HGPS are shown as light blue dots. Pulsars with spin-down power above $10^{35}$ $\mathrm{erg} \mathrm{s}^{-1}$ for which non-detection of PWNe was achieved during the HGPS are also represented as grey squares.

$\mathrm{J} 2111+4606$ was assumed to have $0.15^{\circ}$ radius. Larger extension can be still studied but without other wavelength information that constrains the radius, any other assumption would be purely speculative and could end up on smearing the possible gamma-ray emission by taking a too large extension.

Nevertheless, the scenario of a low target photon medium that decreases the IC interaction chances is reinforced by Figure 9.5. This plot reveals the accumulation of detected PWNe in the spiral arm named Scutum, while no other tendency becomes obvious for the rest of the Galaxy. In order to investigate a speculative relation with the photon field density in each arm, we made use of the computed the FIR field in the Galaxy, by means of the publicly available software 


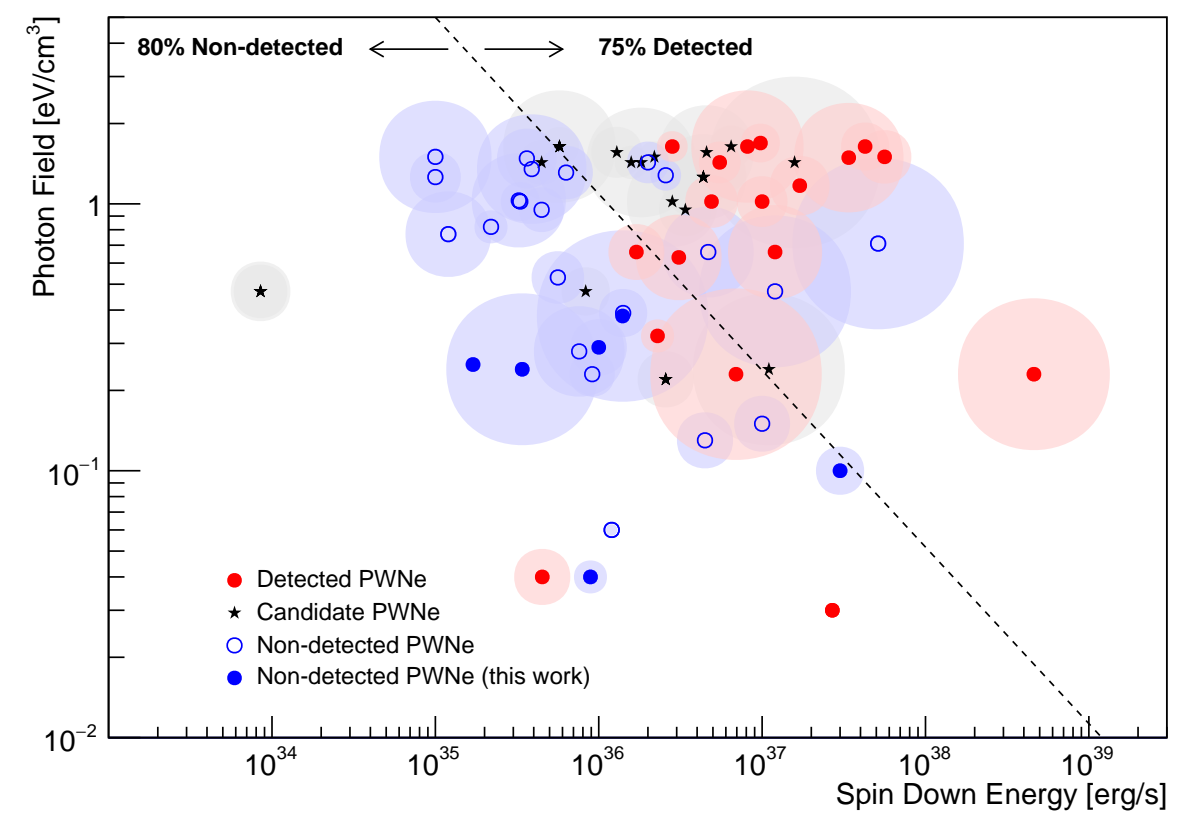

Figure 9.6: FIR energy density photon field as a function of the spin-down power of the pulsars. The detected (red dots), non-detected (blue empty dots) and candidate PWNe (grey stars) included in the HGPS are shown, along with MAGIC candidate sources (blue filled dots) included in this study.

GalPROP The results are shown in Figure 9.6 as a function of the spin-down power of the pulsars. In this figure, along with the PWNe analyzed in this thesis, the detected, non-detected and PWN candidates included in the H.E.S.S. TeV PWN population are shown (H. E. S. S. Collaboration et al. 2017). The semi-transparent colored circles around each dot represent their luminosity. The plot evidences a correlation between these two features: most of the detected PWNe present high spin-down power, while surrounding by a large FIR energy density photon field. This way, it is probed that not only a high $\dot{E}$ is necessary (above $10^{36} \mathrm{erg} \mathrm{s}^{-1}$ ), but a large target photon field would increase the probability of detection. The features of the five PWNeI analyzed place them into the half of the plot where the probability of success on the detection is low: $80 \%$ of the sources with similar characteristics were not detected.

Finally, it is worth to point out the resemblances shown between PSR J2111+4606 and PSR $\mathrm{J} 0007+7303$, the pulsar powering the detected PWN CTA1. Both of them are located at similar distances of $2.7 \mathrm{kpc}$ (assuming pseudo-distance) and $1.4 \mathrm{kpc}$, respectively, and with characteristic ages of $17.5 \mathrm{kyr}$, in the case of PSR J2111+4606, and $13.8 \mathrm{kyr}$ for PSR J0007+7303. Actually, PSR J2111+4606 displays a slightly higher spin-down power of $1.4 \times 10^{36} \mathrm{erg} \mathrm{s}^{-1}$ against $4.5 \times$ $10^{35} \mathrm{erg} \mathrm{s}^{-1}$ shown by PSR J0007+7303. Both PWNe are situated in the same region of the

\footnotetext{
${ }^{1}$ https://galprop.stanford.edu/
} 
Perseus arm and so, the target photon field density is alike (see Figure 9.6). On the other hand, the efficiency of converting the released rotational energy into gamma rays is around one order of magnitude lower in PSR J2111+4606 $\left(4.1 \times 10^{-4}\right.$, see Table 9.5) than in PSR J0007+7303 (around $1.6 \times 10^{-3}$ ). Consequently, the non-detection of PSR J2111+4606 can be also due to this inefficiency or to a larger extension than the $0.15^{\circ}$ radius assumed in this thesis. 


\section{Part IV}

\section{Quality control of LST camera subsystems}

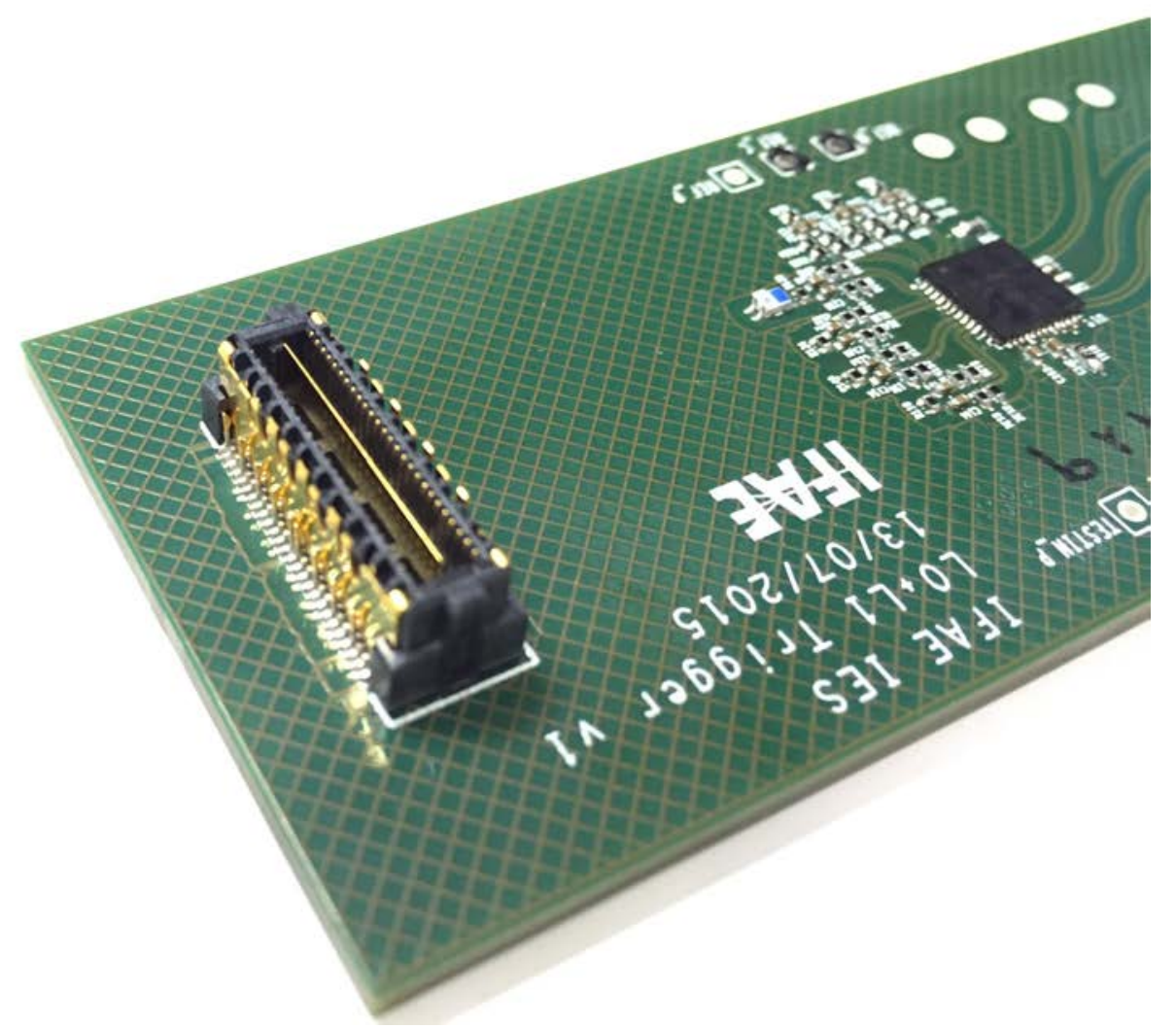

Figure IV: L0+L1 mezzanine designed at IFAE. 



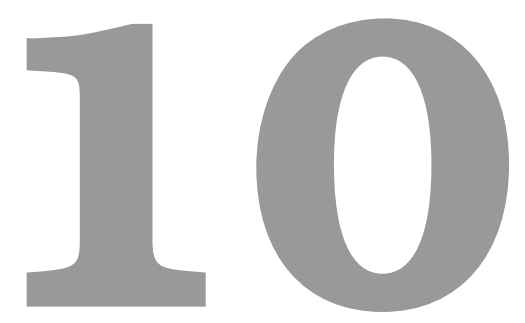

\section{Quality control of LST camera subsystems}

\subsection{Introduction}

The main scientific objective of the LST is to achieve the lowest energies with a 10 times better sensitivity (around $1 \mathrm{TeV}$ ) than the current IACTs. One of the most important systems to success on this purpose is the camera. The reliability of its components depends on the results obtained from the $\mathrm{QC}$, i.e. dedicated tests used to evaluate the functionality of the devices and check, in this way, if they reach the desire specifications to achieve the goals for the LST.

Along my thesis period, I was involved on the QC for several subsystems that composed the future LST prototype, among which the PMTs, the Power Supply Units (PSUs) and the L0+ + L1 trigger mezzanines stand out. In order to set these subsystems in context, in the first section of this chapter I give an overview of the LST camera and its components, including their functionality.

\subsection{Overview of the LST camera}

Figure ?? shows the electrical diagram of the LST camera. The different cabling needed to connect and power all subsystems is classified in seven types, labeled in the image. The camera itself can be split into three sections: front, middle and back. 


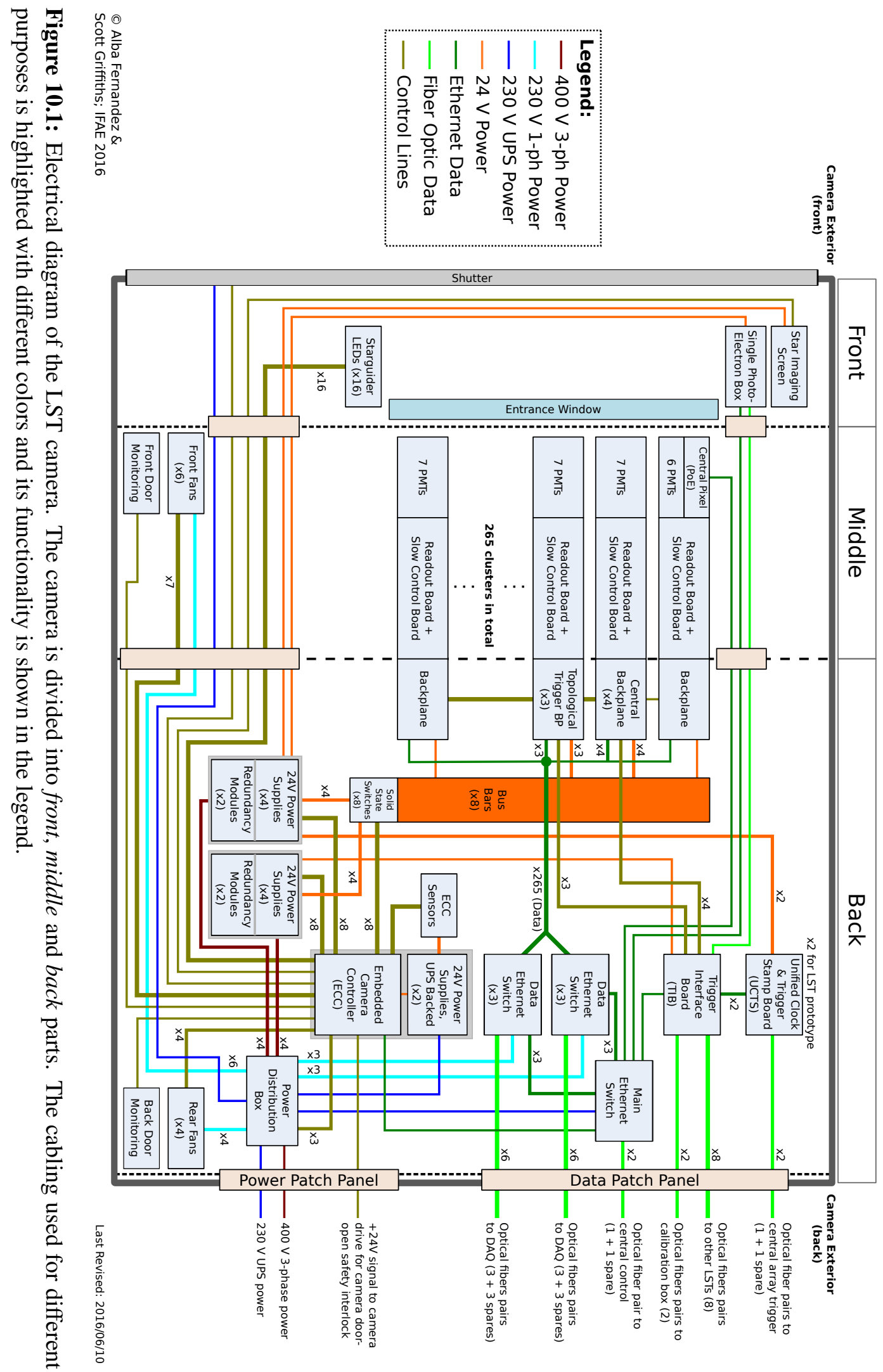




\subsubsection{Front part of the LST camera}

This is the outer part of the camera, the one in contact with the environment. the most external component is the shutter or lids, which prevents light from entering in the camera, protecting this way the PMTs from the daylight. In turn, it provides protection against adverse environmental conditions, such as rain, during the day. The goal of the entrance window, placed after the shutter, is to protect the instrumentation from any aggression (rain, dust, e.g.) during observational time. In this front side, the starguider LED (used to check the pointing of the camera as done in MAGIC, see Chapter 2.4.1.1), the star imaging screen and the Single Photoelectron (SPE) box are also installed. The latter is used for the calibration of the camera (to check e.g. gain or linearity).

\subsubsection{Middle part of the LST camera}

The main body of the camera concentrates in this region. Besides the front fans, designed to cool the system during operation, the middle part of the camera encompasses the 265 clusters, where trigger and readout take place. Figure ?? shows a cluster, where all parts are identified. Each cluster is formed by 7 Hamamatsu PMTs, to which light guides are attached in order to collect the highest amount of photons reflected in the mirrors and excludes, in turn, $\mathrm{NSB}$ light coming at higher angles (see Figure ??). The PMTs work with a $\mathrm{HV}$ of $\sim 1.4 \mathrm{kV}$ provided by the $24 \mathrm{~V}$ DC PSU using the readout board as intermediate. The output signal of each PMT is preamplified with low noise by Pre-Amplifier for the Cherenkov Telescope Array (PACTA), an ApplicationSpecific Integrated Circuit (ASIC) designed by the ICC-UB group. Two paths formed the output of PACTA the low gain and the high gain branch, each of them differential. Having these two paths, a larger dynamic range is possible: linearity in the low gain reaches higher ranges (up to thousand photoelectrons), while the high gain extends linearity at the smallest ones. The PMTs are connected to the readout board through the Slow Control Boards. This device can control the $\mathrm{HV}]$ of the detectors and monitors different parameters as the $\mathrm{DC}$ current, temperature or humidity. Thus, the signal from the seven channels (0-6, one for each PMT) enter in the readout board, which in the case of the LST is called Dragron, and is replicated to reach both readout and trigger subsystems. To the trigger board (L0+L1 mezzanine) only the high gain paths are sent. The L0+L1 mezzanine is an unique Printed Circuit Board (PCB) board composed by the connectors and the corresponding L0 and L1 ASIC, on charge of the main functionality (as accomodate the signal and take the trigger decisions). After entering in the mezzanine, the signals pass through the delay lines, use to compensate the different transit time and synchronize the PMT signal (within a range of 0-5.75 ns). Thus, the signal reaches the L0 ASIC, first step of the trigger system in which individual pixel signals are evaluated. All signals go through an attenuator in order to compensate for different gains. Here, the voltage can be adjusted between 0.6-1.35 times the input voltage (in steps of 0.05). After the attenuator, two trigger options are available: the sum trigger and the majority trigger. 


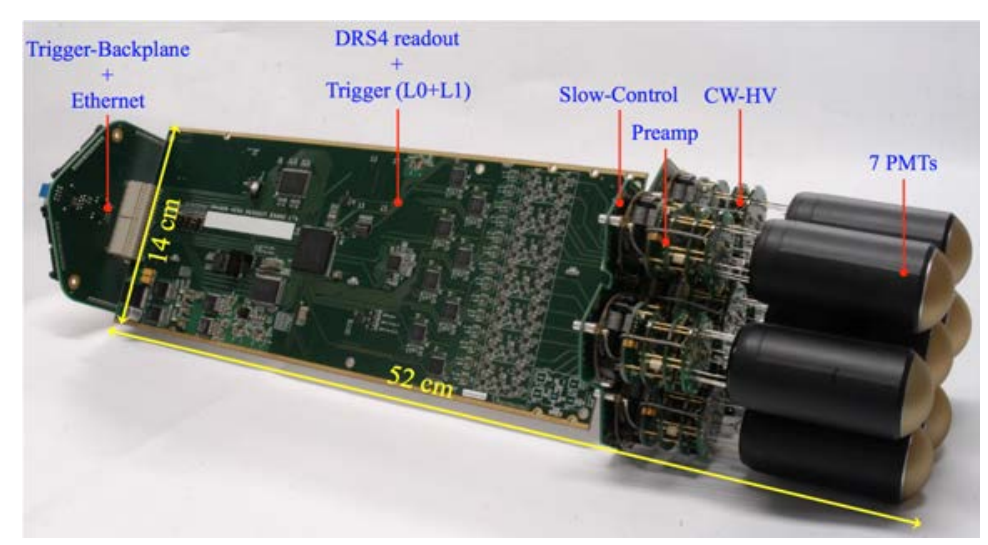

Figure 10.2: LST module with all components highlighted: 7 photodetectors, followed by the HV chip and the preamplifier (PACTA), the Slow Control board, Dragon board in which L0+L1 mezzanine is attached (the trigger board is connected to the opposite side of the board seen in the image) and finally the backplane where the Ethernet switches are placed.

The sum trigger is the one that will continue to the next L1 level inside the trigger subsystem. The concept is as follows: signals above certain threshold are clipped to avoid using spurious signals, like APs. There are three clipping options and each of them can be modulated with 63 finer steps. After clipping, signals from the seven channels are summed, which corresponds to the L0 output. On the other hand, the majority trigger discriminates the signals according to a specific threshold. If the signal overpasses that threshold, a square $100 \mathrm{mV}$ signal is released. The emitted signals for each channel are added and the result would constitute the output of the L0. As mentioned before, the majority output is usually disable and not use in the following steps. Nevertheless, each $100 \mathrm{mV}$ signal is also available as an Low-Voltage Differential Signaling (LVDS) output, from which L0|Individual Pixel Rate (IPR) is obtained. During this thesis, a fast QC of the L0 trigger in 17 mezzanines was performed, making use of an automatic LabView program that modifies the aforementioned parameters (attenuator, clipping, majority discriminator) to cover all possibles values. A proper $\mathrm{QC}$ had been performed and hence, the goal of these tests was to ensure the functionality before integrating the mezzanines on the camera. To do so, we set low DT to accomplish trigger always. We injected a $200 \mathrm{mV}$ signal with $3 \mathrm{~ns}$ of Full Width Half Maximum (FWHM) at $10 \mathrm{kHz}$ and checked the IPR in each channel as well as the output of the signal from the sum trigger path. During these tests, the delay lines were also checked. As result, four mezzanines were detected to be not functional and sent to repair. An afterwards check probed their recover.

Following with the trigger scheme, the output of the L0 decision is sent to the L0 fan-out, place in the backplane board (located already in the back part of the camera, see Figure ??). From there, the L0 output is sent to the six neighbouring clusters and, in turn, it receives the L0 signals from those clusters. Thus, these six signals along with the one of the own cluster is sent to the L1 part of the trigger mezzanine. At the L1 level, a selected combination of L0 signals is 


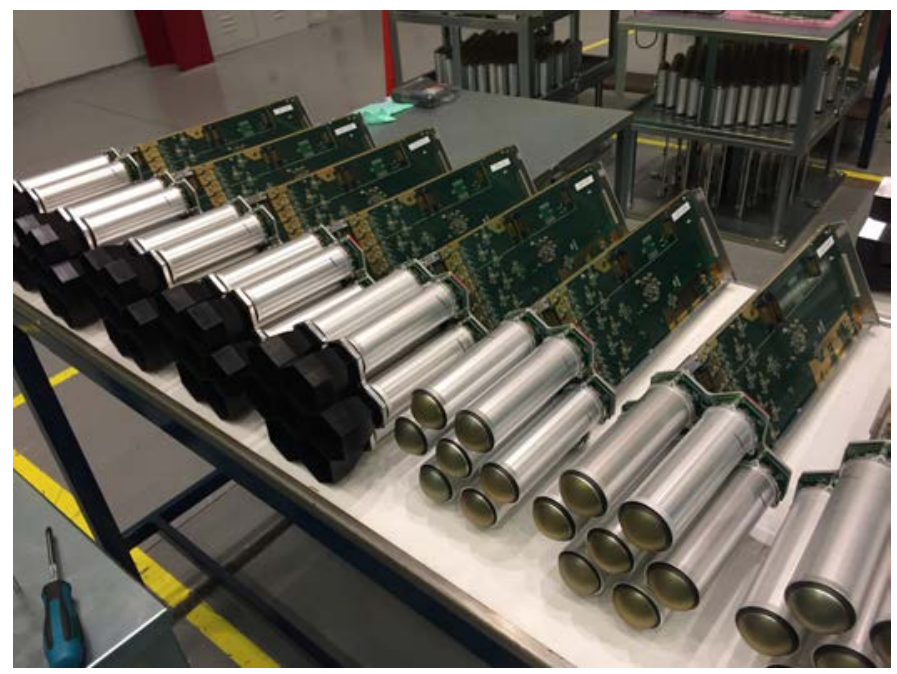

Figure 10.3: LST modules formed by the seven PMTs, the Slow Control board the Dragon board. No trigger mezzanine is connected. The leftmost modules have attached the light guides.

compared to a threshold. In the L1 ASIC there are available three adders, which can be used to test different geometrical combinations of the clusters, although only the output of one of them will be used later on. If the sum of the signals overcomes the threshold, the L1 ASIC sends a LVDS signal to the L1 distribution subsystem (also placed in the backplane). This signal is then transmitted to the central backplane and from there to the Trigger Interface Board (TIB), also located in the back part of the camera and which will be introduced in the next section. A fast QC was also performed on the L1 trigger during this thesis. The test consisted on injecting 1000 events of $300 \mathrm{mV}$ in burst of $12 \mathrm{MHz}$ divided by $10 \mathrm{kHz}$ and scan the signal in each channel and adder from the minimum DT value up to its maximum. We normalized the gain in each channel with respect to a selected reference channel by measuring its value in each channel a priori. The results we wanted to obtain were the $\mathrm{DT}$ at which the rate was half its value (i.e. 500 events). The functionality of the L1 in these 17 mezzanines was probed to be correct and compatible with Centro de Investigaciones Energéticas Medioambientales y Tecnológicas (CIEMAT) results.

Finally, the TIB sends the final command back to the central backplane and from there to the other backplanes to start the readout at the front-end electronics. The signal from each channel is saved in a buffer formed by four DRS4 chips with a longitude of $1 \mu$ s accounting for a readout speed of $1 \mathrm{GSample/s}$. The transmission of the data is then performed by Ethernet cables controlled by a Field Programmable Gate Array (FPGA) situated in the Dragon.

All the 265 modules are placed in a metallic structure prepared at CIEMAT (see Figure ??).

\subsubsection{Back part of the camera}

As seen before, the backplanes and the TIB are placed in this section of the camera. The backplanes are then the connection between the modules and the rest of the subsystems, a part from 


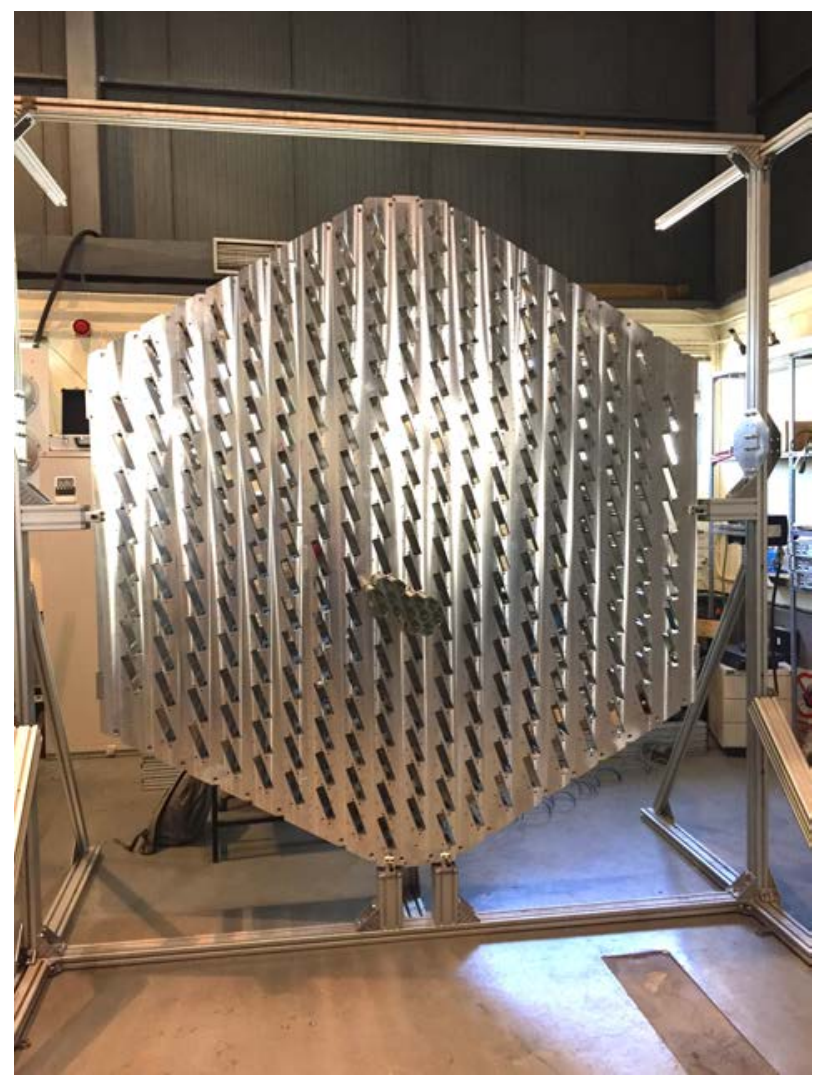

Figure 10.4: Metallic structure holder for the modules of the LST. The module are placed in each hole. In the center, two modules are connected, which have attached the light guides. 


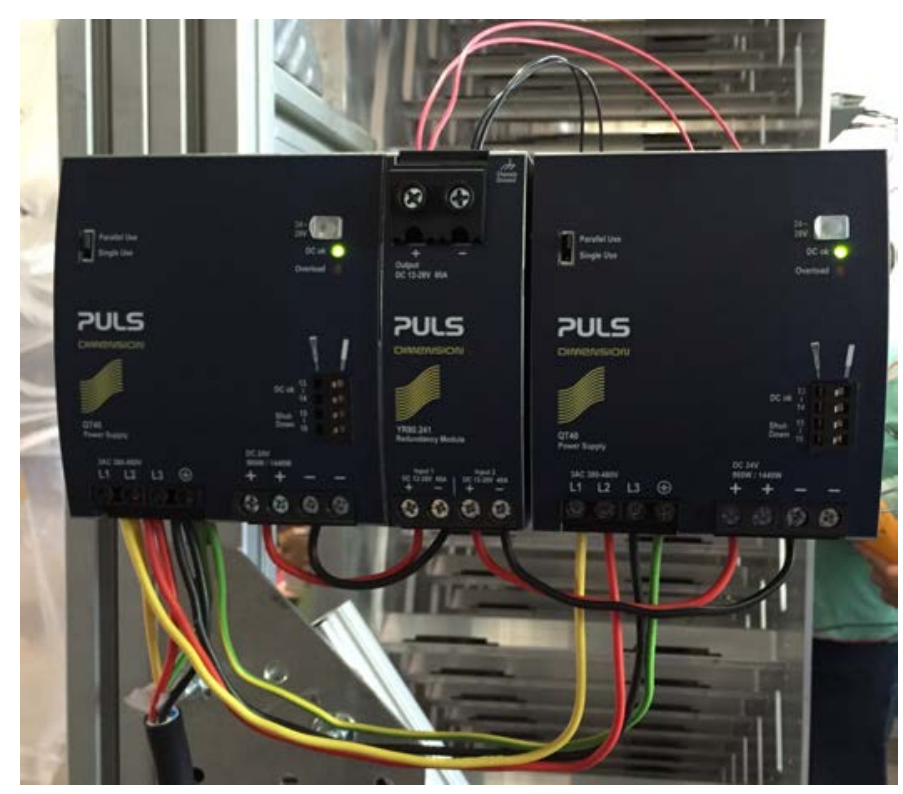

Figure 10.5: $\mathrm{PSU}$ and redundancy modules used in the LST camera.

connecting all modules together: both the $24 \mathrm{~V}$ DC $\overline{\mathrm{PSU}}$ and the Ethernet connection are driven through the backplanes. The power supply is distributed owing to eight bus bars. The $24 \mathrm{~V}$ line is provided by eight PULS QT40.241 PSU at $40 \mathrm{~A}$ (i.e. $960 \mathrm{~W}$ per PSU), as the ones shown in Figure ??. Among them, there are redundancy modules decouple to each pair which provides current for a short time period in case of a failure in any PSU. More information and dedicated $\mathrm{QC}$ of these $\mathrm{PSU}$ are presented in the next section.

Besides these eigth PSU, in the back part there are also two $24 \mathrm{VPSU}$ |Uninterruptible power supply (UPS)-backed, connected to critical devices for the safety and operation of the telescope. These devices are the Embedded Camera Controller (ECC), main brain of the camera, and the ECC sensors. The main aims of these subsystems are to monitor the environment (like temperature or humidity), control auxiliary components (like fans, starguider LED or the lids) and make decisions to ensure the safety of the camera. ECC does not control the modules or the TIB, but it can get information from them (as the $\mathrm{HV}$ or the temperature) to provide reliable information regarding the status inside the camera.

All the $24 \mathrm{VPSU}$ (including the UPS-backed and also the main Ethernet switch) are powered through the Power Distribution Box (PDB), an electrical cabinet located in the back of the camera (see functional diagram in Figure ??). Its functionality is comprehended in four points:

$\odot$ Distribute the incoming $400 \mathrm{~V}$ 3-phases power and $230 \mathrm{~V}$ UPS-backed single-phase power among the subsystems: the $400 \mathrm{~V} 3$-phases branch powers most of the camera, 


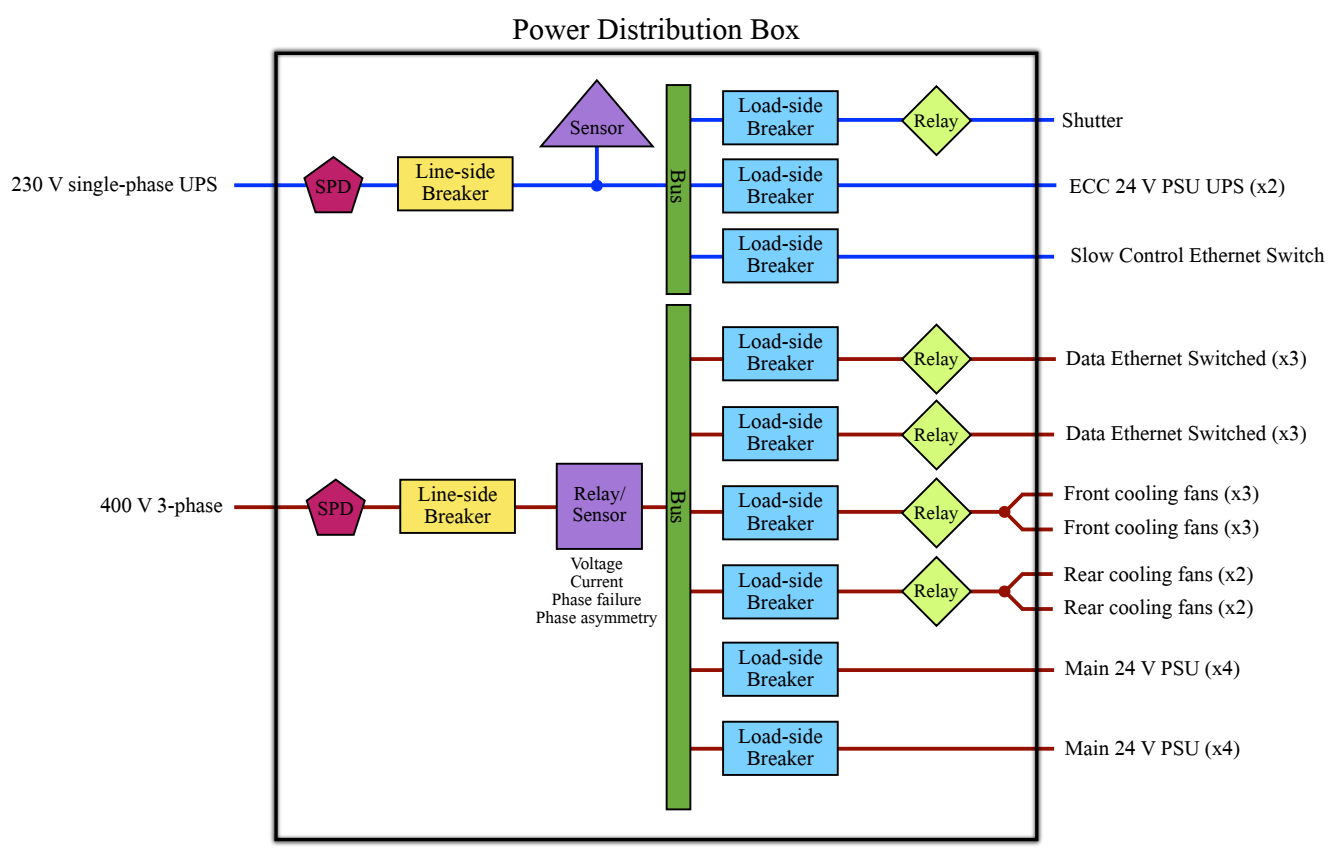

Figure 10.6: Functional diagram of the $\mathrm{PDB}$. The inputs/outputs are listed along the left/right side of the figure.

including the eight $24 \mathrm{~V}$ DCPSU, This branch also supplies the data Ethernet switches and cooling fans. The latter subsystems are powered by $230 \mathrm{~V}$ single-phase. It is possible to power these $230 \mathrm{~V}$ single-phase devices from the $400 \mathrm{~V}$ 3-phases line because the amplitude of each phase is $\sim 230 \mathrm{~V}$. On the other hand, the $230 \mathrm{~V}$ single-phase UPS-backed line powers sensitive devices, as the $24 \mathrm{~V}$ PSU UPS-backed, the shutter or the main Ethernet switch (the link between the ECC and the outside world).

$\odot$ Monitoring the status (voltage, current, etc) of the incoming power lines: Both 400 $\mathrm{V}$ and $230 \mathrm{~V}$ power lines are connected to sensors in order to track any problem with the incoming power lines. Besides the sensor, the $400 \mathrm{~V}$ power line also requires a master control relay, which can be used to cut power in case of maintenance. The $230 \mathrm{~V}$ power line does not incorporate such relay in order to avoid shut down the power accidentally.

$\odot$ Protecting the camera against electrical transients on the incoming power lines (e.g. lightning): This task is carried out internally by Surge Protection Devices (SPDs). Most of the power distribution systems in the LST camera present already some power protection and therefore these SPDs act like an extra protection. On the other side, minor transients in the power lines (like spikes) are handled by the line-side breakers. Finally, the load-side breakers are used to protect the camera subsystems from overcurrents.

$\odot$ Controlling the flow of power to subsystems, either for safety or for powering them ON/OFF. 
All the sensors and relays, along with the SPDs and breakers will be selected at Institut de Física d'Altes Energies (IFAE), accounting for the CTA requirements. The mechanical design of the $\mathrm{PDB}$ was performed between the Laboratoire Leprince-Ringuet and CIEMAT, while the design and assembly of the PDB internals were carried out by MAES Automation in Sabadell.

\subsection{Quality control on individual devices}

In this section, I show the results of the QC performed on different subsystems of the LST camera.

\subsubsection{Power supply noise tests}

DC power supplies ideally supply a constant voltage or current devoid of time-domain fluctuations. In reality, some amount of noise is always generated or relayed by a power supply. Two types of power supply noise are generally considered, depending on how the noise is transmitted:

$\odot$ Conducted emissions: noise from the power supply is conducted through wires to the device it is powering.

$\odot$ Radiated emissions: electromagnetic radiation emitted by the power supply induces noise in nearby devices.

We further characterize these noise sources in the context of the type of power supply being discussed. We consider two general types of power supplies: linear or switch-mode. In linear power supplies, AC mains voltage at $50 \mathrm{~Hz}$ enters the power supply and is rectified to create a positive voltage that fluctuates at a frequency of $\sim 100 \mathrm{~Hz}$. This signal is integrated by large capacitors, regulated to the proper voltage, and filtered to produce a nearly DC signal. A small noise component at $100 \mathrm{~Hz}$, called ripple, always survives this process and is included in the output of the power supply. Moreover, higher frequency noise from the mains power may also be present in the output of a linear power supply. Good linear power supplies generally filter both sources of noise very effectively and radiate little, if at all. Switch-mode or switching power supplies have several advantages over linear power supplies, including higher power output and vastly better efficiency, but are generally more noisy. Switching power supplies operate by rapidly switching power transistors on and off at a characteristic switching frequency. These supplies generate noise at the switching frequency, or a harmonic thereof, which can be either conducted to the output of the supply or radiated into space. Switching frequencies of are generally in the range of $50 \mathrm{kHz}-5 \mathrm{MHz}$. Poorly designed power supplies can be a significant source of noise at these frequencies.

The goal of the tests was to characterize the noise generated by the PULS QT40.241 switchmode power supply, selected to power the cameras the LST and MST as well. Table ?? includes the switching frequencies of the QT40.241. Based on this table, we expect that noise (either conducted or radiated) from the power supply should occur in the $1-300 \mathrm{kHz}$ frequency range, although harmonics could extend this range up to a few $\mathrm{MHz}$. 


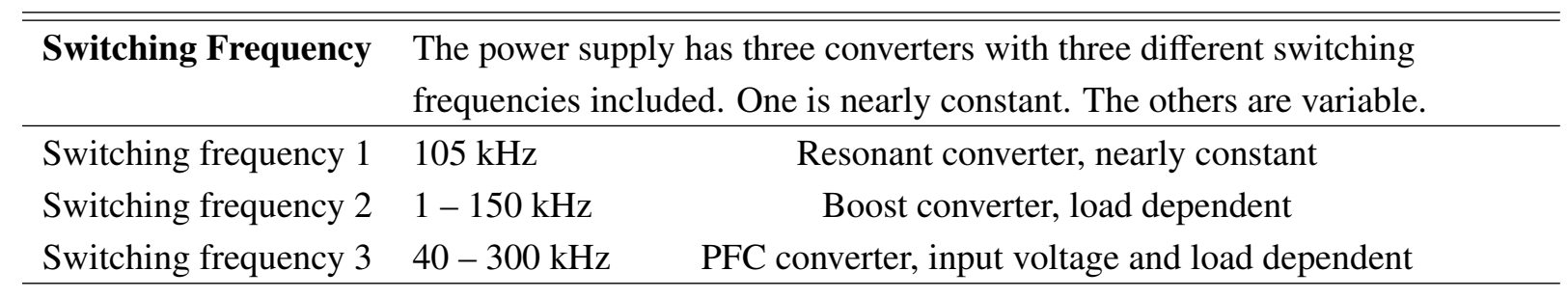

Table 10.1: List of PULS QT40.241 switching frequencies.

The PULS QT40.241 will operate in the vicinity of and provide power to PMTs. Noise at the input of the PACTA will directly affect the noise in the output of the PMT, while noise in the $\mathrm{HV}$ supply or control lines may affect the stability of the PMT output. It is necessary to be able to detect the electrical signal from $[$ SPE $]$ emitted by the PMT photocathode. The amplitude of the PMT output signal generated by a SPE depends on the gain of the PMT, but the height of the pulse should have an approximate amplitude of a few $\mathrm{mV}$. It is therefore important to ensure that the noise is well below this level.

\subsubsection{Equipment and test setup}

The devices used to perform the tests are the following:

$\odot$ PMT: Hamamatsu R11920-100-20 High QE PMT, serial number ZQ6623.

The low voltage input $\left(\mathrm{V}_{c c}\right)$ supplied to the $\mathrm{PMT}$ is $+5 \mathrm{~V}$, while the $\mathrm{HV}$ control supply is variable over a range of $+0.85 \mathrm{~V}$ up to $+1.5 \mathrm{~V}$ and is multiplied by 1000 (yielding an $\mathrm{HV}$ range of $0.85-1.5 \mathrm{kV}$ ). In the tests that follow, unless otherwise noted we use a $\mathrm{HV}$ of $\sim 1.45 \mathrm{kV}$, where noise in the PMT should be most prominent and detectable. An SMA connector attached to the base of the PMT module is used for readout.

\section{$\odot$ Linear Power Supply: Tektronix PS280}

A low-noise, dual output laboratory supply is used to supply power to the PMT to establish a baseline. The $+5 \mathrm{~V}$ and $+0.85-1.5 \mathrm{~V}$ needed by the PMT can be supplied directly to the module from the power supply. Any laboratory-grade dual output supply can be used for this purpose.

$\odot$ Switch-mode Power Supply: PULS QT40.241, serial number 11201661.

This is the device under test. It provides a fixed $+24 \mathrm{~V}$ output, so it cannot directly supply power to the PMT. To power the PMTs with this power supply the Dragon readout board has to be used as an intermediary. The output signal from the PMT can be measured by an oscilloscope or by using the readout board itself.

$\odot$ Readout board: Dragon readout board (v5).

The readout board converts the $+24 \mathrm{~V}$ from the PULS QT40.241 to the necessary voltages for the PMT. The readout board can also be used to record signals from the PMT, which 
can then be analyzed to search for noise. Communication with the readout board requires an Ethernet connection and Cluster Control (ClusCo) or rbcp software.

$\odot$ Oscilloscope: Tektronix TDS3024B digital oscilloscope.

A digital oscilloscope is used to view the output signal from the PMT and to perform a Fast Fourier Transform (FFT) of the PMT signal to search for noise in frequency space. The primary reason for using the TDS3024B is its ability to use a $50 \Omega$ input impedance (typically oscilloscopes have $1 \mathrm{M} \Omega$ input impedance). If using another oscilloscope, ensure correct impedance matching so that feedback oscillations are avoided.

\section{$\odot$ Dark Box:}

Measuring the noise of the $\mathrm{PMT}$ output with the $\mathrm{HV}$ turned on requires that as little light reaches the PMT as possible. The PMT should be placed in a dark box with panel-mounted cable feedthroughs to avoid exposing the PMT to excess light while it is operating. Note that the dark box may provide some EM shielding depending on its construction. The dark box should be large enough to place the PULS QT40.241 inside, next to the PMT.

\subsubsection{Test description}

We evaluate the noise at three different frequency ranges:

$\odot$ Low frequency: $62.5 \mathrm{~Hz}$, searching for $50 \mathrm{~Hz}$ noise (power line noise).

$\odot$ Medium frequency: $2.5 \mathrm{kHz}$, searching for noise at the level of $\mathrm{kHz}$ (pick-up noise).

$\odot$ High frequency: Not fixed value, searching for noise at or above $100 \mathrm{MHz}$.

The results, shown as Voltage vs Time plots, are obtained, in turn, with three different baseline configurations:

$\odot$ All devices powered off.

$\odot$ PMT pre-amplifier powered on (at 5 V), PMT|HV] off.

$\odot$ PMT pre-amplifier and HV powered on.

The power line (conductive) noise tests were performed using both linear PSU and PULS QT40.241 PSU. The noise induced by the CTA PSU, using the Dragon Board as intermediate, was studied through its trigger path (L1 output). In Figure ??, a scheme of the setup used to carry out these measurements is shown. Two configurations were tested: one with the PMT connected to the Slow Control Board, and the other without PMT.

On the other hand, the pick-up (radiative) noise tests were carried out powering the PMT with the linear PSU while the PULS QT40.241 PSU was placed alongside the PMT inside the dark box. The noise was tested under three different test configurations: 

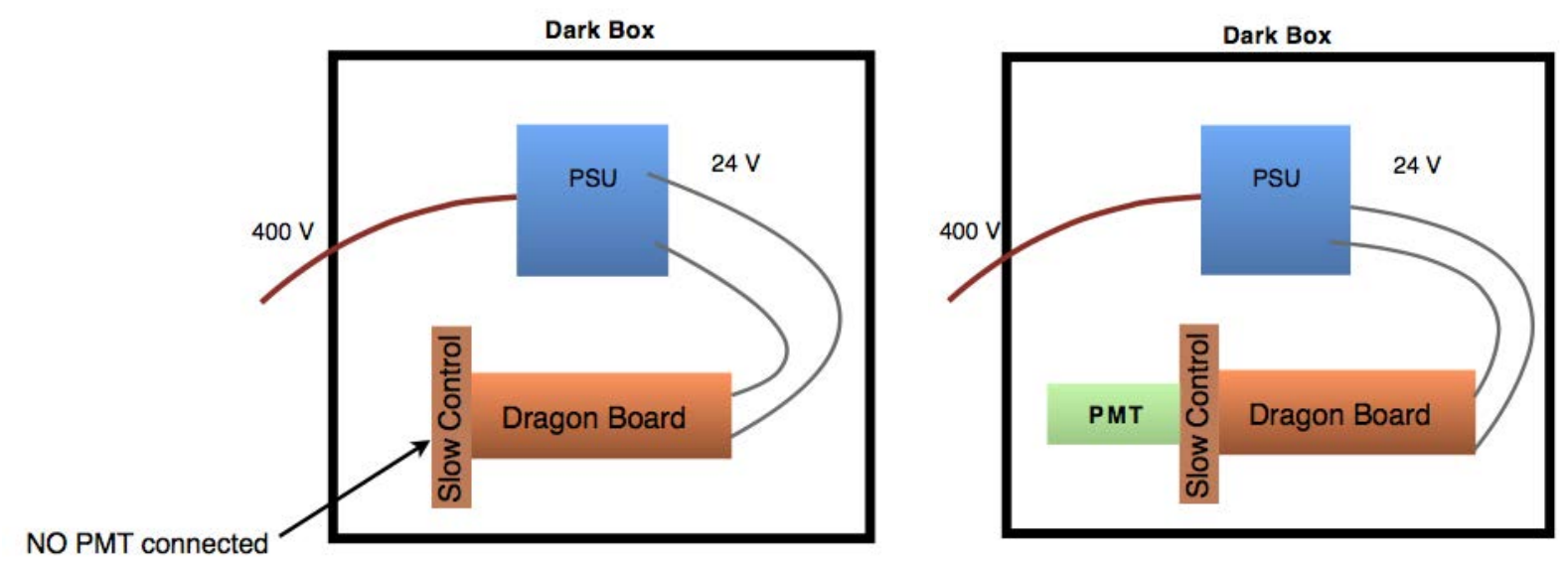

Figure 10.7: Schemes for conducted noise tests when the PMT is powered by the PULS QT40.241 PSU. To perform this measurements the Dragon Board is needed as intermediate between the PSU and the PMT.

$\odot$ PMT powered off, PULS QT40.241 PSU on (unloaded).

$\odot$ PMT powered on, PULS QT40.241 PSU on (unloaded).

$\odot$ PMT powered on, PULS QT40.241 PSU on (loaded).

The load applied to the system was $0.6 \Omega$ in order to obtain the nominal current of $40 \mathrm{~A}$. The scheme in Figure ?? illustrate this configuration.

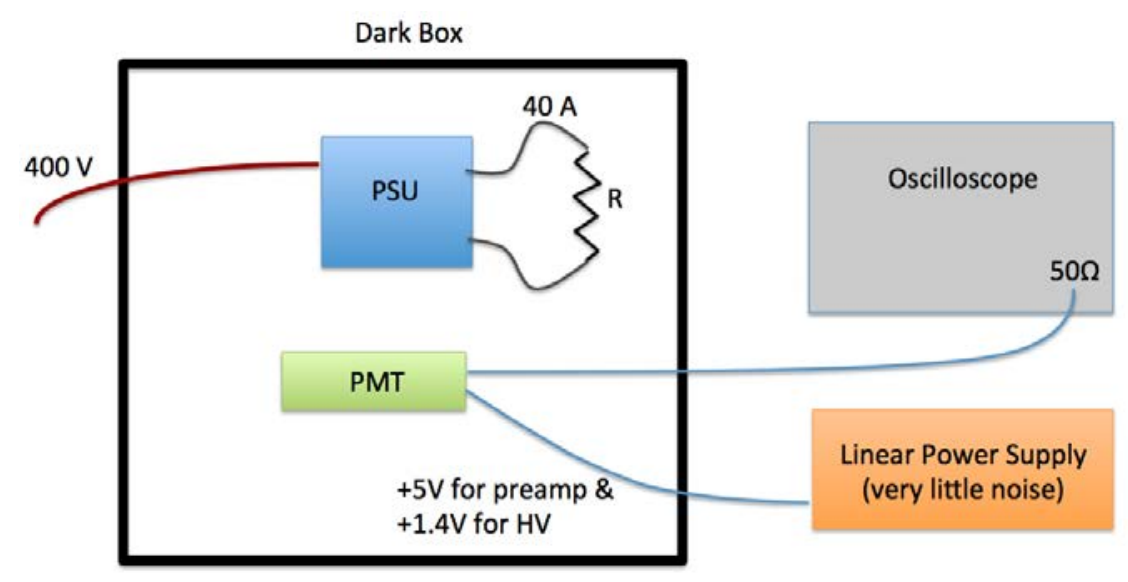

Figure 10.8: Scheme for radiated emission tests. The signal from the PMT powered by the linear PSU, is saved by the oscilloscope. The PULS QT40.241 PSU, device under test, is place close to the PMT inside the dark box. 


\subsubsection{Results}

The test results allow us to conclude that no noise, by conducted or radiated emission, is induced by the linear PSU] or PULS QT40.241 PSU at any possible configuration (see Section ??). For convenience, we only show here the results at which the highest noise is expected, it means, when the PMT is powered on $\left(\mathrm{V}_{c c}=5 \mathrm{~V}\right.$ and $\left.\mathrm{HV}=1.4 \mathrm{kV}\right)$ and the PULS QT40.241 PSU is loaded. In Appendix C you can find the results at different configurations.

\subsubsection{Conducted noise tests}

Linear PSU In Figures ?? and ??, the PMT output signal is shown when it is powered by the linear PSU. The sampling frequency is changed to cover all the above-mentioned range of interest (see Table ??).

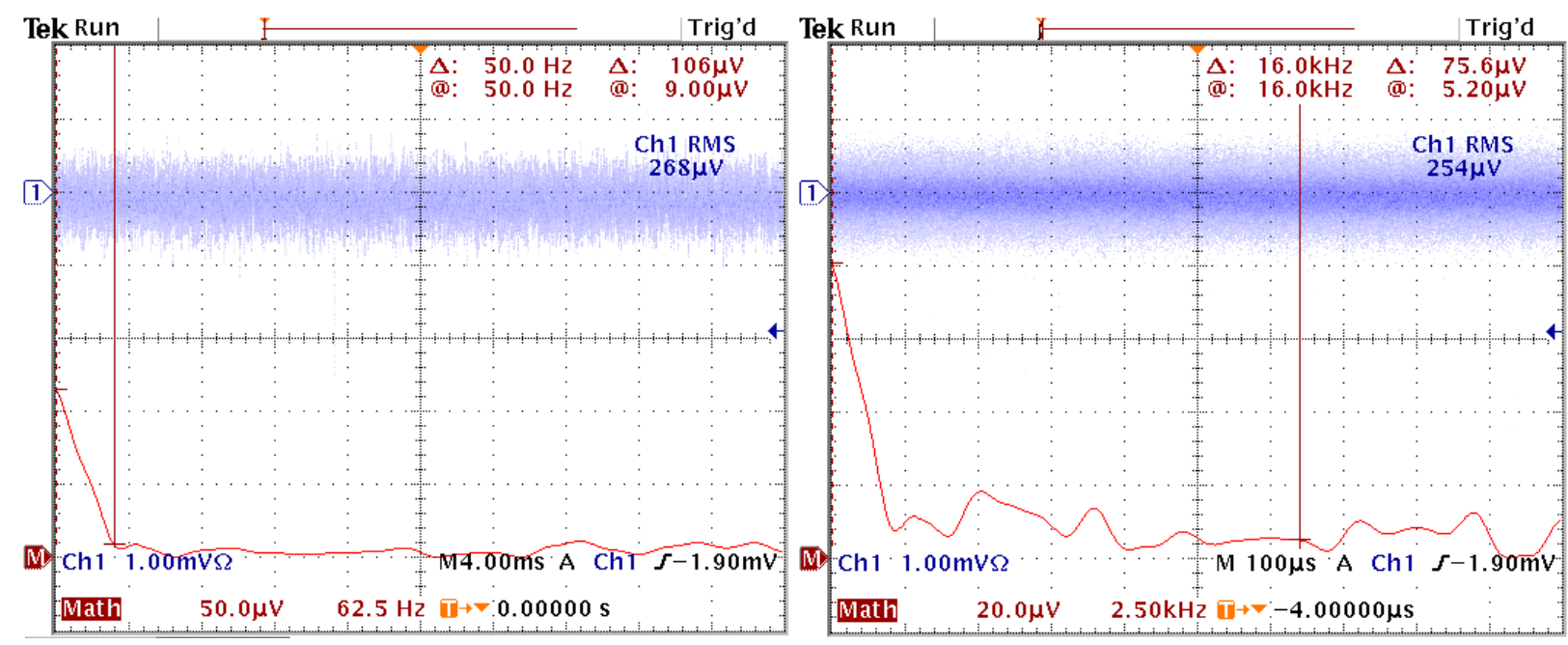

Figure 10.9: Voltage vs Time at different sampling frequency levels of $62.5 \mathrm{~Hz}$ and $2.5 \mathrm{kHz}$, respectively, looking for low and medium frequency noise. The vertical scale is $1 \mathrm{mV} / \mathrm{div}$ for both cases. In red, at the bottom of each plot, the FFT is shown with a vertical scale of $50 \mu \mathrm{V} /$ div and $20 \mu \mathrm{V} /$ div, respectively. For these measurements, all the involved devices were turn on (see Sections ?? and ??). 

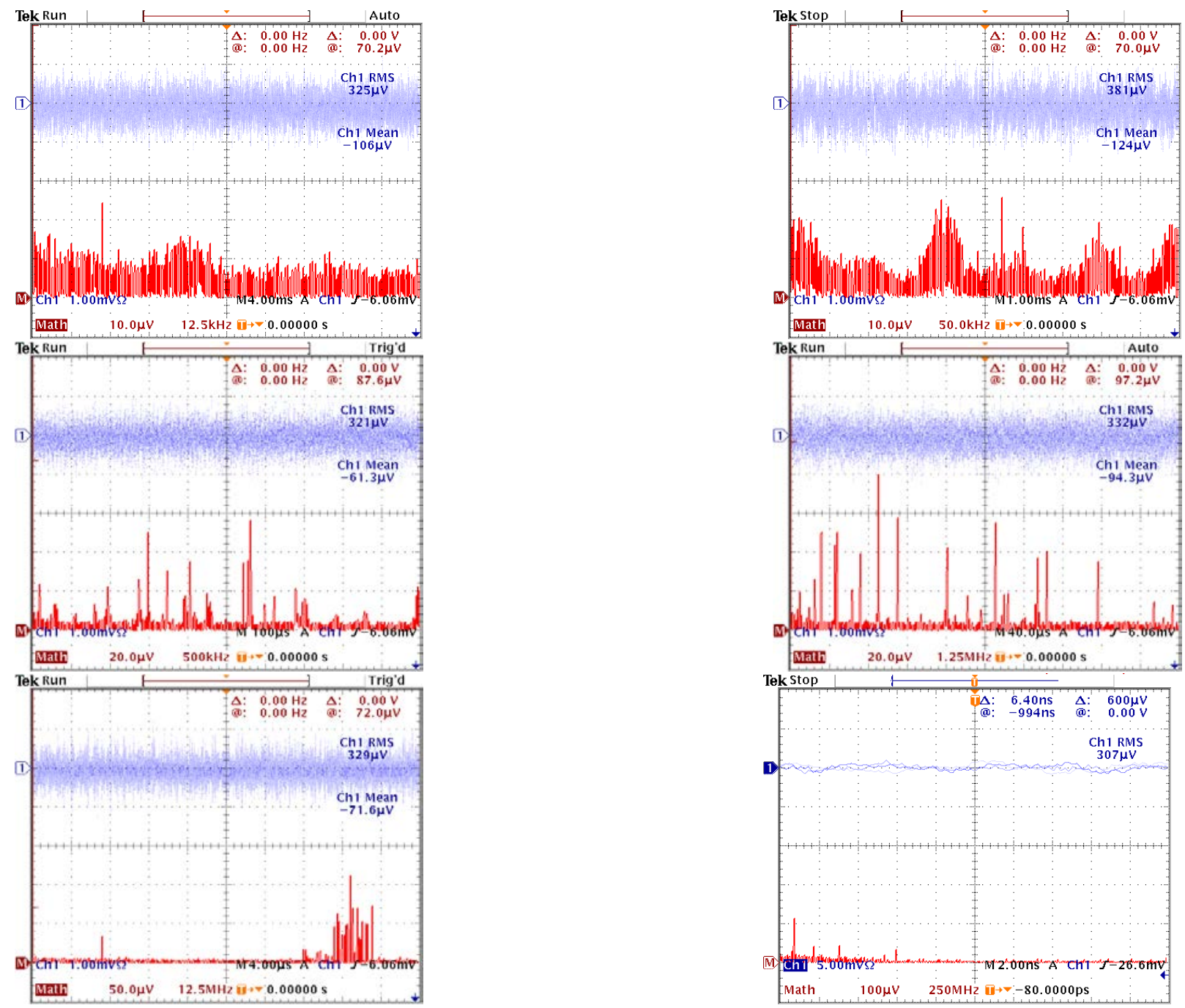

Figure 10.10: Voltage vs Time at different sampling frequency levels of $12.5 \mathrm{kHz}, 50 \mathrm{kHz}, 500 \mathrm{kHz}, 1.25$ $\mathrm{MHz}, 12.5 \mathrm{MHz}$ and $250 \mathrm{MHz}$, respectively, to look for high frequency noise. The vertical scale is 1 $\mathrm{mV} / \mathrm{div}$ for all the plots except for the last one, at $250 \mathrm{MHz}$, whose scale is $5 \mathrm{mV} / \mathrm{div}$. In red, at the bottom of each plot, the FFT is shown with a vertical scale of $10 \mu \mathrm{V} /$ div, $10 \mu \mathrm{V} /$ div, $20 \mu \mathrm{V} /$ div, $20 \mu \mathrm{V} /$ div, 50 $\mu \mathrm{V} / \mathrm{div}$ and $100 \mu \mathrm{V} / \mathrm{div}$, respectively. For these measurements, all the involved devices were turn on (see sections ?? and ??).

PULS QT40.241 PSU The following results were obtained making use of the PULS QT40.241 PSU that powers the readout Dragon Board at which the PMT (through the Slow Control board) is attached (see Section ??). The noise was study from two different ways: obtaining the signal from the trigger path and from the readout path.

$\odot$ Trigger path results: The noise is studied by performing a rate scan at the output of the L1 mezzanine. The rate is the number of events that triggered within a pre-determined time window at a certain DT level. In our study, the width of the time window is $10 \mathrm{~ms}$. 
We change the $\mathrm{DT}$ value from the maximum value at $1.2 \mathrm{~V}$ to $0 \mathrm{~V}$ (negative values are not possible). This decrease is done with two different steps: one of them, the so-called coarse scan, follows steps of $4.8 \mathrm{mV}$ while a more precise one, the fine scan, decreases in two steps of $1.2 \mathrm{mV}$ and one of $2.4 \mathrm{mV}$.

This rate scan test was carried out over 5 different L0+L1 mezzanines. In Figures ??, ??, ??, ?? and ??, we compare the results of the 5 mezzanines applying both coarse and fine scans when using the CTA|PSU and linear PSU to power the Dragon Board. The following results were obtained when no[PMT was attached to the Slow Control board and using the maximum DT L0 value to avoid any incoming signal. For convenience, in Table ??, the results of each configuration are summarized.
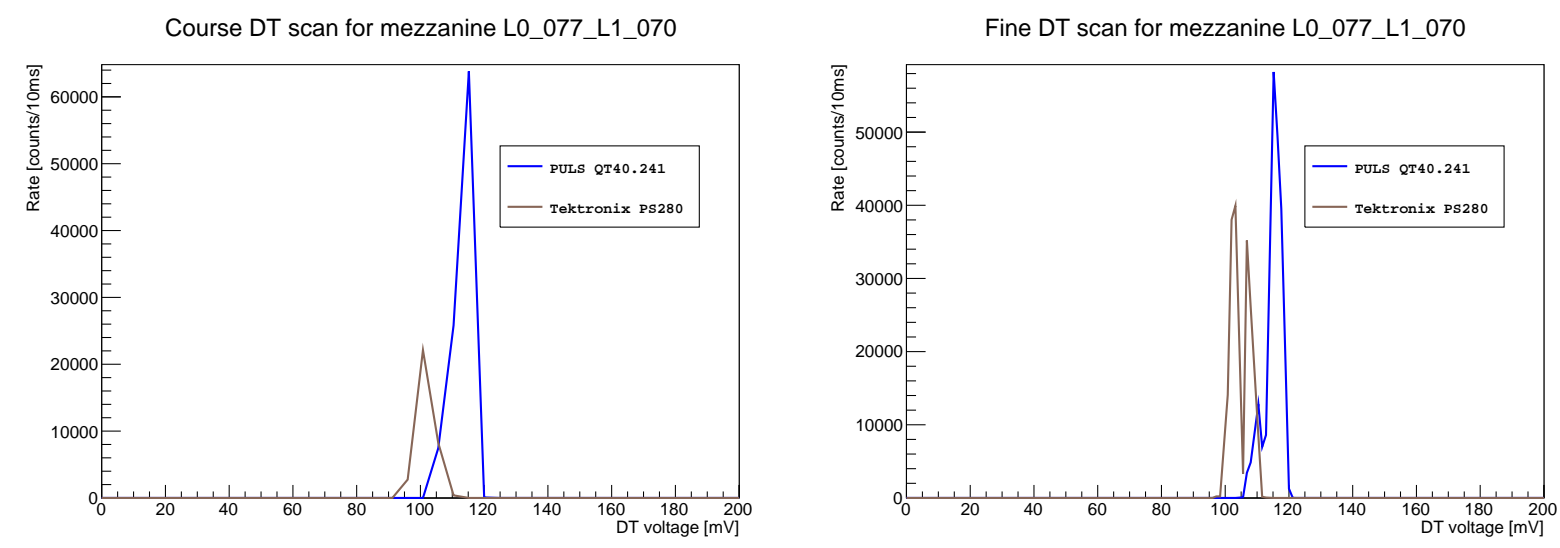

Figure 10.11: glsL1 DT rate scan for the L0+L1 mezzanine L0 077-L1 070. Vertical axis shows rate (counts/10 ms) and X-axis DT voltage in $\mathrm{mV}$. The blue line corresponds to the results from the PULS QT40.241 PSU, the brown one displays linear PSU signal. The leftmost plot shows the results when a course DT scan was perform while the one on the right shows the fine scan.
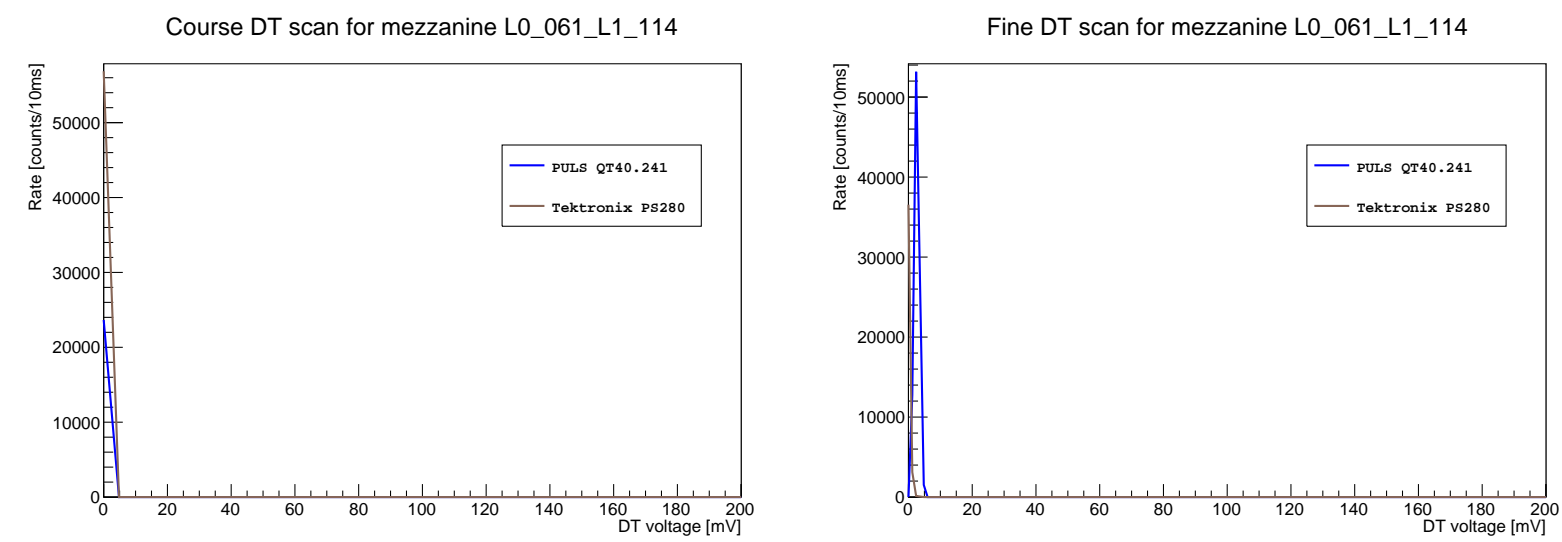

Figure 10.12: Same as Figure ??, but for L0+L1 mezzanine L0 061-L1 114. 

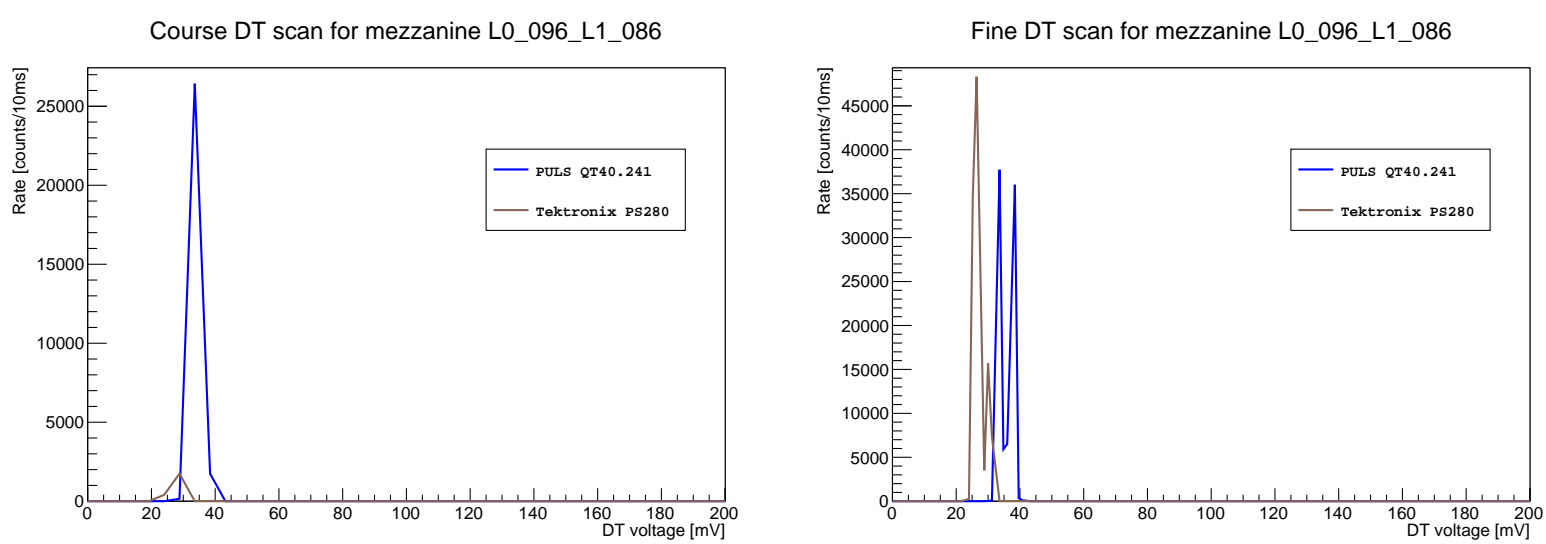

Figure 10.13: Same as Figure ??, but for L0+L1 mezzanine L0 096-L1 086.
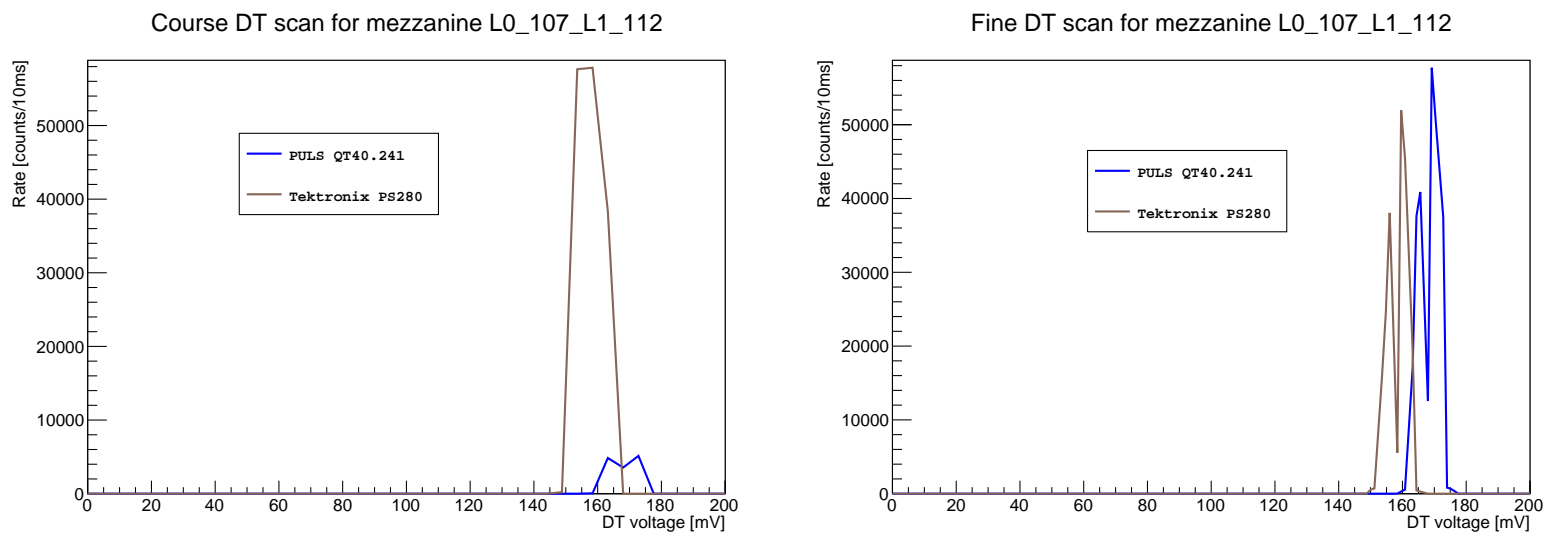

Figure 10.14: Same as Figure ??, but for L0+L1 mezzanine L0 107-L1 112.
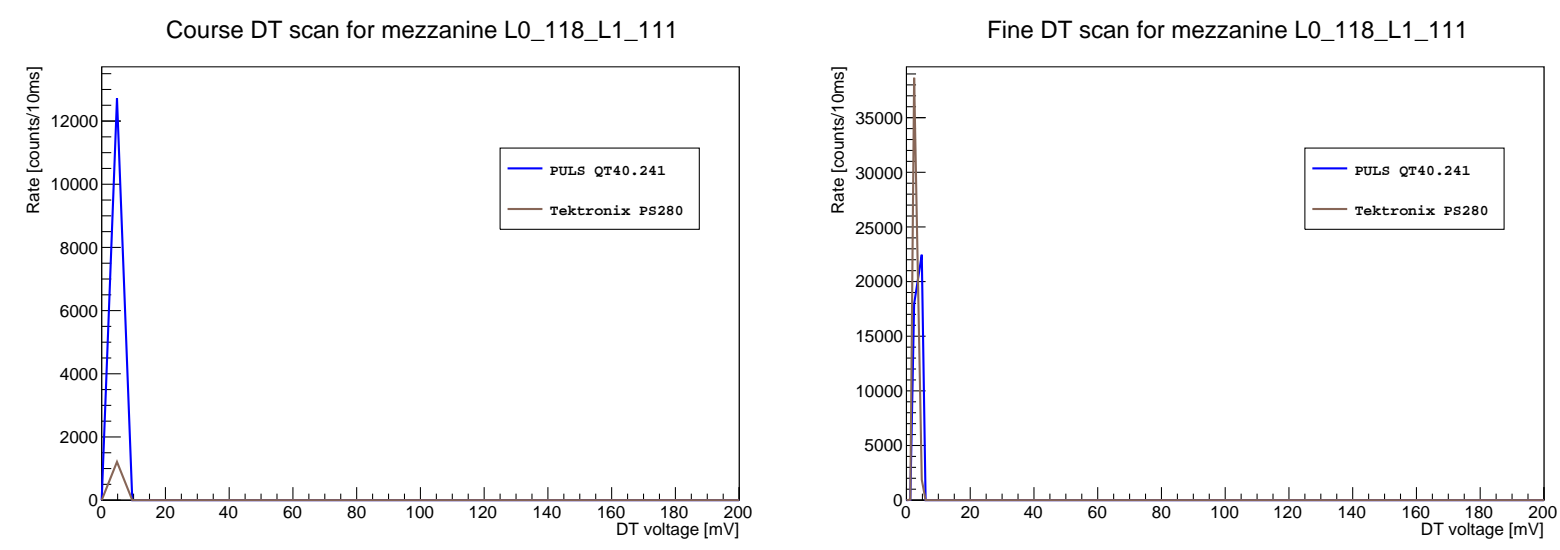

Figure 10.15: Same as Figure ??, but for L0+L1 mezzanine L0 118-L1 111.

There are several things to take into account on these results. First of all, there is only one peak in each case which corresponds to the trigger of the baseline line. The existence on 
only one peak implies that no further noise/signal, besides baseline, triggers. This peak has consistent position in both coarse and fine scans when using different PSU, We can appreciate that it does not peak at $0 \mathrm{mV}$ in all measurements as could be expected. This is due to an offset produced by the electronics (mainly from the ASIC) that will be calibrated during future camera calibration and that does not affect our noise results. Nevertheless, the position of the peak is slightly shifted when the CTA $\mid$ PSU powers the system, which means that a systematic increase of the baseline is produced. Although the origin of this increase is not understood, its value is very low and always below $15 \mathrm{mV}$ (see Table ??).

Another important parameters is the width of the peak that provides information on the amplitude of the noise. In all cases, the width of the peak is narrow, which means that the baseline is confined in short DT range. There are some small differences between the peak's width obtained while powering with the CTA $/$ PSU or linear PSU from one mezzanine to another, but the behavior is not constant or systematic and the difference is always lower than $5 \mathrm{mV}$. This fact allow us to confirm that there is no evidence of a noise increase when we use the CTA|PSU,

In some cases, mainly during the fine scan, we could find double peaks. They are believed to happen because of an overloaded of the rate in a amount of time shorter than the $10 \mathrm{~ms}$. Consequently, the rate is reset to 0 before the integration window finishes giving rise to these structures.

\subsubsection{Radiative noise tests}

In Figure ?? the comparison between the output signal of the PMT when the PULS QT40.241 PSU, placed close by, is powered OFF and ON is shown. We can appreciate that no significant increase of the noise is induced when the PSU is working alongside.

To improve the estimation of the radiated noise, we also obtained a 512 sample averaging signal in the range of $0-500 \mathrm{kHz}$ (see Figure ??). The Y-axis scale for the FFT is $1 \mu \mathrm{V} / \mathrm{div}$. Therefore, the average noise is less than $2 \mu \mathrm{V}$ at most of the frequencies (the noise increases slightly up to $7 \mu \mathrm{V}$ in the range of $0-50 \mathrm{kHz}$ ). 


\begin{tabular}{|c|c|c|c|c|c|}
\hline & \multicolumn{2}{|c|}{ Coarse scan } & \multicolumn{2}{|c|}{ Fine scan } \\
\hline & & Width [mV] & $\begin{array}{c}\text { Voltage at } \\
\text { max. rate }[\mathrm{mV}]\end{array}$ & Width [mV] & $\begin{array}{c}\text { Voltage at } \\
\text { max. rate }[\mathrm{mV}]\end{array}$ \\
\hline \multirow{3}{*}{$\mathrm{L}_{0} 077-\mathrm{L}_{1} 070$} & PULS QT40.421 & 19.2 & 115.2 & 20.4 & 115.2 \\
\hline & Tektronix PS280 & 14.4 & 100.8 & 16.8 & 103.2 \\
\hline & Difference & 4.8 & 14.4 & 3.6 & 12.0 \\
\hline \multirow{3}{*}{$\mathrm{L}_{0} 061-\mathrm{L}_{1} 114$} & PULS QT40.421 & 0 & 0 & 4.8 & 2.4 \\
\hline & Tektronix PS280 & 0 & 0 & 2.4 & 0 \\
\hline & Difference & 0 & 0 & 2.4 & 2.4 \\
\hline \multirow{3}{*}{$\mathrm{L}_{0} 096-\mathrm{L}_{1} 086$} & PULS QT40.421 & 9.6 & 33.6 & 10.8 & 33.6 \\
\hline & Tektronix PS280 & 4.8 & 28.8 & 9.6 & 26.4 \\
\hline & Difference & 4.8 & 4.8 & 1.2 & 7.2 \\
\hline \multirow{3}{*}{$\mathrm{L}_{0} 107 \mathrm{~L}_{1} 112$} & PULS QT40.421 & 14.4 & 172.8 & 16.8 & 169.2 \\
\hline & Tektronix PS280 & 19.2 & 158.4 & 19.2 & 159.6 \\
\hline & Difference & -4.8 & 14.4 & -2.4 & 9.6 \\
\hline \multirow{3}{*}{$\mathrm{L}_{0} 118 \mathrm{~L}_{1} 111$} & PULS QT40.421 & 0 & 4.8 & 3.6 & 4.8 \\
\hline & Tektronix PS280 & 0 & 4.8 & 3.6 & 2.4 \\
\hline & Difference & 0 & 0 & 0 & 2.4 \\
\hline
\end{tabular}

Table 10.2: Conducted noise results for $5 \mathrm{~L} 0+\mathrm{L} 1$ mezzanines. The width of the peak (in $\mathrm{mV}$ ) as well as the DT voltage at which the maximum rate is obtained (also in $\mathrm{mV}$ ) is shown for the two different rate scans, coarse and fine, that differed on the DT steps $(4.8 \mathrm{mV}$ in the former and two steps of $1.2 \mathrm{mV}$ and one of $2.4 \mathrm{mV}$ in the latter). The DT voltage with the maximum rate provides an estimation on the peak position. 

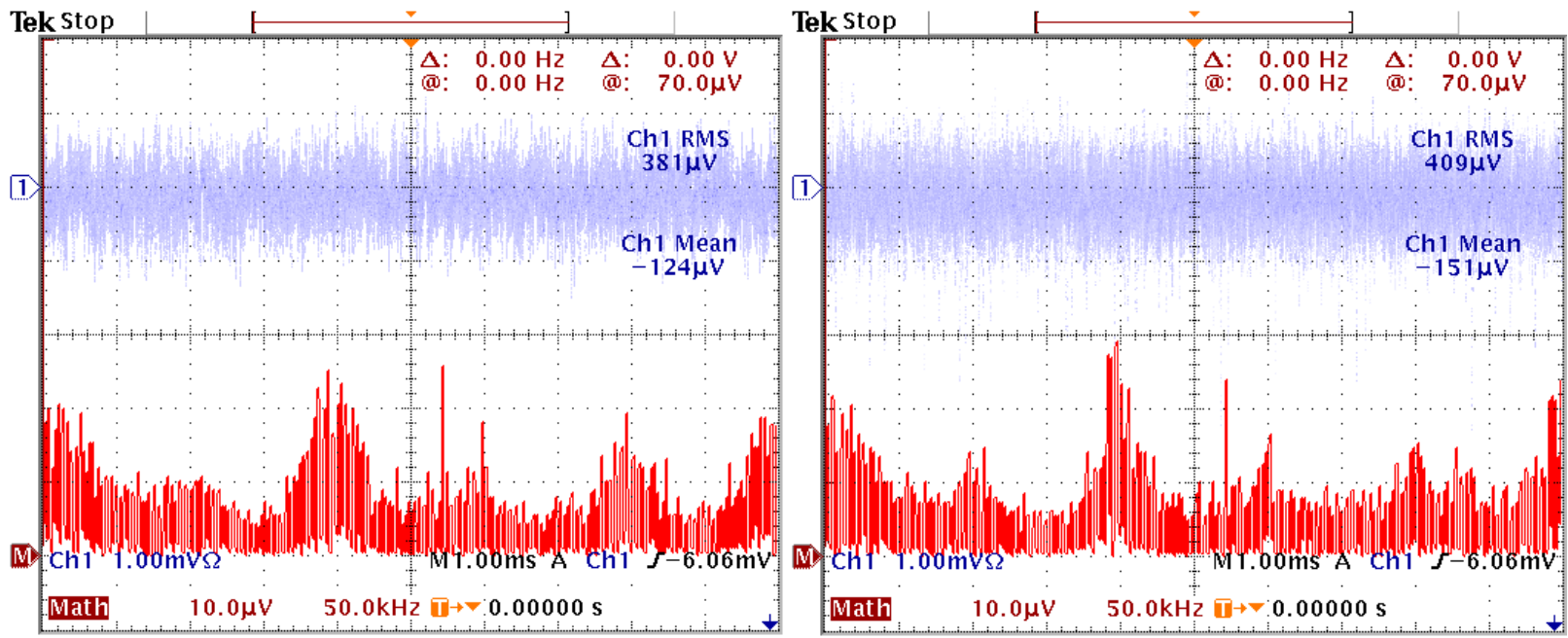

Figure 10.16: Voltage vs Time for a sampling frequency of $50.0 \mathrm{kHz}$. The vertical scale is $1 \mathrm{mV} / \mathrm{div}$. In red, at the bottom of the plot, the FFT is shown with a vertical scale of $10 \mu \mathrm{V} / \mathrm{div}$. For both measurement, the PMT was powered by the linear PSU. On the left, the results obtained with the PULS QT40.241 PSU powered off. On the right, when all the involved devices were turn on (see sections ?? and ??).

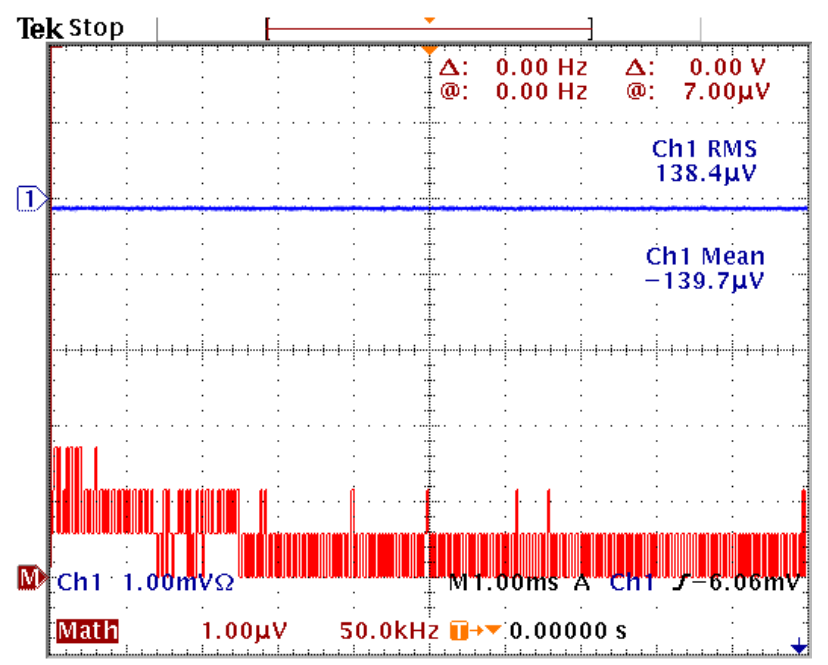

Figure 10.17: Voltage vs Time with 512 sample averaging at a frequency of $50.0 \mathrm{kHz}$. The vertical scale is $1 \mathrm{mV} / \mathrm{div}$. In red, at the bottom of the plot, the FFT is shown with a vertical scale of $1 \mu \mathrm{V} / \mathrm{div}$. For this measurement, all the involved devices were turn on (see sections ?? and ??).

\subsubsection{Conclusions}

No appreciable radiated or conducted noise was detected at the switching frequencies of the PULS QT40.241 PSU. No noise was detected using the linear PSU either. The conducted noise through the Dragon Board's L1 trigger path was analyzed obtaining satisfactory results that yielded to an absence of noise increase when using the PULS QT40.241 PSU. 
10. QUALITY CONTROL OF LST CAMERA SUBSYSTEMS 


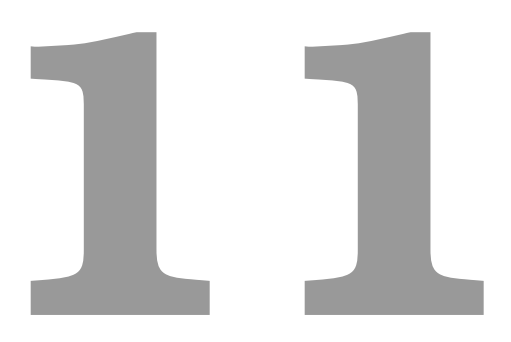

\section{Summary and concluding remarks}

During my thesis, I studied the three best microquasar candidates to emit at VHE Cygnus X-1, Cygnus X-3 and V404 Cygni. For the first time, we firmly detected Cygnus X-1 in the HE regime owing to the analysis of the 7.5 years of Pass 8 Fermi-LAT data. This analysis allowed us to determine that $\mathrm{HE}$ gamma-ray emission only occurs during the $\mathrm{HS}$ and most likely associated to the relativistic jets. In turn, we found a hint of orbital modulation, which could constrain the gamma-ray emission site between $10^{11}-10^{13} \mathrm{~cm}$ from the $\mathrm{BH}$ produced by anisotropic IC on the stellar photon field. The study of this source is complemented by a long-term campaign performed with the MAGIC telescopes for a total of $\sim 100$ hours. No detection was achieved at $\mathrm{VHE}$, which allowed us to discard the jet-medium interaction region as possible production site for VHE gamma rays at the level of MAGIC sensitivity. On the other hand, we observed Cygnus X-3 with MAGIC for a total of $\sim 70$ hours, covering a radio and $\mathrm{HE}$ flare from the beginning until its cessation, whose characteristics at those wavelengths were similar to the outbursts at which the system was detected in the HE regime in 2009. We did not detect the system, possibly related to the unusual high absorption produced by its companion star. Thus, VHElemission, if any, should be produced inside the binary system $\left(<10^{13} \mathrm{~cm}\right.$ from the compact object $)$. Thanks to this deep campaign, we showed that more than even more than 50 hours during flaring activity would be need with the future more sensitive CTA instrument. Finally, we observed the low-mass microquasar V404 Cygni during its outburst period in June 2015, produced after more than 25 years in quiescent state. Observations were carried out during strong X-ray flares and 1 hour simultaneously to a hint in $\mathrm{HE}$. However, detection was not achieved despite the low absorption displayed by the system at distances $>10^{10} \mathrm{~cm}$ from the $\mathrm{BH}$ even during flaring activity. Thus, if the VHE emitter is located in the same region where $\mathrm{HE}$ gamma-ray is produced, our non-detection would imply inefficient particle acceleration inside V404 Cygni jets or not enough 
energetics.

Part of the thesis is also dedicated to the study of PWNe. Here I analyzed 5 promising PWN candidates, given their hosting pulsars' features, observed by MAGIC with no detection or hint of VHE emission. I set these results in context with the deep TeV PWN population study performed by the H.E.S.S. Collaboration, concluding that our candidates are not outliers with respect to the detected sources and hence, gamma-ray emission should be expected. Delving into possible reasons for a non-detection, we conclude that these $5 \mathrm{PWNe}$ are limited by the surrounding low target photon field, which turns the IC mechanism inefficient. We provide a general relation between the target $[\mathrm{IR}$ photon field and the spin-down power of the pulsars for higher probability of VHE gamma-ray detection. In this thesis, it is also shown the first joint work between HAWC Observatory, Fermi-LAT and MAGIC, showing the results for a follow-up studies on detected sources by HAWCWEe investigate the possible $\mathrm{PWN}$ nature of two sources, which allow us to discard any relation between nearby pulsars and the HAWC candidates. MAGIC analysis of these two sources was performed assuming both point-like and extended hypothesis, which provided constraints on a possible radius, likely higher than $0.16^{\circ}$ radius.

I included in my thesis the first VHE gamma-ray results for a Type Ia SN No significant excess was found during the first days after the explosion, which limited the total energy emitted in VHE gamma rays at $<10^{45} \mathrm{erg}$, which is about $10^{-6}$ of the total available energy budget of the $[\mathrm{SN}$ explosion. Making use of a time-dependent flux model for hadronic origin and under certain assumptions, we probed that a power-law density profile is consistent with our results (although more sophisticated theoretical scenarios could shed more light). Following this result, gamma-ray detection is not expected by any current of future generation of IACTs.

Regarding the technical part of my thesis, I worked in the QC tests of several subsystems for the camera of the future LST, mainly on the characterization of PMTs, PSU and trigger mezzanines. With this tests, we were able to probe the high efficiency of the photodetectors and functionality of the trigger mezzanines. Dedicated conductive and radiate noise tests allowed us also to approve the use of the proposed switching $\mathrm{PSU}$ for the LST. 
Appendix 



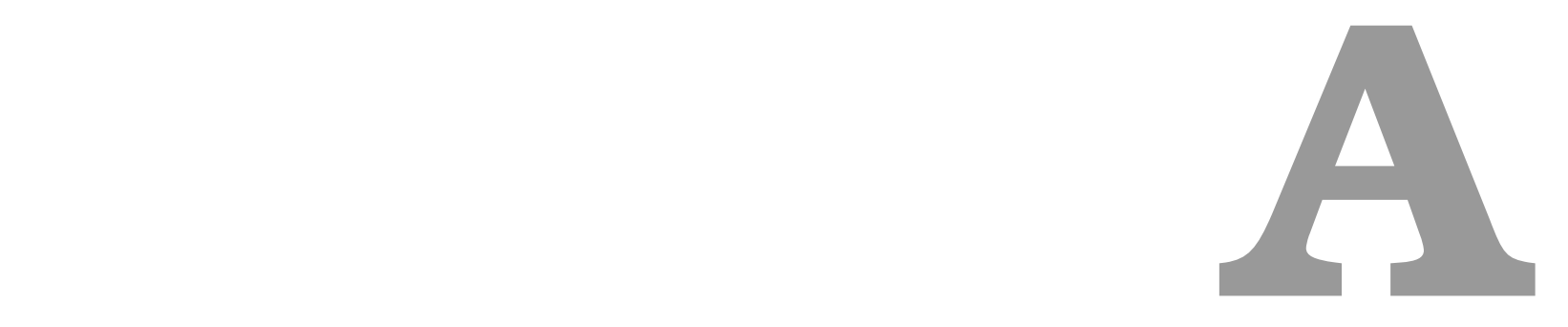

\section{Very-high-energy gamma-ray observations of}

SN 2014J

\section{A.1 The death of a star: types of Supernova}

As shown in Chapter 3.1, after the star reaches a critical mass, known as Chandrasekhar mass $\left(\sim 1.4 \mathrm{M}_{\odot}\right.$; Chandrasekhar 1931), an inevitable explosion that leads to its death takes place. This explosion is the so-called $\mid \mathrm{SN}$ that releases an extremely amount of energy, around $10^{51} \mathrm{erg}$ (Bethe 1993). All this energy, emitted in an unique explosion, corresponds to the totality of energy produce by our Sun in its entire life.

There are several types of $\mathrm{SNe}$, that can be classified according to the amount of $\mathrm{H}$ observed 
in their spectra as well as because of the differences highlighted in their light curves:

$\odot$ Type I SN: The explosions which do not present $\mathrm{H}$ lines in their spectra are classified within this group. In turn, they can be subdivided into three types: Type $I a, I b$ and $I c$. The former display a strong absorption line of ionized Si II (around the wavelength $6510 \AA$ ) close to the maximum. The Type Ib, lacks of such spectral feature of Si II, is characterized by the presence of strong $\mathrm{He}$ lines absorption, while the Type Ic does not show either $\mathrm{Si}$ or $\mathrm{He}$ absorption in their spectrum (see Figure A.1).

$\odot$ Type II SN: Contrary to their counterparts, this type of explosions presents $\mathrm{H}$ line in the spectrum. They can be divided according to their light curves: classified as Type II-L, if after the maximum the luminosity decays linearly, or as Type II-P, if it remains bright (on a plateau) for a few months after reaching the highest luminosity.

Nevertheless, apart from this standard classification, the progenitor, source of energy and remnant of Type Ib and Type Ic $\mathrm{SNe}$ are the same as for Type II SNe, evidencing a clear difference between Type Ia $\mathrm{SNe}$ and their correlative. As a general view, Type II (and consequently, Type $\mathrm{Ib}$ and Ic $\mathrm{SNe}$, originate from the collapse of a massive star once the gravitational force cannot be handle by nuclear reaction in the core: Type Ib loose their $\mathrm{H}$-rich outer layer, revealing the He-rich layer below, whilst Type Ic suffer more mass loss and looses both layers (in all cases, the collapse of an iron core is produced and the differences only concern the layers). All of them leave behind a compact object (either a $\mathrm{NS}$ or a $\mathrm{BH}$ ).

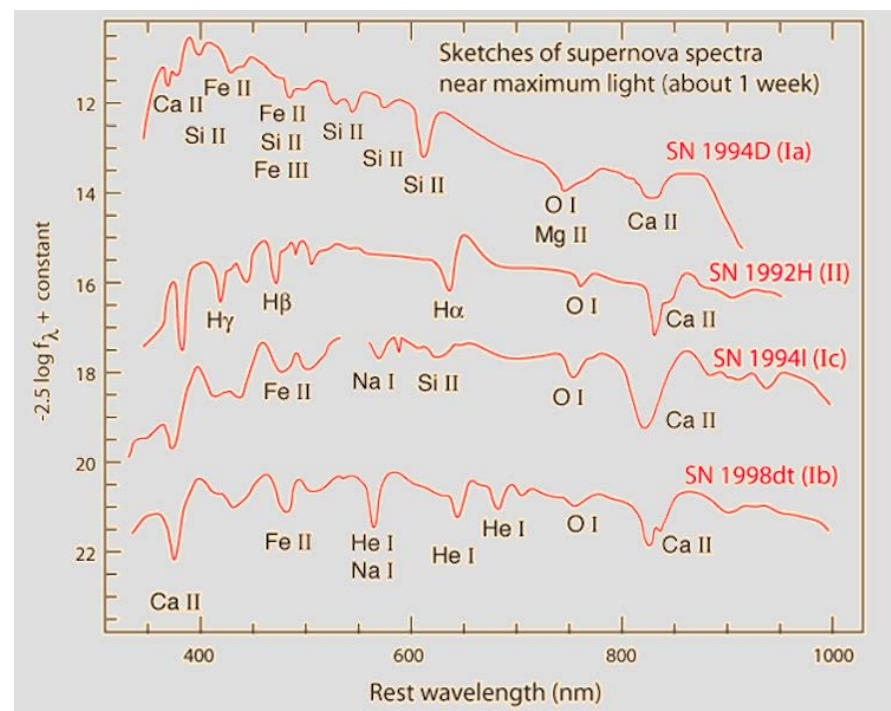

Figure A.1: Sketches of SNe spectra, where the differences on the absorption lines are evidenced between each type (Carroll \& Ostlie 2006, data attributed to Thomas Matheson of National Optical Astronomy Observatory).

On the other hand, Type Ia $\mathrm{SNe}$ originate from the detonation of a primary $\mathrm{WD}$ in a binary 
system that overpasses the Chrandrasekhar mass limit. Unlike the novae ${ }^{1}$, the Type Ia $\mathrm{SNe}$ are not recurrent phenomena: the nuclear energy released in such thermonuclear explosion is that high that no compact remnant is left behind.

There are three types of WDs: $\mathrm{He}, \mathrm{O}, \mathrm{Ne}$ and $\mathrm{C} / \mathrm{O}$ WDs. The former can be excluded as progenitor of Type Ia $\mathrm{SNe}$ as their maximum mass is $\sim 0.45 \mathrm{M}_{\odot}$, otherwise $\mathrm{He}$ would start being burnt in the core. The second type is also excluded because they show Clines in their spectra (which is not a usual feature of these $\mathrm{SNe}$. Therefore, it is believed that the main star of the binary that leads to these thermonuclear explosions are C. $\mathrm{OWDs}$.

The nature of the companion star is still unclear, although two classical scenarios have been promoted: single-degenerate model, in which the WD accretes material from a red giant star (Whelan \& Iben 1973), and the double-degenerate model, in which the explosion is produced by the merging of two WDs (Iben \& Tutukov 1984).

$\odot$ Single-degenerate model: The $\mathrm{WD}$ accretes material from the red giant via Roche lobe overflow or as wind-driven form given the strong wind of the giant companion. This material is $\mathrm{H}$-rich that burns into $\mathrm{He}$ and afterwards into $\mathrm{C}$ increasing the mass of the $\mathrm{WD}$ progressively. However, the accretion rate plays an important role: if it is too low, the $\mathrm{H}$ will be burnt and expelled in the surface giving rise to classical novae; if it is too high, the C will fuse and convert the star into an $\mathrm{O} / \mathrm{Ne} \mid \mathrm{WD}$, which are expected to die as Type II SNe (Saio \& Nomoto 1985). This delicate constraint on the accretion rate decreases the expected number of $\mathrm{SNe}$ arising from this model.

$\odot$ Double-degenerate model: In this scenario, both components of the system are WDs, In this case, the transfer of material does not start due to evolutionary effect but because gravitational energy loss, leading the orbit that the objects follow to shrink and hence, to make them to approach each other. Thus, once one of the WD fills its Roche lobe, the mass transfer begins. As before, the merger rate seems to be insufficient to explain all the Type $\mathrm{Ia}$ SNe,

Still, the evolutionary path that leads to a $\mathrm{C}, \mathrm{OD}$ which exceeds the Chandrasekhar limits is not well understood yet. Moreover, neither the conditions for the successful detonation by assuming any of the scenarios is well-understood. Nevertheless, despite these difficulties, one of the most remarkable features of the sub-class Type Ia $[\mathrm{SNe}$ is that all of them present approximately the same luminosity peak and decay slope, which convert these systems into very interesting objects for cosmological measurements (see, e.g. Carroll \& Ostlie 2006), as the accelerated expansion of our Universe (Perlmutter et al. 1999) or on Galactic chemical evolution (Timmes et al.1995).

All the ejected material by any $\mathrm{SN}$ expands and interacts with the ISM, giving rise to a new astrophysical source known as SNR. SNRs experiment different phases along their evolution. However, the aim of this Appendix is to focus on the results of SN 2014J, a Type Ia SN] observed

\footnotetext{
${ }^{1}$ Novae are abrupt increases of the luminosity on a WD binary system in a very short period time $(\sim$ days $)$, which can be recurrent.
} 
by MAGIC at its very early stage and so, in the following I will only describe the first phase of a SNR. For a more comprehensive and detailed information on this type of sources, the reader is referred to Reynolds (2008).

The first stage of a SNR evolutionary path is the so-call free expansion phase, in which the ejected material expands without being decelerated. This phase is independent of the nature of the $[\mathrm{SN}$ (Type I or II), since the energy and density of the ejected material always exceed the values of the surrounding medium right after the explosion. The wave shock created by the explosion moves within the more or less homogeneous interstellar gas at supersonic velocities $\left(v_{e j} \sim 10^{4}\right.$ $\mathrm{km} / \mathrm{s}$ ). Although ISM can be assumed roughly homogeneous, this is not always true: progenitors of Type II and single-degenerate Type Ia $\mathrm{SNe}$ might create surrounding circumstellar medium (with a density profile of $\rho \propto r^{-2}$ ) due to their strong stellar wind in which the SNR evolves. However, small scales inhomogeneities won't affect the structure of the ejecta. Only major inhomogeneities, like circumstellar medium in equatorial disks or jet-driven $\mathrm{SNe}$, can disturb the symmetry, which are not typical and hence, homogeneous medium are normally assumed in the discussion of SNRs at early phases. Based on this assumption then, during this first stage, the ISM does not influence in the expansion of the shock front, i.e. the pressure applied by the ISM is negligible. As the strong shock front expands, the interstellar gas accumulates, separated from the ejected material by the so-called contact discontinuity ${ }^{1}$. At this point, a reverse shock starts forming behind this contact discontinuity. The classical criterion that terminates this phase is the equality between the mass of the ISM (compressed between the shock front and the contact discontinuity) and the initial ejected stellar mass $\left(M_{e j}\right)$. When this happens the SNR displays the denominated sweep-up radius, $R_{s w}$, which depends on the initial density of the ISM $n_{0}$ :

$$
M_{e j}=\frac{4 \pi}{3}\left(R_{s w}^{3} n_{0}\right) \Rightarrow R_{s w}=\left(\frac{3 M_{e j}}{4 \pi n_{0}}\right)^{1 / 3}
$$

This radius is reached at a time of $t_{s w}=R_{s w} / v_{e j}$, that can expand hundred of years depending on the surrounding medium. At the end of this phase, the reverse shock accumulates enough mass and starts moving into the opposite direction of the ejected material. This inward movement heats the ejected material to high temperatures, a flat pressure structure is developed and therefore, the expansion of the $\mathrm{SNR}$ is produced by the thermal pressure of the hot gas. This is the end of the first stage and the beginning of the so-called Sedov-Taylor phase.

\section{A.2 Introduction to SN 2014J}

On the $21^{\text {st }}$ of January 2014 MJD 56678), SN 2014J was detected by the University College London (UCL) Observatory (Fossey et al. 2014) and classified as a Type Ia SN] with the Dual

\footnotetext{
${ }^{1}$ The contact discontinuity is the the surface between two different materials with similar pressure and velocities but different densities.
} 
Imaging Spectrograph on the Astrophysical Research Consortium (ARC) $3.5 \mathrm{~m}$ telescope (22 $2^{\text {nd }}$ of January; Goobar et al. 2014). It is located in the starburst galaxy M82 at a distance of 3.6 Mpc (Karachentsev \& Kashibadze 2006). Its proximity has granted it the title of the nearest Type Ia SN since 1972 and motivated large multiwavelength follow-up observations from radio to VHE gamma rays.

Deep studies of color excess and reddening estimation were carried out on SN 2014J, phenomena associated to the interstellar extinction of the radiation due to its absorption or scattering by gas or dust in the medium. Type Ia $\mathrm{SNe}$ represent good candidates for reddening studies given their very high luminosity and similarity from one to another. Amanullah et al. (2014) reported, for the first time, a characterization of the reddening of a Type Ia SN] in a full range from $0.2 \mu \mathrm{m}$ to $2 \mu \mathrm{m}$. Their results, with reddening values of $E_{B-V} \sim 1.3$ and $R_{V} \sim 1.4$, are compatible with a powerlaw extinction, expected in the case of multiple scattering scenarios. In the same wavelength band, from UV to NIR, Foley et al. (2014) found reddening parameter values of $E_{B-V} \sim 1.2$ and $R_{V} \sim 1.4$. In this case, the extinction is explained to be caused by a combination of the galaxy dust and a dusty circumstellar medium. However, although compatible with the former extinction law (at a low value of $R_{V} \sim 1.4$ ) and consistent as well with previously mentioned results, Brown et al. (2015), making use of Swift-UVOT data, suggested that most of the reddening is caused by the interstellar dust. Optical and NIR linear polimetric observations of the source presented in Kawabata et al. (2014) supports the scenario where the extinction is mostly produced by the interstellar dust. These evidences favor the double-degenerate scenario for SN 2014J, where less circumstellar dust is expected than in cases with a giant companion star. This type of companion are indeed ruled out by several authors as possible progenitors in SN 2014J, e.g. Pérez-Torres et al. (2014), with the most sensitive study of a Type Ia SN in the radio band, or Margutti et al. (2014), in the X-ray band. The former reported non-detection from the observations performed with eMERLIN and EVN. These results, compared with detailed modeling of the radio emission from the source, allowed them to exclude the single-degenerate scenario in favour of the double-degenerate one with constant density medium of $n \lesssim 1.3 \mathrm{~cm}^{-3}$.

Several authors have speculated about the possibility of $\mathrm{SN}$ explosions being able to produce gamma-ray emission at detectable level by current and/or future telescopes. This gamma-ray emission is associated to the diffuse particle acceleration that the strong shock fronts of extreme detonations like the $\mathrm{SNe}$ produce. This diffuse acceleration is a type of Fermi acceleration that happens in shock waves and hence, would accelerate ambient particles by advection into the shock. However, these models generally consider Type II SNe due to the strong wind of the progenitors which provide larger amount of targets (e.g. Kirk et al. 1995 and Tatischeff 2009). Nevertheless, given the proximity of SN 2014J, this event provides a good exploratory opportunity to probe the eventual production of $\mathrm{VHE}$ gamma rays during the first days after such an explosion. 


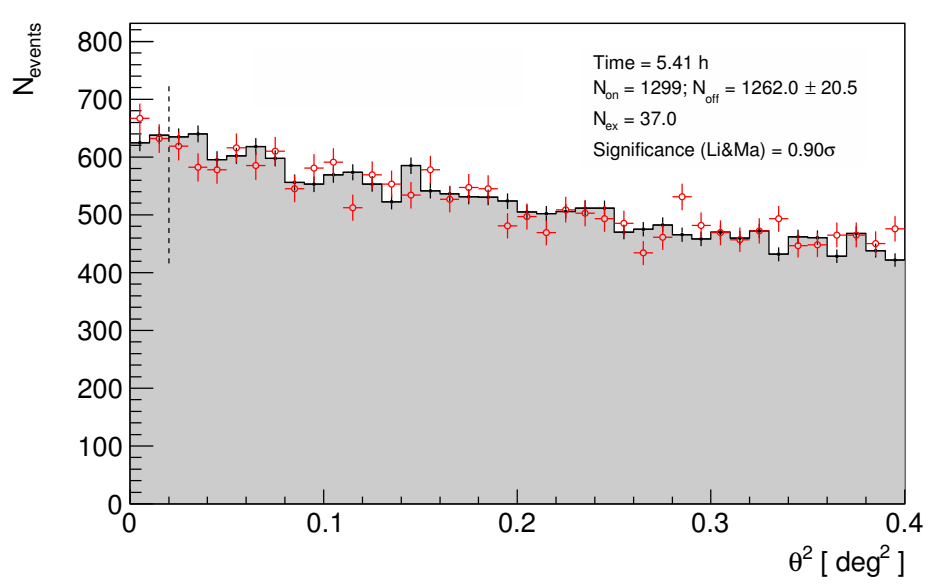

Figure A.2: Distribution of the squared angular distance, $\theta^{2}$, after 5.41 hours of observation between the reconstructed arrival direction of the gamma-ray candidate events and the position of the source in the camera (red empty circles). The $\theta^{2}$ distribution of the background events (black points) is also displayed. The vertical dashed line at $\theta^{2}=0.02 \mathrm{deg}^{2}$ defines the expected signal region.

\section{A.3 MAGIC observations and results}

SN 2014J was observed with the MAGIC telescopes under moderate moonlight conditions from the $27^{\text {th }}$ to the $29^{\text {th }}$ of January and on the $1^{\text {st }}$ and $2^{\text {nd }}$ of February under dark-night conditions at medium zenith angles (from $40^{\circ}$ to $52^{\circ}$ ). Our observations, performed using the wobble-mode (see Section 2.4.2.1), started six days after the first detection by the UCL Observatory because of adverse weather conditions. The complete data set up to $50^{\circ}(\sim 5.5$ hours $)$ was used for the analysis given the overall good quality of the data (concerning weather, light conditions and performance of the system).

The analysis was performed using the analysis pipeline described in Chapter 2.4.3. Figure A.2 shows the $\theta^{2}$ distribution, i.e the squared angular distance between the reconstructed gammaray direction and the position of either SN 2014J (on-source histogram) or the center of the background control region (off-source histogram). Standard FR cuts were used, which means that a selection of $\theta^{2}<0.02 \mathrm{deg}^{2}$, hadronness $<0.28$ and size $>60$ phe (in both telescopes) was applied. The resulting excess of the on-source histogram over the background from the region, where gamma-ray events from SN 2014J are expected is compatible with zero excess. The significance computed using equation 2.14 is $0.90 \sigma$.

ULs on the flux were computed for $95 \%$ C.L. assuming a power-law spectrum, $\mathrm{d} F / \mathrm{d} E \propto$ $E^{-\Gamma}$, with photon index of 2.6. Variations of $\sim 20 \%$ in the photon index produced changes in the integral ULs of less than 5\%, and hence small deviations from the used value do not critically affect the reported ULs. The ULs above $300 \mathrm{GeV}$ and $700 \mathrm{GeV}$ for the single-night observations are reported in Table A.1 and depicted in Figure A.3

After $\sim 5.5$ hours of observations with the MAGIC telescopes, we establish an integral UL 


\begin{tabular}{|c|c|c|c|c|c|}
\hline \multicolumn{2}{|c|}{ Date } & \multirow{2}{*}{$\begin{array}{c}\text { Eff. Time } \\
\text { [hours] }\end{array}$} & \multirow{2}{*}{$\begin{array}{l}\mathrm{Zd} \\
{\left[{ }^{\circ}\right]}\end{array}$} & \multirow{2}{*}{$\begin{array}{l}\mathrm{UL}(E>300 \mathrm{GeV}) \\
\text { [photons } \mathrm{cm}^{-2} \mathrm{~s}^{-1} \text { ] }\end{array}$} & \multirow{2}{*}{$\begin{array}{l}\mathrm{UL}(E>700 \mathrm{GeV}) \\
\text { [photons } \mathrm{cm}^{-2} \mathrm{~s}^{-1} \text { ] }\end{array}$} \\
\hline [yyyy-mm-dd] & |MJD & & & & \\
\hline $2014-01-27$ & 56684.23 & 0.43 & $47-50$ & $1.03 \times 10^{-11}$ & - \\
\hline 2014-01-28 & 56685.06 & 1.41 & $40-43$ & $2.19 \times 10^{-12}$ & $1.55 \times 10^{-12}$ \\
\hline 2014-01-29 & 56686.09 & 1.30 & $40-42$ & $4.55 \times 10^{-12}$ & $5.97 \times 10^{-13}$ \\
\hline 2014-02-01 & 56689.07 & 0.98 & $40-42$ & $3.14 \times 10^{-12}$ & $9.98 \times 10^{-13}$ \\
\hline 2014-02-02 & 56690.08 & 1.30 & $40-42$ & $3.35 \times 10^{-12}$ & $1.76 \times 10^{-12}$ \\
\hline Total & - & 5.41 & $40-50$ & $1.30 \times 10^{-12}$ & $4.10 \times 10^{-13}$ \\
\hline
\end{tabular}

Table A.1: Summary of the MAGIC observations of SN 2014J. From left to right: date of the beginning of the observations, also in MJD, effective time, zenith angle range and integral ULs at 95\% C.L. above 300 and $700 \mathrm{GeV}$. The last row reports the integral ULs derived with the entire data sample. Due to low statistics, no integral $\mathrm{UL}$ was computed for energies above $700 \mathrm{GeV}$ for the first day of observations.

on the gamma-ray flux for energies above $300 \mathrm{GeV}$ of $1.3 \times 10^{-12}$ photons $\mathrm{cm}^{-2} \mathrm{~s}^{-1}$ at $95 \%$ C.L. which corresponds to $1.0 \%$ in units of the Crab Nebula flux (C.U.) in the same energy range. For energies above $700 \mathrm{GeV}$, the integral UL is $4.1 \times 10^{-13}$ photons $\mathrm{cm}^{-2} \mathrm{~s}^{-1}$, corresponding to $1.1 \%$ C.U. at the same C.L. Our ULs for $E>700 \mathrm{GeV}$ are already close to the flux from the host galaxy M82 measured by VERITAS/in the same energy range, $\left(3.7 \pm 0.8_{\text {stat }} \pm 0.7_{\text {syst }}\right) \times$ $10^{-13}$ photons $\mathrm{cm}^{-2} \mathrm{~s}^{-1}$ (VERITAS Collaboration et al. 2009), which constitutes an irreducible background for our measurement. Under the hypothesis that M82 has a gamma-ray spectrum of $\mathrm{d} F / \mathrm{d} E=3 \times 10^{-16}(E / 1000 \mathrm{GeV})^{-2.5}$ photons $\mathrm{cm}^{-2} \mathrm{~s}^{-1} \mathrm{GeV}^{-1}$, as measured by VERITAS, the expected number of excess events in our observations would be 9.4 , with a $95 \%$ C.L. lower limit at -6.1 (obtained by means of the full likelihood method, see Chapter ??). The observed number of excess events by MAGIC is -4.2 , with an associated p-value of $8.4 \times 10^{-2}$, hence consistent with the VHE flux of M82 measured by VERITAS (see Figure A.4).

\section{A.4 Discussion}

As mentioned before, $\mathrm{VHE}$ gamma-ray emission can be expected from the $\mathrm{SN}$ explosion and its remnant by the strong shock front produced. In the literature, both hadronic and leptonic origin have been discussed (Aharonian 2013). In the former, gamma rays result from the decay of neutral pions, $\pi^{0}$, as a consequence of the inelastic collisions between the protons accelerated in the $\mathrm{SN}$ and the ambient atomic nuclei. In the leptonic scenario, the most efficient mechanism to radiate VHE gamma rays seems to be the $\mathrm{IC}$ process of the accelerated electrons on the ambient photons. In both cases, the environment plays an important role for the production of VHE gamma-ray radiation. A near and young supernova ( $\sim 1$ week old) emitting in this energy regime could shed light on the progenitors of these thermonuclear stellar explosions.

Although we did not detect VHE gamma rays right after the explosion, using the known distance of M82 ( $d_{\mathrm{M} 82}=3.6 \mathrm{Mpc}$ Karachentsev \& Kashibadze 2006) and assuming, as before, a 


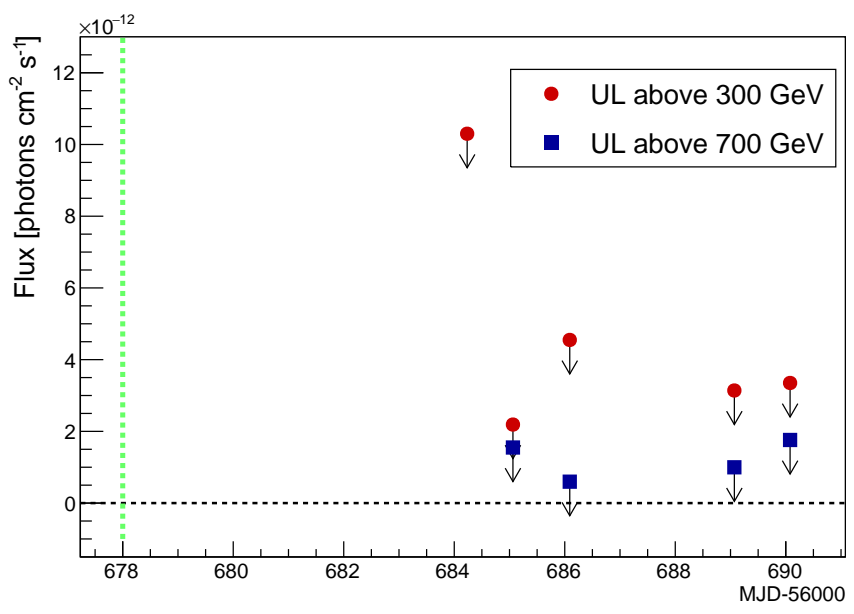

Figure A.3: MAGIC daily integral ULs from the direction of SN 2014J for energies above $300 \mathrm{GeV}$ (red circles) and $700 \mathrm{GeV}$ (blue squares). The integral UL for energies above $700 \mathrm{GeV}$ was not computed for the first night (MJD 56684) due to low statistics (see also Table A.1). The horizontal black dashed line indicates zero flux level and the vertical green line indicates the day of the SN explosion (MJD 56678), just six days before the beginning of the MAGIC observations.

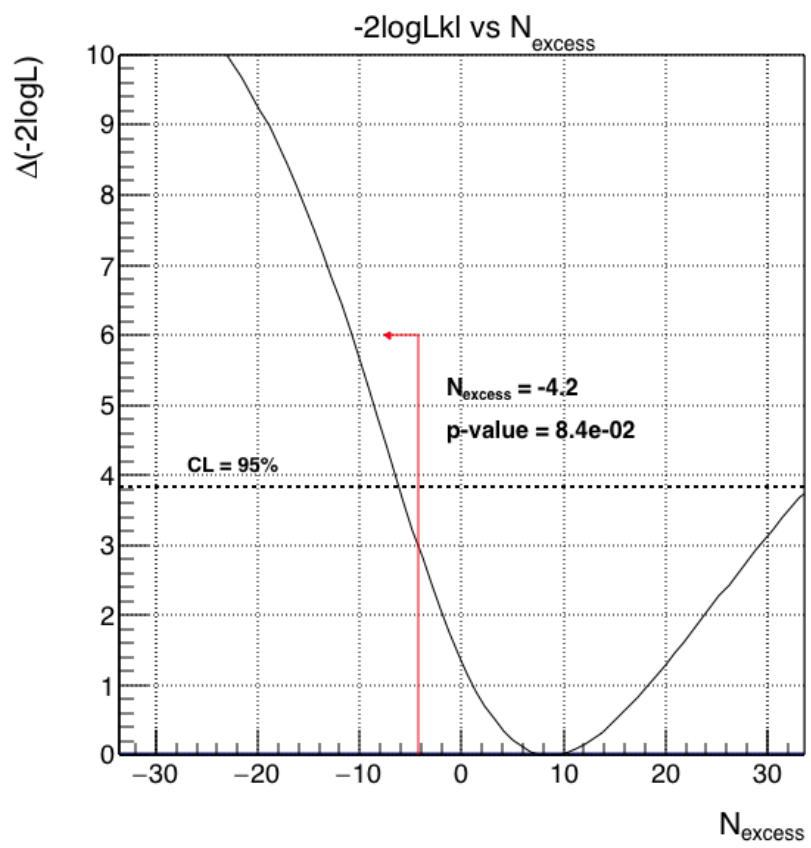

Figure A.4: Full likelihood output for the SN 2014J, assuming the spectral shape measured by VERITAS for the host galaxy M82 and the IRF of MAGIC. The expected number of excess 9.4 has a lower 95\% C.L. of -6.1 events, compatible with the excess of -4.2 events found by MAGIC at energies above $700 \mathrm{GeV}$, making VERITAS and MAGIC results consistent to each other. 
photon index of 2.6, one can convert the measured flux UL into an UL on the power emitted into VHE gamma rays. Therefore, given the integral UL for energies greater than $300 \mathrm{GeV}$, $1.3 \times 10^{-12}$ photons $\mathrm{cm}^{-2} \mathrm{~s}^{-1}$, the resulting UL on the power emitted is of the order of $10^{39} \mathrm{erg} \mathrm{s}^{-1}$. If one now assumes an emission period of the order of 10 days, the total energy emitted in $\mathrm{VHE}$ gamma rays during this period is smaller than $10^{45} \mathrm{erg}$, which is about $10^{-6}$ of the total available energy budget of the $\mathrm{SN}$ explosion $\left(\sim 10^{51} \mathrm{erg}\right.$, Bethe 1993).

Models of the evolution of young SNRs can be used to estimate the expected emission from the region in the future. One of the most important parameters to be assumed is the density profile of the SN ejecta. In this work, we considered a simple power-law density profile, which allow us to use the Dwarkadas (2013) model to obtain an analytic solution for the estimated flux. Other density profiles have been used in the literature: Models like W7 or WDD1, applied by Nomoto et al. (1984) and Iwamoto et al. (1999), are usually utilized in Type Ia SN] studies, but they are based in the single-degenerate scenario. Dwarkadas \& Chevalier (1998) discussed a possible exponential density profile which could represent better the $\mathrm{SN}$ ejecta structure than the power-law one. However, the exponential profile cannot provide an analytic result as the one assumed in this work, which can give a correct solution within the order of magnitude, as explained in Dwarkadas (2013).

Thus, making use of equation 10 in Dwarkadas (2013), one can obtain the time-dependent emission assuming a hadronic origin. Considering only this hadronic origin, we can establish a lower limit on the total gamma-ray radiation. As discussed above, gamma-ray emission can be also expected from $I \mathrm{IC}$ processes. Nevertheless, purely leptonic scenario have been questioned and discarded by several authors, e.g. Völk et al. (2008).

The expected flux depends strongly not only on the assumed density structure of the SNR but also on the density profile of the surrounding ISM. As shown by different authors, we can consider double-degenerate scenario in the case of SN 2014J, i.e. two WDs progenitors. WDS do not suffer wind-driven mass-loss and therefore are not expected to modify the surrounding medium, although different assumptions, from the lack of certainty on the progenitors, have also been studied (see e.g. Dwarkadas 2000). We can then assume that the Type Ia SN explosion took place in a constant density medium. In this work, we used a density of $n=2.2 \times 10^{-24}$ $\mathrm{g} / \mathrm{cm}^{3}$ (Pérez-Torres et al. 2014), assuming that all the content in the hot galaxy of the source, M82, stems from the neutral hydrogen, $\mathrm{H}_{\mathrm{f}}$. This homogeneous medium assumption leads to an increasing flux emission, above a certain gamma-ray energy, with time in the free-expansion SNR stage, as shown below in the expression given by Dwarkadas (2013):

$$
F_{\gamma}(>1 \mathrm{TeV}, t)=\frac{3 q_{\gamma} \xi\left(\kappa C_{1}\right)^{5} m^{3}}{6(5 m-2) \beta \mu m_{p} d^{2}} n^{2} t^{5 m-2}
$$

where the assumed parameters in this work are

$\odot q_{\gamma}=1 \times 10^{-19} \mathrm{~cm}^{3} \mathrm{~s}^{-1} \mathrm{erg}^{-1} \mathrm{H}$-atom ${ }^{-1}$ (for energies greater than $1 \mathrm{TeV}$ ) is the emissivity of gamma rays normalised to the cosmic ray energy density tabulated in Drury et al. (1994). 
This value corresponds to a spectral index of 4.6 of the parent cosmic ray distribution, which was selected according to the assumed spectral index in this work, $\Gamma=2.6$;

$\odot \xi=0.1$ is the fraction of the total $\mathrm{SN}$ explosion energy converted to cosmic ray energy, so an efficient cosmic ray acceleration is assumed;

$\odot \kappa=1.2$ is the ratio between the radius of the forward shock and the contact discontinuity (which separates ejecta and reverse shock);

$\odot C_{1}=1.25 \times 10^{13} \mathrm{~cm} / \mathrm{s}^{m}$ is referred to as a constant related to the kinematics of the $\mathrm{SN}$. This value is calculated from the relation given by Dwarkadas (2013), $R_{\text {shock }}=\kappa C_{1} t^{m}$. In turn, $R_{\text {shock }}$ is obtained from equation 2 in Gabici et al. (2016), by assuming an explosion energy of $10^{51} \mathrm{erg}$, a mass of the ejecta of $1.4 \mathrm{M}_{\odot}$ and a ISM density of $1.3 \mathrm{~cm}^{-3}$, whose value is constrained by Pérez-Torres et al. (2014);

$\odot \beta=0.5$ represents the volume fraction of the already shocked region from which the emission arises ;

$\odot \mu=1.4$ is the mean molecular weight;

$\odot m_{p}=1.6 \times 10^{-24} \mathrm{~g}$ is the proton mass;

$\odot d=3.6 \mathrm{Mpc}$ is the distance to our source;

$\odot t$ is the elapsed time since the explosion; and

$\odot m$ is the expansion parameter.

The expansion parameter varies along the free-expansion phase in different ways according to the assumed model for the density structure of the $\mathrm{SN}$ ejecta after the explosion. For the discussion of this source, I make use of the power-law profile with a density proportional to $R^{-7}$ (Chevalier 1982), where $R$ is the outer radius of the ejecta. The initial value of the expansion parameter is very unalike depending on the density profile assumed, but in all cases evolve to $m=0.40$ (Dwarkadas \& Chevalier 1998). This limit at 0.40 is constrained by the beginning of the Sedov-Taylor phase.

The expansion parameter for the power-law profile keeps constant at 0.57 in the first years of the free-expansion stage. Given this value, the expected flux above $1 \mathrm{TeV}$ (constrained by the emissivity of gamma rays, $q_{\gamma}$, tabulated in Drury et al. 1994) at the time of the MAGIC observations ( $t=6$ days) from equation A.2 is $\approx 10^{-24}$ photons $\mathrm{cm}^{-2} \mathrm{~s}^{-1}$. This flux is consistent with the UL at

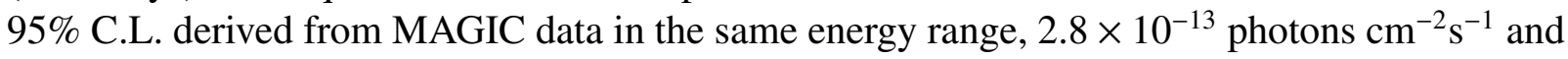
hence, the power-law density profile could be considered as possible model to describe the density structure of SN 2014J, considering all the assumptions and parameters selection discussed above. On the other hand, this model predicts a constant parameter of $m=0.57$ during the first $\sim 300$ years, after which it starts dropping gradually (Dwarkadas \& Chevalier 1998). Although the flux keeps increasing with time according to equation A.2, with this low expansion parameter it will still be about $10^{-21}$ photons $\mathrm{cm}^{-2} \mathrm{~s}^{-1} 100$ years after the $\mathrm{SN}$ occurred, which is well below the sensitivity of the current and planned VHE observatories. 


\section{A.5 Conclusions}

MAGIC observed SN 2014J, the nearest Type Ia SN since 1972, just 6 days after the explosion. Its proximity offered a good chance to probe gamma-ray emission from this kind of source but no gamma-ray excess was found. Integral ULs for energies above $300 \mathrm{GeV}$ and $700 \mathrm{GeV}$ were established at $1.3 \times 10^{-12}$ photons $\mathrm{cm}^{-2} \mathrm{~s}^{-1}$ and $4.1 \times 10^{-13}$ photons $\mathrm{cm}^{-2} \mathrm{~s}^{-1}$, respectively, for a 95\% C.L. and assuming a power-law spectrum. The latter are compatible with the results obtained by VERITAS during their observations on the host galaxy M82.

With the obtained flux UL, we were able to constrained the fraction of energy emitted into VHE gamma rays. Thus, the flux $\mathrm{UL}$ at $E>300 \mathrm{GeV}$ corresponds to an emission power of $<10^{39} \mathrm{erg} \mathrm{s}^{-1}$ or a total maximal emitted VHE gamma-ray energy during the observational period - approximately ten days - of $<10^{45} \mathrm{erg}$, which is about $10^{-6}$ times the total energy budget of a Type Ia SN explosion ( $\left.10^{51} \mathrm{erg}\right)$. Following Dwarkadas (2013) model for hadronic gamma-ray flux, a power-law density profile proportional to $R^{-7}$ is consistent with our ULs, although, due to the uncertainties in several parameters, this cannot exclude other, more sophisticated, theoretical scenarios. Assuming this $\mathrm{SN}$ density profile and a constant density medium, we can estimate an expected emission from the region of the source of $\approx 10^{-25}$ photons $\mathrm{cm}^{-2} \mathrm{~s}^{-1}$. Following these assumptions, this flux would not increase enough in a near future to be detectable by any current or future generation of IACTs. 
A. VERY-HIGH-ENERGY GAMMA-RAY OBSERVATIONS OF SN 2014J 
Appendix 



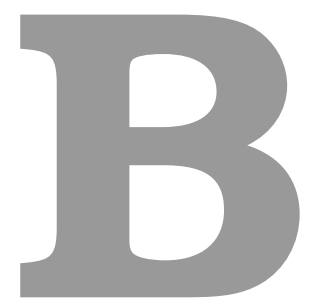

\section{F-factor method}

The number of photons that hits the PMT photocatode follows a Poissonian distribution. This is produced by the fact that only a fraction of the incident photons are actually collected by the photocatode. The emission of phes through the photoelectric effect inside the dynode system is a random binary process. Thus, the number of phes obtained in the PMT would be given by the convolution of these two distributions, which leads to a Poissonian one:

$$
P\left(n ; N_{p h e}\right)=\frac{N_{p h e}^{n}}{n !} e^{-N_{p h e}}
$$

where $P\left(n ; N_{\text {phe }}\right)$ is the probability of observing $n$ phes when the expected value is $N_{\text {phe }}$, defined as $N_{\text {phe }}=N_{\gamma} \cdot Q E$ (where $N_{\gamma}$ is the mean number of photons arriving to the photocathode and $Q E$ is the quantum efficiency of it). Therefore, the mean value is $N_{p h e}$ and the $\operatorname{RMS}$ is $\sqrt{N_{p h e}}$. 
On the other hand, the measured charge (Q) in ADC counts has a mean $\bar{Q}$ and a $\operatorname{RMS}$ of $\sigma_{Q}$ (which is wider than a pure Poissonian RMS). Thus, the relation between both quantities can be expressed as:

$$
F=\frac{1}{\sqrt{N_{p h e}}}=\frac{\sigma_{Q}}{\bar{Q}} \Rightarrow N_{\text {phe }}=\left(\frac{\bar{Q} F}{\sigma_{Q}}\right)^{2}
$$

The F-factor, F, accounts for a broadening of the signal due to the multiplication process inside the dynode system. That is the reason why the F-factor is measured for each PMTseparately before being installed in the telescopes camera. In MAGIC, this value is approximately 1.15 in all cases.

The three $\bar{Q}, \sigma_{Q}$ (from the calibration events, see Chapter X) and F-factor are known, which allows us to compute the mean $N_{p h e}$ from Equation B.2 and to obtain, in turn, the conversion factor, C:

$$
C=\frac{N_{p h e}}{\bar{Q}}=\frac{F^{2} \bar{Q}}{\sigma_{Q}^{2}}
$$

In MAGIC, the values of $\mathrm{C}$ (different for each PMT) are calculated with special calibration events of 4096 events (see Chapter X). During data taking, more calibration flashes are fired (at a frequency of $50 \mathrm{~Hz}$ ) to update them. Afterwards, these constantly updated conversion factors are applied to real data to get the number of phes from the charge, $\mathrm{Q}$, of the ADC 
Appendix 



\section{Power supply noise tests with different setup}

\section{configurations}

In this Appendix, I show plots from all configuration setups presented in Chapter ?? and not included there. For more information about the setup features for each case, please refer to the captions.

\section{C.0.1 Conducted noise tests}

Here I show only conducted noise tests using the linear PSU (Tektronix PS280). The data was recorded using the Tektronix TDS3024B oscilloscope. 

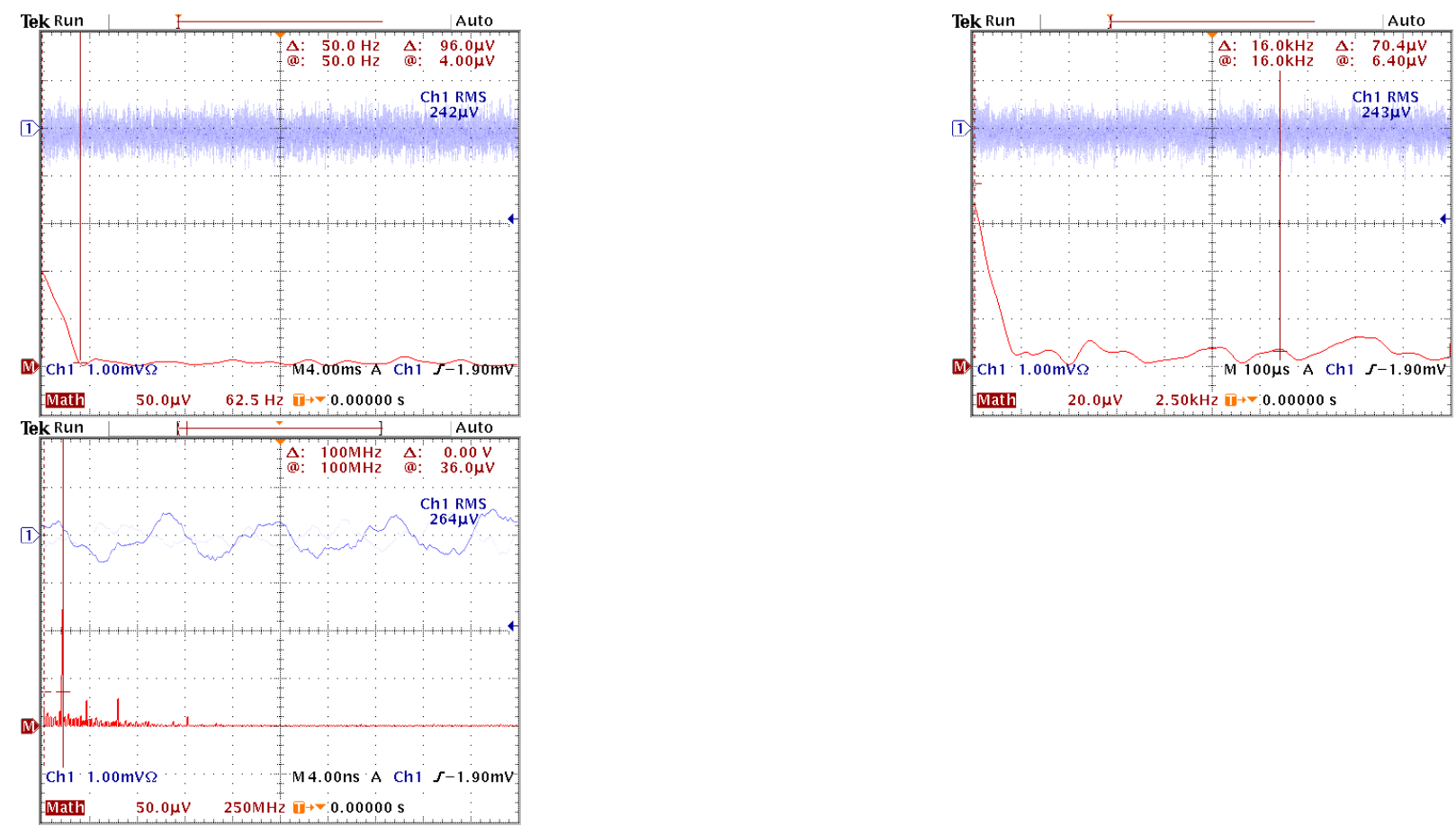

Figure C.1: Voltage vs Time for the three different sampling frequency levels, $62.5 \mathrm{~Hz}, 2.5 \mathrm{kHz}$ and $250 \mathrm{MHz}$, respectively. The vertical scale is $1 \mathrm{mV} / \mathrm{div}$ for all cases. In red, at the bottom of each plot, the FFT is shown with a vertical scale of $50 \mu \mathrm{V} / \mathrm{div}, 20 \mu \mathrm{V} / \mathrm{div}$ and $50 \mu \mathrm{V} / \mathrm{div}$, respectively. For these measurements, all involved devices were turn off ( $\mathrm{V}_{c c}$ and $\mathrm{HV}$ off; see sections ?? and ??).

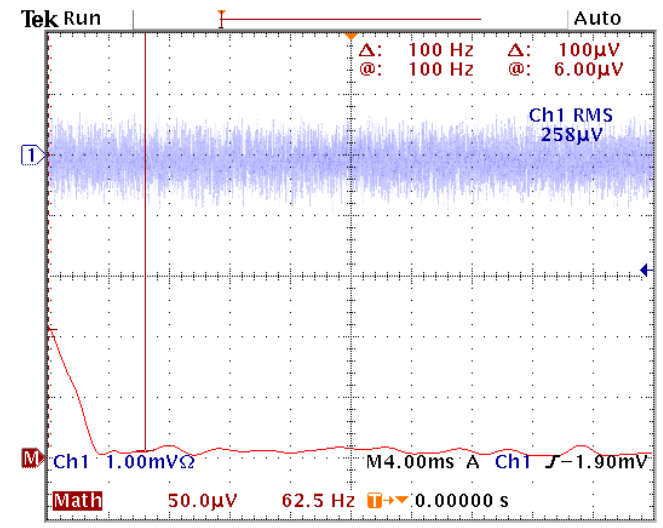

Figure C.2: Voltage vs Time at sampling frequency of $62.5 \mathrm{~Hz}$. The vertical scale is $1 \mathrm{mV} / \mathrm{div}$. In red, at the bottom of the plot, the FFT is shown with a vertical scale of $50 \mu \mathrm{V} /$ div. For this measurement, only $\mathrm{V}_{c c}$ was powered on at the level of $5 \mathrm{~V}$, while the $\mathrm{HV}$ remained powered off (see Sections ?? and ??). 


\section{POWER SUPPLY NOISE TESTS WITH DIFFERENT SETUP}

\section{CONFIGURATIONS}

\section{C.0.2 Radiated noise tests}

For these tests, the power to the PMT (if any) was provided by the linear PSU while the PULS QT40.241 PSU was placed alongside. Data was recorded using the Tektronix TDS3024B oscilloscope. Figures C.3, C.4 and C.5 show the results of the tests with PULS QT40.241 PSU powered off and Figures C.6 and C.7 with PULS QT40.241 PSU powered on (although unloaded).
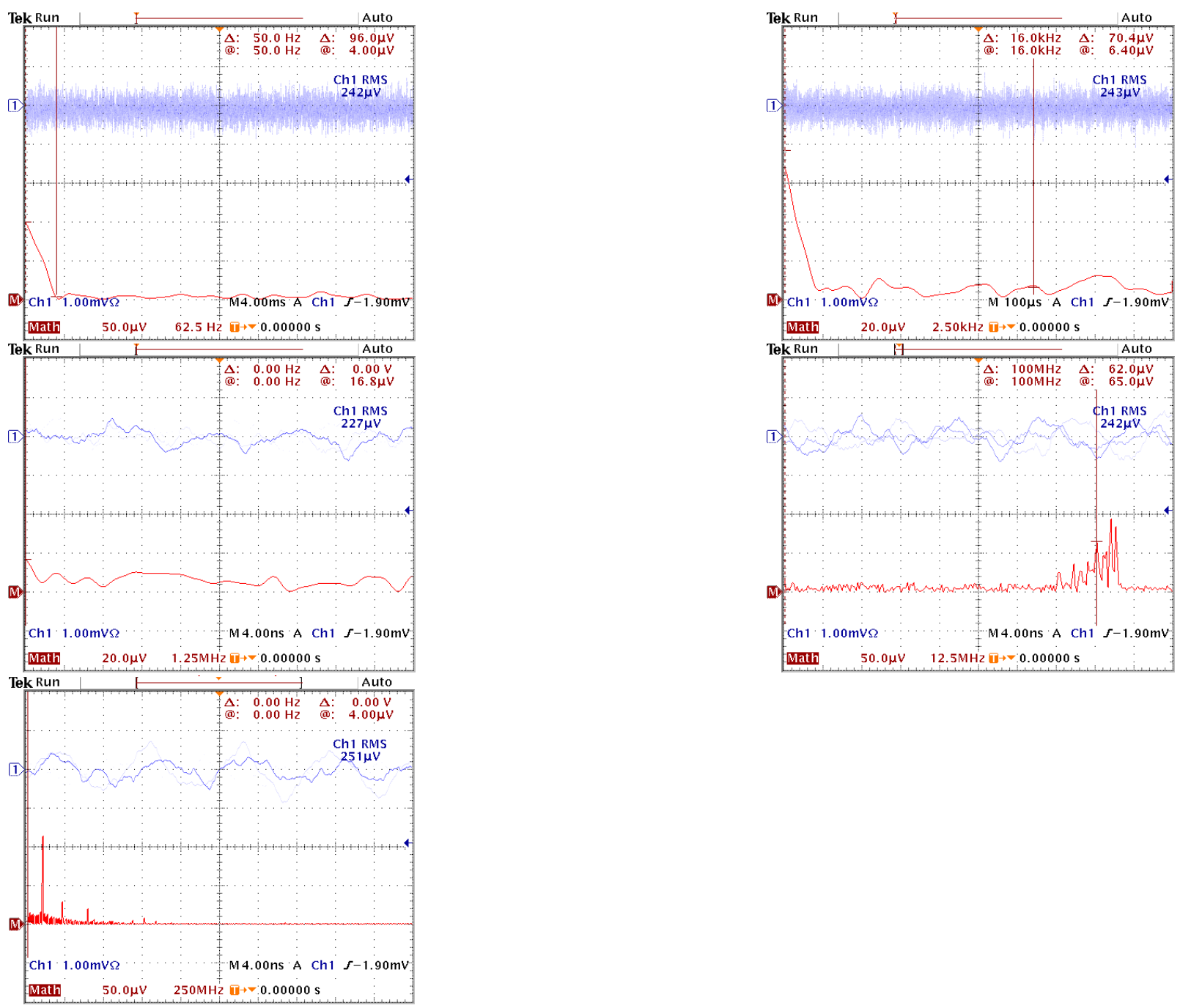

Figure C.3: Voltage vs Time for five different sampling frequency levels, $62.5 \mathrm{~Hz}, 2.5 \mathrm{kHz}, 1.25 \mathrm{MHz}$, $12.5 \mathrm{MHz}$ and $250 \mathrm{MHz}$, respectively. The vertical scale is $1 \mathrm{mV} / \mathrm{div}$ for all cases. In red, at the bottom of each plot, the FFT is shown with a vertical scale of $50 \mu \mathrm{V} / \mathrm{div}, 20 \mu \mathrm{V} / \mathrm{div}, 20 \mu \mathrm{V} / \mathrm{div}, 50 \mu \mathrm{V} / \mathrm{div}$ and $50 \mu \mathrm{V} /$ div, respectively. For these measurements, all involved devices were turn off ( $\overparen{\text { PMT }}$ and PULS QT40.241 PSU off; see Sections ?? and ??). 


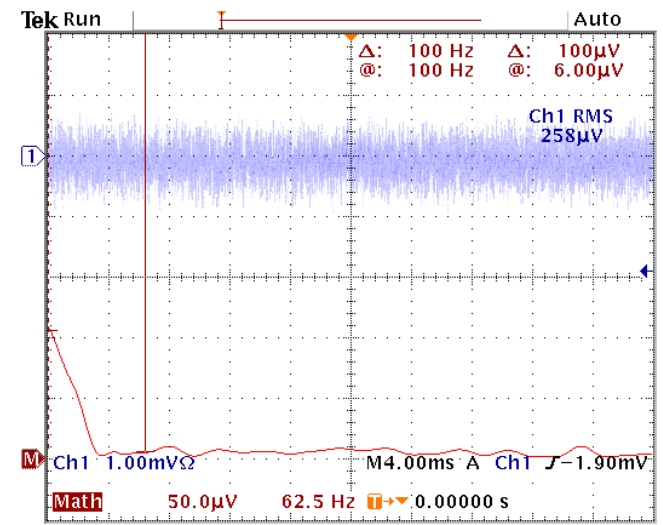

Figure C.4: Voltage vs Time at sampling frequency of $62.5 \mathrm{~Hz}$. The vertical scale is $1 \mathrm{mV} / \mathrm{div}$. In red, at the bottom of the plot, the FFT is shown with a vertical scale of $50 \mu \mathrm{V} /$ div. For this measurement, only $\mathrm{V}_{c c}=5 \mathrm{~V}$ was supplied, while the HV and PULS QT40.241 PSU remained powered off (see Sections ?? and ??).
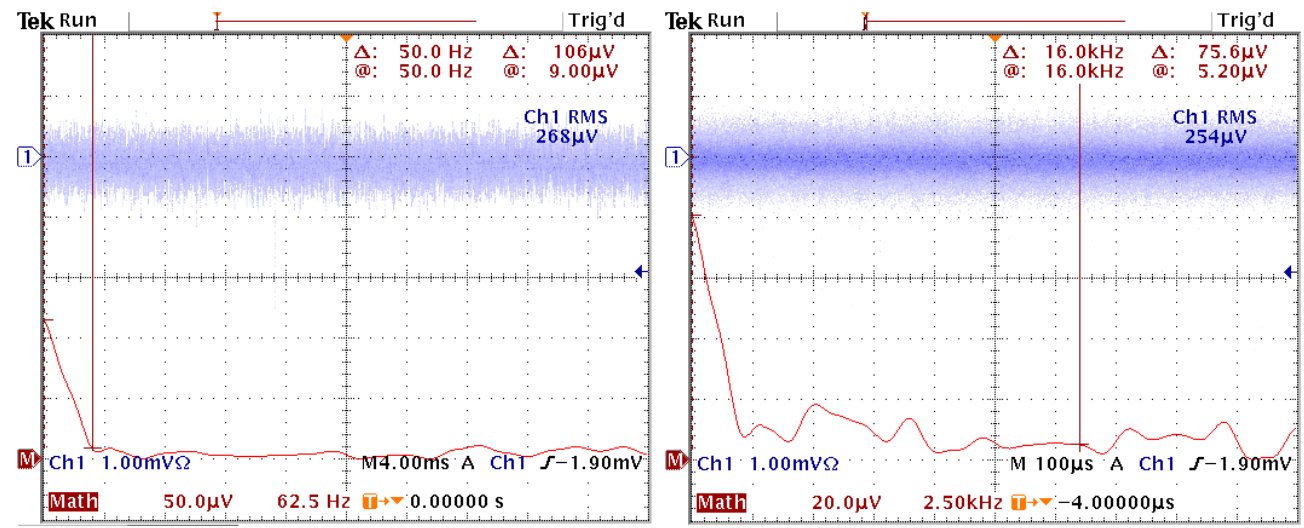

Figure C.5: Voltage vs Time for the two different sampling frequency levels, $62.5 \mathrm{~Hz}$ and $2.5 \mathrm{kHz}$, respectively. The vertical scale is $1 \mathrm{mV} / \mathrm{div}$ in both cases. In red, at the bottom of each plot, the FFT is shown with a vertical scale of $50 \mu \mathrm{V} / \mathrm{div}$ and $20 \mu \mathrm{V} /$ div, respectively. For these measurements, the linear PSU provided $\mathrm{V}_{c c}=5 \mathrm{~V}$ and $\mathrm{HV}=1.4 \mathrm{kV}$ while the PULS QT40.241 $\mathrm{PSU}$ was still turned off; see Sections ?? and ??). 


\section{POWER SUPPLY NOISE TESTS WITH DIFFERENT SETUP}

\section{CONFIGURATIONS}
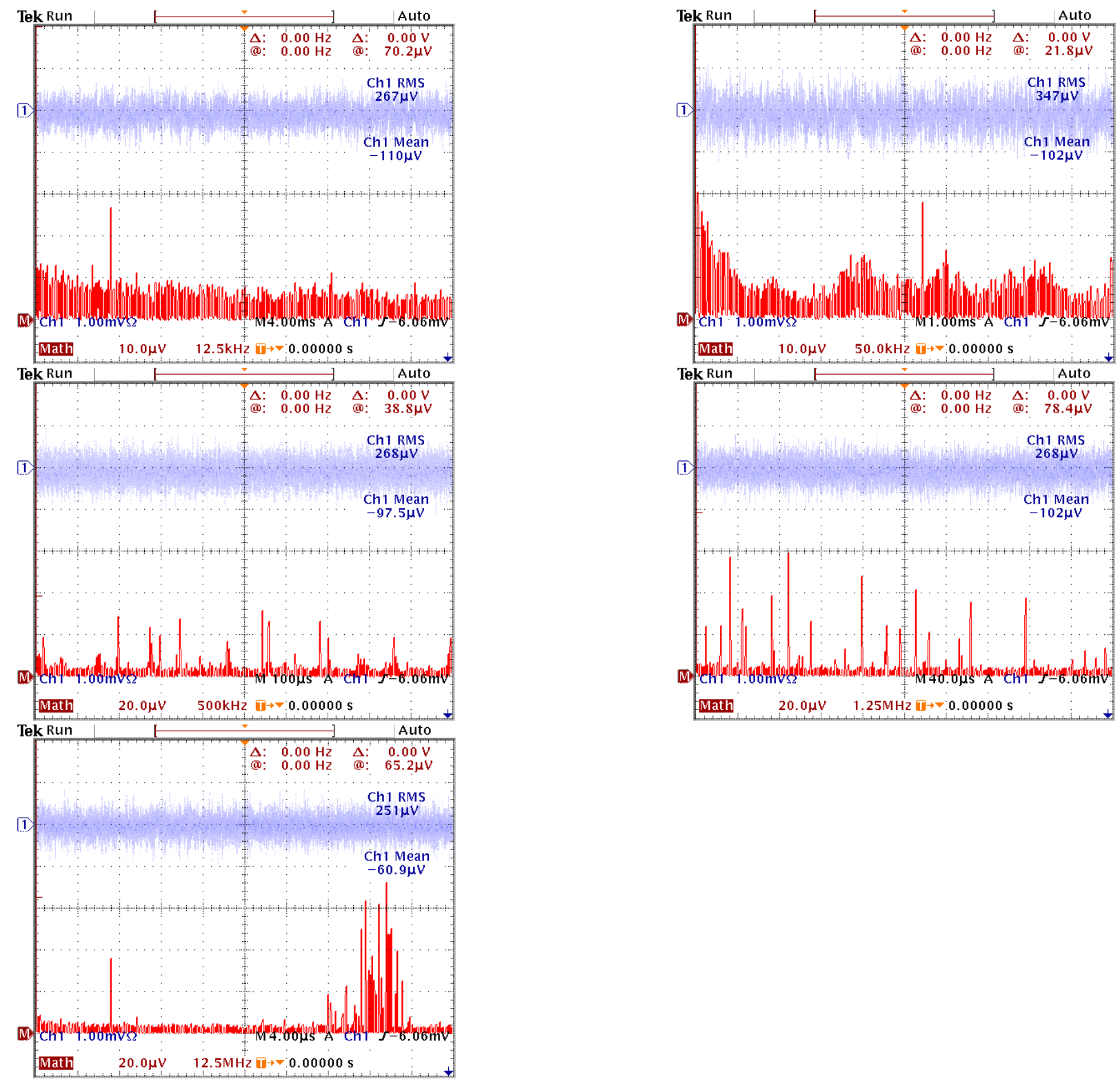

Figure C.6: Voltage vs Time for five different sampling frequency levels, $12.5 \mathrm{kHz}, 50 \mathrm{kHz}, 500 \mathrm{kHz}$, $1.25 \mathrm{MHz}$ and $12.5 \mathrm{MHz}$, respectively. The vertical scale is $1 \mathrm{mV} / \mathrm{div}$ for all cases. In red, at the bottom of each plot, the FFT is shown with a vertical scale of $10 \mu \mathrm{V} /$ div for the two top plots and $20 \mu \mathrm{V} /$ div for the rest. For these measurements, $\mathrm{V}_{c c}$ and $\mathrm{HV}$ were powered off while the PULS QT40.241 PSU] was on although unloaded; see Sections ?? and ??). 

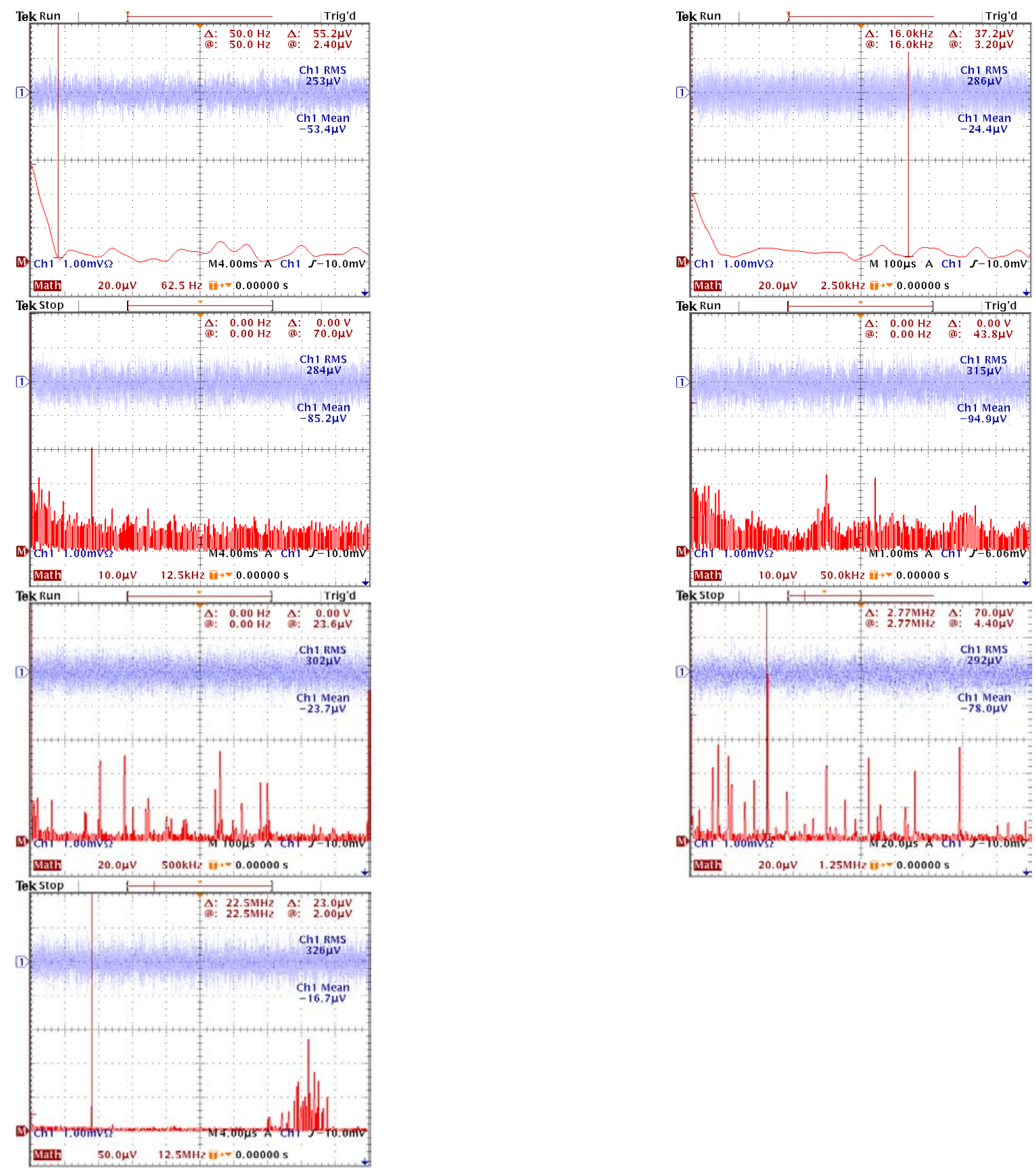

Figure C.7: Voltage vs Time for seven different sampling frequency levels, $62.5 \mathrm{~Hz}, 2.5 \mathrm{kHz}, 12.5 \mathrm{kHz}$, $50 \mathrm{kHz}, 500 \mathrm{kHz}, 1.25 \mathrm{MHz}$ and $12.5 \mathrm{MHz}$, respectively. The vertical scale is $1 \mathrm{mV} / \mathrm{div}$ for all cases. In red, at the bottom of each plot, the FFT is shown with a vertical scale of $20 \mu \mathrm{V} / \mathrm{div}, 20 \mu \mathrm{V} / \mathrm{div}, 10$ $\mu \mathrm{V} /$ div, $10 \mu \mathrm{V} /$ div, $20 \mu \mathrm{V} /$ div, $20 \mu \mathrm{V} /$ div and $50 \mu \mathrm{V} /$ div, respectively. For these measurements, the PMT was powered on with $\mathrm{V}_{c c}=5 \mathrm{~V}$ and $\mathrm{HV}=1.4 \mathrm{kV}$, as well as the PULS QT40.241 PSU]although unloaded; see Sections ?? and ??). 
Aharonian, F. A., et al. 2012, Nature, 482, 507

Aharonian, F. A. 2013, Astroparticle Physics, 43,71

\section{References}

Ahnen, M. L. et al. 2017, A\&A (in preparation)

Akharonian, F. A., et al. 1985, Ap\&SS, 115, 201

Abdo, A. A., et al. 2009a, Science, 325, 840

Abdo, A. A., et al. 2009b, ApJ, 700, L127

Abdo, A. A., et al. 2010, ApJS, 188, 405

Abdo, A. A., et al. 2013, ApJS, 208, 17

Abeysekara, A. U., et al. 2016, ApJ, 817, 3

Abeysekara, A. U., et al. 2017a, ArXiv eprints

Abeysekara, A. U., et al. 2017b, ArXiv eprints

Acero, F., et al. 2015, ApJS, 218, 23

Acharya, B. S., et al. 2013, Astroparticle Physics, 43, 3

Ackermann, M., et al. 2011, Science, 334, 1103

Aharonian, F., et al. 1997, Astroparticle Physics, 6, 343

Aharonian, F., et al. 2005, Science, 309, 746

Aharonian, F. A. et al. 1981, Ap\&SS, 79, 321

Aharonian, F. A. et al. 1998, New A Rev., 42, 579

Aharonian, F. A. 2004, Very high energy cosmic gamma radiation : a crucial window on the extreme Universe (World Scientific Publishing Co)

Albert, J., et al. 2006, Science, 312, 1771

Albert, J., et al. 2007, ApJ, 665, L51

Albert, J., et al. 2008, Nuclear Instruments and Methods in Physics Research A, 588, 424

Aleksić, J., et al. 2010a, ApJ, 721, 843

Aleksić, J., et al. 2010b, ApJ, 721, 843

Aleksić, J., et al. 2010c, ApJ, 725, 1629

Aleksić, J., et al. 2012a, J. Cosmology Astropart. Phys., 10, 032

Aleksić, J., et al. 2012b, Astroparticle Physics, 35, 435

Aleksić, J., et al. 2014a, A\&A, 565, L12

Aleksić, J., et al. 2014b, A\&A, 567, L8

Aleksić, J., et al. 2015, Journal of High Energy Astrophysics, 5, 30

Aleksić, J., et al. 2016a, Astroparticle Physics, 72, 61

Aleksić, J., et al. 2016b, Astroparticle Physics, 72, 76

Aliu, E., et al. 2008a, Science, 322, 1221

Aliu, E., et al. 2008b, Science, 322, 1221 
Aliu, E., et al. 2009a, Astroparticle Physics, 30, 293

Aliu, E., et al. 2009b, Astroparticle Physics, 30,293

Amanullah, R., et al. 2014, ApJ, 788, L21

Ansoldi, S., et al. 2016, A\&A, 585, A133

Antoni, T., et al. 2005, Astroparticle Physics, 24,1

Archambault, S., et al. 2013, ApJ, 779, 150

Archer, A., et al. 2016, ApJ, 831, 113

Arons, J. et al. 1979, ApJ, 231, 854

Arons, J. 2002, in Astronomical Society of the Pacific Conference Series, Vol. 271, Neutron Stars in Supernova Remnants, ed. P. O. Slane \& B. M. Gaensler, 71

Arons, J. 2009, in Astrophysics and Space Science Library, Vol. 357, Astrophysics and Space Science Library, ed. W. Becker, 373

Arumugasamy, P., et al. 2014, ApJ, 790, 103

Arzoumanian, Z., et al. 2002, ApJ, 568, 289

Arzoumanian, Z., et al. 2011, ApJ, 739, 39

Atoyan, A. M. et al. 1999, MNRAS, 302, 253

Bally, J., et al. 1989, ApJ, 338, L65

Barthelmy, S. D., et al. 2015, GRB Coordinates Network, 17929

Becklin, E. E., et al. 1973, Nature, 245, 302

Bednarek, W. et al. 2007, A\&A, 464, 437

Bell, A. R. 1991, International Cosmic Ray Conference, 2, 420
Belloni, T., et al. 2000, A\&A, 355, 271

Bertero, M. 1989, Linear inverse and illposed problems, Vol. 75 (Academic Press, New York), 1-120

Bethe, H. A. 1993, ApJ, 412, 192

Bhat, C. L., et al. 1986, ApJ, 306, 587

Bitossi, M. 2009, Ultra-fast Sampling and Readout for the MAGIC-II Telescope Data Acquisition System, PhD. thesis

Blandford, R. D. 1976, MNRAS, 176, 465

Blandford, R. D. et al. 1977, MNRAS, 179, 433

Blandford, R. D. et al. 1982, MNRAS, 199, 883

Bodaghee, A., et al. 2013, ApJ, 775, 98

Bolton, C. T. 1972, Nature, 235, 271

Bondi, H. 1952, MNRAS, 112, 195

Bordas, P., et al. 2009, A\&A, 497, 325

Bosch-Ramon, V., et al. 2006, A\&A, 447, 263

Bosch-Ramon, V., et al. 2008, A\&A, 489, L21

Bosch-Ramon, V. et al. 2009, International Journal of Modern Physics D, 18, 347

Bretz, T., et al. 2009, Astroparticle Physics, 31,92

Brocksopp, C., et al. 1999, A\&A, 343, 861

Brocksopp, C., et al. 1999, Mon.Not.Roy.Astron.Soc., 309, 1063

Brown, P. J., et al. 2015, ApJ, 805, 74 
Brun, P. 2014, in Very high energy phenomena in the universe, Very high energy phenomena in the universe

Bucciantini, N., et al. 2006, MNRAS, 368, 1717

Bulgarelli, A., et al. 2010, The Astronomer's Telegram, 2512

Camenzind, M. 2007, Compact objects in astrophysics : white dwarfs, neutron stars, and black holes

Cameron, P. B. et al. 2007, ApJ, 665, L135

Carpano, S., et al. 2007, A\&A, 461, L9

Carrigan, S., et al. 2013, ArXiv e-prints

Carroll, B. W. et al. 2006, An introduction to modern astrophysics and cosmology

Casares, J., et al. 1992, Nature, 355, 614

Casares, J. et al. 1994, MNRAS, 271, L5

Chandrasekhar, S. 1931, ApJ, 74, 81

Cheng, K. S., et al. 1986, ApJ, 300, 500

Cherenkov, P. 1934, C. R. (Doklady) Akad. Sci. URSS, 2, 451

Cheung, C. C. et al. 2016, The Astronomer's Telegram, 9502

Chevalier, R. A. 1982, ApJ, 258, 790

Coppi, P. S. 1999, in Astronomical Society of the Pacific Conference Series, Vol. 161, High Energy Processes in Accreting Black Holes, ed. J. Poutanen \& R. Svensson, 375

Corbel, S., et al. 2012, MNRAS, 421, 2947

Curtis, H. D. 1918, Publications of Lick Observatory, 13,31 de Jager, O. C. et al. 1992, ApJ, 396, 161

Di Salvo, T., et al. 2001, ApJ, 547, 1024

Drury, L. O., et al. 1994, Astron.Astrophys., 287, 959

Dwarkadas, V. V. et al. 1998, ApJ, 497, 807

Dwarkadas, V. V. 2000, ApJ, 541, 418

Dwarkadas, $\quad$ V. $\quad$ V. 2013, Mon.Not.Roy.Astron.Soc., 434, 3368

Elterman, L. 1964, Appl. Opt., 3, 745

Esin, A. A., et al. 1998, ApJ, 505, 854

Fender, R. P., et al. 2004, MNRAS, 355, 1105

Fender, R. P., et al. 2006, Mon.Not.Roy.Astron.Soc., 369, 603

Fermi, E. 1949, Physical Review, 75, 1169

Fermi LAT Collaboration, et al. 2009a, Science, 326, 1512

Fermi LAT Collaboration, et al. 2009b, Science, 326, 1512

Ferrigno, C., et al. 2015, The Astronomer's Telegram, 7662

Foley, R. J., et al. 2014, MNRAS, 443, 2887

Fomin, V. P., et al. 1994, Astroparticle Physics, 2, 137

Fossey, S. J., et al. 2014, Central Bureau Electronic Telegrams, 3792

Fruck, C., et al. 2014, arXiv:1403.3591

Gabici, S. et al. 2007, ApJ, 665, L131

Gabici, S., et al. 2016, ArXiv e-prints

Gaensler, B. M. et al. 2006, ARA\&A, 44, 17

Gallo, E., et al. 2003, MNRAS, 344, 60 
Gallo, E., et al. 2005, Nature, 436, 819

Gallo, E., et al. 2012, MNRAS, 423, 590

García, J. R., et al. 2014, arXiv:1404.4219

Georganopoulos, M., et al. 2002, A\&A, 388, L25

Giacconi, R., et al. 1962, Physical Review Letters, 9, 439

Giacconi, R., et al. 1967, ApJ, 148, L119

Gies, D. R., et al. 2008, ApJ, 678, 1237

Gilfanov, M. 2010, in Lecture Notes in Physics, Berlin Springer Verlag, Vol. 794, Lecture Notes in Physics, Berlin Springer Verlag, ed. T. Belloni, 17

Gini, C. 1921, Economic Journal, 31, 22

Ginzburg, V. L. et al. 1964, The Origin of Cosmic Rays

Goldreich, P. et al. 1969, ApJ, 157, 869

Goobar, A., et al. 2014, ApJ, 784, L12

Gosnell, N. M., et al. 2012, ApJ, 745, 57

Green, D. A. 1986, MNRAS, 219, 39P

Gregory, P. C., et al. 1972, Nature Physical Science, 239, 114

Grimm, H.-J., et al. 2002, A\&A, 391, 923

Grinberg, V., et al. 2013, A\&A, 554, A88

Guenette, R. et al. 2009, ArXiv [0908.0714]

Gursky, H. 1966, S\&T, 32

H. E. S. S. Collaboration, et al. 2017, ArXiv e-prints
Hanlon, W. $\quad$ F. 2010, http://www.physics.utah.edu/ whanlon/spectrum.html

Hanson, M. M., et al. 2000, ApJ, 541, 308

Harding, A. K. 2013, Frontiers of Physics, 8, 679

Heck, D., et al. 1998, CORSIKA: a Monte Carlo code to simulate extensive air showers. (Forschungszentrum Karlsruhe $\mathrm{GmbH})$

Heger, A., et al. 2003, ApJ, 591, 288

Hess, V. F. 1912, Z. Phys., 13, 1084

Hester, J. J., et al. 1996, ApJ, 456, 225

Hester, J. J., et al. 2002, ApJ, 577, L49

Hester, J. J. 2008, ARA\&A, 46, 127

Hewish, A., et al. 1968, Nature, 217, 709

Hillas, A. M. 1984, ARA\&A, 22, 425

Hirotani, K. 2008, ArXiv e-prints

Hjalmarsdotter, L., et al. 2008, MNRAS, 384,278

Hobbs, G., et al. 2005, MNRAS, 360, 974

Hofmann, W., et al. 1999, Astroparticle Physics, 12, 135

Iben, I., Jr. et al. 1984, ApJS, 54, 335

Iwamoto, K., et al. 1999, ApJS, 125, 439

Jackson, J. C. 1972, Nature Physical Science, 236, 39

Jamil, O., et al. 2010, MNRAS, 401, 394

Jourdain, E., et al. 2012, ApJ, 761, 27 
Jourdain de Muizon, M., et al. 1988, A\&A, 193, 248

Kaiser, C. R., et al. 2000, A\&A, 356, 975

Kampert, K.-H. et al. 2014, Comptes Rendus Physique, 15, 318

Karachentsev, I. D. et al. 2006, Astrophysics, 49,3

Kawabata, K. S., et al. 2014, ApJ, 795, L4

Kennel, C. F. et al. 1984, ApJ, 283, 710

Khangulyan, D., et al. 2008, MNRAS, 383, 467

Khangulyan, D., et al. 2014, ApJ, 783, 100

Khargharia, J., et al. 2010, ApJ, 716, 1105

Kirk, J. G., et al. 1995, A\&A, 293

Krimm, H. A., et al. 2013, ApJS, 209, 14

Kulkarni, S. R., et al. 1992, Nature, 360, 139

Lachowicz, P., et al. 2006, MNRAS, 368, 1025

Laurent, P., et al. 2011, in X-ray Astrophysics up to $511 \mathrm{keV}$

Lessard, R. W., et al. 2001, Astroparticle Physics, 15, 1

Levinson, A. et al. 2001, Physical Review Letters, 87, 171101

Lewin, W. H. G., et al. 1995, X-ray Binaries

Li, T.-P. et al. 1983, Astrophys.J., 272, 317

Loh, A., et al. 2016, MNRAS, 462, L111

Lombardi, S. 2011, International Cosmic Ray Conference, 3, 266
Longair, M. S. 2011, High Energy Astrophysics

López-Coto, R. 2015, Very-high-energy $\gamma$ ray observations of pulsar wind nebulae and catacysmic variable stars with MAGIC and development of trigger systems for IACTs, $\mathrm{PhD}$. thesis

López-Coto, R., et al. 2016, Journal of Instrumentation, 11, P04005

Lyubarskii, Y. E. 1996, A\&A, 311, 172

Maccarone, T. J. 2003, A\&A, 409, 697

MAGIC Collaboration, et al. 2017, ArXiv eprints

Majumdar, P., et al. 2005, International Cosmic Ray Conference, 5, 203

Makino, F., et al. 1989, IAU Circ., 4786

Malyshev, D., et al. 2013a, MNRAS, 434, 2380

Malyshev, D., et al. 2013b, MNRAS, 434, 2380

Malzac, J., et al. 2008, A\&A, 492, 527

Manchester, R. N. et al. 1977, Pulsars

Manchester, R. N., et al. 2005, AJ, 129, 1993

Margon, B. 1984, ARA\&A, 22, 507

Margutti, R., et al. 2014, Astrophys.J., 790, 52

Markoff, S., et al. 2001, A\&A, 372, L25

Martí, J., et al. 2001, A\&A, 375, 476

Matsuoka, M., et al. 2009, PASJ, 61, 999

Mazin, M. 2007, A study of very high energy gamma-ray emission from AGNs and constraints on the extragalactic background light, PhD. thesis 
McCollough, M. L., et al. 2016, ApJ, 830, L36

McConnell, M. L., et al. 2002, ApJ, 572, 984

Meier, D. 1996, ApJ, 459, 185

Meier, D. L., et al. 2001, Science, 291, 84

Miller-Jones, J. C. A., et al. 2004, ApJ, 600, 368

Miller-Jones, J. C. A., et al. 2009, MNRAS, 394,1440

Millikan, R. A. et al. 1926, Physical Review, 28,851

Mirabel, I. F., et al. 1992, Nature, 358, 215

Mirabel, I. F. et al. 1998, Nature, 392, 673

Mirabel, I. F. et al. 1999, ARA\&A, 37, 409

Mirabel, I. F. 2006, Science, 312, 1759

Mirzoyan, R. 1997, International Cosmic Ray Conference, 7, 265

Moderski, R., et al. 2005, MNRAS, 363, 954

Mooley, K., et al. 2015, The Astronomer's Telegram, 7658

Moralejo, A. 2000, Búsqueda de fuentes cósmicas de radiación gamma de muy alta energía con el detector AIROBICC, PhD. thesis

Munar-Adrover, P., et al. 2016, ApJ, 829, 101

Nakajima, D., et al. 2013, Proceedings of the ICRC 2013, id 787

Nice, D. J., et al. 2013, ApJ, 772, 50

Nomoto, K., et al. 1984, ApJ, 286, 644

Orellana, M., et al. 2007, A\&A, 476, 9
Orosz, J. A., et al. 2011, ApJ, 742, 84

Ostriker, J. P. et al. 1969, ApJ, 157, 1395

Owocki, S. P., et al. 2009, ApJ, 696, 690

Pacini, F. 1967, Nature, 216, 567

Parsignault, D. R., et al. 1972, Nature Physical Science, 239, 123

Pelling, R. M., et al. 1987, ApJ, 319, 416

Pepe, C., et al. 2015, A\&A, 584, A95

Pérez-Torres, M. A., et al. 2014, ApJ, 792, 38

Perlmutter, S. et al. 1999, Astrophys.J., 517, 565

Perucho, M. et al. 2008, A\&A, 482, 917

Piano, G., et al. 2016, The Astronomer's Telegram, 9429

Pletsch, H. J., et al. 2012, ApJ, 744, 105

Porth, O., et al. 2013, MNRAS, 431, L48

Poutanen, J., et al. 2008, MNRAS, 389, 1427

Prestwich, A. H., et al. 2007, ApJ, 669, L21

Priedhorsky, W. C., et al. 1983, ApJ, 270, 233

Ptuskin, V. S., et al. 1993, A\&A, 268, 726

Rees, M. J. 1984, ARA\&A, 22, 471

Rees, M. J. 1998, in Black Holes and Relativistic Stars, ed. R. M. Wald, 79

Reich, W., et al. 1985, A\&A, 151, L10

Reid, M. J., et al. 2011, ApJ, 742, 83

Reig, P. et al. 1999, MNRAS, 306, 100

Reynolds, S. P. 2008, ARA\&A, 46, 89

Rico, J. 2008, ApJ, 683, L55 
Rissi, M., et al. 2009, IEEE Transactions on Nuclear Science, 56, 3840

Rodriguez, J., et al. 2015a, A\&A, 581, L9

Rodriguez, J., et al. 2015b, ApJ, 807, 17

Rolke, W. A., et al. 2005, Nuclear Instruments and Methods in Physics Research A, 551,493

Romero, G. E., et al. 2002, A\&A, 393, L61

Romero, G. E., et al. 2003, A\&A, 410, L1

Romero, G. E. et al. 2008, A\&A, 485, 623

Romero, G. E., et al. 2014, A\&A, 562, L7

Ruderman, M. A. et al. 1975, ApJ, 196, 51

Rushton, A., et al. 2012a, MNRAS, 419, 3194

Rushton, A., et al. 2012b, MNRAS, 419, 3194

Russell, D. M. et al. 2010, ArXiv e-prints

Sabatini, S., et al. 2010a, ApJ, 712, L10

Sabatini, S., et al. 2010b, ApJ, 712, L10

Sabatini, S., et al. 2013a, ApJ, 766, 83

Sabatini, S., et al. 2013b, ApJ, 766, 83

Saio, H. et al. 1985, A\&A, 150, L21

Savonije, J. 1983, in Accretion-Driven Stellar X-ray Sources, ed. W. H. G. Lewin \& E. P. J. van den Heuvel, 343-366

Saz Parkinson, P. M., et al. 2010, ApJ, 725, 571

Scargle, J. D., et al. 2013, ApJ, 764, 167
Schmelling, M. 1994, Nuclear Instruments and Methods in Physics Research A, 340, 400

Sedov, L. I. 1959, Similarity and Dimensional Methods in Mechanics

Shahbaz, T., et al. 1994, MNRAS, 271, L10

Shakura, N. I. et al. 1973, A\&A, 24, 337

Shaw, A. W., et al. 2015, The Astronomer's Telegram, 7738

Siegert, T., et al. 2016, Nature, 531, 341

Singh, N. S., et al. 2002, A\&A, 392, 161

Sitarek, J., et al. 2013a, Nuclear Instruments and Methods in Physics Research A, 723, 109

Sitarek, J., et al. 2013b, Nuclear Instruments and Methods in Physics Research A, 723, 109

Spitkovsky, A. et al. 2004, ApJ, 603, 669

Stark, M. J. et al. 2003, ApJ, 587, L101

Stirling, A. M., et al. 2001, Mon.Not.Roy.Astron.Soc., 327, 1273

Sturrock, P. A. 1971, ApJ, 164, 529

Szostek, A. et al. 2007, MNRAS, 375, 793

Szostek, A., et al. 2008, MNRAS, 388, 1001

Tanaka, Y. T., et al. 2016, ApJ, 823, 35

Tatischeff, V. 2009, A\&A, 499, 191

Tavani, M., et al. 2009a, Nature, 462, 620

Tavani, M., et al. 2009b, Nature, 462, 620

Taylor, G. 1950, Proceedings of the Royal Society of London Series A, 201, 159 
Tescaro, D., et al. 2009, arXiv:0907.0466

Tescaro, D., et al. 2013, arXiv:1310.1565

Tian, W. W. et al. 2006, A\&A, 455, 1053

Tikhonov, A. et al. 1977, Solutions of illposed problems, Scripta series in mathematics (Winston)

Timmes, F. X., et al. 1995, ApJS, 98, 617

Trushkin, S. A., et al. 2015a, The Astronomer's Telegram, 7716

Trushkin, S. A., et al. 2015b, The Astronomer's Telegram, 7667

Trushkin, S. A., et al. 2016a, The Astronomer's Telegram, 9416

Trushkin, S. A., et al. 2016b, ArXiv e-prints

Uchida, Y. et al. 1985, PASJ, 37, 515

Uchida, Y. et al. 1986, Canadian Journal of Physics, 64, 507

van der Laan, H. 1966, Nature, 211, 1131

van Kerkwijk, M. H., et al. 1992, Nature, 355,703

van Leeuwen, F. 2007, A\&A, 474, 653

VERITAS Collaboration, et al. 2009, Nature, 462,770

Vieyro, F. L. et al. 2012, A\&A, 542, A7

Vila, G. S. et al. 2008, International Journal of Modern Physics D, 17, 1903

Vladimirsky, B. M., et al. 1973, International Cosmic Ray Conference, 1, 456

Völk, H. J., et al. 2008, A\&A, 490, 515
Wagner, R. 2006, Measurement of VHE $\gamma$-ray emission from four blazars using the MAGIC telescope and a comparative blazar study., $\mathrm{PhD}$. thesis

Waltman, E. B., et al. 1994, AJ, 108, 179

Waltman, E. B., et al. 1995, AJ, 110, 290

Waltman, E. B., et al. 1996, AJ, 112, 2690

Wen, L., et al. 1999, ApJ, 525, 968

Whelan, J. et al. 1973, ApJ, 186, 1007

Yao, W.-M., et al. 2006, Journal of Physics G Nuclear Physics, 33, 1

Yoon, D., et al. 2016, MNRAS, 456, 3638

Younes, G. 2015, GRB Coordinates Network, 17932

Younk, P. W., et al. 2015, ArXiv e-prints

Yuan, F., et al. 2005, ApJ, 620, 905

Zabalza, V. 2015, ArXiv e-prints

Zanin, R. 2011, Observation of the Crab pulsar wind nebula and micro quasar candidates with MAGIC, PhD. thesis

Zanin, R., et al. 2013, in Proc. of the 33st International Cosmic Ray Conference, Rio de Janeiro, Brasil

Zanin, R., et al. 2016, A\&A, 596, A55

Zdziarski, A. A., et al. 2011, MNRAS, 412, 1985

Zdziarski, A. A., et al. 2012, MNRAS, 423, 663

Zdziarski, A. A. et al. 2013, MNRAS, 436, 2950

Zdziarski, A. A., et al. 2016a, ArXiv e-prints 


\section{REFERENCES}

Zdziarski, A. A., et al. 2016b, ArXiv e-prints

Zdziarski, A. A., et al. 2016c, MNRAS, 455, 1451

Zhang, J.-F., et al. 2015, ApJ, 806, 168

Ziółkowski, J. 2014, MNRAS, 440, L61 
REFERENCES 


\section{Acronyms and Abbreviations}

$L_{\text {Edd }} \quad$ Eddington luminosity.

CALLISTO CALibrate LIght Signals and Time Offsets.

CORSIKA COsmic Ray SImulations for KAscade.

Flute FLUx vs. Time and Energy.

Fluxlc FLUX and Light Curve.

MERPP MERging and Prepoccesing Program.

SORCERER Simple, Outright Raw Calibration; Easy, Reliable Extraction Routines.

1FGL First Fermi-LAT catalog.

2FGL Second Fermi-LAT catalog.

3FGL Third Fermi LAT catalog.

a.s.l. $\quad$ above sea level.

ADC Analog-to-Digital Converter.

AGILE Astrorivelatore Gamma ad Imagini LEggero.

AGN Active Galactic Nuclei.

AMC Active Mirror Control.

AP After Pulse.

Ar Argon.

ARC Astrophysical Research Consortium.

ASIC Application-Specific Integrated Circuit.

ATNF Australia Telescope National Facility.

Az Azimuth.

BH Black Hole.

C Carbon.

C.L. Confidence Level. 


$\begin{array}{ll}\text { C.U. } & \text { Crab Units. } \\ \text { CC } & \text { Central Control. } \\ \text { CCD } & \text { Charge-Coupled Device. } \\ \text { CH } & \text { Counting House. } \\ \text { CIEMAT } & \text { Centro de Investigaciones Energéticas Medioam- } \\ & \text { bientales y Tecnológicas. } \\ \text { ClusCo } & \text { Cluster Control. } \\ \text { CMB } & \text { Cosmic Microwave Background. } \\ \text { CR } & \text { Cosmic Ray. } \\ \text { CTA } & \text { Cherenkov Telescope Array. } \\ \text { CV } & \text { Cataclysmic Variable. } \\ & \\ \text { DAQ } & \text { Data AcQuisition. } \\ \text { DC } & \text { Direct Current. } \\ \text { DRS } & \text { Domino Ring Sampler. } \\ \text { DRS2 } & \text { Domino Ring Sampler version 2. } \\ \text { DRS4 } & \text { Domino Ring Sampler version 4. } \\ \text { DT } & \text { Discriminator Threshold. } \\ & \\ \text { EAS } & \text { Extended Air Shower. } \\ \text { EBL } & \text { Extragalactic Background Light. } \\ \text { ECC } & \text { Embedded Camera Controller. } \\ \text { EM } & \text { Electromagnetic. } \\ & \\ \end{array}$

FADC Flash Analog-to-Digital Converter.

Fe Iron.

FFT Fast Fourier Transform.

FILAR Four Input Links for ATLAS Readout.

FIR Far Infrared.

FoV Field of View.

FPGA Field Programmable Gate Array.

FR Full Range Energy.

FWHM Full Width Half Maximum.

GBM GLAST Burst Monitor.

GCN Gamma-ray Coordinate Network.

GPS Global Positioning System.

GRB Gamma-Ray Burst. 
GZK Greisen-Zatsepin-Kuzmin.

H Hydrogen.

H.E.S.S. High Energy Stereoscopic System.

HAWC High Altitude Water Cherenkov.

HE High Energy.

He Helium.

HGPS H.E.S.S. Galactic Plane Survey.

HID Hardness-Intensity Diagram.

HMXB High-Mass X-ray Binary.

HOLA High Optical Link ATLAS.

HPD Hybrid Photo Detector.

HS Hard State.

HV High Voltage.

IACT Imaging Atmospheric Cherenkov Telescope.

IC Inverse Compton.

IFAE Institut de Física d'Altes Energies.

IPR Individual Pixel Rate.

IPRC Individual Pixel Rate Control.

IR Infrared.

IRF Instrument Response Function.

IS Intermediate State.

ISM Interstellar Medium.

KoP Key Observation Program.

LO Level 0.

L1 Level 1.

L3 Level 3.

LAT Large Area Telescope.

LE Low Energy.

LED Light-Emitting Diode.

LGS-AO Keck Observatory's Laser Guide Star Adaptive Optics.

LIDAR LIght Detection And Ranging.

LMXB Low-Mass X-ray Binary.

LST Large Size Telescope. 
LUT Look-Up Table.

LVDS Low-Voltage Differential Signaling.

MAGIC Major Atmospheric Gamma-ray Imaging Cherenkov.

MARS MAGIC Analysis and Reconstruction Software.

MC Monte Carlo.

MHD Magnetohydrodynamic.

MJD Modified Julian Day.

MOLA MAGIC OnLine Analysis.

MoU Memorandum of Understanding.

MST Medium Size Telescope.

Ne Neon.

NIR Near-infrared.

NN Next Neighbour.

NS Neutron Star.

NSB Night Sky Background.

O Oxygen.

OfWP Off from Wobble Partner.

OG Outer Gap.

OVRO Owens Valley Radio Observatory.

PACTA Pre-Amplifier for the Cherenkov Telescope Array.

PC Polar Cap.

PCB Printed Circuit Board.

PCI Peripheral Component Interconnect.

PDB Power Distribution Box.

phe photoelectron.

PMT Photomultiplier Tube.

PSF Point Spread Function.

PSU Power Supply Unit.

PULSAR PULSer And Recorder.

PWN Pulsar Wind Nebula.

QC Quality Control. 
QE Quantum Efficiency.

RF Random Forest.

RMS Root Mean Square.

RoI Region of Interest.

RPP Rotation-Powered Pulsar.

SDM Standard Disk Model.

SED Spectral Energy Distribution.

SG Slot Gap.

Si Silicon.

SiPM Silicon PhotoMultiplier.

SN Supernova.

SNR Supernova Remnant.

SPD Surge Protection Device.

SPE Single Photoelectron.

SS Soft State.

SSC Synchrotron Self Compton.

SST Small Size Telescope.

TIB Trigger Interface Board.

TPU Trigger Processing Unit.

TS Test Statistics.

UB Universitat de Barcelona.

UCL University College London.

UHE Ultra-High-Energy.

UL Upper limit.

UPS Uninterruptible power supply.

UV Ultraviolet.

VCSEL Vertical Cavity Surface Emitting Laser.

VERITAS Very Energetic Radiation Imaging Telescope Array System.

VHE Very-High-Energy.

WCD Water Cherenkov Detector.

WD White Dwarf. 
WR Wolf Rayet.

Zd Zenith distance. 



\section{Acknowledgements}

Xa quedou máis do que queda. Con estas palabras me animaba siempre mi padre cuando la tensión, el cansancio o el agobio se apoderaban de mí y creía que no iba a ser capaz de dar un poco más. Pero lo fui, papá. Y ahora, ya no queda nada. Esta etapa ha llegado a su fin.

Es difícil agradecer en pocas líneas a toda la gente que ha estado a mi lado durante este proceso. Quizás lo más justo es mirar hacia atrás y agradecer a todos los que estuvisteis apoyándome a lo largo de todo el camino.

Todo comenzó, sin lugar a dudas, en una academia de Betanzos, donde mi tío Sito hacía fácil lo que en aquel momento parecía tan complicado. Tío Sito, aunque nunca te lo dije, este amor por la física comenzó contigo.

En mi viaje hacia la vida universitaria, y durante los primeros años de ésta, tuve que vivir una etapa negra. Pero de todo se aprende en esta vida, y ahora sé que esos años me hicieron más fuerte y me ayudaron a valorar la felicidad y a las personas. Personas como Antonio y Miguel, que estuvieron siempre a mi lado. Gracias de todo corazón por haber estado ahí, por haberme acompañado esos años, por ser mis amigos.

En la universidad me encontré con el mejor grupo de actores que la física puede dar. Juan, Mónica, Tamara, María, Aida, Díaz, y tú también Miguel, tranquilos, si no triunfamos en la física, podemos vivir de la interpretación! Entre las aulas de Santiago, tuve también el gran placer de hacerme amiga del dúo inseparable, Víctor e Izan. Espero siempre con impaciencia nuestras reuniones anuales frente a un café, una copa o un cacaolat para ponernos al día sobre nuestras vidas. Y en estas líneas tienen que estar aquellos junto a quienes compartí tantas horas en la facultad o en "la Conchi", bailes en Retablo, cenas en casa y muchos muchos cafés (o mentapoleas con hielo y sacarina) en la cafetería de la facultad. Gara, Pau, Diego, Sergio, siempre seréis mis reyes de Santiago. En este recuerdo a Santiago, no puede faltar mis más sincero agradecimiento a José Edelstein y Juan Antonio Garzón Heydt por la confianza que depositaron en mí. José, Hans, sin vuestro apoyo no habría podido escribir esta tesis.

Y desde Santiago llegé a Barcelona, donde empezaría el máster en la UB. Ahí tuve la suerte de empezar a adentrarme en el mundo de la física de muy altas energías de la mano de Josep María Paredes, Marc Ribó y Valentí Bosch-Ramón, de los cuales seguiría aprendiendo durante el doctorado. También tengo que agradecerle a Andreu Sanuy su paciencia en el laboratorio. Gracias a toda la gente del máster que hicisteis 
de ése, un año maravilloso. Pero por supuesto, tengo que destacar a los primeros master and commanders: Andrea, Víctor, Alfonso (El Guapo), Ari y Adri.

En febrero del 2013 se me abrirían las puertas de IFAE, donde comenzaría el gran viaje del doctorado un 1 de septiembre. Sonrío al pensar en toda la gente que apareció en esta etapa de mi vida, a la que tengo tanto que agradecer.

Pero, como no puede ser de otra manera, empezaré con mi supervisor, con quien he tenido el enorme placer de haber trabajado estos cuatro años. Oscar, muchísimas gracias por todo lo que me has enseñado, por tu paciencia y dedicación, por tu empatía, por no poner nunca mala cara ante una duda y hacer siempre un hueco para discutir nuestros mil proyectos. No podría haber tenido un mejor director, así que espero de verdad haber estado a la altura como estudiante.

A Javi quiero agradecerle toda la ayuda que me brindó cuando tenía un problema, aunque me delatase como almost senior student. A Abelardo quiero darle las gracias por ser tan intenso y realista en los group meetings, una forma maravillosa de aprender lo bueno y lo malo de este campo. A Juan-SeniorPrize2015 le agradezco que nos dijese sin tapujos cuando "it is all wrong", incitándonos así a mejorar. Al Manel li vull agrair les seves llions de hardware, en que siempre aprenda alguna cosa nova. A Matteo y Ramón les quiero agradecer su ayuda como directores de IFAE y a Enrique como tutor.

En IFAE he compartido despacho, ideas y dudas, cafés y chupitos, risas y lágrimas con gente maravillosa que ha hecho de estos años una etapa absolutamente inolvidable. Comienzo con los postdocs que van y vienen de IFAE, pero a los que yo siempre recuerdo con mucho cariño. Julian, I do not have enough lines here to thank you for all your help, always with a smile in your face. JohnE, qué pasa contigo, tio?, I want to thank you for all the talks we had, with the future (or should I say reality?) as the main topic. Paolo, el domingo vermouth con Dani y Quique a las 12. A parte de eso, gracias por tu entusiasmo, por no fallar nunca a un plan y por haber recorrido Barcelona a pie y metro con una silla al lado. Tarek, a ti y a tu políticamente incorrectas frases ya os conocía, pero voy a echar de menos que alguien toque a la puerta de mi despacho tan característicamente para tomar el café de la mañana. Scott, thank you very much for all your help in the lab and everything I learned during our talks. Koji, trips did not allow me to work with you too much, but I hope your stay at IFAE is as good as it was mine.

$Y$ en dos despachos unidos por una puerta que raramente se cierra, he conocido gente a la que llevaré siempre en el corazón. Adiv, mi compañero de enfrente durante dos años, gracias por todos los paseos domingueros por Barcelona y por las sesiones de fotos. Leyre, la meva companya de català, y la mejor compañera de viajes que podía tener, tronca!. Daniele, Merve and Elia, it was a pleasure to spend time with you at IFAE, I hope you follow our old traditions in the office. And the special ones: Dani, Quim, con vosotros comencé el doctorado y con vosotros lo voy a acabar. Ha sido un enorme placer teneros de compañeros y, por supuesto, de amigos. Dani, 
gracias por brindarnos tranquilidad y parsimonia, excepto cuando hay un "ashuwea" de por medio. Quim, el mejor compañero de despacho que podría haber tenido. Echaré mucho de menos nuestras discusiones de pizarra y mandarnos vídeos que nos alegran la hora del café. Gracias también al MAGIC-like group, más conocido por DES: Carlos, Pauline, Judit, Alex, Marcos, un placer haber compartido estos años y cafés con vosotros. Y a Jelena, por aguantar esos cafés al otro lado de la pared.

A los ingenieros de IFAE, Oscar Martínez, Juan Boix, Pepe Illa, Oscar Abril, gracias por vuestra ayuda y dedicación todos estos años. Y cómo no, gracias a Cristina por mantenernos siempre al tanto de todo y sobre todo por preocuparse de nosotros. Els teus nens es fan grans...

Y así pasó mi tiempo en IFAE, entre trabajo y amigos. Entre un teléfono falso en Madrid, la azotea de un piso en Roma desde donde se veía toda la ciudad, el inolvidable viaje a Florencia, el vino encima de un muro imposible de escalar, el pueblo perdido de Bulgaria donde el dueño de la casa hablaba "nuestro" idioma (Ciao, come stai?), Sofia y el fatídico destino de la furgoneta (a pesar de la alarma que indicaba que todo iba bien), PLOVEdiv y su flan, y su increíble bar en el segundo piso donde regalan sombreros de pirata, el karaoke de Berlín donde Twist and Shout se convirtió en nuestra canción, los shifts que coinciden con Los Indianos. Y cómo no, los vermouths, los mojitos, las mejores bravas de Barcelona aunque "ya no las hacen como antes", el bus 11, las cenas en mi casa, el Delicias, los vídeos de las tesis, las listas y los emails con los nombres en negrita. Dejo IFAE con millones de recuerdos a mis espaldas. Por todos ellos, muchísimas gracias.

I would also like to thank the people that formed MAGIC and make it run: Martin and Javi, for your priceless help in La Palma, all the galactic conveners I could work with, Pratik, Ignasi, Taka, Christian, Emma and Roberta (aunque ellas se merecen un párrafo a parte), all the people I shared shifts with and work in the multiple projects, specially Daniela, David Carreto and Galindo, always a pleasure working with you.

Roberta, Emma, gracias de verdad por toda vuestra ayuda y por todas esas discusiones en las que siempre aprendía algo nuevo. Espero de corazón que nuestros proyectos se extiendan muchos años más y poder así seguir aprendiendo y descubriendo cosas nuevas con vosotras.

Afortunadamente el doctorado me ha permitido viajar a lo largo del mundo y trabajar con diversos grupos de los cuales he aprendido mucho. First, I would like to thank Petra Huentemeyer, who opened the doors of the HAWC Collaboration to me. During my stay in Michigan, I could learn about HAWC thanks to her, Michele, Hugo and Hao. Along with all of them, Dustin, Anna and John made those two months a lovely period. Un año después volvía a EE.UU., esta vez para trabajar, no sólo en HAWC, sino también en Fermi. A Pepa y a Daniel Castro quiero agradecerles su ayuda esos tres meses. Pepa, te prometo que algún día te prepararé las croquetas andantes que te debo. Esa etapa en Goddard la disfruté también con Mireia, quien me 
arrancaba del despacho para tomar un café. I also want to thank Jordan Goodman, Andrew Smith, Colas, Dan and Israel, the great HAWC group at the University of Maryland for making me feel welcome and for letting me get deeper in the knowledge of HAWC. Thank you to the MPIK group as well, for your kindness during my stay in Heidelberg.

Gracias a Rose, ma petite, por salvar al panda con un cappuccino o una pantalla de por medio. Da igual lo lejos que estemos, you $\mathcal{E}$ me siempre sonará en nuestra radio.

Pero sobre todo, gracias a TI. Que empezaste siendo mi compañero de despacho, $m i$ cadete, y te acabaste convirtiendo en una parte esencial de mí y de mi vida. Que siempre estás ahí, con tu cariño y tu sonrisa, apoyándome en los mejores y peores momentos. Porque no hay palabras en este mundo para expresar lo que siento por ti y lo feliz que me haces. Por todo lo que hemos vivido juntos y lo que nos queda por vivir, gracias, Rubén. Te quiero con todo mi corazón.

Gracias a mi familia, a mis tíos, mis padrinos y mis primos, por estar siempre ahí. A mi abuelo Paco, que soñó con una nieta de pelo negro y vestido azul, a la que después protegía de camino a casa; a mi abuelo Ramos, por todos los viajes en coche y los partidos de fútbol con botas de goma; a mi abuela Carmen, la persona más alegre que jamás conoceré, por llevarme al Cantón desde pequeñita, por cuidarme todos los años que pudiste. Os echo mucho de menos. Á miña avoa Maruxa, polo seu cariño incondicional. Y Martín, Carlos, Lara, no pierdo la esperanza de que algún día leáis estas palabras y os queráis convertir en astronauta como la tía. Finalmente, gracias mamá, papá, por todos los sacrificios que tuvisteis que hacer. No ha sido fácil, pero lo hemos conseguido, nos hemos doctorado. Gracias por enseñarme que el trabajo duro y la perseverancia dan siempre sus frutos. Y gracias Brais, por tu alegría, por saber escucharme, por ser el mejor hermano mayor que nadie podría tener. No creo que pueda nunca agradeceros a ninguno de los tres todo lo que habéis hecho para que hoy llegue a escribir estas palabras. Sólo espero que os sintáis orgullosos de lo que hemos conseguido y que sepáis lo mucho que os quiero. 